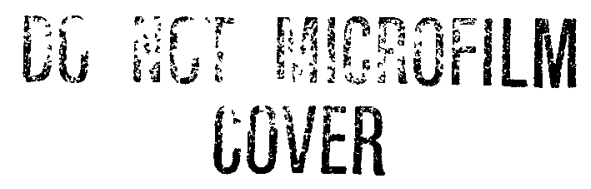

\title{
Hanford Wells
}

\author{
V. L. McGhan
}

June 1989

Prepared for the U.S. Department of Energy under Contract DE-AC06-76RLO 1830

Pacific Northwest Laboratory

Operated for the U.S. Department of Energy by Battelle Memorial Institute 


\section{DISCLAIMER}

This program was prepared as an account of work sponsored by an agency of the United States Government. Neither the United States Government nor any agency thereof, nor Battelle Memorial Institute, nor any of their employees, makes any warranty, express or implied, or assumes any legal liability or responsibility for the accuracy, completeness, or usefulness of any information, apparatus, product, or process disclosed, or represents that its use would not infringe privately owned rights. Reference herein to any specific commerical product, process, or service by trade name, trademark, manufacturer, or otherwise, does not necessarily constitute or imply its endorsement, recommendation, or favoring by the United States Government or any agency thereof, or Battelle Memorial Institute. The views and opinions of authors expressed herein do not necessarily state or reflect those of the United States Government or any agency thereof.

\section{PACIFIC NORTHWEST LABORATORY operated by \\ BATTELLE MEMORIAL INSTITUTE for the \\ UNITED STATES DEPARTMENT OF ENERGY under Contract DE-AC06-76RLO 1830}

\section{DO HOT MICROFILM THIS PAGE}

\author{
Printed in the United States of America \\ Available from \\ National Technical Information Service \\ United States Department of Commerce \\ 5285 Port Royal Road \\ Springfield, Virginia 22161 \\ NTIS Price Codes \\ Microfiche A01 \\ Printed Copy
}

$\begin{array}{cc}\text { Pages } & \begin{array}{c}\text { Price } \\ \text { Codes } \\ 001-025\end{array} \\ 026-050 & \text { A02 } \\ 051-075 & \text { A03 } \\ 076-100 & \text { A04 } \\ 101-125 & \text { A05 } \\ 126-150 & \text { A06 } \\ 151-175 & \text { A07 } \\ 176-200 & \text { A08 } \\ 201-225 & \text { A09 } \\ 226-250 & \text { A10 } \\ 251-275 & \text { A11 } \\ 276-300 & \text { A12 } \\ & \text { A13 }\end{array}$




\section{DISCLAIMER}

This report was prepared as an account of work sponsored by an agency of the United States Government. Neither the United States Government nor any agency Thereof, nor any of their employees, makes any warranty, express or implied, or assumes any legal liability or responsibility for the accuracy, completeness, or usefulness of any information, apparatus, product, or process disclosed, or represents that its use would not infringe privately owned rights. Reference herein to any specific commercial product, process, or service by trade name, trademark, manufacturer, or otherwise does not necessarily constitute or imply its endorsement, recommendation, or favoring by the United States Government or any agency thereof. The views and opinions of authors expressed herein do not necessarily state or reflect those of the United States Government or any agency thereof. 


\section{DISCLAIMER}

Portions of this document may be illegible in electronic image products. Images are produced from the best available original document. 
HANFORD WELLS

V. L. McGhan

June 1989

Prepared for

the U.S. Department of Energy

under Contract DE-ACO6-76RLO 1830

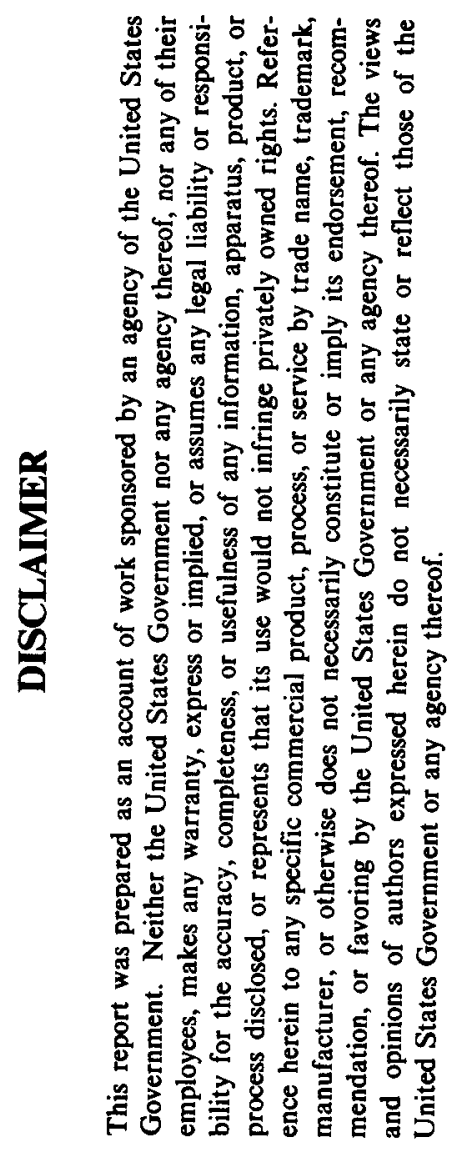

Pacific Northwest Laboratory

Richland, Washington 99352 



\section{SUMMARY}

The Site Characterization and Assessment Section of the Geosciences Department at Pacific Northwest Laboratory (PNL) has compiled a 1 ist of wells located on or near the Hanford Site. Information has been updated on wells existing from the days before construction of the Hanford Works to the present. This work was funded by the U.S. Department of Energy (DOE). The list of wells will be used by DOE contractors who need condensed, tabular information on well location, construction, and completion dates. This report does not include data on 1ithologic logs and ground-water contamination. Moreover, the completeness of this list is limited because of new well construction and existing well modifications, which are continually under way.

Despite these limitations, this list represents the most complete description possible of data pertaining to wells on or adjacent to the Hanford Site. 


\section{ACKNOWLEDGMENTS}

The author wishes to acknowledge the contributions made by J. T. Rieger for updated data base information, J. K. Merz for data entry and retrieval, and D. J. Bates for programming so the well numbers would come out in the right order. Thanks also to the editors and word processors who were involved in updating this document. 
- 


\section{CONTENTS}

SUMMARY

$i \mathrm{i}$

ACKNOWLEDGMENTS . . . . . . . . . . . . . . . . . . . . . v v

INTRODUCTION . . . . . . . . . . . . . . . . . . . . . 1

WELL NUMBERING SYSTEM . . . . . . . . . . . . . . . . . 5

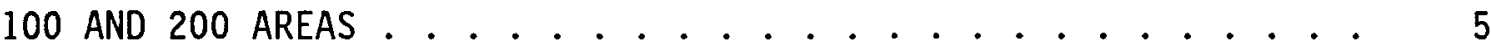

300 AREA ......................... . . . . 6

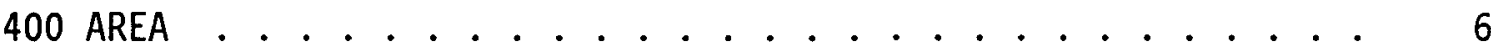

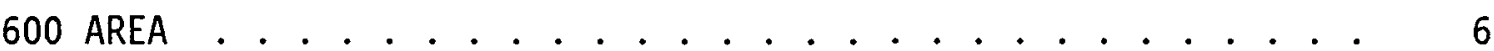

HANFORD TOWNSITE ...................... 6

1100 AND 3000 AREAS . . . . . . . . . . . . . . . . 7

PIEZOMETER TUBES . . . . . . . . . . . . . . . . 7

ENVIRONMENTAL MONITORING ANALYSIS NUMBERS . . . . . . . . . 7

WESTINGHOUSE HANFORD COMPANY FILE NUMBER . . . . . . . . . . . 7

DESCRIPTION OF WELL DATA LISTING . . . . . . . . . . . . . . . . 9

COLUMN 1 - WELL DESIGNATION ................ . . . 9

COLUMN 2 - COORDINATES .......................... 9

COLUMN 3 - ELEVATION OF CASING ................... 9

COLUMN 4 - DRILLED DEPTH . . . . . . . . . . . . . . . . 9

COLUMN 5 - INITIAL DEPTH TO WATER . . . . . . . . . . . 9

COLUMN 6 - CASING DIAMETER . . . . . . . . . . . . . . 10

COLUMN 7 - DEPTH TO BOTTOM OF THE WELL . . . . . . . . . . . . 10

COLUMN 8 - MINIMUM AND MAXIMUM DEPTH TO PERFORATIONS . . . . . 10

COLUMN 9 - DATE COMPLETED .................. 11

COLUMN 10 - FORMER NAME ................... 11

vi i 
COLUMN 11 - COMMENTS ............................. 11

MAPS ........................ . . . 12

REFERENCES . . . . . . . . . . . . . . . . . . . . . . R.1

APPENDIX A - BWIP CORE HOLES - - NAME CROSS REFERENCE . . . . . . . . . A.1

APPENDIX B - HANFORD TOWNSITE WELLS . . . . . . . . . . . . . . B.1

APPENDIX C - ENVIRONMENTAL MONITORING ANALYSIS NUMBERS • • • • • . • C.1

APPENDIX D - WESTINGHOUSE COMPUTER FILE NUMBERS . . . . . . . . . . D. 0.1

APPENDIX E - WELL LIST . . . . . . . . . . . . . . . . . . E.1

APPENDIX F - ABBREVIATIONS AND ACRONYMS . . . . . . . . . . . . . F.1

Plate 1 - 600-Area Map .................. . Back Pocket 


\section{FIGURES}

1 Map of the Hanford Site................ 2

2 Water We11 Location Map -- 200-East Area . . . . . . . . 13

3 Water Well Location Map -- 200-West Area . . . . . . . . . 15

\section{TABLES}

1 Hanford Wells.................. 3

2 Total Wells by Area .................. 4 


\section{INTRODUCTION}

The Hanford Site (Figure 1) contains over 3500 wells constructed before the operation of the Hanford Works to the present. The more than 200 preHanford Works wells were mostly hand-dug farm wells, many of which have been backfilled or are no longer usable (Jenkins 1922). The newer wells are cased we1ls that were either churn drilled, drive-barrel drilled, or rotary drilled. The wells drilled within the Hanford Site were either constructed by contract drillers, drilling crews employed by DOE prime contractors, or by the U.S. Geological Survey (USGS) (Newcomb, Strand, and Frank 1972).

Wells on the Hanford Site have various purposes. Some were constructed originally to provide sanitary water for outlying installations such as U.S. Army facilities (Walters and Grolier 1960). Other wells are used by the Ground-Water Monitoring Program in determining what effect Hanford Site operations may have on the ground water and subsurface formations in the vicinity of waste disposal facilities. Still others are used to obtain data for a geological survey of the region. Several deep wells were drilled by the Basalt Waste Isolation Project (BWIP) (see Appendix A) to assess the feasibility of using the deep basalts beneath the Hanford Site for permanent disposal of nuclear wastes (Fecht and Lillie 1982).

A cross reference for the well numbers drilled by BWIP to Hanford coordinates can be found in Appendix A. Site investigations of nuclear power plants have necessitated the drilling of many wells to characterize the underlying formations (Fecht and Lillie 1982). Some of these wells have been destroyed during construction of new facilities or blasted to destruction during seismic refraction surveys, but are included on the master record to identify data obtained from them. More recent drilling has been done to achieve monitoring compliance at the Hanford Site in accordance with the Resource Conservation and Recovery Act (RCRA) as described in 40 CFR 265 (1988), Subpart F, and the Washington Administrative Code [1984 (WAC 173303)]. Table 1 contains a summary of well use.

As of January 1989, over 2900 wells still exist. Table 2 contains a summary of their status. About 1990 of these existing wells were drilled to 


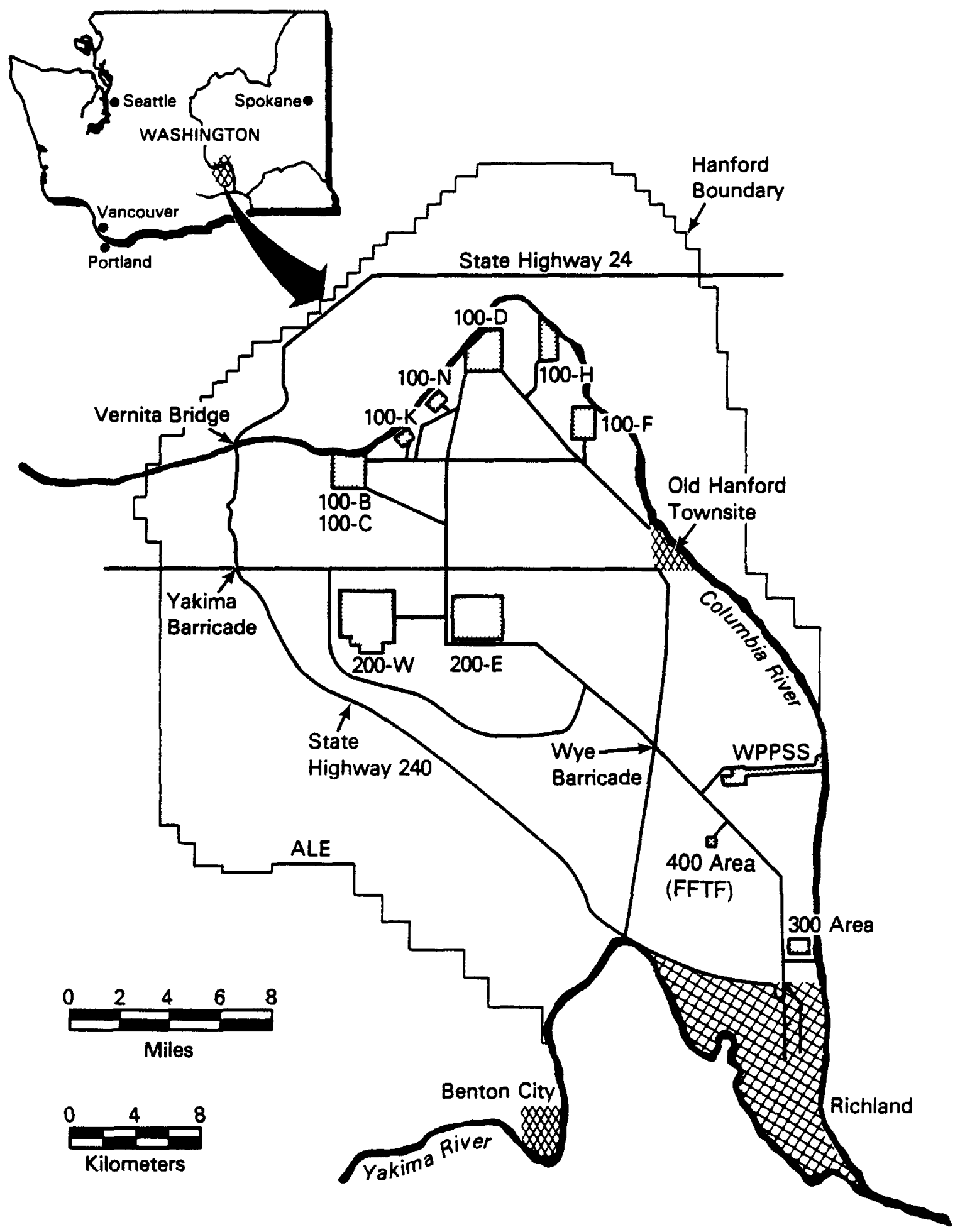

FIGURE 1. Map of the Hanford Site 
TABLE 1. Hanford Wells

\begin{tabular}{lr} 
Well Use & $\begin{array}{c}\text { Number } \\
\text { of Wells }\end{array}$ \\
\hline Ground-Water Contamination Surveillance & 546 \\
Ground-Water Hydrological Data Collection & 213 \\
Dry Wells for Monitoring Waste Management Facilities & 1196 \\
Basalt Stratigraphy Characterization & 241 \\
Water Supply Wells & 13 \\
Wells for Geologic and Seismic Studies & 611 \\
RCRA Wells & 121
\end{tabular}

TABLE 2. Total Wells by Area

\begin{tabular}{lcc} 
Area & Constructed & $\begin{array}{c}\text { Existing } \\
\text { (April 1988) }\end{array}$ \\
\cline { 2 - 3 } 100 & 208 & 174 \\
200 & 1883 & 1652 \\
300 & 62 & 55 \\
400 & 19 & 16 \\
600 & 1227 & 988 \\
1100 (Richland) & 105 & 39 \\
3000 (N. Richland) & 18 & 12 \\
Total Wel1s & 3522 & 2936
\end{tabular}

the ground-water table. About 1470 of these wells (including about 24 farm wel1s) still contain water. The others have become dry through infiltration of sediments or a general lowering of the water table in their vicinity.

This report provides complete documentation of wells on the Hanford Site, including some adjacent wells, and supersedes all previous compilations of Hanford Wells [e.g., PNL-5397 (February 1985)]. Not included in this report are those destroyed farm we17s that were never surveyed into the Hanford coordinate system nor used for water table measurements or water sample collection. The available data on these wells can be found in Jenkins (1922). 
While considerable effort has been made to obtain the most accurate and complete tabulation possible of the Hanford Site wells, omissions and errors may exist; e.g., wells drilled or modified by other contractors and not reported to the Pacific Northwest Laboratory (PNL) will not be found in this report. 


\section{WELL NUMBERING SYSTEM}

Well structures are identified by their location on the Hanford Site. The designation includes three groups of symbols. The first number identifies the area in which the well is located: 1 (100 Areas); 2 (200 Areas); 3 (300 Area); 4 (400 Area); 6 (600 Area); 11 (1100 Area) and 30 (3000 Area). Further explanation for the second and third groups of symbols are given below.

\section{AND 200 AREAS}

Wells located in the 100 and 200 Areas are further identified in the second group of symbols according to the designation of the particular area $(E, W, B, C, D, K, H, F, N)$, followed by the number of the sheet map encompassing that portion of the area in which the well is located. The sheet maps are shown on official second-level maps for each area; e.g., the first two-symbol groups for a well located within the area described by Sheet Map 24 of the 200-E Area would be 2-E24. The 100-K Area and the 100-N Area second-level maps are not further divided into sheet maps; thus, the second group of symbols for these areas are simply $K$ and $N$. The third-symbol group in the well number identifies the specific structure within the sheet map areas. In some cases, the numbering system is arbitrary, but where practical, numbers were chosen in accordance with a previous numbering system; e.g., the well formerly designated 361-B-므 now has the number 2-E28- $\underline{6}$.

Some of the monitoring wells in the 200 Areas are dry wells (i.e., wells that do not extend to the water table). Dry wells have been differentiated from deeper wells by numbering all of these shallow wells with the thirdgroup numbers greater than 50. In some cases, wells have been designated with 100- and 200-Area numbers even though they are actually located outside the area fence. These structures are monitoring wells adjacent to underground disposal facilities that are located outside the area. Monitoring wells adjacent to the $B C$-Cribs are examples of this situation. 
300 AREA

Wells in the 300 Area are designated in a manner similar to that described for the 100 and 200 Areas. The single difference occurs in the second symbol group in which no area designation is given, but which simply consists of the 300-Area sheet map number (e.g., 3-5-2).

\section{AREA}

Most of the wells in the 400 Area were drilled before construction started and were included in the 600-Area numbering system. Well numbers in this area are now prefixed by 4 . The second and third groups of numbers are plant coordinates and are explained in the following paragraph.

\section{AREA}

The 600 Area includes all of the Hanford Site outside the limited access areas. Well numbers in this area are prefixed by 6 . The second and third groups of numbers for 600-Area wells consist of the north and west $\mathrm{plant}$ coordinates, respectively, rounded off to the nearest $1000 \mathrm{ft}$. For example, a well located at plant coordinates N25665, W14554, would be designated 6-26-15. If the well is located south or east of the plant coordinate origin, an $S$ or $E$ is used with the appropriate number. Some 600-Area wells are located within $1000 \mathrm{ft}$ of each other; these have letters ( $A, B, C$, etc.) following the numbers for unique identification. A few wells located outside the Hanford Site boundaries have also been given 600-Area designations.

\section{HANFORD TOWNSITE}

The wells in the old Hanford townsite are designated as 600-Area wells with HAN as the second number group and the well number as the third number group. These wells were originally surveyed into the Hanford townsite grid, which was established during reactor construction. A conversion to plant coordinates has been made. Appendix B lists Hanford townsite wells and some miscellaneous offsite wells. 


\section{AND 3000 AREAS}

We11 numbers in the 1100 Area (City of Richland) and the 3000 Area (north of Richland) have 11 and 30 prefixes, respectively. The second and third number groups are the Richland coordinates rounded to the nearest 1000 $\mathrm{ft}$. These wells were located in the Richland coordinate system because the plant grid had not been extended this far south when they were surveyed. New wells within the Hanford Site boundaries in the 1100 and 3000 Areas are located and named in the 600-Area plant grid system. However, wells drilled since 1961 within the Richland city limits are not included in the 1 isting.

\section{PIEZOMETER TUBES}

Piezometer tubes were installed in a number of wells to measure potentials and sample the aquifer at various depths. The presence of these tubes is indicated by a letter $(0, P, Q, R, S$, or $T)$ after the well number; these tubes are listed with the corresponding well in this tabulation. Six other sets of piezometer tubes are individually drilled several feet apart and are al so designated by the sequence of letters $P, Q$, R, etc. Each tube of this type is regarded as a separate well in the tabulation.

\section{ENVIRONMENTAL MONITORING ANALYSIS NUMBERS}

The Environmental Monitoring Analysis (EMA) number identifies the well for the computer program that processes sampling schedules and results. A11 wells assigned an EMA number have been or are currently in the routine ground-water sampling schedule. Environmental Monitoring Analysis numbers followed by an "R" are on the Westinghouse Hanford Company's Environmental Surveillance sampling schedule. Some wells, if sampled for both contractors, have both PNL and Westinghouse EMA numbers. Appendix C lists EMA numbers.

\section{WESTINGHOUSE HANFORD COMPANY FILE NUMBER}

The Westinghouse Hanford Company file numbers pertain to tank farm wells. The first number is the tank farm number, the second is the tank number, and the third is the position of the well around the tank. Each tank is treated as a clock face with numbers located in hour quadrants. Numbers run 1 to 12 , with number 12 due north on the tank's center line. Wells 
outside the tank area are numbered 13 to 24 , with number 24 due north on the tank's center line. Appendix D lists this file. 


\section{DESCRIPTION OF WELL DATA LISTING}

The tabutation of existing wells and others for which data are recorded is comprised of sections, each of which reports wells from one of the areas and was prepared from a computer listing of the master well information file maintained by PNL's Geosciences Department. The listing format consists of the columns described below. Appendix E contains the well listing.

\section{COLUMN 1 - WELL DESIGNATION}

All of the existing wells that can be located and others for which data have been reported are designated in Column 1 by the system described earlier. Well designations can change when wells are surveyed into a coordinate system or when a resurvey is made.

\section{COLUMN 2 - COORDINATES}

Well coordinates are listed in Column 2 when this information is available. Plant coordinates are designated PN and PW (Plant North and Plant West); Richland coordinates are designated RN and RW (Richland North and Richland West). For certain wells, the coordinates have been estimated.

\section{COLUMN 3 - ELEVATION OF CASING}

The elevation of the top of the well casing is reported in feet above mean sea level. Some values have been estimated.

\section{COLUMN 4 - DRILLED DEPTH}

The depth of the well, as determined at the time the well was completed, is 1 isted in feet below the ground surface.

\section{COLUMN 5 - INITIAL DEPTH TO WATER}

The depth from the top of the casing to the water table is listed in feet for wells in which this distance has been measured. In some cases, these are initial depths to water when the well was first drilled; in others, the depth is updated with more recent information. These depths to water can 
fluctuate over several feet, particularly for wells near the Columbia River. The Hanford Ground-Water Data Base maintained by PNL's Geosciences Department located in the Sigma $V$ building should be consulted for complete water-level histories.

Several old farm wells that are not currently being used for water table elevation measurements are designated "wet" or "dry" as appropriate. In addition, numerous wells used by Washington Public Power Supply System as seismic shot holes are listed as "dry." The same "dry" listing applies to wells drilled by Golder Associates for Puget Sound Power and Light Company's Skagit/Hanford Nuclear Project.

\section{COLUMN 6 - CASING DIAMETER}

The diameter of the casing used in construction of the well is given in inches. A few of the wells constructed at the Hanford Site required casing of more than one size, and for these, only the diameter of the access hole is recorded. The length of the shortest side is listed for rectangularly shaped farm wells.

\section{COLUMN 7 - DEPTH TO BOTTOM OF THE WELL}

The most recent measurement of the bottom depth of the well is reported. Generally, wells become partially filled as sediments enter the well through the perforations. The depths shown in this column are updated from data obtained when remedial work is done or geophysical logs are run.

\section{COLUMN 8 - MINIMUM AND MAXIMUM DEPTH TO PERFORATIONS}

The depths to the top and bottom of the casing perforations are expressed in feet below the top of the well casing. However, in some wells alternating perforated and unperforated sections may be between the depths listed. Wells that were completed with well screens have no perforation depths listed. The screen depths are listed under the Comments Column. 


\section{COLUMN 9 - DATE COMPLETED}

This column records the date, to the nearest month where possible, on which the well was completed. In some cases, only the year the well was drilled is known. A 0-0 date indicates that the well has been completed, but the date is unknown.

\section{COLUMN 10 - FORMER NAME}

Column 10 lists the designations that have been used to identify the well in the past. These include local Hanford Site names or names applied by the agency responsible for constructing the well, such as the USGS designations. The USGS we11 location code numbers are based on the legal rectangular subdivision of public lands, with reference to the Willamette base 1 ine and meridian (e.g., 2/3-13E1). The two numbers preceding the hyphen indicate the township north and range east (T.2N., R.3E.) of the Willamette base 1 ine and meridian, respectively. Because the entire state of Washington is north of the Willamette base line, the letters denoting directions north and south are often omitted. The Hanford Site is east of the Willamette meridian so the letter $E$ is omitted. The first number after the hyphen indicates the section (Section 13), and the letter $E$ is the 40 -acre subdivision of the section. The last number, 1 , is the well number within the particular 40-acre tract. Therefore, well T2N/R3E-13E1 or equivalently 2/3-13E1 is in the southeast quarter of the northwest quarter of Section 13, Township 2 North, Range 3 East, and is the first well recorded in that tract.

The reference numbers used in this column refer to the reference listing for this report. The abbreviations and acronyms used in the former designations column are in Appendix F.

\section{COLUMN 11 - COMMENTS}

Noted in this column are changes made to the wells during routine maintenance or sample pump installation, seismic study wells, water supply wells, and wells that have had screens installed. Wells that have not been located and have probably been destroyed are so indicated in this column. 


\section{MAPS}

Maps of the 200-East and 200-West Areas (Figures 2 and 3) are included on the following pages, and a 600-Area map is in the binder. Only the wells that extend to ground water are shown in the 200-Area maps. The 600-Area map shows all wells currently available for water-level measurements and groundwater sampling. 


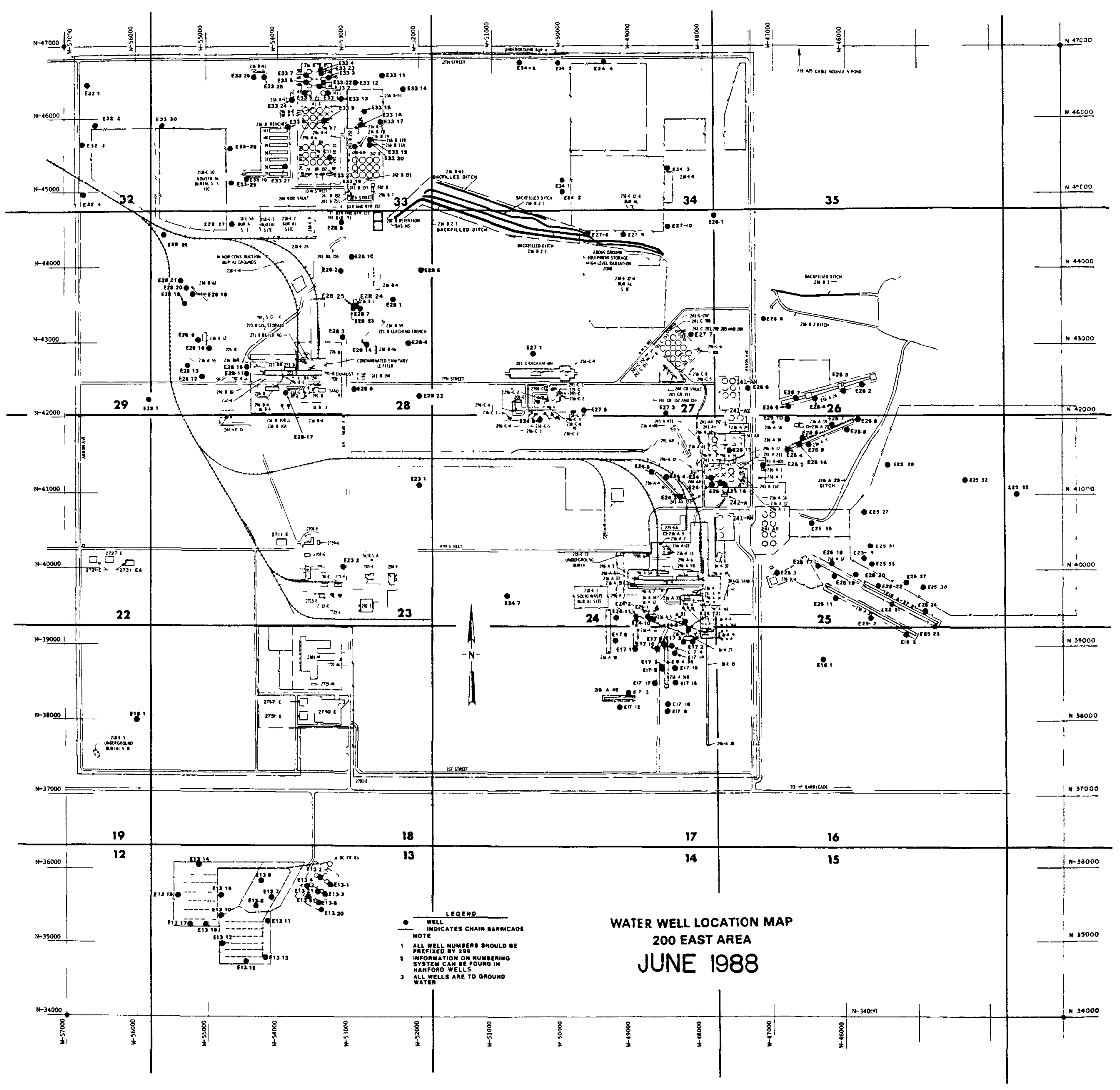

FIGURE_2. Water We17 Location Map -- 200-East Area 



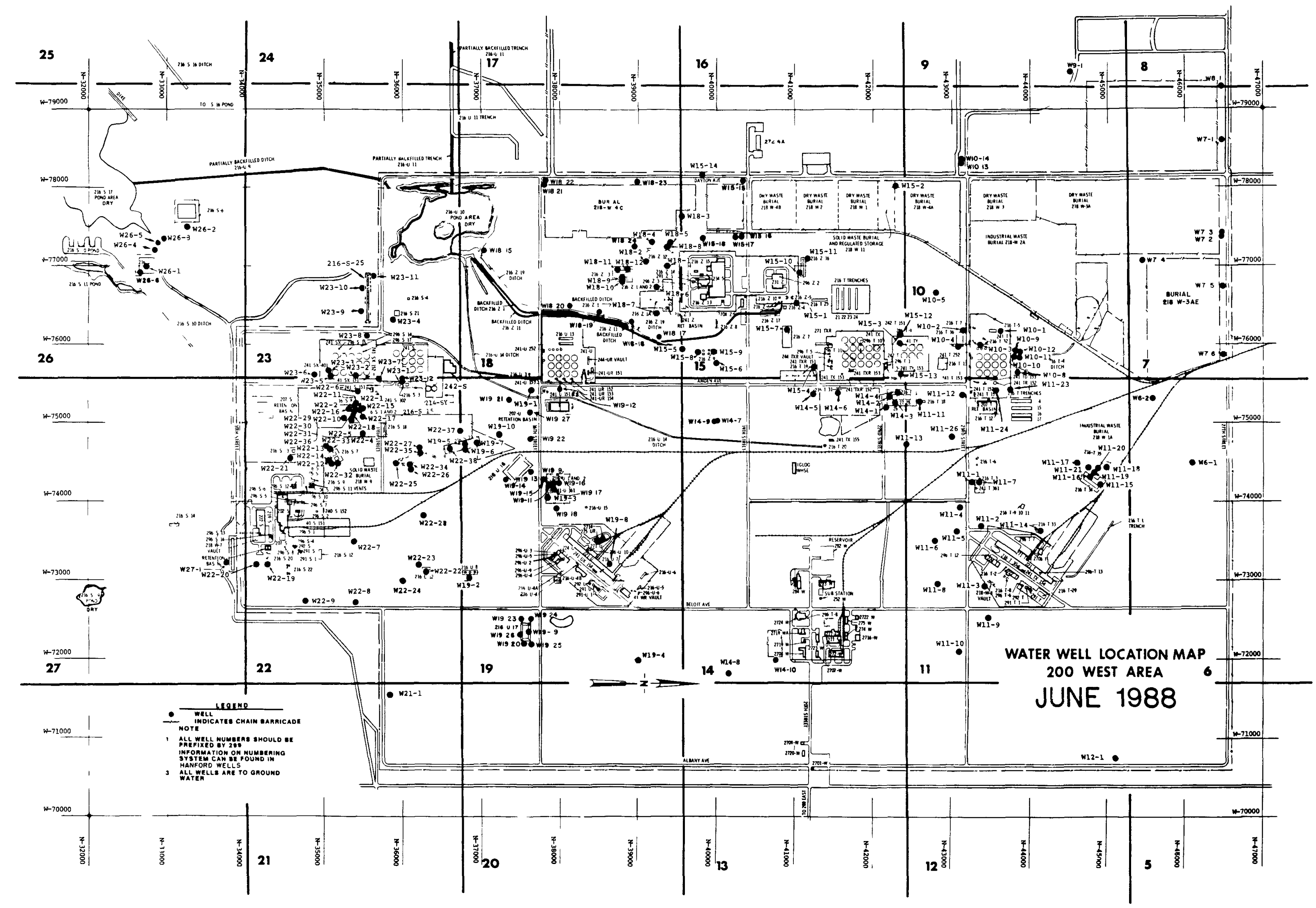

FIGURE 3. Water Well Location Map -- 200-West Area 



\section{REFERENCES}

Fecht, K. R., and J. T. Lillie. 1982. A Catalog of Borehole Logs from the 600 Area, Hanford Site. RHO-LD-158, Rockwell Hanford Operations, Richland, Washington.

40 CFR 265. 1988. U.S. Environmental Protection Agency, "Interim Status Standards for Owners and Operators of Hazardous Waste Treatment, Storage, and Disposal Facilities." U.S. Code of Federal Regulations.

Jenkins, 0. P. 1922. Underground Water Supply of the Region About White Bluffs and Hanford. State of Washington Department of Conservation and Development, Division of Geology, Bulletin No. 26, Olympia, Washington.

McGhan, V. L., P. J. Mitchel1, and R. S. Argo. 1985. Hanford Wells. PNL5397, Pacific Northwest Laboratory, Richland, Washington.

Newcomb, R. C., J. R. Strand, and F. J. Frank. 1972. Geology and GroundWater Characteristics of the Hanford Reservation of the U.S. Atomic Energy Commission. Washington. Geological Survey Professional Paper 717.

U.S. Government Printing Office, Washington, D.C.

Walters, K. L., and M. J. Grolier. 1960. Geology and Groundwater Resources of the Columbia Basin Project Area, Washington. State of Washington Water Supply Bulletin No. 8. Vol. 1. Washington State Department of Conservation, 01ympia, Washington.

Washington Administrative Code (WAC). 1985. "Dangerous Waste Regulations." WAC-173-303, 01ympia, Washington. 



\section{APPENDIX A}

BASALT WASTE ISOLATION PROJECT CORE HOLES -- NAME CROSS REFERENCE 
APPENDIX A

BASALT WASTE ISOLATION PROJECT CORE HOLES -- NAME CROSS REFERENCE

BWIP Core Holes - - Name Cross Reference

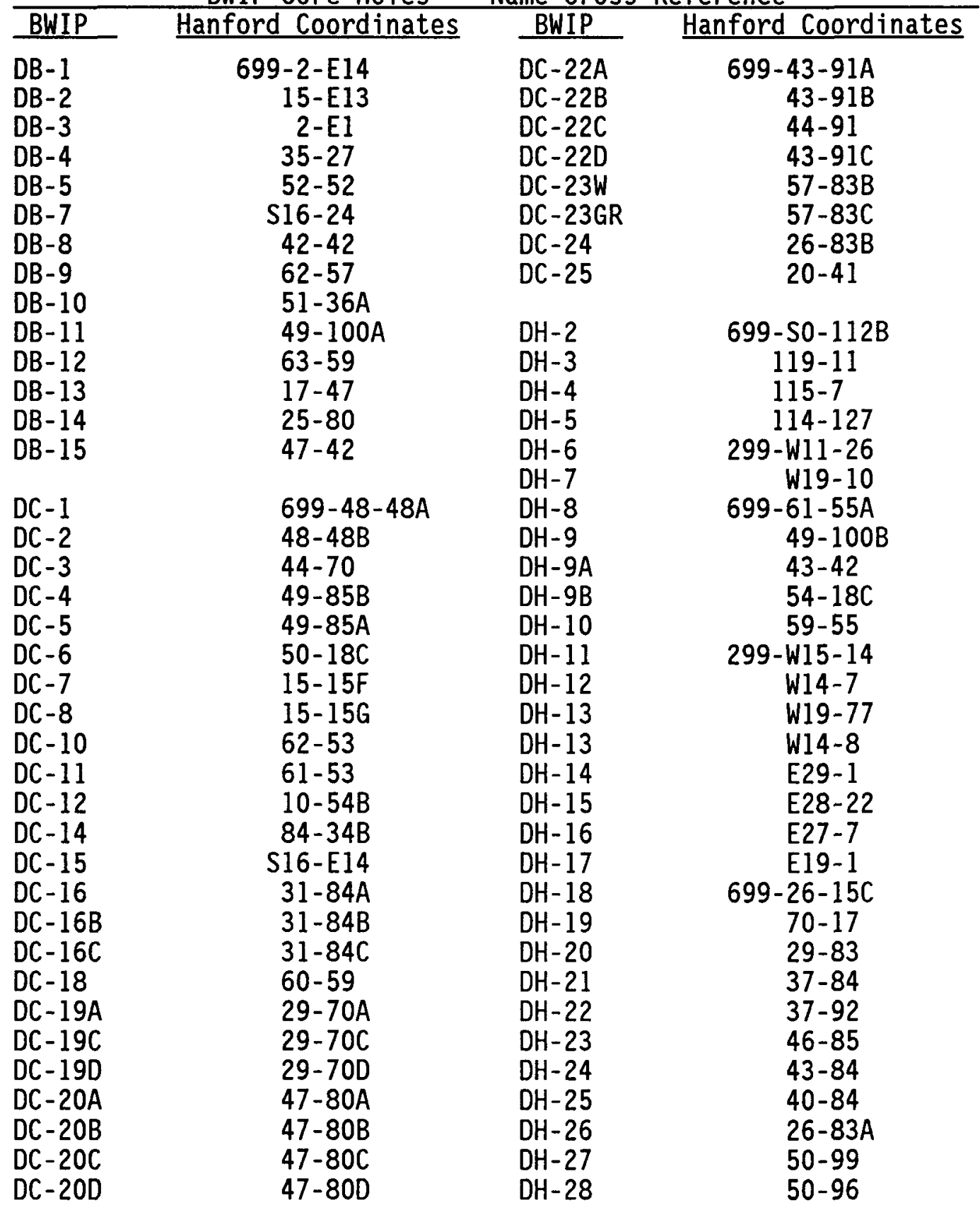


BWIP Core Holes -- Name Cross Reference

\begin{tabular}{lc}
\hline BWIP & Hanford Coordinat \\
\cline { 2 - 2 } DH-29 & $699-50-98$ \\
DH-33 & $34-98$ \\
& \\
DDH-1 & $699-S 17-30 A$ \\
DDH-2 & S17-30B \\
DDH-3 & S17-30C \\
DDH-4 & S17-28 \\
DDH-5 & S17-25 \\
DDH-6 & S17-24 \\
& \\
RRL-1 & \\
RRL-2 & \\
RRL-2B & $699-33-84$ \\
RRL-2C & $339-84 A$ \\
RRL-3 & $39-84 B$ \\
RRL-4 & $39-84 C$ \\
RRL-5 & $45-78$ \\
RRL-6A & $35-78 B$ \\
RRL-6B & $42-88$ \\
RRL-7 & $34-89 A$ \\
RRL-8 & $34-89 B$ \\
RRL-9 & $46-85 B$ \\
RRL-10 & $47-92$ \\
RRL-11 & $40-80$ \\
RRL-12 & $37-89$ \\
RRL-13 & $35-94$ \\
RRL-14 & $32-83$ \\
RRL-16 & $29-70 A$ \\
RRL-17 & $41-91$ \\
& $37-83$ \\
& $43-81$
\end{tabular}




\section{APPENDIX B}

HANFORD TOWNSITE WELLS 



\section{APPENDIX B}

HANFORD TOWNSITE WELLS

\begin{tabular}{|c|c|c|}
\hline ANFORD TOWNSITE GRID & PLANT COORDINATES & OTHER DESIGNATION \\
\hline $\begin{array}{l}\text { 699-HAN-1 } \\
\text { HAN-2 } \\
\text { HAN-3 } \\
\text { HAN-4 } \\
\text { HAN-5 } \\
\text { HAN-6 } \\
\text { HAN-7 } \\
\text { HAN-8 } \\
\text { HAN-9 } \\
\text { HAN-10 } \\
\text { HAN-11 } \\
\text { HAN-12 } \\
\text { HAN-13 } \\
\text { HAN-14 } \\
\text { HAN-15 } \\
\text { HAN-16 } \\
\text { HAN-17 } \\
\text { HAN-18 } \\
\text { HAN-19 } \\
\text { HAN }-20 \\
\text { HAN-21 } \\
\text { HAN-22 } \\
\text { HAN-23 } \\
\text { HAN-24 } \\
\text { HAN-25 } \\
\text { HAN-26 } \\
\text { HAN-27 } \\
\text { HAN-28 } \\
\text { HAN-29 } \\
\text { HAN-30 } \\
\text { HAN-31 } \\
\text { HAN-32 } \\
\text { HAN-33 } \\
\text { HAN-34 } \\
\text { HAN-35 } \\
\text { HAN-36 }\end{array}$ & $\begin{array}{c}699-53-13 \\
52-14 B \\
52-13 \\
52-14 A \\
50-10 \\
48-7 \\
57-16 \\
54-19 \\
54-18 \\
49-7 B \\
49-7 A \\
51-13 \\
\\
52-18 A \\
52-18 B \\
49-10 \\
49-13 \\
9-13 A \\
49-13 E \\
49-13 B \\
49-13 C \\
49-12 B \\
49-12 A \\
48-19 \\
48-17 \\
52-18 \\
52-19 \\
50-14\end{array}$ & $\begin{array}{l}\text { HD-1W } \\
\text { HD-2W } \\
\text { HD-3W } \\
\text { HD-4W } \\
\text { HD-5W } \\
\text { HD-6W } \\
\text { HD-7W } \\
\text { HD-8W } \\
\text { HD-9W } \\
\text { HD-10W } \\
\text { HI-11W } \\
\text { HD-12W } \\
\text { HI-9TH } \\
\text { HD-14W } \\
\text { HD-14TH } \\
\text { HD-15TH } \\
\text { HD-16W } \\
\text { HD-16TH } \\
\text { HD-17W } \\
\text { HD-17TH } \\
\text { HD-18TH } \\
\text { HD-19TH } \\
\text { HD-20TH } \\
\text { HD-21TH } \\
\text { HD-22TH } \\
\text { HD-23W } \\
\text { HD-24W } \\
\text { HANFORD CITY WELL } \\
\text { HANFORD HIGHSCHOOL } \\
\text { U.S.E.D. L-5 } \\
\text { HI-10W } \\
\text { 13/27-23R1 } \\
\text { REF. 2 NO. 30 } \\
\text { WHITWAR FARM } \\
\text { REF.2 } 2 \text { NO. } 177 \\
13 / 28-31 F 2 \\
\text { HD }\end{array}$ \\
\hline
\end{tabular}

\section{MISCELLANEOUS OFFSITE WELLS}
WBR RCH
W 15
WIT BLF
VAL RCH
RCH CEM
ALY MNR

B. 1 


$$
\text { . }
$$




\section{APPENDIX C}

ENVIRONMENTAL MONITORING ANALYSIS (EMA) NUMBERS 
APPENDIX C

ENVIRONMENTAL MONITORING ANALYSIS (EMA) NUMBERS

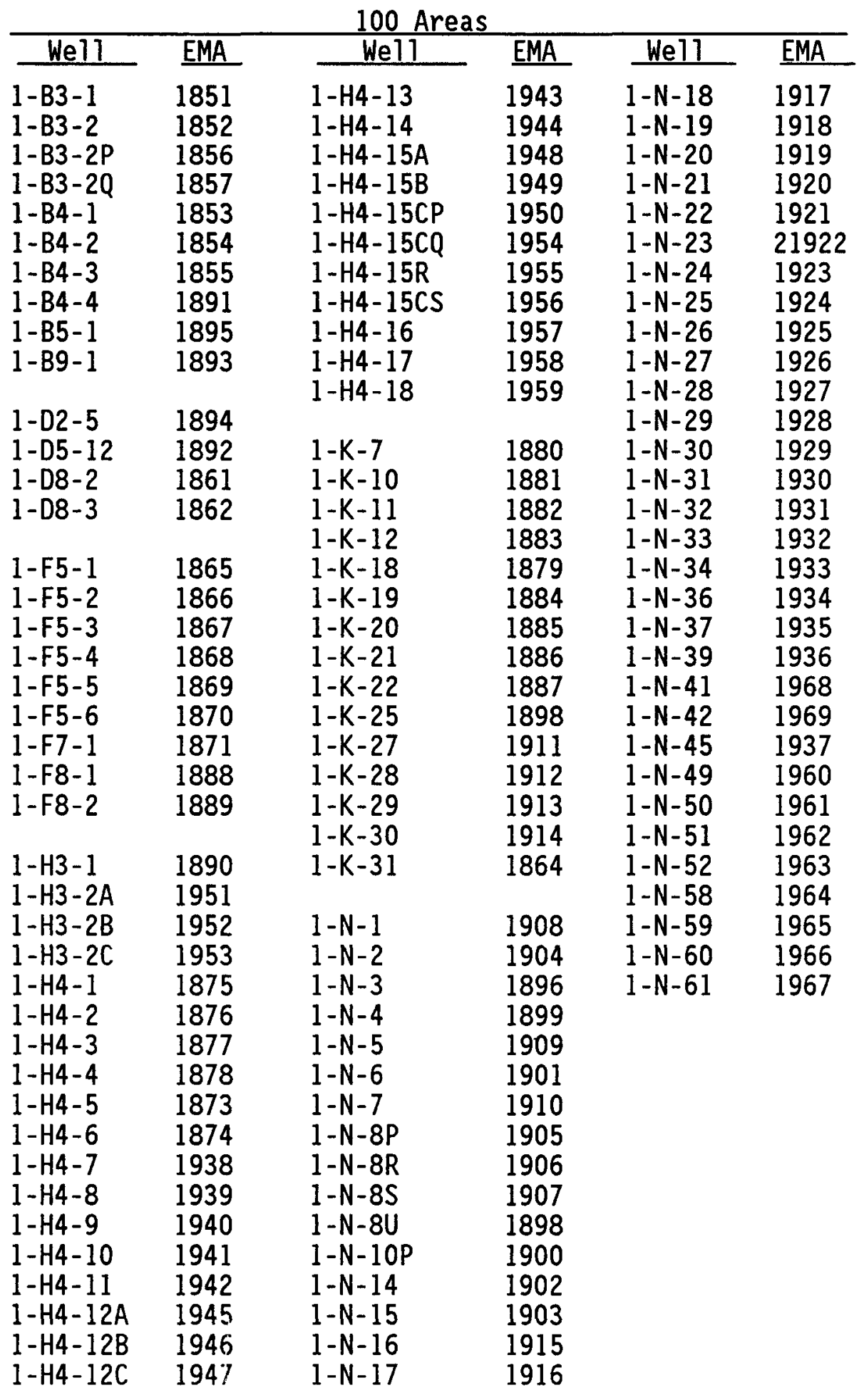

C. 1 
200-East Areas

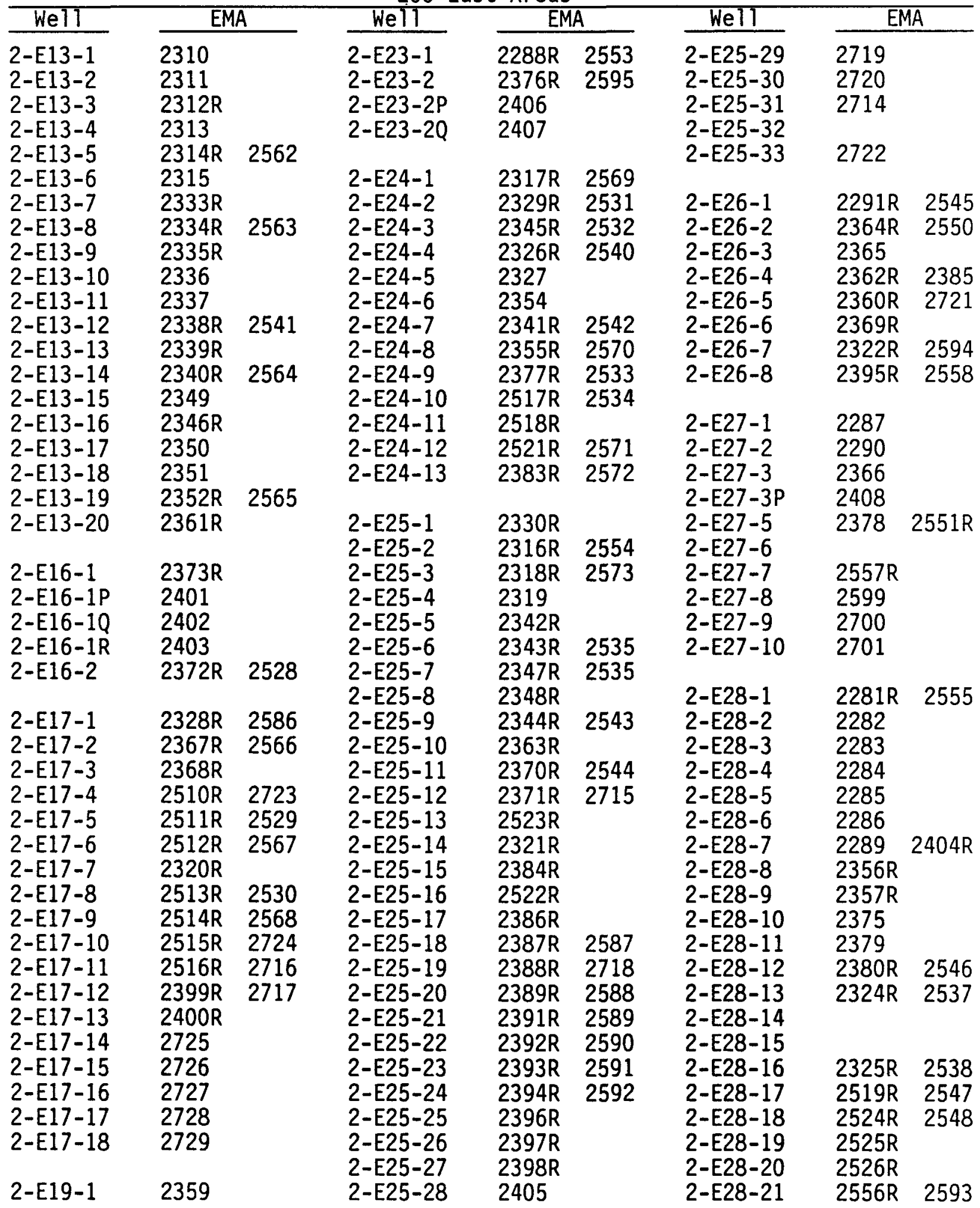


200-East Area (contd)

\begin{tabular}{|c|c|c|}
\hline Well & \multicolumn{2}{|c|}{ EMA } \\
\hline $\begin{array}{l}2-E 28-22 \\
2-E 28-23 \\
2-E 28-24 \\
2-E 28-25 \\
2-E 28-26 \\
2-E 28-27\end{array}$ & $\begin{array}{l}2390 R \\
2560 R \\
2561 R \\
2706 \\
2707\end{array}$ & $\begin{array}{l}2574 \\
2596\end{array}$ \\
\hline $\begin{array}{l}2-E 32-1 \\
2-E 32-2 \\
2-E 32-3 \\
2-E 32-4\end{array}$ & $\begin{array}{l}2358 \mathrm{R} \\
2711 \\
2712 \\
2713\end{array}$ & 2597 \\
\hline $2-E 33-1 A$ & $2301 R$ & 2575 \\
\hline 2-E33-3 & $2303 R$ & 2552 \\
\hline $\begin{array}{l}2-E 33-5 \\
2-E 33-6\end{array}$ & $\begin{array}{l}2308 \mathrm{R} \\
2307\end{array}$ & 2576 \\
\hline $\begin{array}{l}2-E 33-7 \\
2-E 33-8 \\
2-E 33-9 \\
2-E 33-10 \\
2-E 33-11\end{array}$ & $\begin{array}{l}2305 R \\
2300 R \\
2299 R \\
2306 R \\
2298\end{array}$ & $\begin{array}{l}2577 \\
2578 \\
2579 \\
2580\end{array}$ \\
\hline $\begin{array}{l}2-E 33-12 \\
2-E 33-13 \\
2-E 33-14 \\
2-E 33-15 \\
2-E 33-16 \\
2-E 33-17\end{array}$ & $\begin{array}{l}2294 \mathrm{R} \\
2296 \\
2297 \\
2293 \\
2292 \\
2295\end{array}$ & 2559 \\
\hline $\begin{array}{l}2-E 33-18 \\
2-E 33-19\end{array}$ & $\begin{array}{l}2309 R \\
2331\end{array}$ & 2581 \\
\hline $\begin{array}{l}2-E 33-20 \\
2-E 33-21 \\
2-E 33-22\end{array}$ & $\begin{array}{l}2332 R \\
2353 R\end{array}$ & $\begin{array}{l}2582 \\
2583\end{array}$ \\
\hline $\begin{array}{l}2-E 33-23 \\
2-E 33-24 \\
2-E 33-25 \\
2-E 33-26\end{array}$ & $\begin{array}{l}2323 R \\
2520 R \\
2381 R \\
2382 R\end{array}$ & $\begin{array}{l}2584 \\
3539\end{array}$ \\
\hline $\begin{array}{l}2-E 33-27 \\
2-E 33-28 \\
2-E 33-29 \\
2-E 33-30\end{array}$ & $\begin{array}{l}2527 R \\
2708 \\
2709 \\
2710\end{array}$ & 2585 \\
\hline $\begin{array}{l}2-E 34-1 \\
2-E 34-2 \\
2-E 34-3 \\
2-E 34-4 \\
2-E 34-5 \\
2-E 34-6\end{array}$ & $\begin{array}{l}2374 R \\
2598 \\
2702 \\
2703 \\
2704 \\
2705\end{array}$ & 2549 \\
\hline
\end{tabular}

200-West Area

\begin{tabular}{|c|c|c|c|c|c|}
\hline WelT & \multicolumn{2}{|c|}{ EMA } & Well & \multicolumn{2}{|c|}{ EMA } \\
\hline $\begin{array}{l}2-W 6-1 \\
2-w 6-2\end{array}$ & $\begin{array}{l}2927 R \\
2692\end{array}$ & 2990 & $\begin{array}{l}2-W 11-14 \\
2-W 11-15 \\
2-W 11-16\end{array}$ & $\begin{array}{l}2944 \\
2961 R \\
2959\end{array}$ & 2998 \\
\hline $\begin{array}{l}2-W 7-1 \\
2-W 7-2 \\
2-W 7-3\end{array}$ & $\begin{array}{l}2686 \\
2687 \\
2688\end{array}$ & & $\begin{array}{l}2-W_{1} 1-17 \\
2-W_{1} 1-18 \\
2-W_{1} 1-19\end{array}$ & $\begin{array}{l}2962 R \\
2699\end{array}$ & $\begin{array}{l}2981 \\
22963 R\end{array}$ \\
\hline $\begin{array}{l}2-w 7-4 \\
2-w 7-5 \\
2-w 7-6\end{array}$ & $\begin{array}{l}2689 \\
2690 \\
2691\end{array}$ & & $\begin{array}{l}2-W 11-20 \\
2-W 11-21 \\
2-w 11-23 \\
2-w 11-24\end{array}$ & $\begin{array}{l}2976 \mathrm{R} \\
2973 \mathrm{R} \\
2616 \mathrm{R} \\
3010 \mathrm{R}\end{array}$ & \\
\hline $2-w 8-1$ & 2676 & & $2-W 11-26$ & & \\
\hline
\end{tabular}

2-W9-1 2686

2-W10-1 2634

2-W10-2

2-W10-3

$2-W 10-4$

2-W10-5

$2-W 10-6$

2-W10-7

$2-W 10-8$

2-W10-9

2-W10-10

2-W10-11

2-W10-12

2-W10-13

2-W10-14

2683

2684

2-W11-1 2873

2-W11-2 2874

2-W11-2P 2601

2-W11-2Q 2602

2-W11-2R 2603

2-W11-2S 2604

2-W11-2T 2605

2-W11-3

$2875 \mathrm{R}$

2-W11-4 2876

2-W11-5 2877

2-W11-6 2878

$2-W 11-7$

2-W11-8

$2695 \mathrm{R}$

2880

2-W11-9 2881

2-W11-10 2882

2-W11-11 2887R

2-W11-12 2888

$2-W 11-13 \quad 2638$

2-W11-13P 2945

2-W11-13Q 2950
2-W12-1 2883

2892R

2893R

$2886 R$

2-W14-1

2-W14-2

2-W14-3

$2-W 14-3 P$

2-W14-4

2-W14-5

2-W14-6

$\begin{array}{ll}2997 & 2-W 14-7 \\ 3009 R & 2-W 14-8\end{array}$

2-W14-8A

2-W14-9

2-W14-10

$28023018 R$

2-W15-1 2897R

2-W15-2 2697R 2891

2-W15-3 2894R

2-W15-4 2642

2-W15-5 2922R

2-W15-6 2655

$2-W 15-7$

$2-W 15-8$

2960R 2982

2-W15-9 2975R

2-W15-10 2609R

2-W15-11

2-W15-12

2610R

2698R

$3012 R$

2884

2680

2681

2682

2677

$2942 R$

2-W18-1 2935R

2-W18-2 2932R

2-W18-3 2936R

3011
$2885 \mathrm{R}$ 
200-West Area (contd)

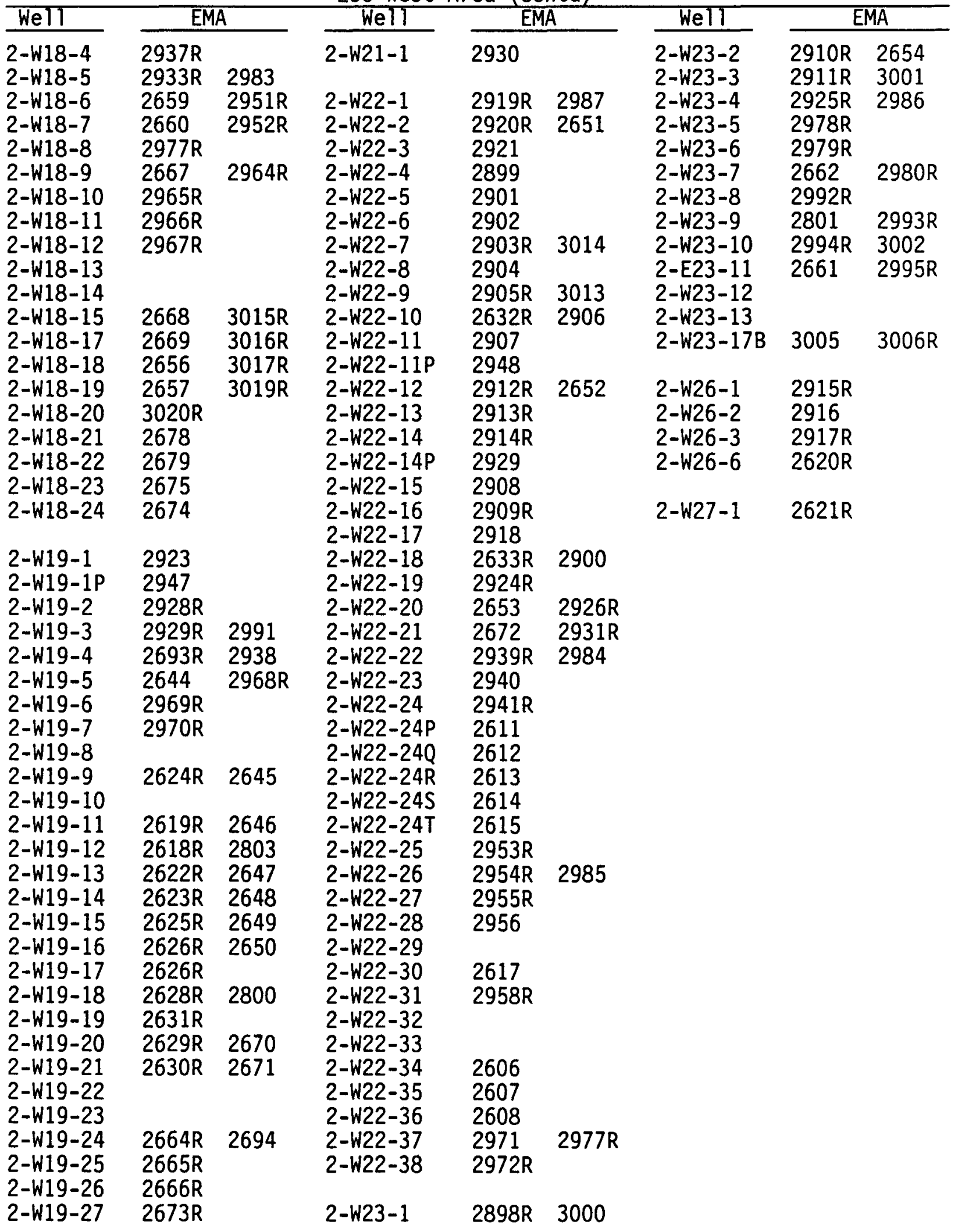

C. 4 


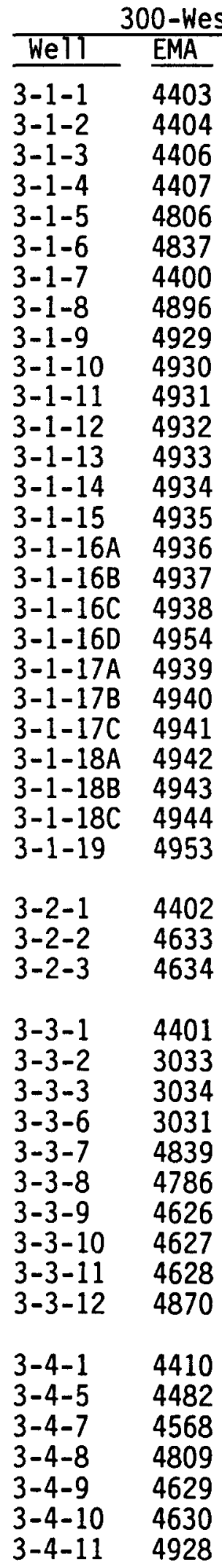

\begin{tabular}{|c|c|c|c|}
\hline \multicolumn{2}{|l|}{ Area } & \multicolumn{2}{|c|}{400 Area } \\
\hline Well & EMA & Well & EMA \\
\hline $\begin{array}{l}3-5-1 \\
3-5-2\end{array}$ & $\begin{array}{l}4411 \\
4575\end{array}$ & $\begin{array}{l}4-S 1-7 A \\
4-S 1-7 B \\
4-S 1-7 C\end{array}$ & $\begin{array}{l}4801 \\
4819 \\
4877\end{array}$ \\
\hline $3-6-1$ & 4409 & & $\begin{array}{l}4878 \\
4879\end{array}$ \\
\hline $\begin{array}{l}3-8-1 \\
3-8-2 \\
3-8-3\end{array}$ & $\begin{array}{l}4405 \\
4412 \\
4412\end{array}$ & $\begin{array}{l}4- \\
4-\end{array}$ & $\begin{array}{l}4956 \\
4897\end{array}$ \\
\hline & 4865 & $\begin{array}{l}4-50-7 \\
4-50-8\end{array}$ & $\begin{array}{l}4817 \\
4818\end{array}$ \\
\hline
\end{tabular}

\begin{tabular}{|c|c|c|}
\hline 600 & Area & \\
\hline WeाT & \multicolumn{2}{|c|}{$\overline{E M A}$} \\
\hline $6-541-13 C$ & $\overline{4900}$ & \\
\hline $6-\$ 41-15$ & 4903 & \\
\hline $6-\$ 39-16 C$ & 4904 & \\
\hline $6-536-13 B$ & 4905 & \\
\hline $6-\$ 34-15$ & 4901 & \\
\hline $6-S 32-E 13 A$ & 4906 & \\
\hline $6-\$ 31-E 13$ & 4902 & \\
\hline $6-531-1$ & 4606 & \\
\hline $6-S 31-1 P$ & 4745 & \\
\hline $6-S 30-E 14$ & 4582 & \\
\hline $6-S 30-E 15 B$ & 4805 & \\
\hline $6-S 29-E 12$ & 4803 & \\
\hline $6-528-E 0$ & $4764 \mathrm{R}$ & 4952 \\
\hline $6-S 27-E 14$ & 4413 & \\
\hline $6-\$ 24-19$ & 4510 & \\
\hline $6-\$ 23-26$ & 4807 & \\
\hline $19-E 13$ & 4802 & \\
\hline $19-11$ & 4780 & \\
\hline 18-E2A & 4572 & \\
\hline 18-E2AP & 4746 & \\
\hline-51 & 4852 & \\
\hline $6-516-24$ & 4911 & \\
\hline $4-20 A$ & 4535 & \\
\hline 4-20AP & 4662 & \\
\hline $6-S 12-3$ & 4424 & \\
\hline
\end{tabular}

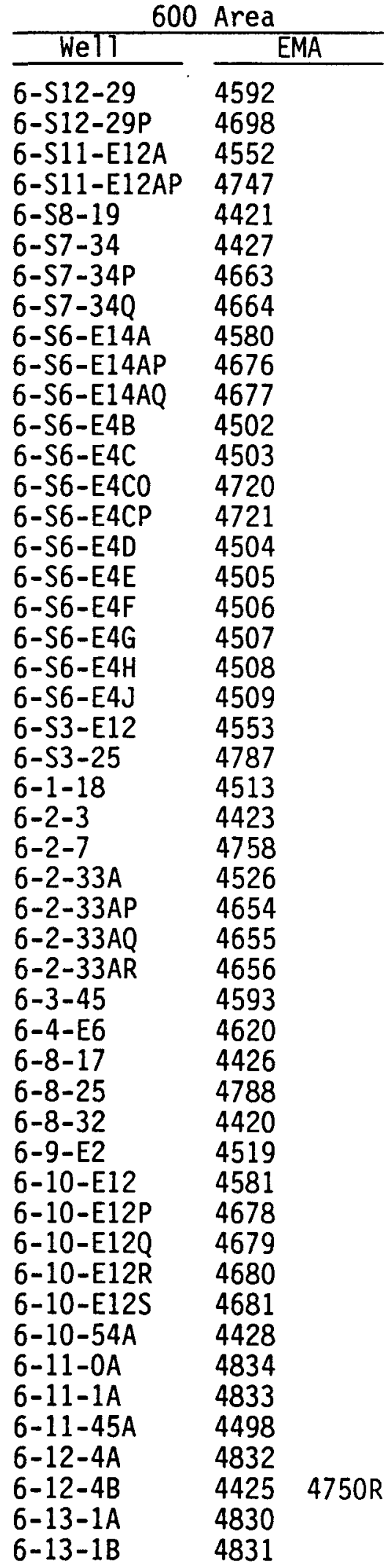


600 Area (contd)

\begin{tabular}{|c|c|c|c|c|c|c|c|c|}
\hline WelT & \multicolumn{2}{|c|}{ EMA } & Well & \multicolumn{2}{|c|}{ EMA } & WelT & \multicolumn{2}{|c|}{ EMA } \\
\hline $\begin{array}{l}6-13-1 C \\
6-13-64 \\
6-14-E 6 P \\
6-14-E 6 Q \\
6-14-E 6 R \\
6-14-E 6 S \\
6-14-E 6 T \\
6-14-38 \\
6-14-38 P \\
6-14-38 Q \\
6-14-38 R \\
6-14-38 S \\
6-14-47 \\
6-15-15 A \\
6-15-15 A P \\
6-15-15 A Q \\
6-15-15 A R \\
6-15-15 A S \\
6-15-15 A T \\
6-15-15 B \\
6-15-26 \\
6-17-5 \\
6-17-47 \\
6-17-70 \\
6-19-43 \\
6-19-47 A \\
6-19-58 \\
6-19-58 P \\
6-19-88 \\
6-20-E 12 \\
6-20-E 12 P \\
6-20-E 12 Q \\
6-20-E 12 R \\
6-20-E 12 S \\
6-20-E 5 A \\
6-20-E 5 P \\
6-20-E 5 Q \\
6-20-E 5 R \\
6-20-E 5 S \\
6-20-E 5 T \\
6-20-20 \\
6-20-39 \\
6-20-39 P \\
6-20-39 R \\
6-20-39 T \\
6-20-82 \\
6-21-6 \\
6-22-70 \\
\end{array}$ & $\begin{array}{l}4854 \\
4429 \\
4700 \\
4701 \\
4702 \\
4703 \\
4766 \\
4527 \\
4616 \\
4617 \\
4618 \\
4619 \\
4608 \\
4551 \\
4601 \\
4602 \\
4603 \\
4604 \\
4605 \\
4810 \\
4464 \\
4422 \\
4530 \\
4531 \\
4417 \\
4609 \\
4528 \\
4672 \\
4522 \\
4567 \\
4611 \\
4612 \\
4613 \\
4614 \\
4838 \\
4705 \\
4706 \\
4707 \\
4708 \\
4765 \\
4418 \\
4559 \\
4782 \\
4725 \\
4726 \\
4529 \\
4855 \\
4595\end{array}$ & $4888 R$ & $\begin{array}{l}6-22-70 P \\
6-22-70 Q \\
6-23-34 \\
6-24-1 P \\
6-24-10 \\
6-24-1 R \\
6-24-15 \\
6-24-1 T \\
6-24-33 \\
6-24-34 A \\
6-24-34 B \\
6-24-34 C \\
6-24-35 \\
6-24-46 \\
6-25-33 A \\
6-25-33 B \\
6-25-33 B P \\
6-25-33 B Q \\
6-25-34 A \\
6-25-34 B \\
6-25-34 C \\
6-25-55 \\
6-25-70 \\
6-25-80 \\
6-26-15 A \\
6-26-33 \\
6-26-34 \\
6-26-35 A \\
6-26-35 B \\
6-26-35 C \\
6-26-35 D \\
6-26-35 D P \\
6-26-350 Q \\
6-26-89 \\
6-27-8 \\
6-28-40 \\
6-28-40 P \\
6-28-40 Q \\
6-28-40 R \\
6-28-40 S \\
6-28-52 A \\
6-28-55 \\
6-29-4 \\
6-29-78 \\
6-31-31 \\
6-31-31 P \\
6-31-31 Q \\
6-31-31 R\end{array}$ & $\begin{array}{l}4598 \\
4557 \\
4481 \\
4754 \\
4755 \\
4736 \\
4737 \\
4521 \\
4840 \\
4857 \\
4594 \\
4471 \\
4738 \\
4757 \\
4739\end{array}$ & $4891 R$ & $\begin{array}{l}6-31-31 S \\
6-31-53 B \\
6-31-53 B P \\
6-31-53 B Q \\
6-31-65 \\
6-32-22 \\
6-32-42 \\
6-32-43 \\
6-32-62 \\
6-32-62 P \\
6-32-70 \\
6-32-72 \\
6-32-77 \\
6-33-42 \\
63356 \\
6-34-39 A \\
6-34-41 B \\
6-34-42 \\
6-34-51 \\
6-34-88 \\
6-35-9 \\
6-35-66 \\
6-35-70 \\
6-35-78 A \\
6-36-46 P \\
6-36-46 Q \\
6-36-46 R \\
6-36-46 S \\
6-36-61 A \\
6-36-61 B \\
6-36-61 B P \\
6-36-93 \\
6-37-E 4 \\
6-37-43 \\
6-37-43 P \\
6-37-43 Q \\
6-37-82 A \\
6-37-82 A P \\
6-37-82 A Q \\
6-37-82 A R \\
6-37-82 A S \\
6-37-82 B \\
6-37-82 B P \\
6-37-82 B Q \\
6-37-82 B R \\
6-37-82 B S \\
6-38-15 \\
6-38-65\end{array}$ & $\begin{array}{l}4740 \\
4520 \\
4659 \\
4660 \\
4495 \\
4794 \\
4777 \\
4778 \\
4550 \\
4783 \\
4492 \\
4491 \\
4446 \\
4779 \\
4523 \\
4448 \\
4789 \\
4790 \\
4414 \\
4439 \\
4419 \\
4494 \\
4441 \\
4445 \\
4751 \\
4752 \\
4753 \\
4767 \\
4447 \\
4549 \\
4784 \\
4579 \\
4876 \\
4480 \\
4748 \\
4749 \\
4554 \\
4621 \\
4622 \\
4623 \\
4624 \\
4555 \\
4685 \\
4685 \\
4687 \\
4688 \\
4880 \\
4546\end{array}$ & $\begin{array}{l}4957 R \\
4869 R\end{array}$ \\
\hline
\end{tabular}

C. 6 
600 Area (contd)

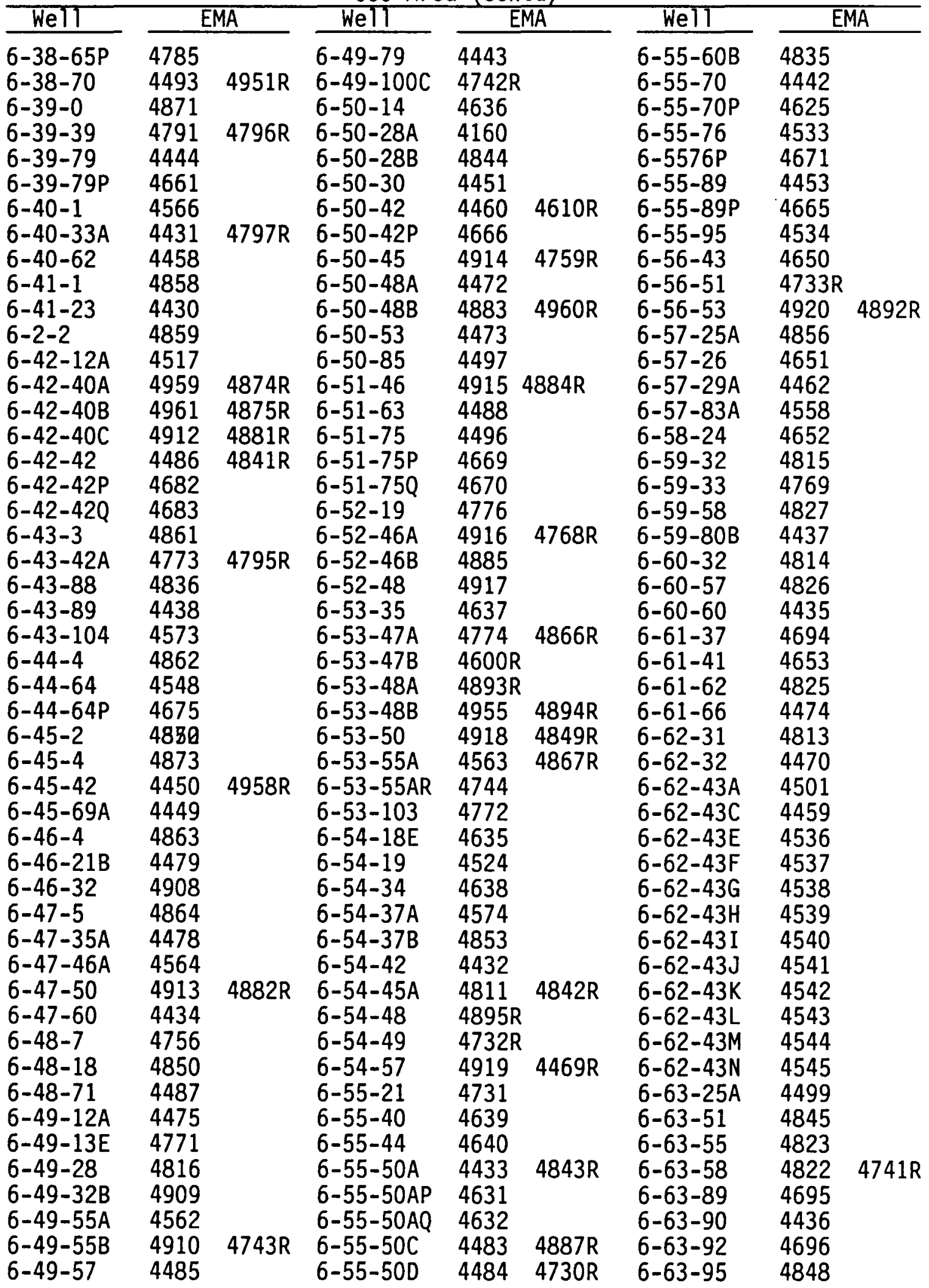

C.7 
600 Area (contd)

\begin{tabular}{|c|c|c|c|c|c|c|}
\hline Well & EMA & Well & EMA & WeTT & & EMA \\
\hline $\begin{array}{l}6-64-27 \\
6-64-62 \\
6-65-22 \\
6-65-23 \\
6-65-38 \\
6-65-50 \\
6-65-59 A \\
6-65-59 A P \\
6-65-72 \\
6-65-83 \\
6-65-95 \\
6-65-114 A \\
6-65-114 B \\
6-66-23 \\
6-66-23 P \\
6-66-38 \\
6-66-38 P \\
6-66-38 Q \\
6-66-39 \\
6-66-58 \\
6-66-64 \\
6-66-91 \\
6-66-103 \\
6-67-51 \\
6-67-51 P \\
6-67-51 Q \\
6-67-77 \\
6-67-86 \\
6-67-86 P \\
6-67-860 \\
6-67-86 R \\
6-67-865 \\
6-67-98 \\
6-68-105 \\
6-69-38 \\
6-69-45 \\
6-69-45 P \\
6-69-45 Q \\
6-69-45 R \\
6-70-23 \\
6-70-37 \\
6-70-68 \\
6-71-30 \\
6-71-52 \\
6-71-77 \\
6-71-85 \\
6-72-73 \\
6-72-88 \\
6-72-98 \\
6\end{array}$ & $\begin{array}{l}4599 \\
4824 \\
4715 \\
4851 \\
4716 \\
4477 \\
4532 \\
4667 \\
4468 \\
4775 \\
4847 \\
4798 \\
4799 \\
4547 \\
4668 \\
4586 \\
4689 \\
4690 \\
4812 \\
4821 \\
4820 \\
4697 \\
4587 \\
4561 \\
4691 \\
4692 \\
4467 \\
4585 \\
4650 \\
4651 \\
4652 \\
4653 \\
4556 \\
4588 \\
4461 \\
4560 \\
4641 \\
4642 \\
4643 \\
4718 \\
4808 \\
4455 \\
4490 \\
4454 \\
4584 \\
4466 \\
4569 \\
4465 \\
4463\end{array}$ & $\begin{array}{l}6-72-92 P \\
6-72-92 Q \\
6-72-98 \\
6-73-61 \\
6-74-44 \\
6-74-48 \\
6-74-48 P \\
6-77-36 \\
6-77-44 \\
6-77-54 \\
6-78-36 \\
6-78-62 \\
6-80-43 P \\
6-80-43 Q \\
6-80-43 R \\
6-80-43 S \\
6-81-38 \\
6-81-58 \\
6-81-58 P \\
6-82-45 A \\
6-83-32 \\
6-83-47 \\
6-84-35 A \\
6-84-35 A 0 \\
6-84-35 A P \\
6-84-35 A Q \\
6-84-35 A R \\
6-84-35 A S \\
6-85-40 A \\
6-86-42 \\
6-86-60 \\
6-87-42 A \\
6-87-55 \\
6-89-35 \\
6-90-45 \\
6-90-47 \\
6-91-37 \\
6-92-14 \\
6-92-49 \\
6-93-37 \\
6-96-49 \\
6-96-49 P \\
6-97-43 \\
6-97-43 P \\
6-97-48 \\
6-97-51 A \\
6-97-51 B \\
6-101-48 A \\
6-101-48 B\end{array}$ & $\begin{array}{l}4657 \\
4658 \\
4463 \\
4583 \\
4516 \\
4589 \\
4644 \\
4500 \\
4800 \\
4512 \\
4489 \\
4511 \\
4760 \\
4761 \\
4762 \\
4763 \\
4722 \\
4597 \\
4684 \\
4717 \\
4719 \\
4515 \\
4596 \\
4596 \\
4646 \\
4647 \\
4648 \\
4649 \\
4860 \\
4723 \\
4570 \\
4793 \\
4792 \\
4571 \\
4770 \\
4457 \\
4724 \\
4828 \\
4727 \\
4476 \\
4591 \\
4645 \\
4590 \\
4693 \\
4456 \\
4728 \\
4729 \\
4735 \\
4846\end{array}$ & $\begin{array}{l}\text { 6-115-61 } \\
6-\text { WBR RCH } \\
6-W 15 \\
6-W I T \text { BLF } \\
6-\text { VAL RCH } \\
6-\text { RCH CEM } \\
6-A L Y \text { MNR } \\
\frac{3000 \text { Ar }}{\text { We } 11} \\
30-42-16 \\
30-45-16 \\
30-45-18 A \\
30-47-18 B \\
3000 N \\
\frac{1100 \text { Ar }}{4 \text { We } 11} \\
11-34-15 \\
11-39-16 \mathrm{C} \\
11-41-13 \mathrm{C} \\
11-41-15\end{array}$ & $\begin{array}{l} \\
\\
\\
\text { EMA } \\
4607 \\
4578 \\
4577 \\
4576 \\
4907 \\
\text { EMA } \\
4901 \\
4904 \\
4900 \\
4903\end{array}$ & $\begin{array}{l}4829 \\
3500 \\
3501 \\
3502 \\
3503 \\
3504 \\
3505\end{array}$ \\
\hline
\end{tabular}

C. 8 
APPENDIX D

WESTINGHOUSE COMPUTER FILE NUMBERS 

APPENDIX D

WESTINGHOUSE COMPUTER FILE NUMBERS

241-A Tank Farm

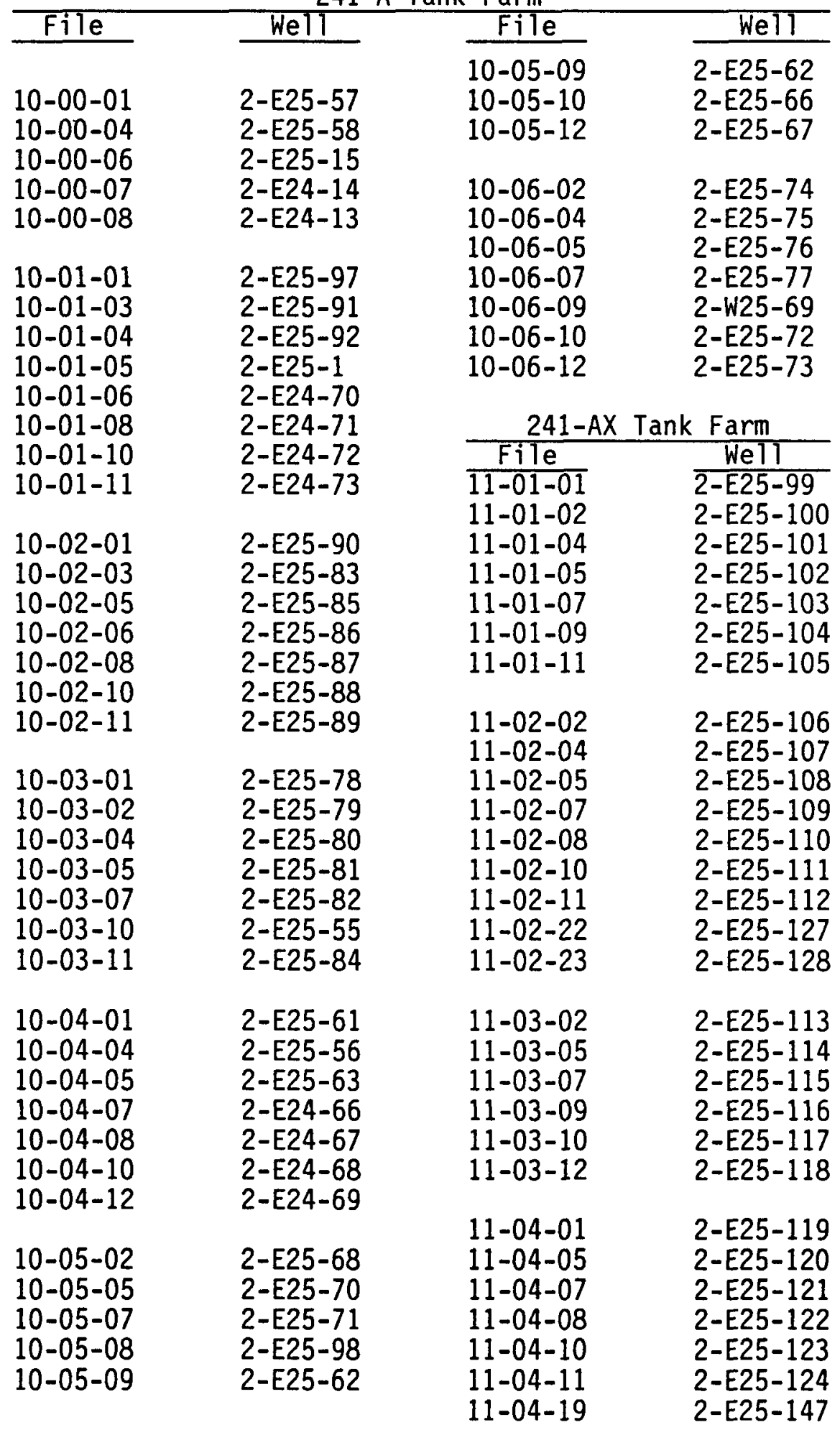

0.1 
241-B Tank Farm

\begin{tabular}{|c|c|c|c|}
\hline \multirow[b]{2}{*}{$\begin{array}{c}\text { File } \\
20-00-01 \\
20-00-02 \\
20-00-05 \\
20-00-07 \\
20-00-09 \\
20-00-11\end{array}$} & Well & File & Well \\
\hline & $\begin{array}{l}2-E 33-53 \\
2-E 33-51 \\
2-E 33-52 \\
2-E 33-56 \\
2-E 33-57 \\
2-E 33-55\end{array}$ & $\begin{array}{l}20-10-02 \\
20-10-07 \\
20-10-09 \\
20-10-12 \\
20-11-09\end{array}$ & $\begin{array}{l}2-E 33-213 \\
2-E 33-216 \\
2-E 33-215 \\
2-E 33-214 \\
2-E 33-217\end{array}$ \\
\hline $20-01-03$ & $2-E 33-220$ & $\begin{array}{l}20-12-02 \\
20-12-03\end{array}$ & $\begin{array}{l}2-E 33-201 \\
2-E 33-199\end{array}$ \\
\hline $\begin{array}{l}20-02-03 \\
20-02-05 \\
20-02-07 \\
20-02-09 \\
20-02-11\end{array}$ & $\begin{array}{l}2-E 33-179 \\
2-E 33-180 \\
2-E 33-181 \\
2-E 33-182 \\
2-E 33-183\end{array}$ & $\begin{array}{l}20-12-06 \\
20-12-07 \\
20-12-11\end{array}$ & $\begin{array}{l}2-E 33-202 \\
2-E 33-203 \\
2-E 33-204\end{array}$ \\
\hline $\begin{array}{l}20-03-02 \\
20-03-03 \\
20-03-06 \\
20-03-09 \\
20-03-11\end{array}$ & $\begin{array}{l}2-E 33-184 \\
2-E 33-185 \\
2-E 33-186 \\
2-E 33-187 \\
2-E 33-188\end{array}$ & & \\
\hline $\begin{array}{l}20-04-03 \\
20-04-06\end{array}$ & $\begin{array}{l}2-E 33-219 \\
2-E 33-221\end{array}$ & & \\
\hline $20-05-06$ & $2-E 33-218$ & & \\
\hline $\begin{array}{l}20-06-02 \\
20-06-03 \\
20-06-06 \\
20-06-11\end{array}$ & $\begin{array}{l}2-E 33-189 \\
2-E 33-190 \\
2-E 33-191 \\
2-E 33-192\end{array}$ & & \\
\hline $\begin{array}{l}20-07-02 \\
20-07-05 \\
20-07-08 \\
20-07-11\end{array}$ & $\begin{array}{l}2-E 33-212 \\
2-E 33-149 \\
2-E 33-148 \\
2-E 33-147\end{array}$ & & \\
\hline $\begin{array}{l}20-08-02 \\
20-08-03 \\
20-08-05 \\
20-08-07 \\
20-08-09\end{array}$ & $\begin{array}{l}2-E 33-192 \\
2-E 33-193 \\
2-E 33-194 \\
2-E 33-195 \\
2-E 33-196\end{array}$ & & \\
\hline $\begin{array}{l}20-09-02 \\
20-09-06 \\
20-09-11\end{array}$ & $\begin{array}{l}2-E 33-197 \\
2-E 33-198 \\
2-E 33-200\end{array}$ & & \\
\hline
\end{tabular}

D.2 
241-BX Tank Farm

\begin{tabular}{|c|c|c|c|}
\hline File & Well & File & Well \\
\hline $\begin{array}{l}21-00-01 \\
21-00-02 \\
21-00-03 \\
21-00-05 \\
21-00-07 \\
21-00-09 \\
21-00-11 \\
21-00-21 \\
21-00-22\end{array}$ & $\begin{array}{l}2-E 33-63 \\
2-E 33-142 \\
2-E 33-140 \\
2-E 33-62 \\
2-E 33-77 \\
2-E 33-92 \\
2-E 33-65 \\
2-E 33-78 \\
2-E 33-93\end{array}$ & $\begin{array}{l}21-27-01 \\
21-27-02 \\
21-27-06 \\
21-27-07 \\
21-27-08 \\
21-27-09 \\
21-27-10 \\
21-27-11\end{array}$ & $\begin{array}{l}2-E 33-141 \\
2-E 33-138 \\
2-E 33-139 \\
2-E 33-134 \\
2-E 33-146 \\
2-E 33-133 \\
2-E 33-132 \\
2-E 33-61\end{array}$ \\
\hline $\begin{array}{l}21-01-01 \\
21-01-02\end{array}$ & $\begin{array}{l}2-E 33-144 \\
2-E 33-135\end{array}$ & & \\
\hline $\begin{array}{l}21-02-01 \\
21-01-03 \\
21-02-06 \\
21-02-07 \\
21-02-11\end{array}$ & $\begin{array}{l}2-E 33-129 \\
2-E 33-145 \\
2-E 33-143 \\
2-E 33-130 \\
2-E 33-101\end{array}$ & & \\
\hline $\begin{array}{l}21-05-02 \\
21-05-03 \\
21-05-06 \\
21-05-10 \\
21-05-12\end{array}$ & $\begin{array}{l}2-E 33-158 \\
2-E 33-159 \\
2-E 33-161 \\
2-E 33-162 \\
2-E 33-157\end{array}$ & & \\
\hline $\begin{array}{l}21-06-01 \\
21-06-02 \\
21-06-05 \\
21-06-10\end{array}$ & $\begin{array}{l}2-E 33-163 \\
2-E 33-164 \\
2-E 33-165 \\
2-E 33-166\end{array}$ & & \\
\hline $\begin{array}{l}21-08-02 \\
21-08-06 \\
21-08-07\end{array}$ & $\begin{array}{l}2-E 33-64 \\
2-E 33-151 \\
2-E 33-152\end{array}$ & & \\
\hline $\begin{array}{l}21-10-01 \\
21-10-05 \\
21-10-07 \\
21-10-11\end{array}$ & $\begin{array}{l}2-E 33-167 \\
2-E 33-168 \\
2-E 33-169 \\
2-E 33-170\end{array}$ & & \\
\hline $\begin{array}{l}21-11-04 \\
21-11-05 \\
21-11-07 \\
21-11-10 \\
21-11-11\end{array}$ & $\begin{array}{l}2-E 33-173 \\
2-E 33-171 \\
2-E 33-172 \\
2-E 33-174 \\
2-E 33-175\end{array}$ & & \\
\hline $\begin{array}{l}21-12-02 \\
21-12-05 \\
21-12-07 \\
21-12-10\end{array}$ & $\begin{array}{l}2-E 33-153 \\
2-E 33-154 \\
2-E 33-155 \\
2-E 33-156\end{array}$ & & \\
\hline
\end{tabular}

D. 3 
241-BY Tank Farm

\begin{tabular}{|c|c|c|c|}
\hline File & Well & File & Well \\
\hline $\begin{array}{l}22-00-01 \\
22-00-03\end{array}$ & $\begin{array}{l}2-E 33-85 \\
2-E 33-84\end{array}$ & $\begin{array}{l}22-10-05 \\
22-10-10\end{array}$ & $\begin{array}{l}2-E 33-124 \\
2-E 33-125\end{array}$ \\
\hline $\begin{array}{l}22-00-05 \\
22-00-10\end{array}$ & $\begin{array}{l}2-E 33-83 \\
2-E 33-88\end{array}$ & $\begin{array}{l}22-11-01 \\
22-11-05 \\
22-11-09\end{array}$ & $\begin{array}{l}2-E 33-126 \\
2-E 33-127 \\
2-E 33-128\end{array}$ \\
\hline $\begin{array}{l}22-01-01 \\
22-01-04 \\
22-01-07\end{array}$ & $\begin{array}{l}2-E 33-176 \\
2-E 33-177 \\
2-E 33-178\end{array}$ & $\begin{array}{l}22-12-01 \\
22-12-03\end{array}$ & $\begin{array}{l}2-E 33-100 \\
2-E 33-94\end{array}$ \\
\hline $\begin{array}{l}22-02-01 \\
22-02-02 \\
22-02-05 \\
22-02-07\end{array}$ & $\begin{array}{l}2-E 33-101 \\
2-E 33-227 \\
2-E 33-228 \\
2-E 33-102\end{array}$ & $\begin{array}{l}22-12-06 \\
22-12-07 \\
22-12-09 \\
22-12-10\end{array}$ & $\begin{array}{l}2-E 33-96 \\
2-E 33-97 \\
2-E 33-98 \\
2-E 33-99\end{array}$ \\
\hline $\begin{array}{l}22-03-01 \\
22-03-04\end{array}$ & $\begin{array}{l}2-E 33-104 \\
2-E 33-211\end{array}$ & $\frac{241-C \text { Tank }}{\text { File }}$ & $\begin{array}{l}\text { Farm } \\
\text { Well }\end{array}$ \\
\hline $\begin{array}{l}22-03-05 \\
22-03-06 \\
22-03-09\end{array}$ & $\begin{array}{l}2-E S 3-103 \\
2-E 33-210 \\
2-E 33-105\end{array}$ & $\begin{array}{l}30-00-01 \\
30-00-03\end{array}$ & $\begin{array}{l}2-E 27-56 \\
2-E 27-54\end{array}$ \\
\hline $\begin{array}{l}22-04-01 \\
22-04-05 \\
22-04-09\end{array}$ & $\begin{array}{l}2-E 33-106 \\
2-E 33-107 \\
2-E 33-108\end{array}$ & $\begin{array}{l}30-00-10 \\
30-00-12\end{array}$ & $\begin{array}{l}2-E 27-53 \\
2-E 27-52\end{array}$ \\
\hline $\begin{array}{l}22-05-01 \\
22-05-05 \\
22-05-09\end{array}$ & $\begin{array}{l}2-E 33-109 \\
2-E 33-110 \\
2-E 33-111\end{array}$ & $\begin{array}{l}30-01-01 \\
30-01-06 \\
30-01-09 \\
30-01-12\end{array}$ & $\begin{array}{l}2-E 27-60 \\
2-E 27-59 \\
2-E 27-58 \\
2-E 27-61\end{array}$ \\
\hline $\begin{array}{l}22-06-01 \\
22-06-05 \\
22-06-07 \\
22-06-09\end{array}$ & $\begin{array}{l}2-E 33-112 \\
2-E 33-113 \\
2-E 33-86 \\
2-E 33-114\end{array}$ & $\begin{array}{l}30-04-02 \\
30-04-08 \\
30-04-12\end{array}$ & $\begin{array}{l}2-E 27-67 \\
2-E 27-66 \\
2-E 27-65\end{array}$ \\
\hline $\begin{array}{l}22-07-01 \\
22-07-02 \\
22-07-05\end{array}$ & $\begin{array}{l}2-E 33-115 \\
2-E 33-206 \\
2-E 33-116\end{array}$ & $\begin{array}{l}30-05-02 \\
30-05-04 \\
30-05-10\end{array}$ & $\begin{array}{l}2-E 27-70 \\
2-E 27-69 \\
2-E 27-68\end{array}$ \\
\hline $22-07-09$ & 2-E33-117 & $\begin{array}{l}30-06-02 \\
30-06-04\end{array}$ & $\begin{array}{l}2-E 27-72 \\
2-E 27-73\end{array}$ \\
\hline $\begin{array}{l}22-08-01 \\
22-08-02\end{array}$ & $\begin{array}{l}2-E 33-118 \\
2-E 33-208\end{array}$ & $30-06-10$ & $2-W 27-71$ \\
\hline $\begin{array}{l}22-08-05 \\
22-08-06\end{array}$ & $\begin{array}{l}2-E 33-119 \\
2-E 33-207\end{array}$ & $30-08-03$ & $2-E 27-51$ \\
\hline $\begin{array}{l}22-08-07 \\
22-08-09 \\
22-08-12\end{array}$ & $\begin{array}{l}2-E 33-87 \\
2-E 33-120 \\
2-E 33-209\end{array}$ & $\begin{array}{l}30-11-10 \\
30-11-06 \\
30-11-09\end{array}$ & $\begin{array}{l}2-E 27-63 \\
2-E 27-64 \\
2-E 27-62\end{array}$ \\
\hline $\begin{array}{l}22-09-01 \\
22-09-05 \\
22-09-08\end{array}$ & $\begin{array}{l}2-E 33-123 \\
2-E 33-122 \\
2-E 33-121\end{array}$ & & \\
\hline
\end{tabular}


241-S Tank Farm

\begin{tabular}{|c|c|c|c|}
\hline File & Well & File & Well \\
\hline $\begin{array}{l}40-00-02 \\
40-00-04 \\
40-00-06\end{array}$ & $\begin{array}{l}2-W 23-51 \\
2-W 23-1 \\
2-W 23-56\end{array}$ & $\begin{array}{l}40-07-01 \\
40-07-04 \\
40-07-06 \\
40-07-08\end{array}$ & $\begin{array}{l}2-W 23-160 \\
2-W 23-185 \\
2-W 23-161 \\
2-W 23-186\end{array}$ \\
\hline $\begin{array}{l}40-01-01 \\
40-01-04 \\
40-01-06\end{array}$ & $\begin{array}{l}2-W 23-145 \\
2-W 23-179 \\
2-W 23-146\end{array}$ & $\begin{array}{l}40-07-10 \\
40-07-11\end{array}$ & $\begin{array}{l}2-W 23-162 \\
2-W 23-187\end{array}$ \\
\hline $\begin{array}{l}40-01-08 \\
40-01-10\end{array}$ & $\begin{array}{l}2-w 23-147 \\
2-W 23-180\end{array}$ & $\begin{array}{l}40-08-06 \\
40-08-08\end{array}$ & $\begin{array}{l}\text { 2-W23-163 } \\
\text { 2-W23-202 }\end{array}$ \\
\hline $\begin{array}{l}40-02-01 \\
40-02-02\end{array}$ & $\begin{array}{l}2-W 23-148 \\
2-W 23-188\end{array}$ & $40-08-12$ & $2-W 23-216$ \\
\hline $\begin{array}{l}40-02-03 \\
40-02-05 \\
40-02-07 \\
40-02-08 \\
40-02-10 \\
40-02-11\end{array}$ & $\begin{array}{l}2-W 23-52 \\
2-W 23-181 \\
2-W 23-149 \\
2-W 23-189 \\
2-W 23-150 \\
2-W 23-182\end{array}$ & $\begin{array}{l}40-09-02 \\
40-09-05 \\
40-09-06 \\
40-09-08 \\
40-09-09\end{array}$ & $\begin{array}{l}2-W 23-165 \\
2-W 23-57 \\
2-W 23-166 \\
2-W 23-203 \\
2-W 23-167\end{array}$ \\
\hline $\begin{array}{l}40-03-01 \\
40-03-03 \\
40-03-05 \\
40-03-06 \\
40-03-08 \\
40-03-09 \\
40-03-11\end{array}$ & $\begin{array}{l}2-W 23-151 \\
2-W 23-212 \\
2-W 23-53 \\
2-W 23-152 \\
2-W 23-183 \\
2-W 23-153 \\
2-W 23-184\end{array}$ & $\begin{array}{l}40-10-01 \\
40-10-03 \\
40-10-05 \\
40-10-06 \\
40-10-08 \\
40-10-09 \\
40-10-13\end{array}$ & $\begin{array}{l}2-W 23-12 \\
2-W 23-168 \\
2-W 23-204 \\
2-W 23-169 \\
2-W 23-205 \\
2-W 23-170 \\
2-W 23-218\end{array}$ \\
\hline $\begin{array}{l}40-04-01 \\
40-04-05 \\
40-04-07 \\
40-04-08\end{array}$ & $\begin{array}{l}2-W 23-123 \\
2-W 23-124 \\
2-W 23-122 \\
2-W 23-177\end{array}$ & $\begin{array}{l}40-11-01 \\
40-11-05 \\
40-11-06 \\
40-11-08 \\
40-11-09\end{array}$ & $\begin{array}{l}2-W 23-171 \\
2-W 23-206 \\
2-W 23-172 \\
2-W 23-207 \\
2-W 23-173\end{array}$ \\
\hline $\begin{array}{l}40-05-03 \\
40-05-05 \\
40-05-05 \\
40-05-07 \\
40-05-08 \\
40-05-10\end{array}$ & $\begin{array}{l}2-W 23-154 \\
2-W 23-54 \\
2-W 23-178 \\
2-W 23-155 \\
2-W 23-199 \\
2-W 23-156\end{array}$ & $\begin{array}{l}40-12-02 \\
40-12-04 \\
40-12-06 \\
40-12-07 \\
40-12-09\end{array}$ & $\begin{array}{l}2-W 23-174 \\
2-W 23-220 \\
2-W 23-175 \\
2-W 23-208 \\
2-W 23-176\end{array}$ \\
\hline $\begin{array}{l}40-06-02 \\
40-06-04 \\
40-06-05 \\
40-06-05 \\
40-06-06 \\
40-06-08 \\
40-06-09\end{array}$ & $\begin{array}{l}2-W 23-157 \\
2-W 23-213 \\
2-W 23-55 \\
2-W 23-200 \\
2-W 23-158 \\
2-W 23-201 \\
2-W 23-159\end{array}$ & & \\
\hline
\end{tabular}

D.5 
241-SX Tank Farm

\begin{tabular}{|c|c|c|c|}
\hline File & Well & File & Well \\
\hline $\begin{array}{l}41-00-02 \\
41-00-03 \\
41-00-04 \\
41-00-05 \\
41-00-08\end{array}$ & $\begin{array}{l}2-W 23-61 \\
2-W 23-2 \\
2-W 23-5 \\
2-W 23-3 \\
2-W 23-65\end{array}$ & $\begin{array}{l}41-07-02 \\
41-07-03 \\
41-07-05 \\
41-07-07 \\
41-07-08 \\
41-07-10\end{array}$ & $\begin{array}{l}2-W 23-74 \\
2-W 23-75 \\
2-W 23-76 \\
2-W 23-77 \\
2-W 23-78 \\
2-W 23-79\end{array}$ \\
\hline $\begin{array}{l}41-01-01 \\
41-01-04\end{array}$ & $\begin{array}{l}2-W 23-132 \\
2-W 23-190\end{array}$ & $41-07-12$ & $2-W 23-73$ \\
\hline $\begin{array}{l}41-01-06 \\
41-01-07 \\
41-01-08 \\
41-01-10 \\
41-01-11\end{array}$ & $\begin{array}{l}2-W 23-133 \\
2-W 23-60 \\
2-W 23-134 \\
2-W 23-191 \\
2-W 23-192\end{array}$ & $\begin{array}{l}41-08-02 \\
41-08-03 \\
41-08-04 \\
41-08-06 \\
41-08-07 \\
41-08-11\end{array}$ & $\begin{array}{l}2-W 23-102 \\
2-W 23-103 \\
2-W 23-98 \\
2-W 23-99 \\
2-W 23-100 \\
2-W 23-101\end{array}$ \\
\hline $\begin{array}{l}41-02-02 \\
41-02-05 \\
41-02-07 \\
41-02-08 \\
41-02-11\end{array}$ & $\begin{array}{l}2-W 23-135 \\
2-W 23-223 \\
2-W 23-59 \\
2-W 23-136 \\
2-W 23-193\end{array}$ & $\begin{array}{l}41-09-02 \\
41-09-03 \\
41-09-04 \\
41-09-06 \\
41-09-07\end{array}$ & $\begin{array}{l}2-W 23-110 \\
2-W 23-104 \\
2-W 23-105 \\
2-W 23-106 \\
2-W 23-107\end{array}$ \\
\hline $\begin{array}{l}41-03-02 \\
41-03-05 \\
41-03-06\end{array}$ & $\begin{array}{l}2-W 23-194 \\
2-W 23-195 \\
2-W 23-138\end{array}$ & $\begin{array}{l}41-09-09 \\
41-09-11\end{array}$ & $\begin{array}{l}2-W 23-108 \\
2-W 23-109\end{array}$ \\
\hline $\begin{array}{l}41-03-09 \\
41-03-10 \\
41-03-12\end{array}$ & $\begin{array}{l}2-W 23-139 \\
2-W 23-196 \\
2-W 23-137\end{array}$ & $\begin{array}{l}41-10-01 \\
41-10-02 \\
41-10-03 \\
41-10-05\end{array}$ & $\begin{array}{l}2-W 23-80 \\
2-W 23-66 \\
2-W 23-81 \\
2-W 23-82\end{array}$ \\
\hline $\begin{array}{l}41-04-01 \\
41-04-03 \\
41-04-05 \\
41-04-07 \\
41-04-08\end{array}$ & $\begin{array}{l}2-W 23-140 \\
2-W 23-197 \\
2-W 23-198 \\
2-W 23-62 \\
2-W 23-225\end{array}$ & $\begin{array}{l}41-10-06 \\
41-10-08 \\
41-10-10 \\
41-10-11\end{array}$ & $\begin{array}{l}2-W 23-83 \\
2-W 23-84 \\
2-W 23-67 \\
2-W 23-85\end{array}$ \\
\hline $41-04-11$ & $2-W 23-141$ & $\begin{array}{l}41-11-02 \\
41-11-03\end{array}$ & $\begin{array}{l}2-W 23-96 \\
2-W 23-97\end{array}$ \\
\hline $\begin{array}{l}41-05-02 \\
41-05-03 \\
41-05-05 \\
41-05-07 \\
41-05-08 \\
41-05-10\end{array}$ & $\begin{array}{l}2-W 23-130 \\
2-W 23-131 \\
2-W 23-125 \\
2-W 23-125 \\
2-W 23-127 \\
2-W 23-128\end{array}$ & $\begin{array}{l}41-11-05 \\
41-11-06 \\
41-11-08 \\
41-11-09 \\
41-11-10\end{array}$ & $\begin{array}{l}2-W 23-92 \\
2-W 23-93 \\
2-W 23-94 \\
2-W 23-95 \\
2-W 23-64\end{array}$ \\
\hline $41-05-12$ & 2-W23-129 & $\begin{array}{l}41-12-02 \\
41-12-03\end{array}$ & $\begin{array}{l}2-W 23-111 \\
2-W 23-112\end{array}$ \\
\hline $\begin{array}{l}41-06-02 \\
41-06-05 \\
41-06-06 \\
41-06-09 \\
41-06-11 \\
41-06-23\end{array}$ & $\begin{array}{l}2-W 23-142 \\
2-W 23-143 \\
2-W 23-226 \\
2-W 23-144 \\
2-W 23-58 \\
2-W 23-227\end{array}$ & $\begin{array}{l}41-12-04 \\
41-12-06 \\
41-12-07 \\
41-12-09 \\
41-12-10\end{array}$ & $\begin{array}{l}2-W 23-68 \\
2-W 23-113 \\
2-W 23-114 \\
2-W 23-115 \\
2-W 23-116\end{array}$ \\
\hline
\end{tabular}

0.6 
241-SX Tank Farm (contd)

\begin{tabular}{|c|c|c|c|}
\hline File & WeIT & File & Well \\
\hline $41-13-10$ & $2-W 23-72$ & $\begin{array}{l}41-15-02 \\
41-15-03\end{array}$ & $\begin{array}{l}2-w 23-117 \\
2-W 23-118\end{array}$ \\
\hline $\begin{array}{l}41-14-02 \\
41-14-03 \\
41-14-04 \\
41-14-06 \\
41-14-08 \\
41-14-09 \\
41-14-11\end{array}$ & $\begin{array}{l}2-W 23-91 \\
2-W 23-86 \\
2-W 23-69 \\
2-W 23-87 \\
2-W 23-88 \\
2-W 23-89 \\
2-W 23-90\end{array}$ & $\begin{array}{l}41-15-05 \\
41-15-07 \\
41-15-09 \\
41-15-10\end{array}$ & $\begin{array}{l}2-W 23-119 \\
2-W 23-70 \\
2-23-120 \\
2-W 23-12\end{array}$ \\
\hline
\end{tabular}

0.7 
241-T Tank Farm

\begin{tabular}{|c|c|c|c|}
\hline File & Well & File & Well \\
\hline $\begin{array}{l}50-00-01 \\
50-00-02 \\
50-00-03 \\
50-00-05\end{array}$ & $\begin{array}{l}2-W 10-8 \\
2-W 11-23 \\
2-W 11-51 \\
2-W 11-53\end{array}$ & $\begin{array}{l}50-07-03 \\
50-07-07 \\
50-07-08\end{array}$ & $\begin{array}{l}2-w 10-149 \\
2-w 10-142 \\
2-w 10-150\end{array}$ \\
\hline $\begin{array}{l}50-00-06 \\
50-00-08 \\
50-00-10 \\
50-00-12\end{array}$ & $\begin{array}{l}2-W 10-55 \\
2-W 10-52 \\
2-W 10-54 \\
2-W 10-53\end{array}$ & $\begin{array}{l}50-08-05 \\
50-08-07 \\
50-08-08 \\
50-08-09 \\
50-08-19\end{array}$ & $\begin{array}{l}2-W 10-143 \\
2-W 10-133 \\
2-w 10-176 \\
2-W 10-112 \\
2-w 10-178\end{array}$ \\
\hline $\begin{array}{l}50-01-02 \\
50-01-06 \\
50-01-09 \\
50-01-12\end{array}$ & $\begin{array}{l}2-W 10-101 \\
2-W 10-103 \\
2-W 10-104 \\
2-W 10-105\end{array}$ & $\begin{array}{l}50-09-01 \\
50-09-02 \\
50-09-05\end{array}$ & $\begin{array}{l}2-W 10-164 \\
2-W 10-166 \\
2-W 10-134\end{array}$ \\
\hline $\begin{array}{l}50-02-02 \\
50-02-05 \\
50-02-08\end{array}$ & $\begin{array}{l}2-W 10-122 \\
2-W 10-123 \\
2-W 10-116\end{array}$ & $50-09-09$ & $\begin{array}{l}2-W 10-120 \\
2-W 10-114\end{array}$ \\
\hline $\begin{array}{l}50-02-09 \\
50-02-10 \\
50-02-12\end{array}$ & $\begin{array}{l}2-W 10-119 \\
2-W 10-124 \\
2-w 10-125\end{array}$ & $\begin{array}{l}50-10-05 \\
50-10-07 \\
50-10-08 \\
50-10-10\end{array}$ & $\begin{array}{l}2-w 10-135 \\
2-W 10-136 \\
2-w 10-151 \\
2-W 10-137\end{array}$ \\
\hline $\begin{array}{l}50-03-01 \\
50-03-04 \\
50-03-05 \\
50-03-06 \\
50-03-08 \\
50-03-10\end{array}$ & $\begin{array}{l}2-W 10-126 \\
2-W 10-118 \\
2-W 10-117 \\
2-W 10-145 \\
2-W 10-127 \\
2-W 10-128\end{array}$ & $\begin{array}{l}50-11-05 \\
50-11-07 \\
50-11-08 \\
50-11-10 \\
50-11-11\end{array}$ & $\begin{array}{l}2-W 10-138 \\
2-W 10-152 \\
2-W 10-139 \\
2-W 10-153 \\
2-W 10-177\end{array}$ \\
\hline $\begin{array}{l}50-04-03 \\
50-04-05 \\
50-04-07 \\
50-04-08 \\
50-04-10\end{array}$ & $\begin{array}{l}2-W 10-146 \\
2-W 10-129 \\
2-W 10-147 \\
2-W 10-148 \\
2-W 10-130\end{array}$ & $\begin{array}{l}50-12-05 \\
50-12-07 \\
50-12-10\end{array}$ & $\begin{array}{l}2-W 10-154 \\
2-W 10-140 \\
2-W 10-141\end{array}$ \\
\hline $\begin{array}{l}50-05-06 \\
50-05-07 \\
50-05-11\end{array}$ & $\begin{array}{l}2-W 10-113 \\
2-w 10-165 \\
2-W 10-121\end{array}$ & & \\
\hline $\begin{array}{l}50-06-02 \\
50-06-03 \\
50-06-04 \\
50-06-05 \\
50-06-06 \\
50-06-08 \\
50-06-11 \\
50-06-16 \\
50-06-17\end{array}$ & $\begin{array}{l}2-w 10-108 \\
2-w 10-107 \\
2-W 10-110 \\
2-W 10-111 \\
2-W 10-106 \\
2-W 10-109 \\
2-W 10-115 \\
2-W 10-167 \\
2-W 10-162\end{array}$ & & \\
\hline
\end{tabular}

D.8 
241-TX Tank Farm

\begin{tabular}{|c|c|c|c|}
\hline File & Well & File & Well \\
\hline $\begin{array}{l}51-00-03 \\
51-00-05 \\
51-00-06 \\
51-00-07 \\
51-00-09 \\
51-00-10\end{array}$ & $\begin{array}{l}2-W 15-67 \\
2-W 15-70 \\
2-W 15-69 \\
2-W 15-73 \\
2-W 15-74 \\
2-W 15-75\end{array}$ & $\begin{array}{l}51-07-11 \\
51-07-18 \\
51-08-05 \\
51-08-09 \\
51-08-11\end{array}$ & $\begin{array}{l}2-W 15-132 \\
2-W 15-195 \\
2-W 15-136 \\
2-W 15-137 \\
2-W 15-135\end{array}$ \\
\hline $\begin{array}{l}51-01-02 \\
51-01-04 \\
51-01-06 \\
51-01-08 \\
51-01-09\end{array}$ & $\begin{array}{l}2-W 15-166 \\
2-W 15-167 \\
2-W 15-168 \\
2-W 15-169 \\
2-W 15-155\end{array}$ & $\begin{array}{l}51-09-03 \\
51-09-04 \\
51-09-08 \\
51-09-10 \\
51-09-12\end{array}$ & $\begin{array}{l}2-W 15-139 \\
2-W 15-188 \\
2-W 15-140 \\
2-W 15-189 \\
2-W 15-138\end{array}$ \\
\hline $\begin{array}{l}51-02-02 \\
51-02-05 \\
51-02-07 \\
51-02-09 \\
51-02-12\end{array}$ & $\begin{array}{l}2-W 15-170 \\
2-W 15-143 \\
2-W 15-172 \\
2-W 15-142 \\
2-W 15-141\end{array}$ & $\begin{array}{l}51-10-01 \\
51-10-04 \\
51-10-08 \\
51-10-12 \\
51-10-13 \\
51-10-25\end{array}$ & $\begin{array}{l}2-W 15-176 \\
2-W 15-103 \\
2-W 15-104 \\
2-W 15-105 \\
2-W 15-196 \\
2-W 15-197\end{array}$ \\
\hline $\begin{array}{l}51-03-01 \\
51-03-02 \\
51-03-06 \\
51-03-09 \\
51-03-11\end{array}$ & $\begin{array}{l}2-W 15-192 \\
2-W 15-71 \\
2-W 15-127 \\
2-W 15-128 \\
2-W 15-191\end{array}$ & $\begin{array}{l}51-11-01 \\
51-11-02 \\
51-11-03 \\
51-11-07 \\
51-11-10\end{array}$ & $\begin{array}{l}2-W 15-177 \\
2-W 15-72 \\
2-W 15-106 \\
2-W 15-107 \\
2-W 15-108\end{array}$ \\
\hline $\begin{array}{l}51-04-02 \\
51-04-05 \\
51-04-06 \\
51-04-08 \\
51-04-10 \\
51-04-12\end{array}$ & $\begin{array}{l}2-W 15-153 \\
2-W 15-130 \\
2-W 15-154 \\
2-W 15-131 \\
2-W 15-156 \\
2-W 15-129\end{array}$ & $\begin{array}{l}51-12-01 \\
51-12-04 \\
51-12-05 \\
51-12-07 \\
51-12-10 \\
51-12-11\end{array}$ & $\begin{array}{l}2-W 15-159 \\
2-W 15-109 \\
2-W 15-179 \\
2-W 15-110 \\
2-W 15-180 \\
2-W 15-111\end{array}$ \\
\hline $\begin{array}{l}51-05-01 \\
51-05-03 \\
51-05-05 \\
51-05-07 \\
51-05-08 \\
51-05-10\end{array}$ & $\begin{array}{l}2-W 15-173 \\
2-W 15-174 \\
2-W 15-145 \\
2-W 15-171 \\
2-W 15-146 \\
2-W 15-144\end{array}$ & $\begin{array}{l}51-13-05 \\
51-13-08 \\
51-13-12 \\
51-14-04\end{array}$ & $\begin{array}{l}2-W 15-112 \\
2-W 15-113 \\
2-W 15-114 \\
2-W 15-115\end{array}$ \\
\hline $\begin{array}{l}51-06-02 \\
51-06-04 \\
51-06-08 \\
51-06-10 \\
51-06-12\end{array}$ & $\begin{array}{l}2-W 15-148 \\
2-W 15-149 \\
2-W 15-175 \\
2-W 15-147 \\
2-W 15-147\end{array}$ & $\begin{array}{l}51-14-11 \\
51-15-04 \\
51-15-07 \\
51-15-09 \\
51-15-11\end{array}$ & $\begin{array}{l}2-W 15-118 \\
2-W 15-119 \\
2-W 15-160 \\
2-W 15-120\end{array}$ \\
\hline $\begin{array}{l}51-07-01 \\
51-07-03 \\
51-07-04 \\
51-07-06\end{array}$ & $\begin{array}{l}2-W 15-178 \\
2-W 15-187 \\
2-W 15-133 \\
2-W 15-193\end{array}$ & $\begin{array}{l}51-16-04 \\
51-16-07 \\
51-16-11\end{array}$ & $\begin{array}{l}2-W 15-161 \\
2-W 15-163 \\
2-W 15-162\end{array}$ \\
\hline
\end{tabular}

0.9 
241-TX Tank Farm (contd)

\begin{tabular}{c}
\hline File \\
\hline $51-07-07$ \\
$51-07-09$ \\
$51-17-02$ \\
$51-17-03$ \\
$51-17-10$ \\
$51-17-11$ \\
$51-18-01$ \\
$51-18-03$ \\
$51-18-05$ \\
$51-18-07$ \\
$51-18-09$ \\
$51-18-10$ \\
$51-18-11$
\end{tabular}

well

2-W15-190

2-W15-134

2-W15-121

2-W15-68

2-W15-122

2-W15-164

2-W15-181

2-W15-123

2-W15-182

2-W15-124

2-W15-183

2-W15-125

2-W15-165
241-TY Tank Farm

\begin{tabular}{|c|c|}
\hline File & Well \\
\hline $\begin{array}{l}52-01-01 \\
52-01-05 \\
52-01-09\end{array}$ & $\begin{array}{l}2-W 10-88 \\
2-W 10-89 \\
2-W 10-90\end{array}$ \\
\hline $\begin{array}{l}52-02-01 \\
52-02-05 \\
52-02-09\end{array}$ & $\begin{array}{l}2-W 10-91 \\
2-W 10-92 \\
2-W 10-93\end{array}$ \\
\hline
\end{tabular}

52-03-03

52-03-06

52-03-12

52-04-02

52-04-03

52-04-06

52-04-09

52-04-10

52-05-07

52-06-02

52-06-04

52-06-05

52-06-06

52-06-07
2-W10-95

2-E10-96

2-W10-94

2-W10-97

$2-W 10-83$

$2-W 10-98$

2-W10-99

2-W10-82

2-W15-186

2-W10-100

2-W15-185

$2-W 15-79$

2-W15-184

2-W15-3 
241-U Tank Farm

\begin{tabular}{|c|c|c|c|}
\hline File & Well & File & Welा \\
\hline $\begin{array}{l}60-00-02 \\
60-00-05 \\
60-00-06 \\
60-00-08\end{array}$ & $\begin{array}{l}2-W 19-54 \\
2-W 19-53 \\
2-W 18-51 \\
2-W 18-55\end{array}$ & $\begin{array}{l}60-09-01 \\
60-09-07 \\
60-09-08 \\
60-09-10\end{array}$ & $\begin{array}{l}2-W 18-120 \\
2-W 18-121 \\
2-W 18-122 \\
2-W 18-123\end{array}$ \\
\hline $60-00-11$ & $2-W 18-52$ & $\begin{array}{l}60-10-01 \\
60-10-02\end{array}$ & $\begin{array}{l}2-W 18-100 \\
2-W 19-75\end{array}$ \\
\hline $\begin{array}{l}60-01-08 \\
60-01-10\end{array}$ & $\begin{array}{l}2-W 18-135 \\
2-W 18-136\end{array}$ & $\begin{array}{l}60-10-05 \\
60-10-07\end{array}$ & $\begin{array}{l}2-W 18-104 \\
2-W 18-148\end{array}$ \\
\hline $\begin{array}{l}60-02-01 \\
60-02-05 \\
60-02-06 \\
60-02-07 \\
60-02-10 \\
60-02-11\end{array}$ & $\begin{array}{l}2-W 18-137 \\
2-W 18-138 \\
2-W 18-140 \\
2-W 18-139 \\
2-W 18-141 \\
2-W 18-142\end{array}$ & $\begin{array}{l}60-11-02 \\
60-11-03 \\
60-11-05 \\
60-11-06 \\
60-11-07 \\
60-11-12\end{array}$ & $\begin{array}{l}2-W 18-105 \\
2-W 18-102 \\
2-W 18-109 \\
2-W 18-101 \\
2-W 18-110 \\
2-W 18-105\end{array}$ \\
\hline $\begin{array}{l}60-03-01 \\
60-03-05 \\
60-03-08 \\
60-03-10 \\
60-03-11\end{array}$ & $\begin{array}{l}2-W 18-143 \\
2-W 18-144 \\
2-W 18-145 \\
2-W 18-146 \\
2-W 18-147\end{array}$ & $\begin{array}{l}60-12-01 \\
60-12-03 \\
60-12-05 \\
60-12-07 \\
60-12-10 \\
60-12-12\end{array}$ & $\begin{array}{l}2-W 18-113 \\
2-W 18-103 \\
2-W 18-92 \\
2-W 18-90 \\
2-W 18-91 \\
2-W 18-105\end{array}$ \\
\hline $\begin{array}{l}60-04-03 \\
60-04-08 \\
60-04-10 \\
60-04-12\end{array}$ & $\begin{array}{l}2-W 19-76 \\
2-W 18-124 \\
2-W 18-125 \\
2-W 18-126\end{array}$ & & \\
\hline $\begin{array}{l}60-05-04 \\
60-05-05 \\
60-05-07 \\
60-05-08 \\
60-05-10\end{array}$ & $\begin{array}{l}2-W 18-176 \\
2-W 18-127 \\
2-W 18-128 \\
2-W 18-129 \\
2-18-130\end{array}$ & & \\
\hline $\begin{array}{l}60-06-07 \\
60-06-08 \\
60-06-10\end{array}$ & $\begin{array}{l}2-W 18-131 \\
2-W 18-132 \\
2-W 18-133\end{array}$ & & \\
\hline $\begin{array}{l}60-07-01 \\
60-07-02 \\
60-07-10 \\
60-07-11\end{array}$ & $\begin{array}{l}2-W 18-114 \\
2-19-74 \\
2-W 18-116 \\
2-W 18-117\end{array}$ & & \\
\hline $\begin{array}{l}60-08-08 \\
60-08-10 \\
60-08-11\end{array}$ & $\begin{array}{l}2-W 18-118 \\
2-W 18-119 \\
2-W 18-54\end{array}$ & & \\
\hline
\end{tabular}


APPENDIX E

WELL LIST 



\section{APPENDIX E}

\section{WELL LIST}

This appendix contains a listing of all existing Hanford wells and others for which data are recorded. A description by column of the data listed can be found in the body of this report beginning on page 9 .

\footnotetext{
E. $1 / \bar{E} \cdot 2$
} 
blank

DO NOT MICROFIIM
THIS PAGE

an 


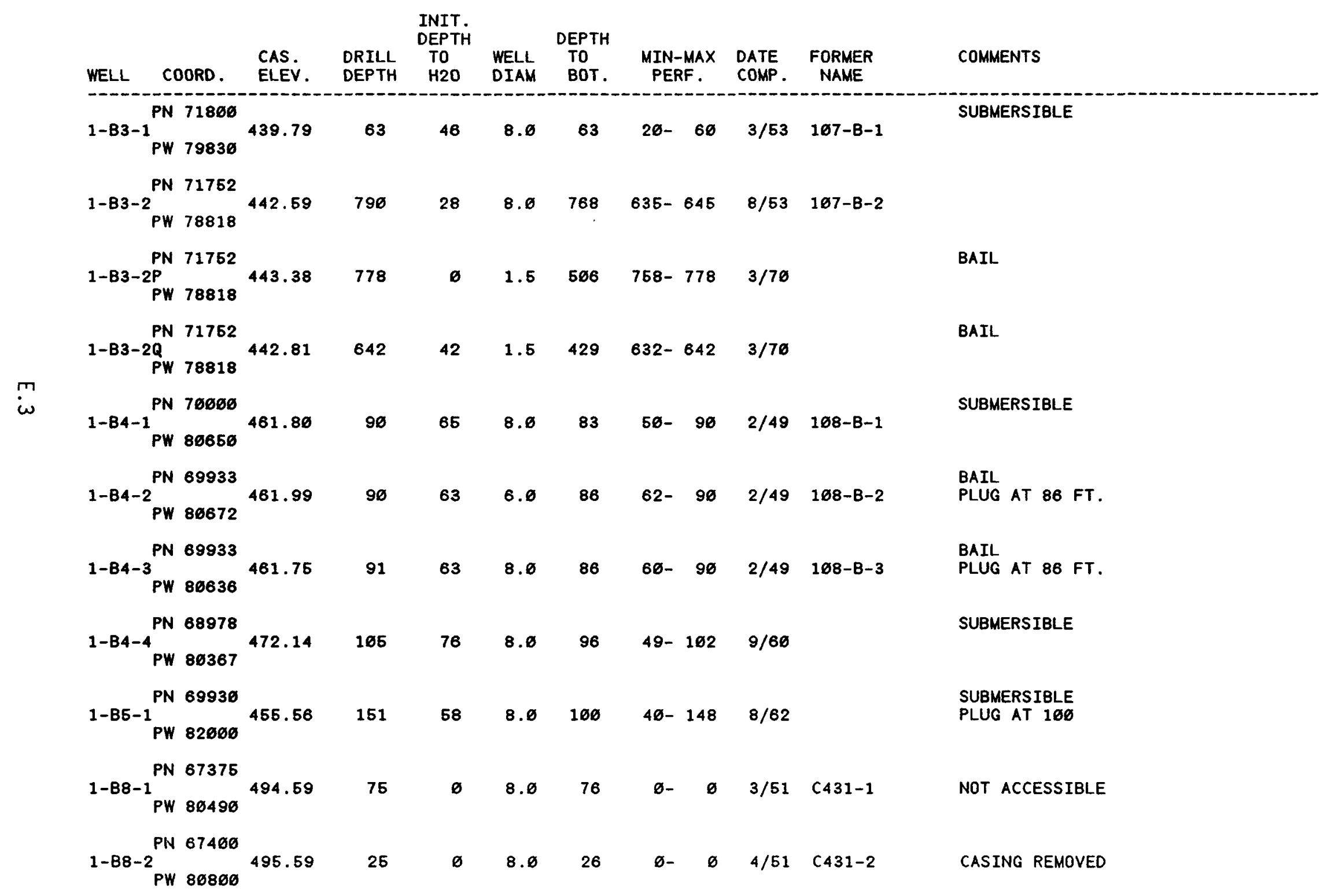




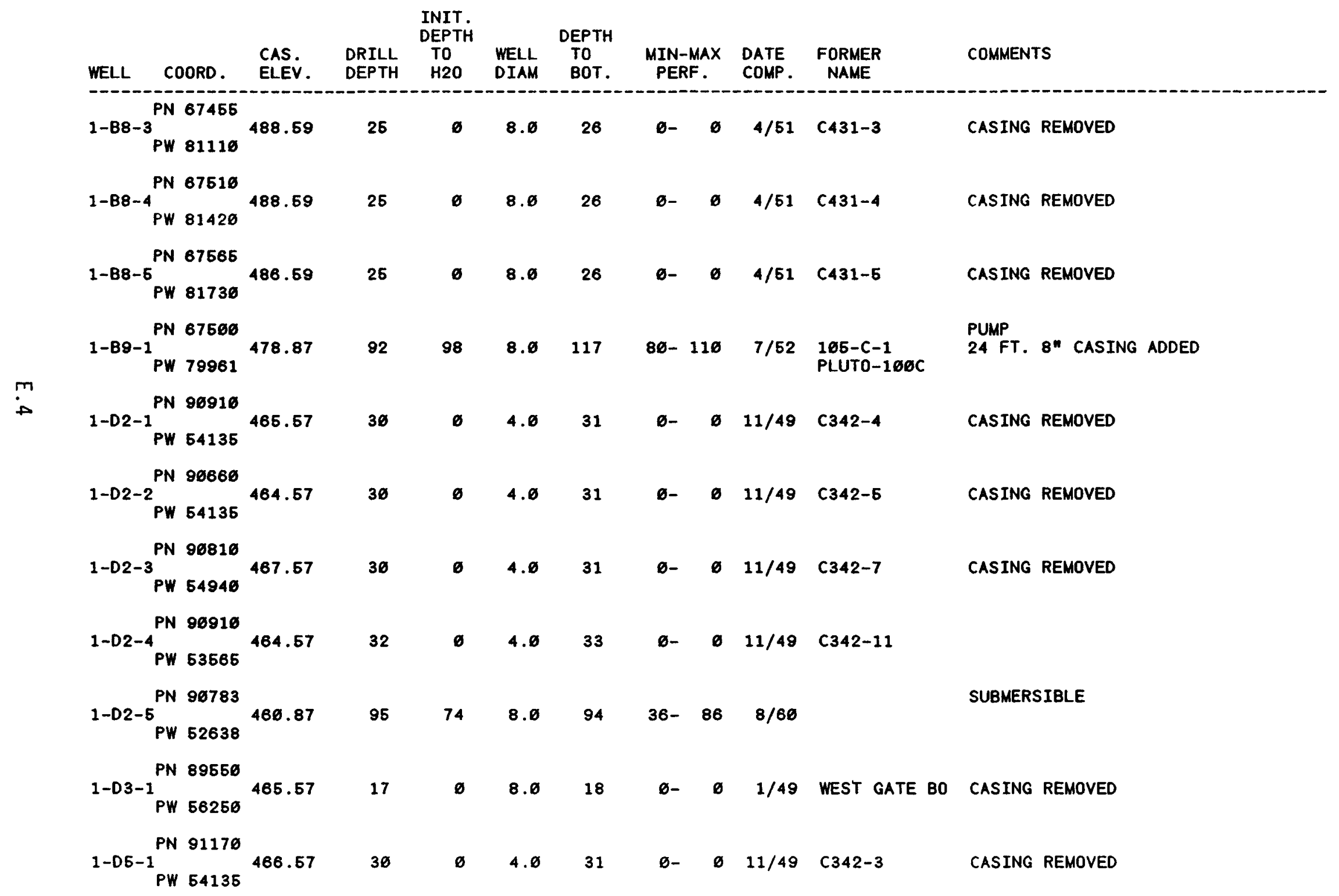




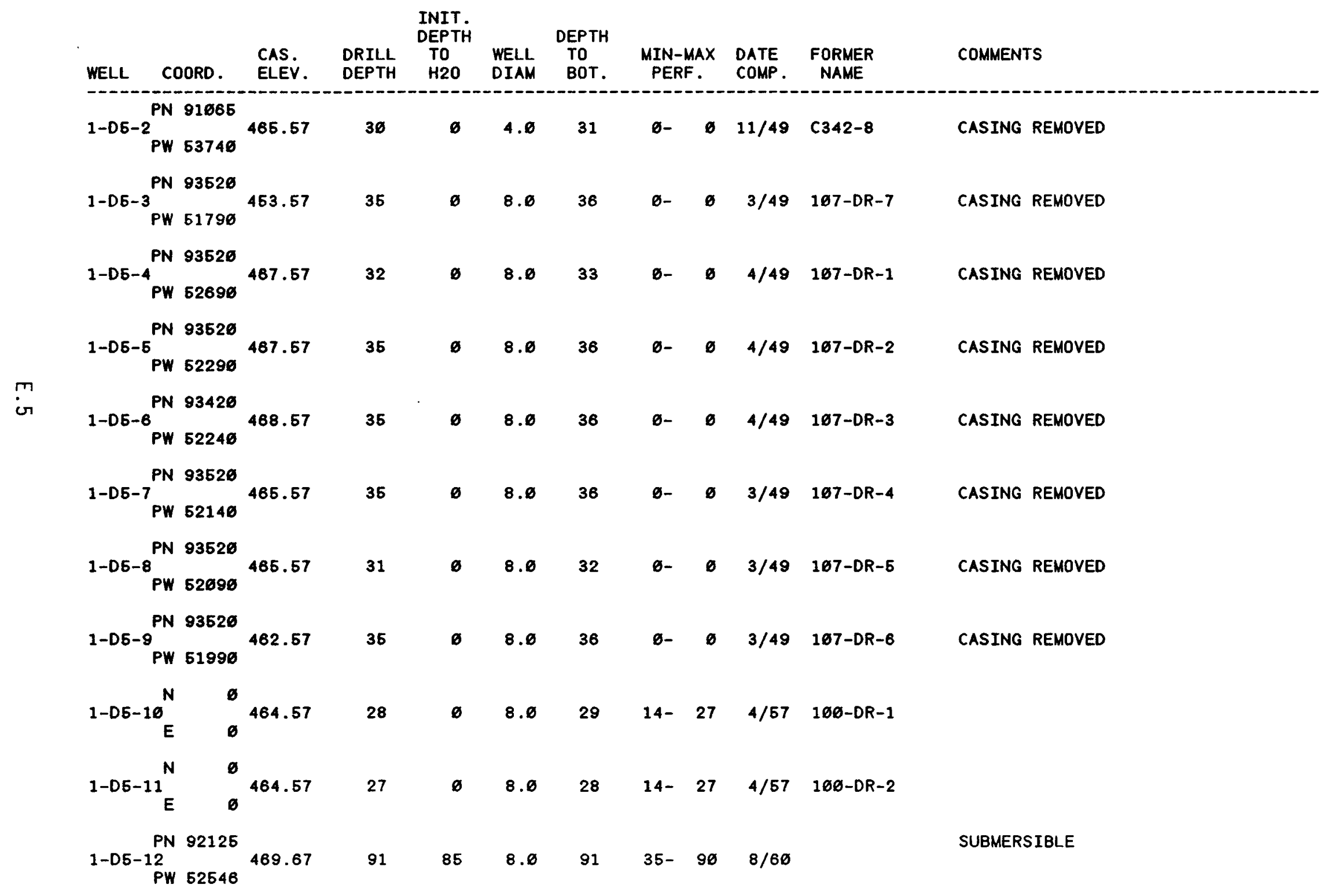




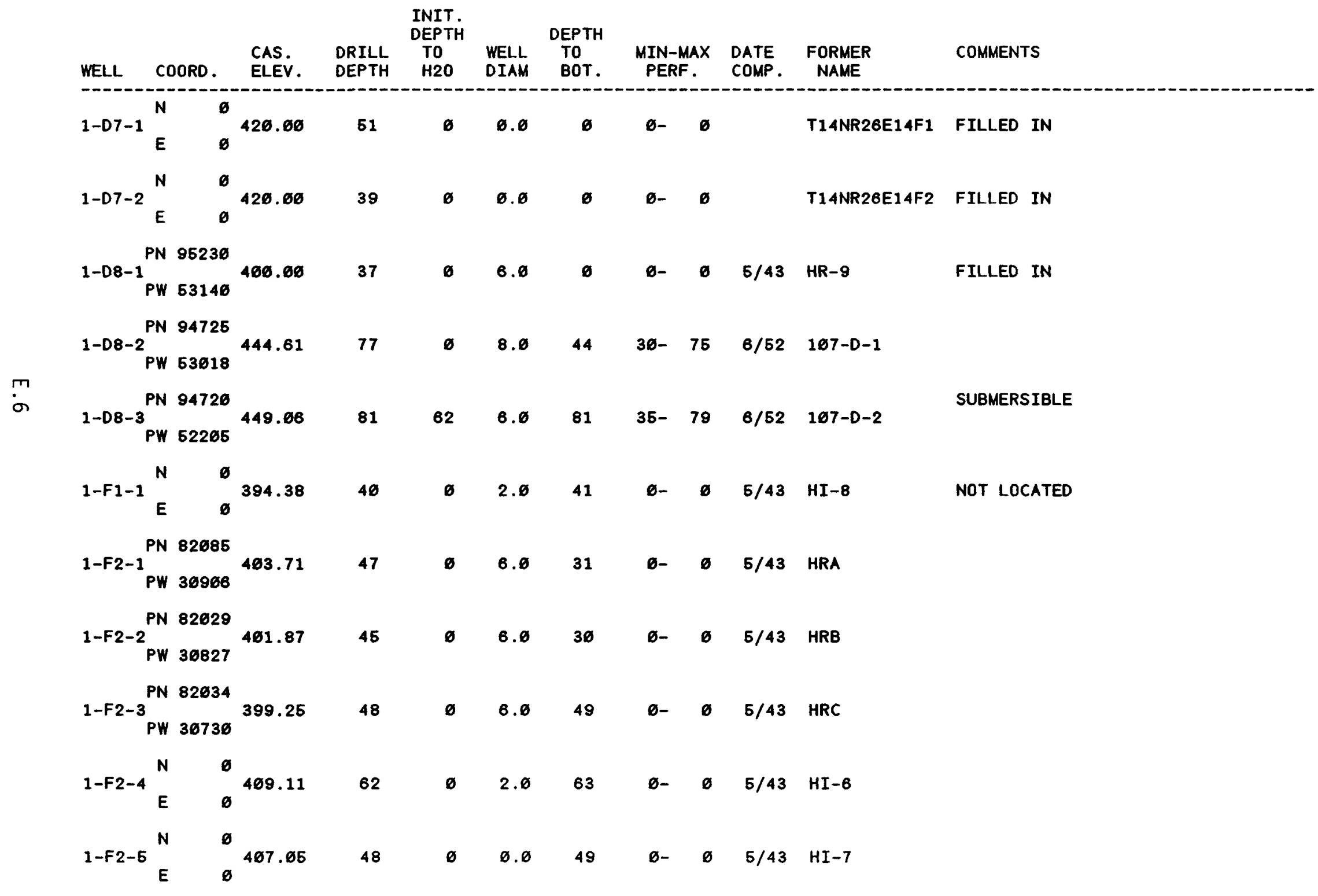




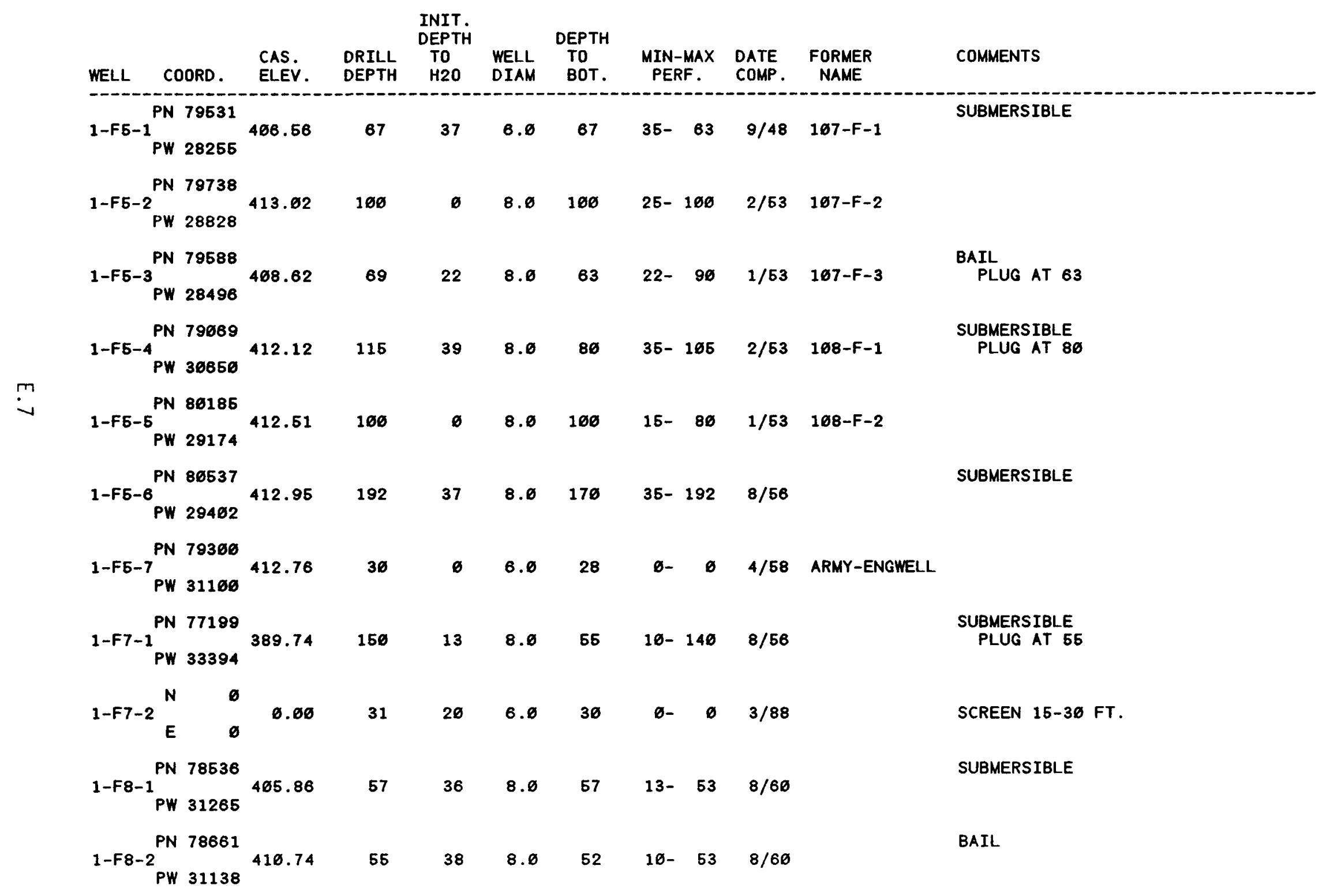




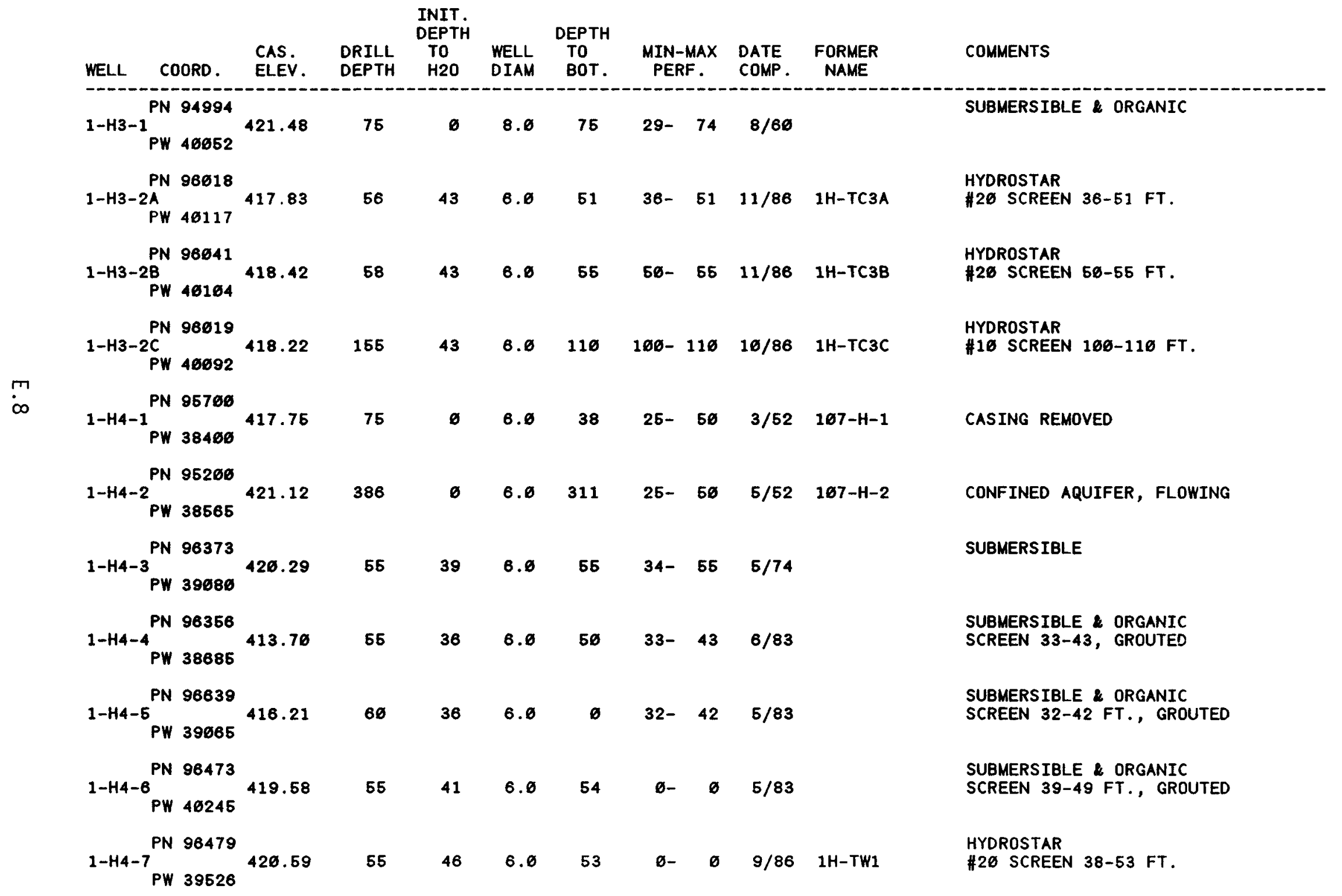




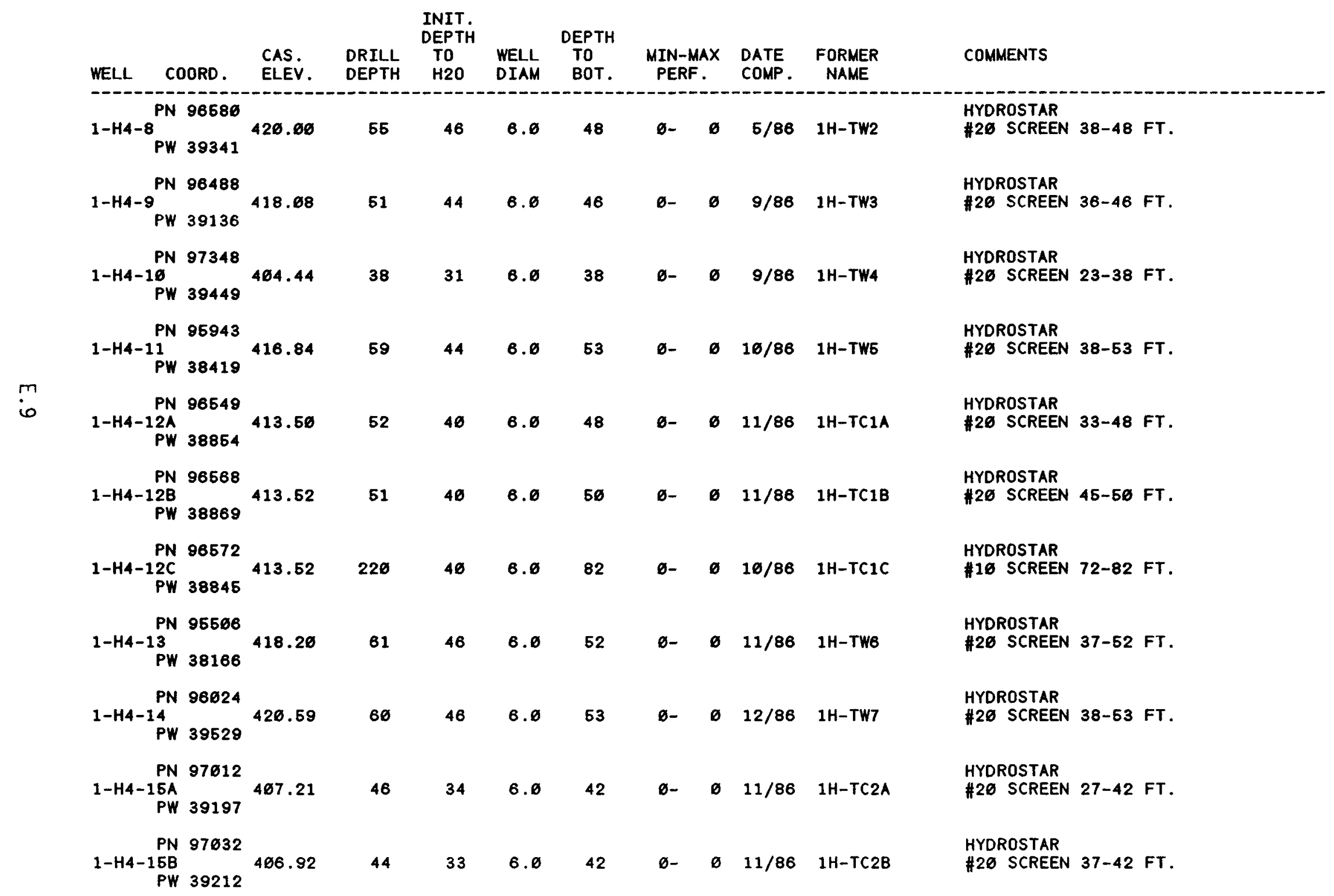




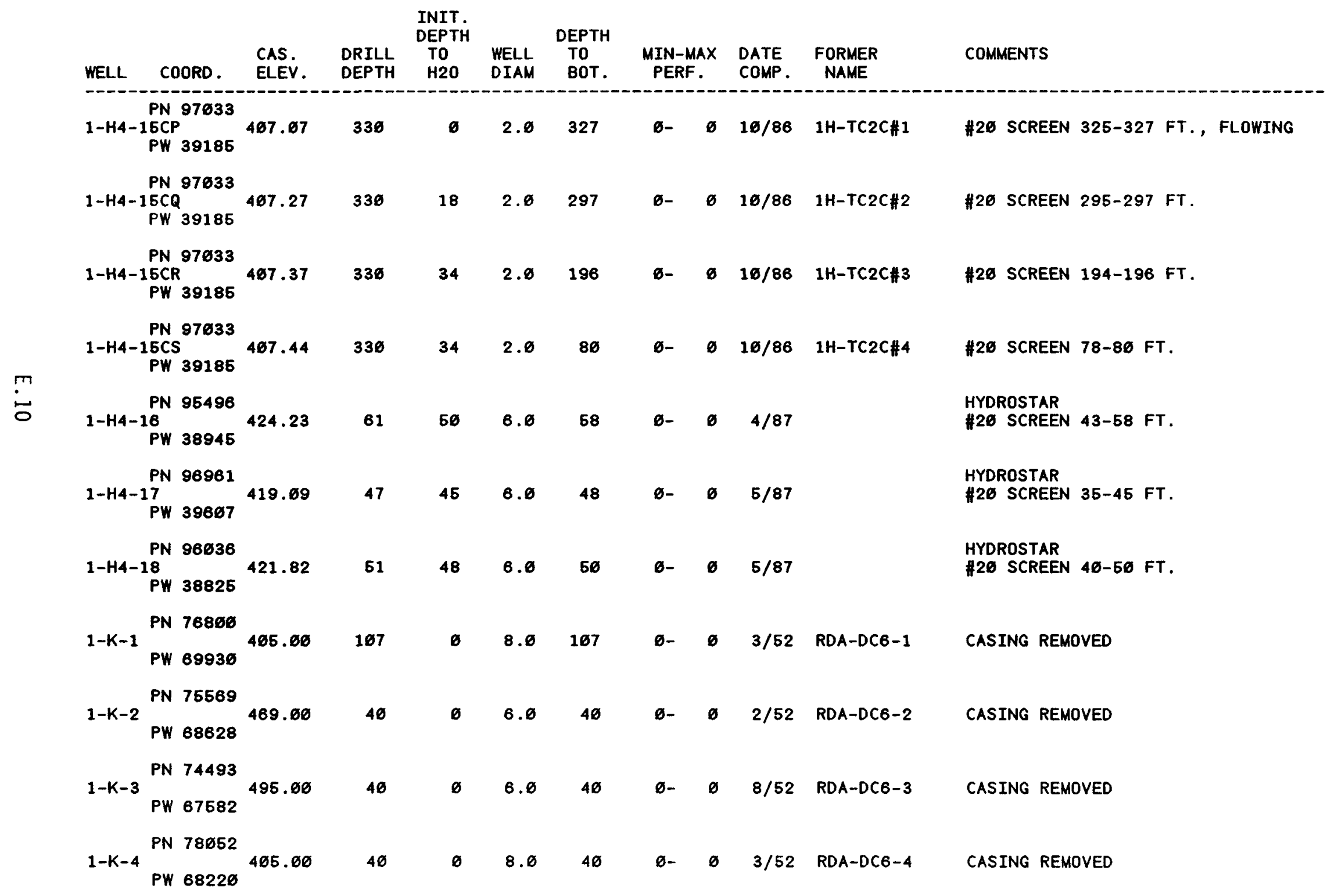




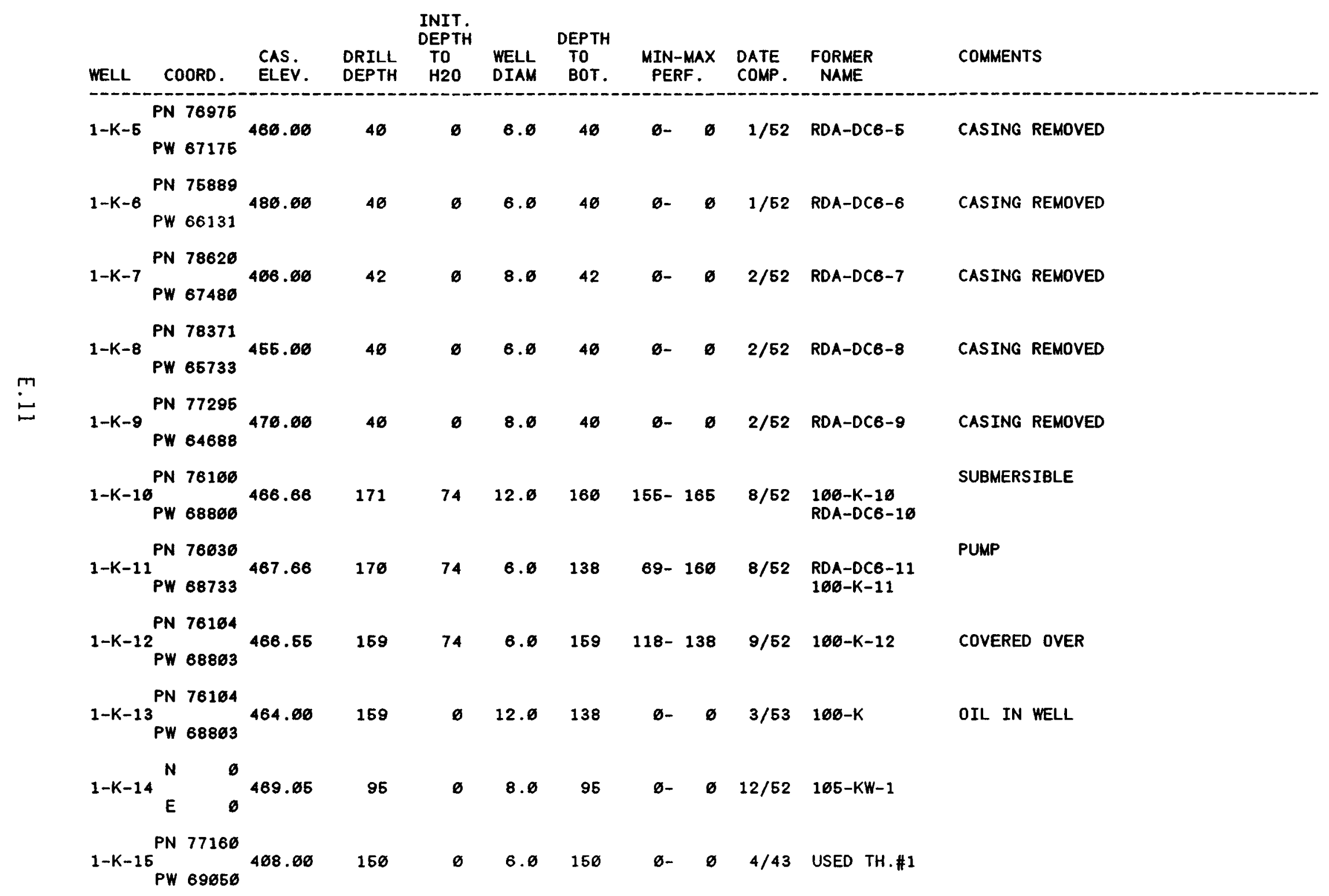




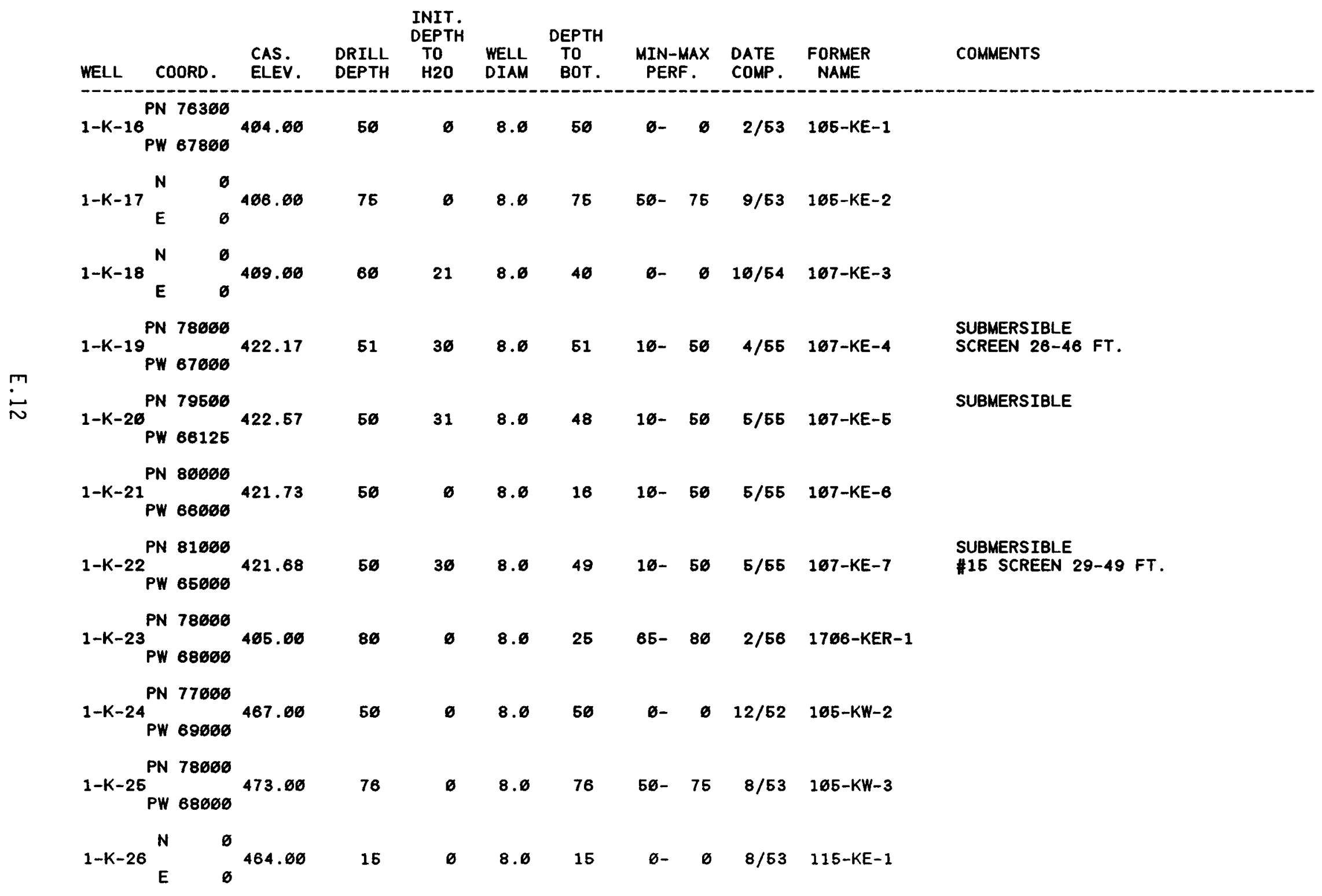




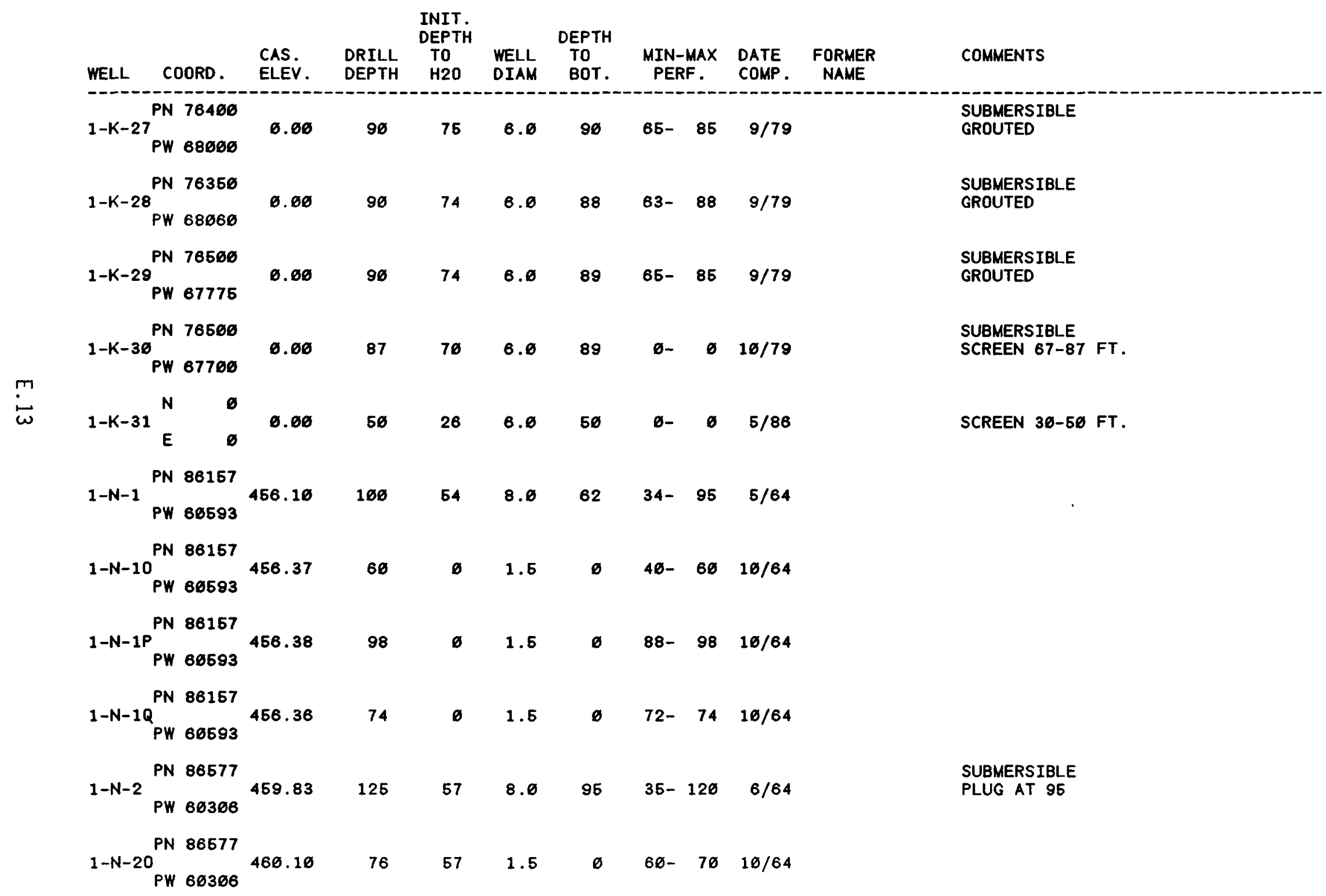




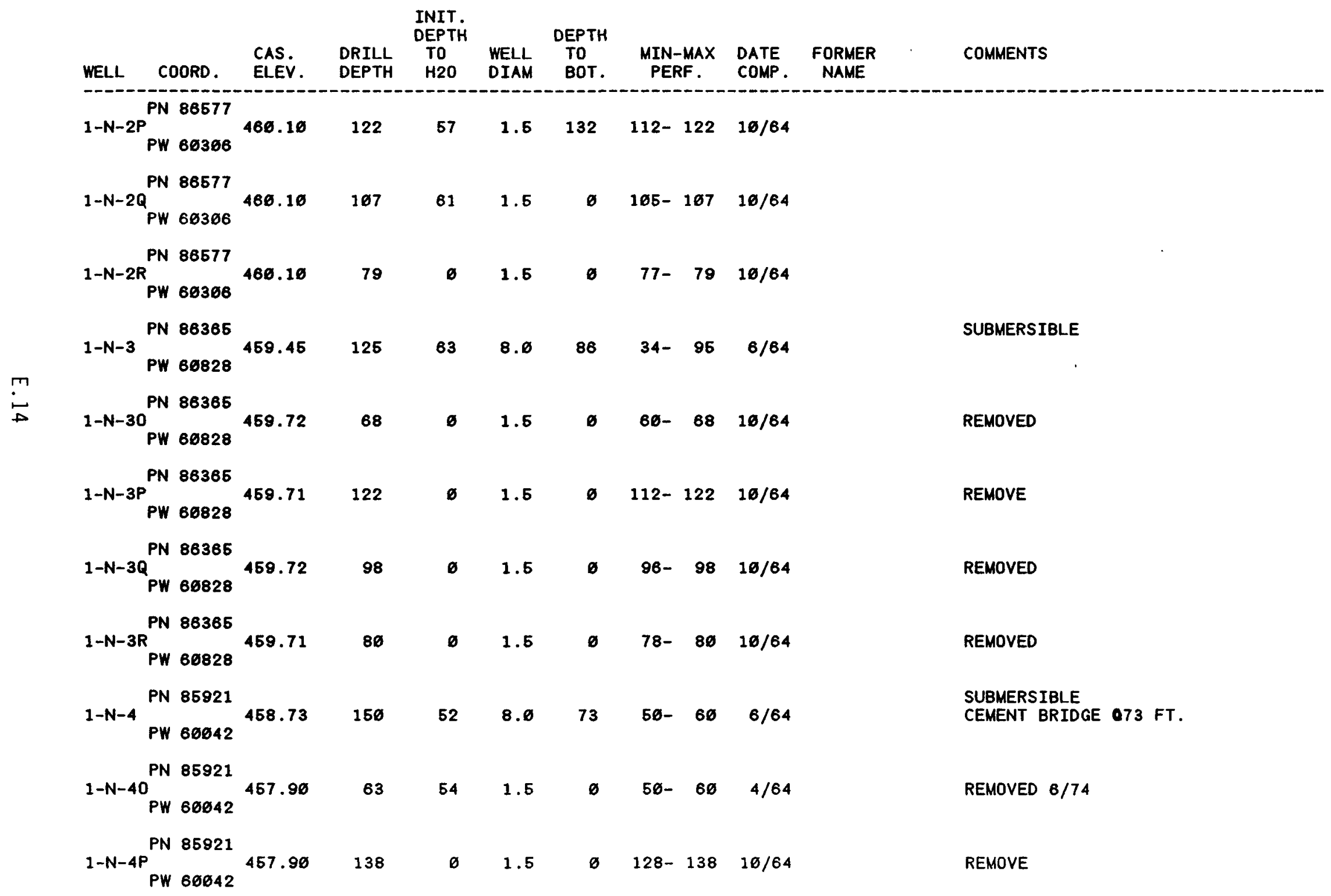




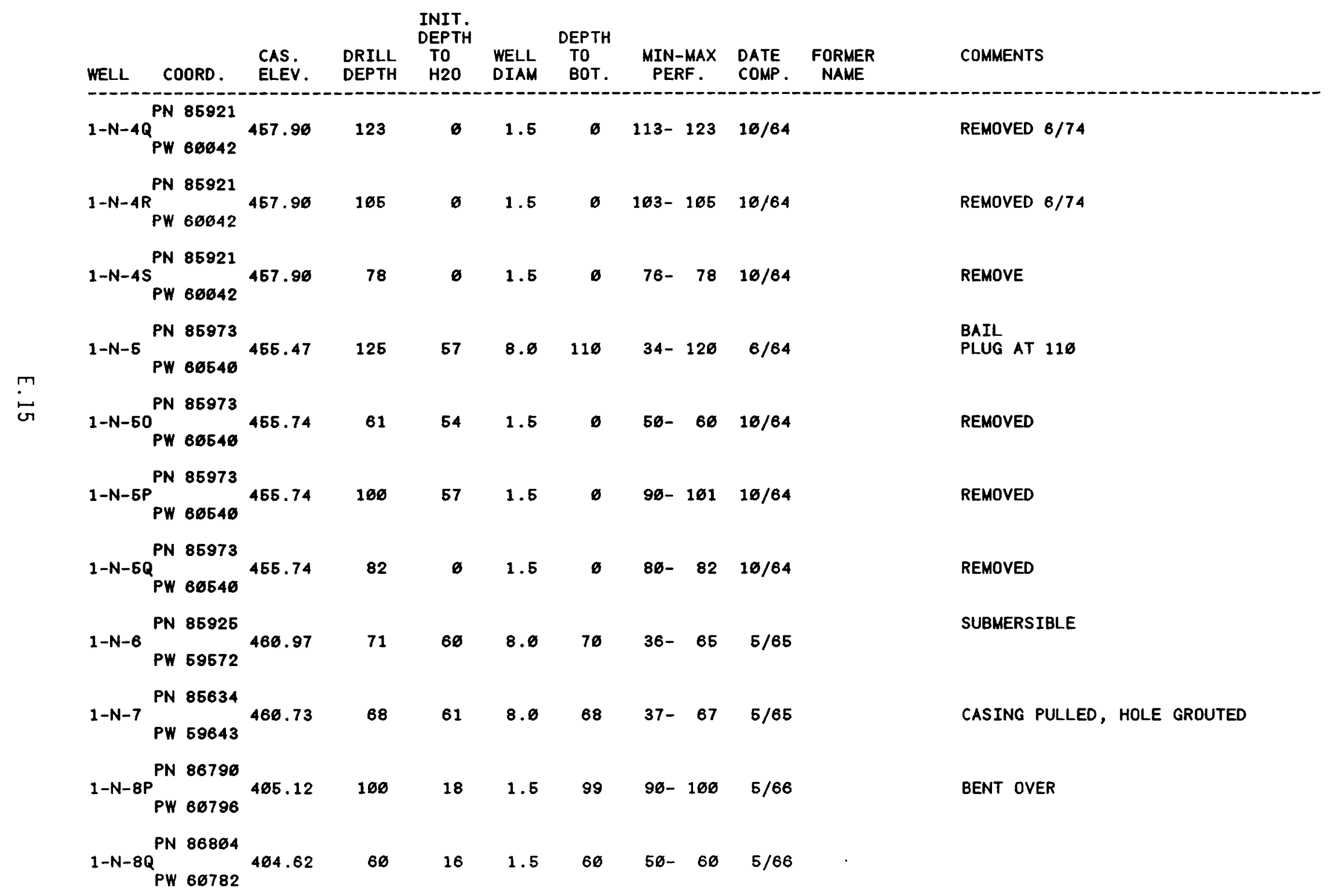




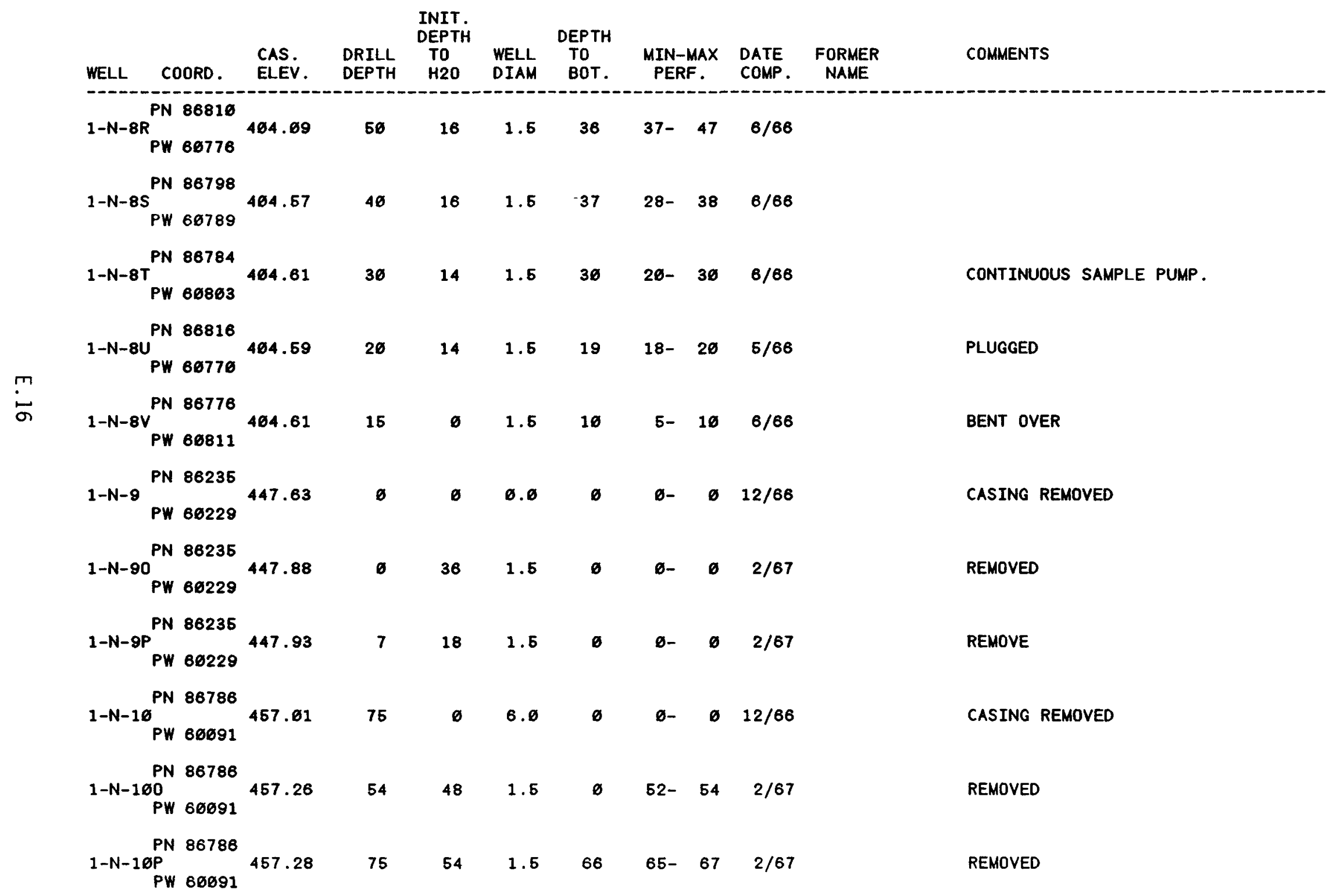




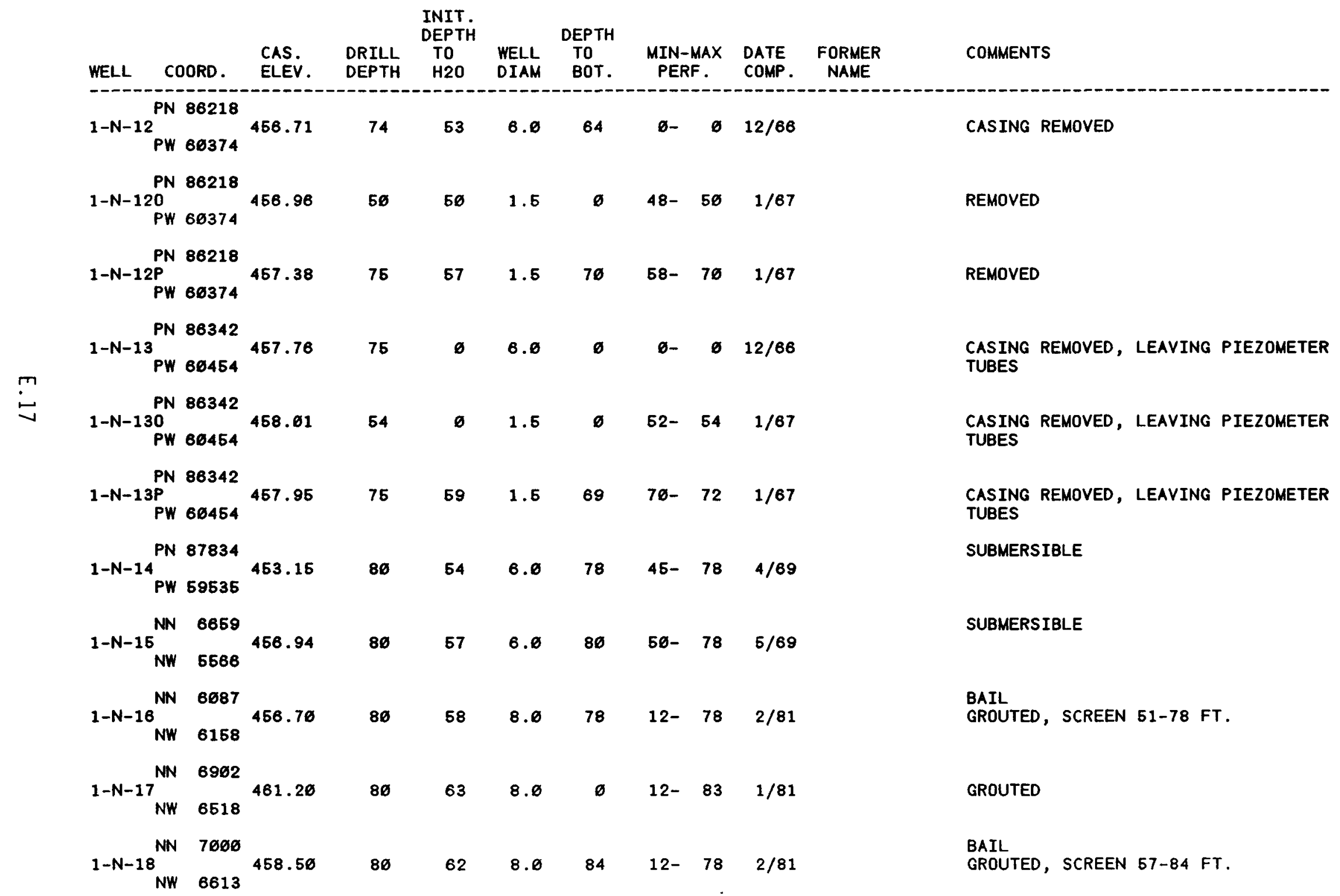




\begin{tabular}{|c|c|c|c|c|c|c|c|c|c|c|c|c|}
\hline WELL & & ORD. & $\begin{array}{l}\text { CAS. } \\
\text { ELEV. }\end{array}$ & $\begin{array}{l}\text { DRILL } \\
\text { DEPTH }\end{array}$ & $\begin{array}{l}\text { INIT. } \\
\text { DEPTH } \\
\text { TO } \\
\text { H2O }\end{array}$ & $\begin{array}{l}\text { WELL } \\
\text { DIAM }\end{array}$ & $\begin{array}{l}\text { DEPTH } \\
\text { TO } \\
\text { BOT. }\end{array}$ & $\begin{array}{l}\text { MIN- } \\
\text { PER }\end{array}$ & $\begin{array}{l}\operatorname{MAX} \\
\text { F. }\end{array}$ & $\begin{array}{l}\text { DATE } \\
\text { COMP. }\end{array}$ & $\begin{array}{l}\text { FORMER } \\
\text { NAME }\end{array}$ & COMMENTS \\
\hline $1-N-15$ & $9^{\mathrm{NN}}$ & $\begin{array}{l}6783 \\
6769\end{array}$ & 453.96 & 78 & ø & 8.0 & 78 & ס- & ø & $1 / 81$ & & BAIL \\
\hline $1-N-20$ & ${ }^{N W}$ & $\begin{array}{l}6646 \\
6680\end{array}$ & 455.90 & 80 & 62 & 8.0 & 80 & $12-$ & 78 & $1 / 81$ & & $\begin{array}{l}\text { BAIL } \\
\text { GROUTED }\end{array}$ \\
\hline $1-N-21$ & $1^{N N}$ & $\begin{array}{l}6522 \\
6767\end{array}$ & 467.08 & 80 & 60 & 8.0 & 79 & $12-$ & 78 & $1 / 81$ & & $\begin{array}{l}\text { BAIL } \\
\text { GROUTED }\end{array}$ \\
\hline $1-N-22$ & $2^{N W}$ & $\begin{array}{l}6346 \\
6762\end{array}$ & 467.16 & 79 & 62 & 8.0 & 80 & $12-$ & 77 & $1 / 81$ & & $\begin{array}{l}\text { BAIL } \\
\text { GROUTED, SCREEN 58-8ø FT. }\end{array}$ \\
\hline $1-N-23$ & $3^{\mathrm{NN}}$ & $\begin{array}{l}6232 \\
6784\end{array}$ & 466.38 & 80 & 59 & 8.0 & 80 & $12-$ & 78 & $1 / 81$ & & $\begin{array}{l}\text { BAIL } \\
\text { GROUTED }\end{array}$ \\
\hline $1-N-24$ & ${ }_{4}^{N N}$ & $\begin{array}{l}6049 \\
6892\end{array}$ & 432.58 & 55 & 34 & 8.0 & 55 & $12-$ & 54 & $1 / 81$ & & $\begin{array}{l}\text { BAIL } \\
\text { GROUTED }\end{array}$ \\
\hline $1-N-2 E$ & ${ }_{6}^{N W}$ & $\begin{array}{l}6923 \\
6910\end{array}$ & 425.86 & 49 & 35 & 8.0 & 48 & $12-$ & 47 & $1 / 81$ & & $\begin{array}{l}\text { BAIL } \\
\text { GROUTED, SCREEN 26-48 FT. }\end{array}$ \\
\hline $1-N-26$ & ${ }^{N N}$ & $\begin{array}{l}5765 \\
6797\end{array}$ & $465.8 \varnothing$ & 79 & 63 & 8.0 & $\emptyset$ & $12-$ & 77 & $1 / 81$ & & GROUTED, SCREEN 60-81 FT. \\
\hline $1-N-27$ & $7^{N N}$ & $\begin{array}{l}7728 \\
4163\end{array}$ & 449.68 & 69 & 49 & 8.0 & 69 & $\emptyset-$ & D & $8 / 83$ & & $\begin{array}{l}\text { SUBMERSIBLE } \\
\text { SCREEN 32-69, GROUTED }\end{array}$ \\
\hline $1-N-28$ & $8^{N N}$ & $\begin{array}{l}7050 \\
4165\end{array}$ & 464.24 & 83 & 63 & 8.6 & 83 & D- & $\theta$ & $9 / 83$ & & $\begin{array}{l}\text { SUBMERSIBLE } \\
\text { SCREEN } 47-83\end{array}$ \\
\hline $1-N-2 s$ & $9^{\mathrm{NN}}$ & $\begin{array}{l}6943 \\
4525\end{array}$ & 465.25 & 84 & 64 & 8.0 & 84 & D- & g & $8 / 83$ & & $\begin{array}{l}\text { SUBMERSIBLE } \\
\text { SCREEN 47-84, GROUTED }\end{array}$ \\
\hline
\end{tabular}




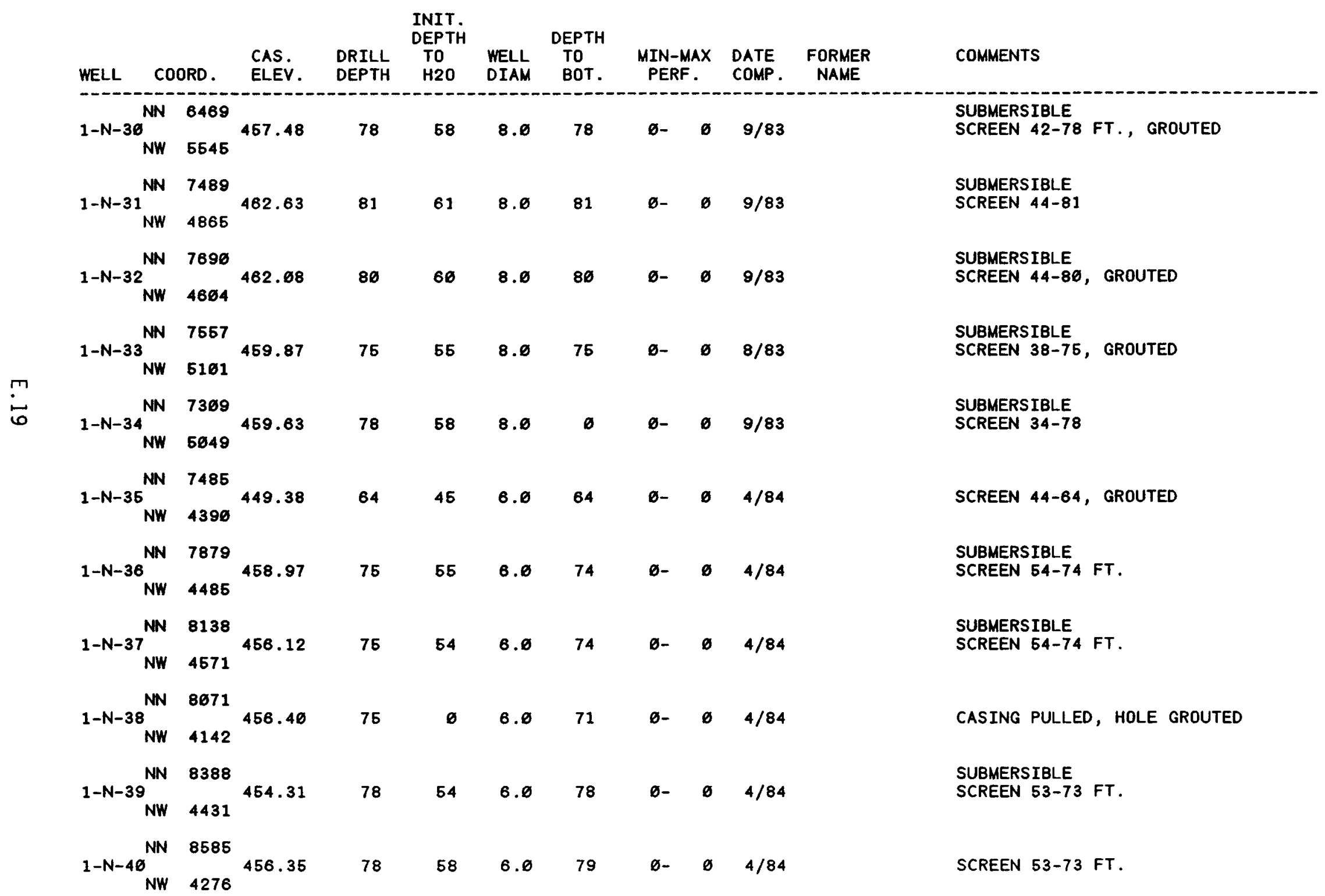




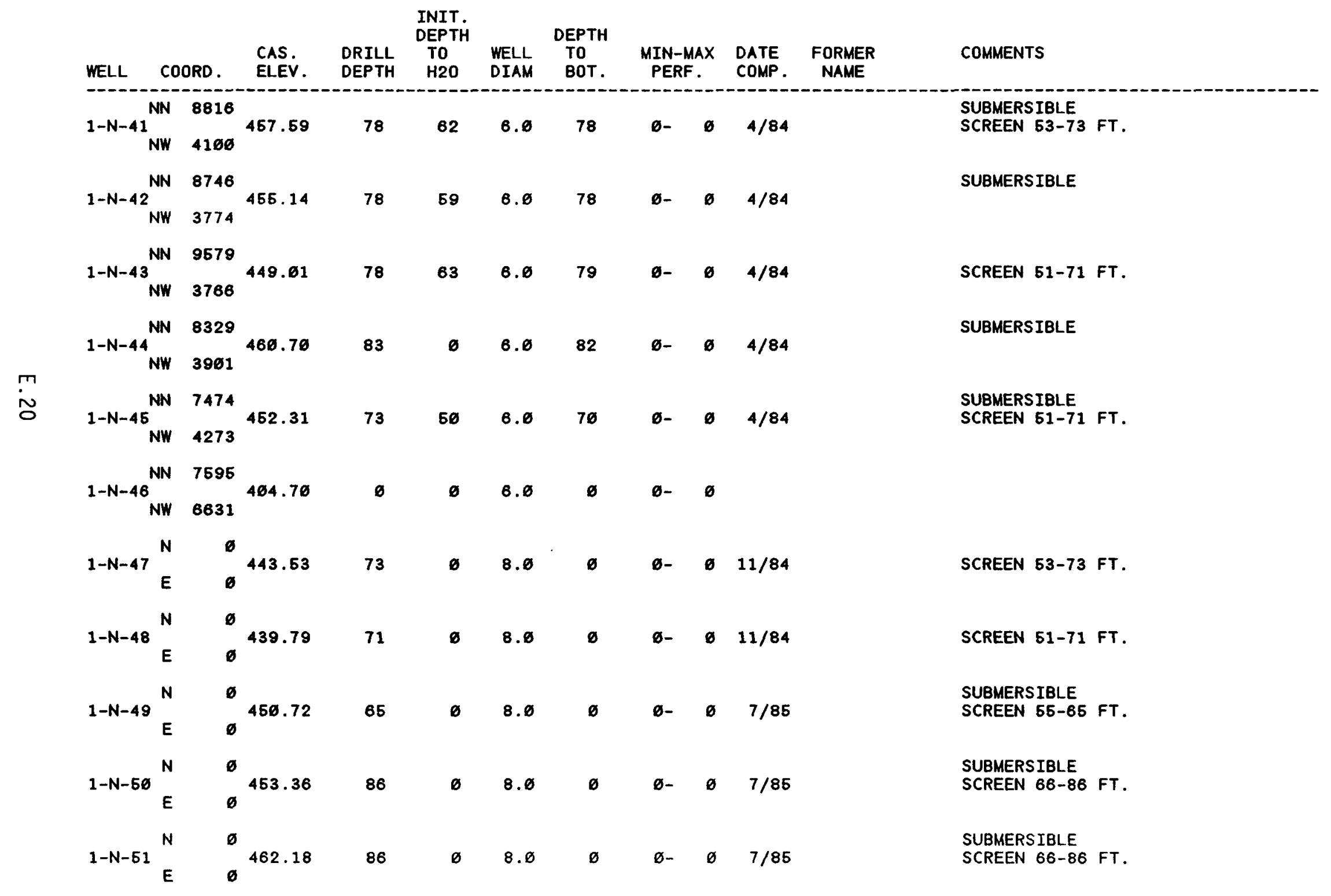




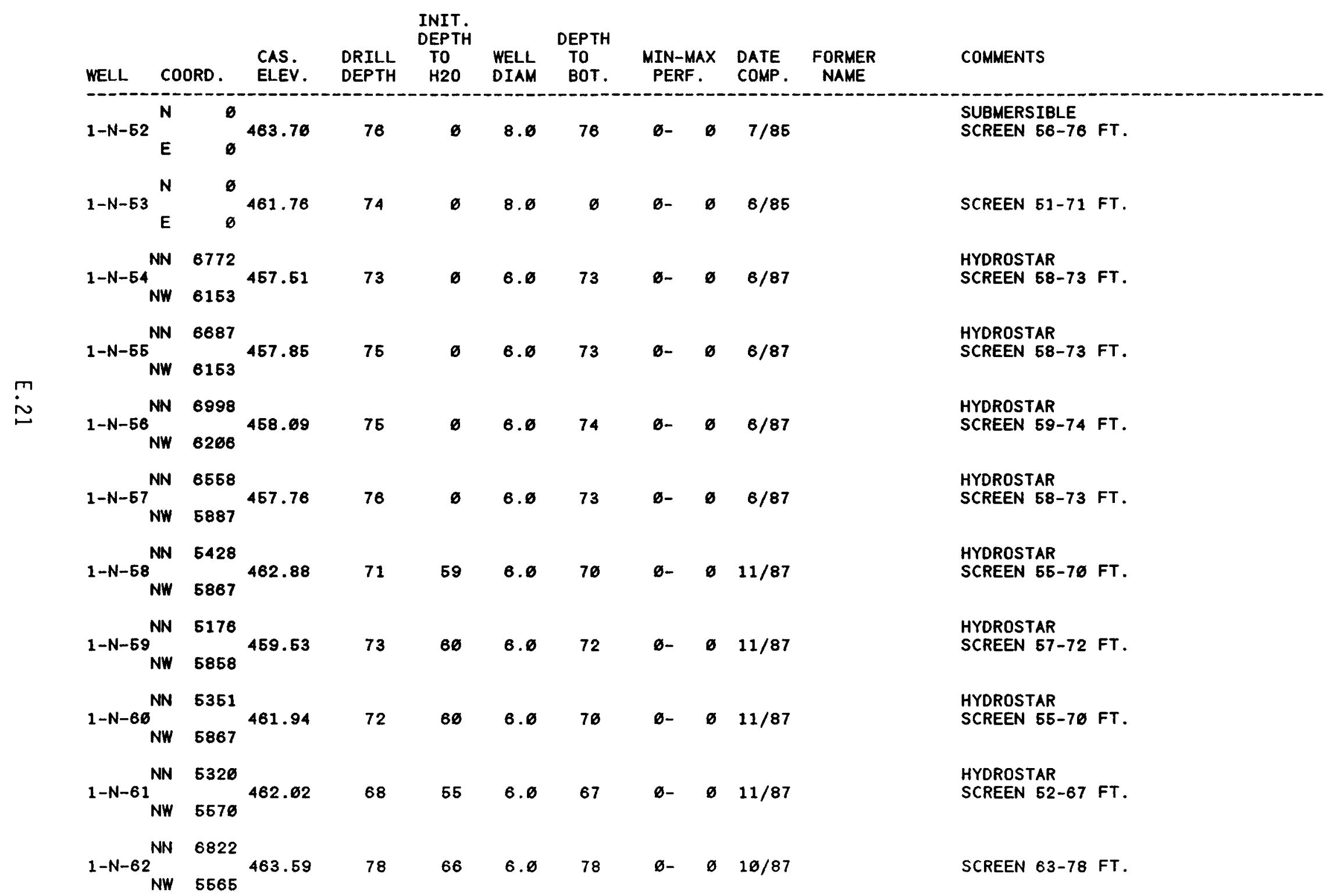




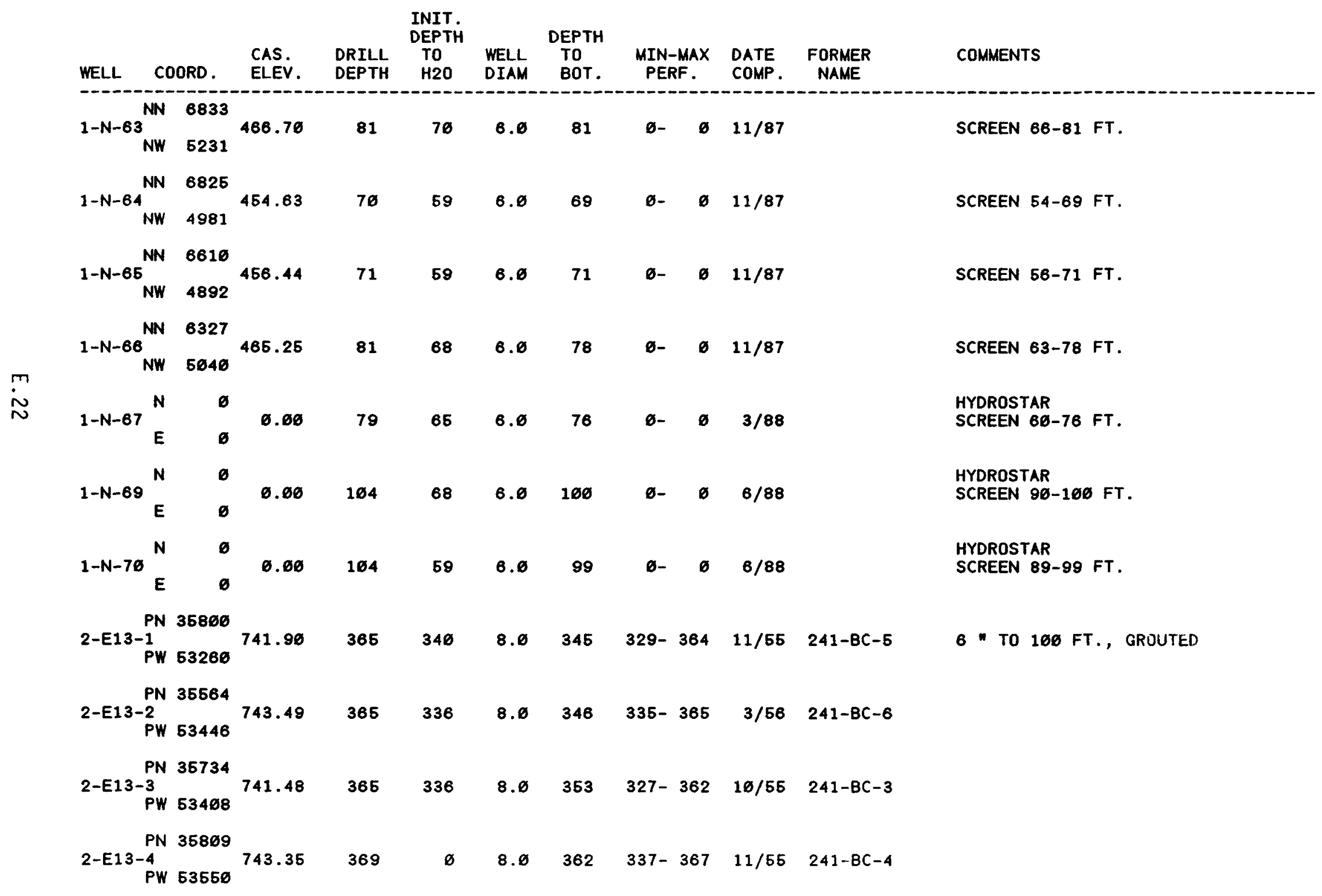




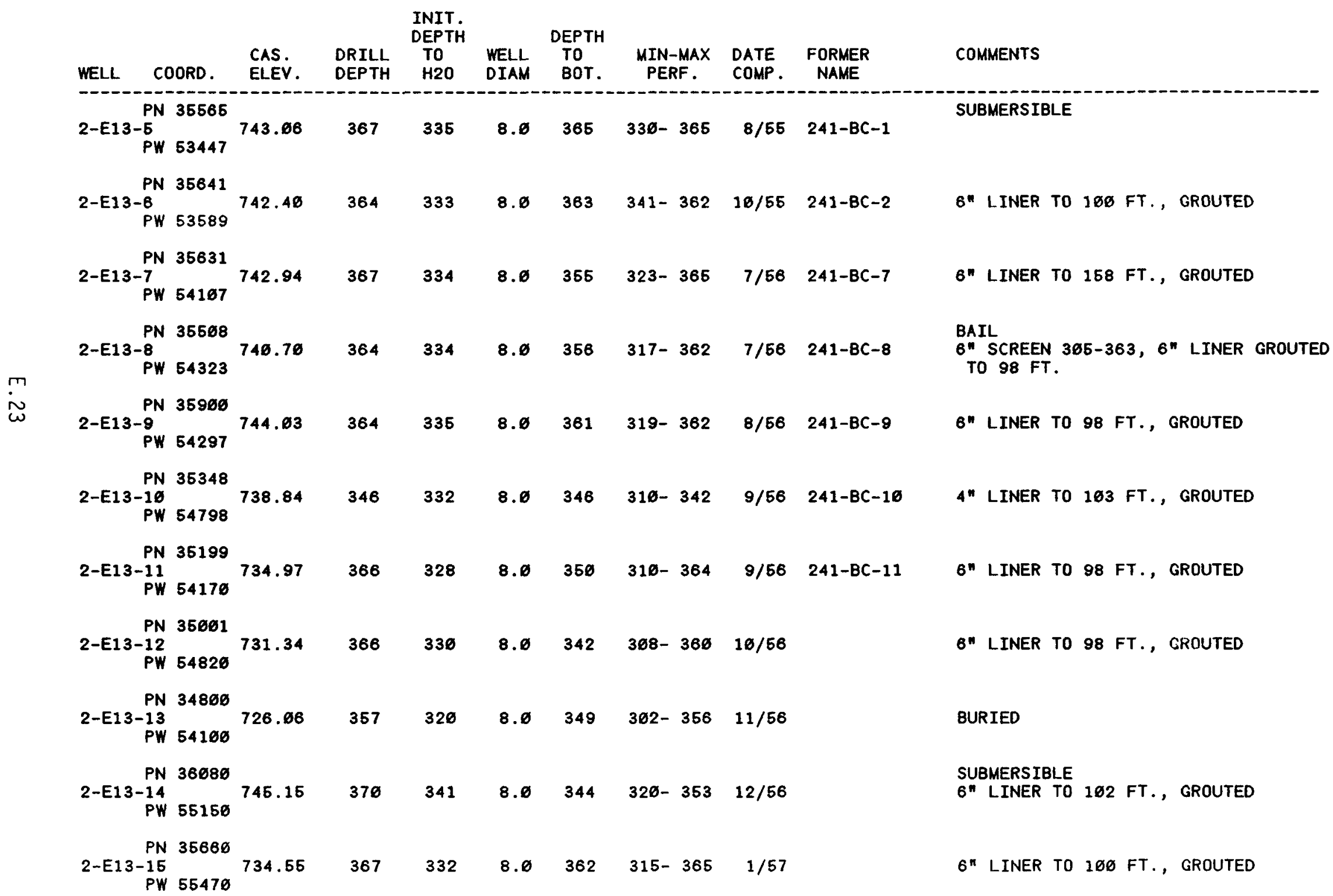




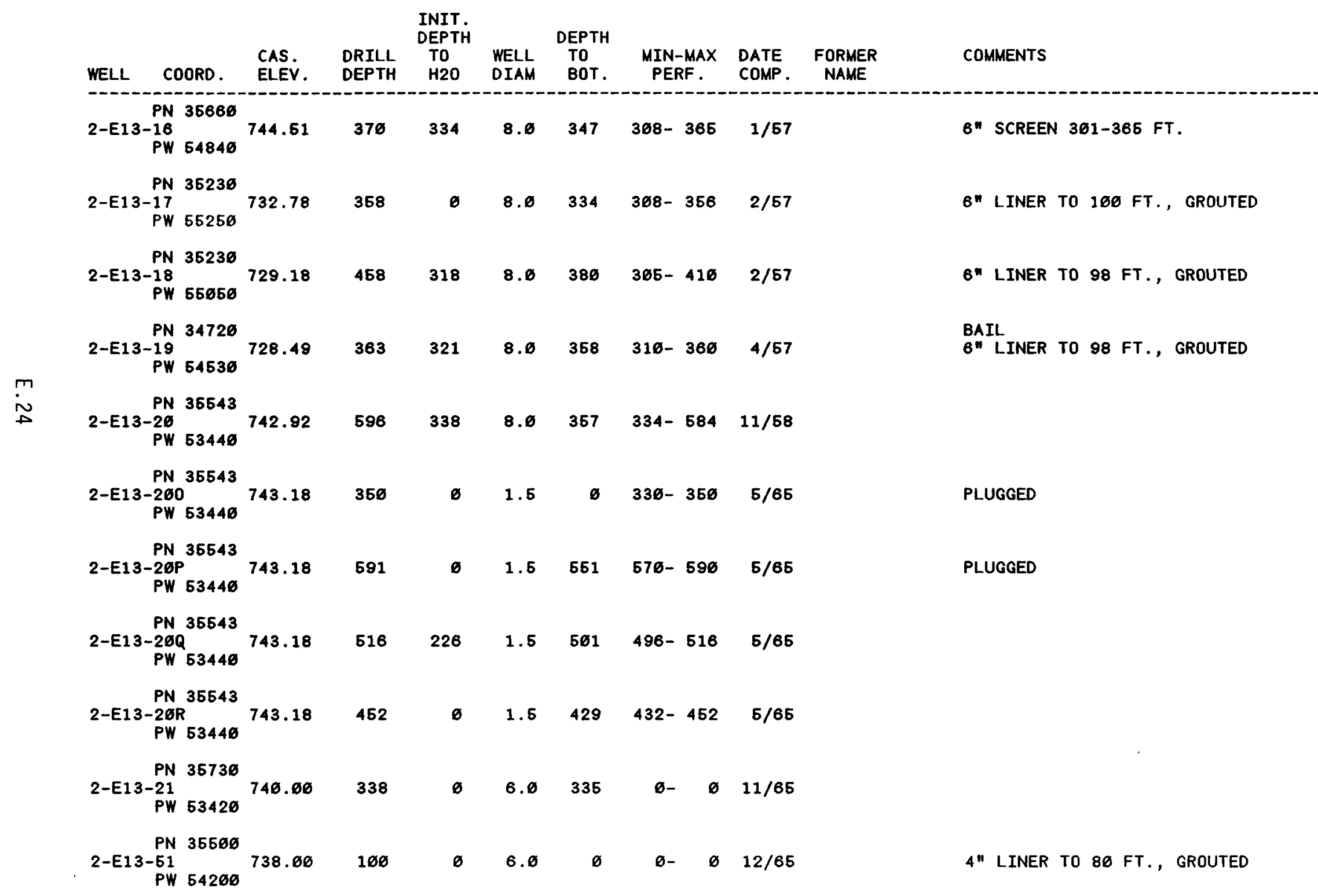




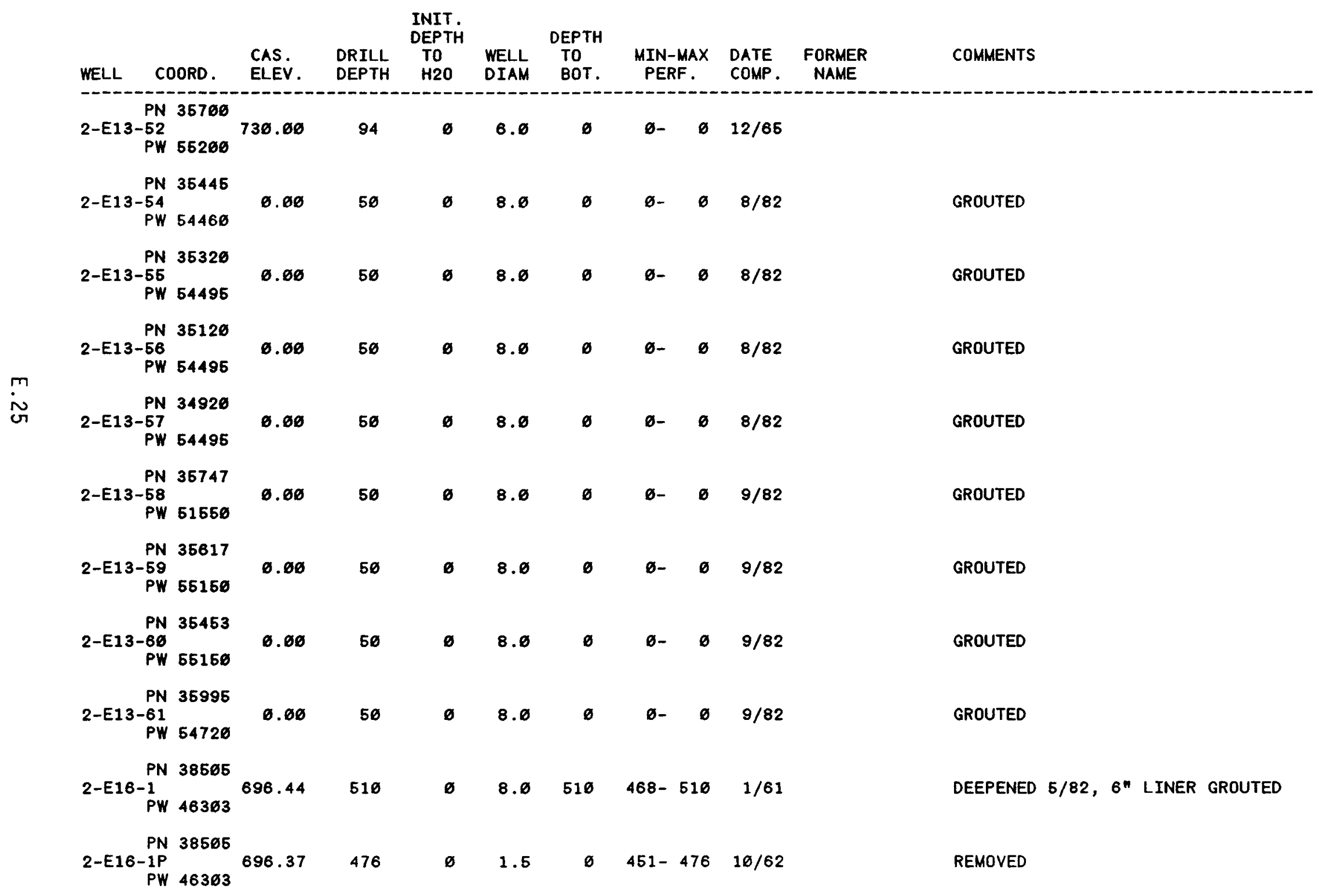




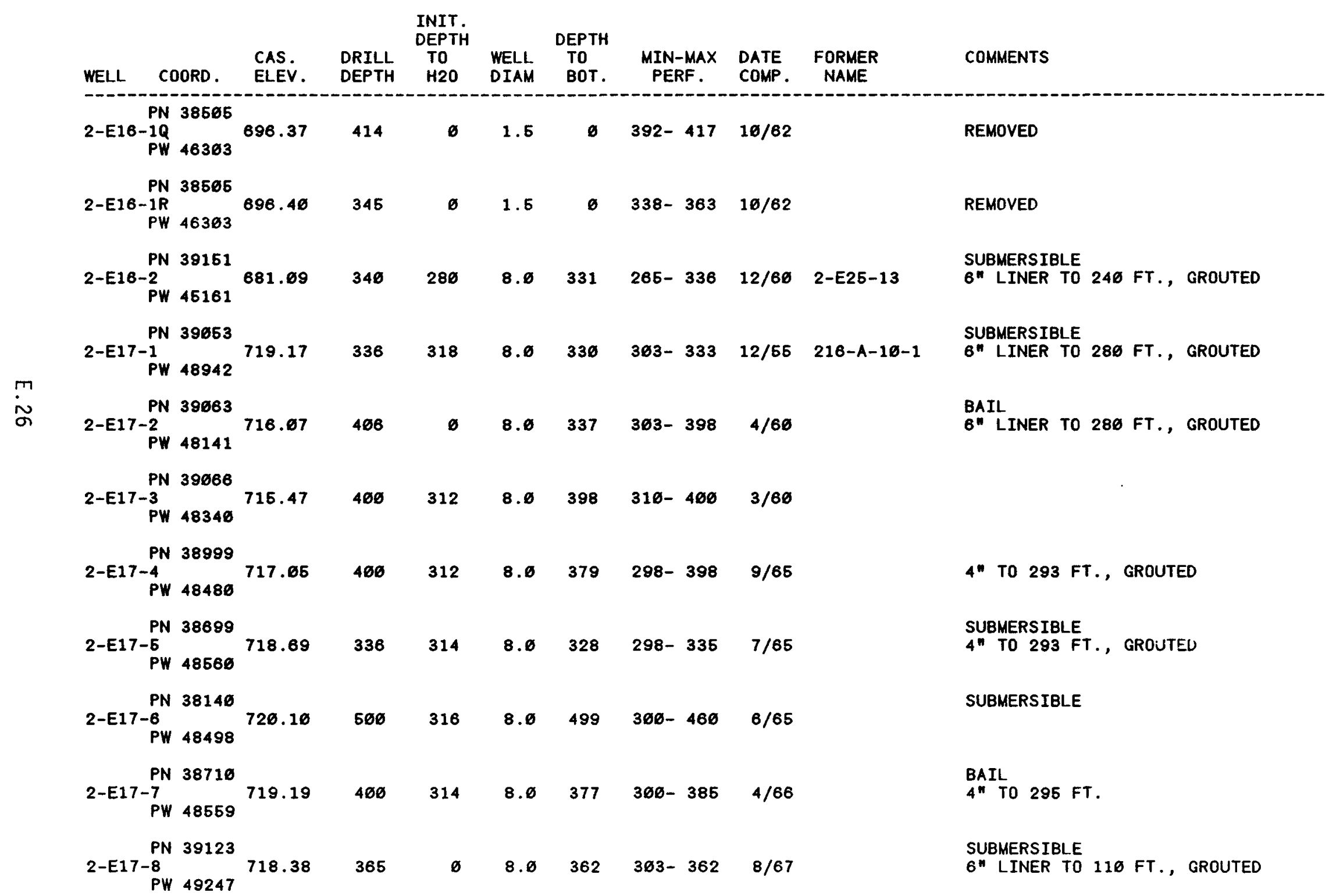




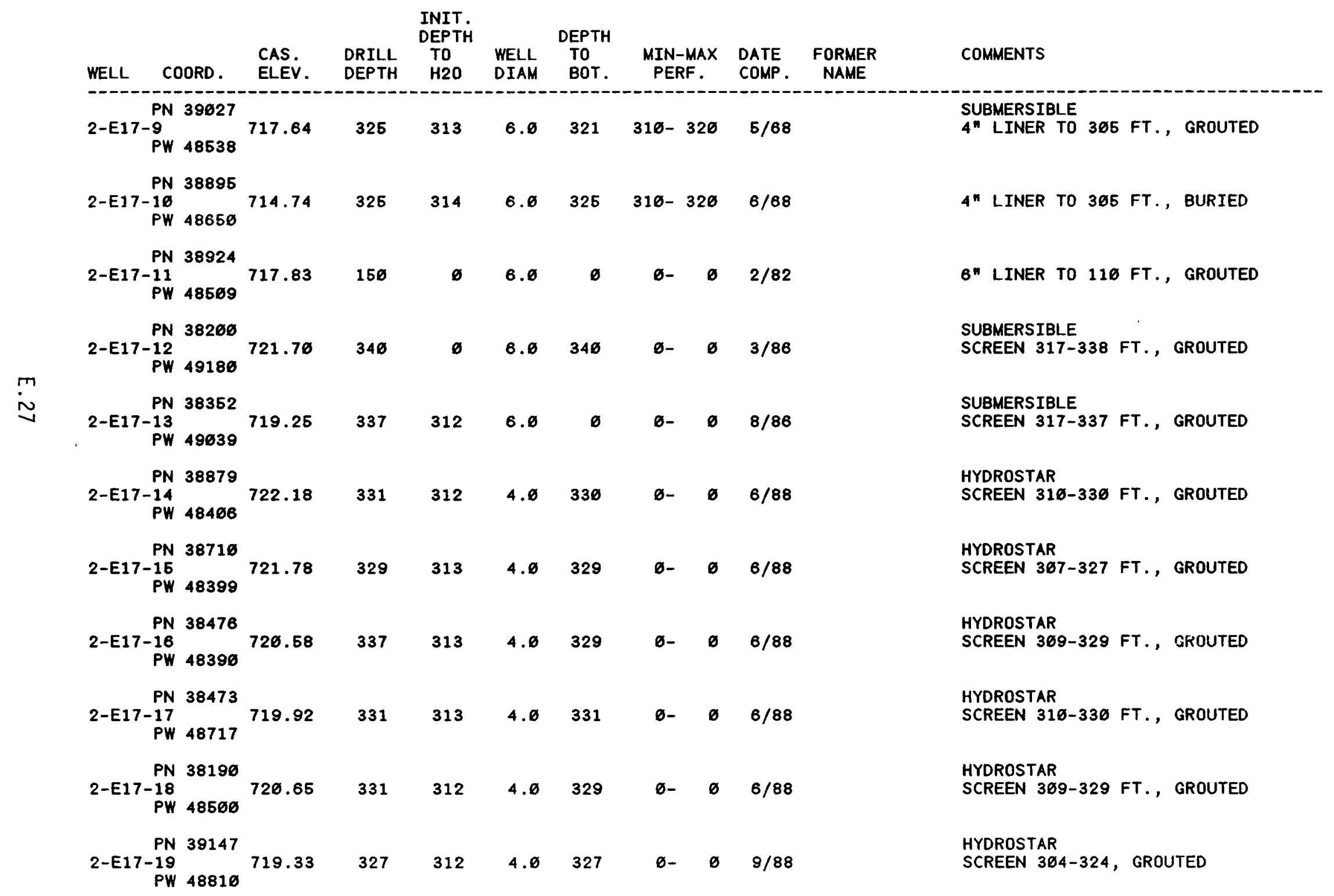




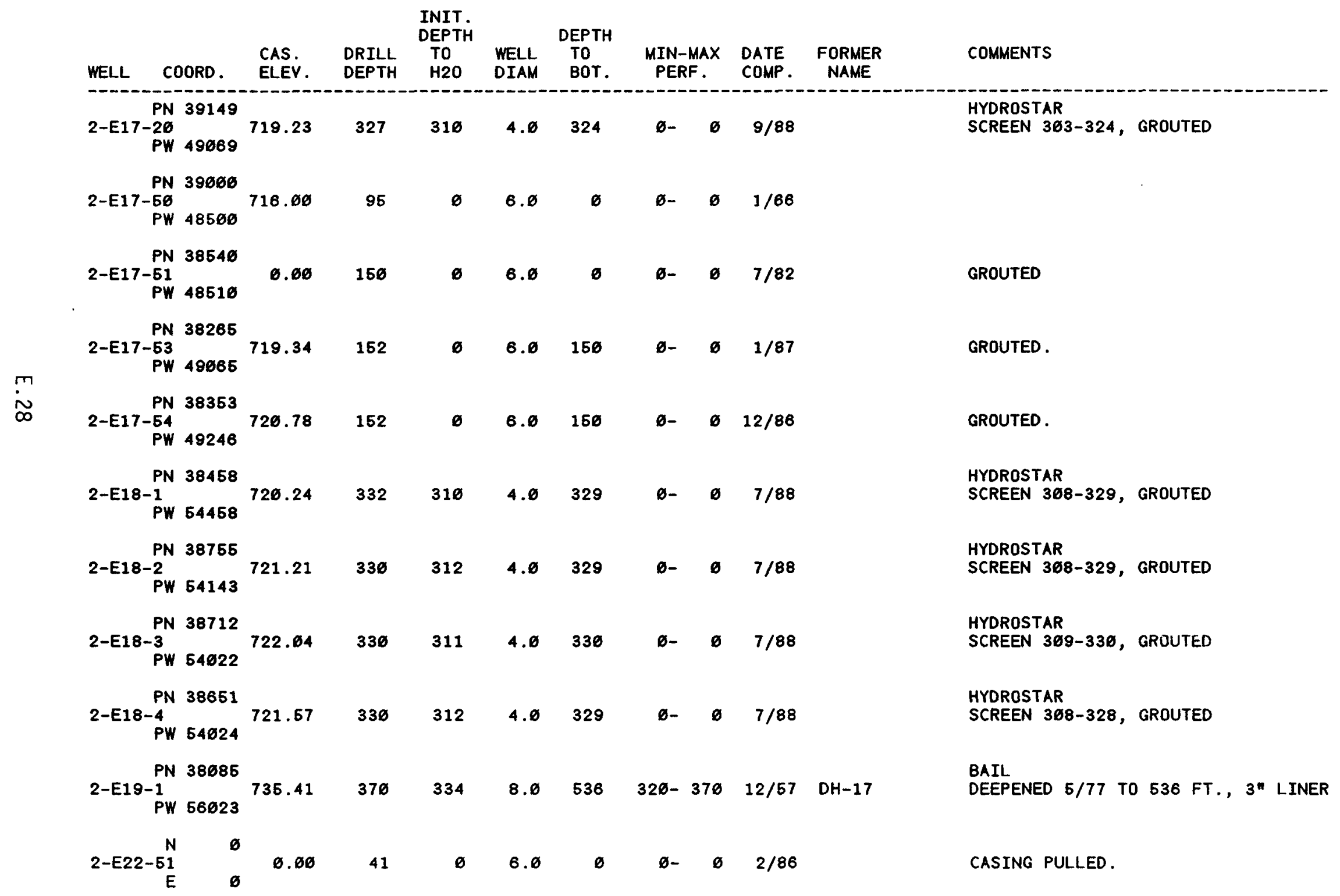




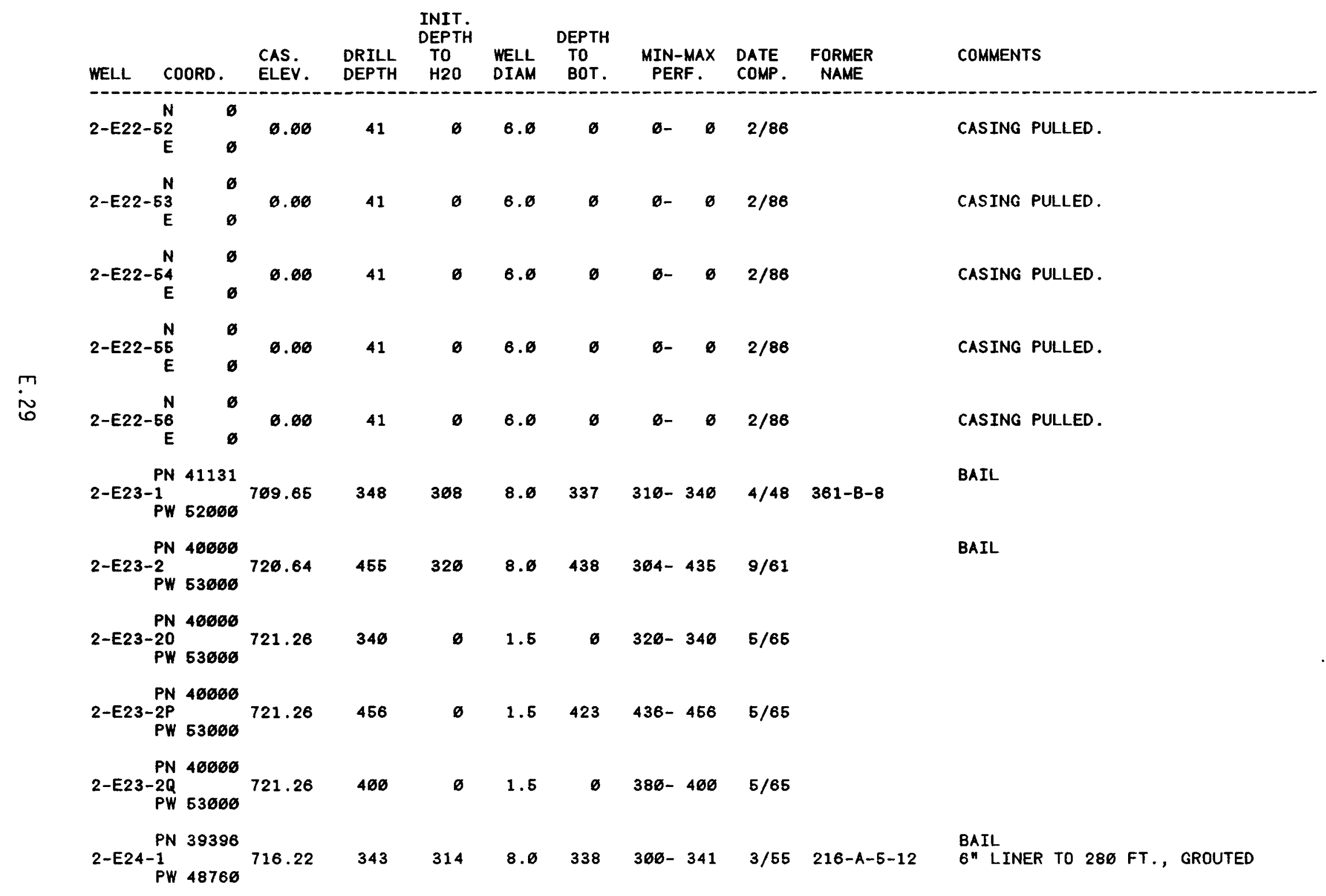




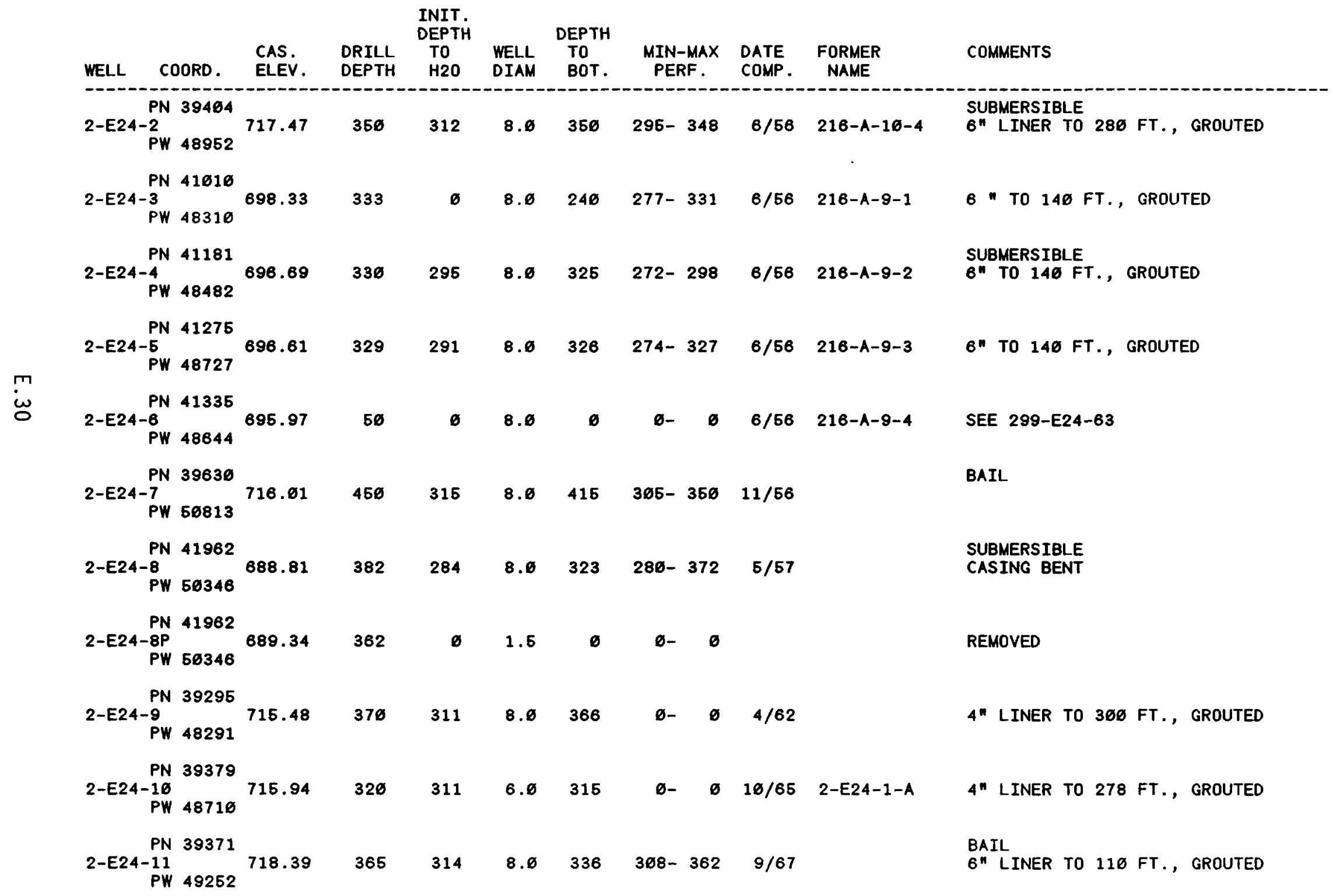




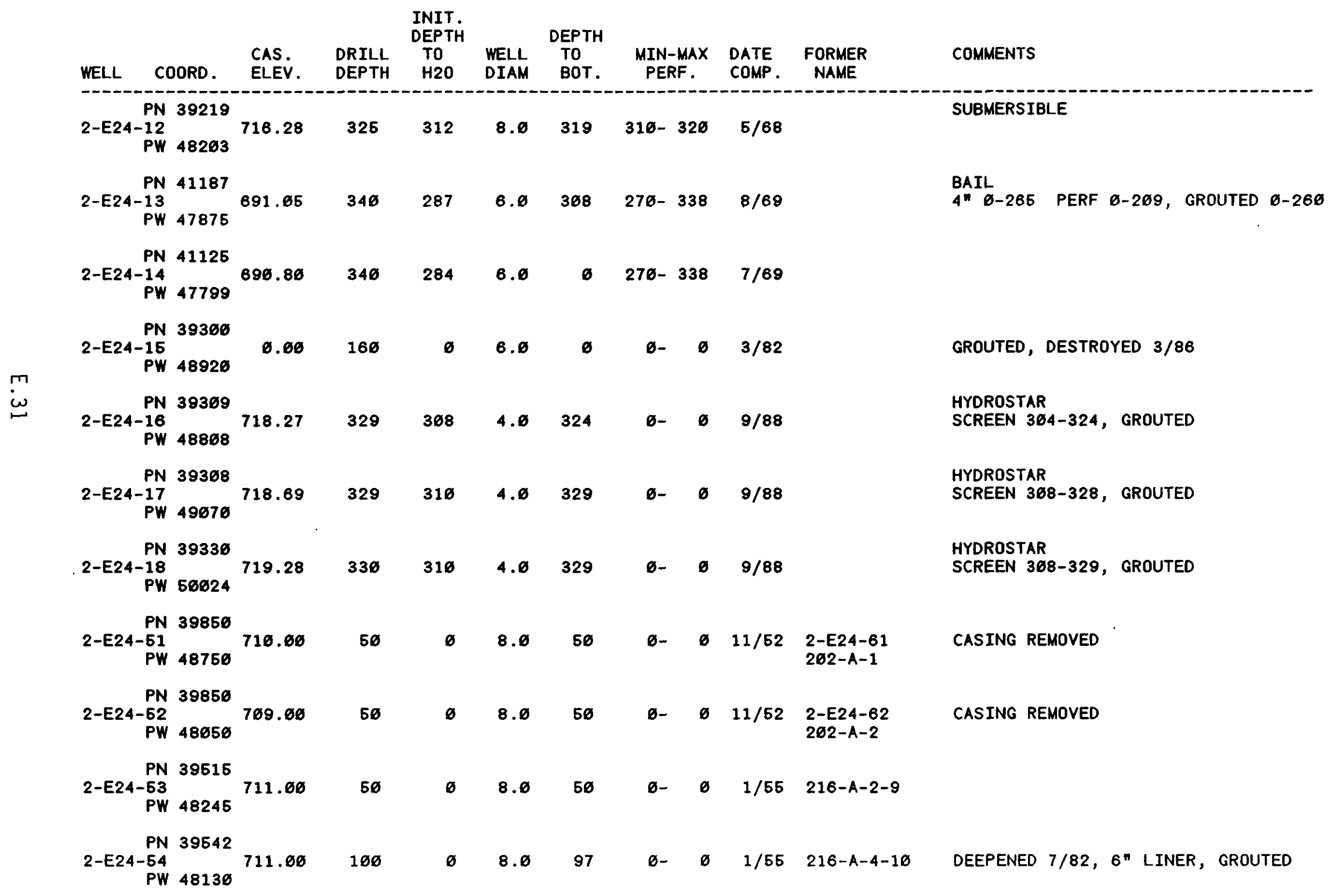




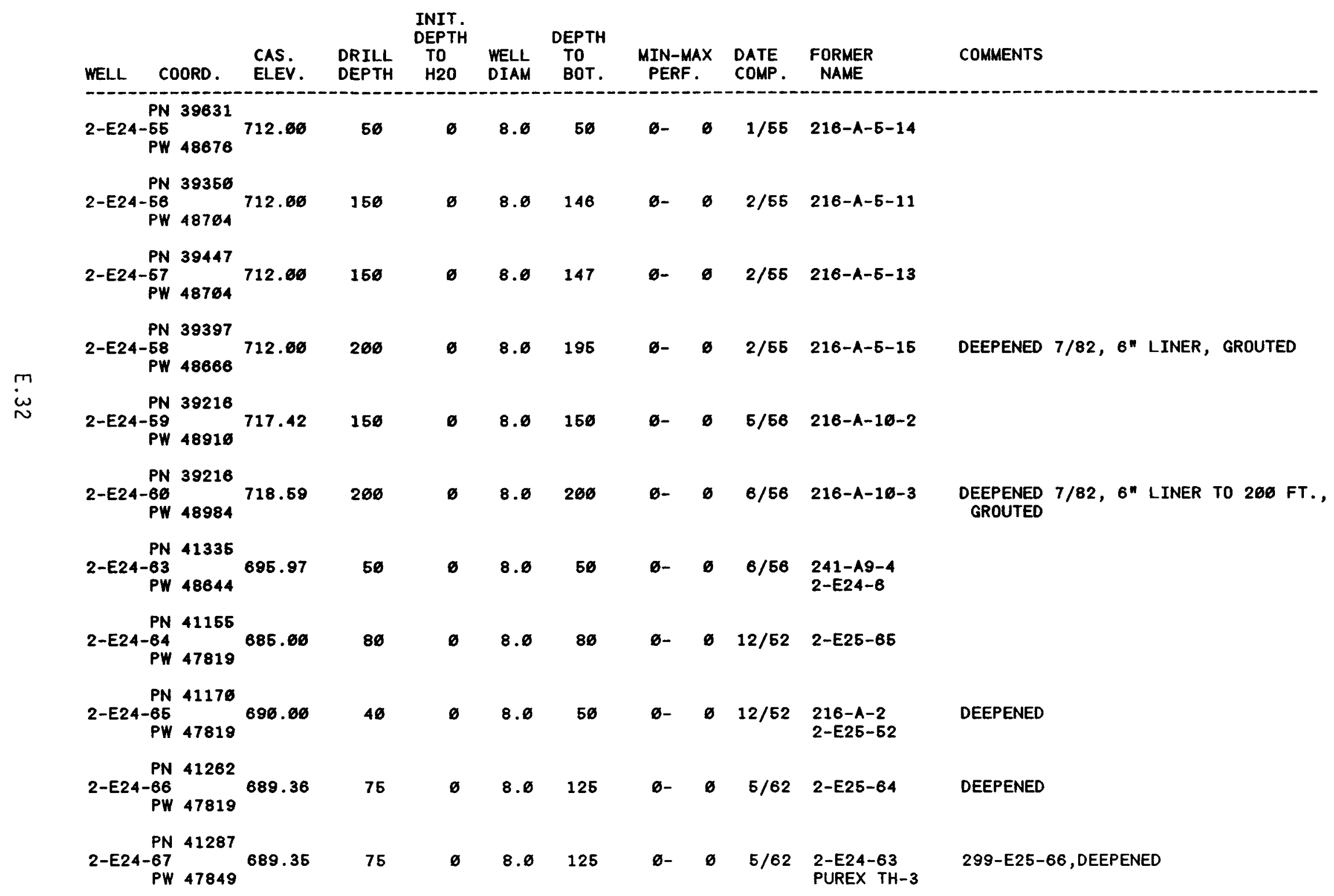




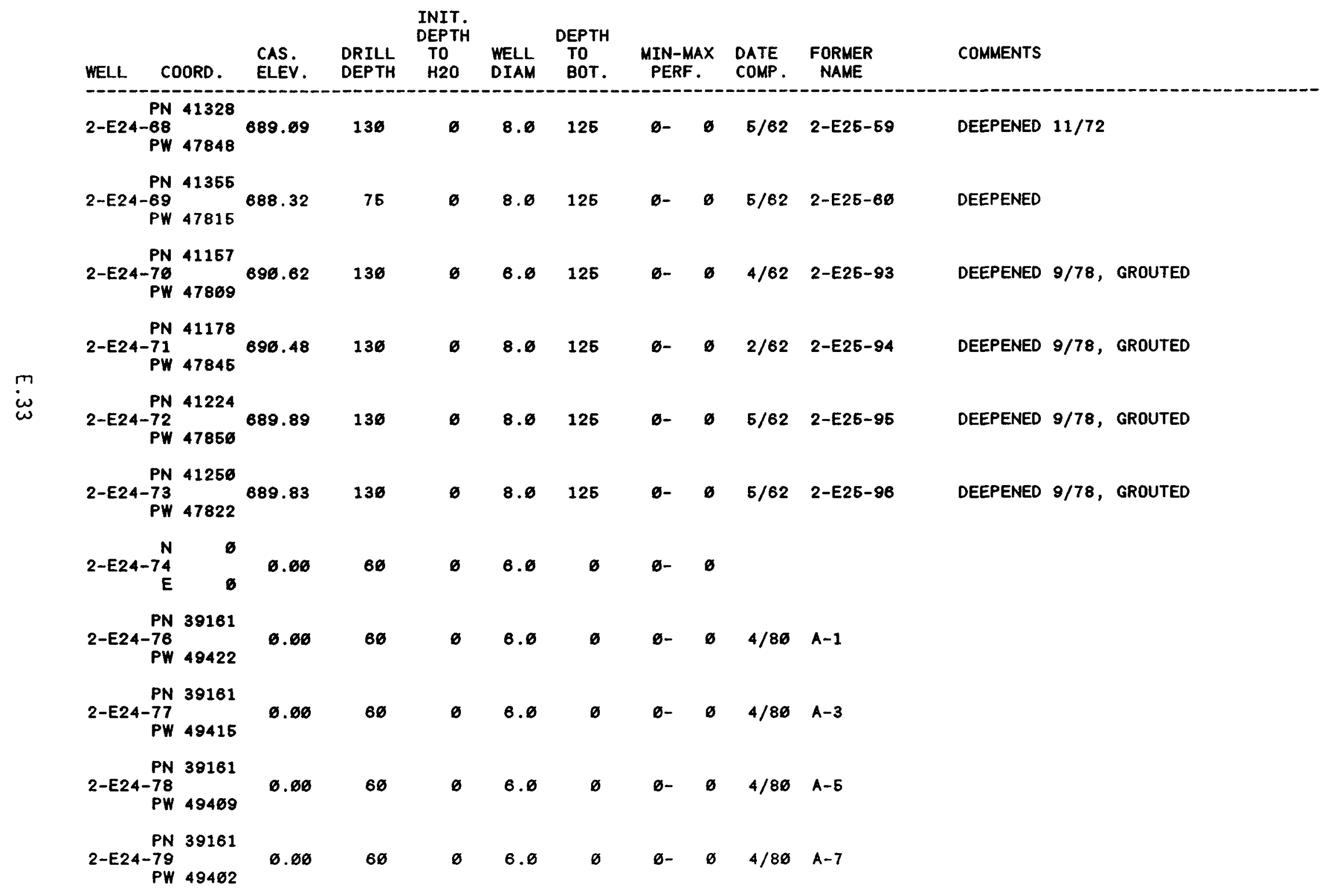




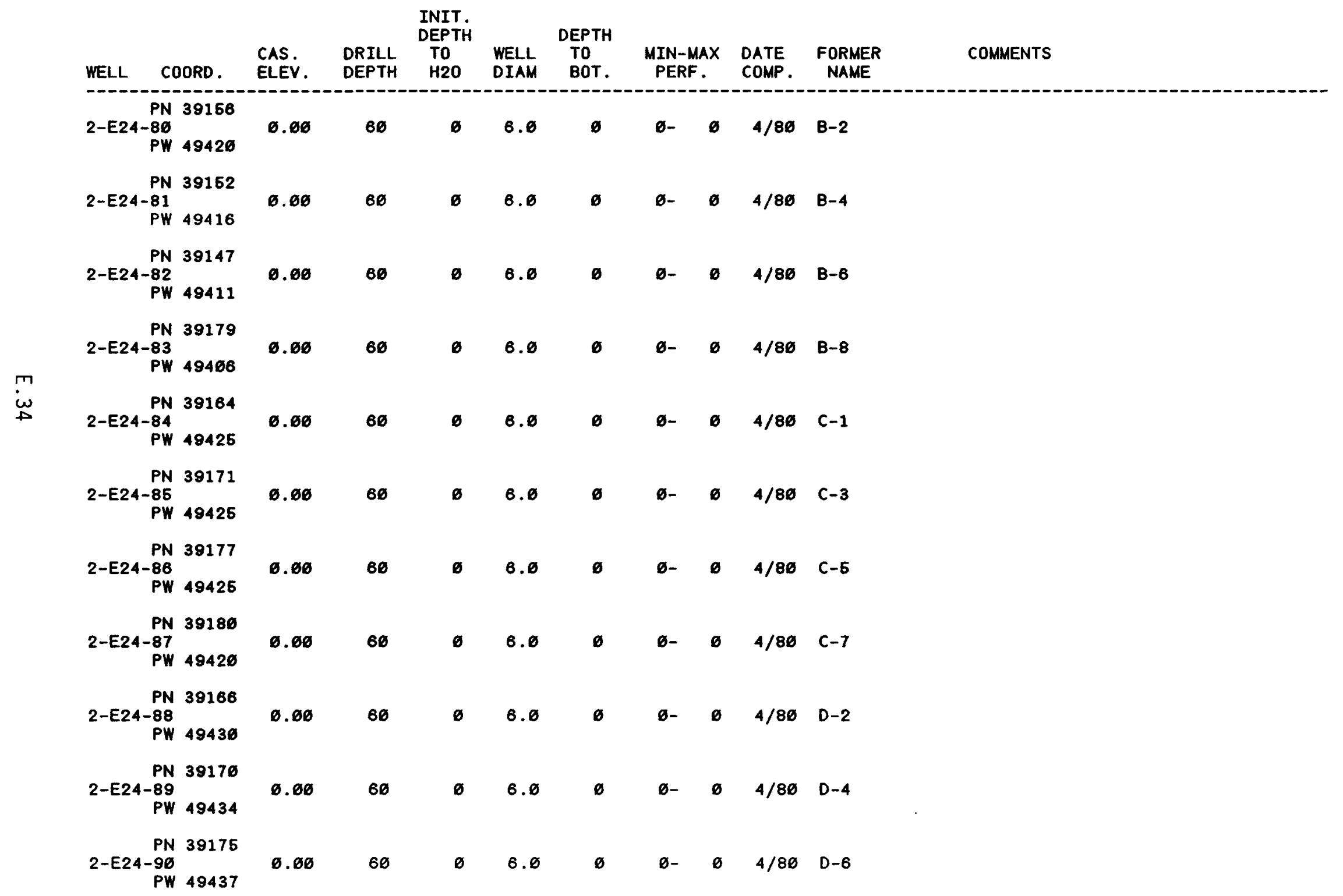




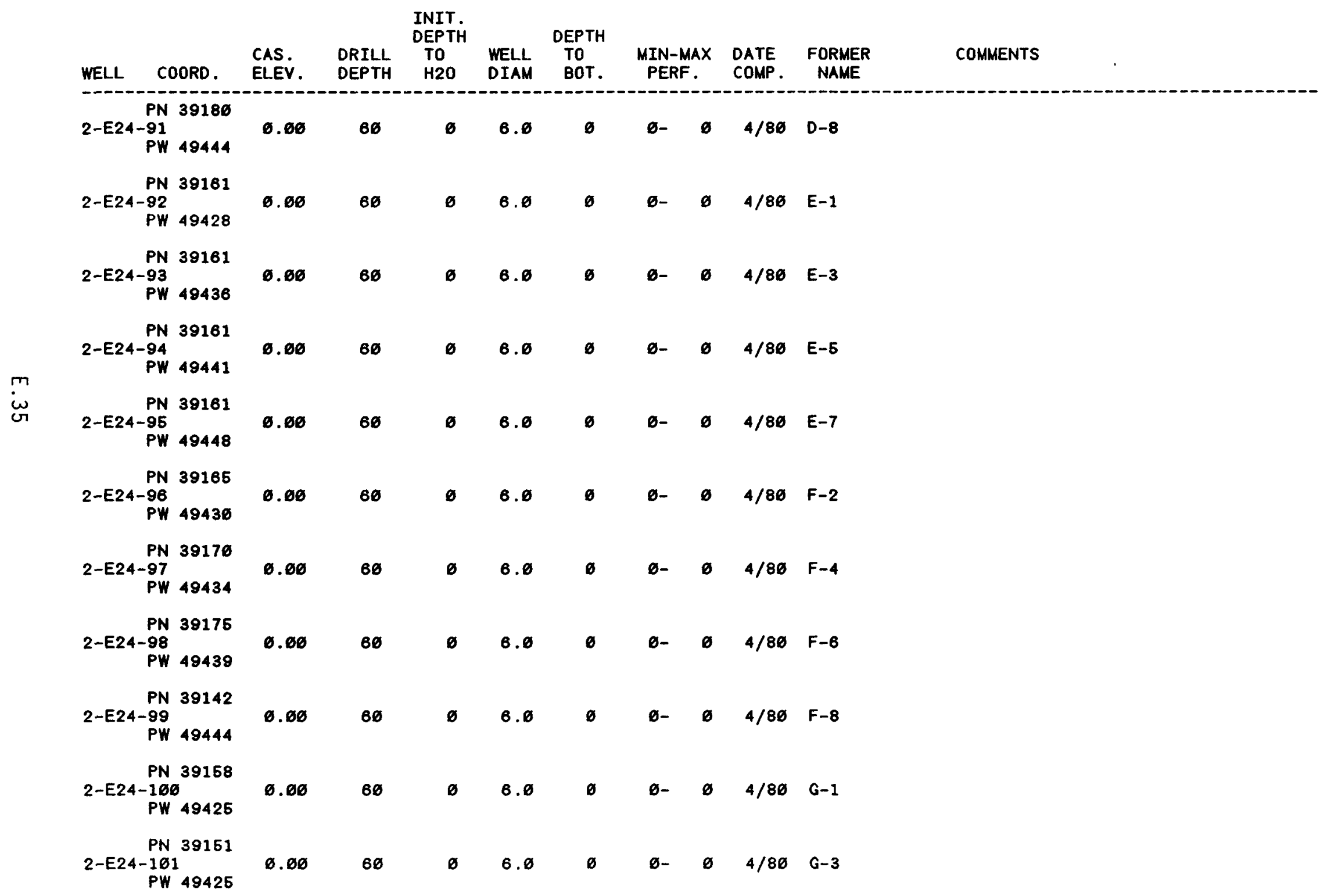




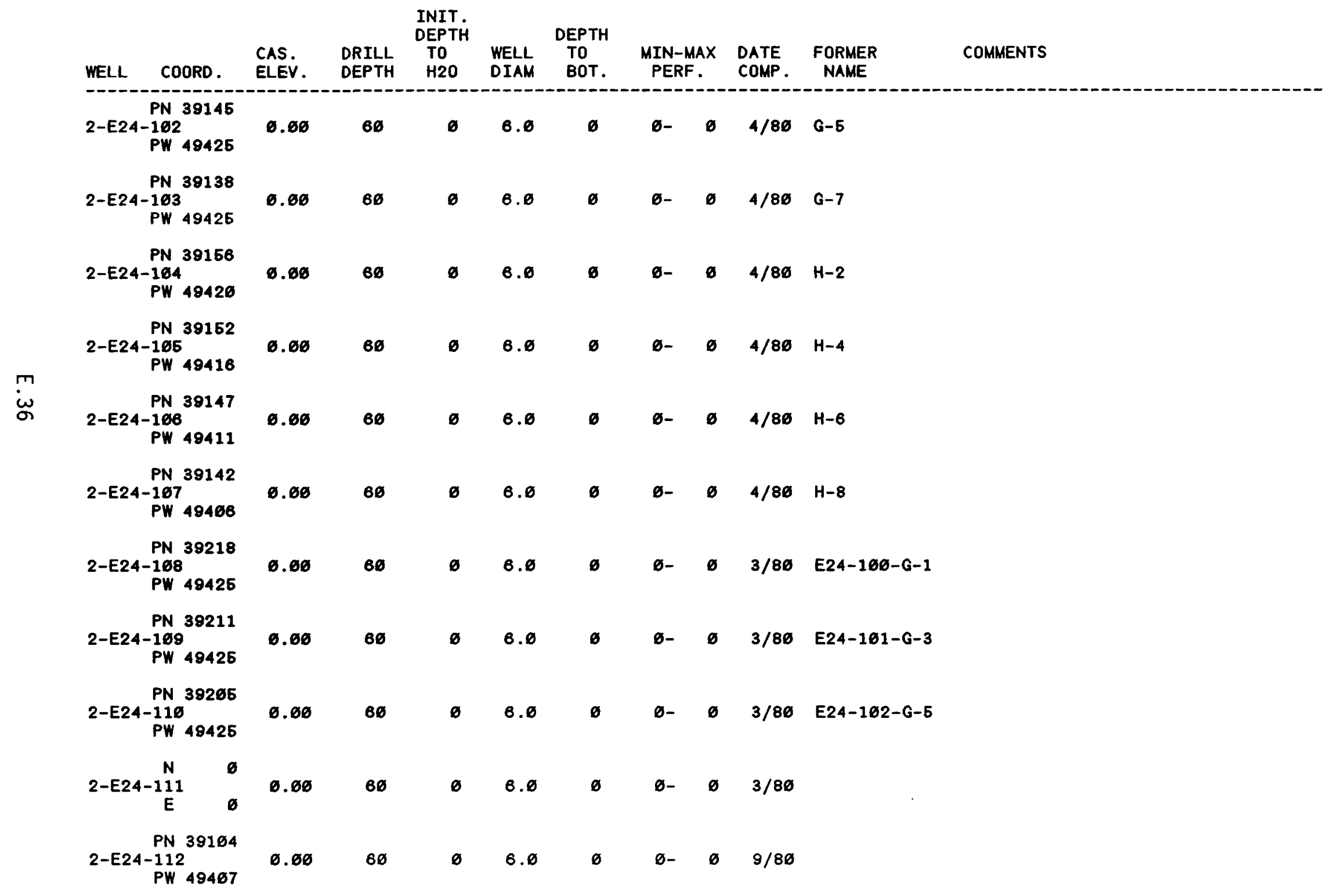




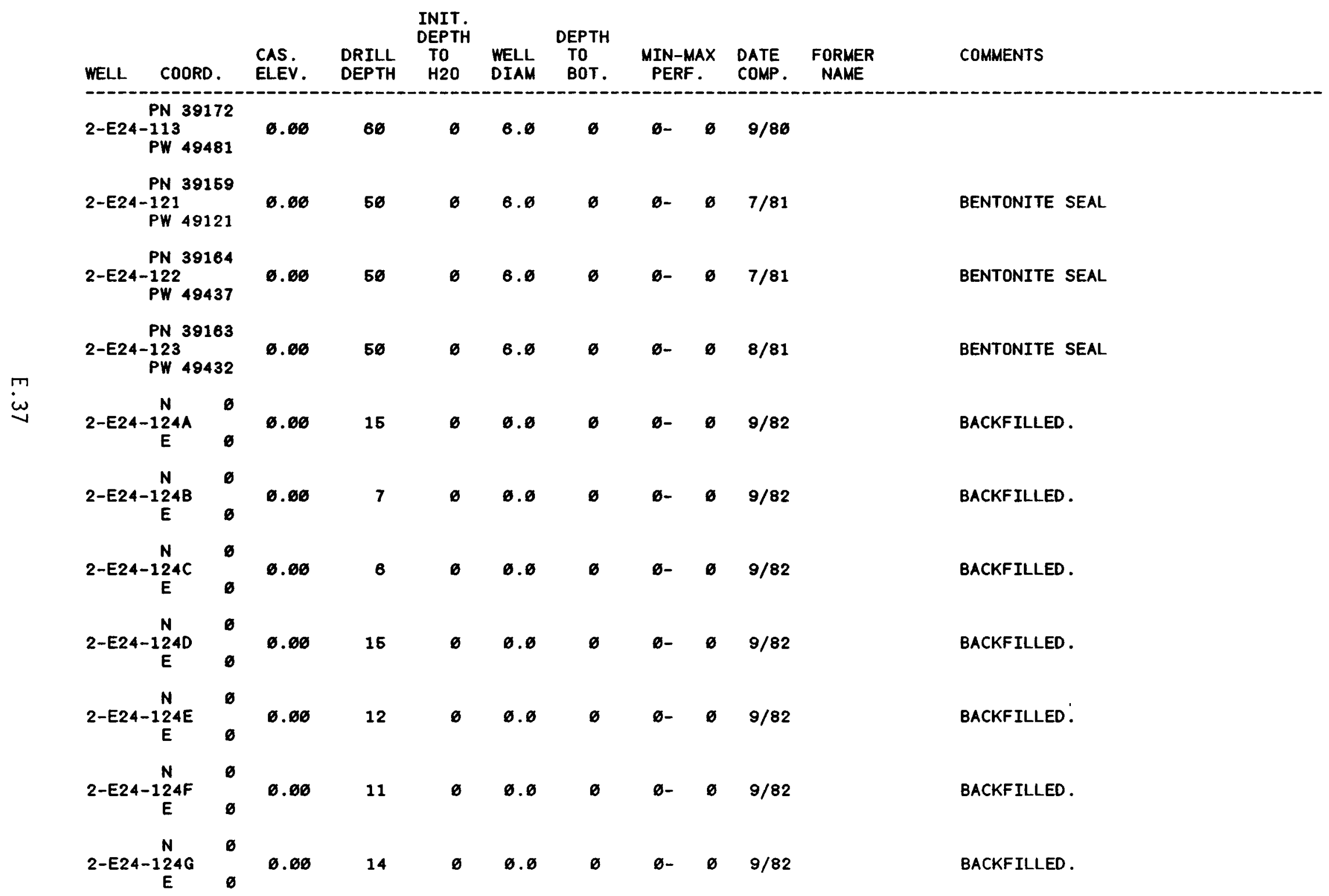




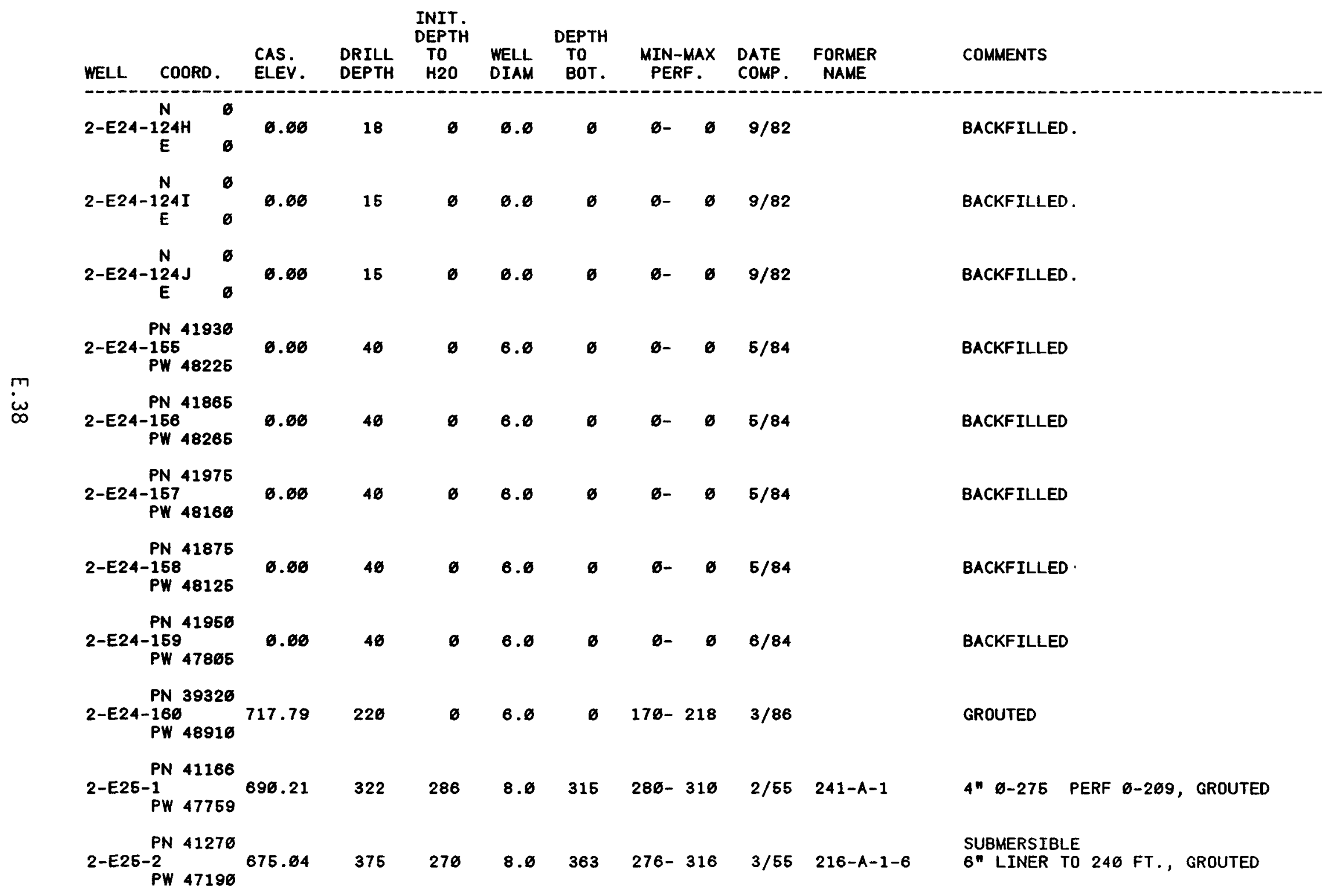




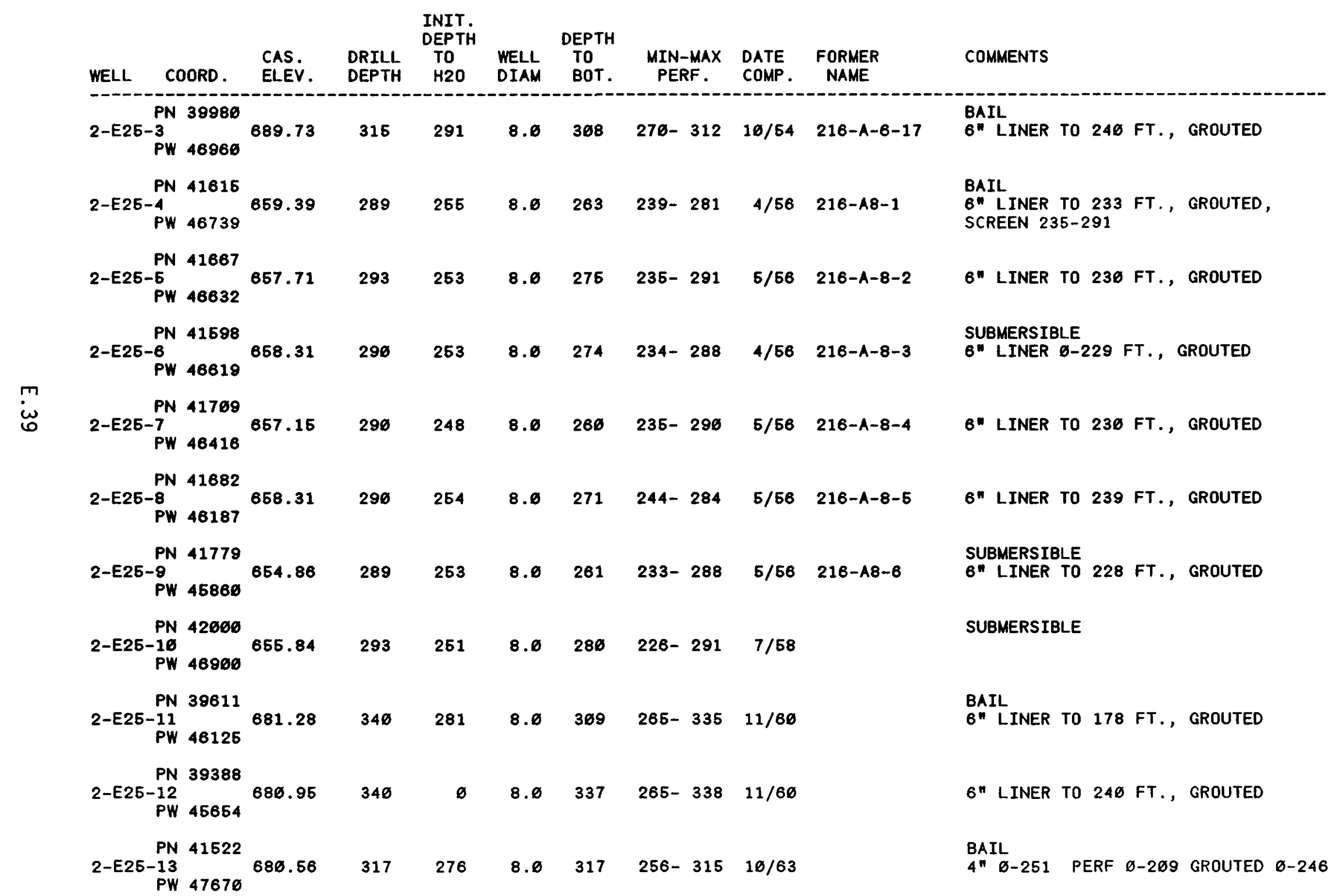




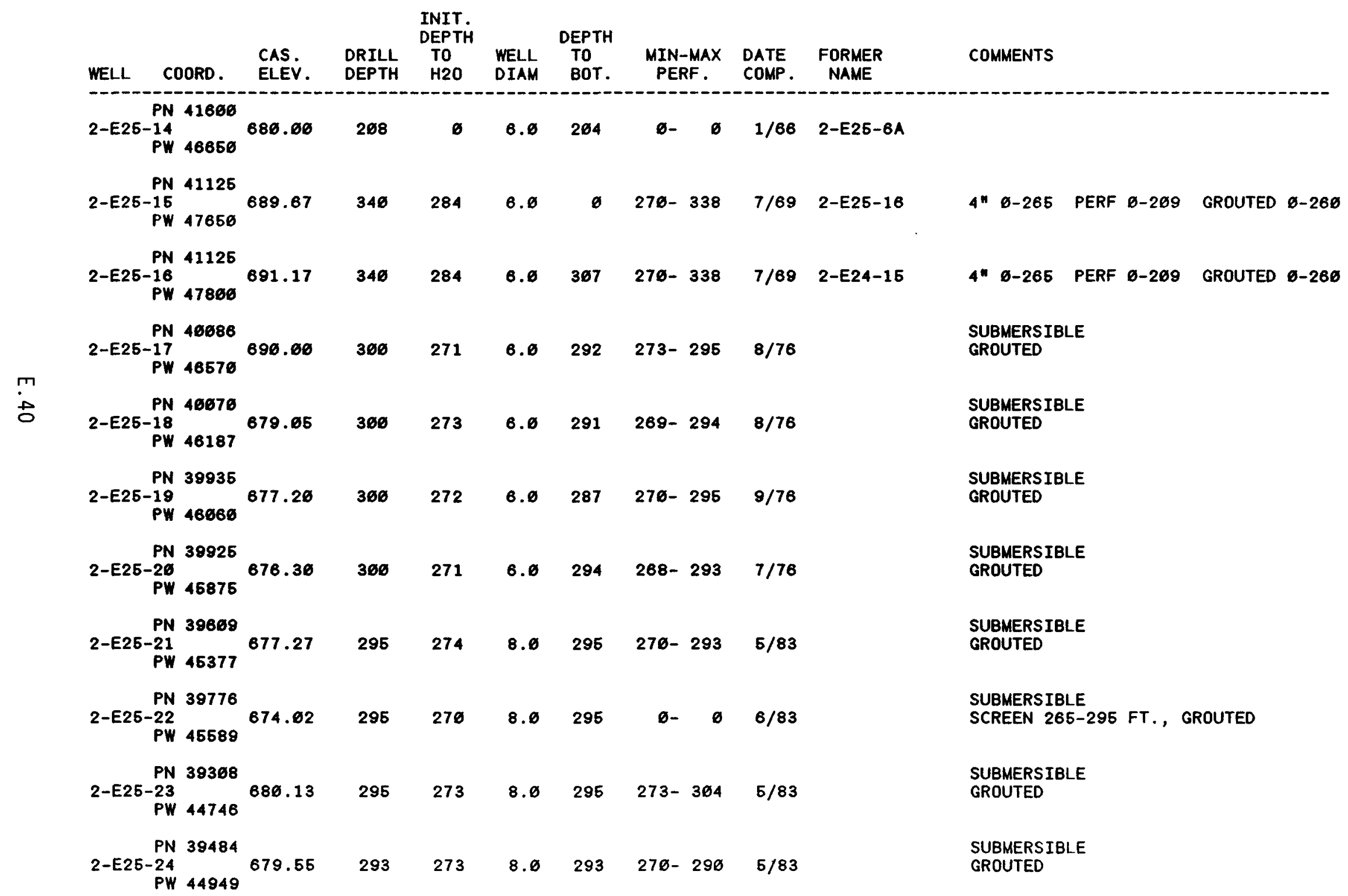




\begin{tabular}{|c|c|c|c|c|c|c|c|c|c|c|}
\hline WELL & COORD. & $\begin{array}{l}\text { CAS. } \\
\text { ELEV. }\end{array}$ & $\begin{array}{l}\text { DRILL } \\
\text { DEPTH }\end{array}$ & $\begin{array}{l}\text { INIT. } \\
\text { DEPTH } \\
\text { TO } \\
\text { H2O }\end{array}$ & $\begin{array}{l}\text { WELL } \\
\text { DIAM }\end{array}$ & $\begin{array}{l}\text { DEPTH } \\
\text { TO } \\
\text { BOT. }\end{array}$ & $\begin{array}{l}\text { MIN-MA } \\
\text { PERF. }\end{array}$ & & $\begin{array}{ll}\text { DATE } & \text { FORMER } \\
\text { COMP. } & \text { NAME }\end{array}$ & COMMENTS \\
\hline 2-E25- & $\begin{array}{l}\text { PN } 41062 \\
25 \\
\text { PW } 43648\end{array}$ & 689.42 & 288 & 263 & 6.0 & 288 & g- & $\emptyset$ & $4 / 85$ & $\begin{array}{l}\text { HYDROSTAR } \\
\text { SCREEN } 269-289 \text { FT. }\end{array}$ \\
\hline 2-E25 & $\begin{array}{ll}\text { PN } & 46773 \\
26 & \\
\text { PW } & 45884\end{array}$ & 688.65 & 290 & 263 & 6.0 & 289 & 6- & $\emptyset$ & $4 / 85$ & $\begin{array}{l}\text { SUBMERSIBLE } \\
\text { SCREEN } 27 \theta-290 \mathrm{FT} .\end{array}$ \\
\hline 2-E25 & $\begin{array}{ll}\text { PN } & 39856 \\
-27 & \\
\text { PW } & 45136\end{array}$ & 676.10 & 294 & 270 & 6.0 & 292 & ø- & 6 & $5 / 85$ & $\begin{array}{l}\text { HYDROSTAR } \\
\text { SCREEN 274-294 FT. }\end{array}$ \\
\hline 2-E25- & $\begin{array}{ll}\text { PN } & 41424 \\
28 & \\
\text { PW } & 45541\end{array}$ & 682.44 & 340 & 0 & 6.0 & 335 & ס- & 6 & $4 / 88$ & $\begin{array}{l}\text { SUBMERSIBLE } \\
\text { SCREEN } 32 \varnothing-34 \varnothing \mathrm{FT} .\end{array}$ \\
\hline 2-E25- & $\begin{array}{ll}\text { PN } & 46169 \\
-29 & \\
\text { PW } & 46734\end{array}$ & 672.84 & 6 & D & $\varnothing . \varnothing$ & 330 & - & $\emptyset$ & & HYDROSTAR \\
\hline 2-E25- & $\begin{array}{l}\text { PN } 39710 \\
-36 \\
\text { PW } \\
44960\end{array}$ & 678.15 & B & 0 & 0.0 & 6 & ø- & $\varnothing$ & & HYDROSTAR \\
\hline 2-E25- & $\begin{array}{ll}\text { PN } & 46311 \\
-31 & \\
\text { PW } & 45752\end{array}$ & 672.53 & б & D & 6.0 & 298 & 6- & $\emptyset$ & & HYDROSTAR \\
\hline 2-E25 & $\begin{array}{ll}P N & 11206 \\
-32 & \\
P W & 44325\end{array}$ & 669.19 & 6 & ø & 0.0 & 351 & 6- & ø & & HYOROSTAR \\
\hline 2-E25- & $\begin{array}{l}\text { PN } 46116 \\
-33 \\
\text { PW } 456099\end{array}$ & 674.97 & 400 & $\emptyset$ & 10.0 & 397 & a- & 0 & $1 / 88$ & HYDROSTAR \\
\hline 2-E25- & $\begin{array}{c}\text { PN } 41385 \\
-34 \\
\text { PW } \\
45616\end{array}$ & 662.87 & 276 & 256 & 4.0 & 272 & 6- & 0 & $9 / 88$ & $\begin{array}{l}\text { HYDROSTAR } \\
\text { SCREEN 252-272 FT GROUTED }\end{array}$ \\
\hline $2-E 25$ & $\begin{array}{ll}\text { PN } & 46616 \\
-35 & \\
\text { PW } & 46538\end{array}$ & 674.39 & 285 & 264 & 4.6 & 281 & $0-$ & 0 & $7 / 88$ & $\begin{array}{l}\text { HYDROSTAR } \\
\text { SCREEN } 26 \varnothing-281 \text { FT GROUTED }\end{array}$ \\
\hline
\end{tabular}




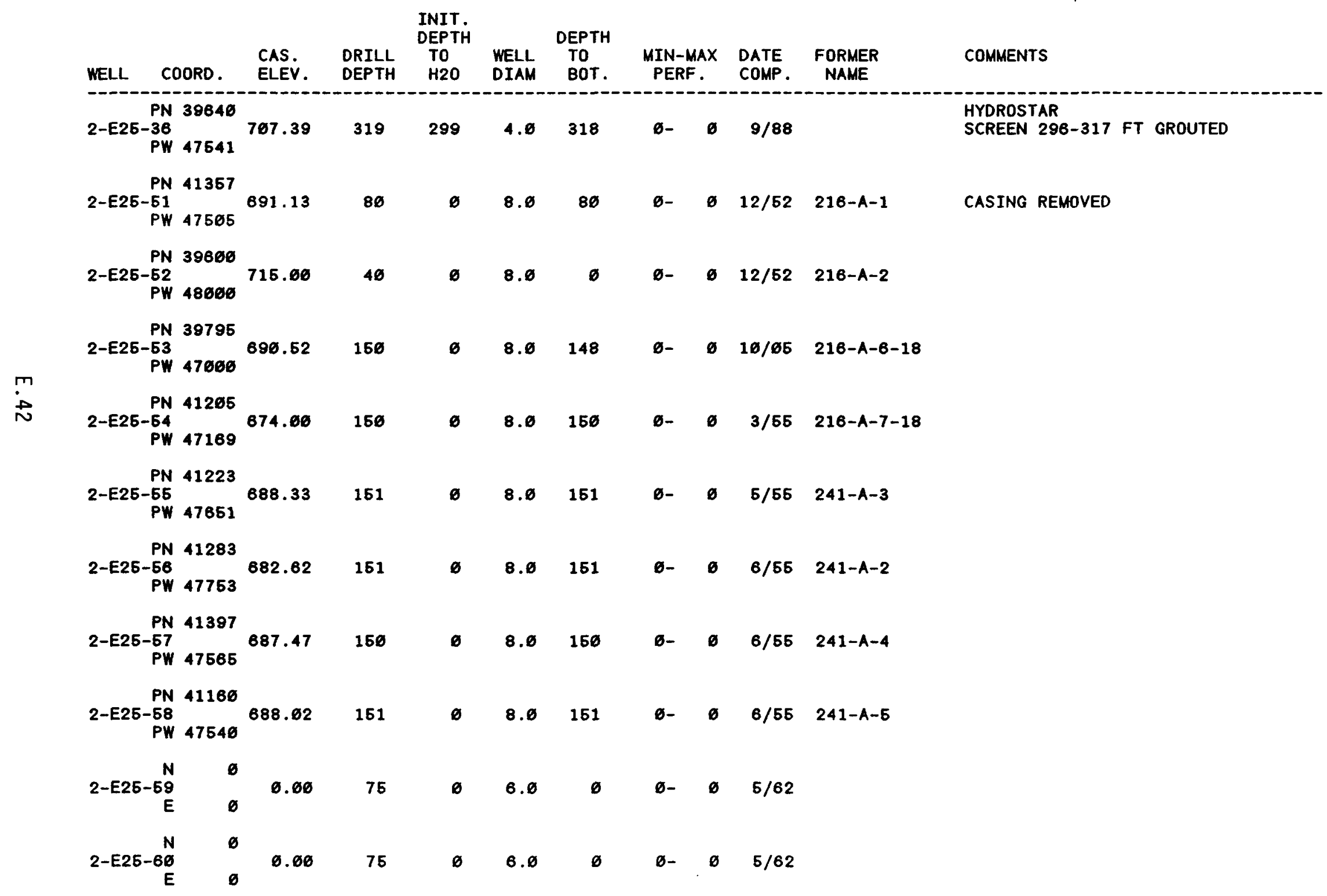




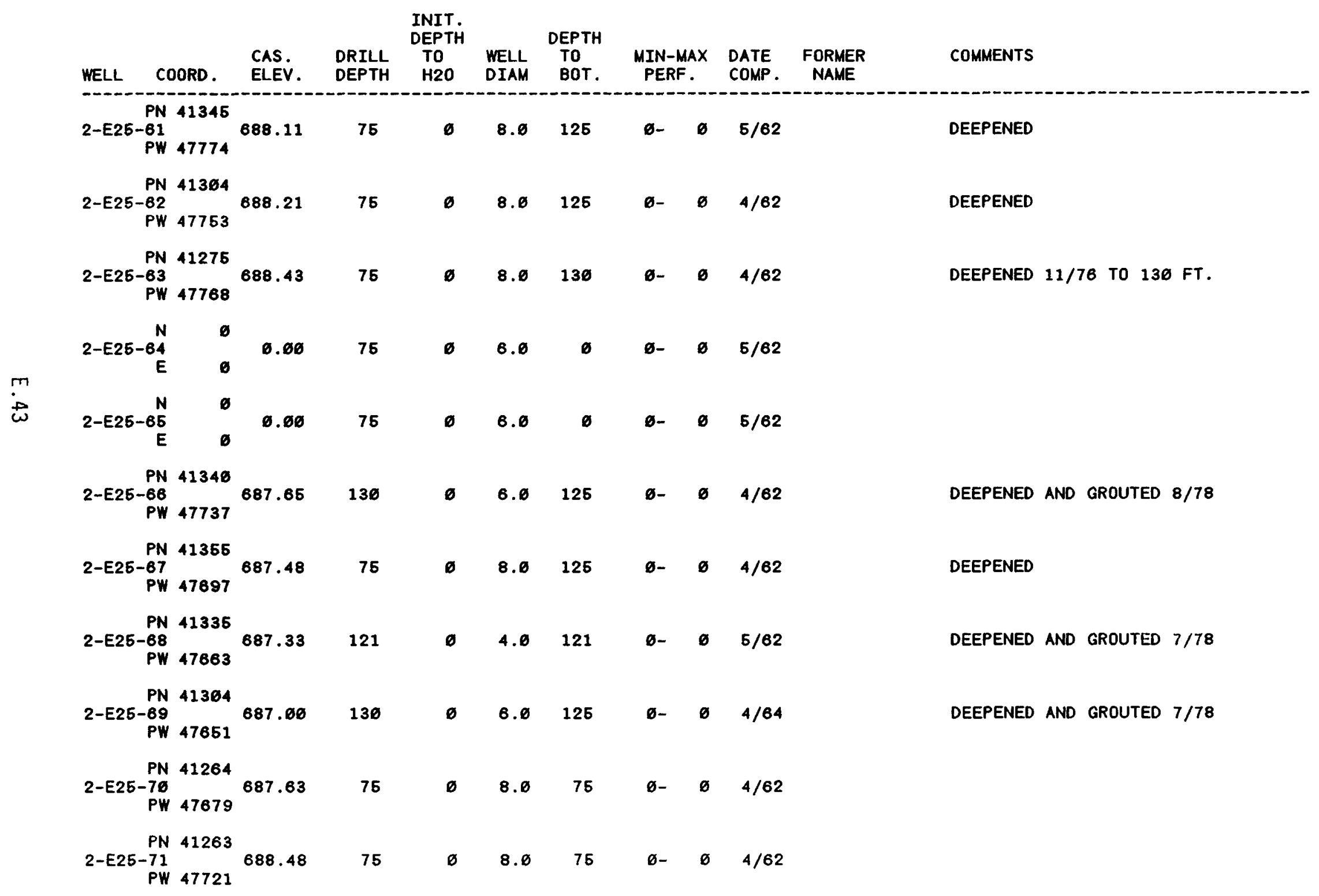




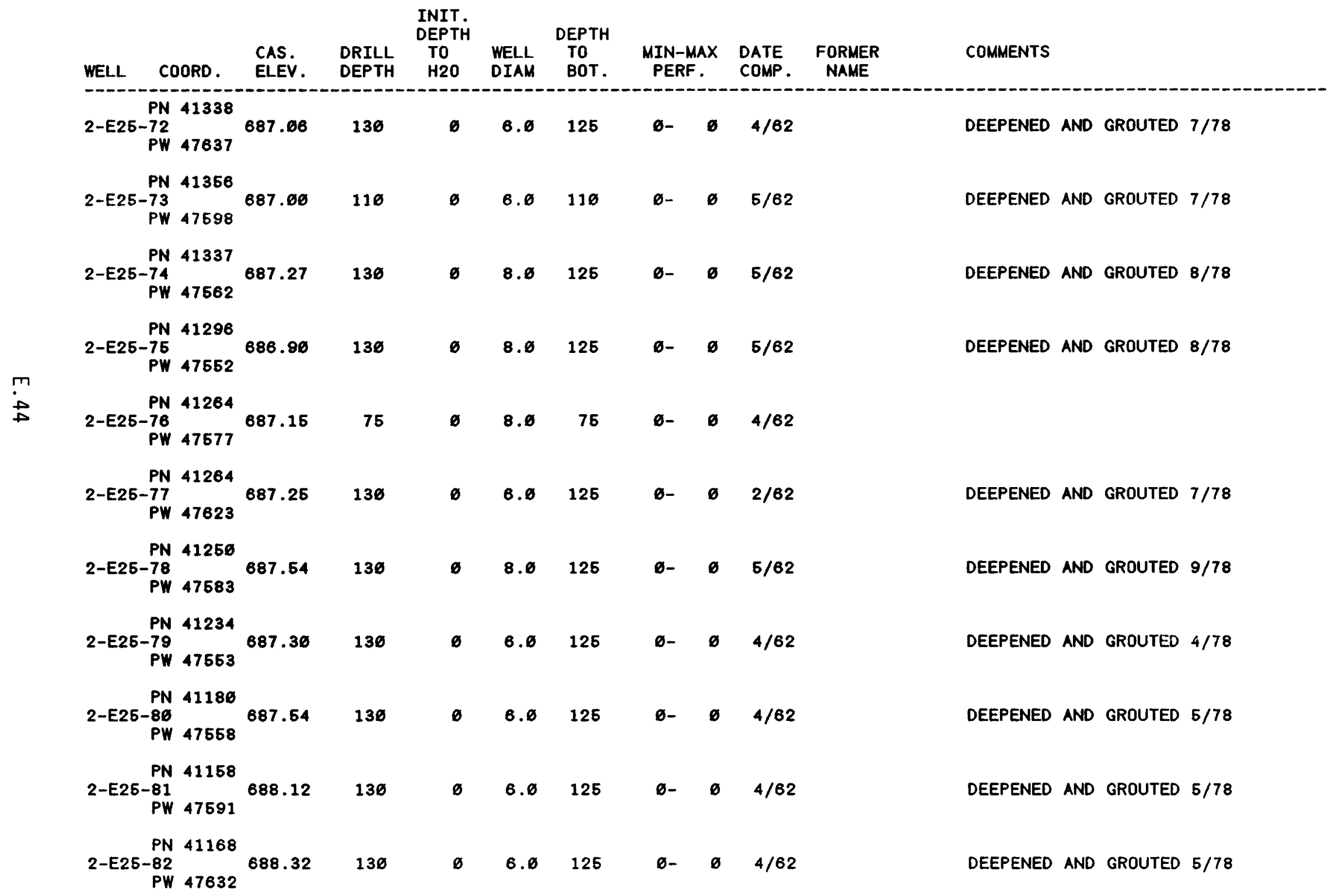




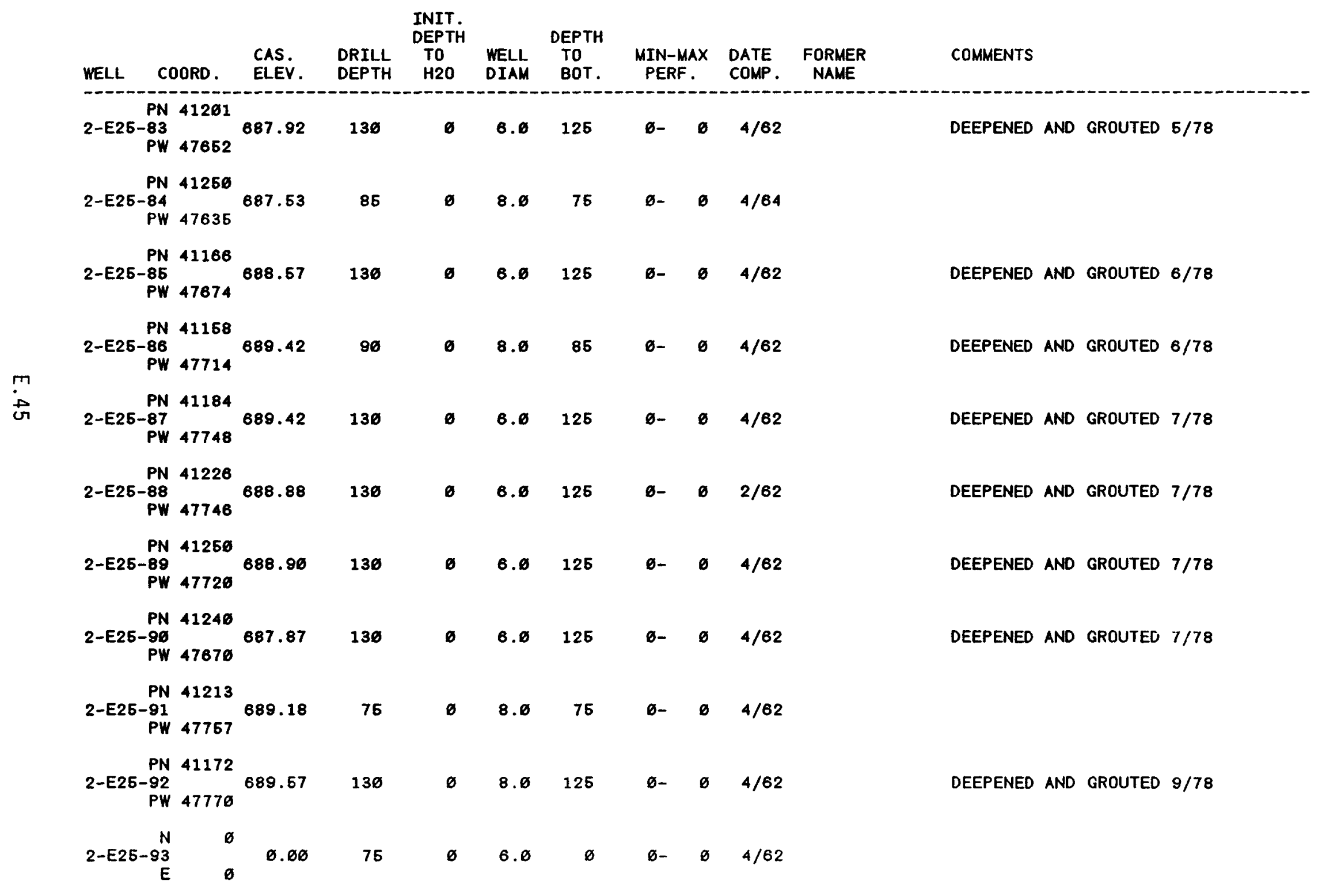




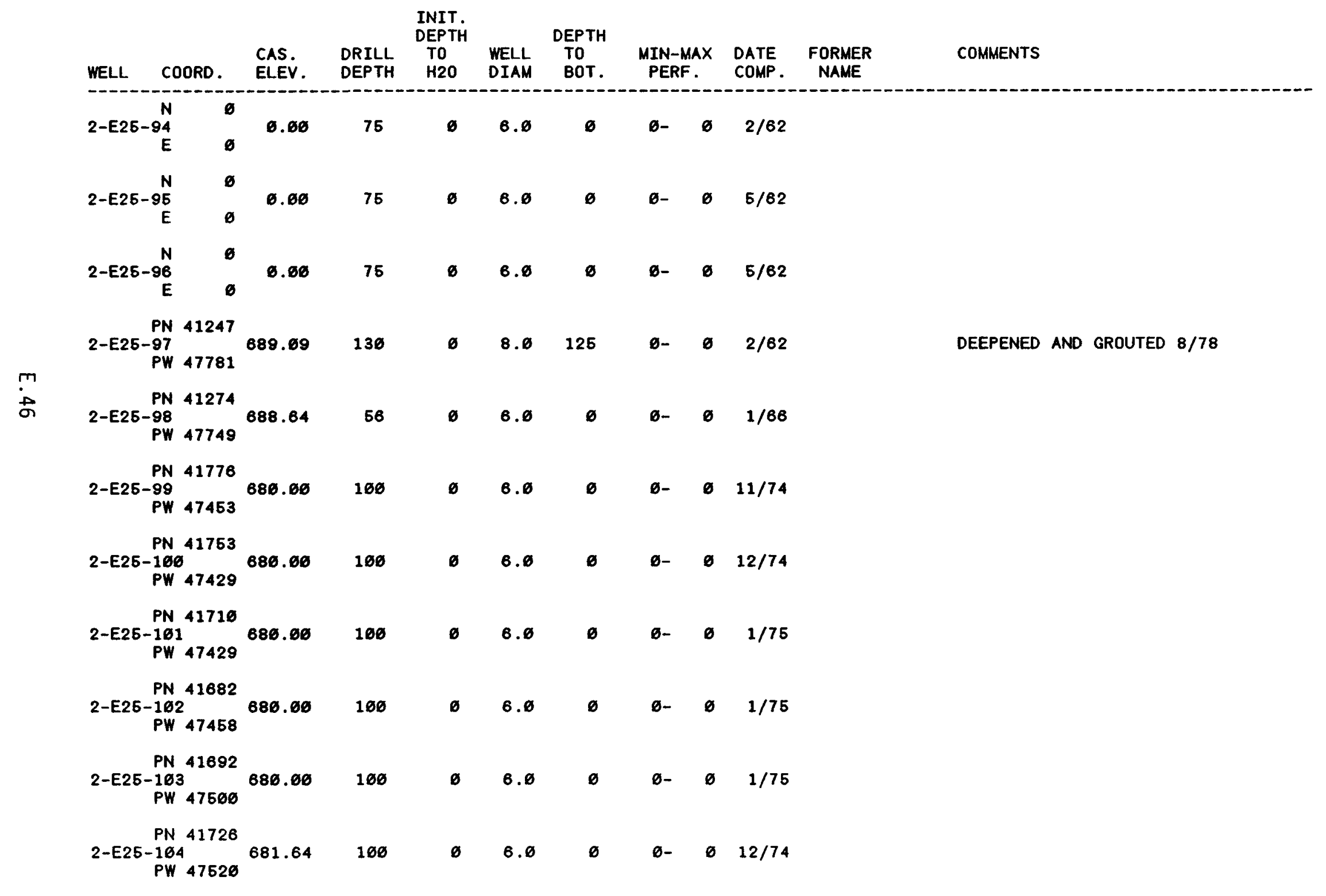




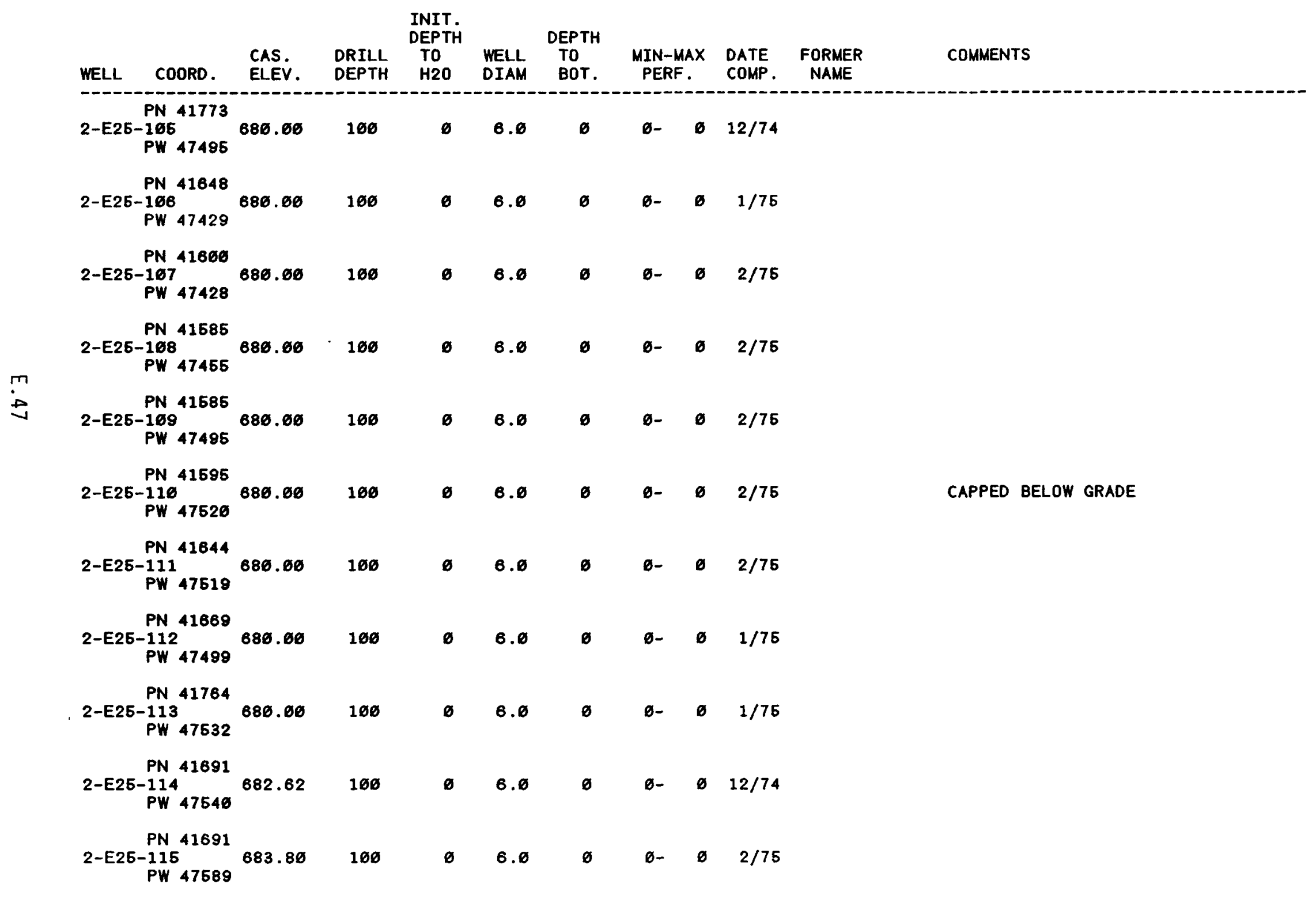




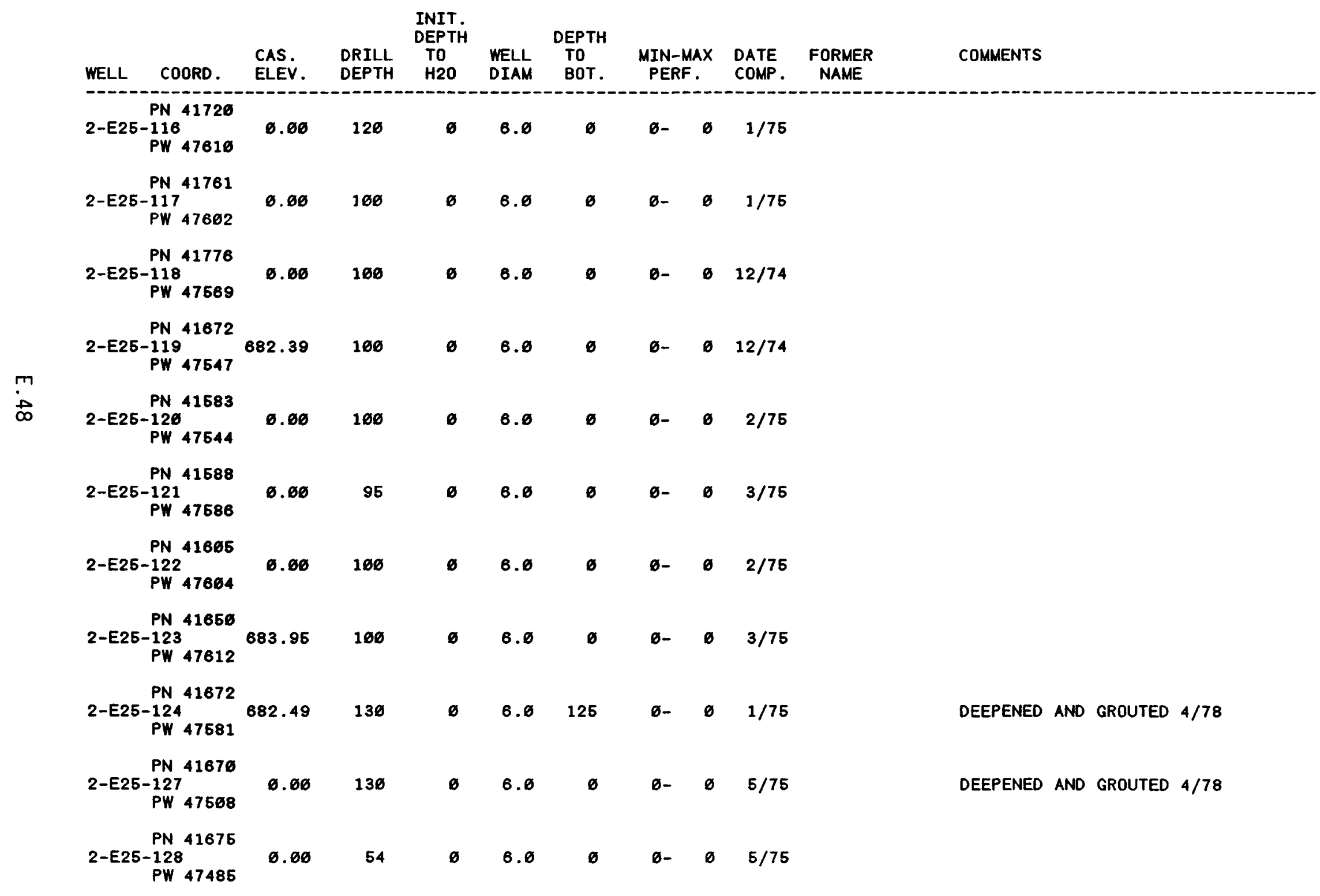




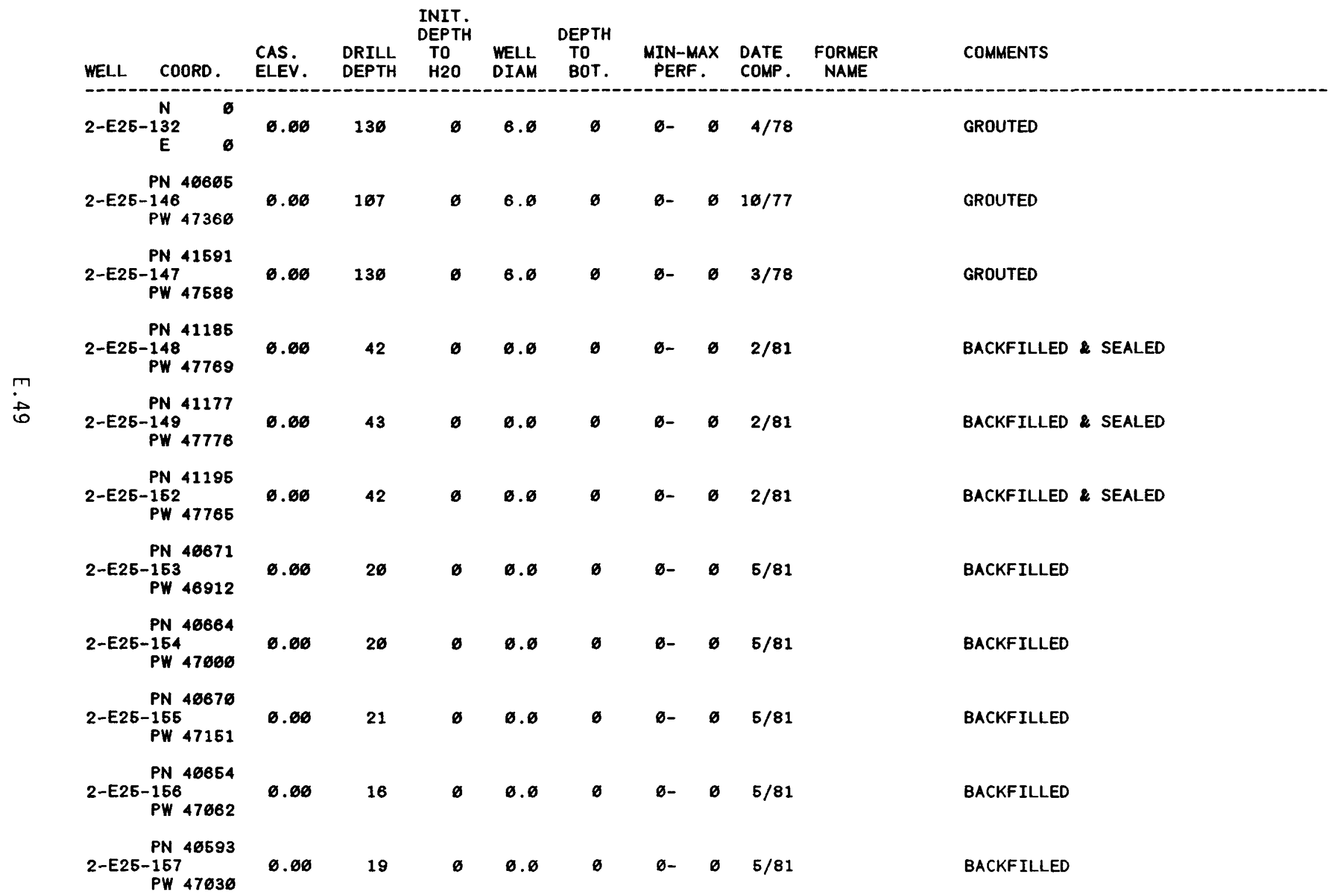




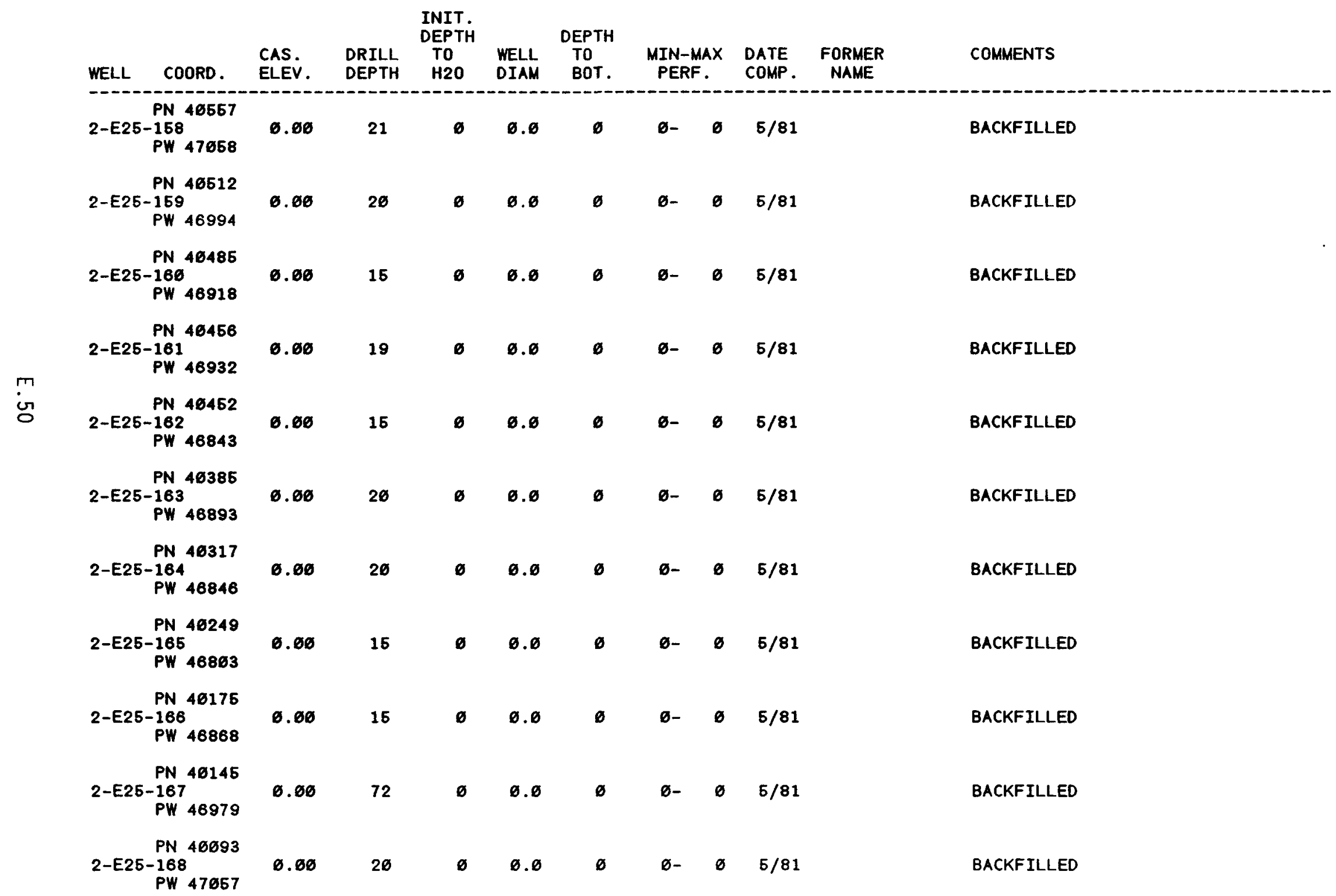




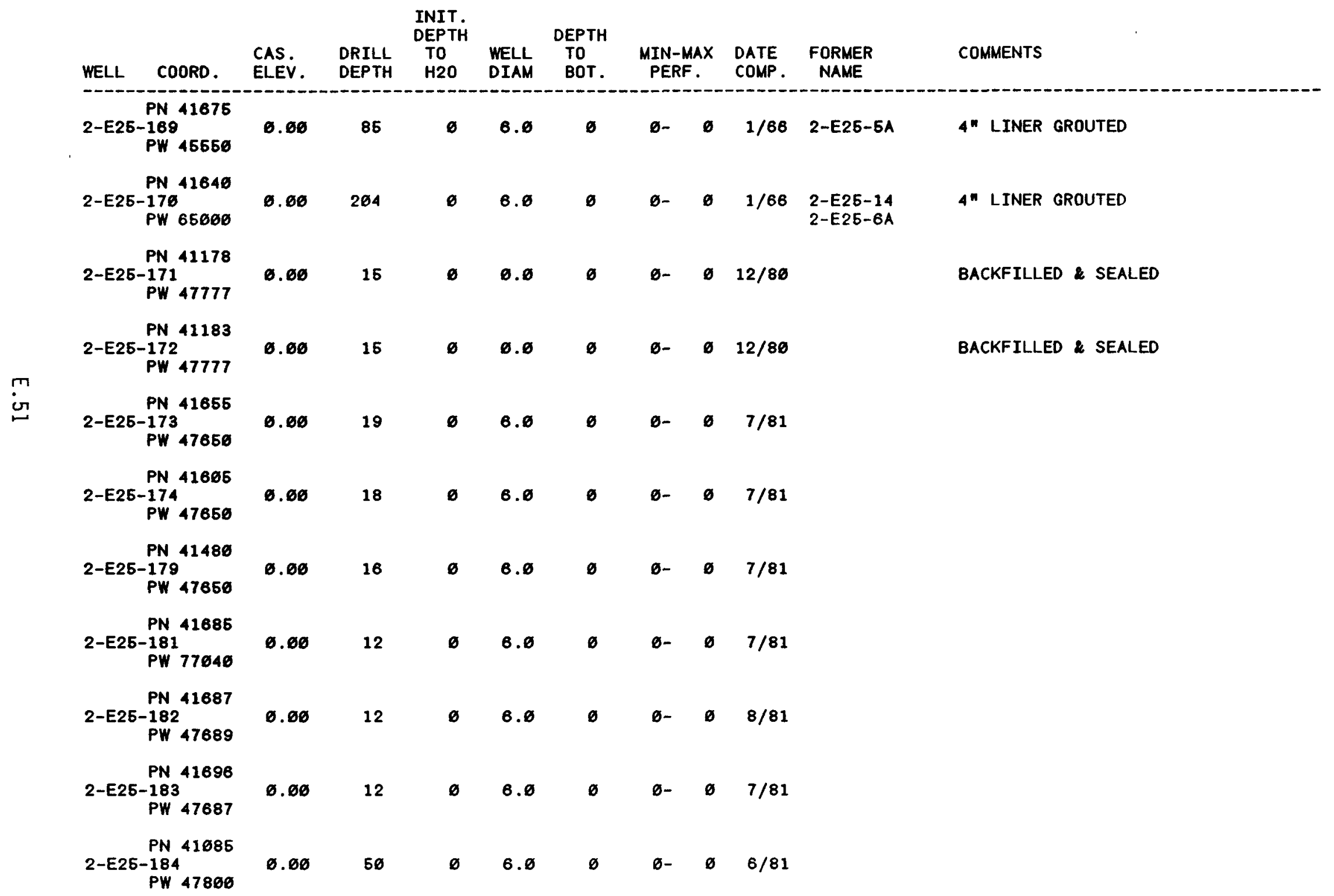




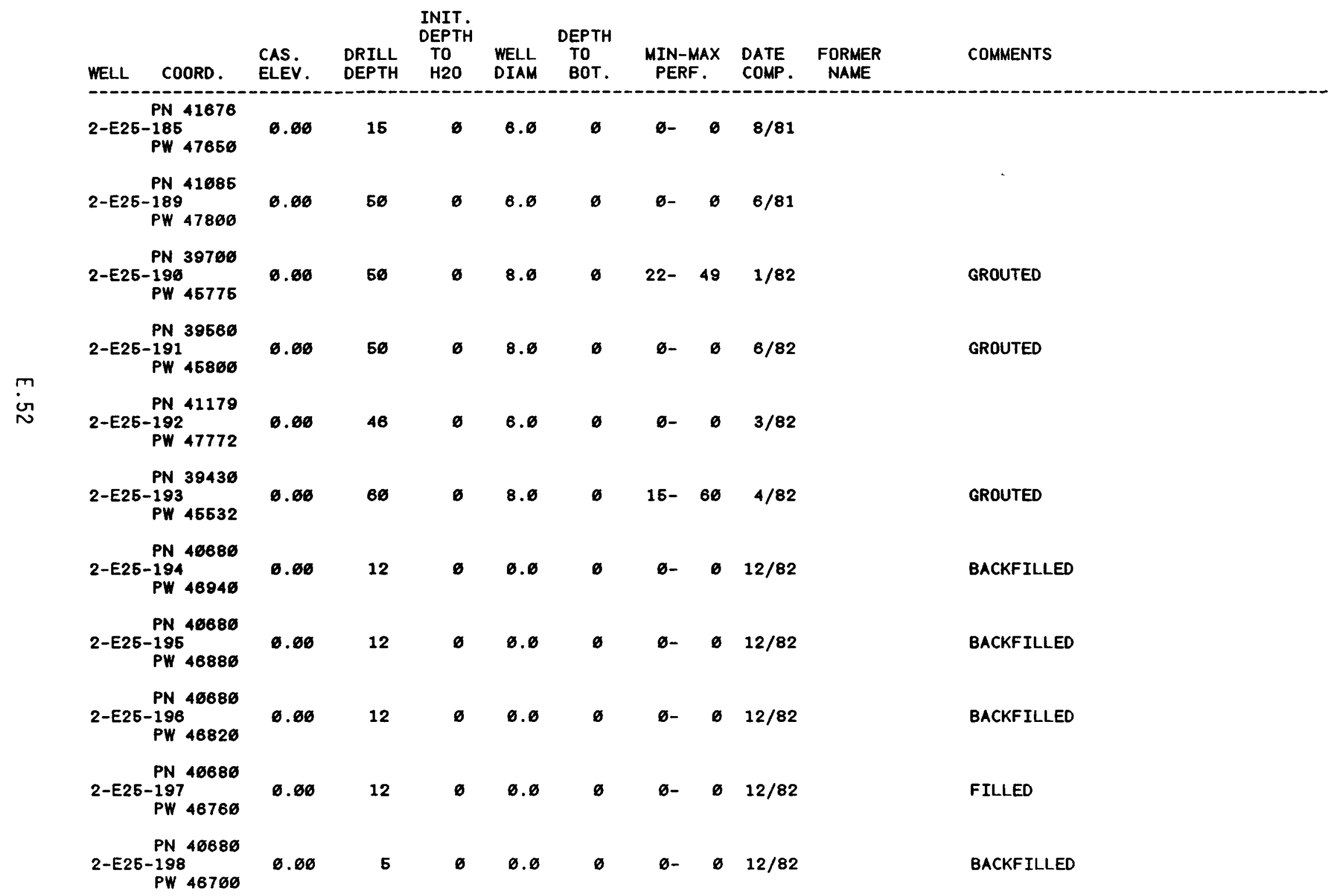




\begin{tabular}{|c|c|c|c|c|c|c|c|c|c|c|c|}
\hline WELL & COORD. & $\begin{array}{l}\text { CAS. } \\
\text { ELEV. }\end{array}$ & $\begin{array}{l}\text { DRILL } \\
\text { DEPTH }\end{array}$ & $\begin{array}{l}\text { INIT. } \\
\text { DEPTH } \\
\text { TO } \\
\text { H2O }\end{array}$ & $\begin{array}{l}\text { WELL } \\
\text { DIAM }\end{array}$ & $\begin{array}{l}\text { DEPTH } \\
\text { TO } \\
\text { BOT. }\end{array}$ & $\begin{array}{l}\text { MIN-MA } \\
\text { PERF. }\end{array}$ & & $\begin{array}{l}\text { DATE } \\
\text { COMP. }\end{array}$ & $\begin{array}{c}\text { FORMER } \\
\text { NAME }\end{array}$ & COMMENTS \\
\hline 2-E25 & $\begin{array}{l}\text { PN } 40690 \\
199 \\
\text { PW } 46940\end{array}$ & 0.06 & 12 & $\varnothing$ & 0.6 & b & $0-$ & 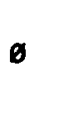 & $12 / 82$ & & BACKFILLED \\
\hline 2-E25. & $\begin{array}{l}P N 46890 \\
262 \\
P W{ }^{26760}\end{array}$ & 0.00 & 12 & 6 & 6.6 & $\sigma$ & $0-$ & $\sigma$ & $12 / 82$ & & BACKFILLED \\
\hline 2-E25. & $\begin{array}{l}\text { PN } 46670 \\
263 \\
P W \quad 46706\end{array}$ & 0.60 & 12 & 6 & $\varnothing .6$ & ø & $0-$ & $\sigma$ & $12 / 82$ & & BACKF ILLED \\
\hline 2-E25 & $\begin{array}{l}\text { PN } 41187 \\
204 \\
\text { PW } 47768\end{array}$ & 0.00 & 45 & $\emptyset$ & 6.0 & $\varnothing$ & D- & o & $1 / 84$ & & GROUTED \\
\hline 2-E25. & $\begin{array}{l}\text { PN } 41490 \\
285 \\
P W 46880\end{array}$ & 0.00 & 25 & 0 & 6.0 & $\emptyset$ & $0-$ & $\sigma$ & $2 / 84$ & & BACKFILLED \\
\hline 2-E25. & $\begin{array}{l}\text { PN } 41580 \\
266 \\
P W 46200\end{array}$ & 0.66 & 25 & 6 & 6.6 & 6 & D- & $\varnothing$ & $2 / 84$ & & BACKFILLED \\
\hline 2-E25 & $\begin{array}{l}P N 41636 \\
-267 \\
P W 46925\end{array}$ & 0.60 & 25 & 6 & 0.0 & $\varnothing$ & B- & 6 & $2 / 84$ & & BACKF ILLED \\
\hline 2-E25 & $\begin{array}{l}\text { PN } 41160 \\
208 \\
\text { PW } 46050\end{array}$ & 0.00 & 46 & ø & 0.0 & D & 6- & 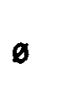 & $2 / 84$ & & BACKF ILLED \\
\hline 2-E25. & $\begin{array}{l}\text { PN } 468000 \\
-269 \\
P W 46600\end{array}$ & 0.00 & 25 & b & 0.0 & $\varnothing$ & 8- & 0 & $2 / 84$ & & BACKFILLED \\
\hline 2-E25 & $\begin{array}{l}\text { PN } 46540 \\
-216 \\
P W 46180\end{array}$ & 6.06 & 25 & 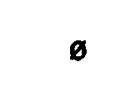 & 0.8 & $\varnothing$ & B- & $\theta$ & $1 / 84$ & & BACKFILLED \\
\hline 2-E25 & $\begin{array}{l}P N 41675 \\
-211 \\
P W{ }_{45000}\end{array}$ & 0.00 & 25 & $\varnothing$ & 0.0 & $\emptyset$ & 6- & 6 & $2 / 84$ & & BACKFILLED \\
\hline
\end{tabular}




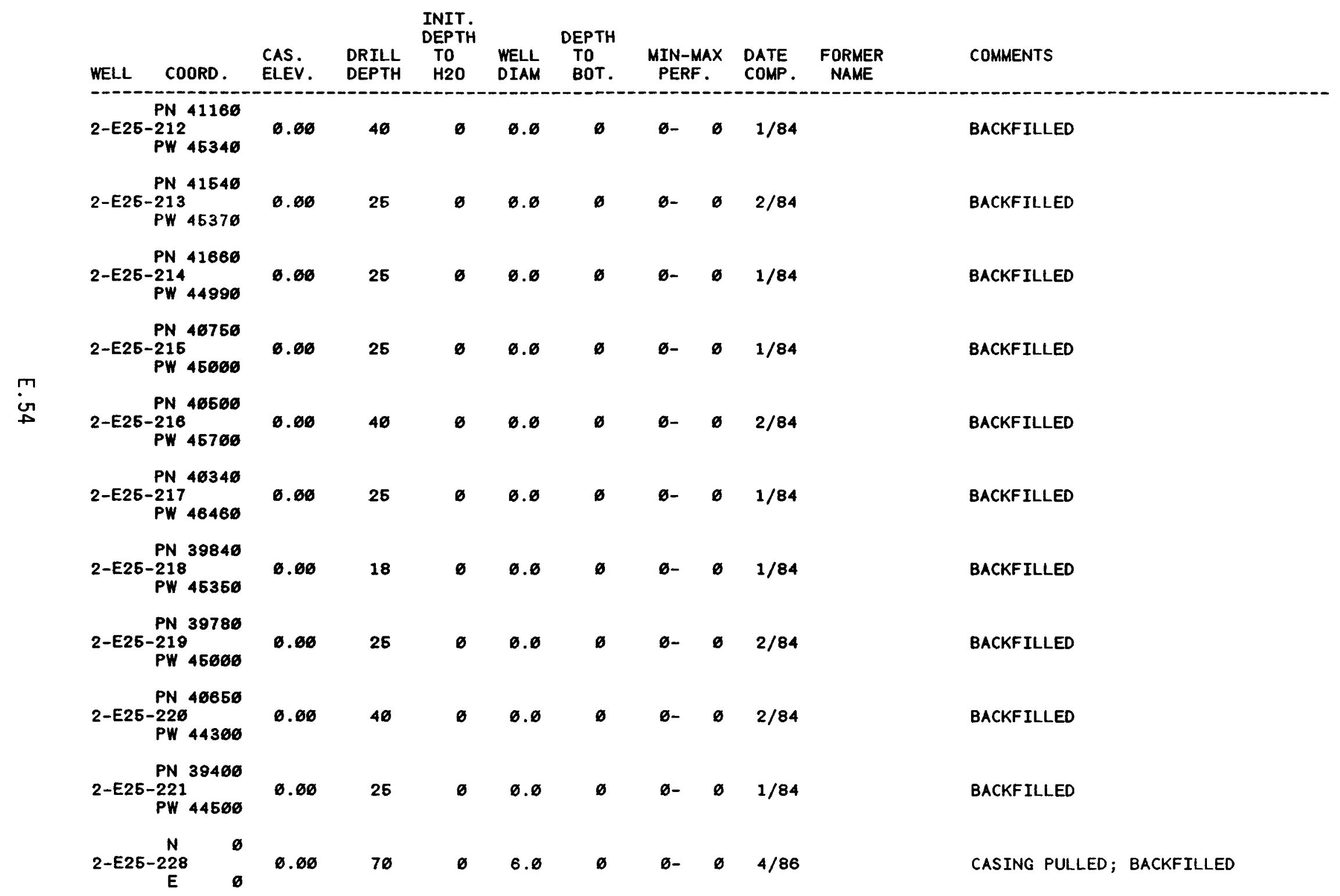




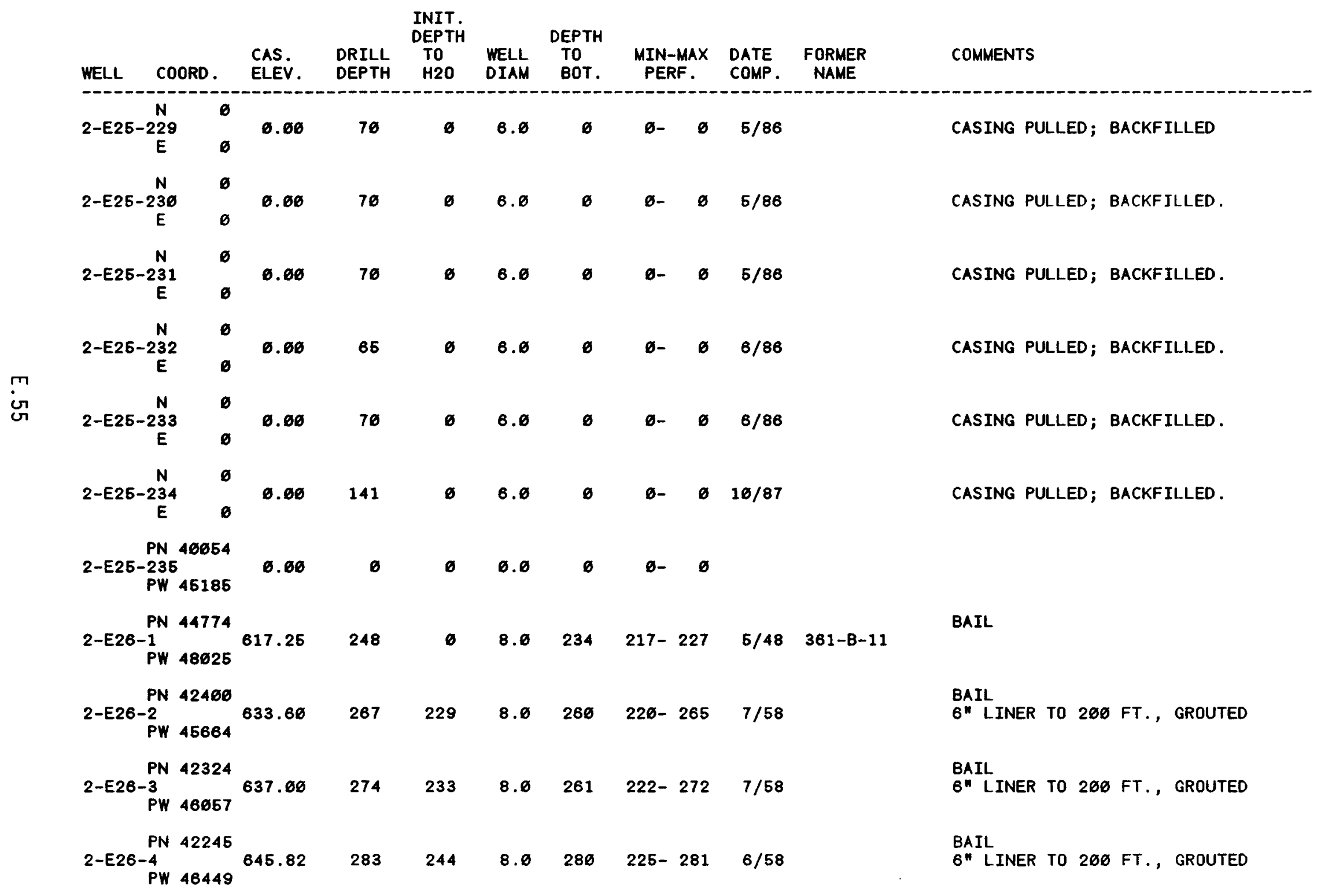




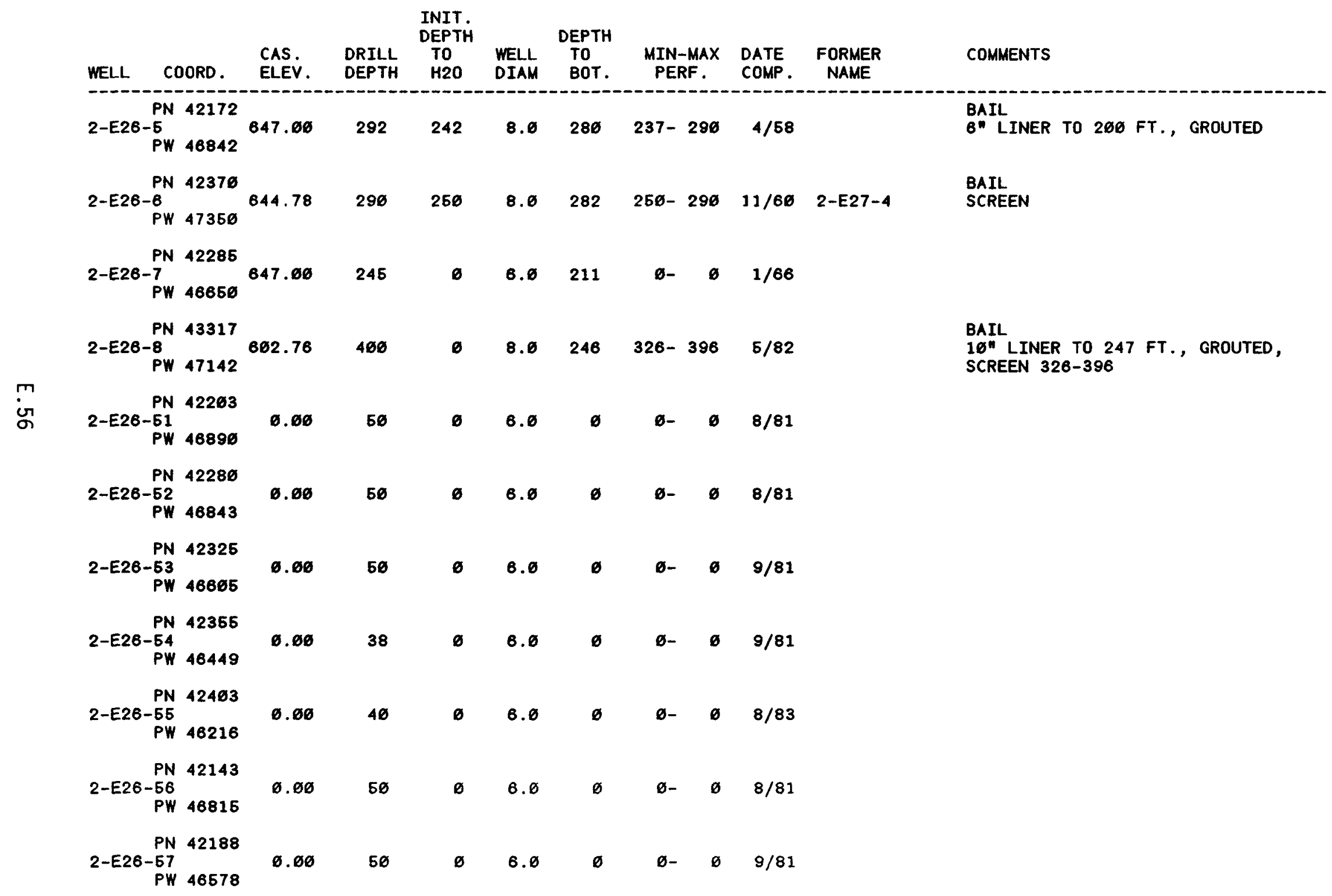




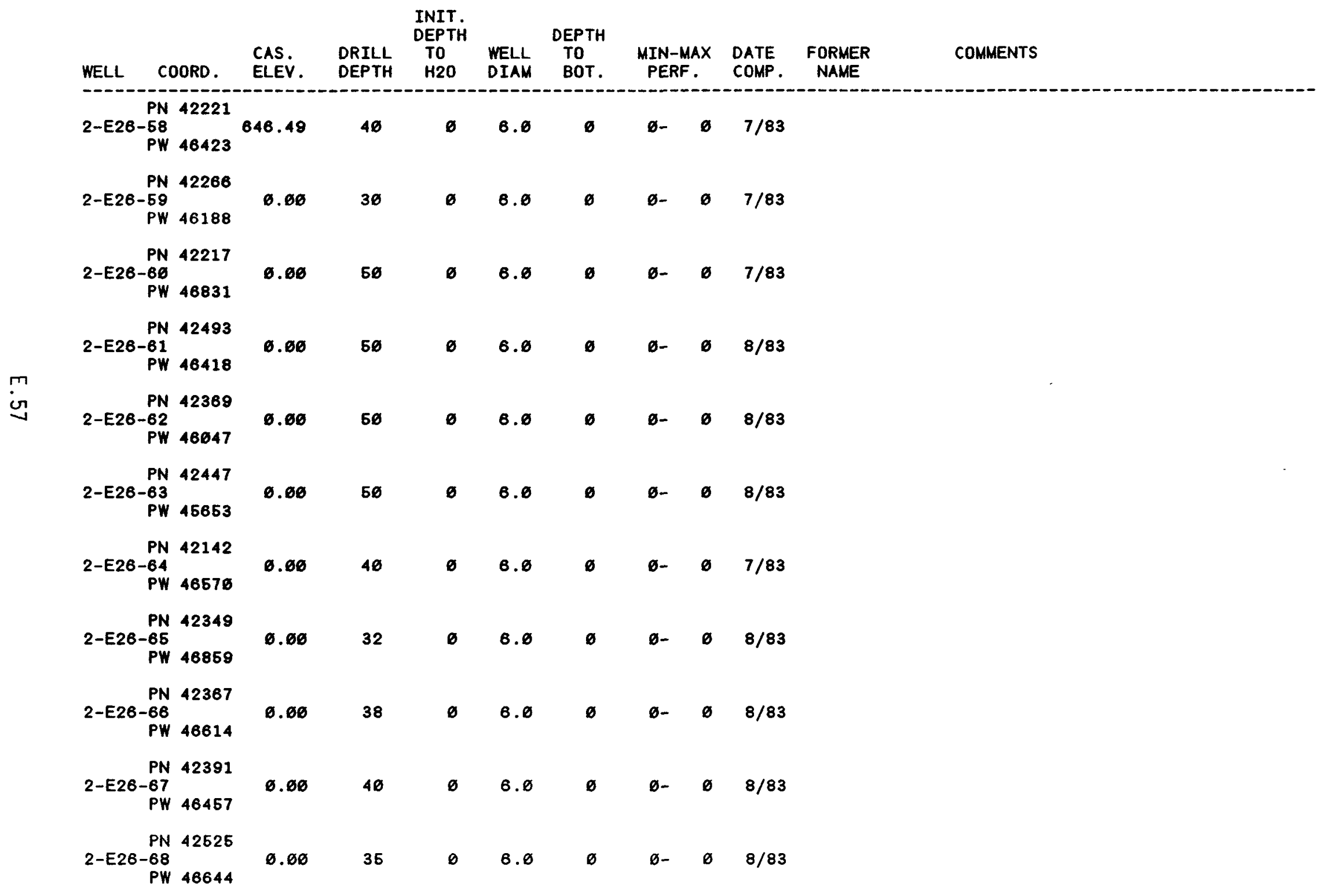




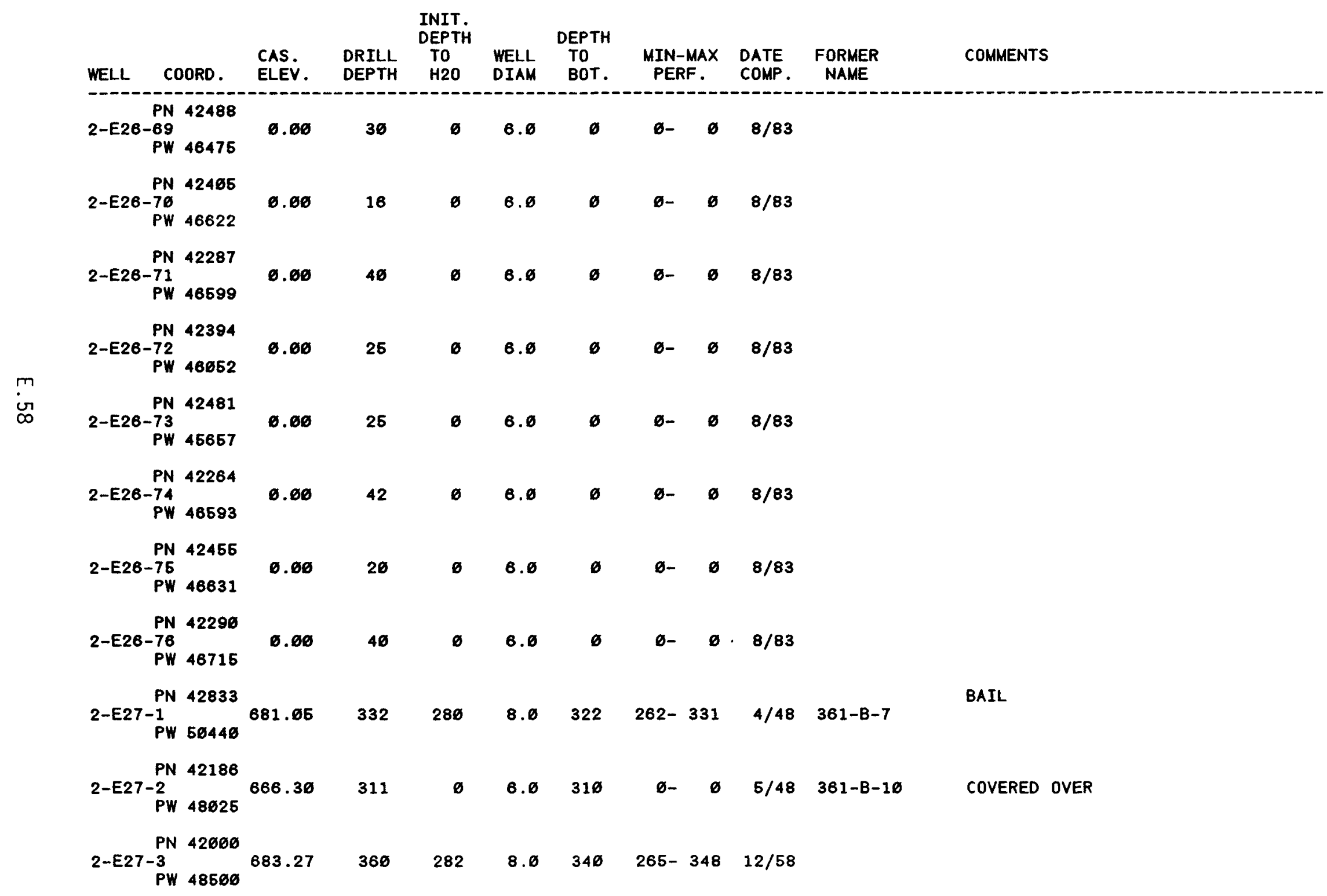




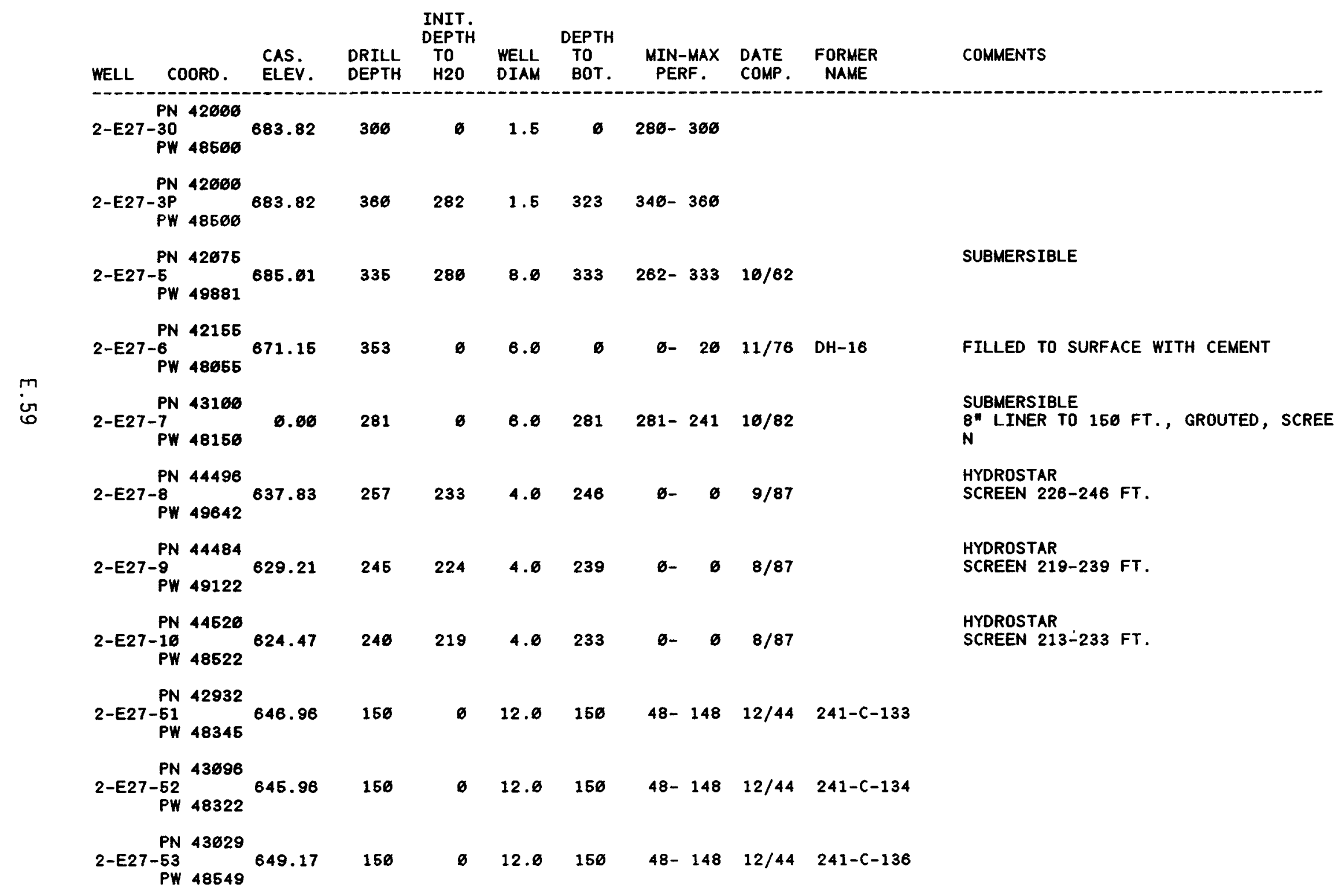




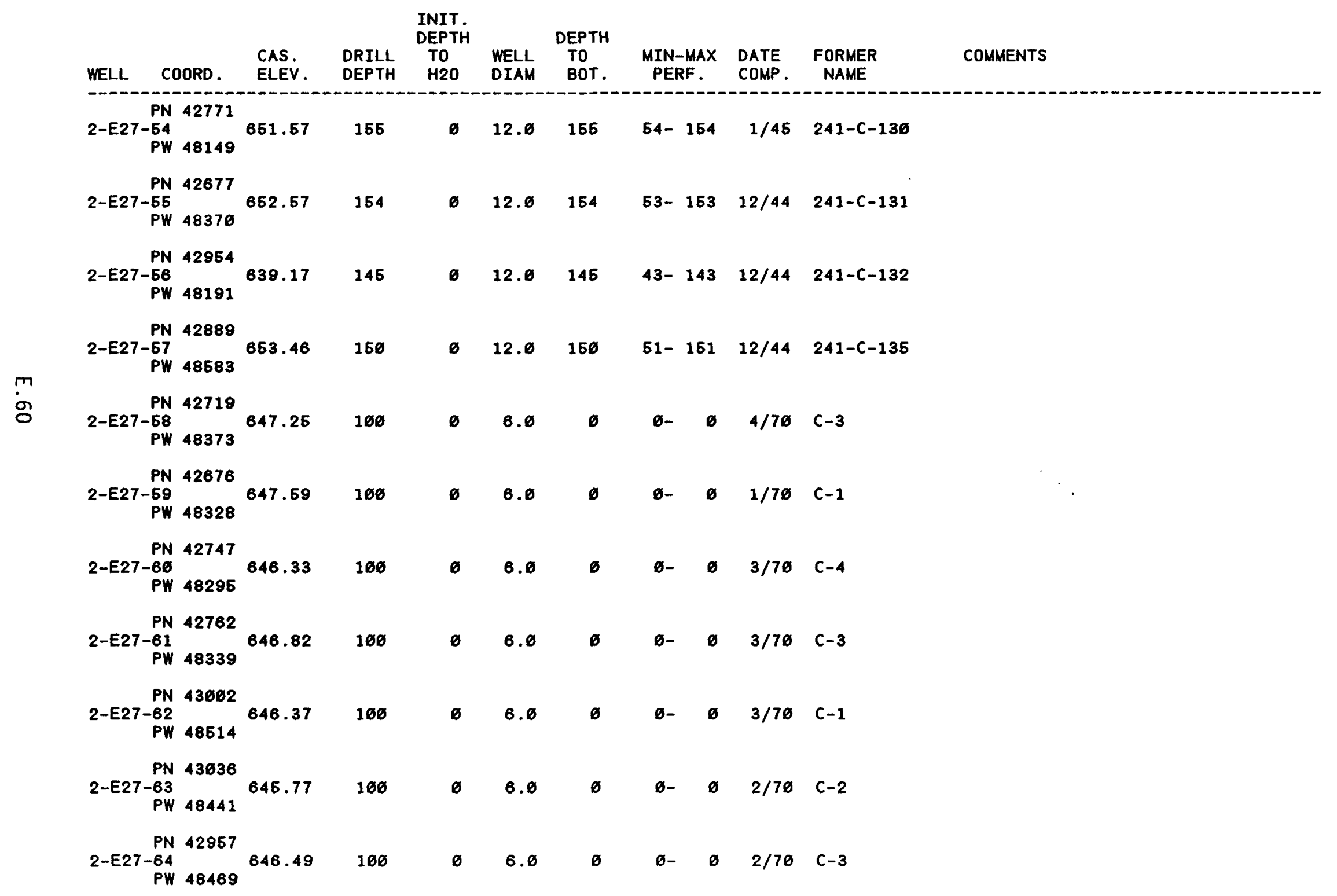




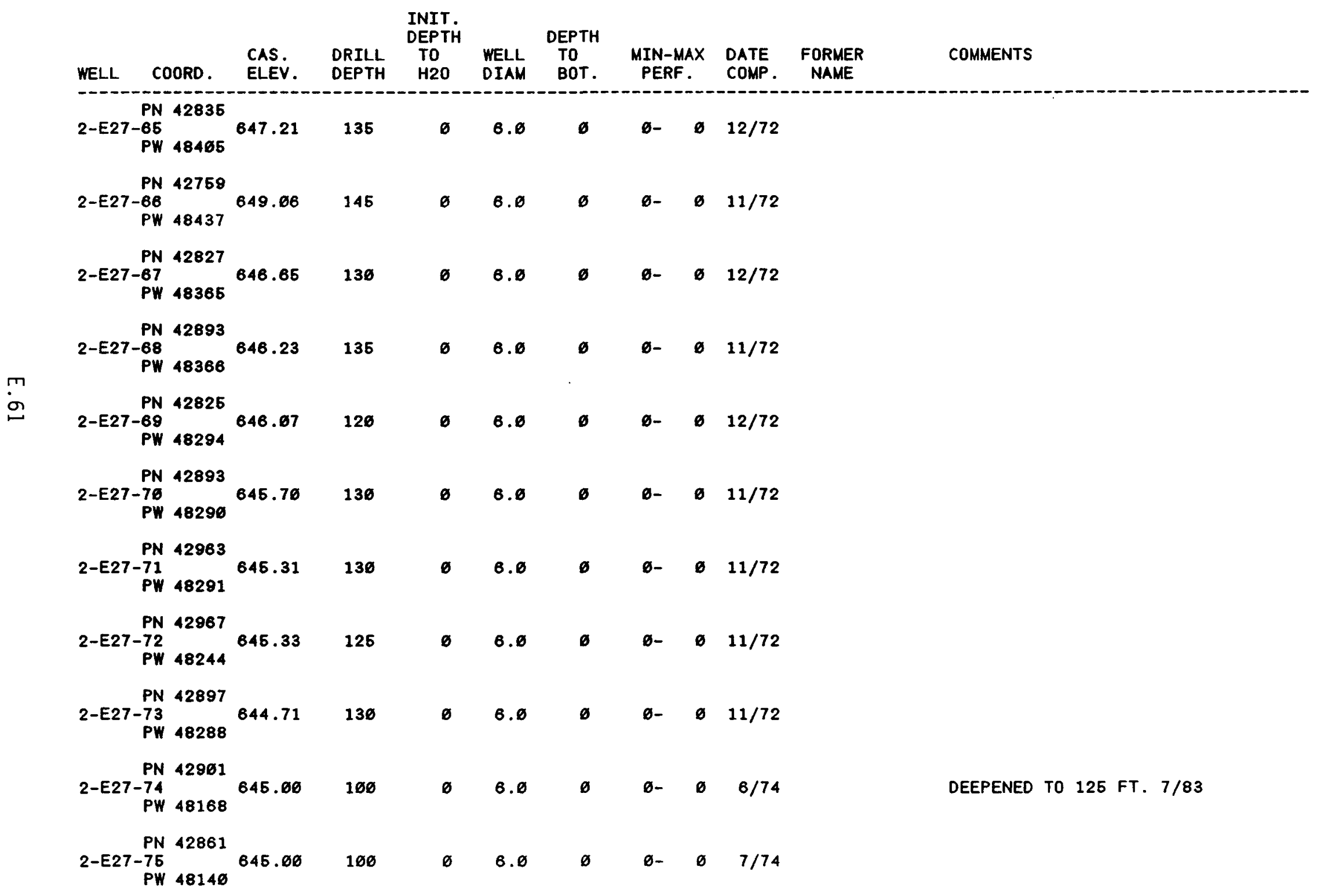




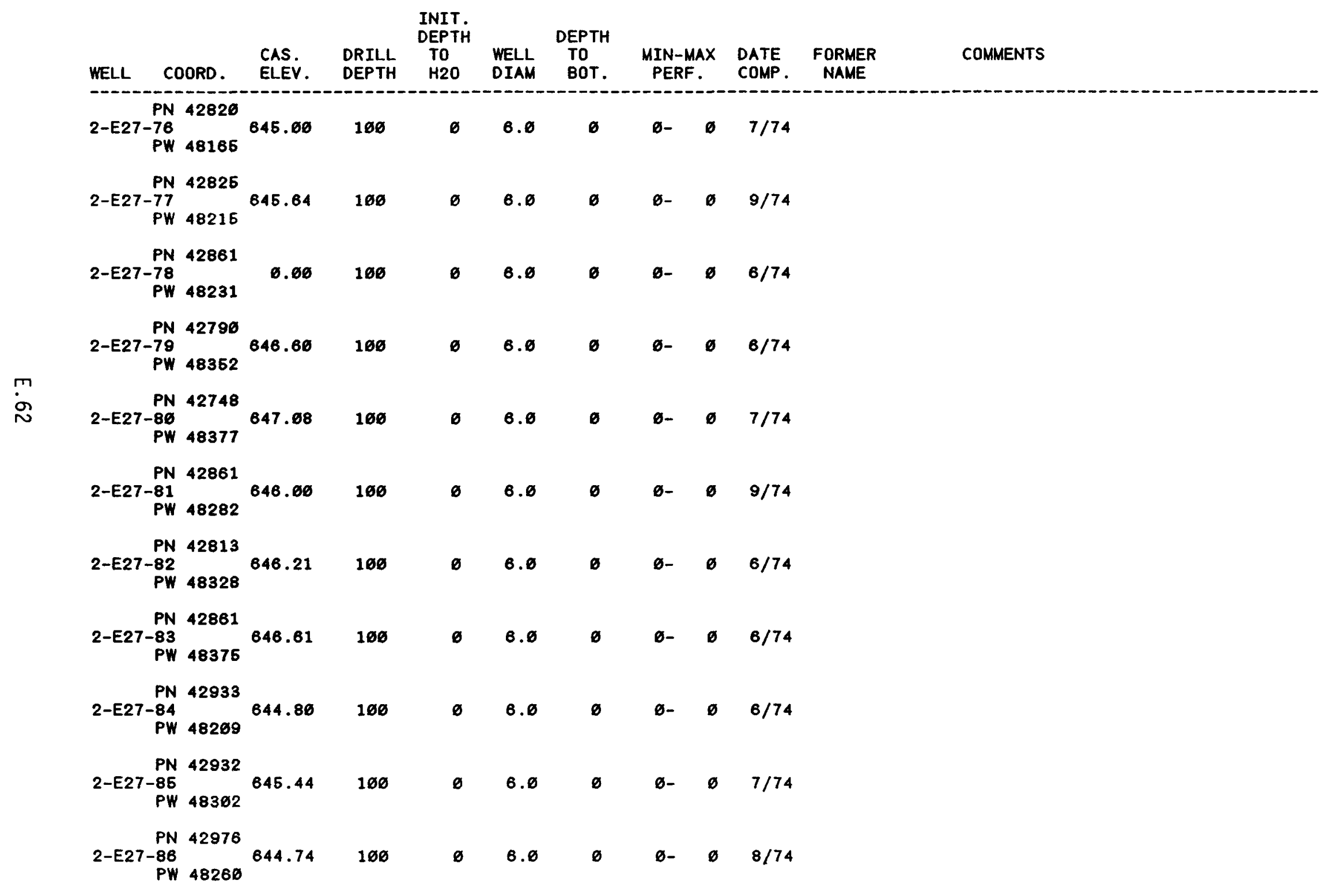




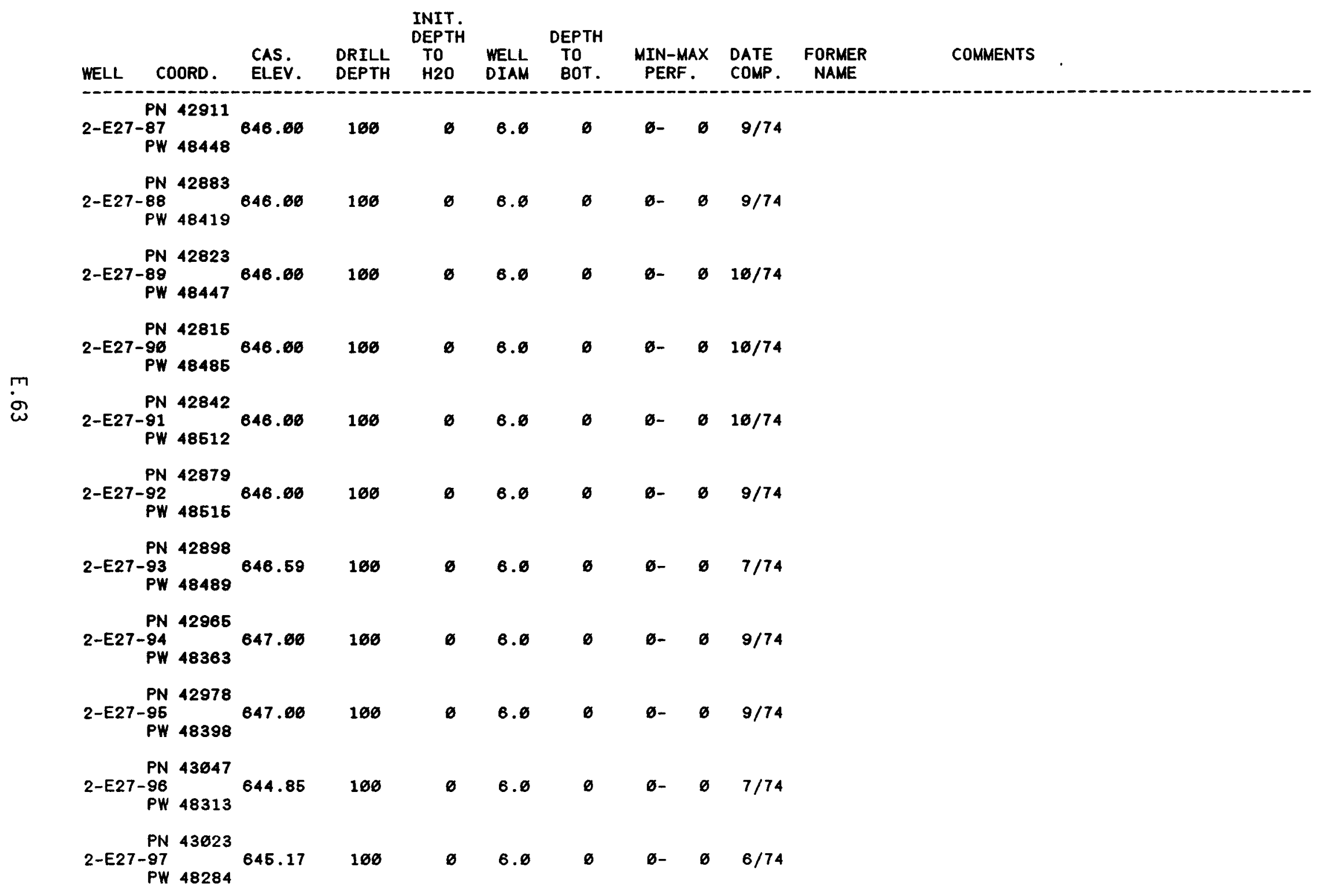




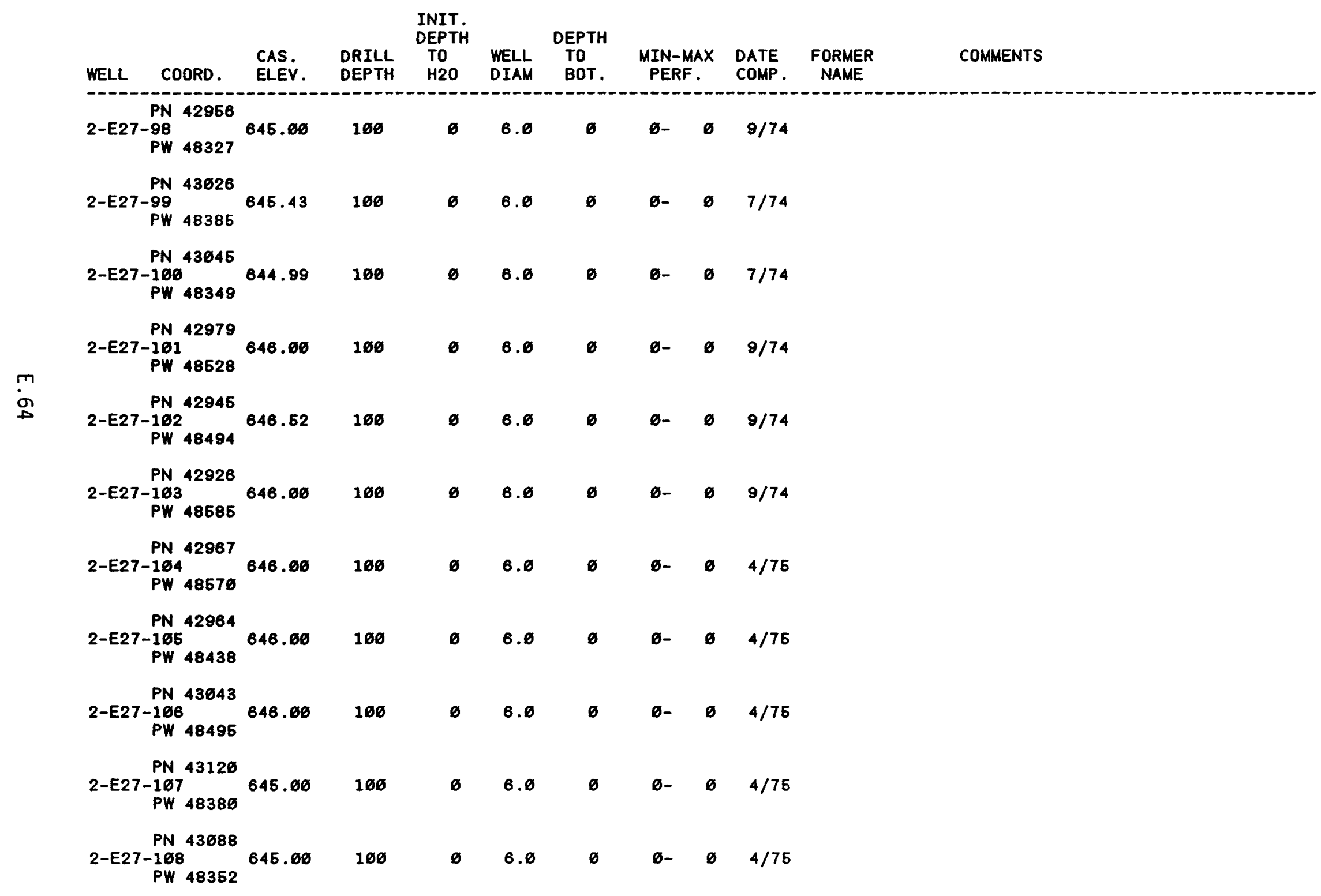




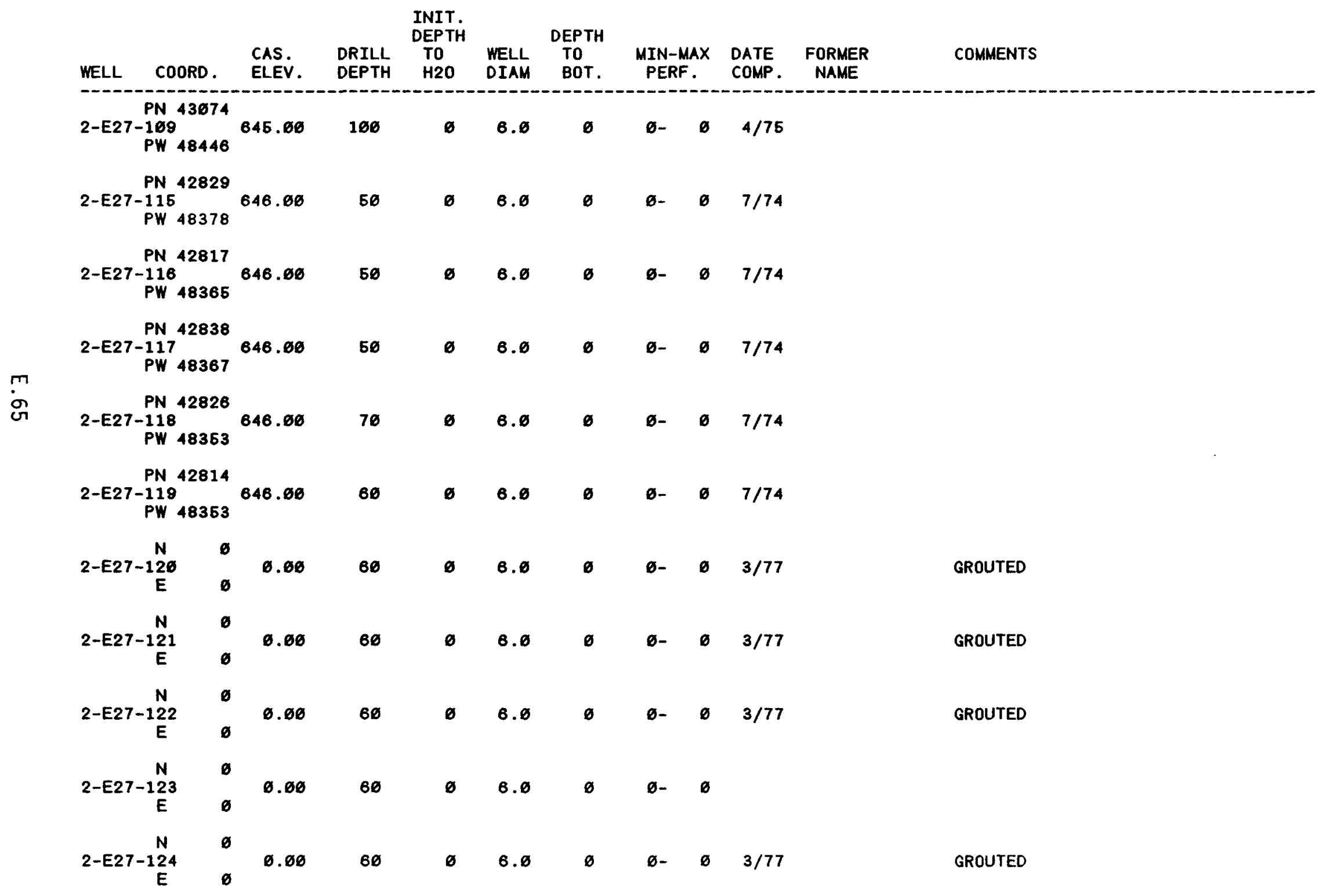




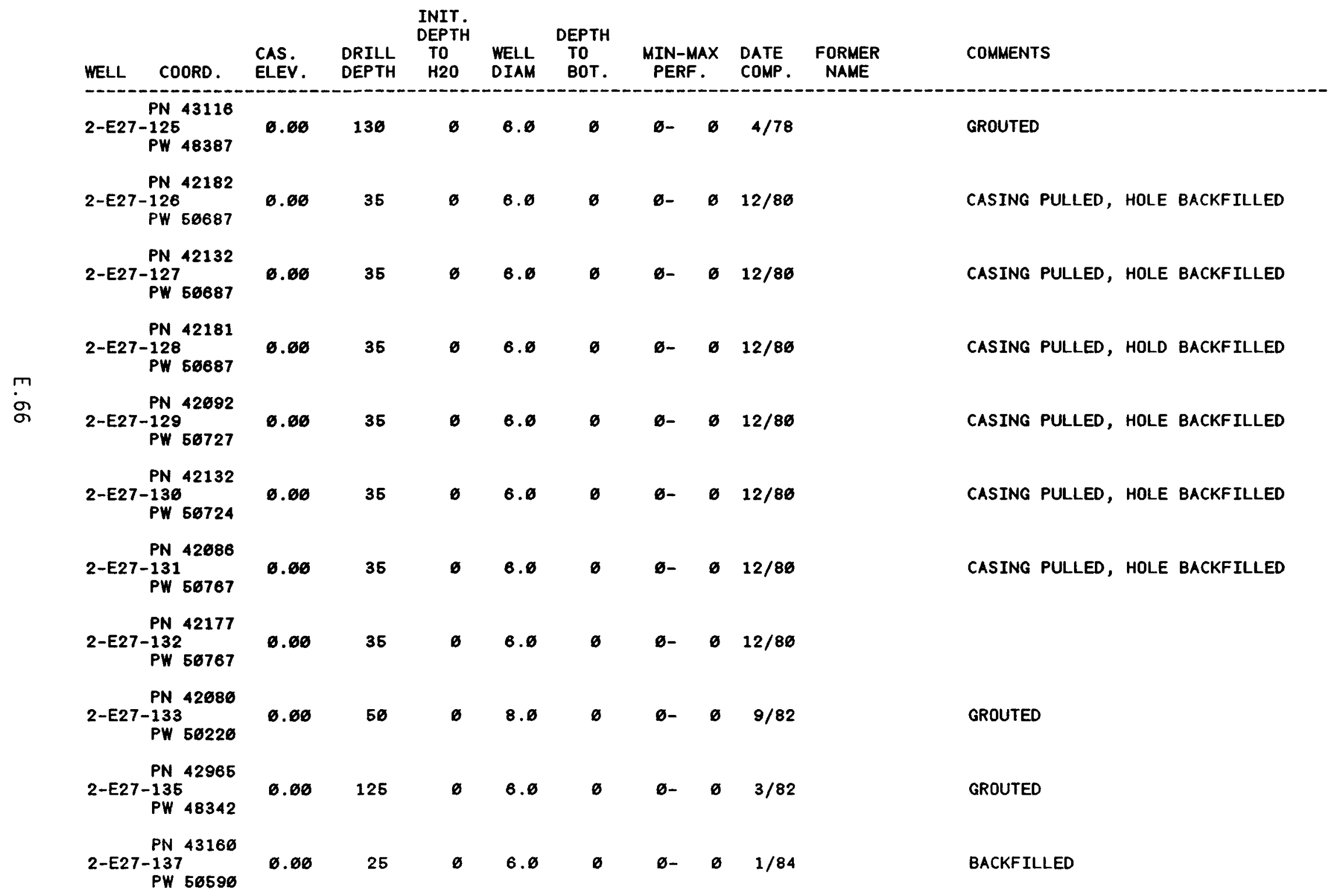




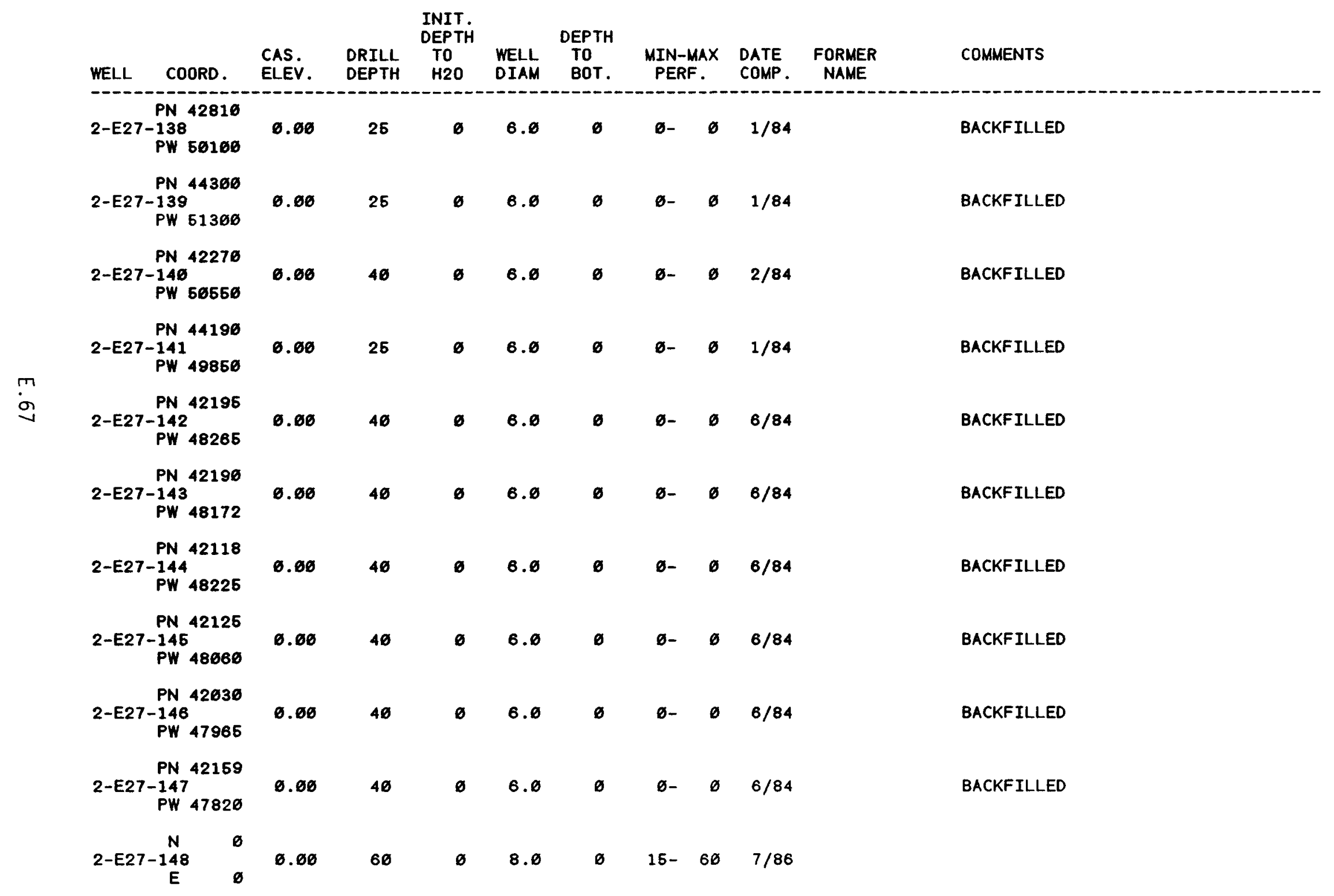




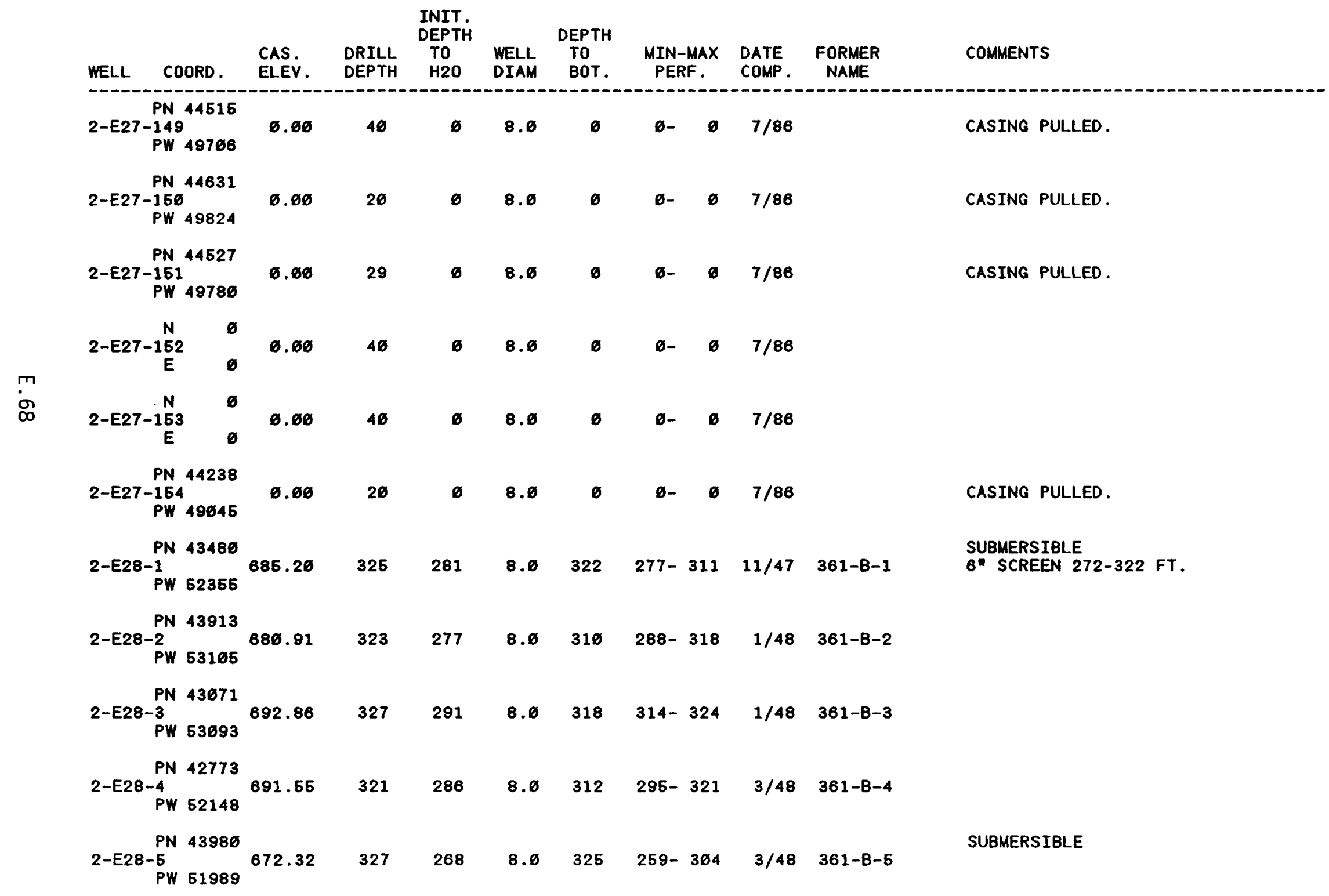




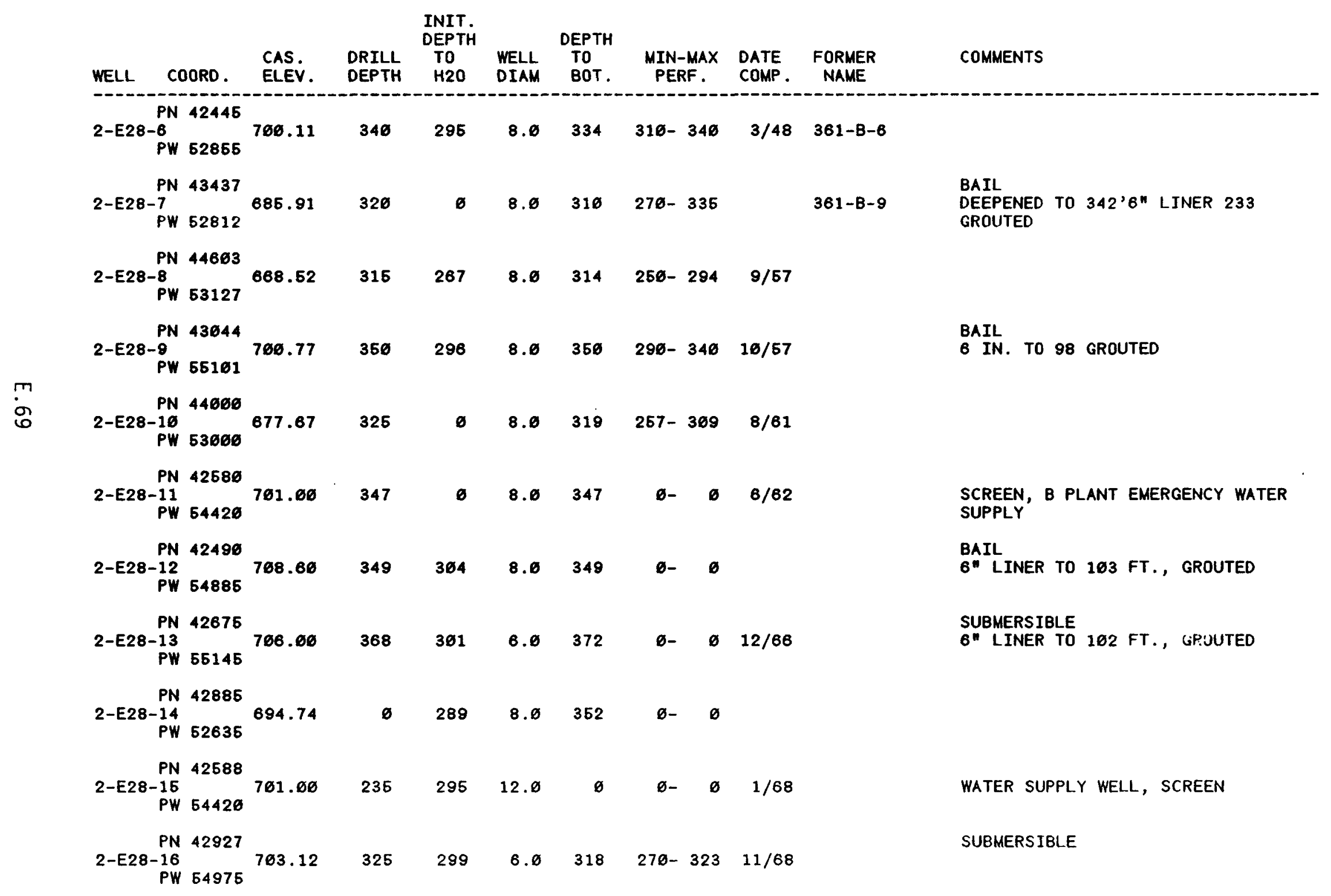




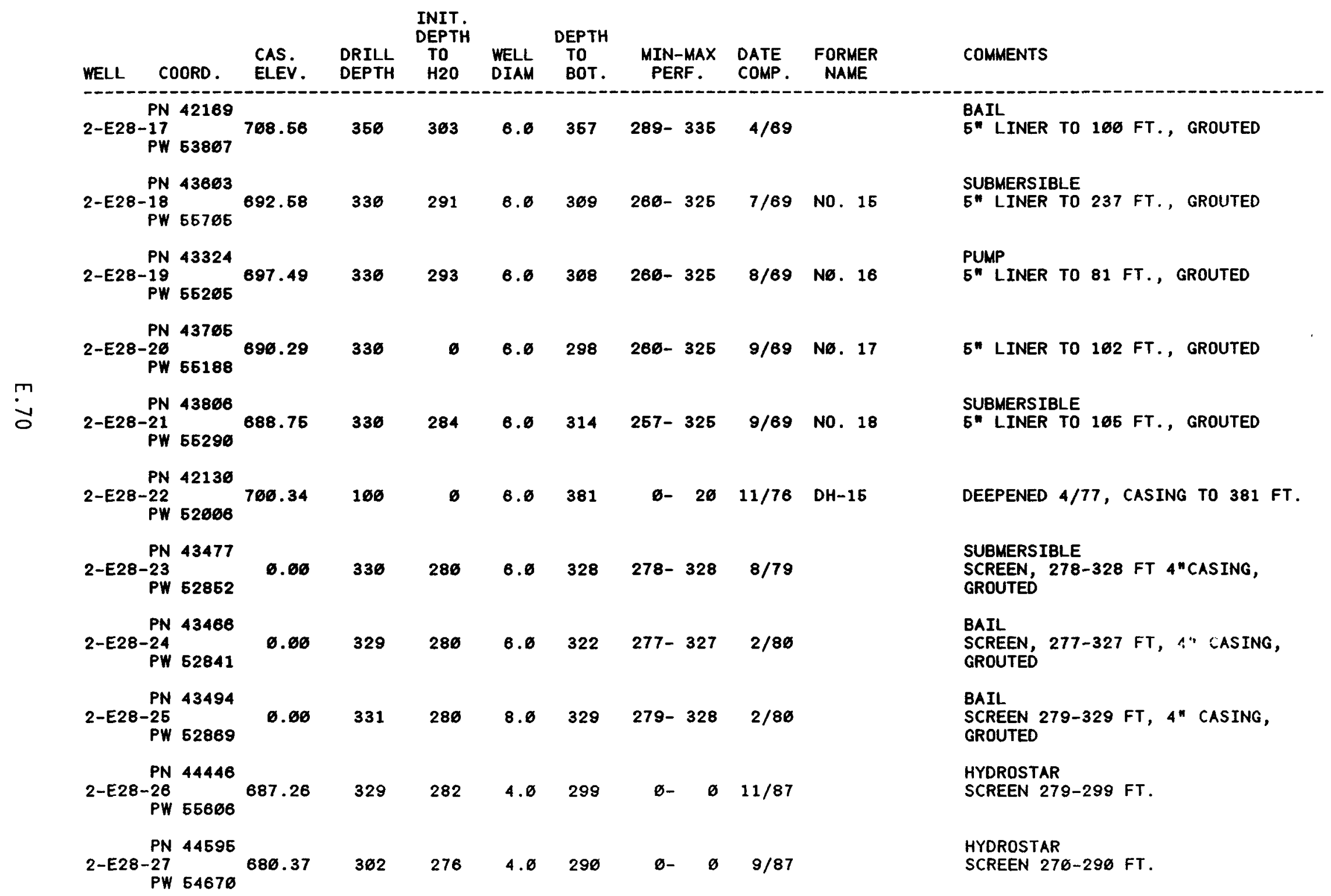




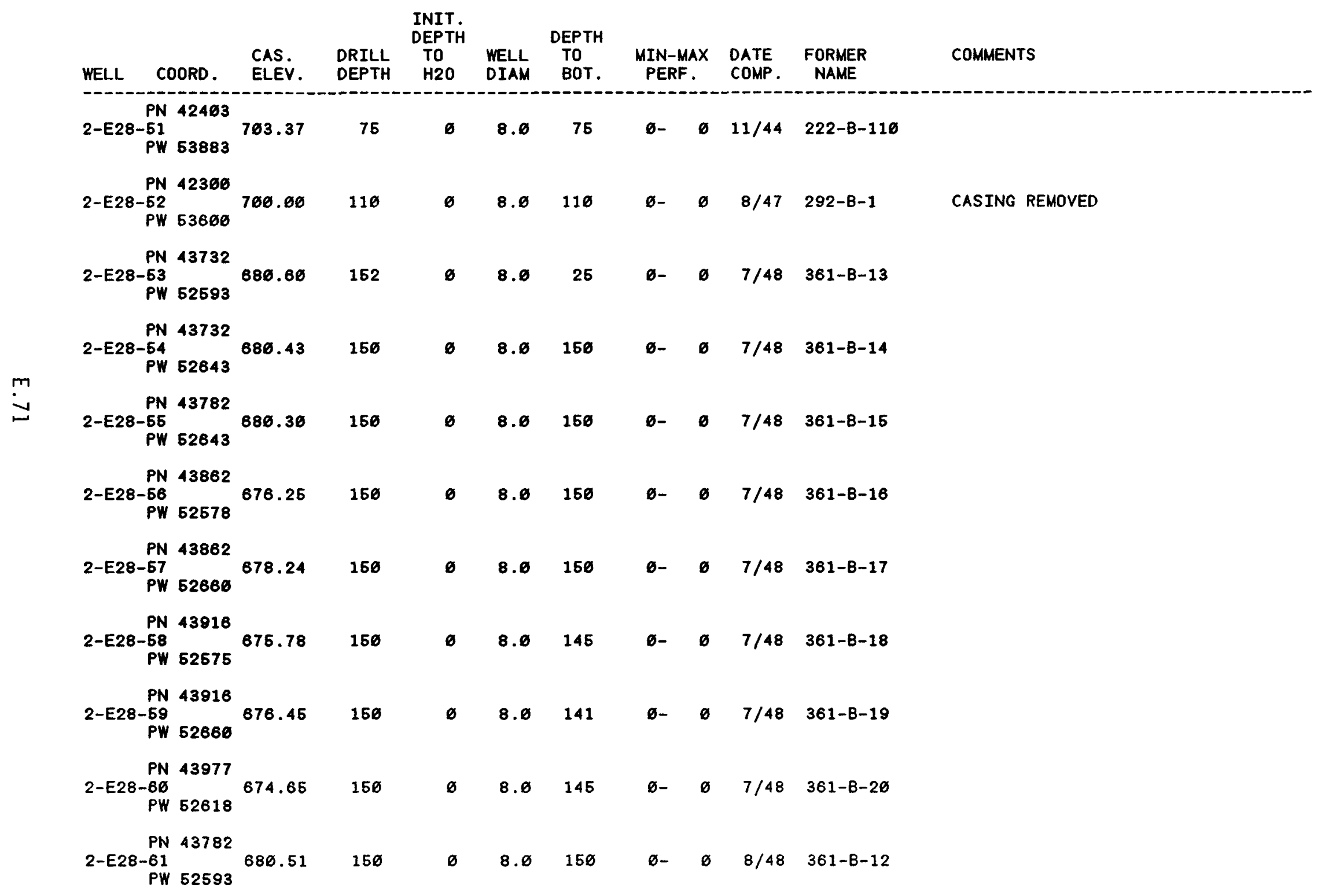




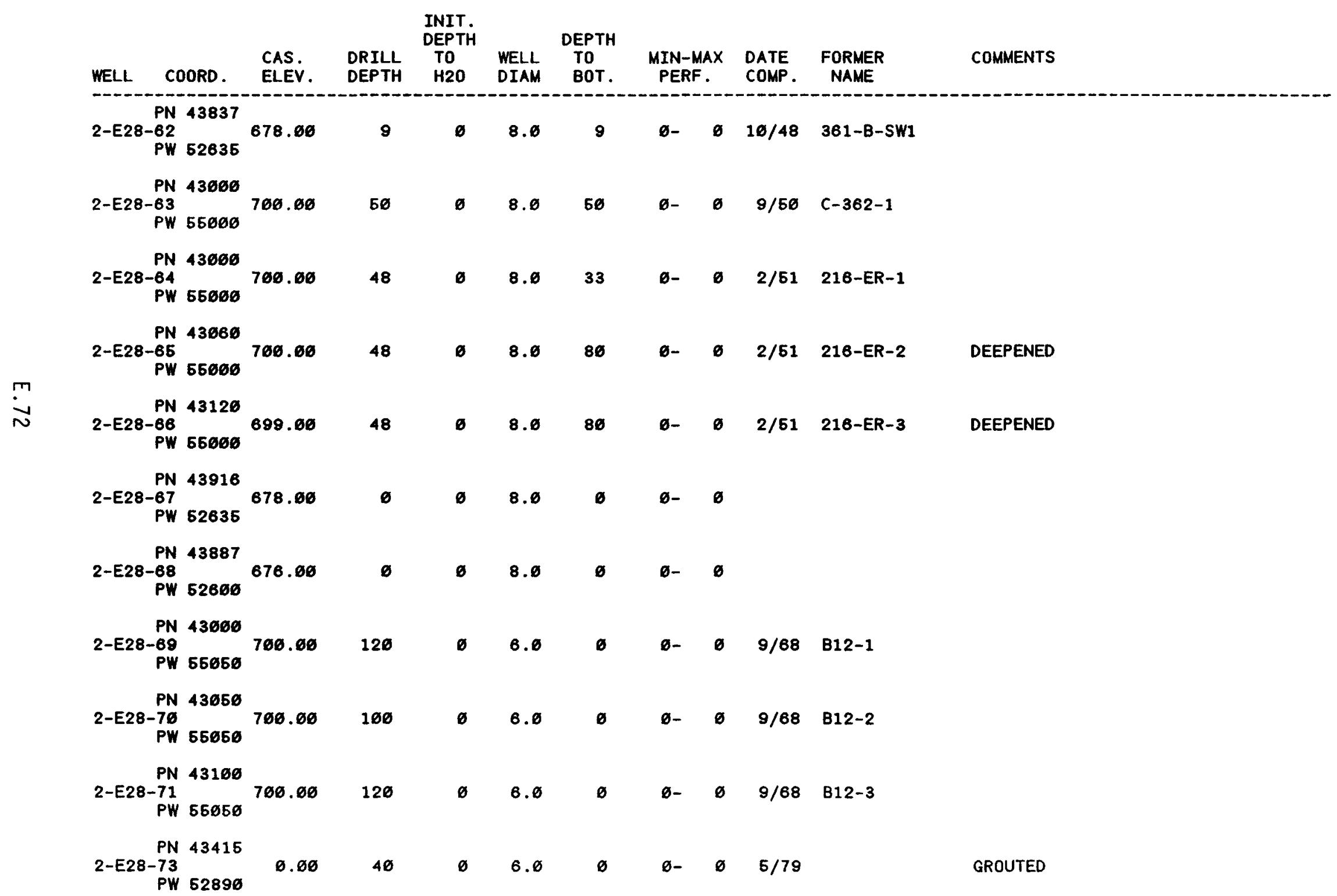




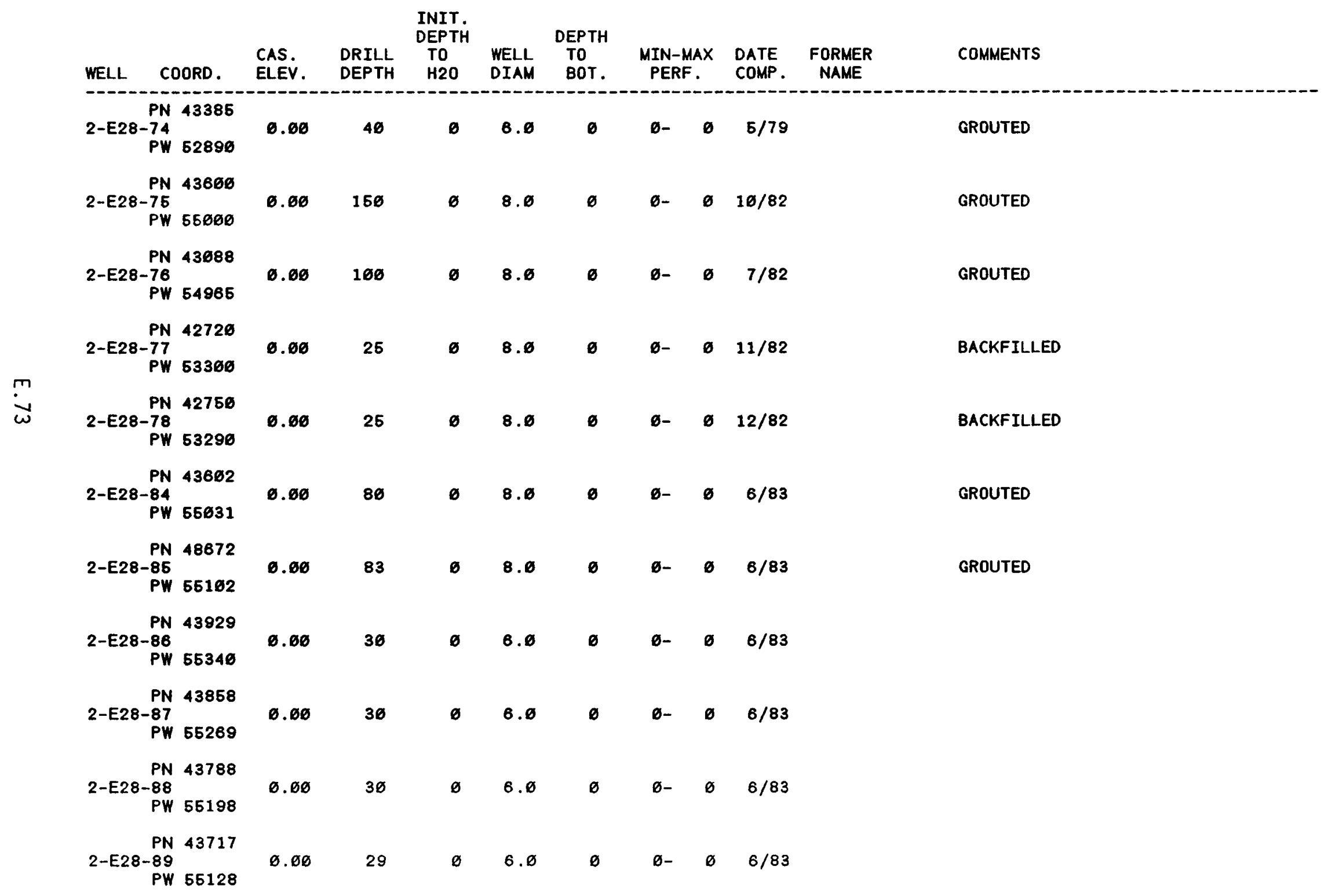




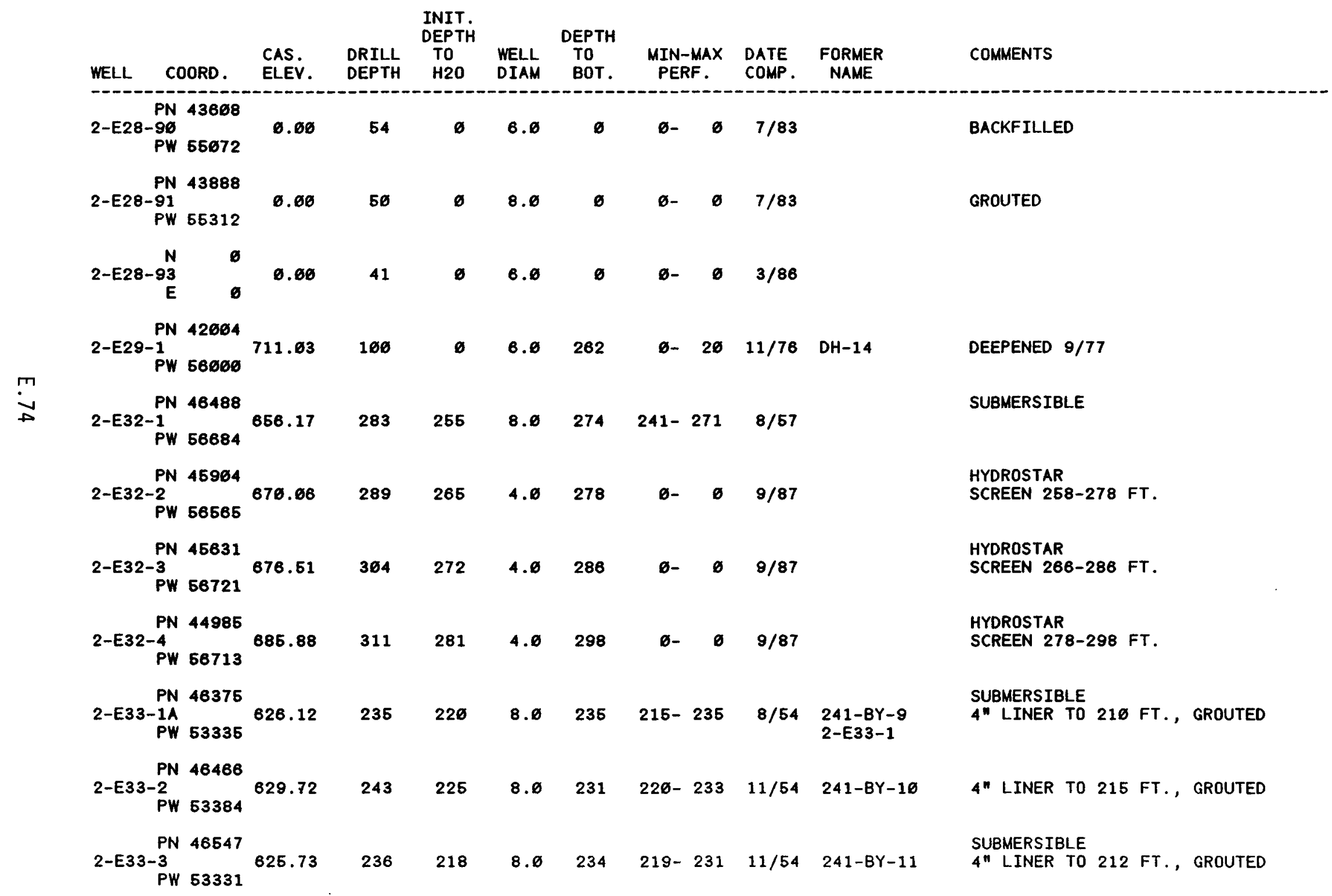




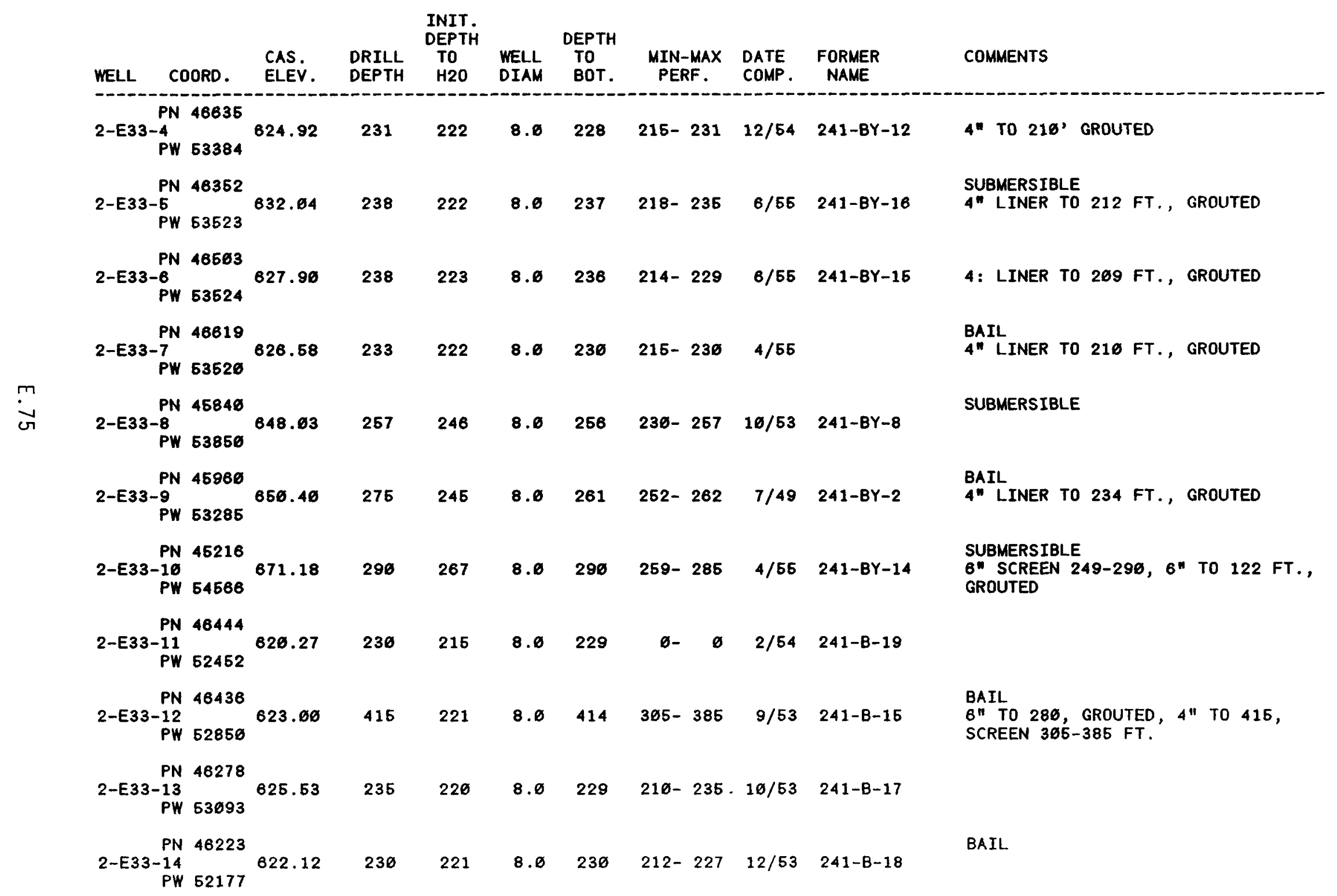




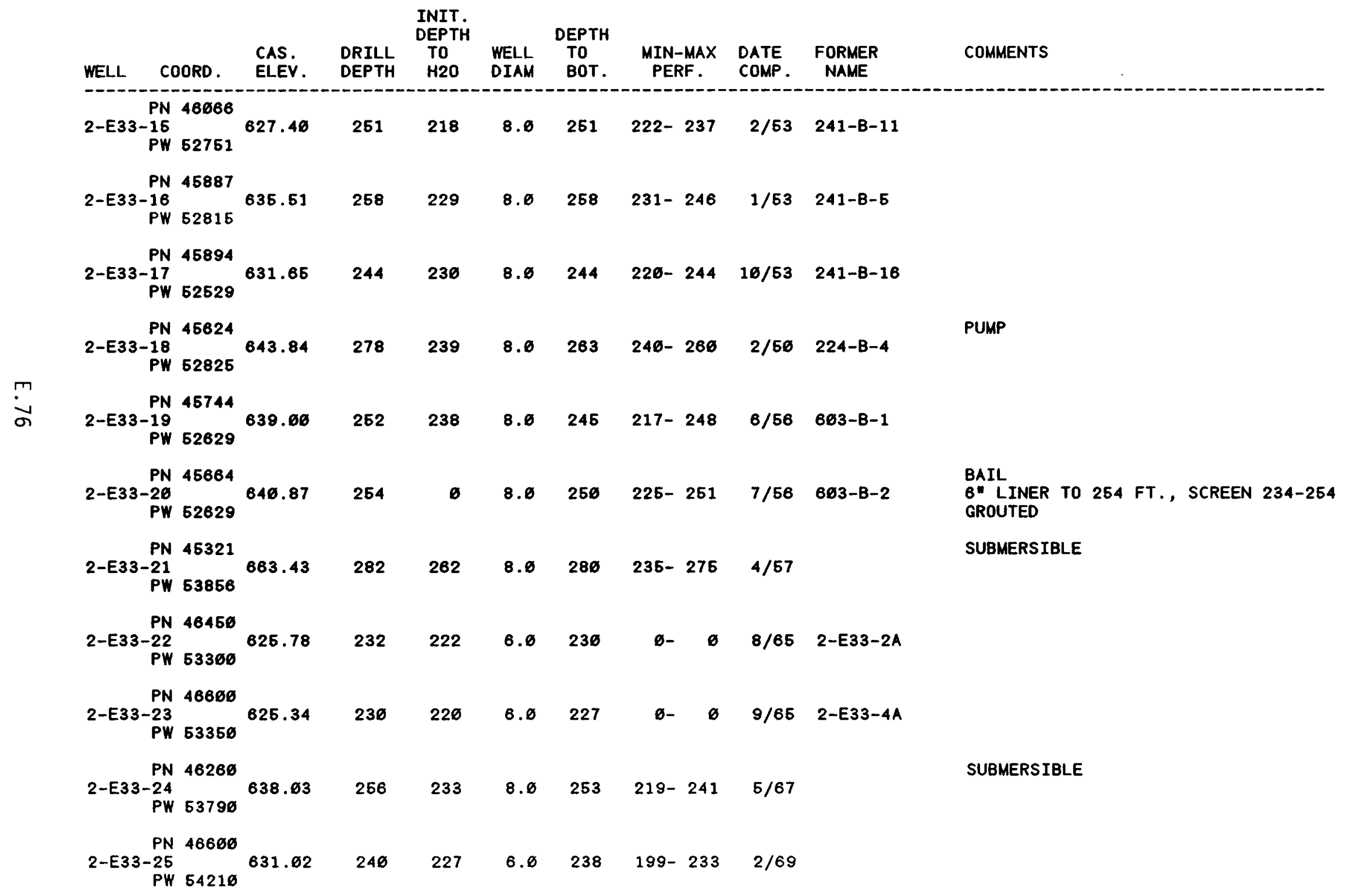




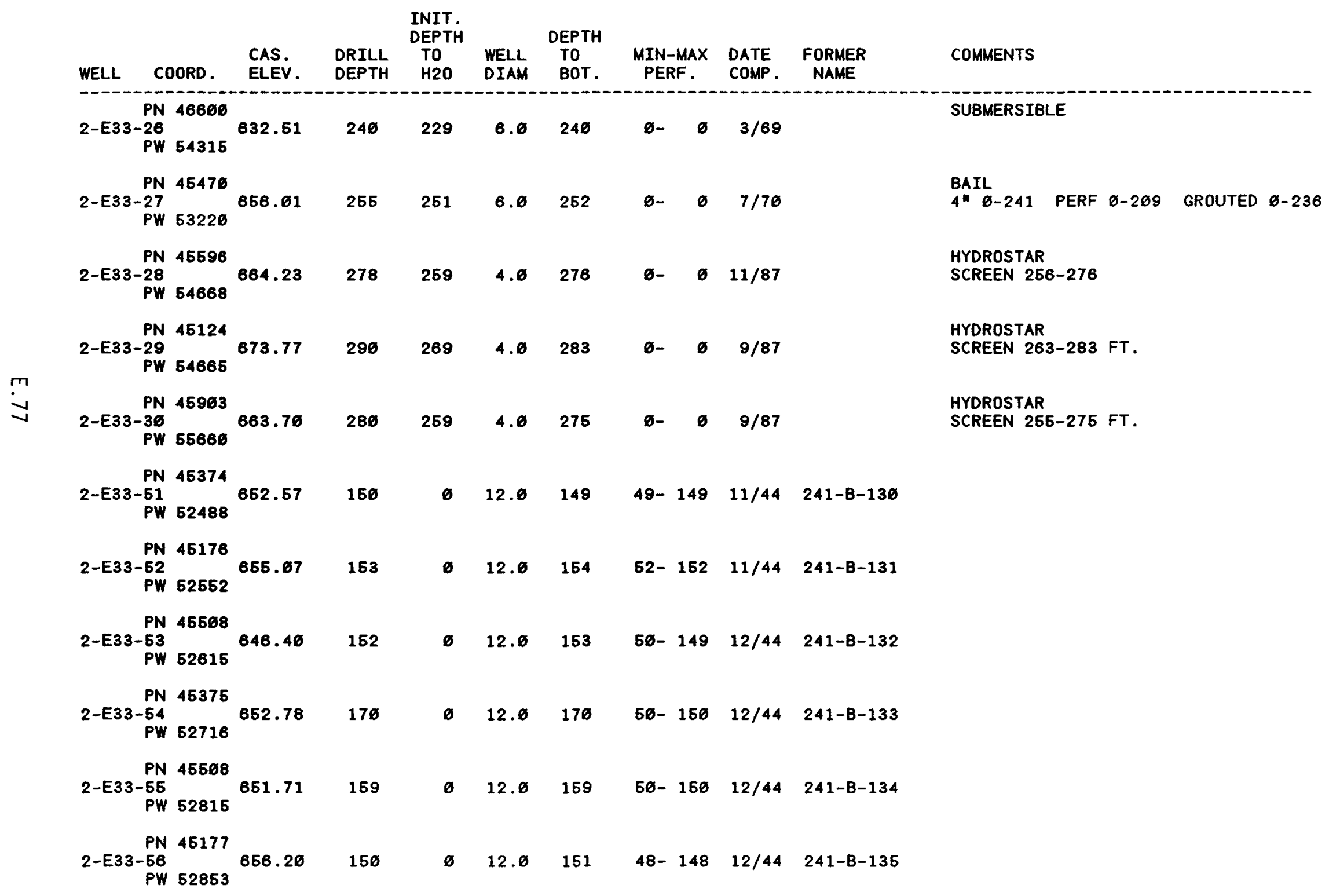




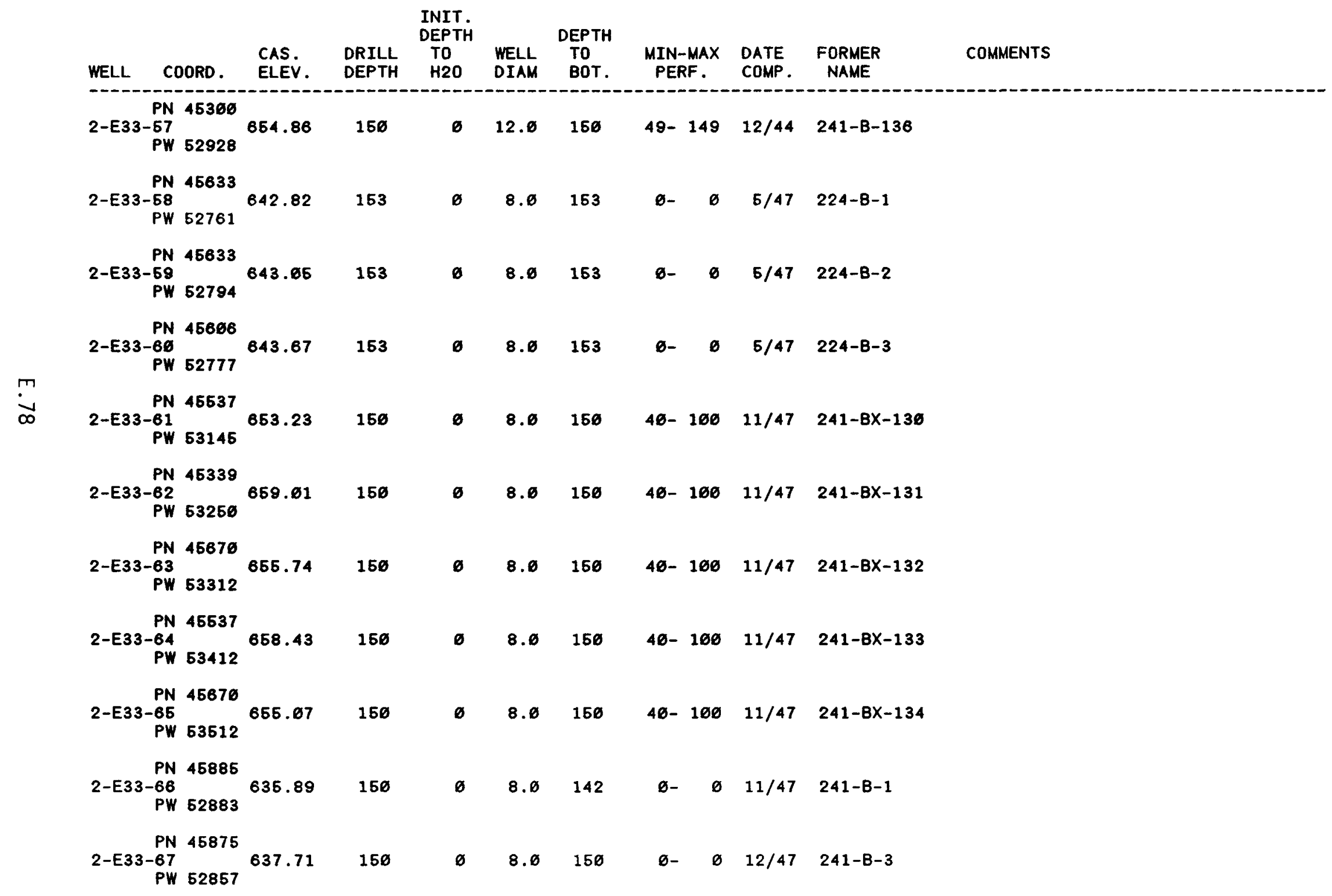




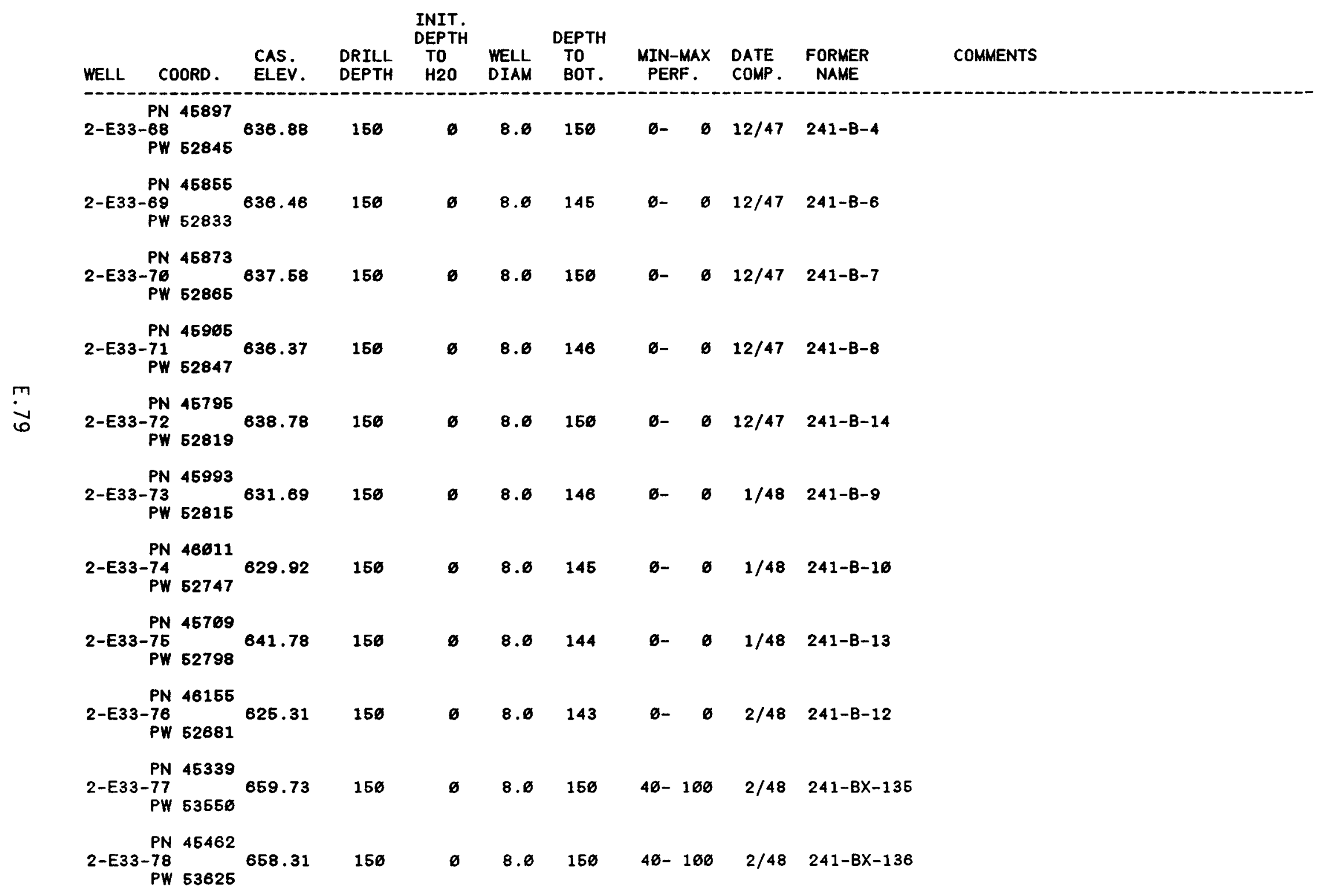




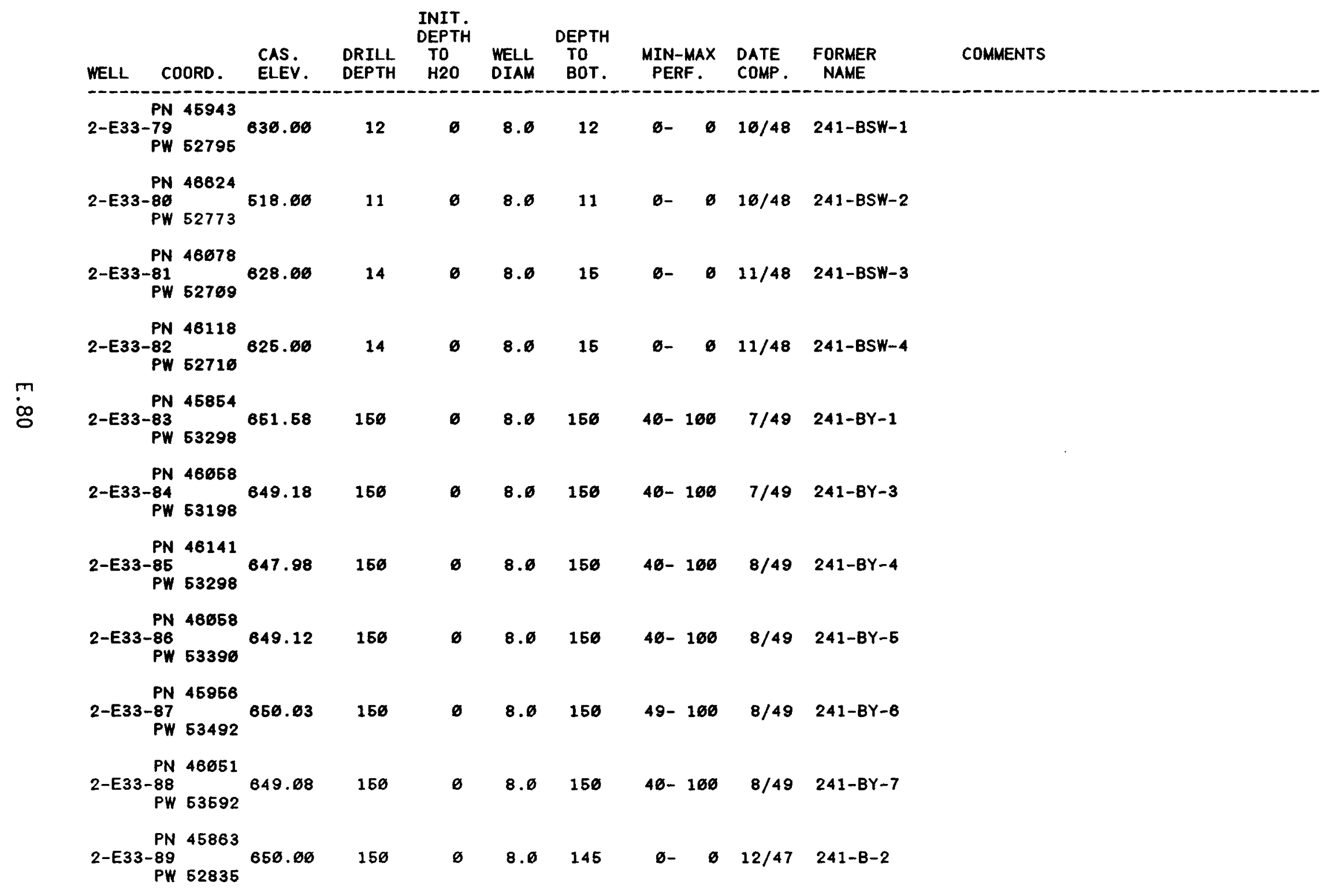




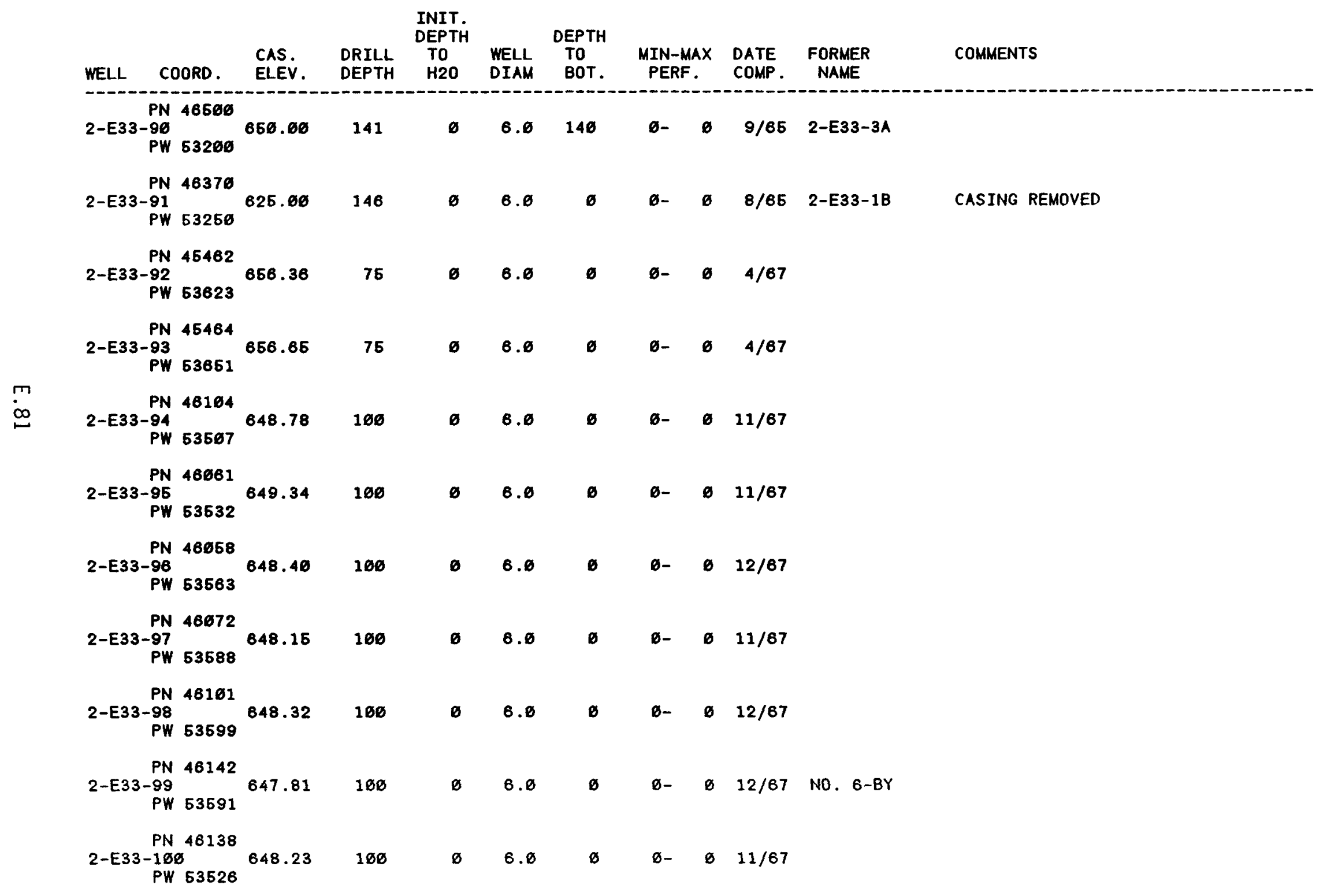




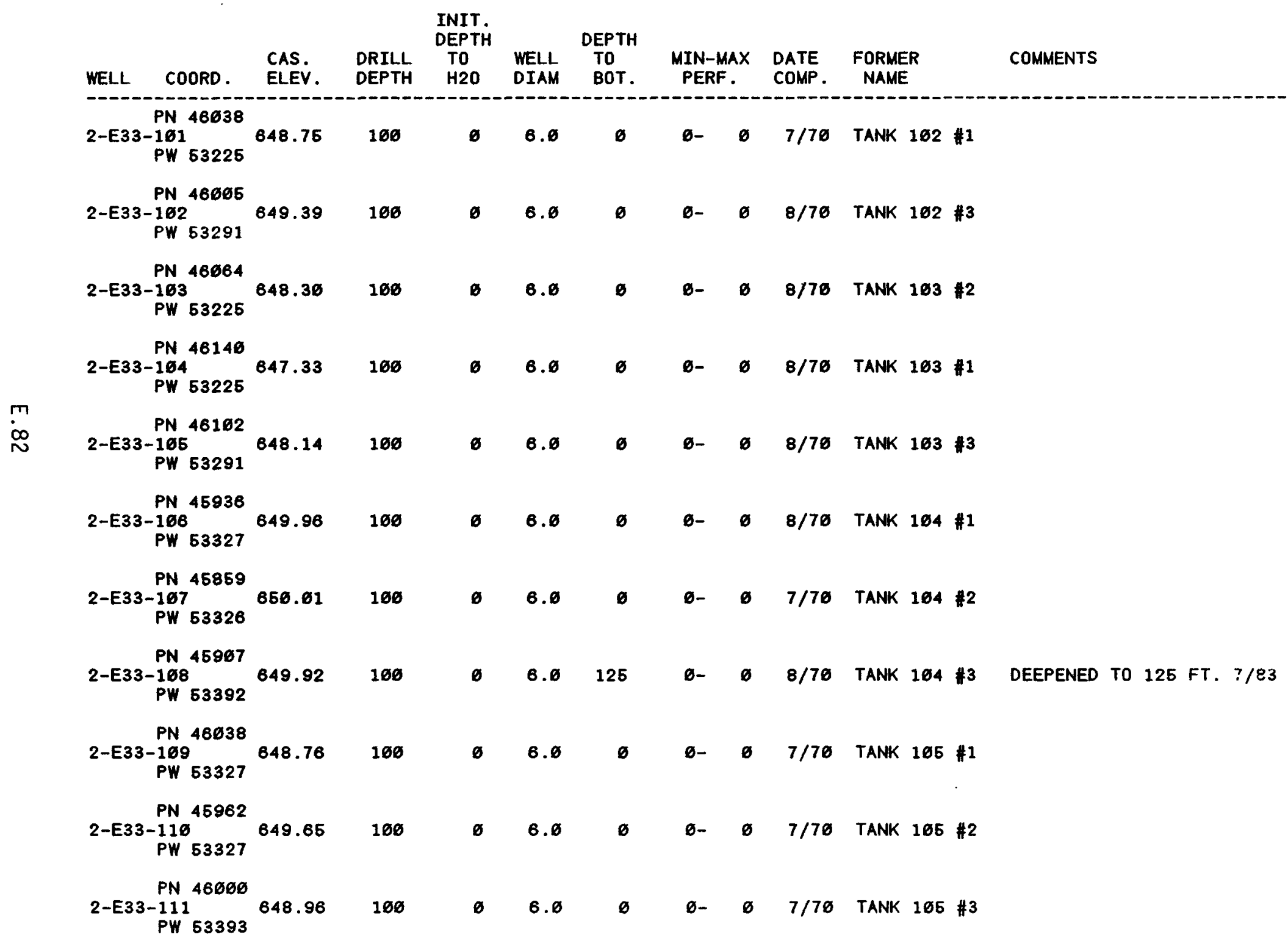




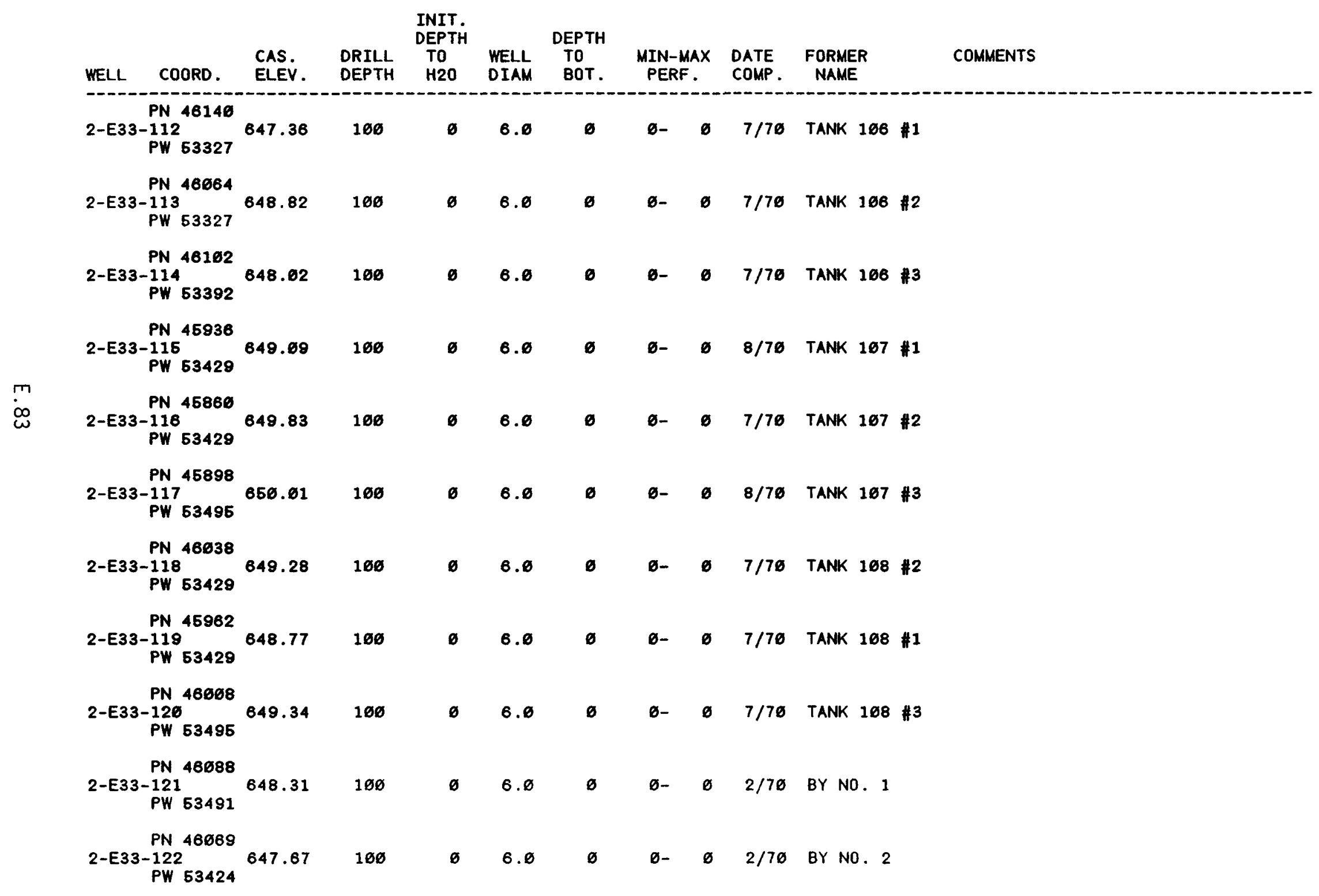




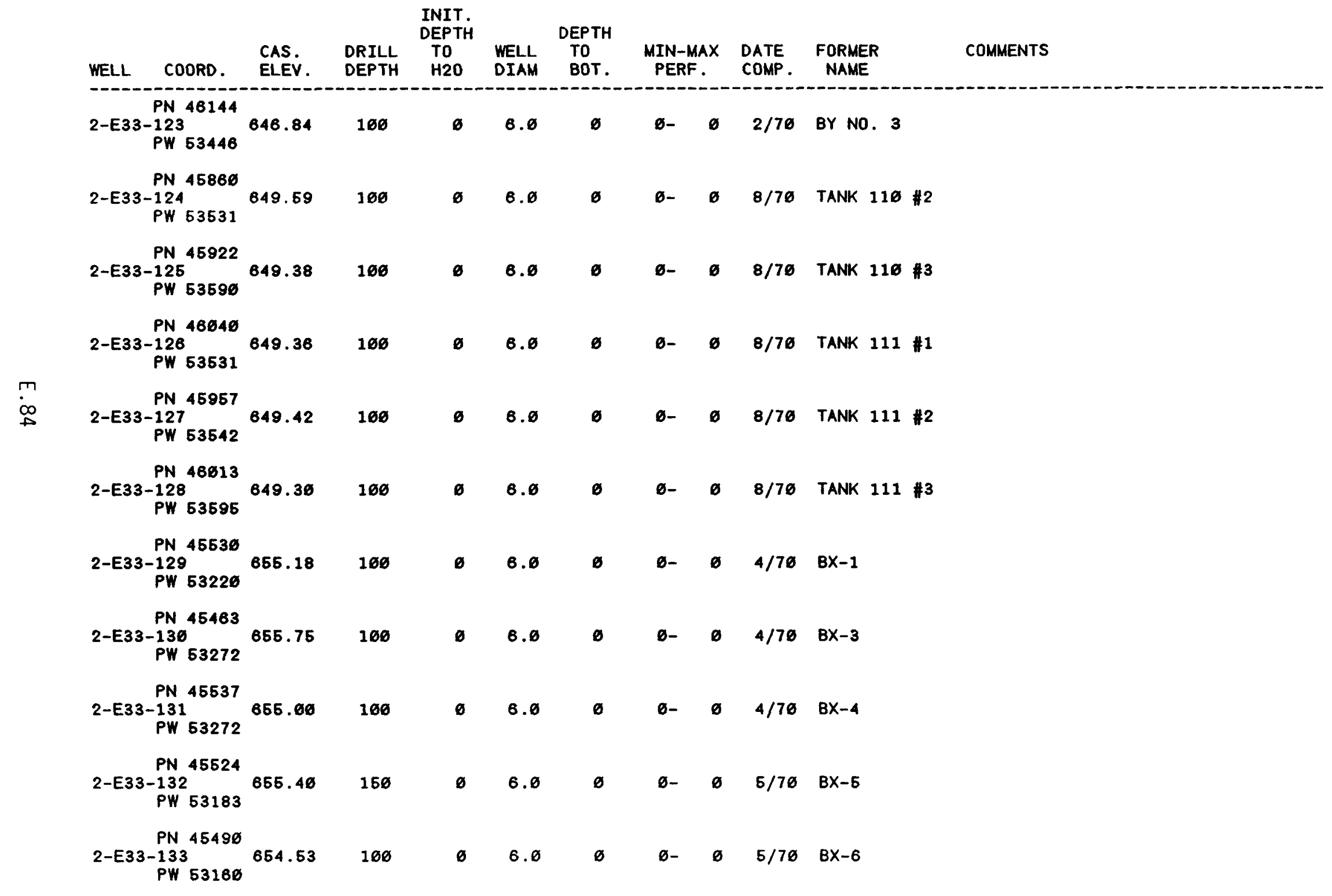




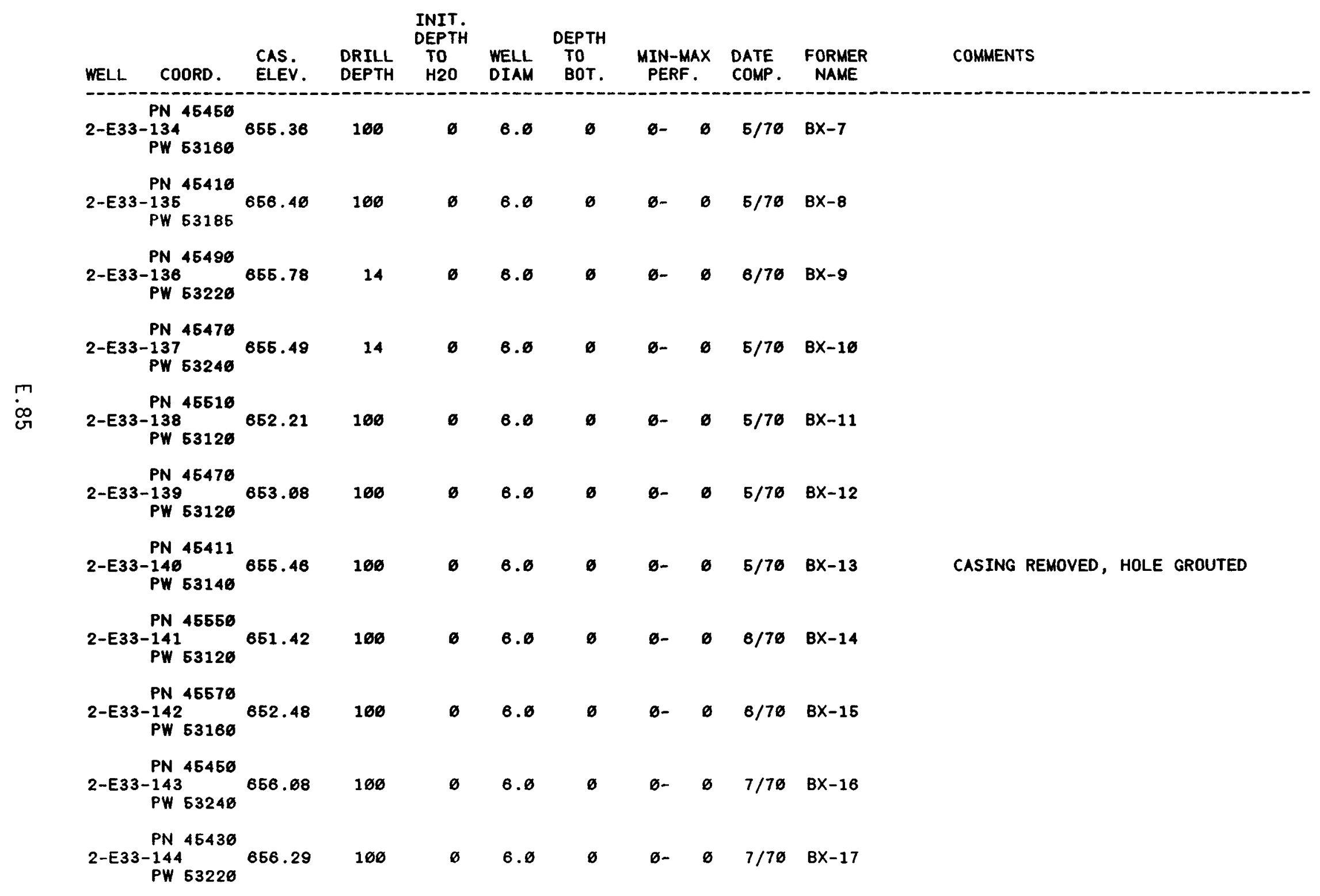




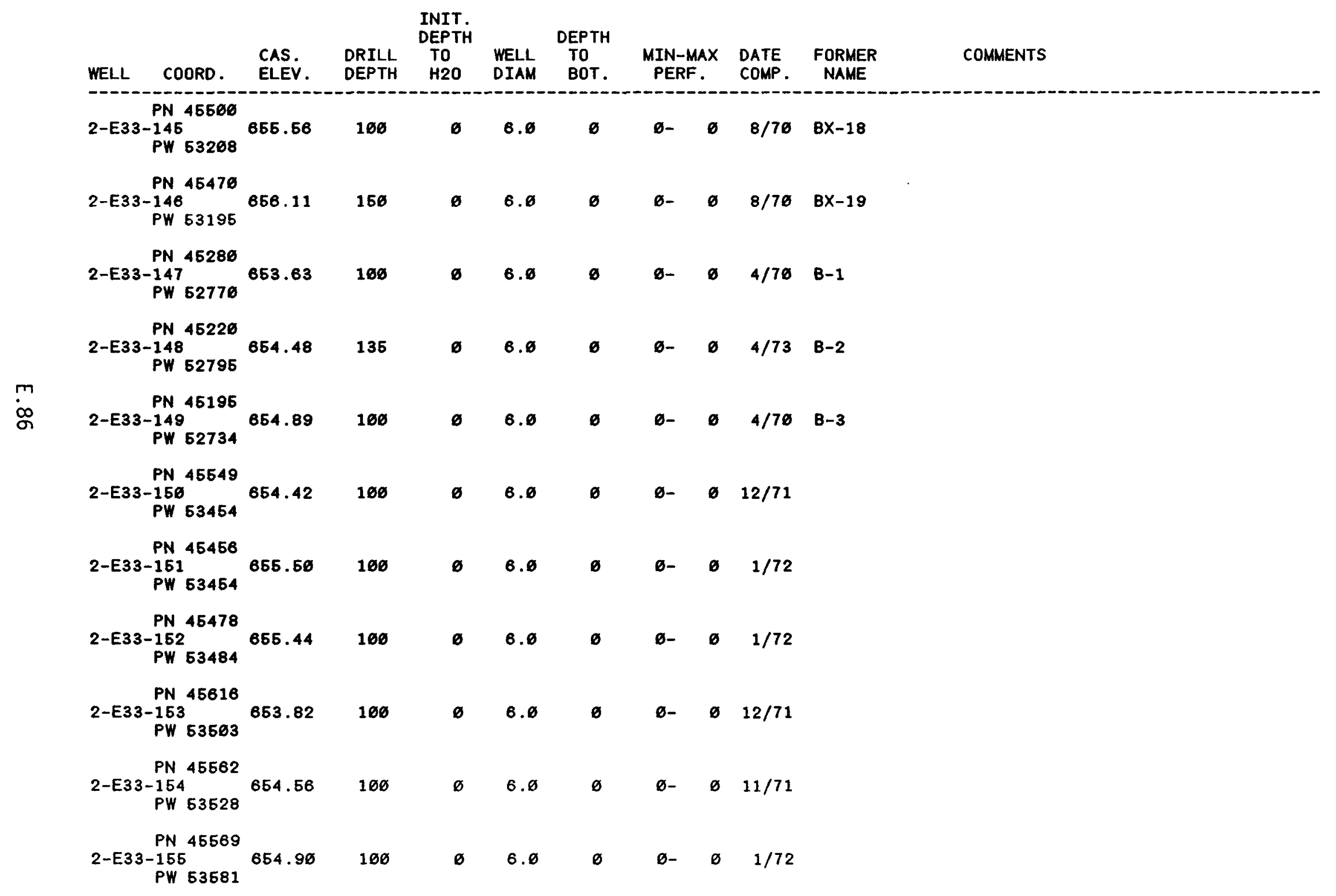




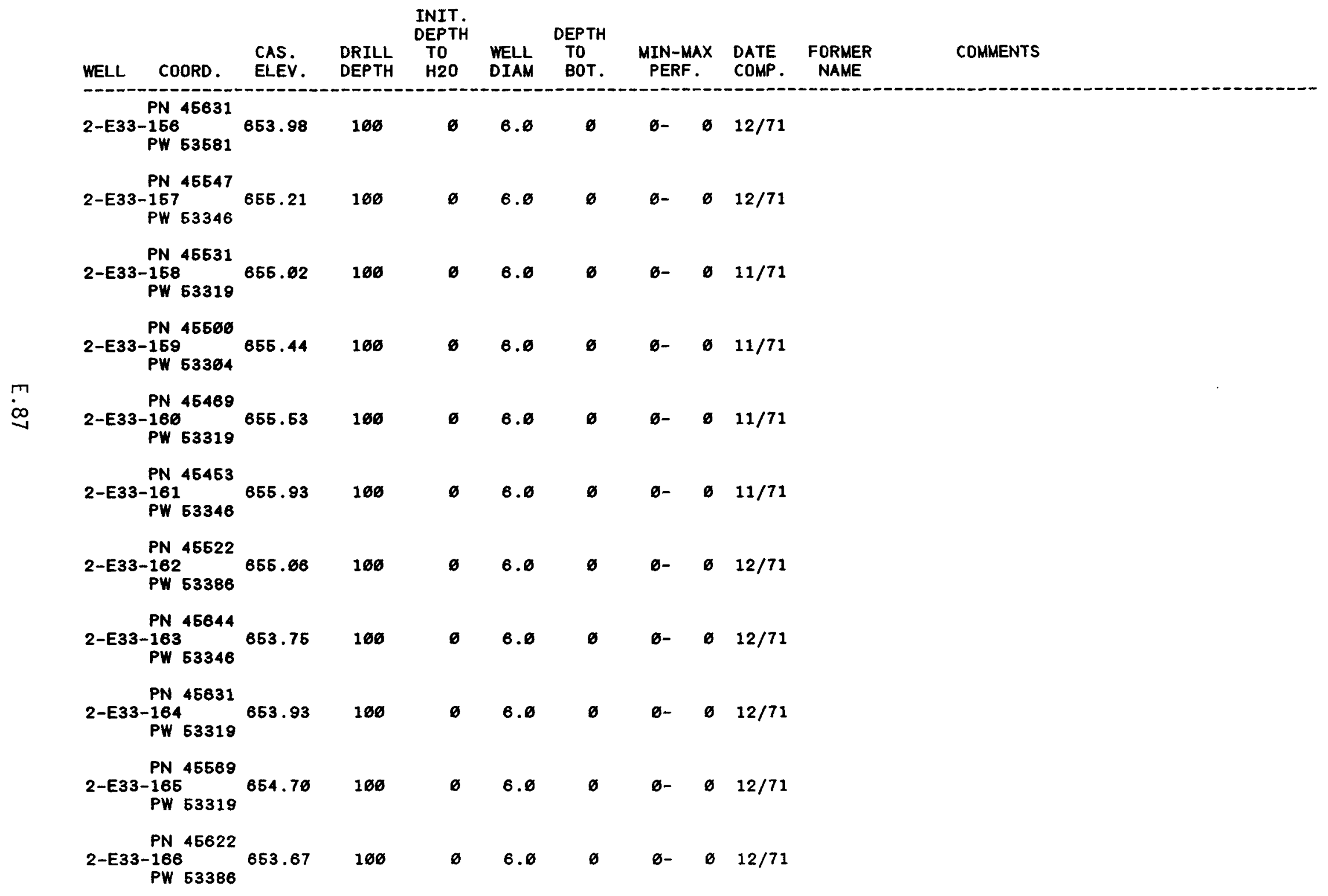




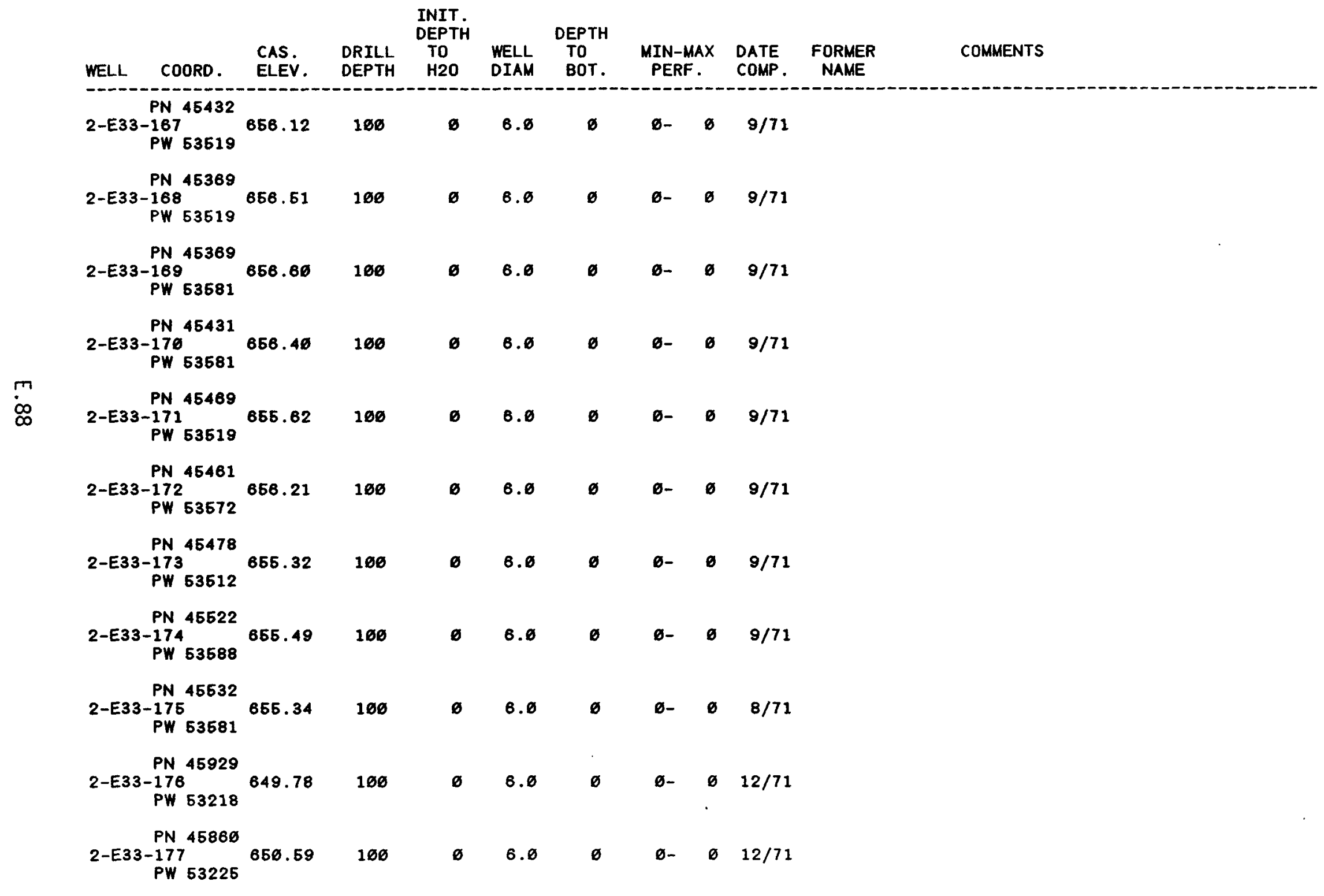




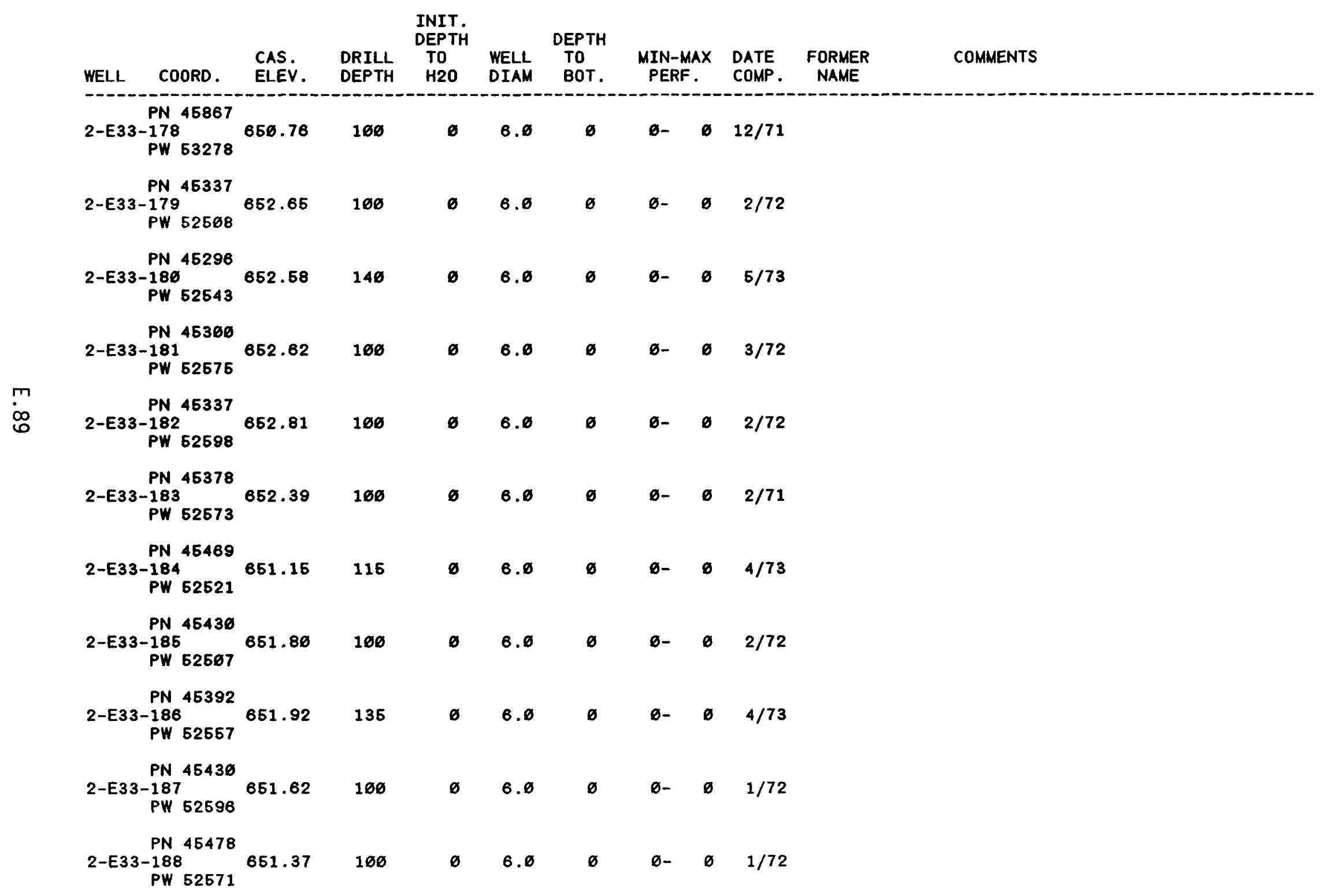




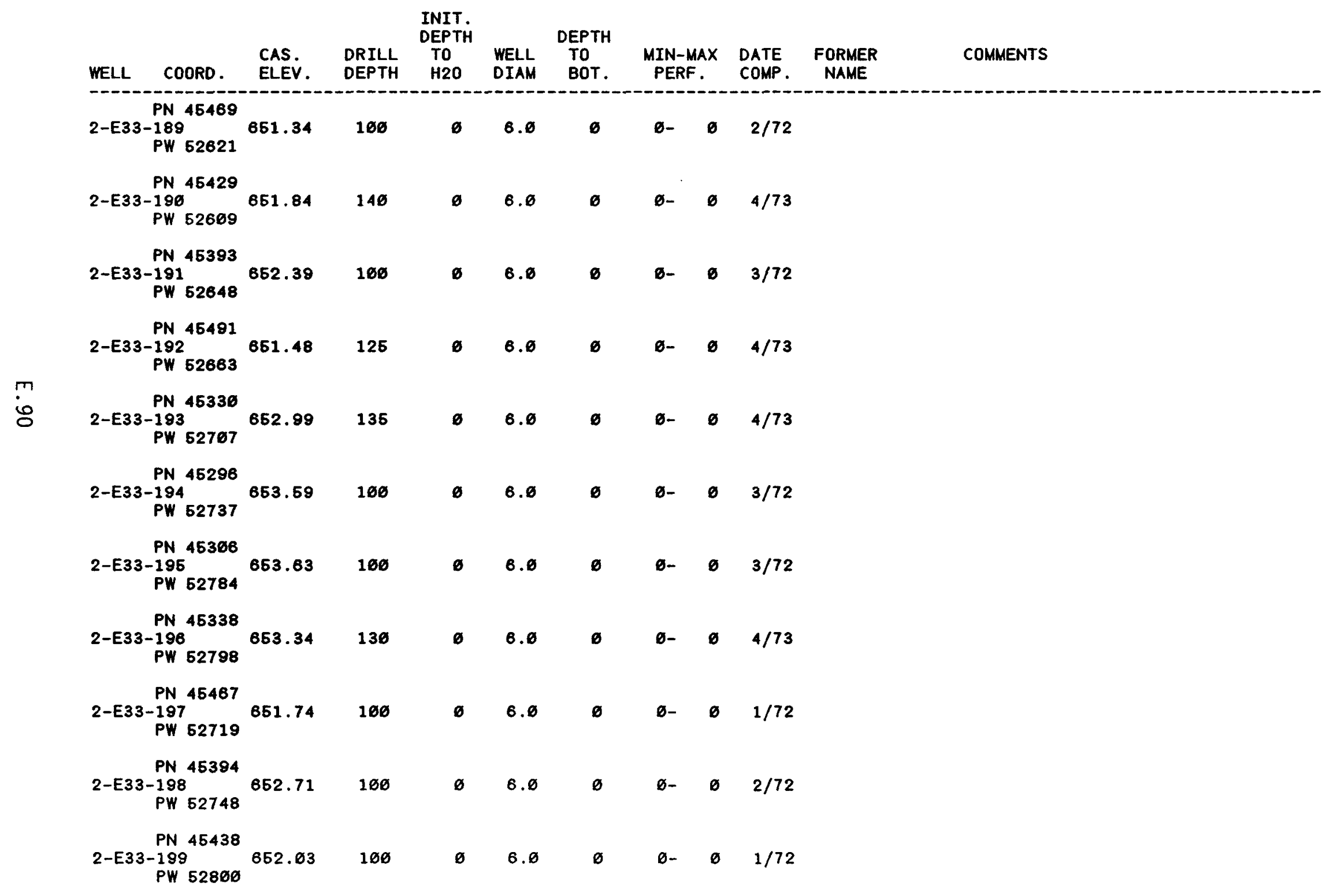




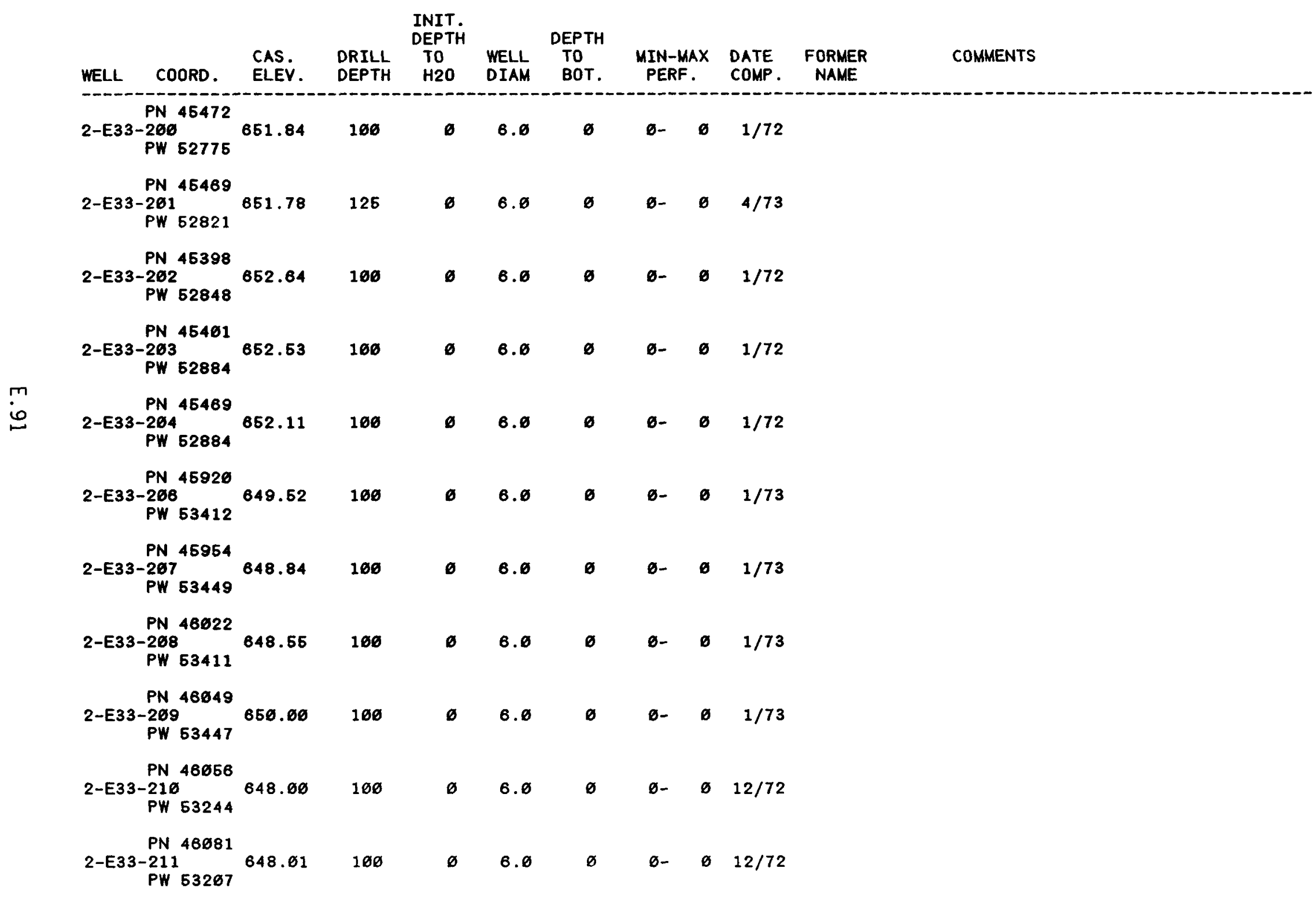




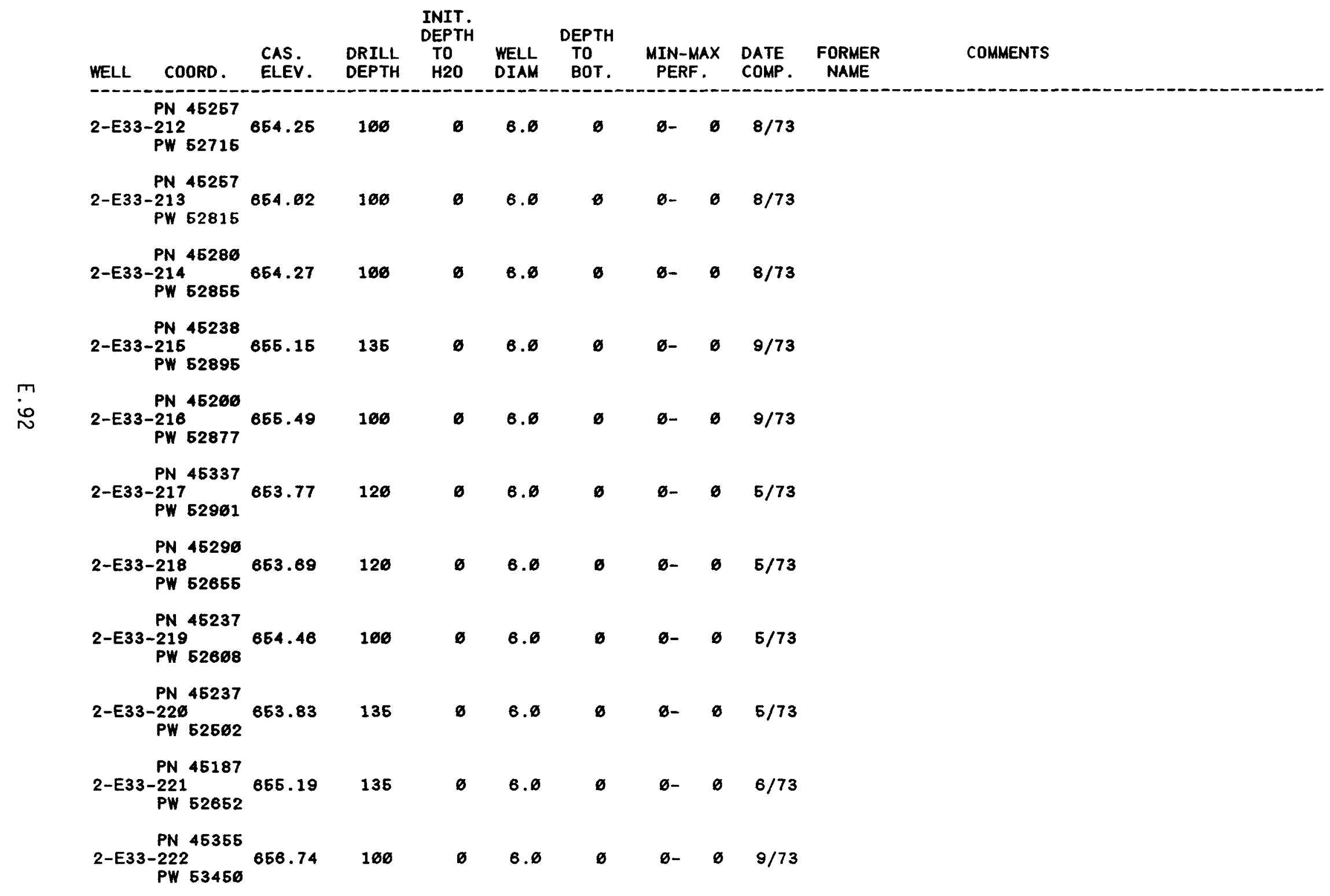




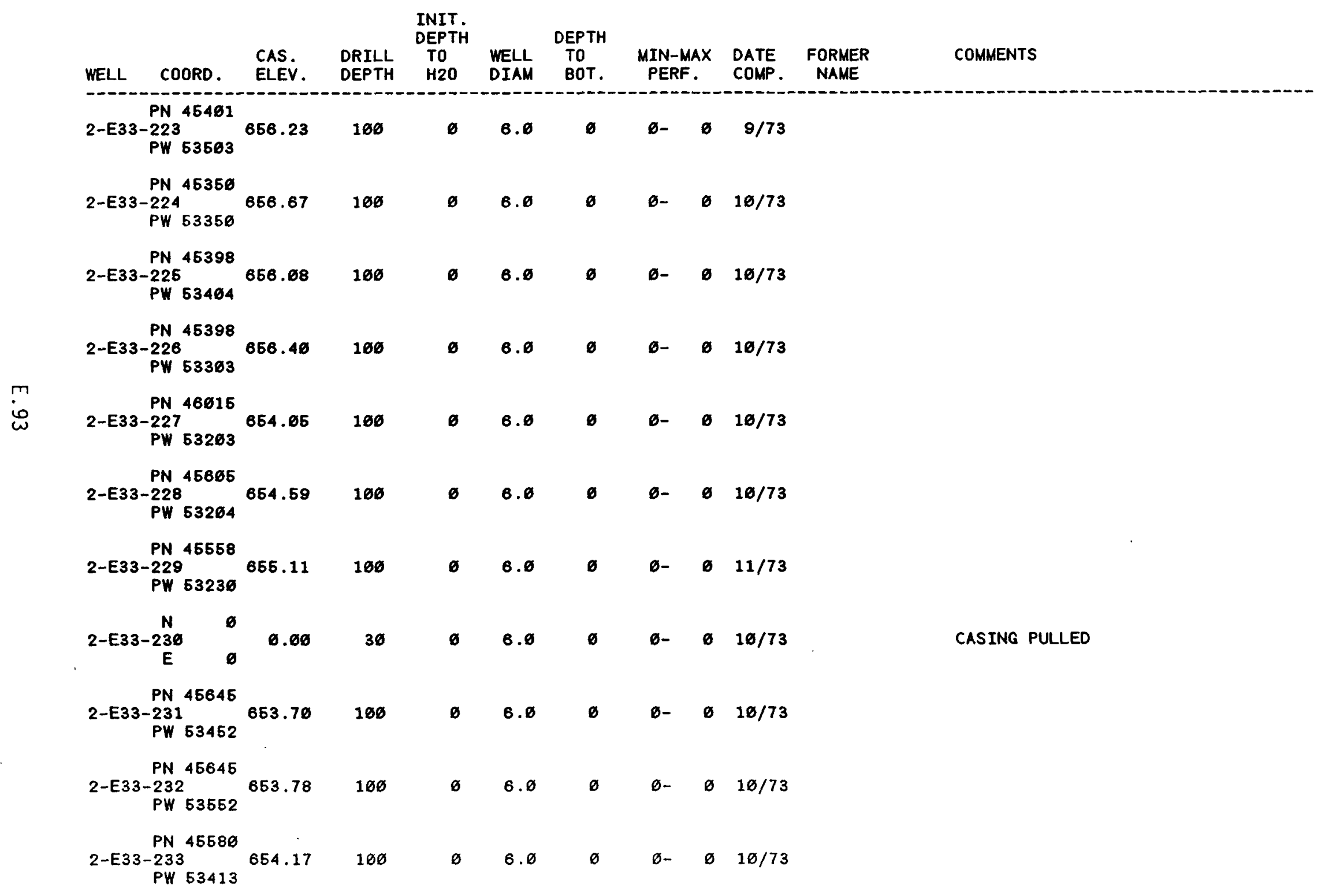




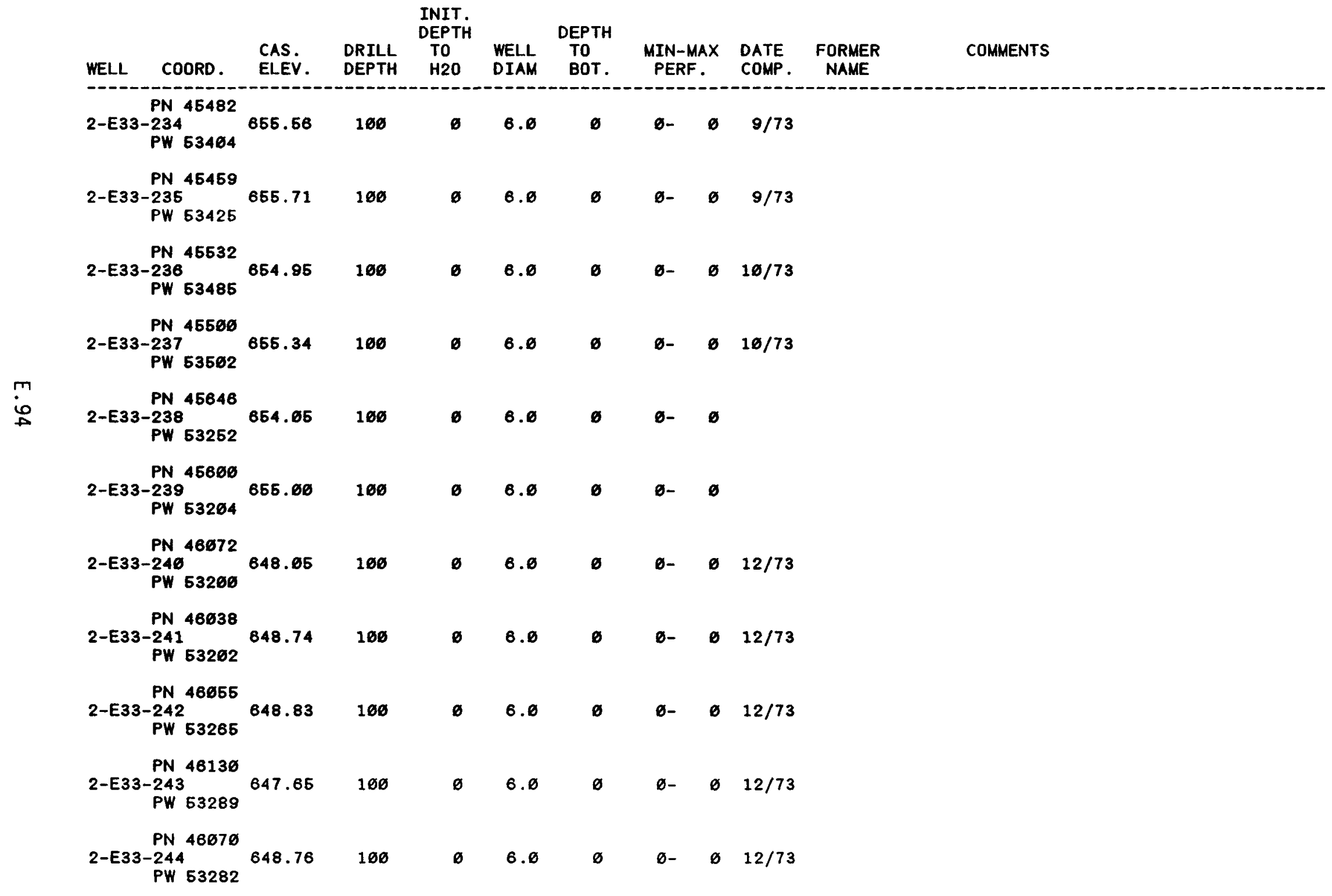




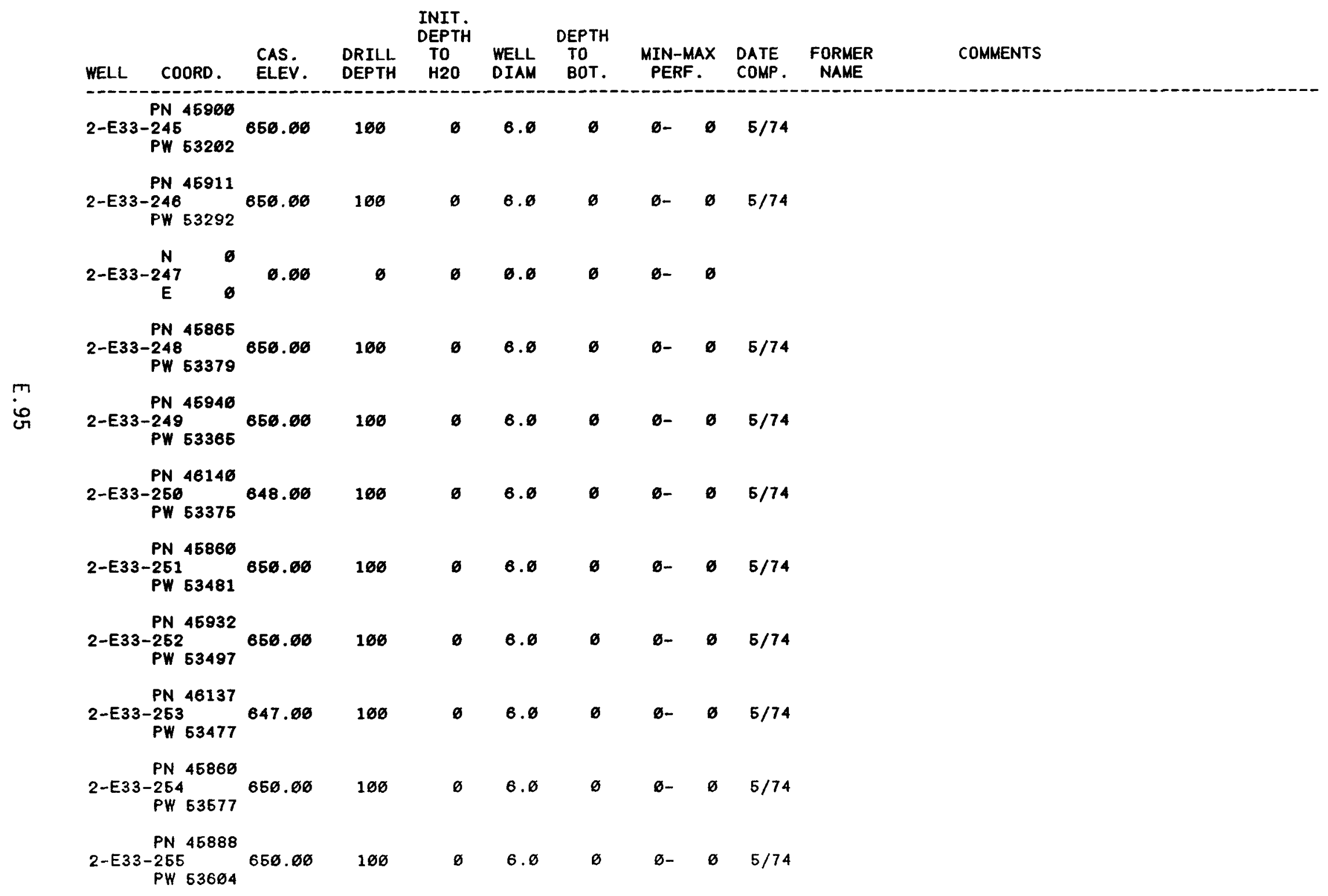




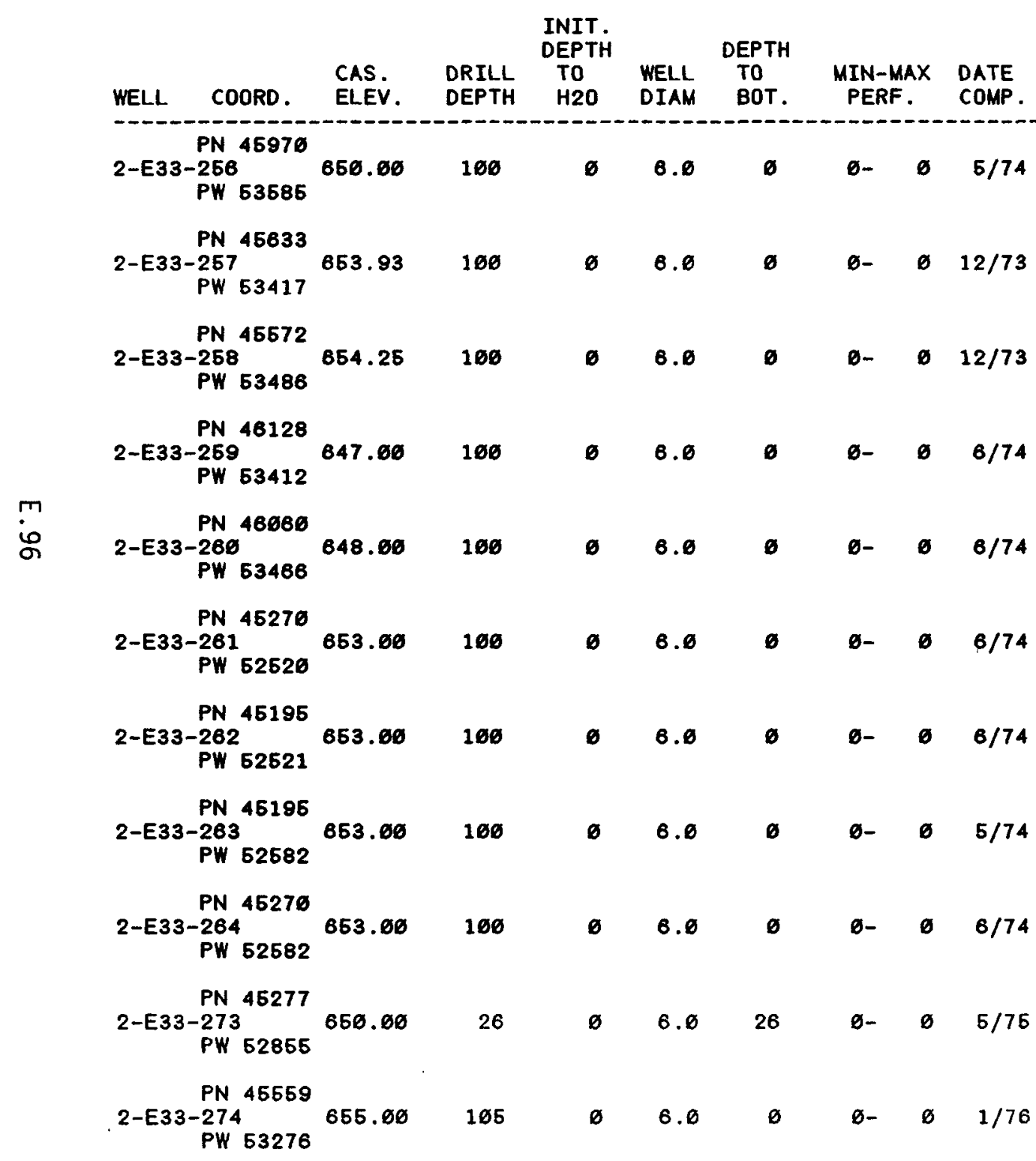




\begin{tabular}{|c|c|c|c|c|c|c|c|c|c|c|c|c|}
\hline WELL & $\mathrm{CO}$ & & $\begin{array}{l}\text { CAS. } \\
\text { ELEV. }\end{array}$ & $\begin{array}{l}\text { DRILL } \\
\text { DEPTH }\end{array}$ & $\begin{array}{l}\text { INIT. } \\
\text { DEPTH } \\
\text { TO } \\
\text { H2O }\end{array}$ & $\begin{array}{l}\text { WELL } \\
\text { DIAM }\end{array}$ & $\begin{array}{c}\text { DEPTH } \\
\text { TO } \\
\text { BOT. }\end{array}$ & $\begin{array}{r}\text { MIN-M } \\
\text { PERF }\end{array}$ & & $\begin{array}{l}\text { DATE } \\
\text { COMP. }\end{array}$ & $\begin{array}{c}\text { FORMER } \\
\text { NAME }\end{array}$ & COMMENTS \\
\hline $2-E 33$ & $\begin{array}{l}\text { PN } \\
-277 \\
\text { PW }\end{array}$ & & 656.00 & 105 & $\varnothing$ & 6.0 & $\emptyset$ & 0- & $\emptyset$ & $1 / 76$ & & \\
\hline $2-E 33$ & $\begin{array}{l}\text { PN } \\
-286 \\
\text { PW }\end{array}$ & & $\varnothing . \varnothing 0$ & Eש & E & 8.6 & 50 & ø- & ø & $8 / 82$ & & GROUTED \\
\hline 2-E3: & $\begin{array}{l}P N \\
-287 \\
P W\end{array}$ & & 0.00 & $5 \varnothing$ & $\varnothing$ & 8.6 & 56 & b- & ø & $8 / 82$ & & GROUTED \\
\hline 2-E3: & $\begin{array}{l}P N \\
-288 \\
P W\end{array}$ & & 0.06 & 56 & $\varnothing$ & 8.0 & 49 & ø- & $\emptyset$ & $8 / 82$ & & GROUTED \\
\hline $2-E 3$ & $\begin{array}{l}\text { PN } \\
-289 \\
\text { PW }\end{array}$ & & 0.00 & 56 & 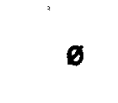 & 8.0 & 49 & ø- & $\emptyset$ & $8 / 82$ & & GROUTED \\
\hline $2-E 3$ & $\begin{array}{l}\mathrm{PN} \\
-296 \\
\mathrm{PW}\end{array}$ & & 0.00 & 50 & 0 & 8.0 & ø & b- & $\emptyset$ & $8 / 82$ & & GROUTED \\
\hline $2-E 3$ & $\begin{array}{r}N \\
-291 \\
E\end{array}$ & 0 & $\varnothing . \varnothing 0$ & 11 & ø & 2.0 & ø & 6- & ø & $4 / 88$ & & SCREEN 6-11 FT. \\
\hline $2-E 3$ & $\begin{array}{c}N \\
-292 \\
E\end{array}$ & 0 & $\varnothing . \varnothing 0$ & 11 & $\boldsymbol{D}$ & 2.0 & ø & ø- & $\emptyset$ & $4 / 88$ & & SCREEN 6-11 FT. \\
\hline $2-E 3$ & $\begin{array}{c}N \\
-293 \\
E\end{array}$ & 0 & 0.00 & 15 & 0 & 2.0 & $\varnothing$ & ø- & $\emptyset$ & $4 / 88$ & & SCREEN $1 \varnothing-15 \mathrm{FT}$. \\
\hline $2-E 3$ & $\begin{array}{c}N \\
-294 \\
E\end{array}$ & $\begin{array}{l}\emptyset \\
\emptyset\end{array}$ & $\varnothing . \varnothing 0$ & 4 & D & 0.0 & $\varnothing$ & ø- & $\emptyset$ & $4 / 88$ & & ABANDONED \\
\hline $2-E 3$ & $\begin{array}{c}N \\
-295 \\
E\end{array}$ & 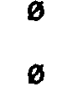 & $\varnothing . \varnothing 0$ & 16 & $\varnothing$ & 2.0 & ø & ø- & 0 & $4 / 88$ & & SCREEN 11-16 FT. \\
\hline
\end{tabular}




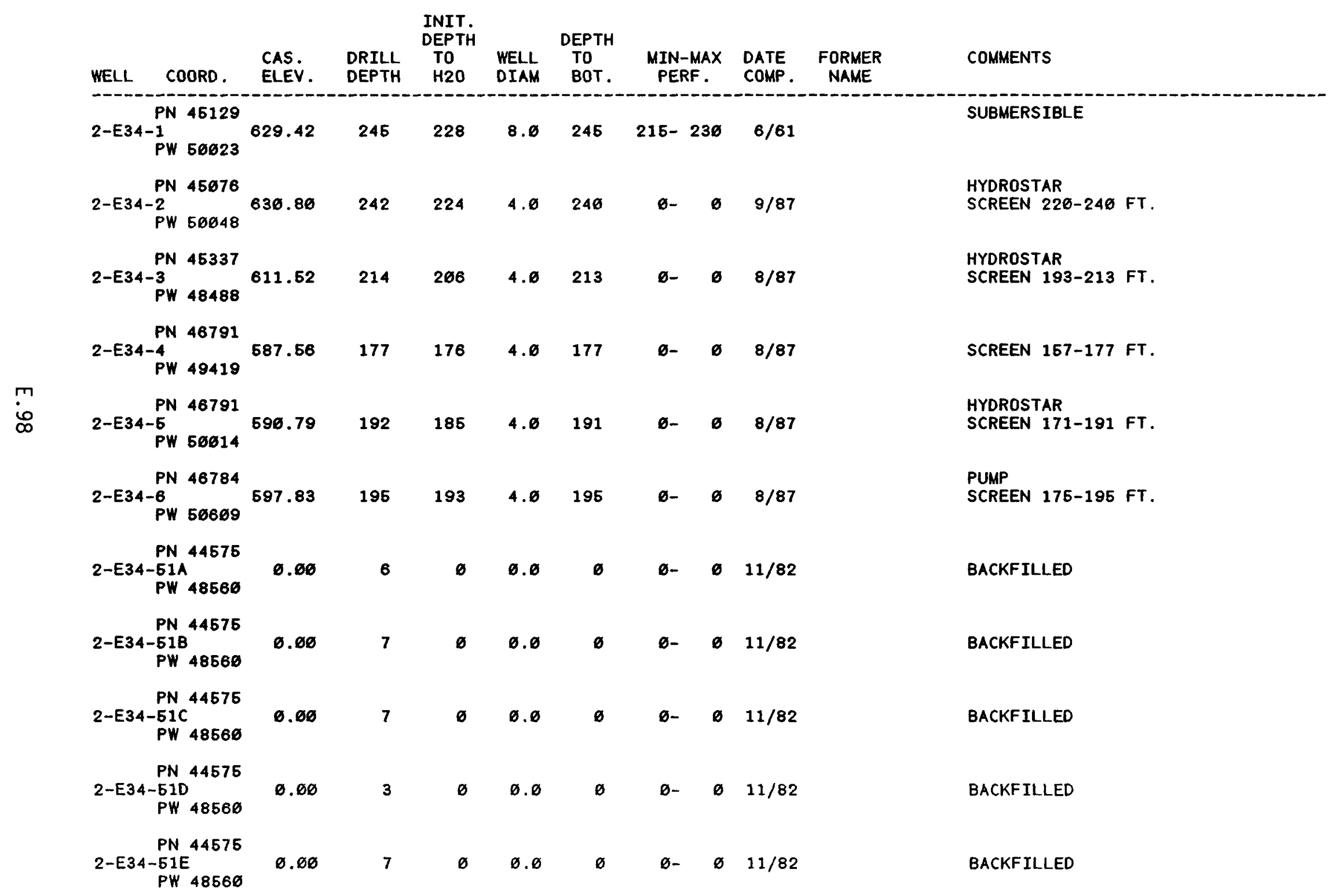




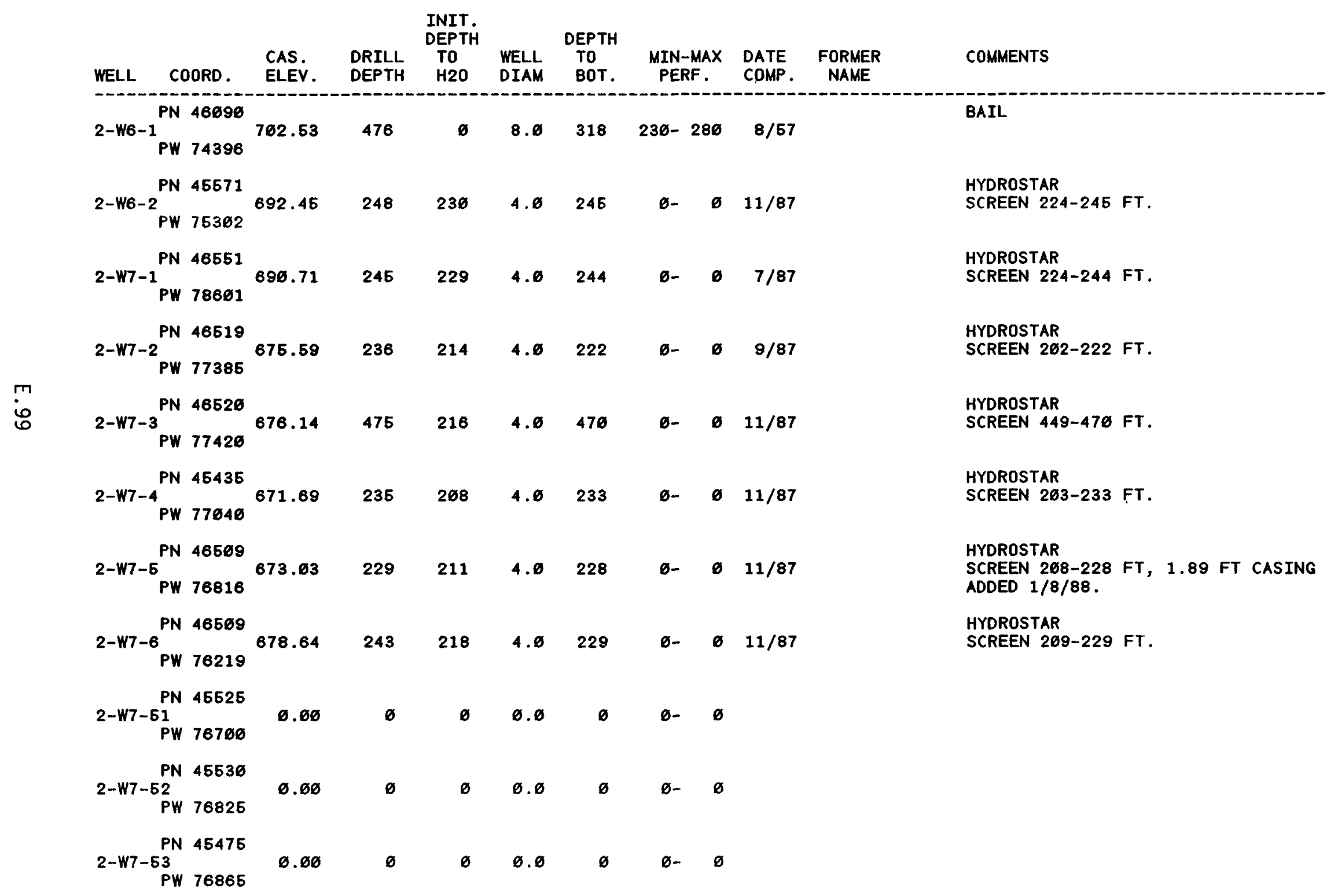




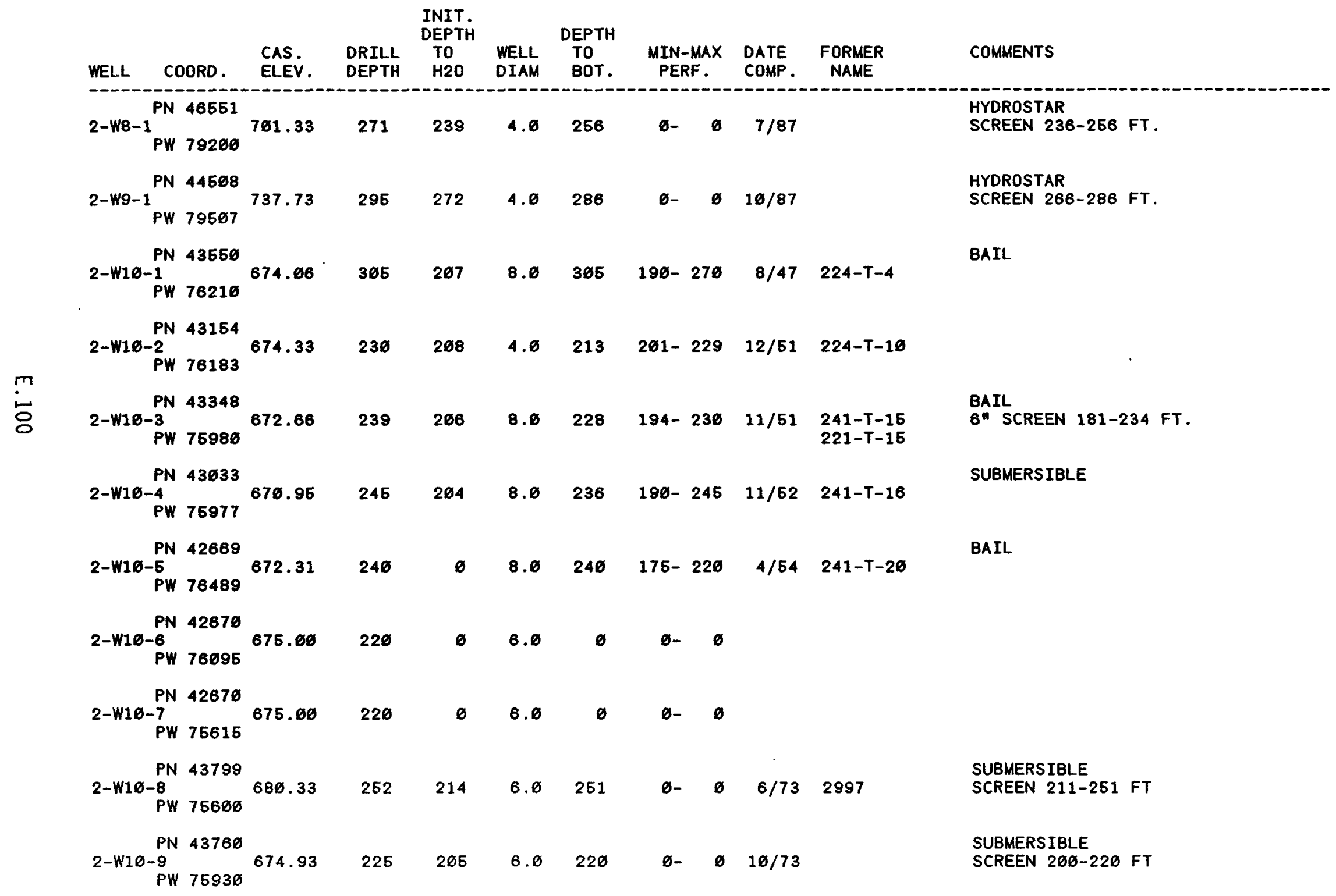




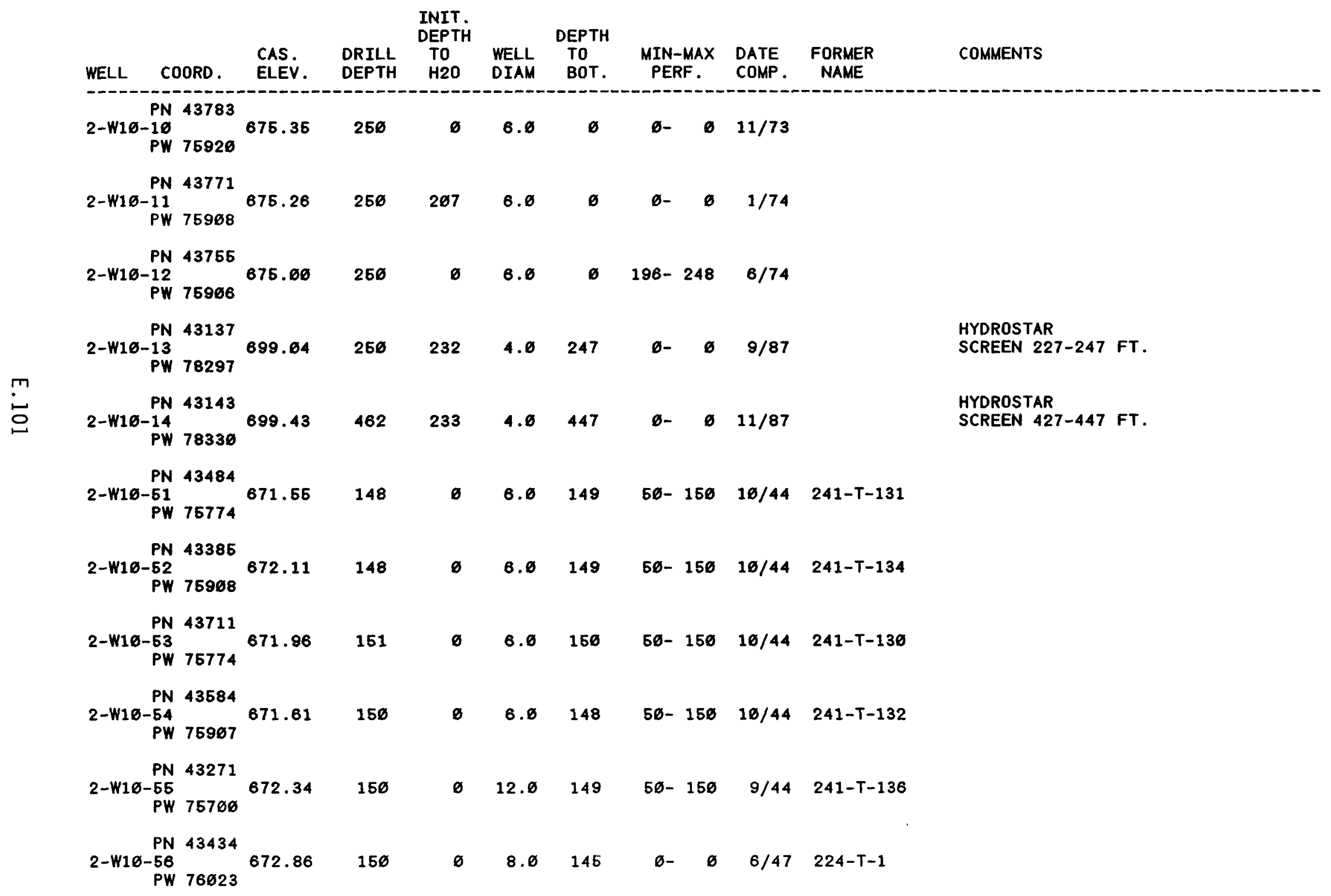




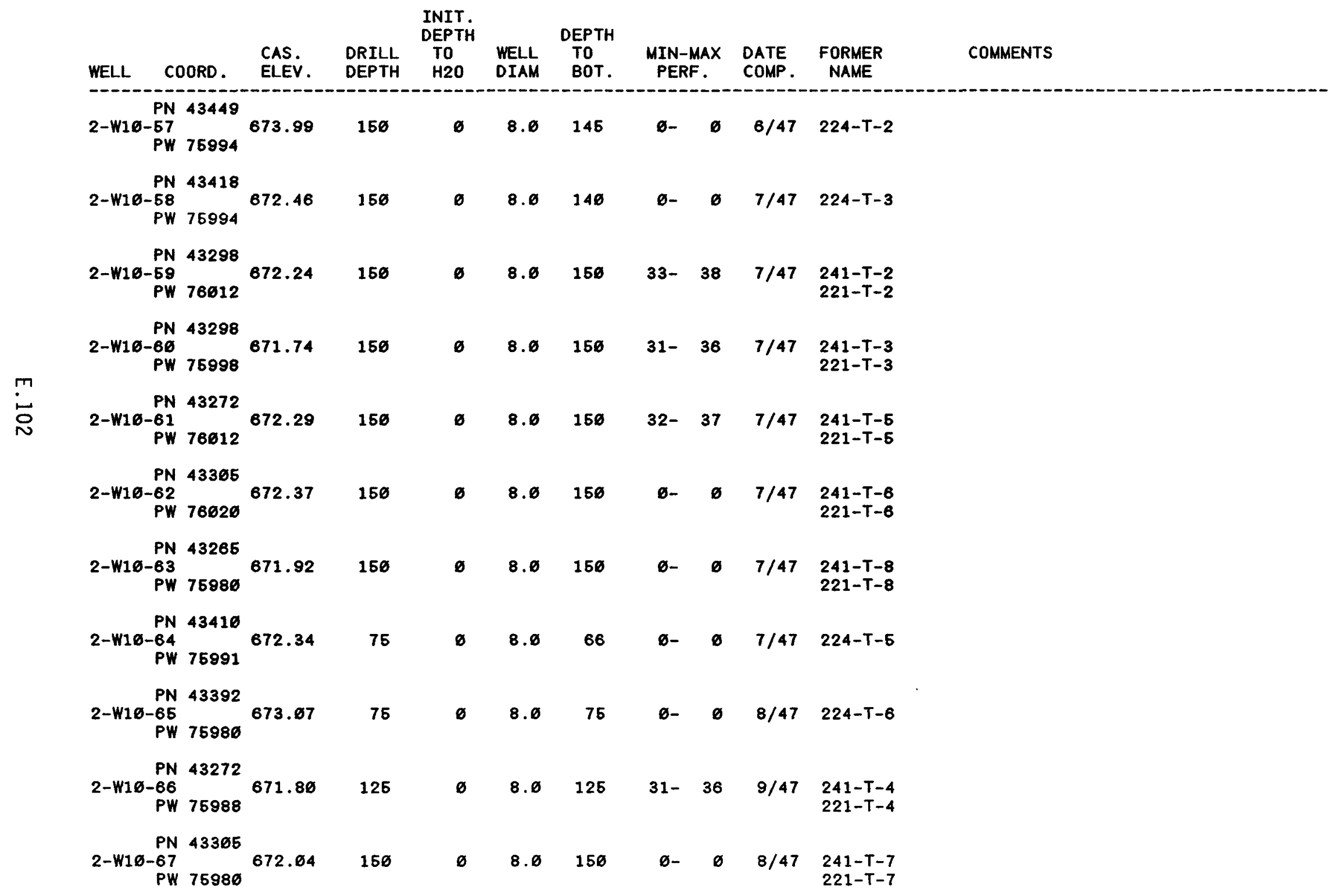




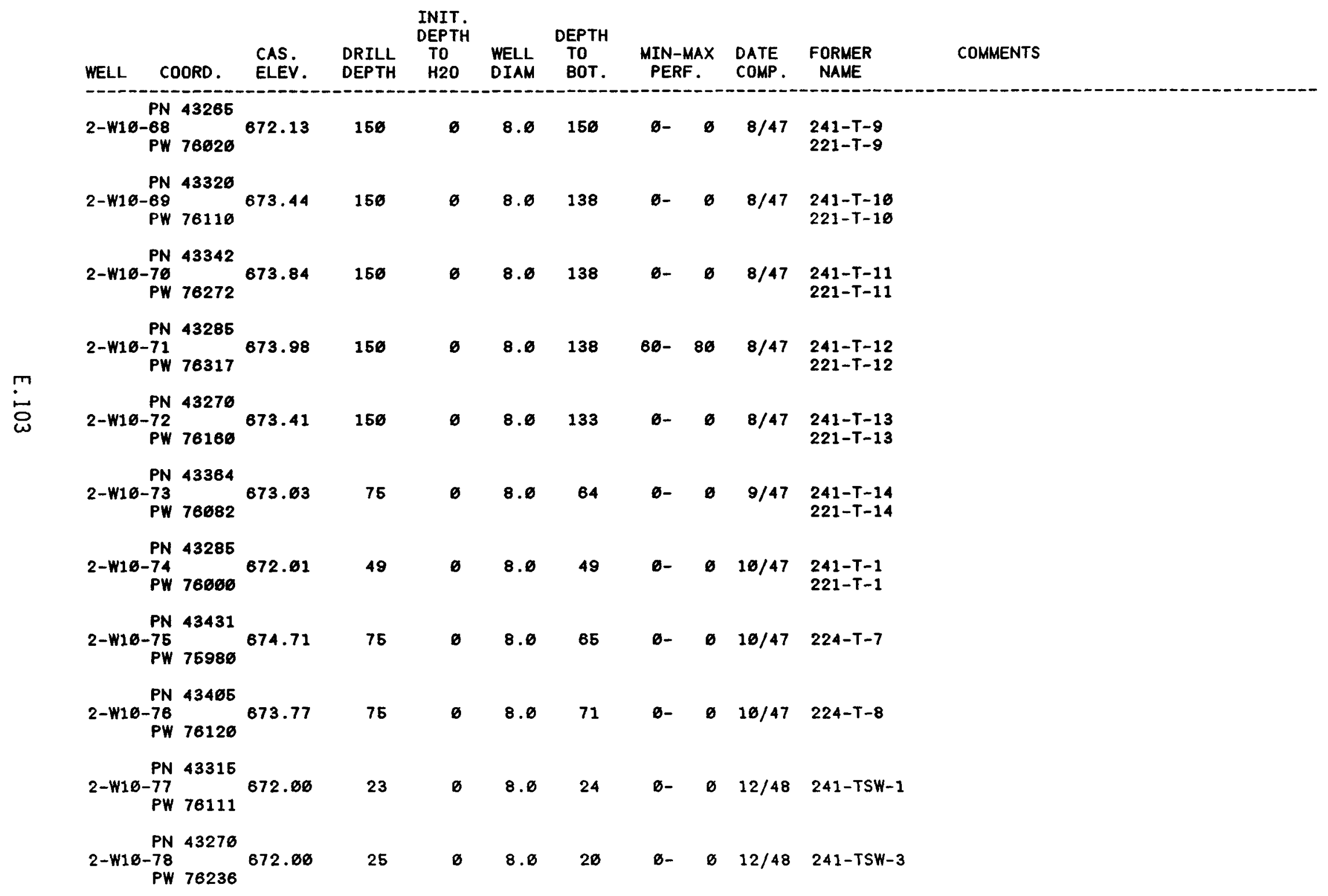




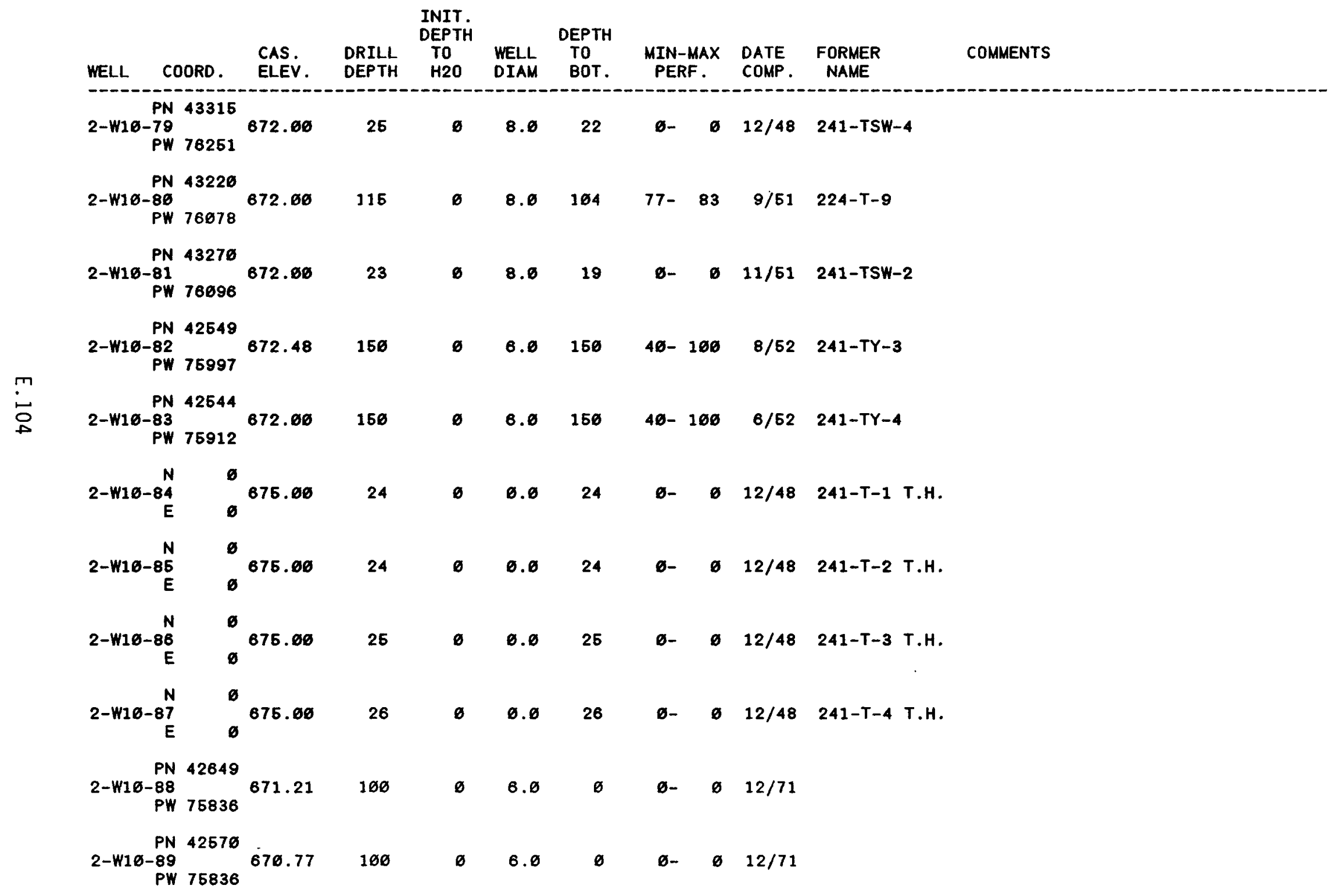




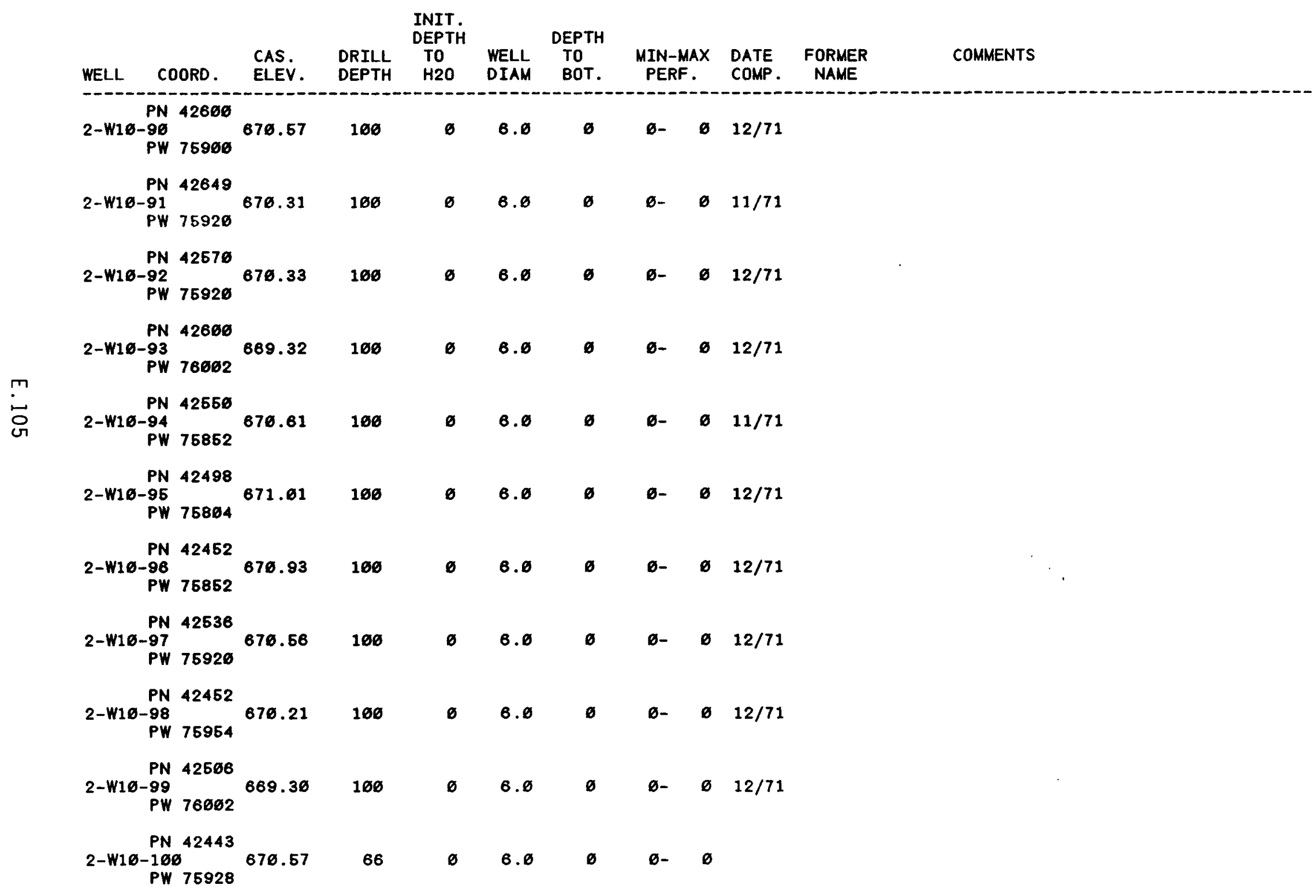




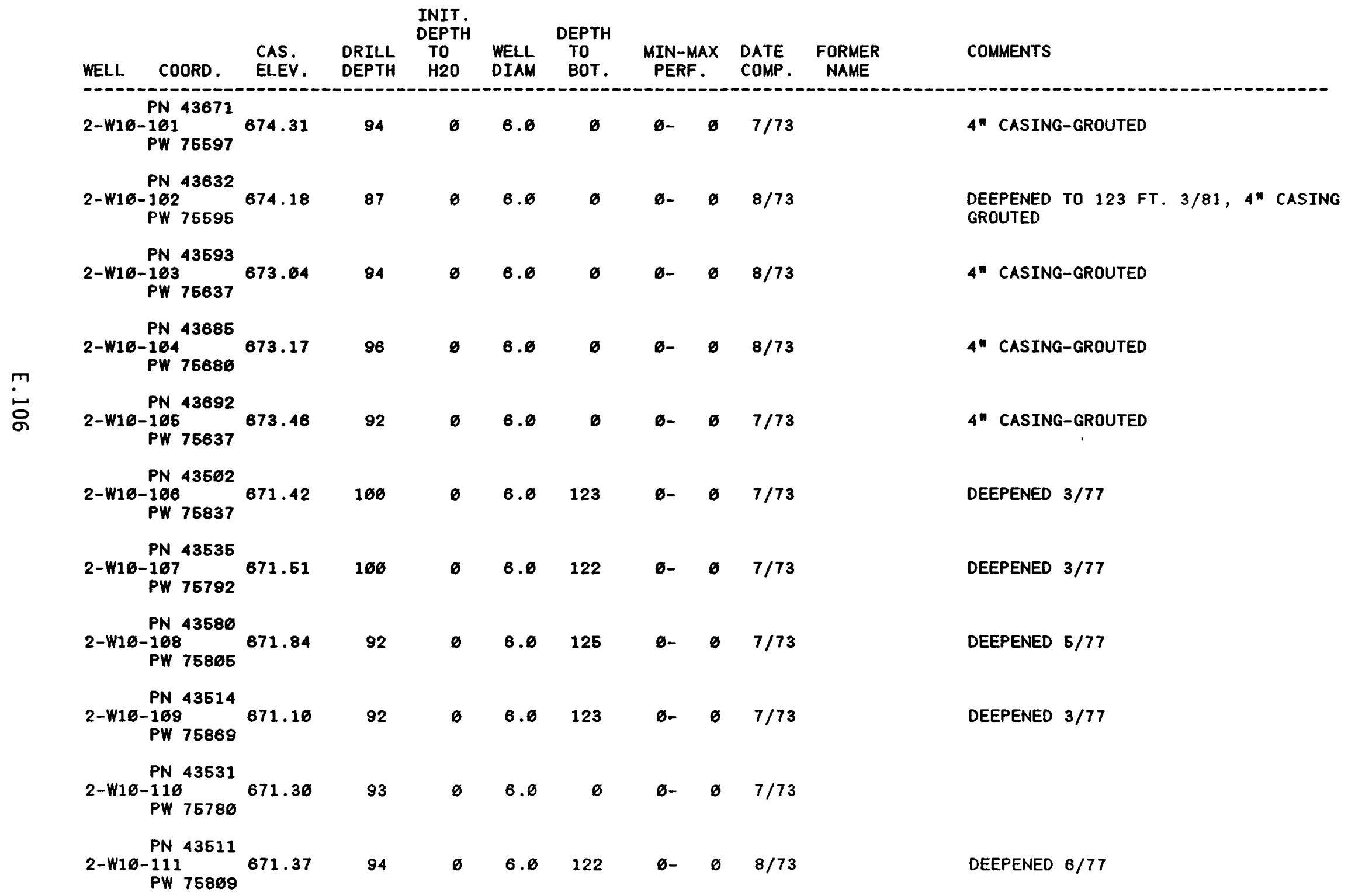




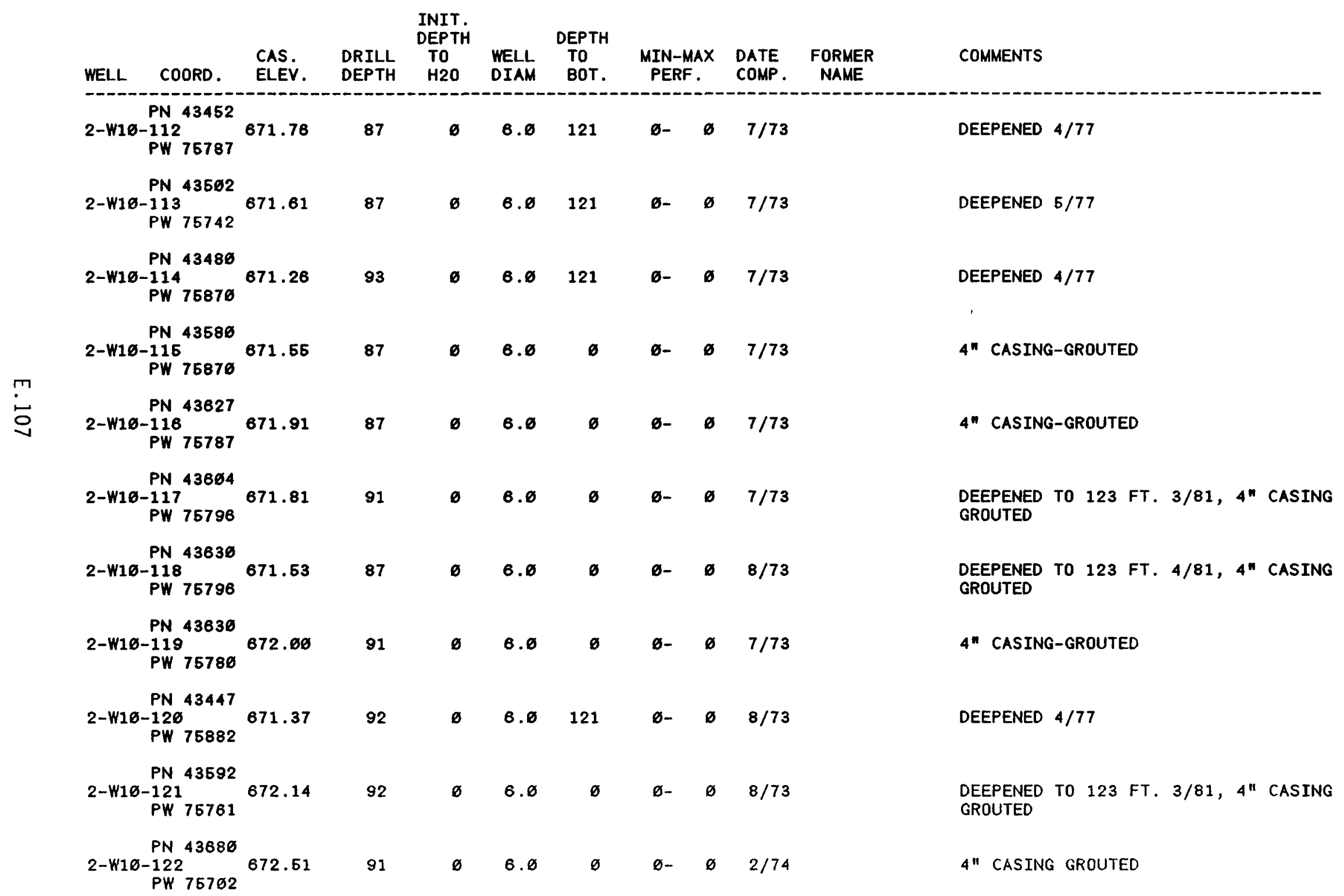




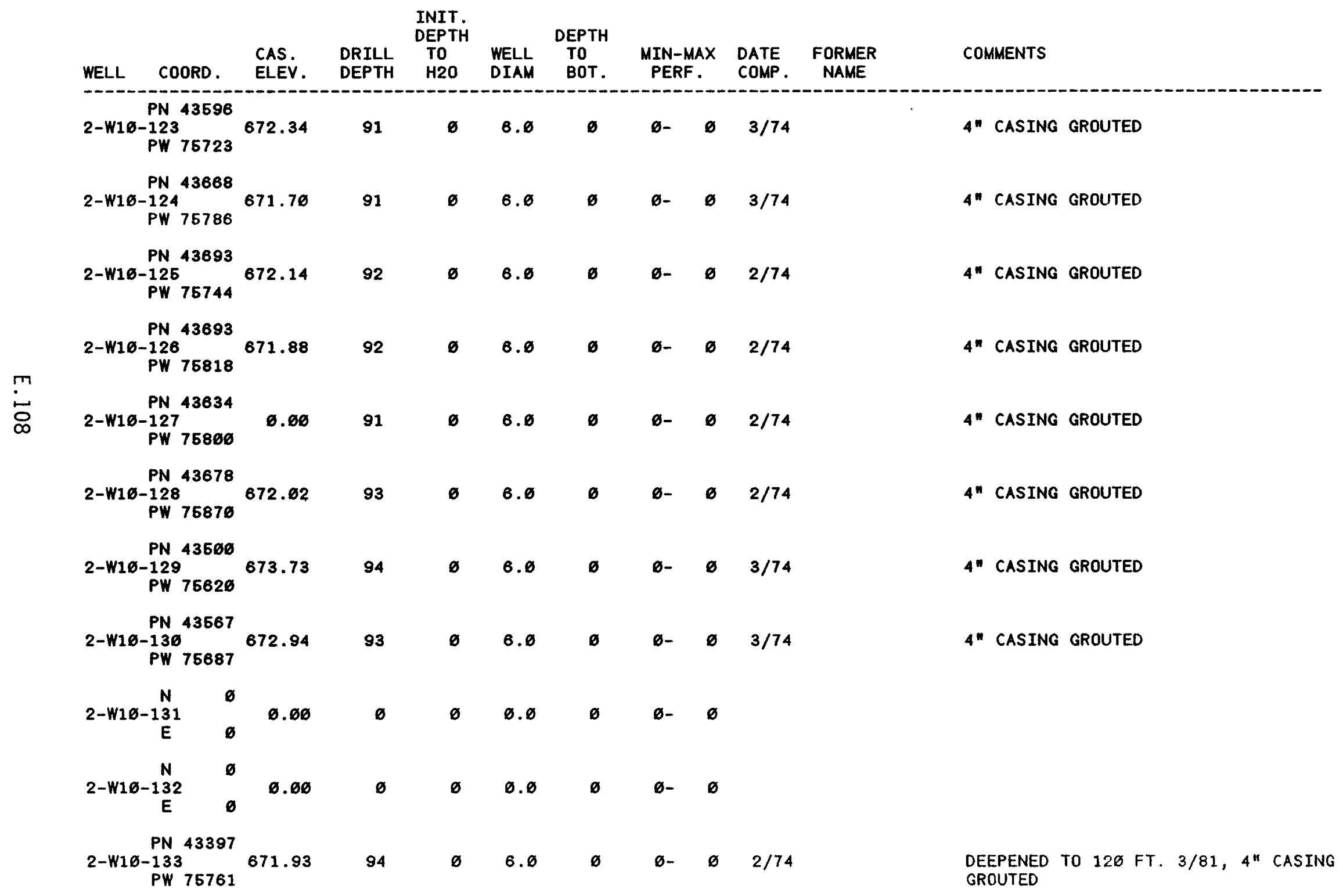




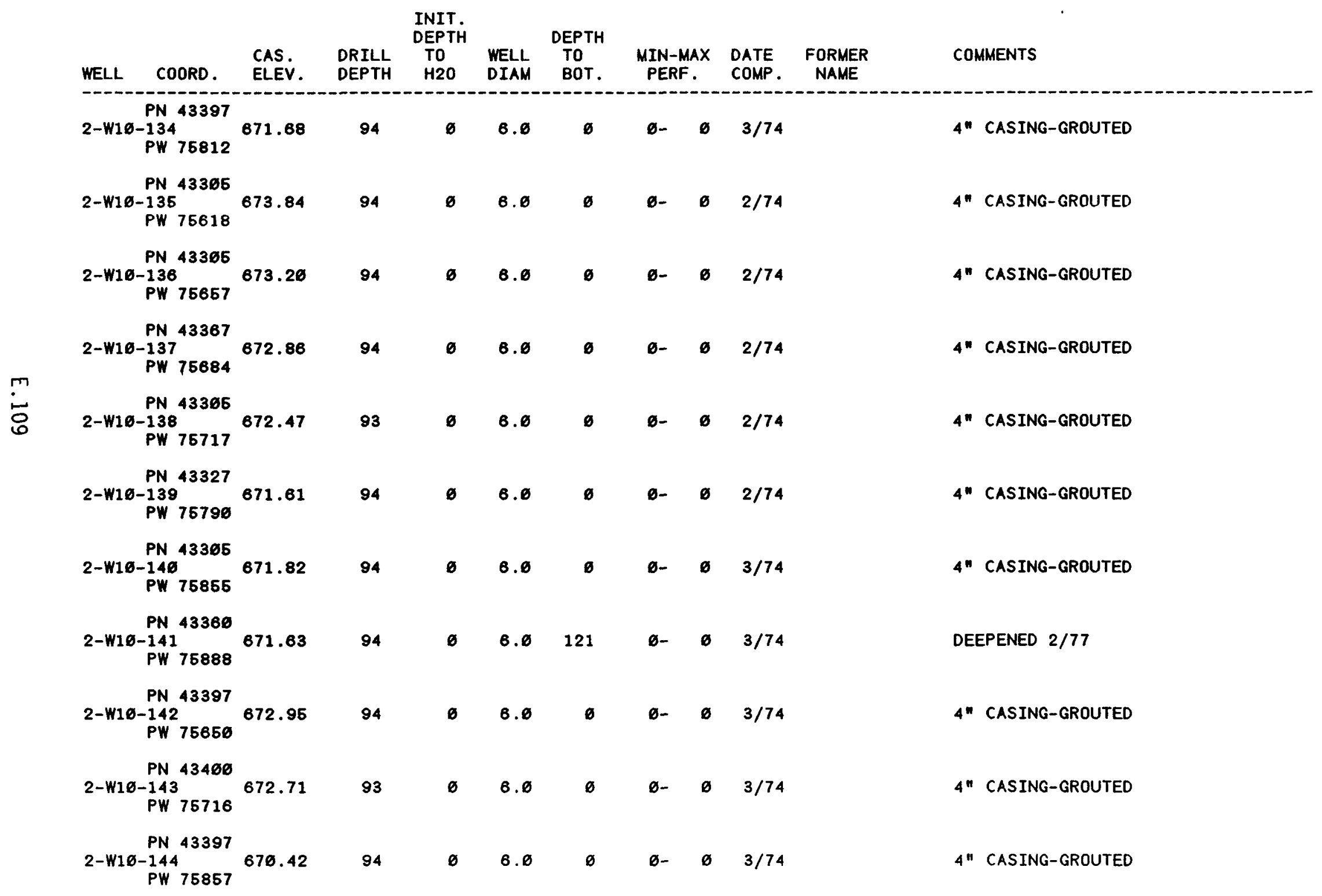




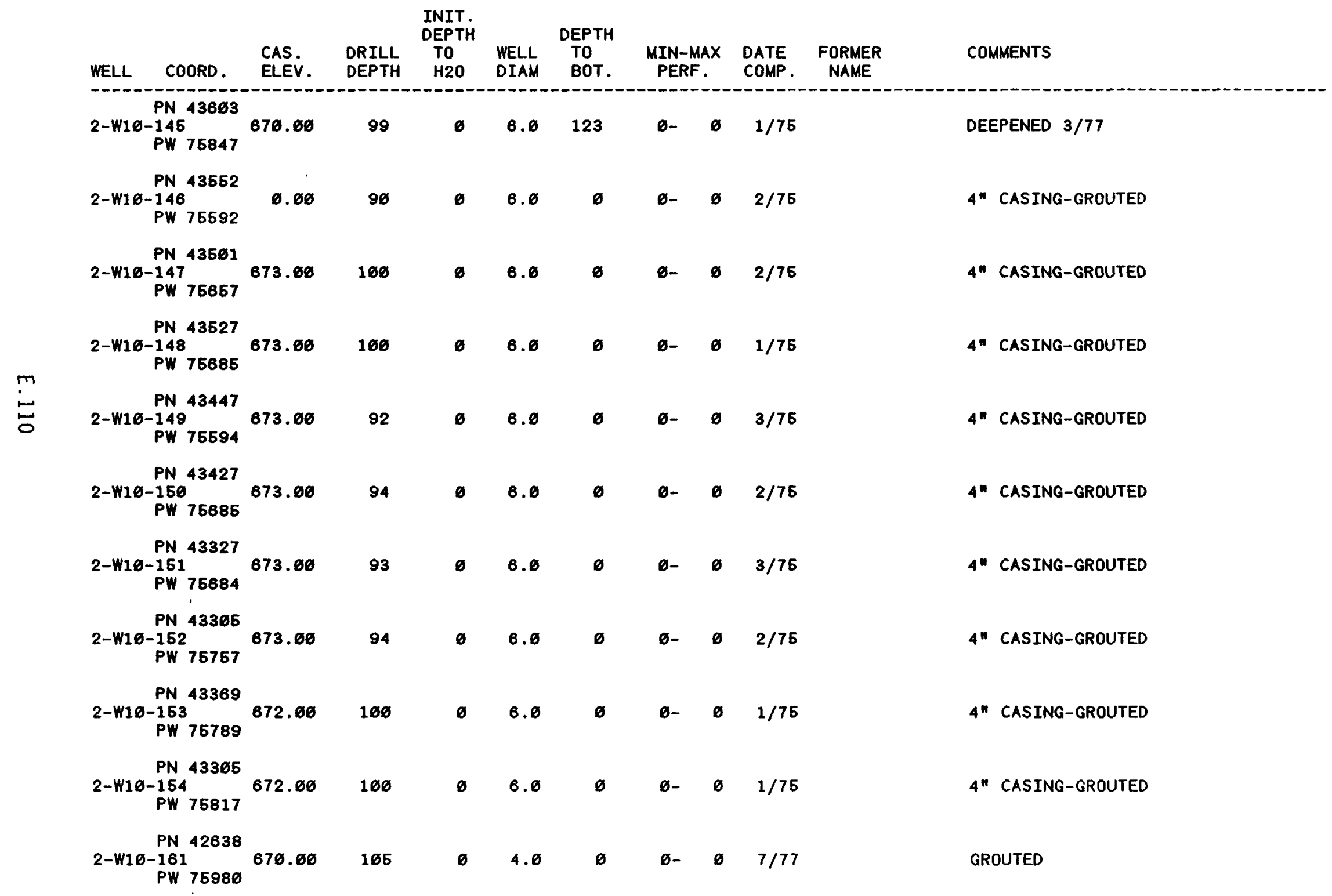




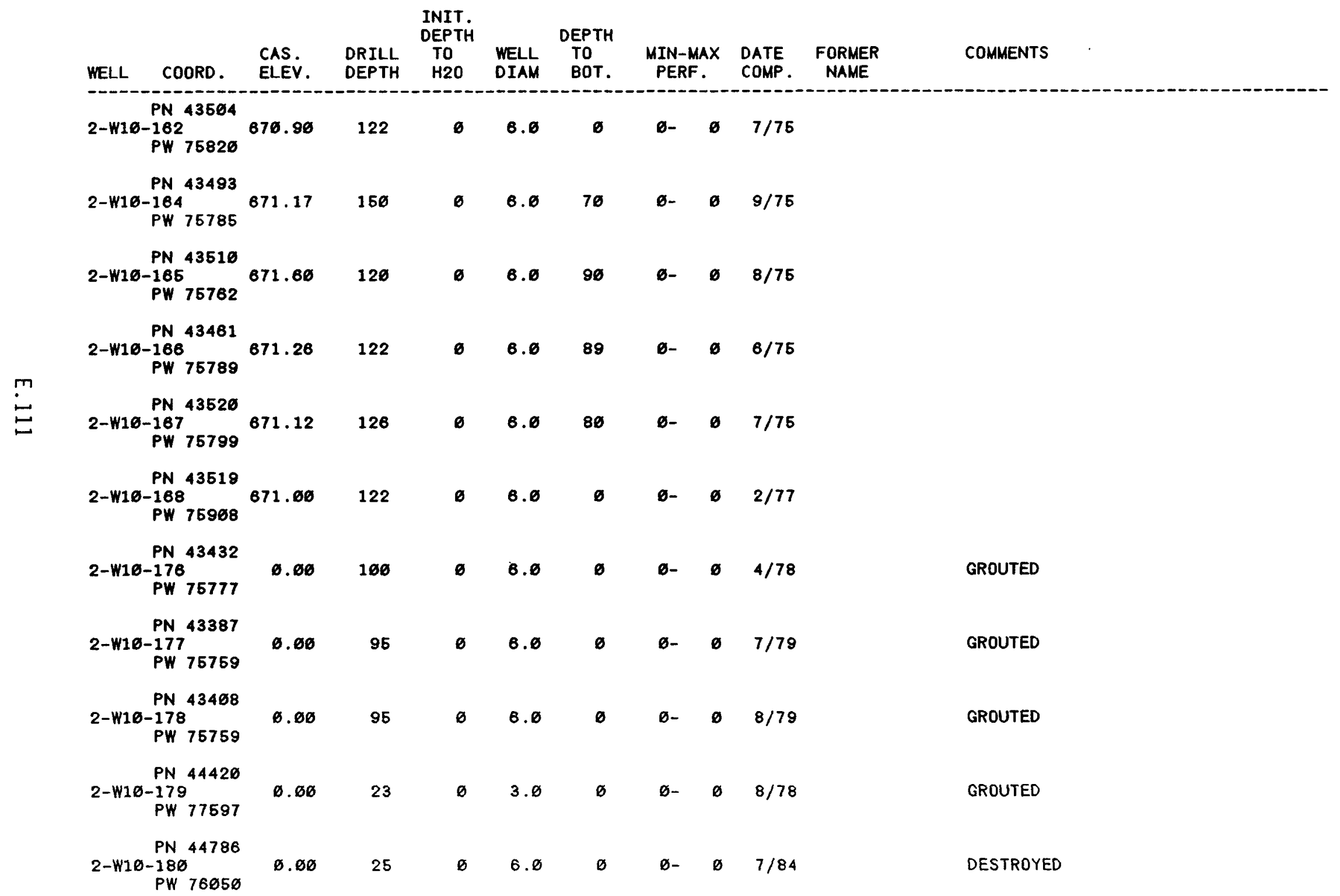




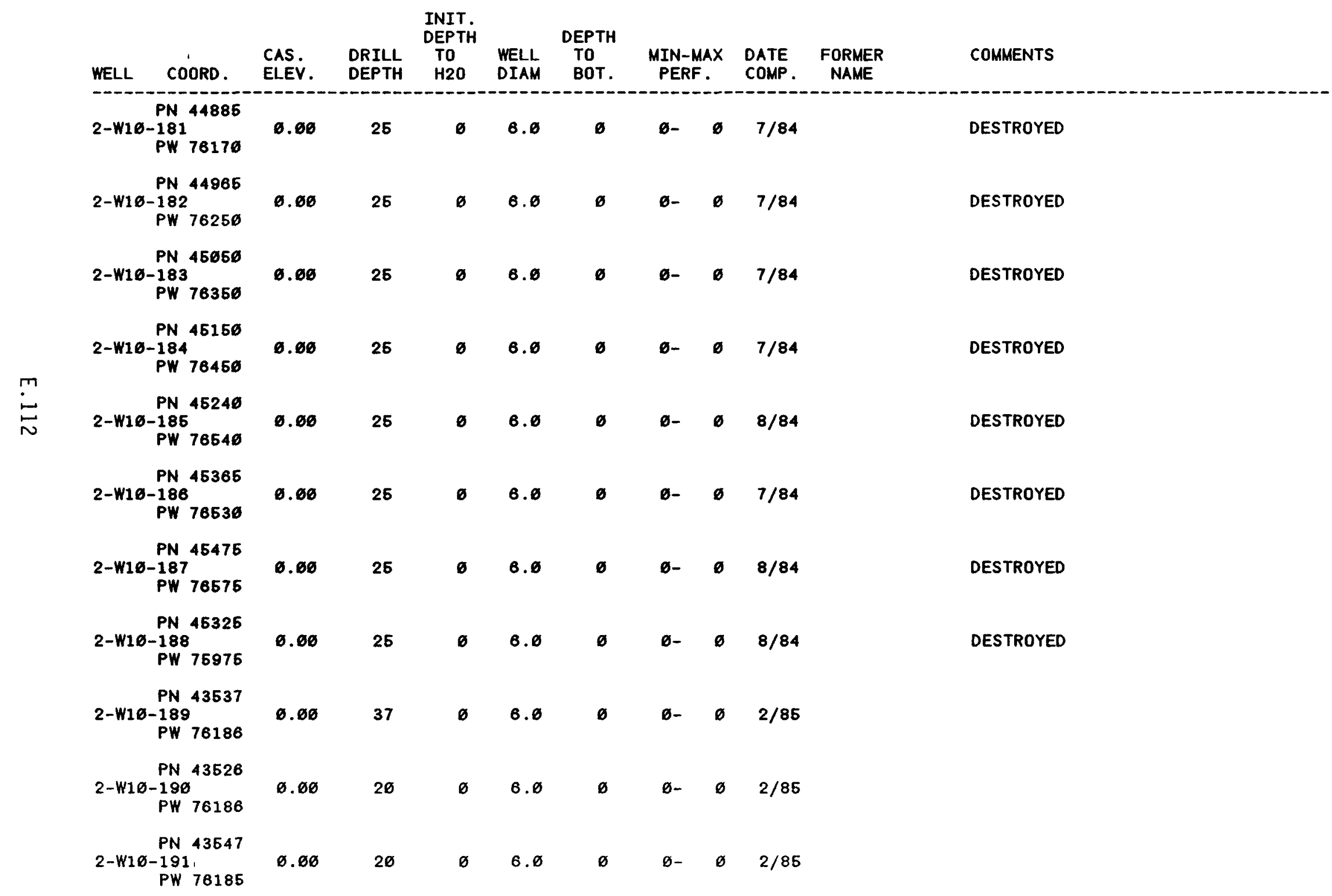




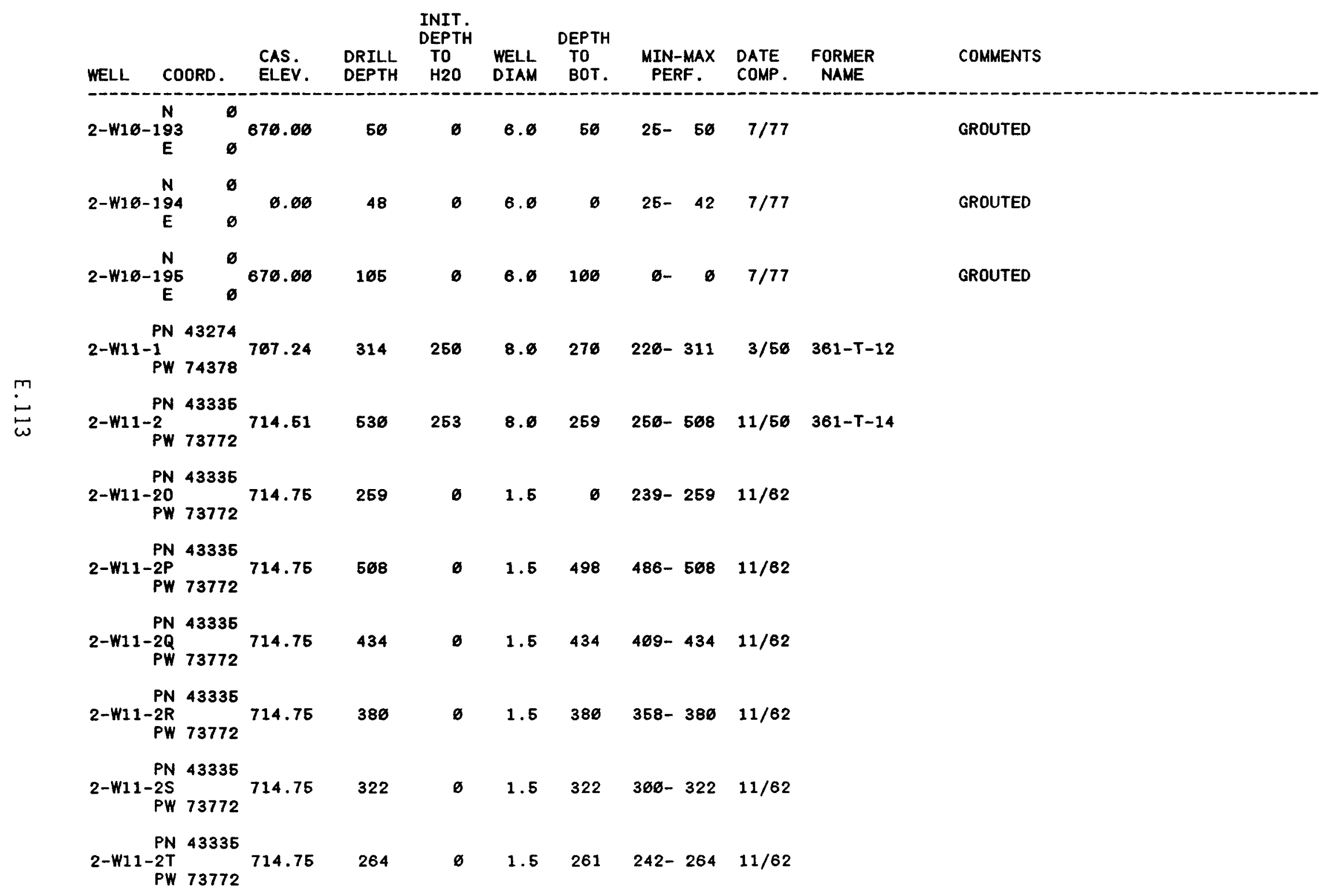




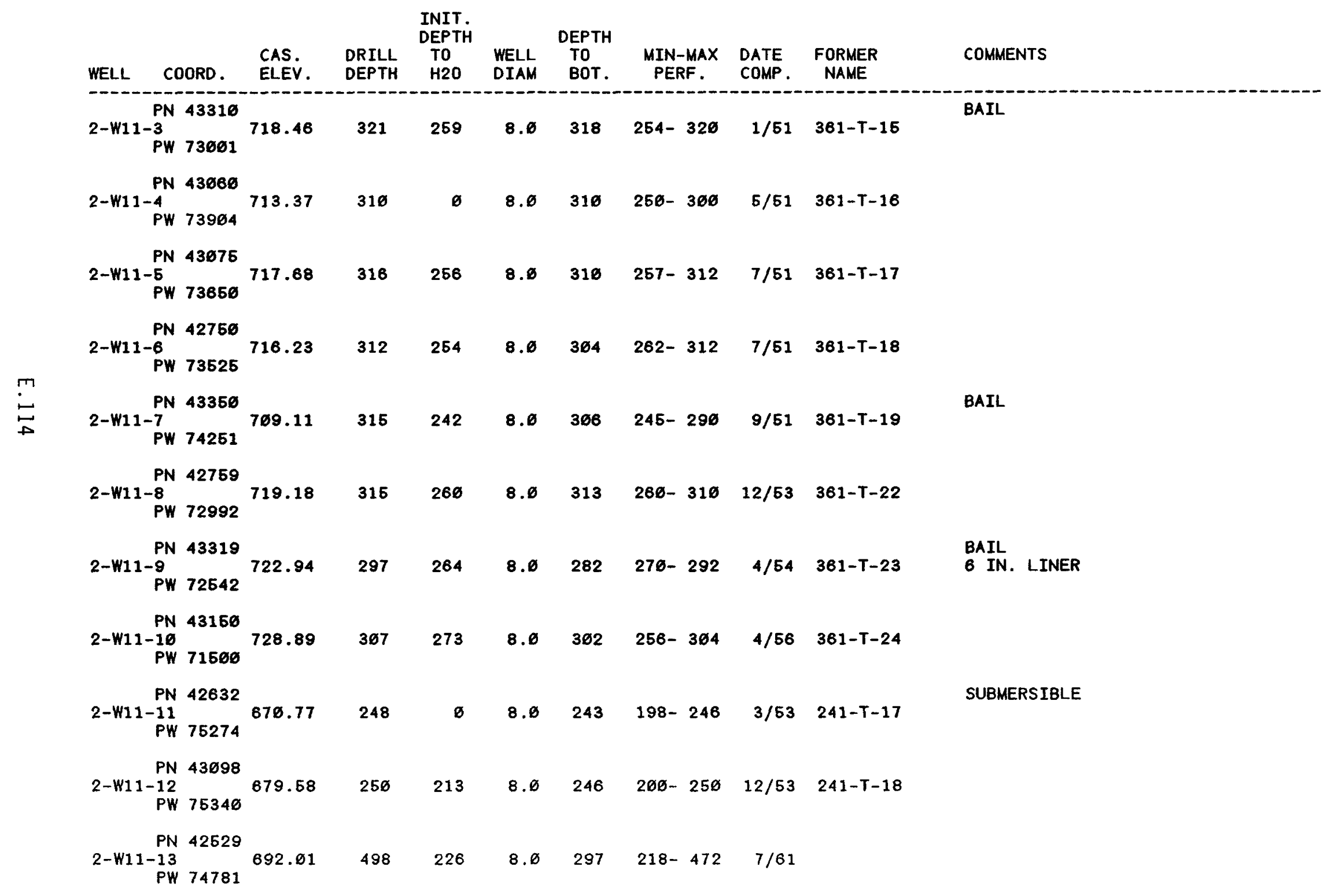




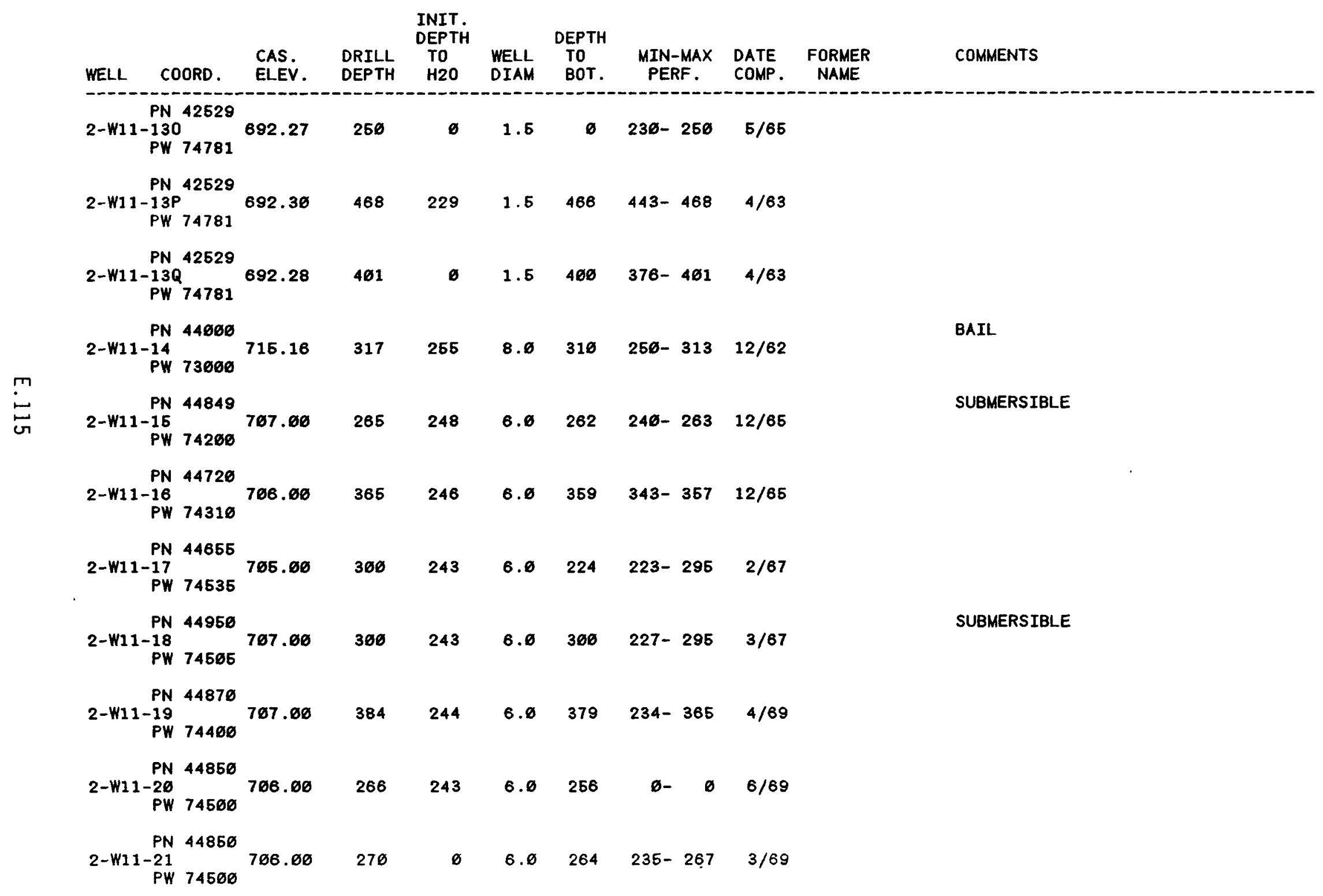




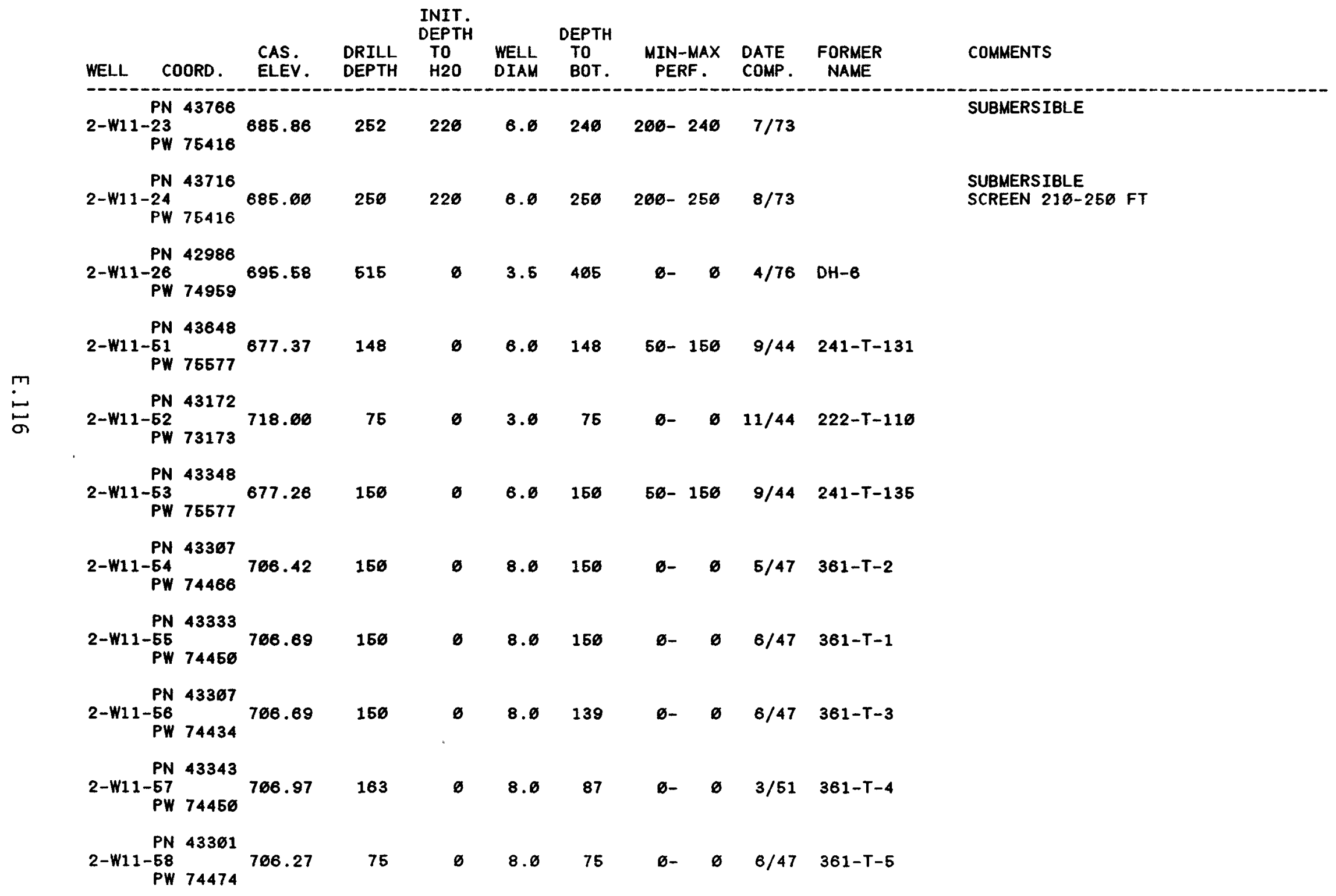




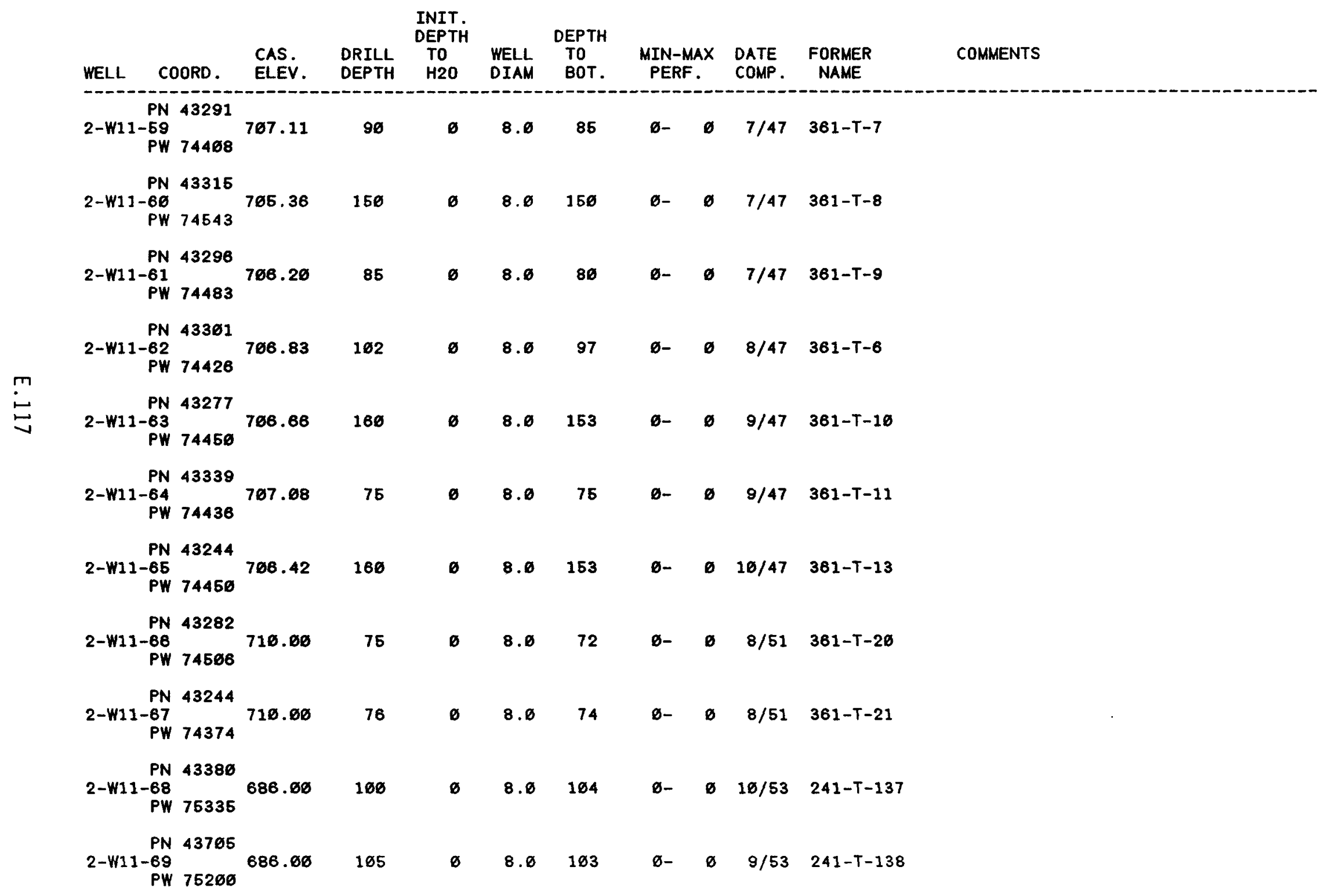




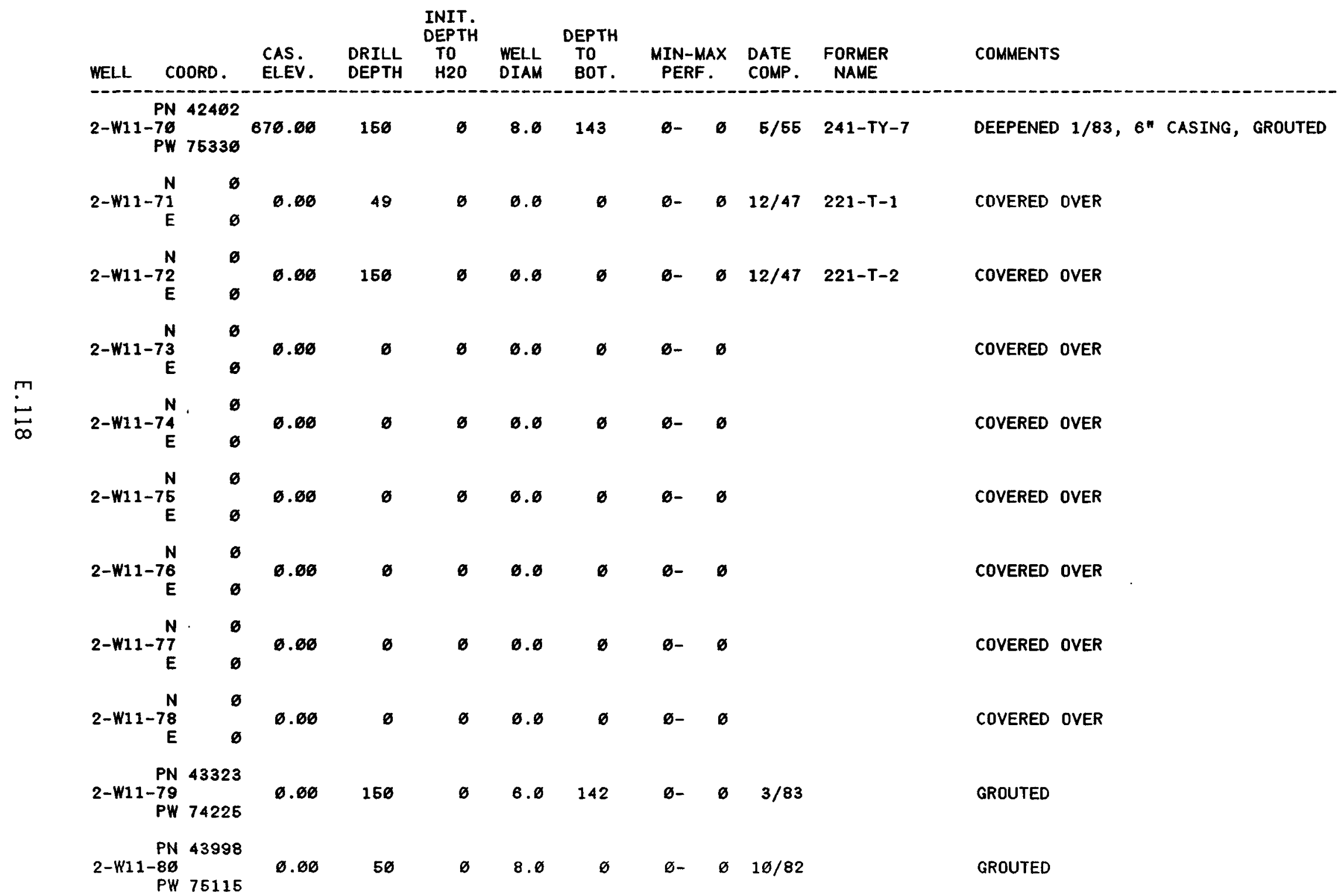




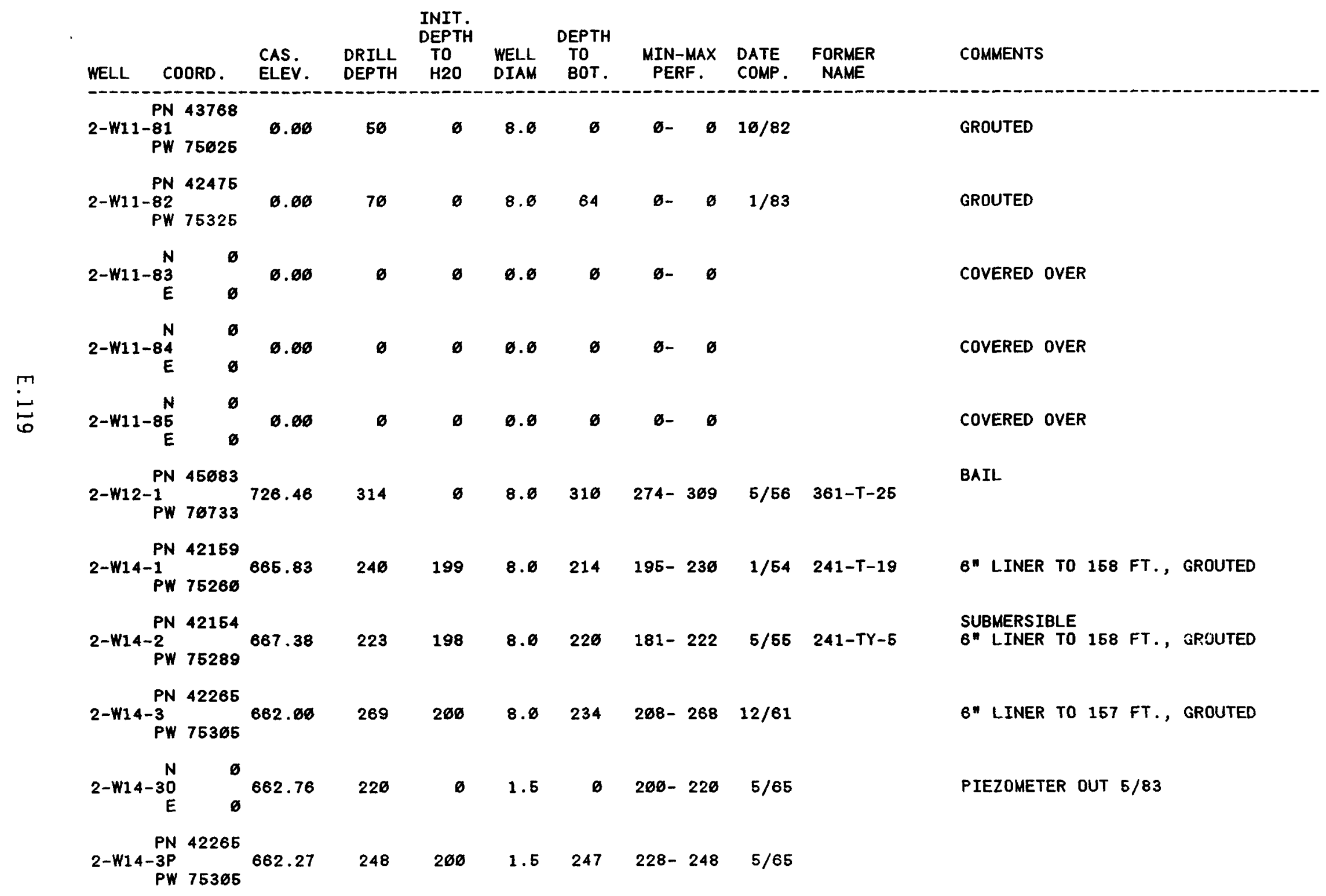




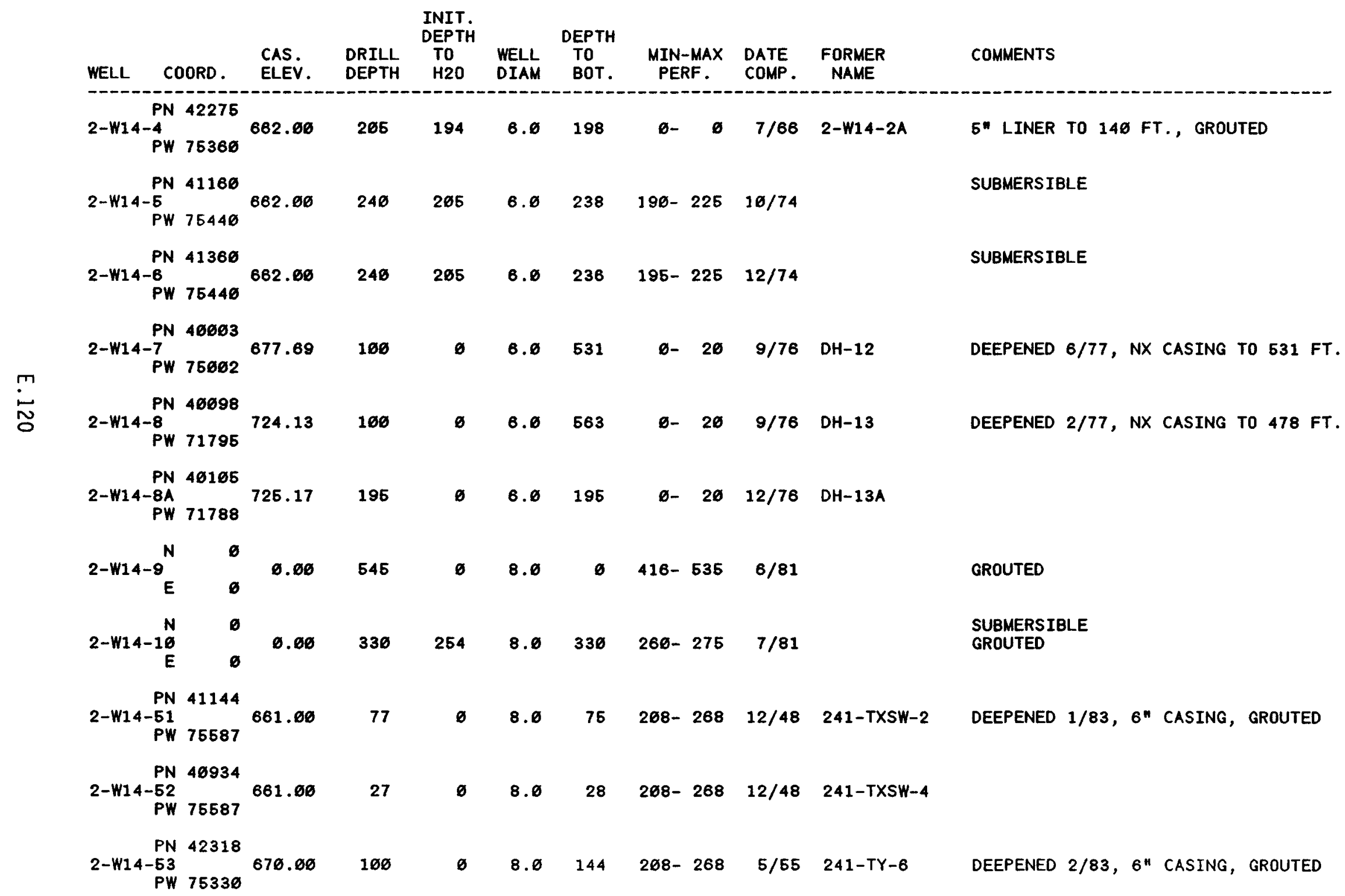




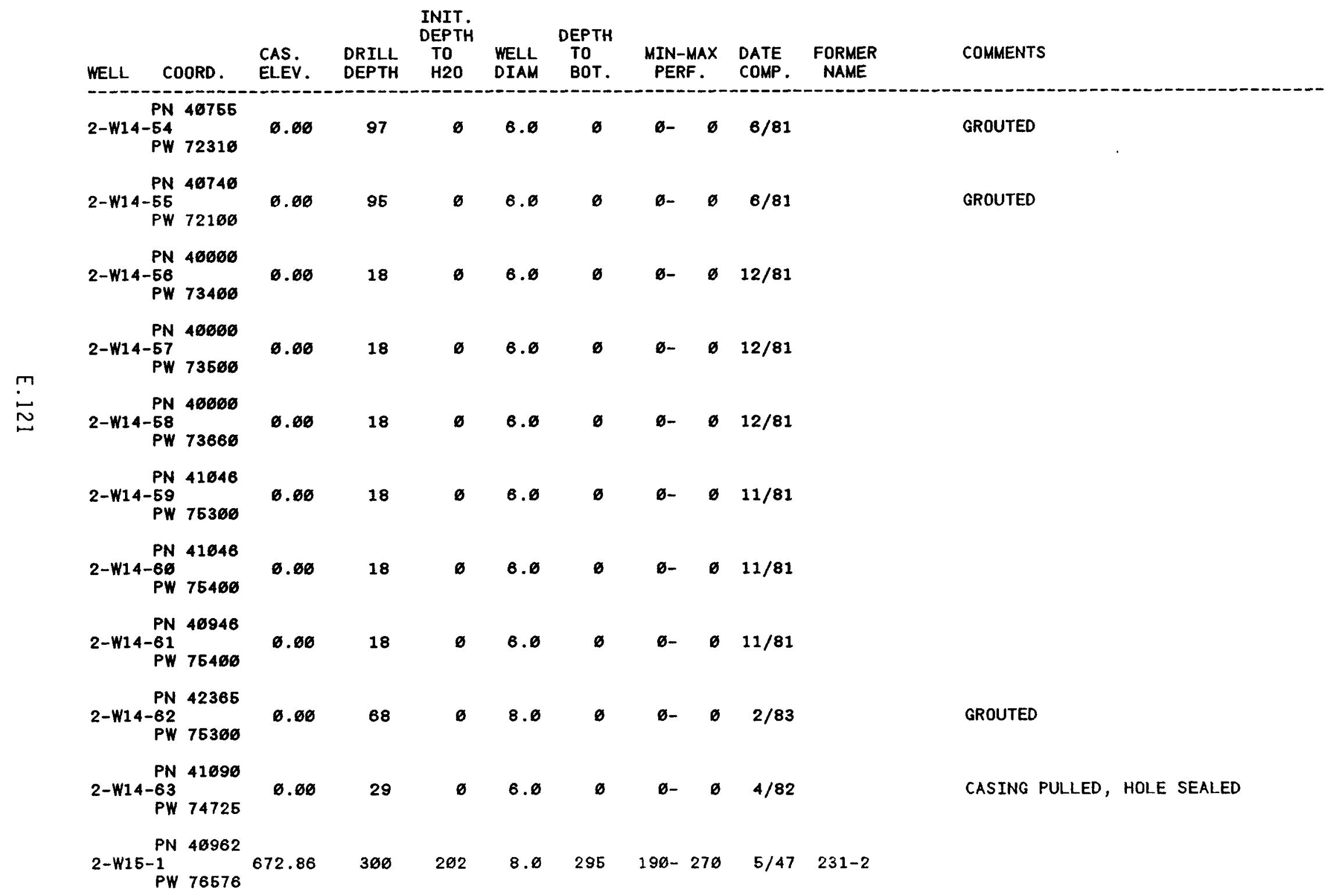




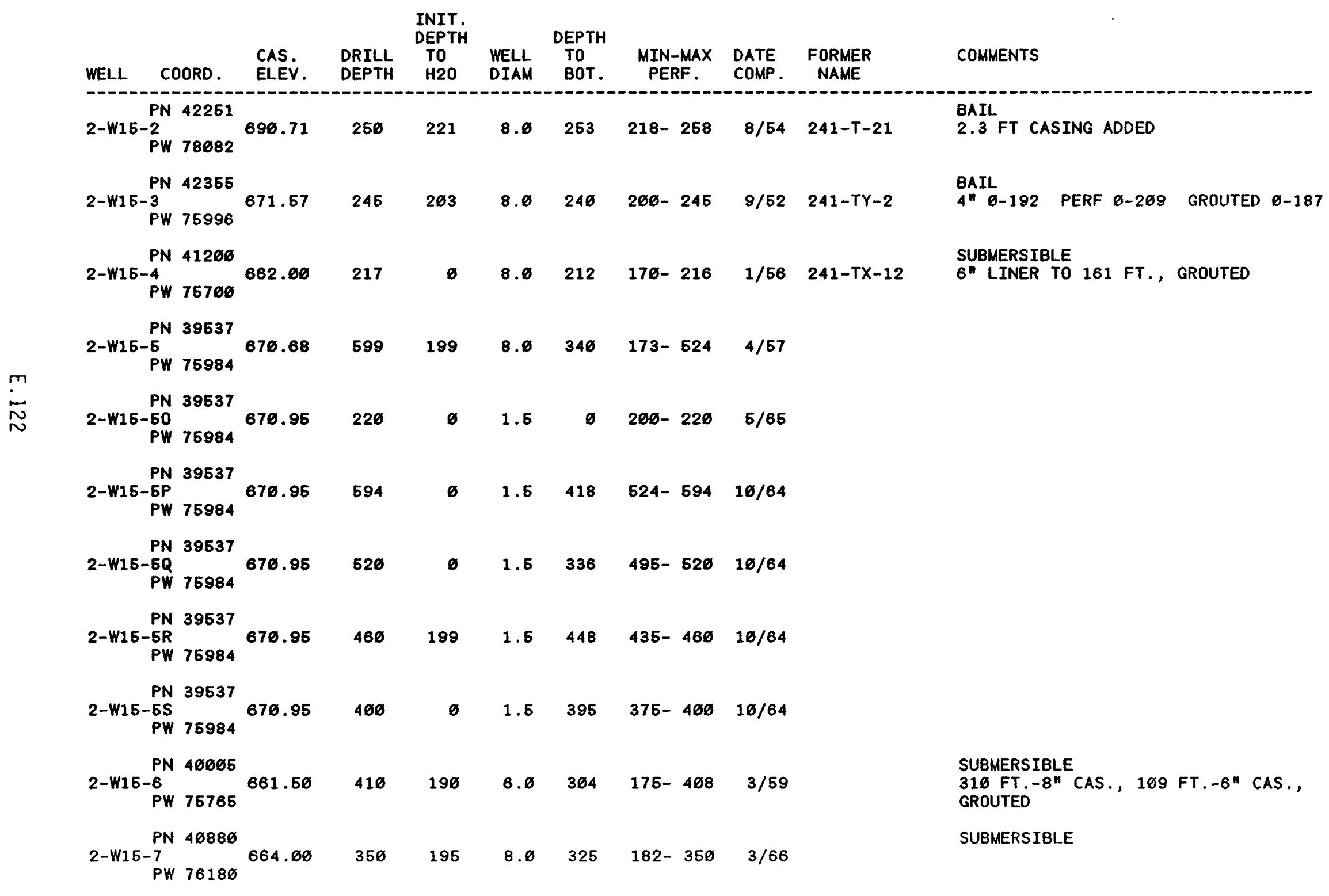




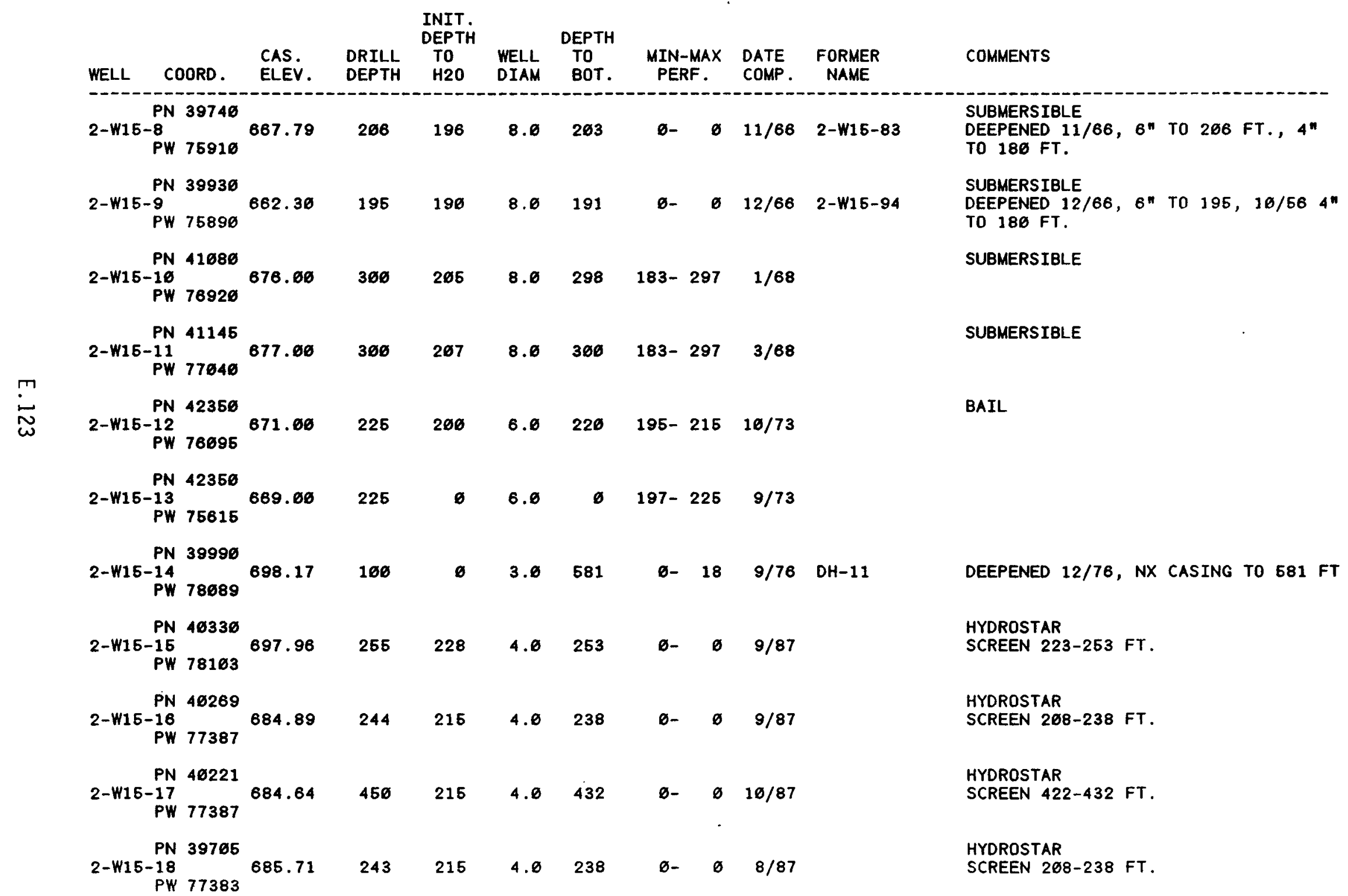




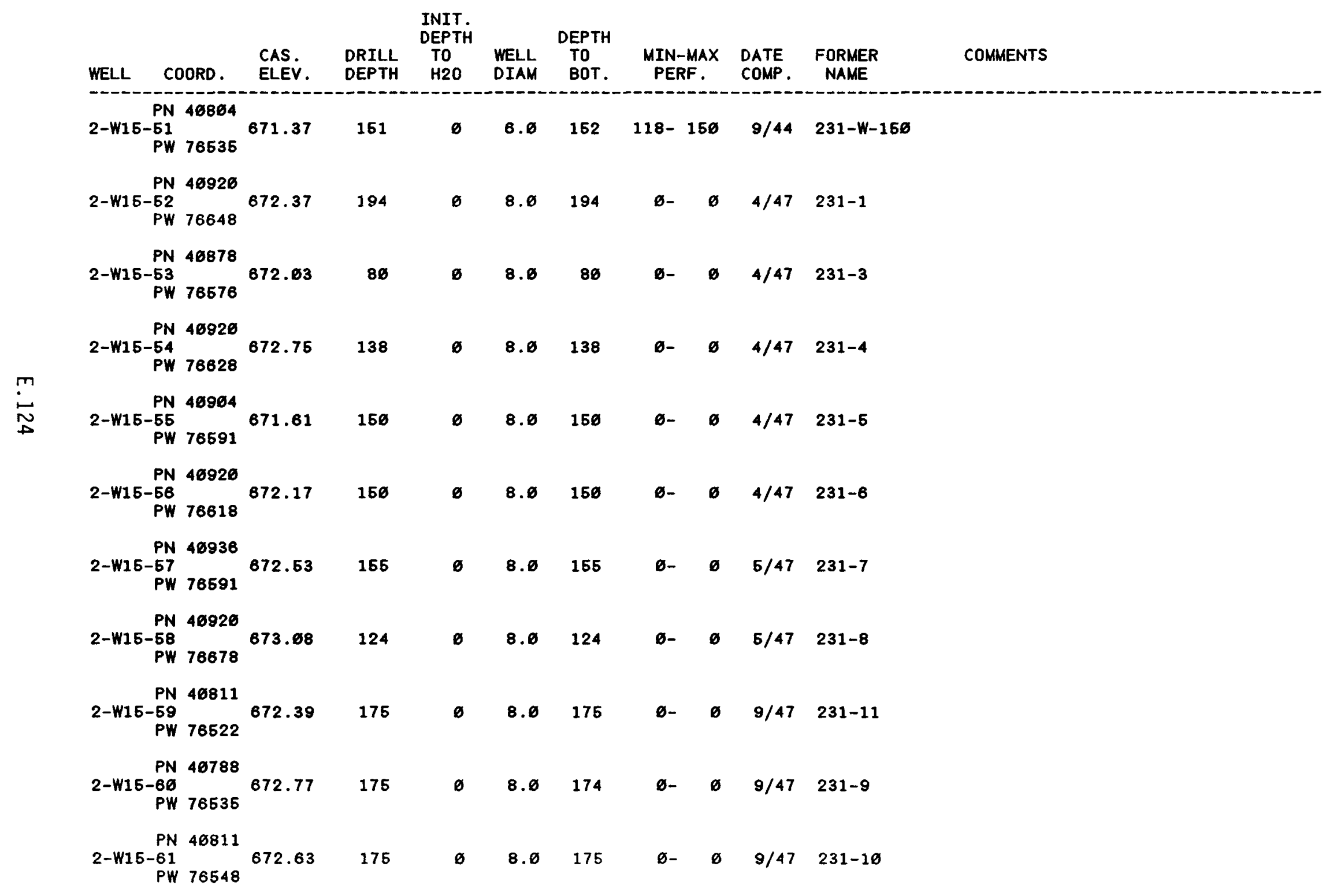




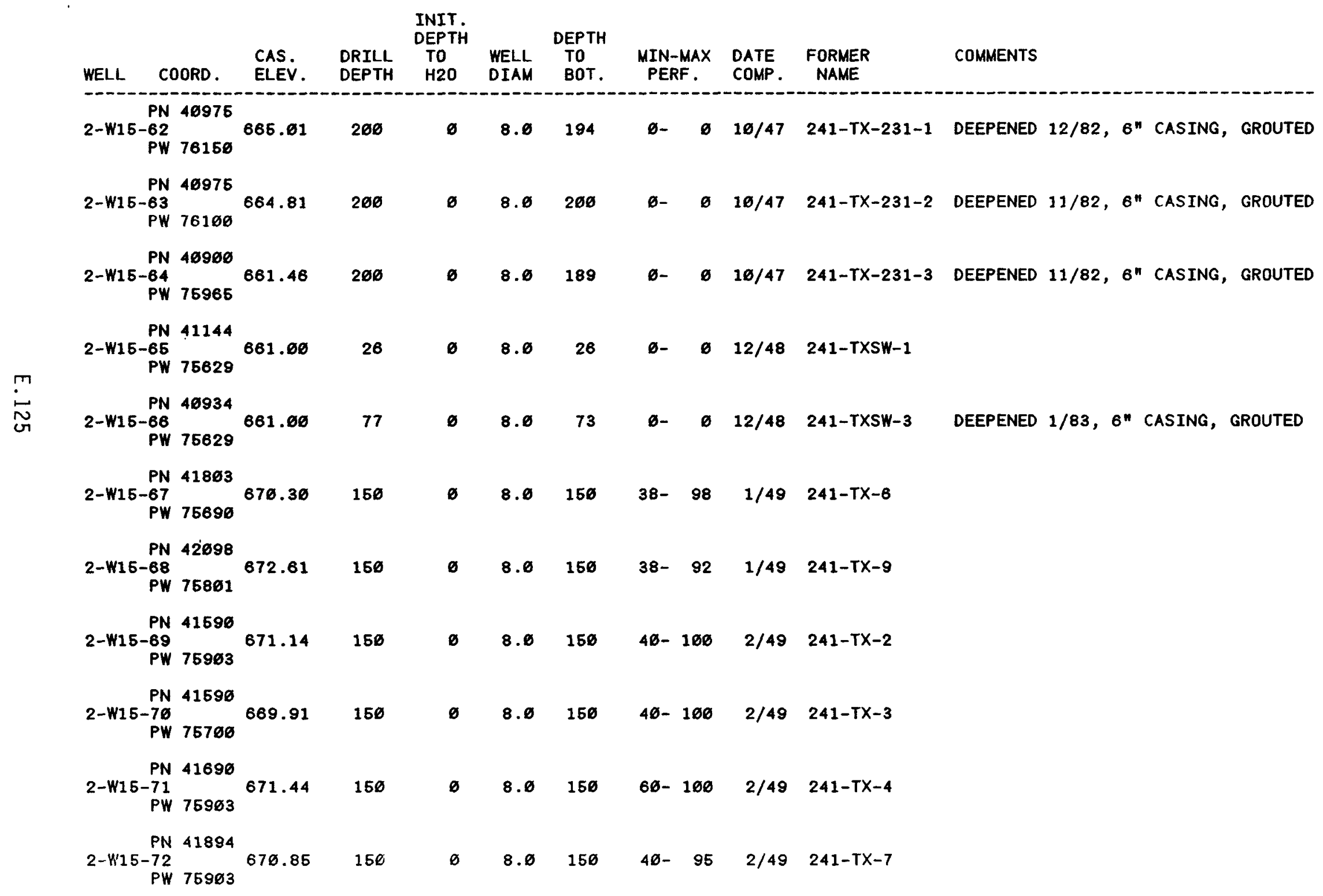




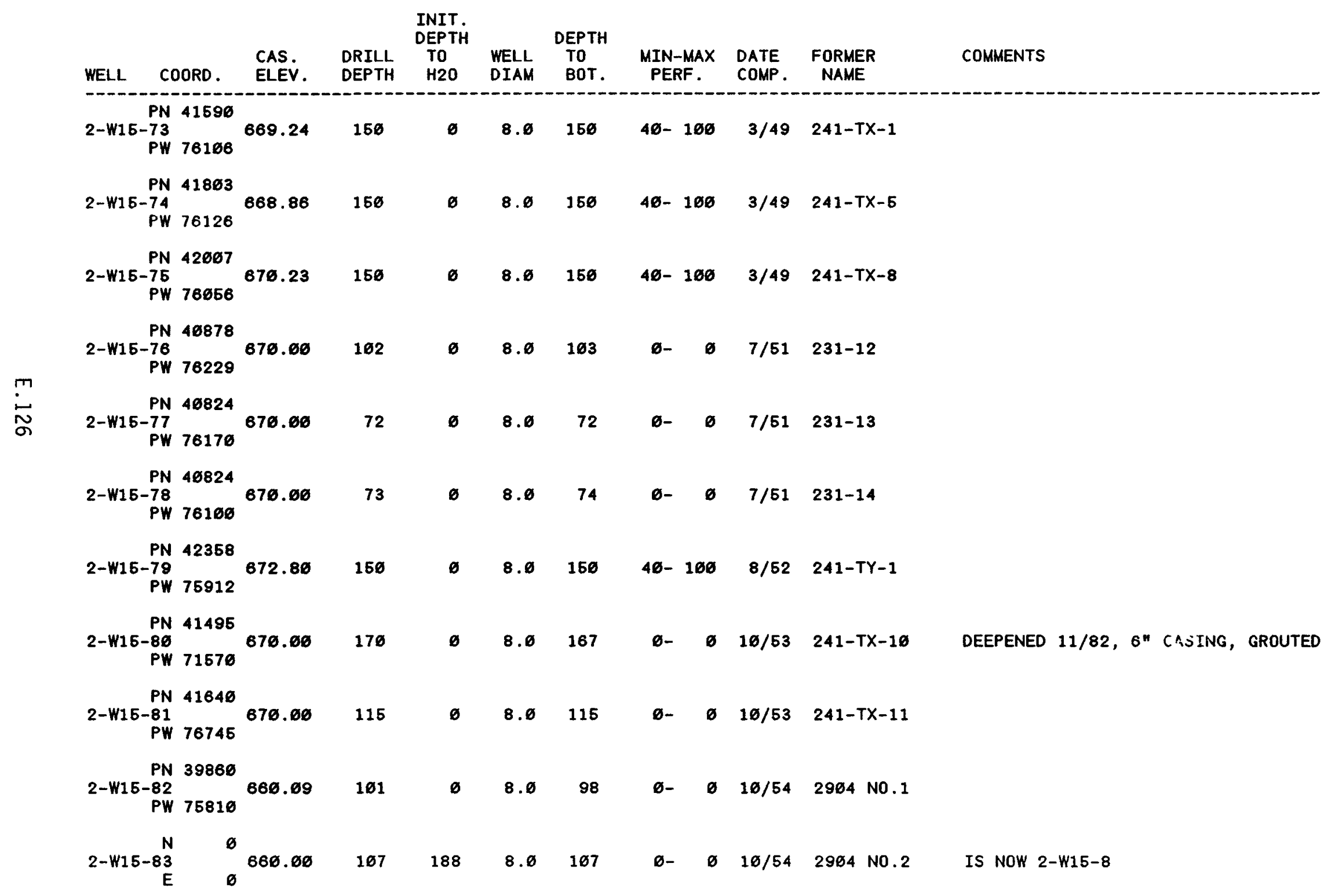




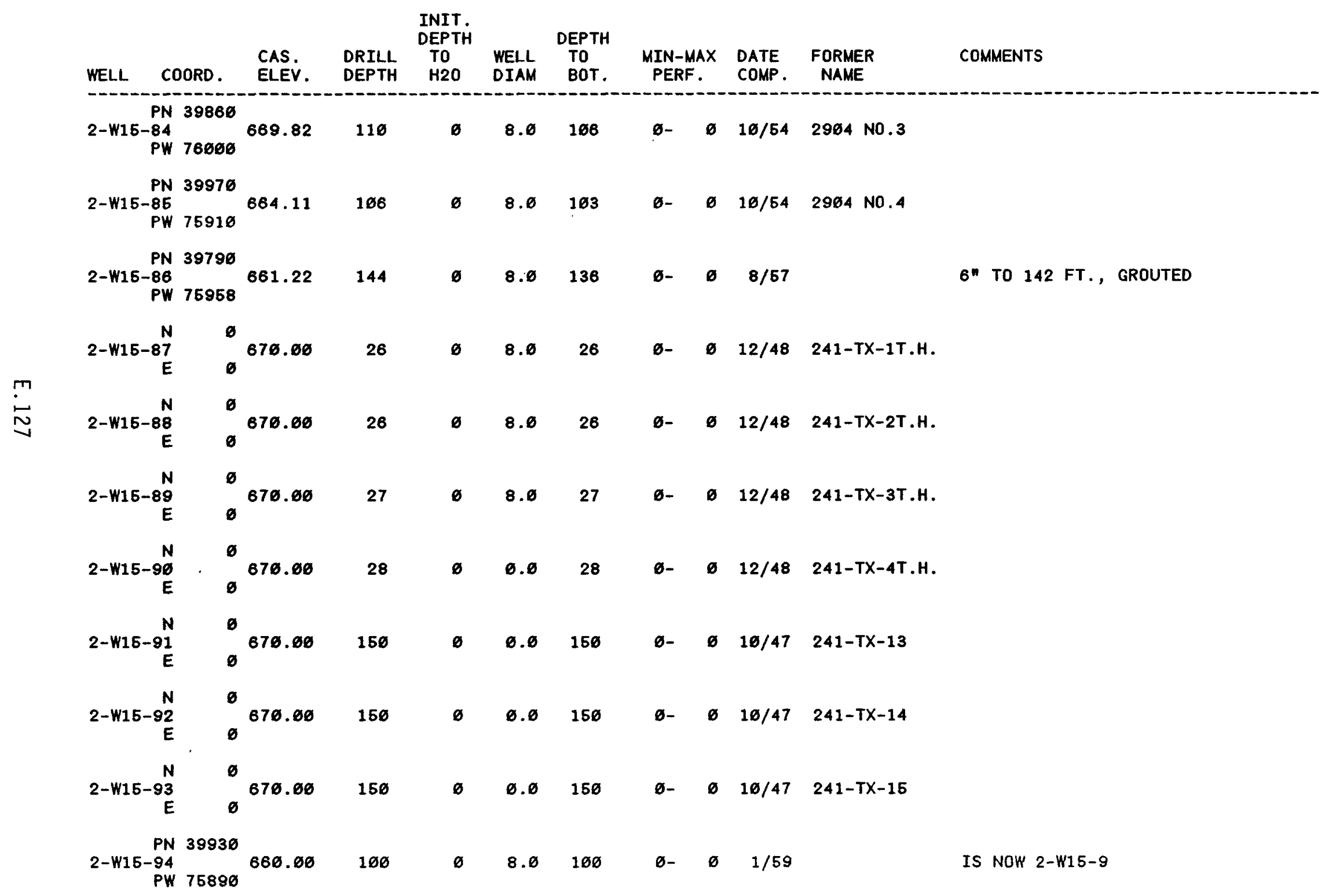




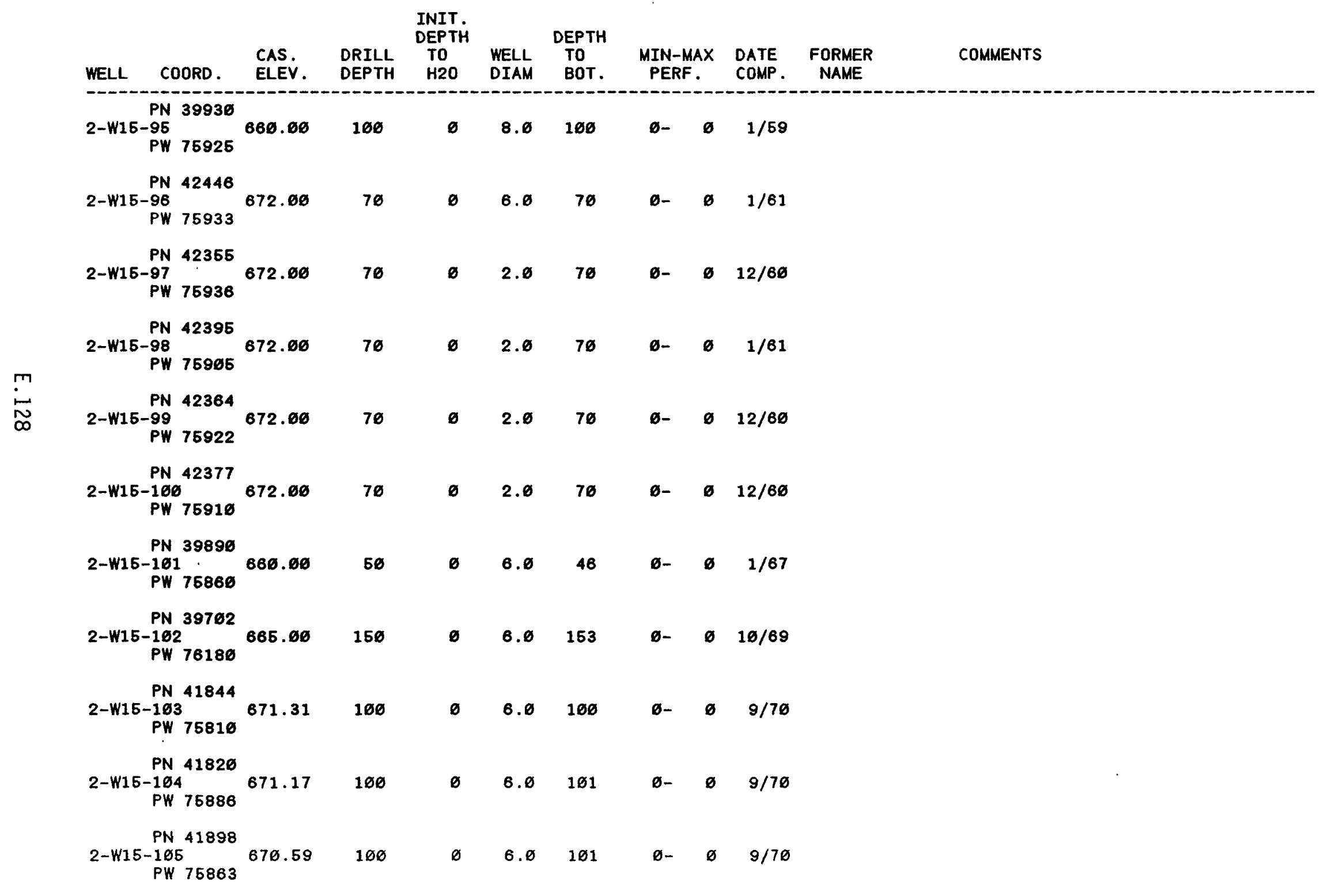




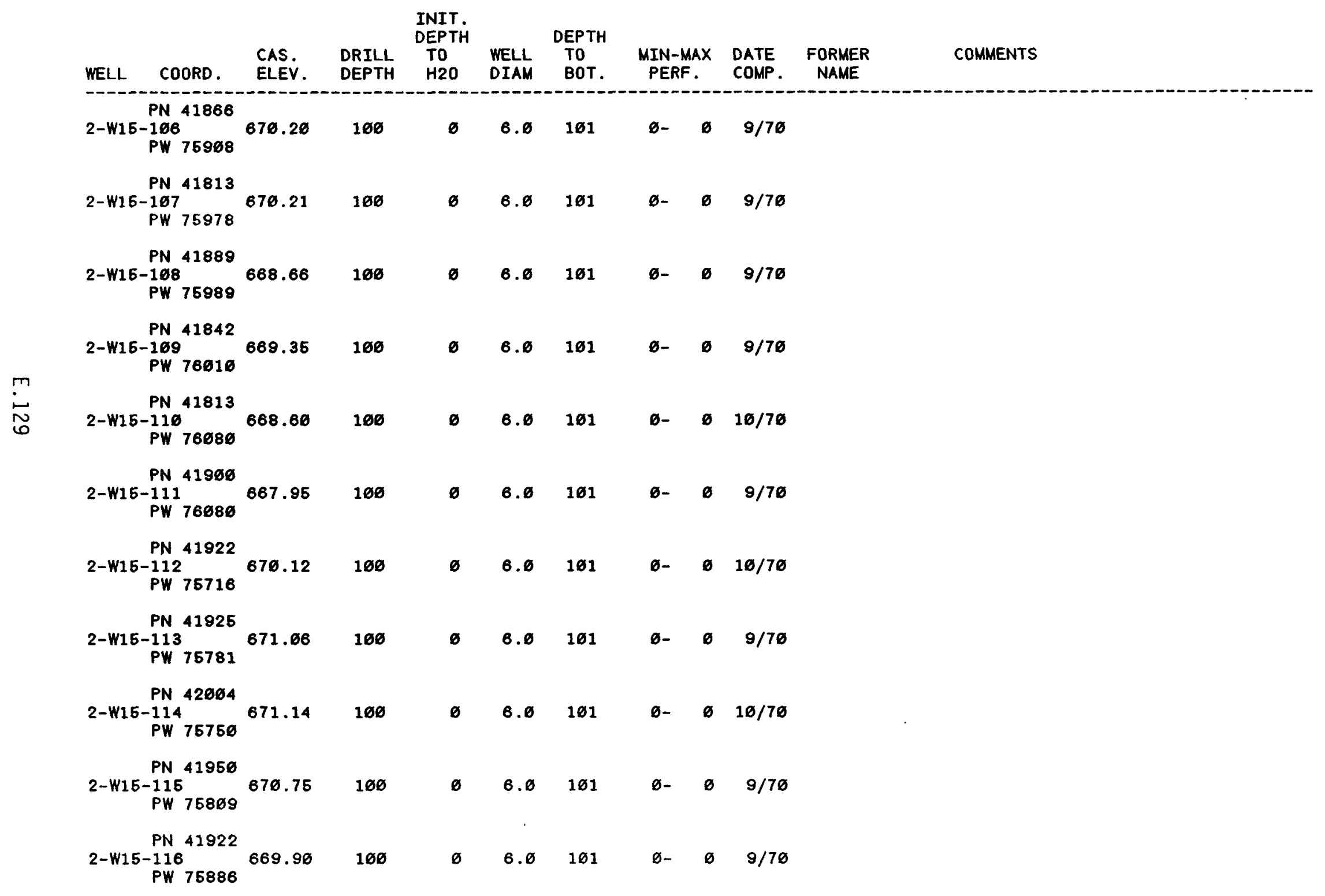




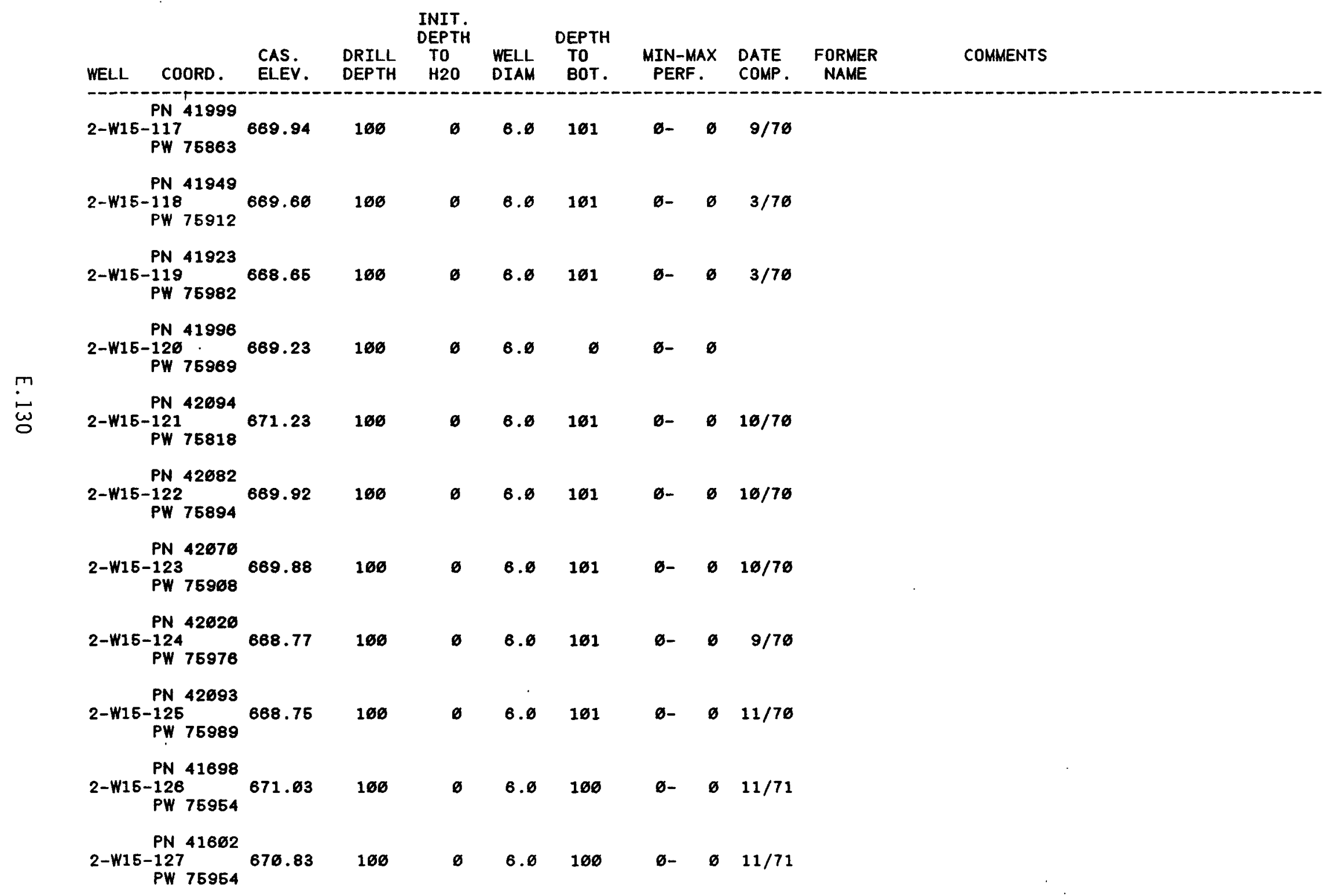




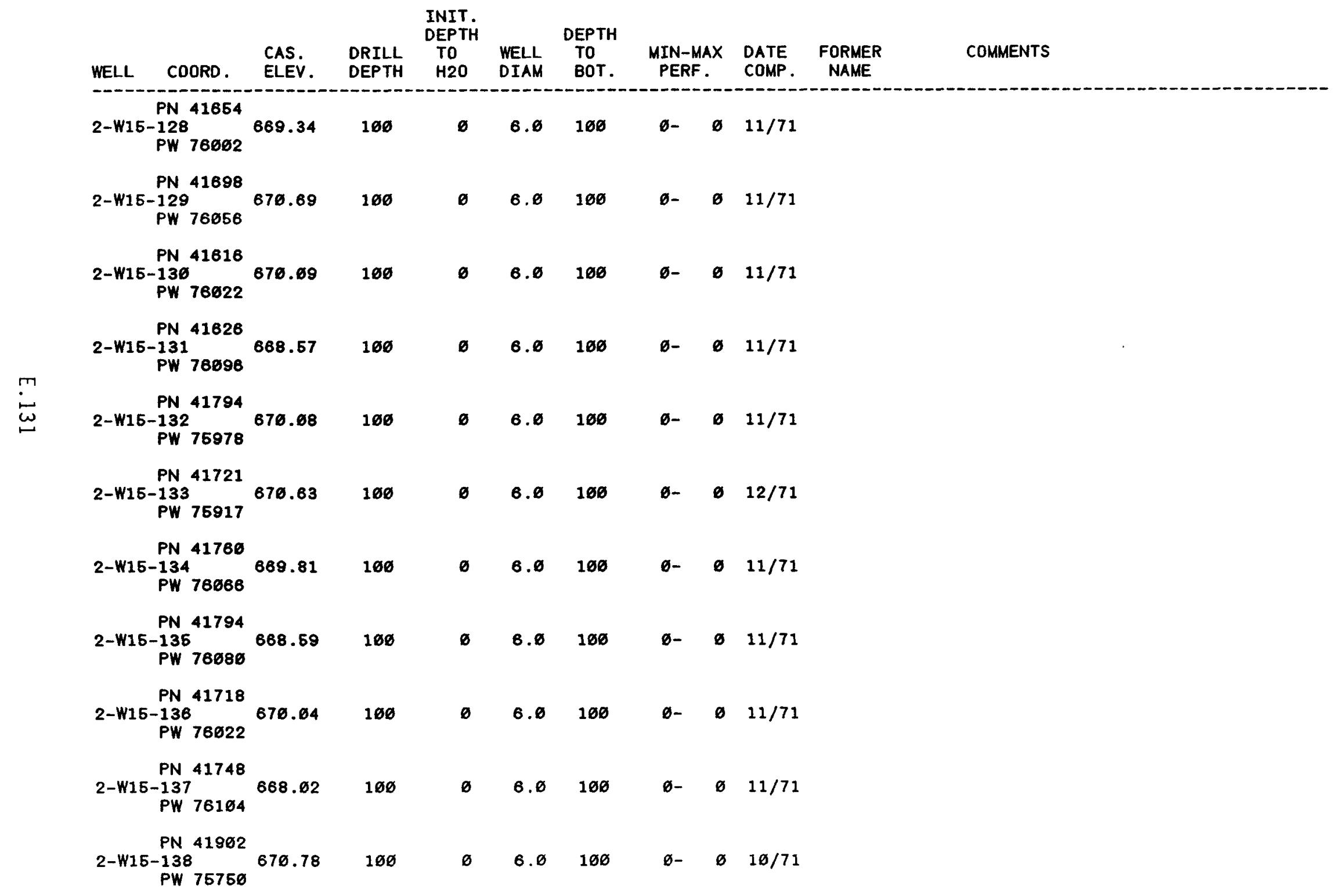




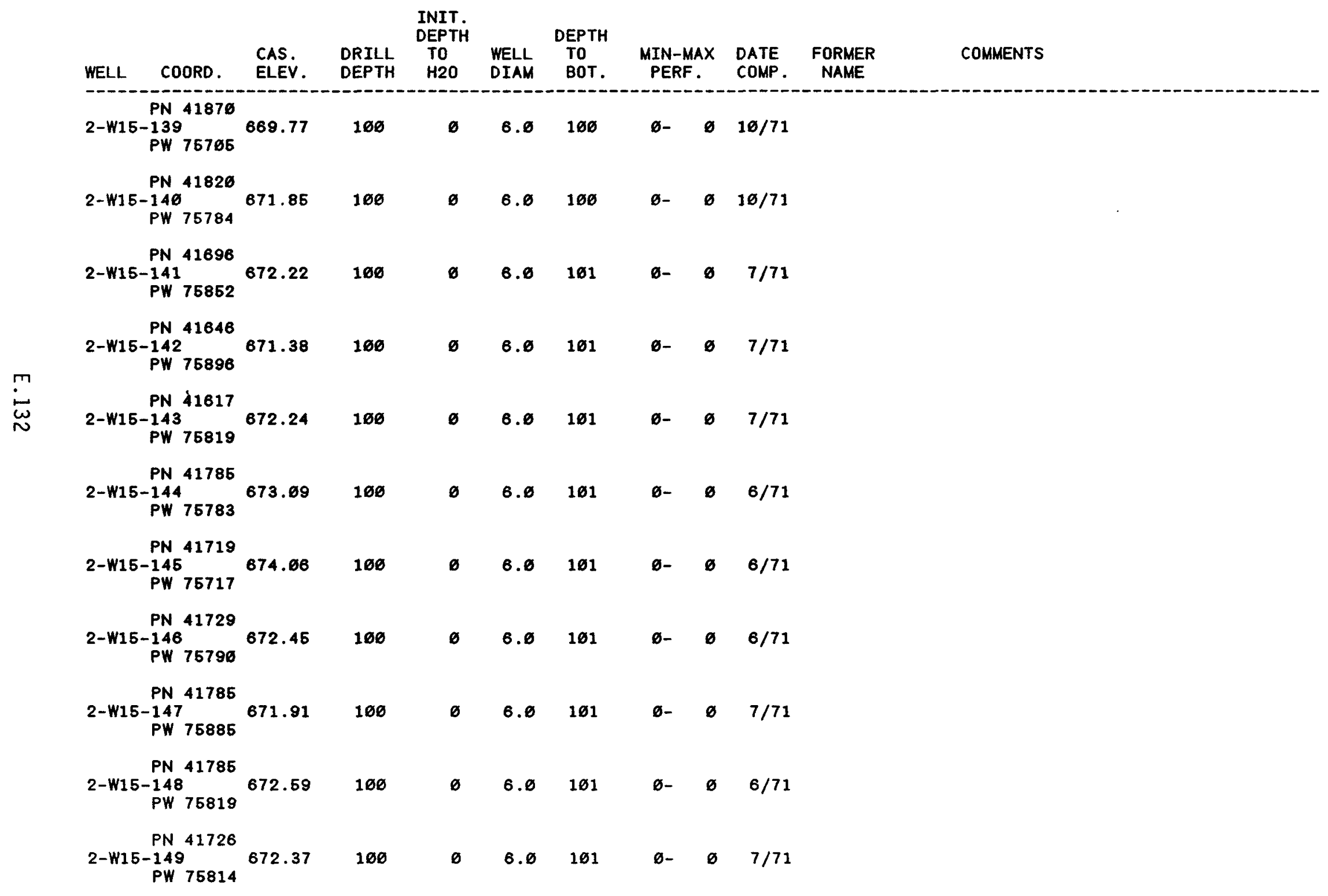




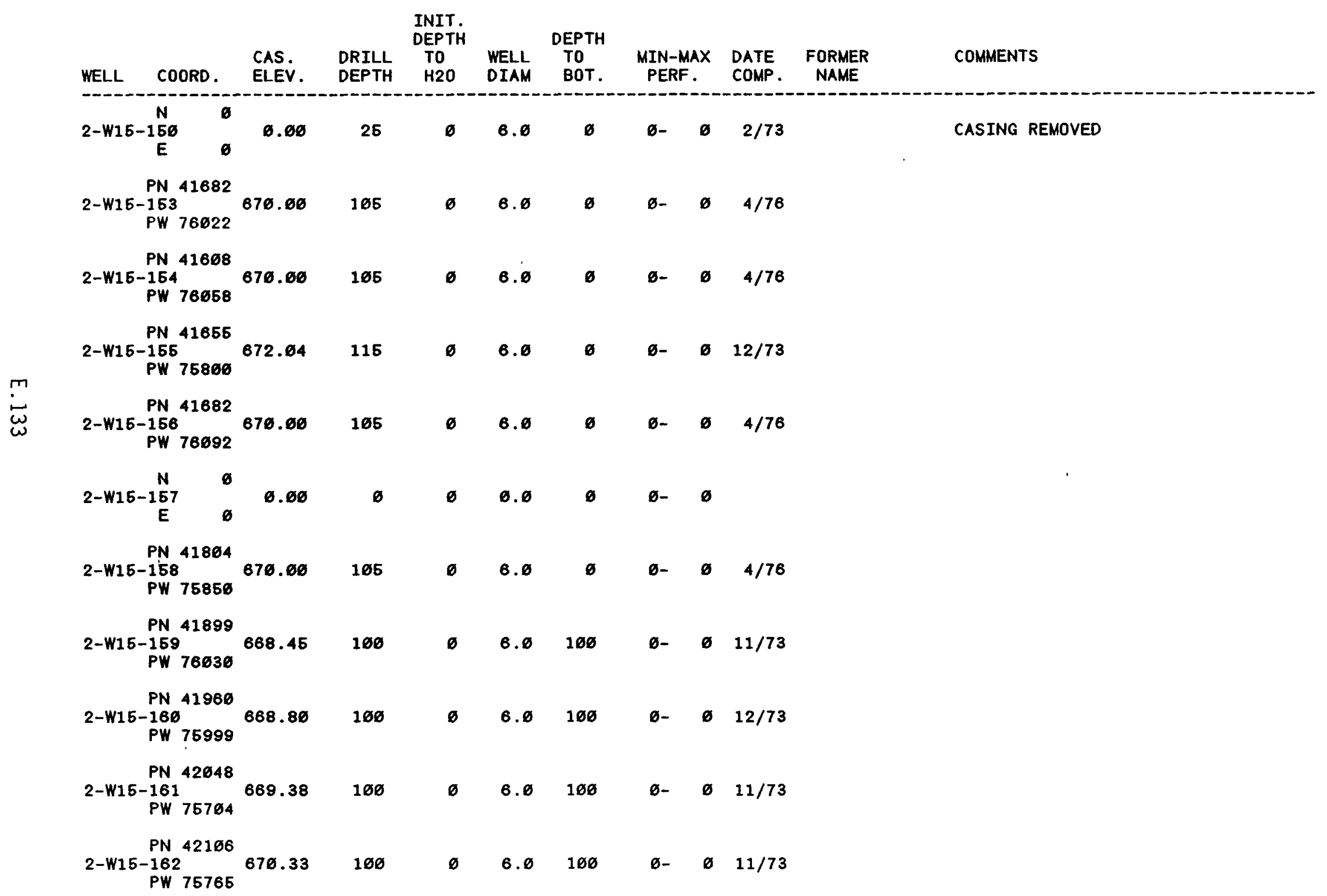




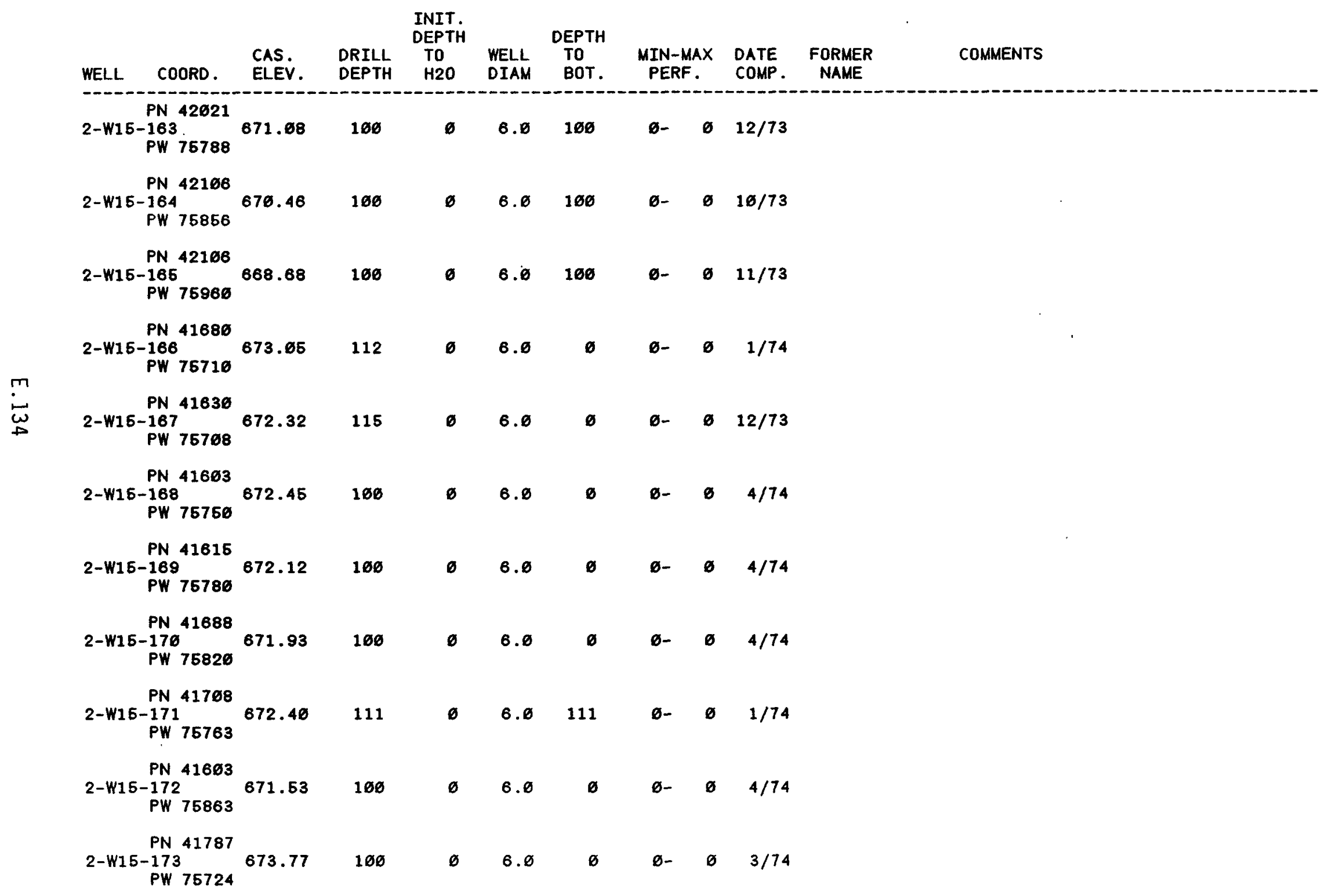




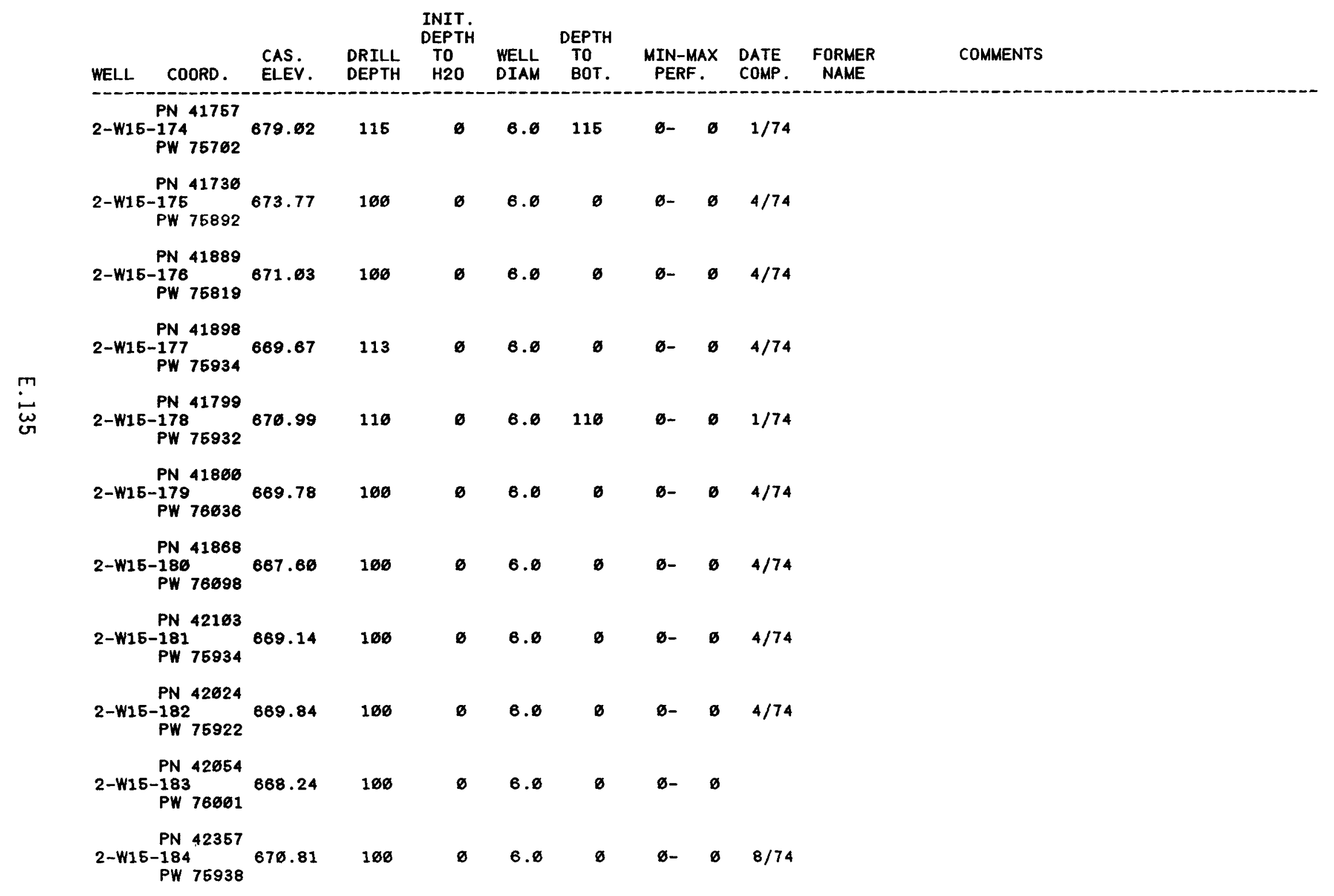




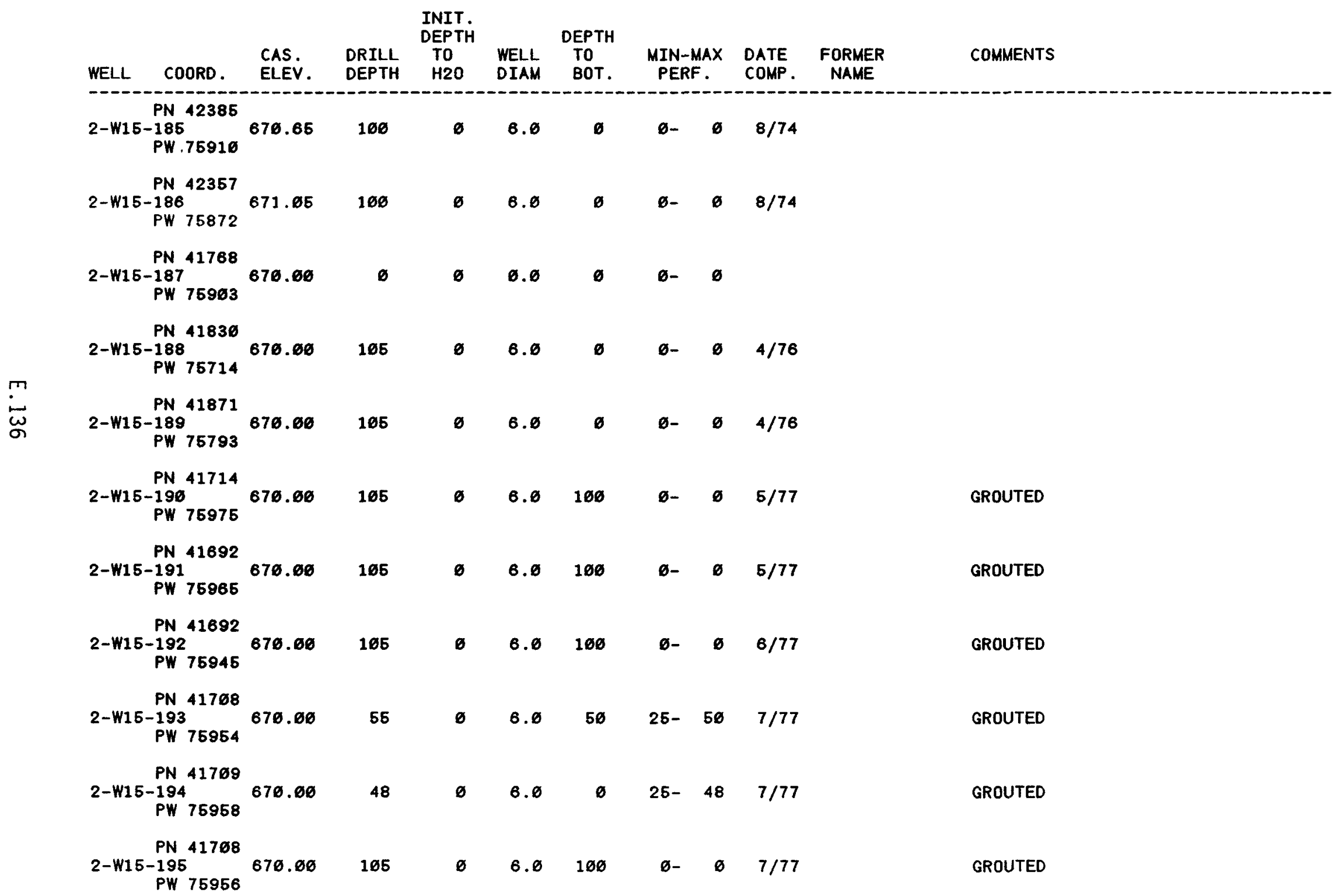




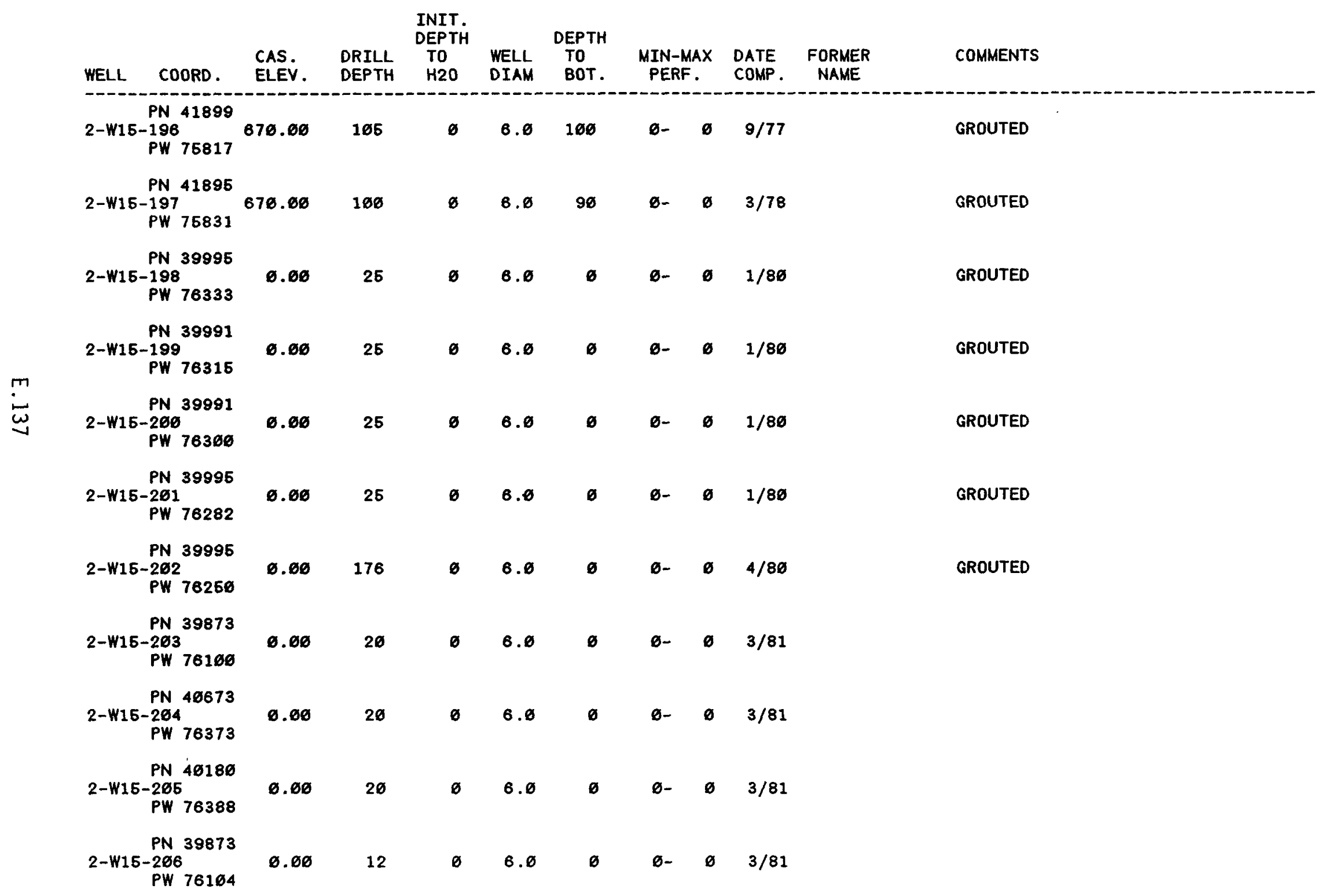




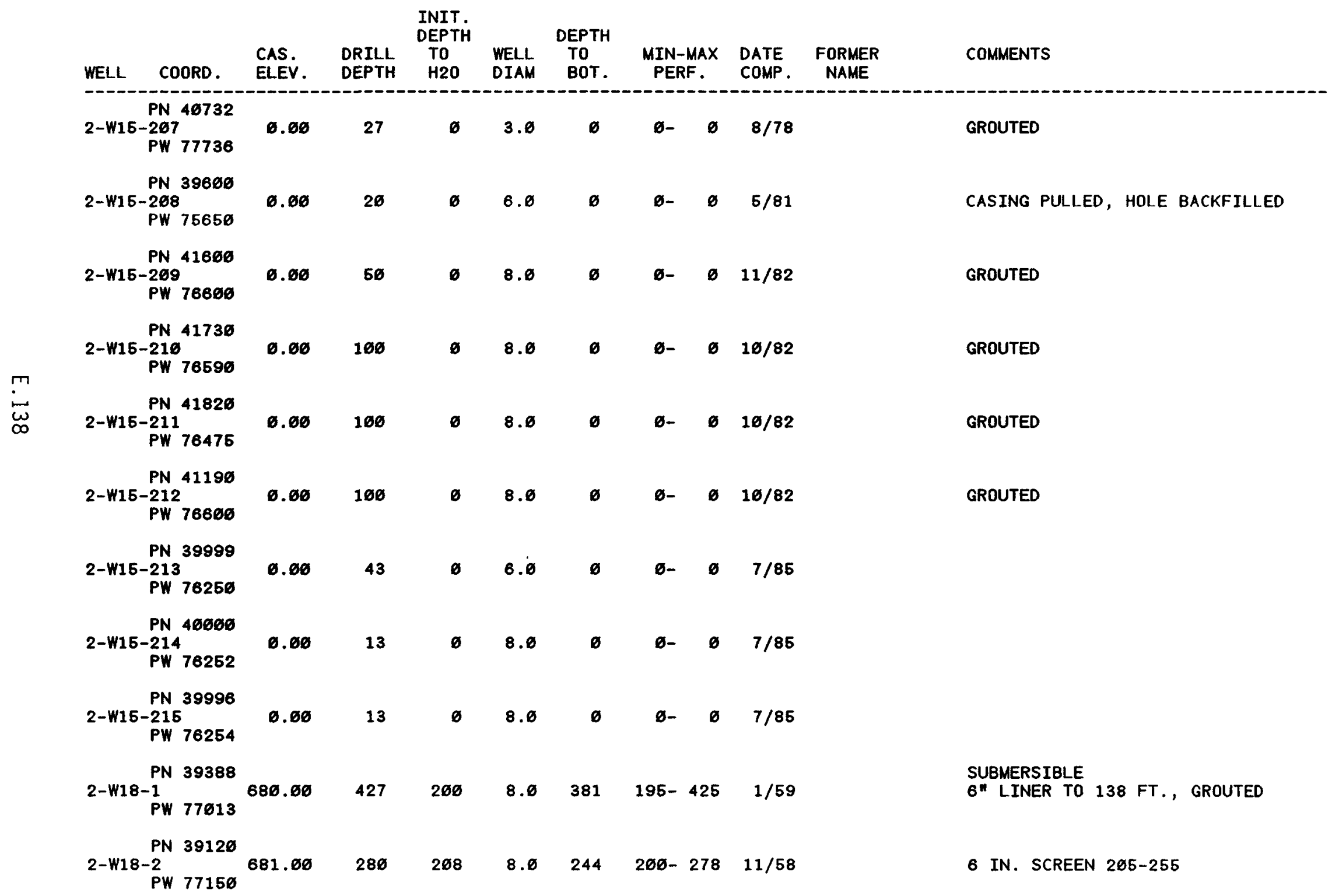




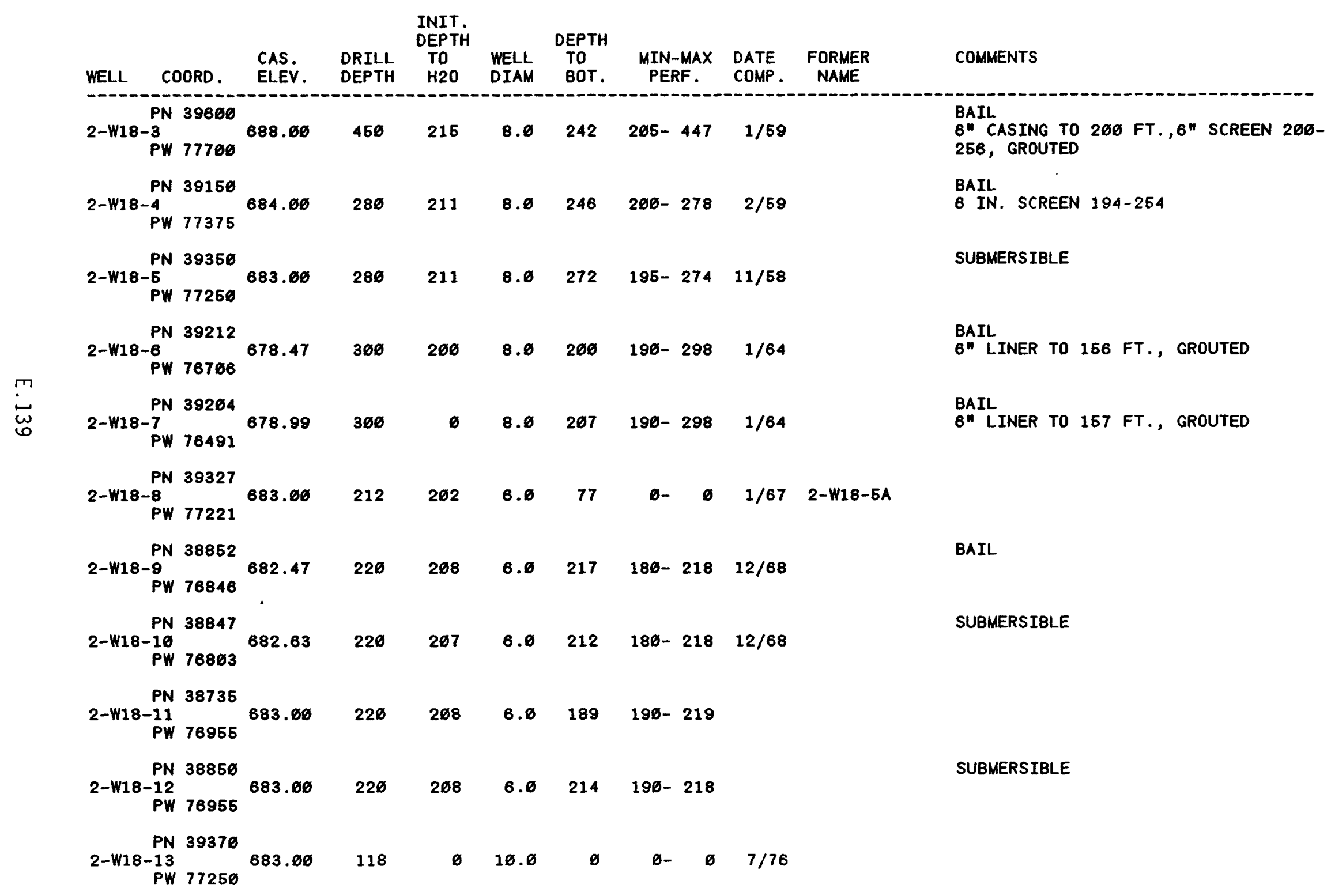




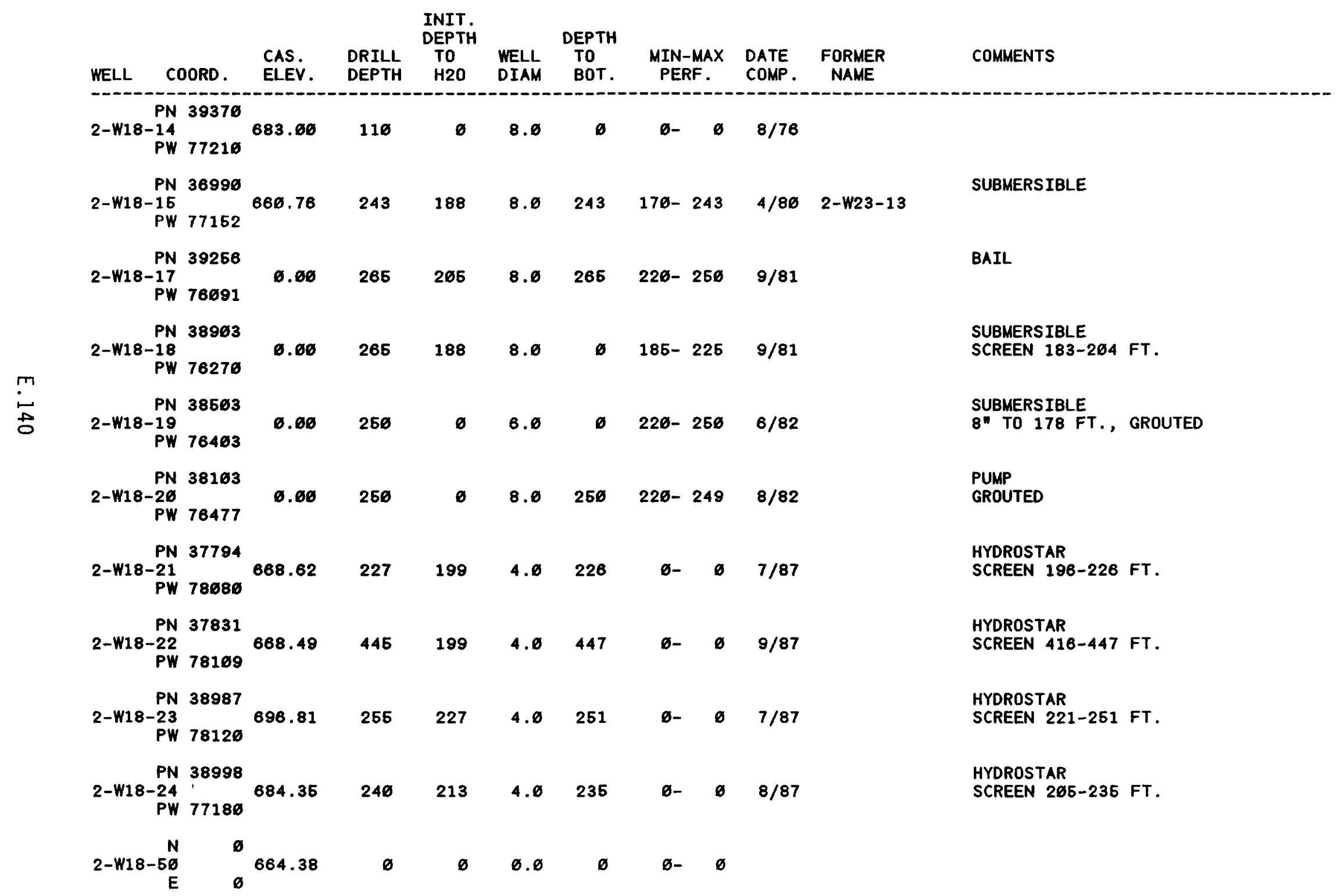




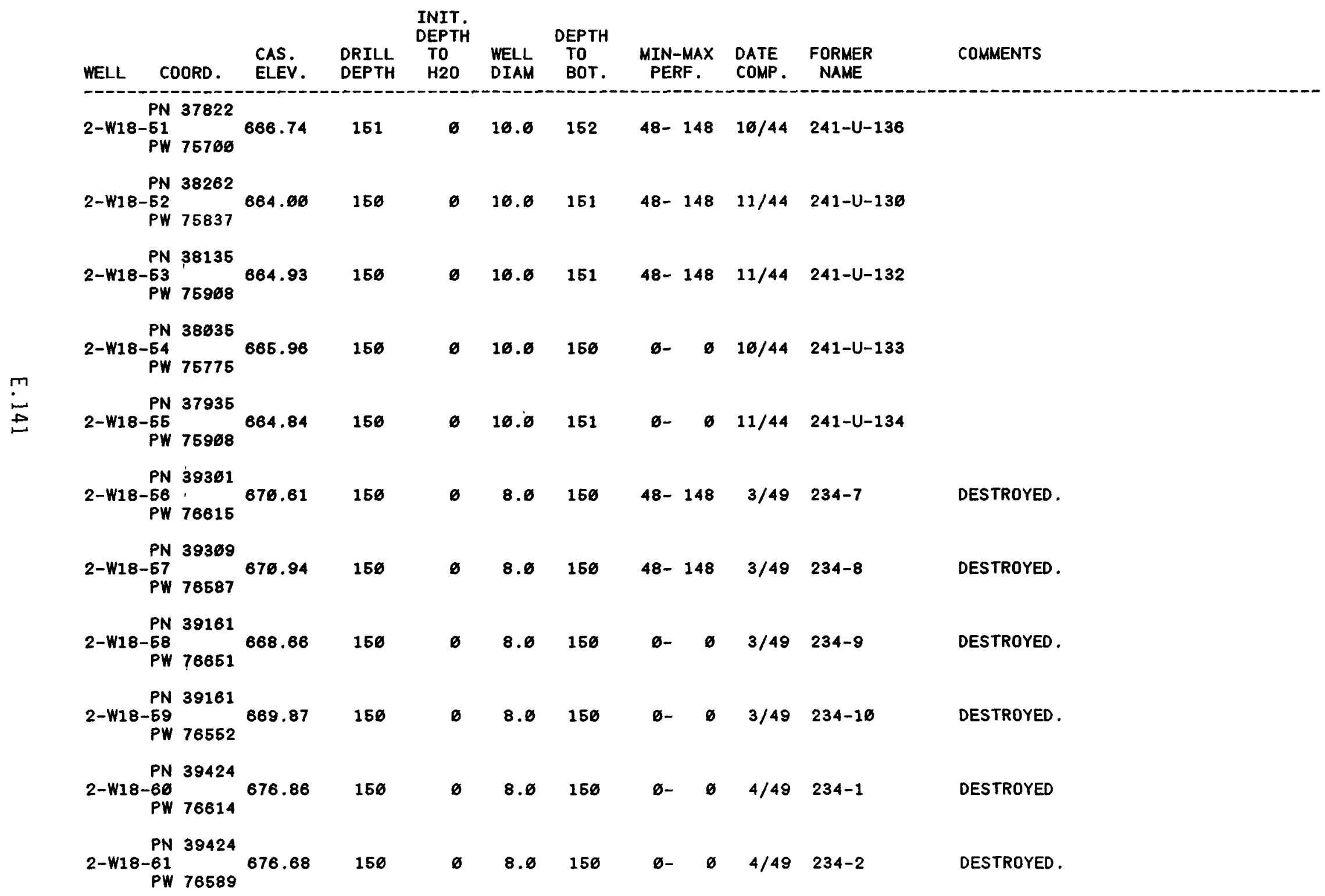




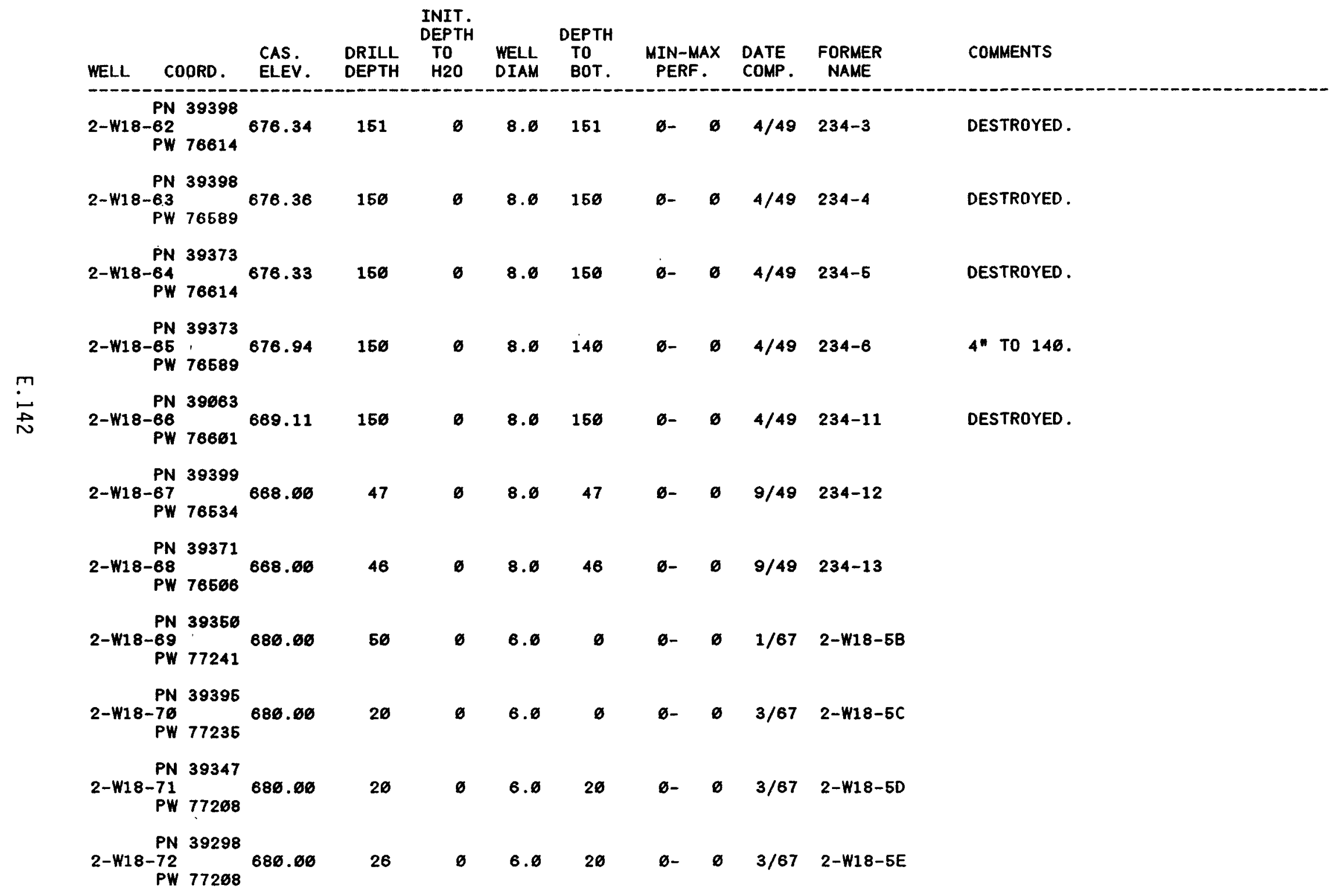




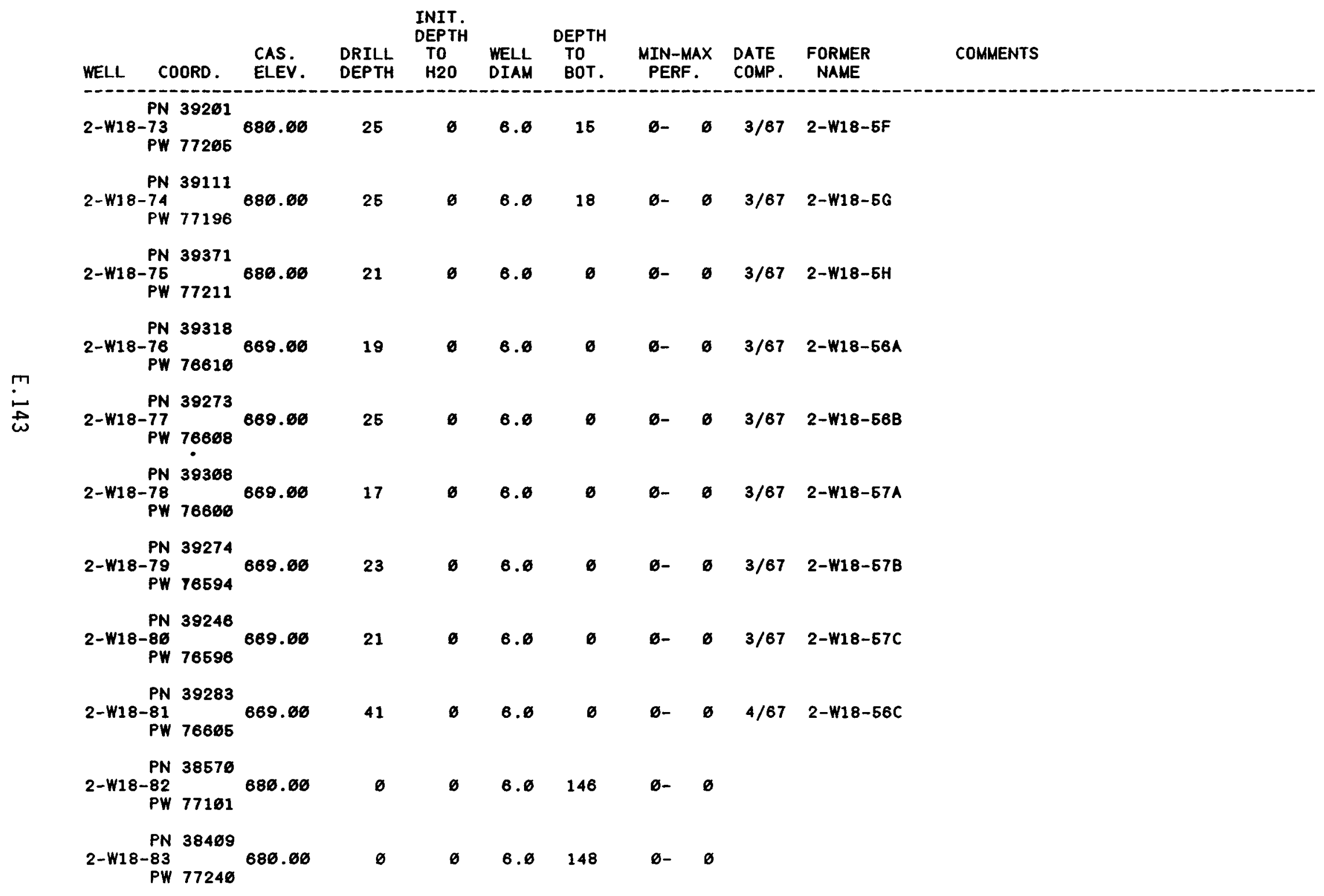




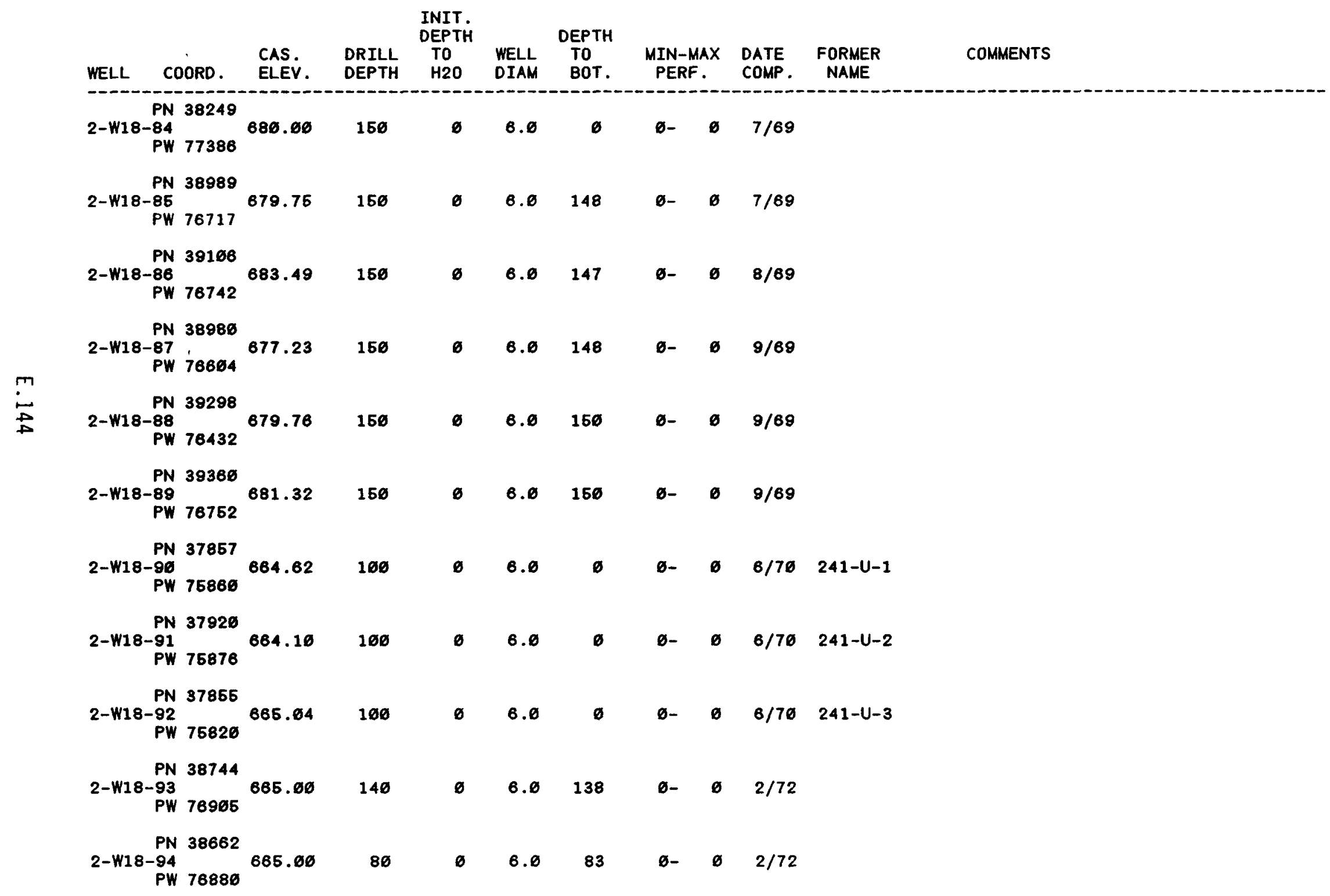




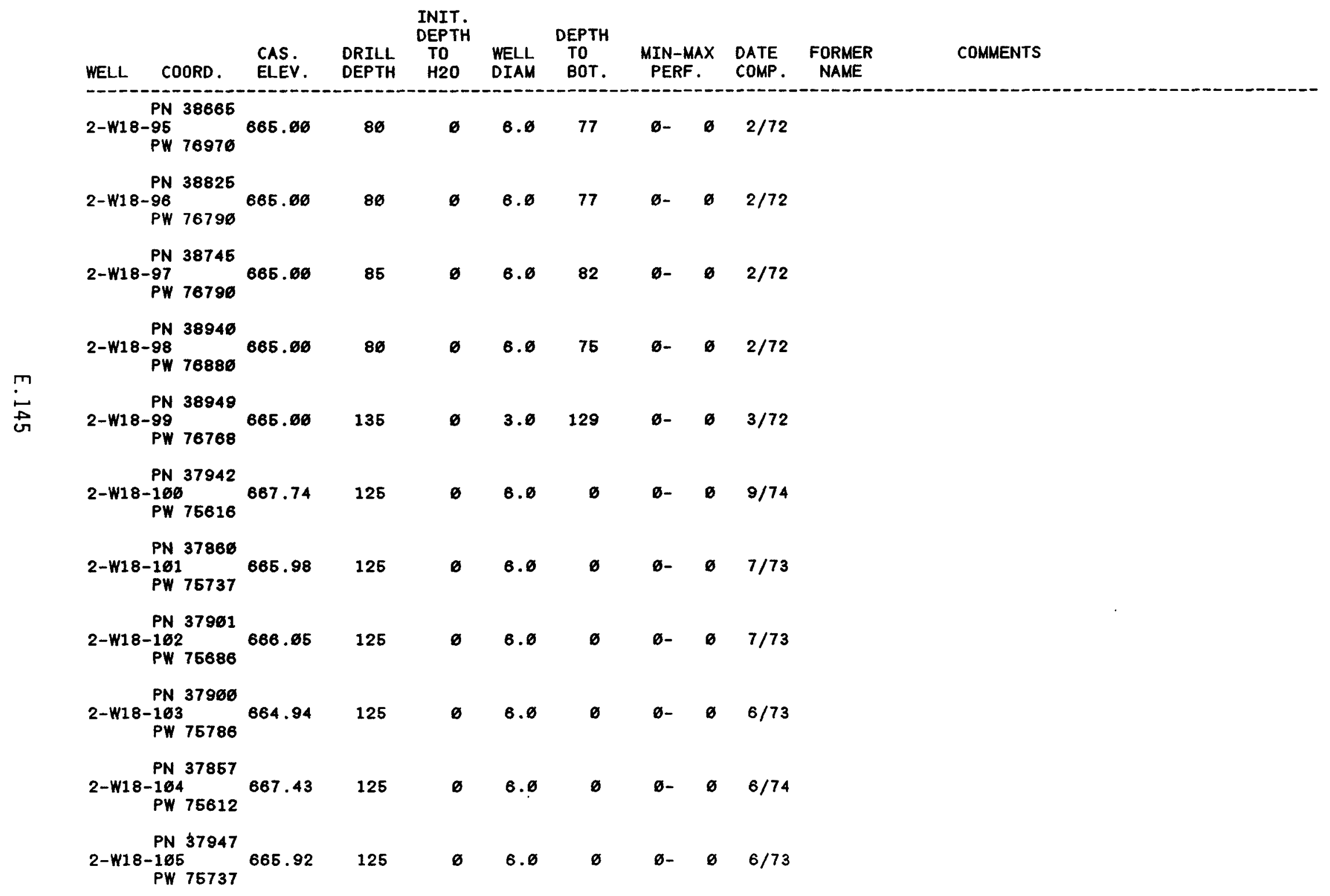




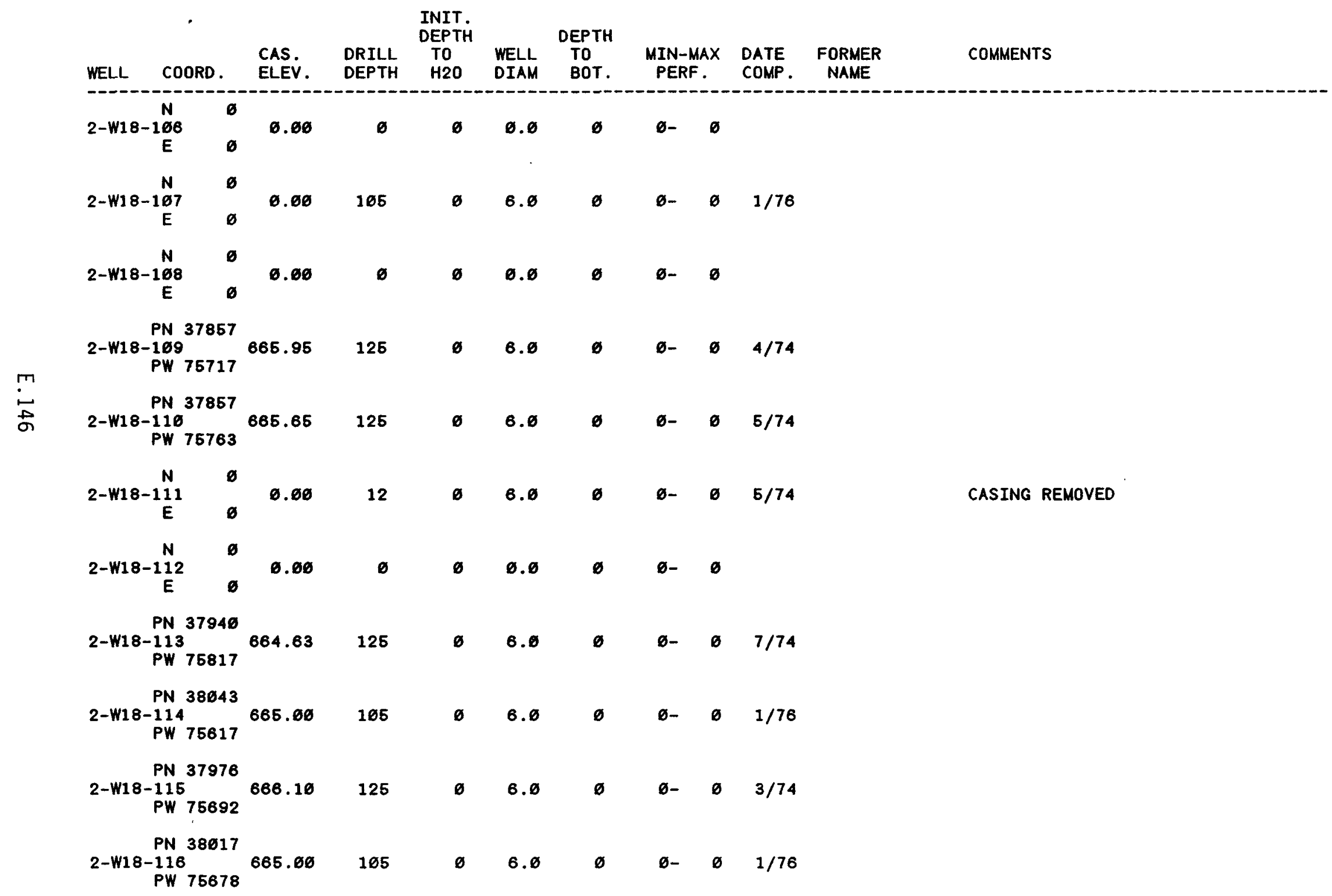




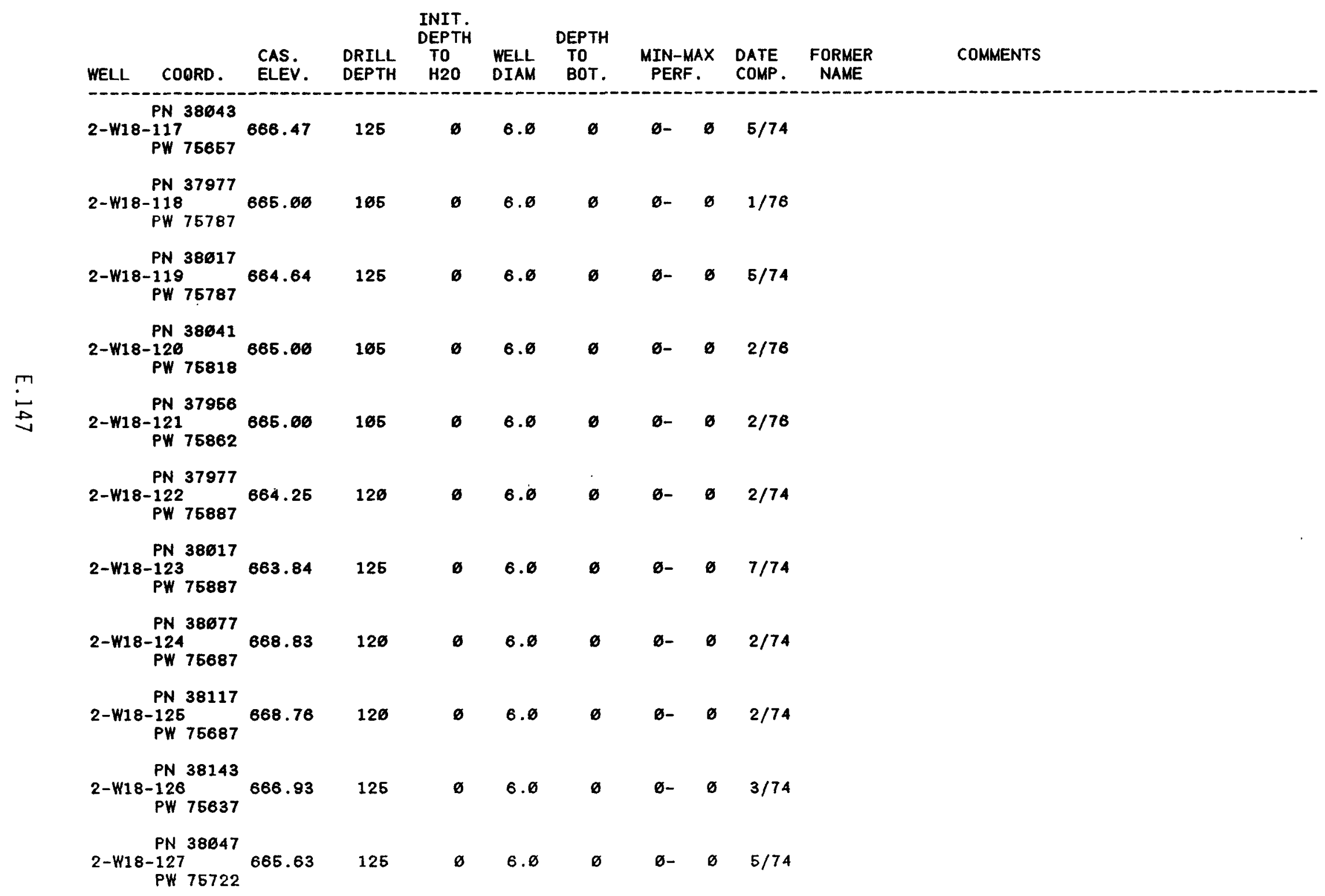




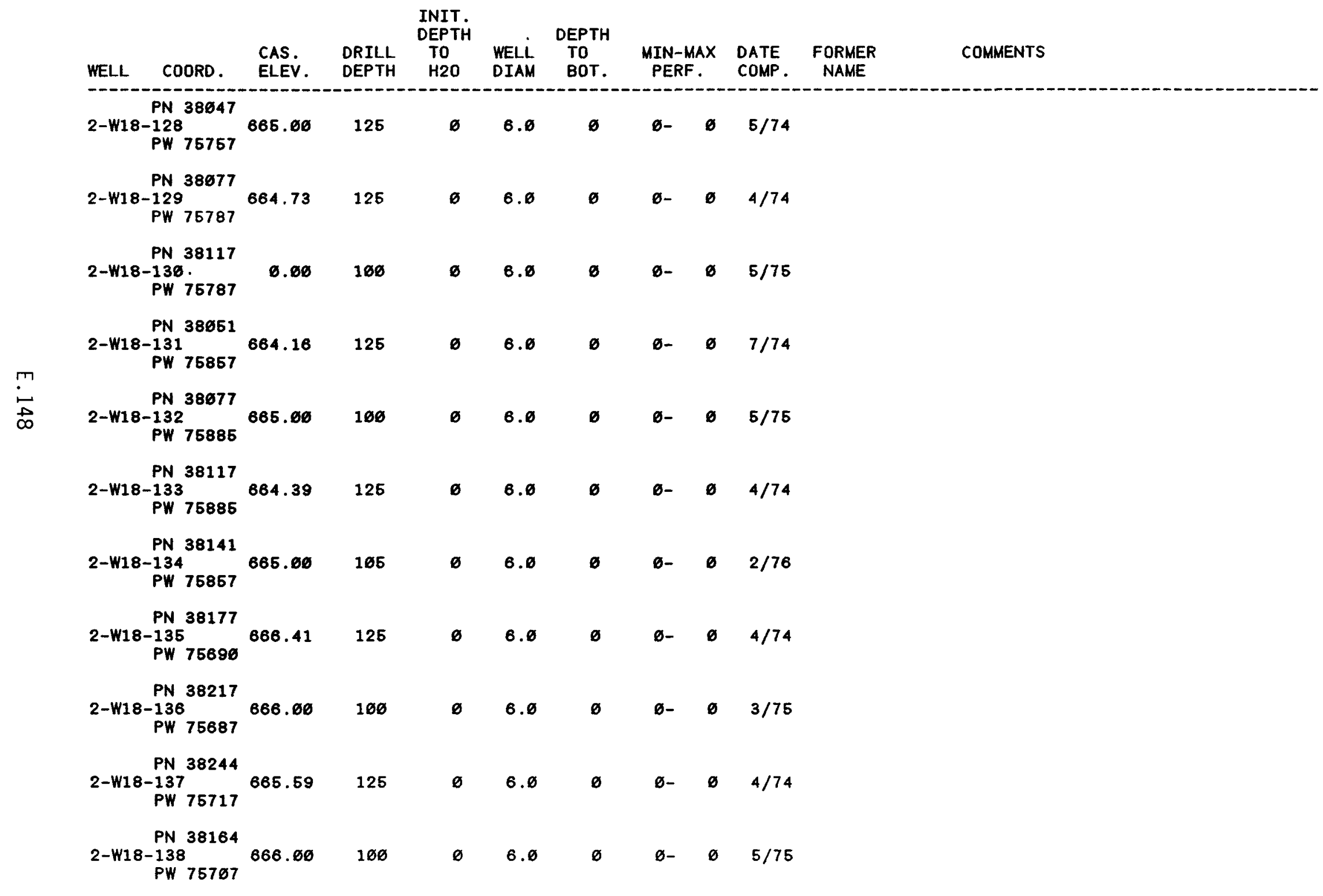




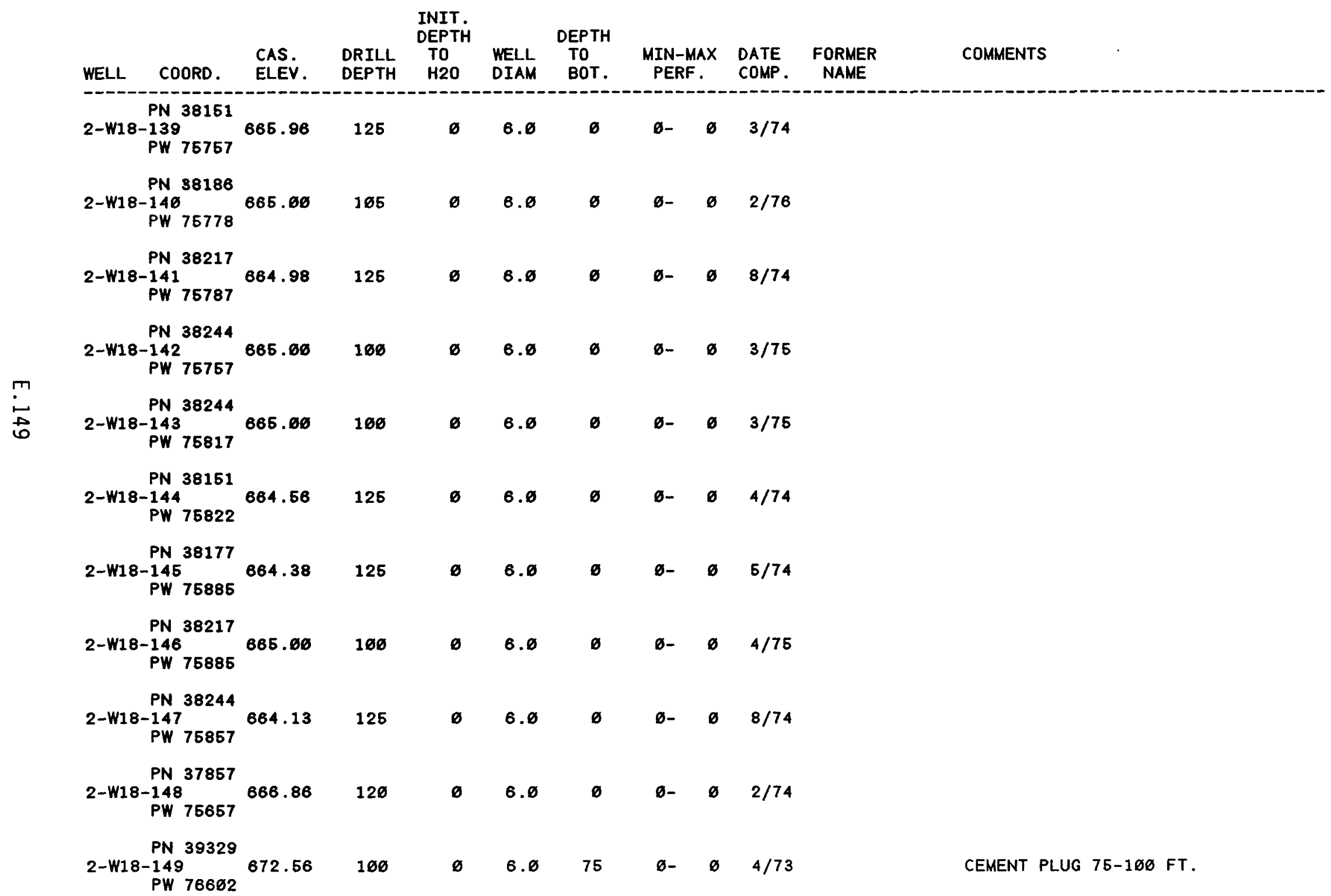




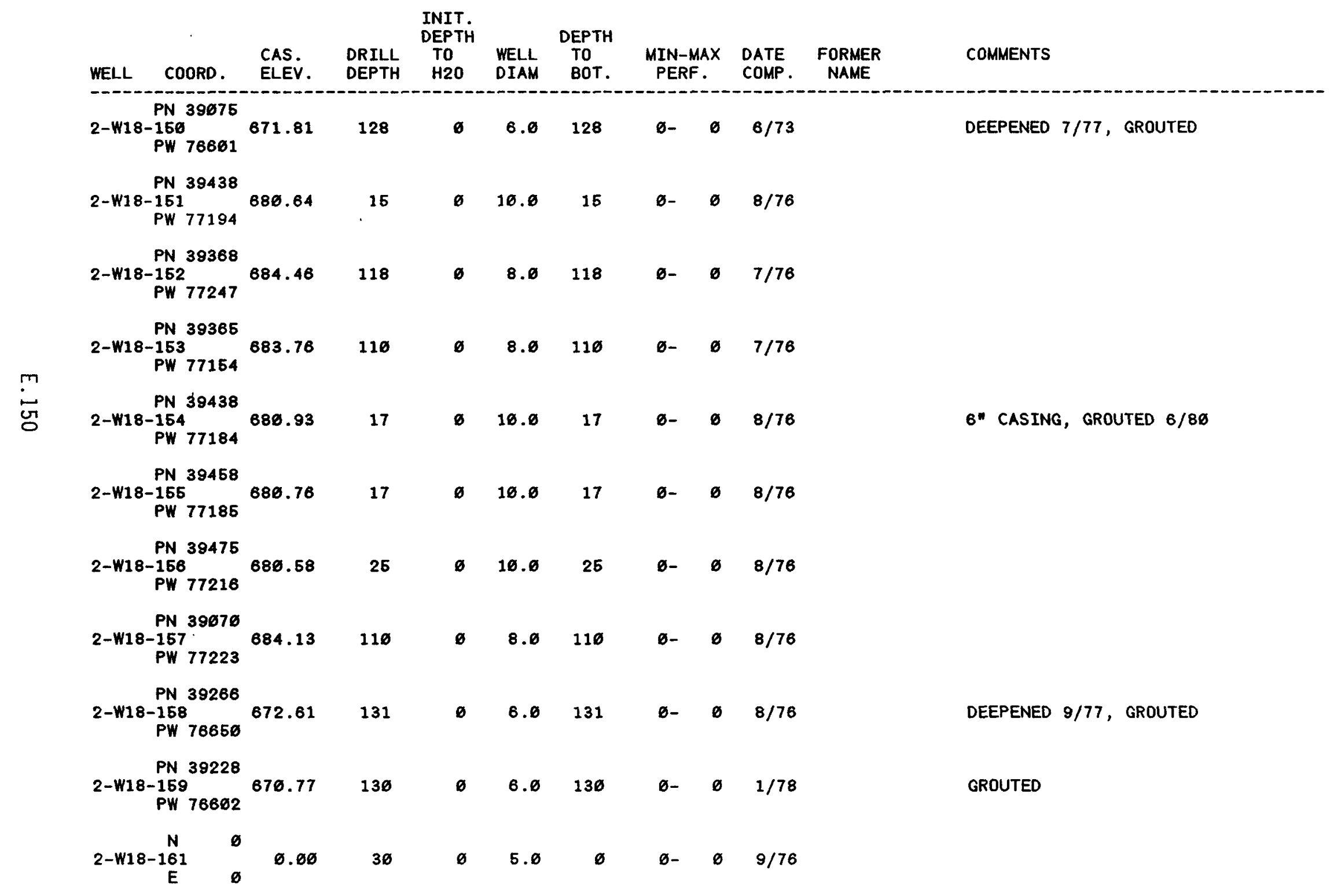




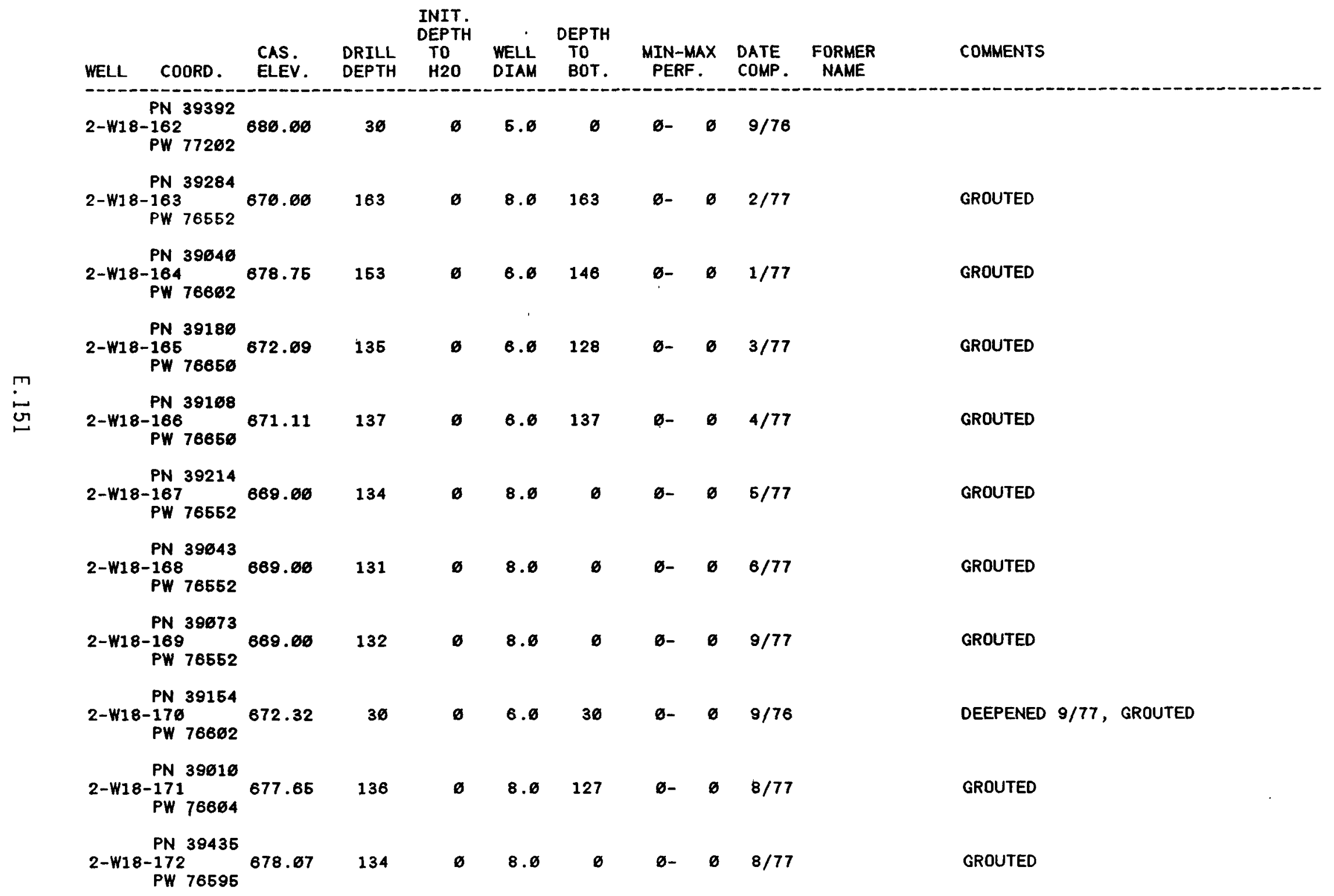




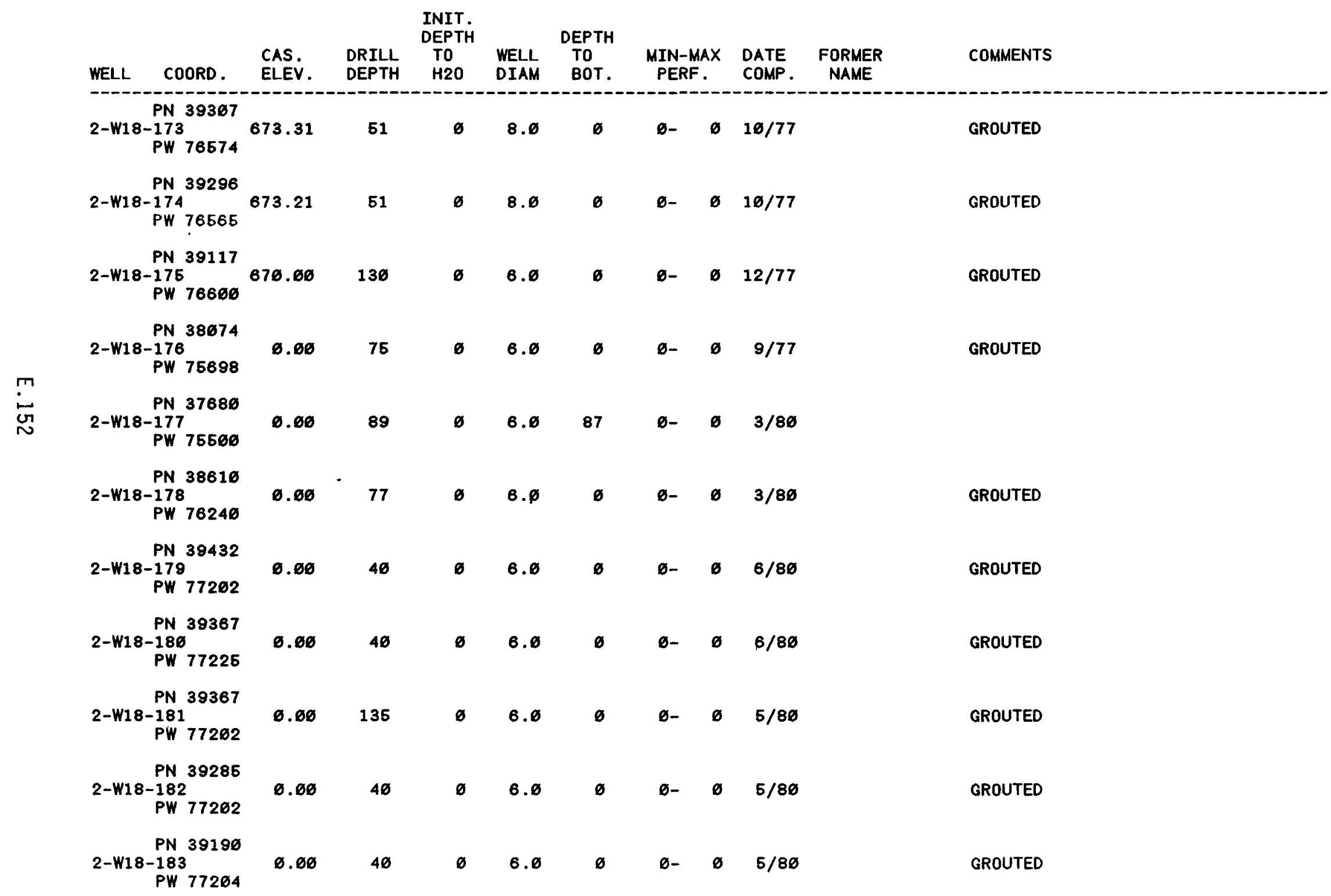




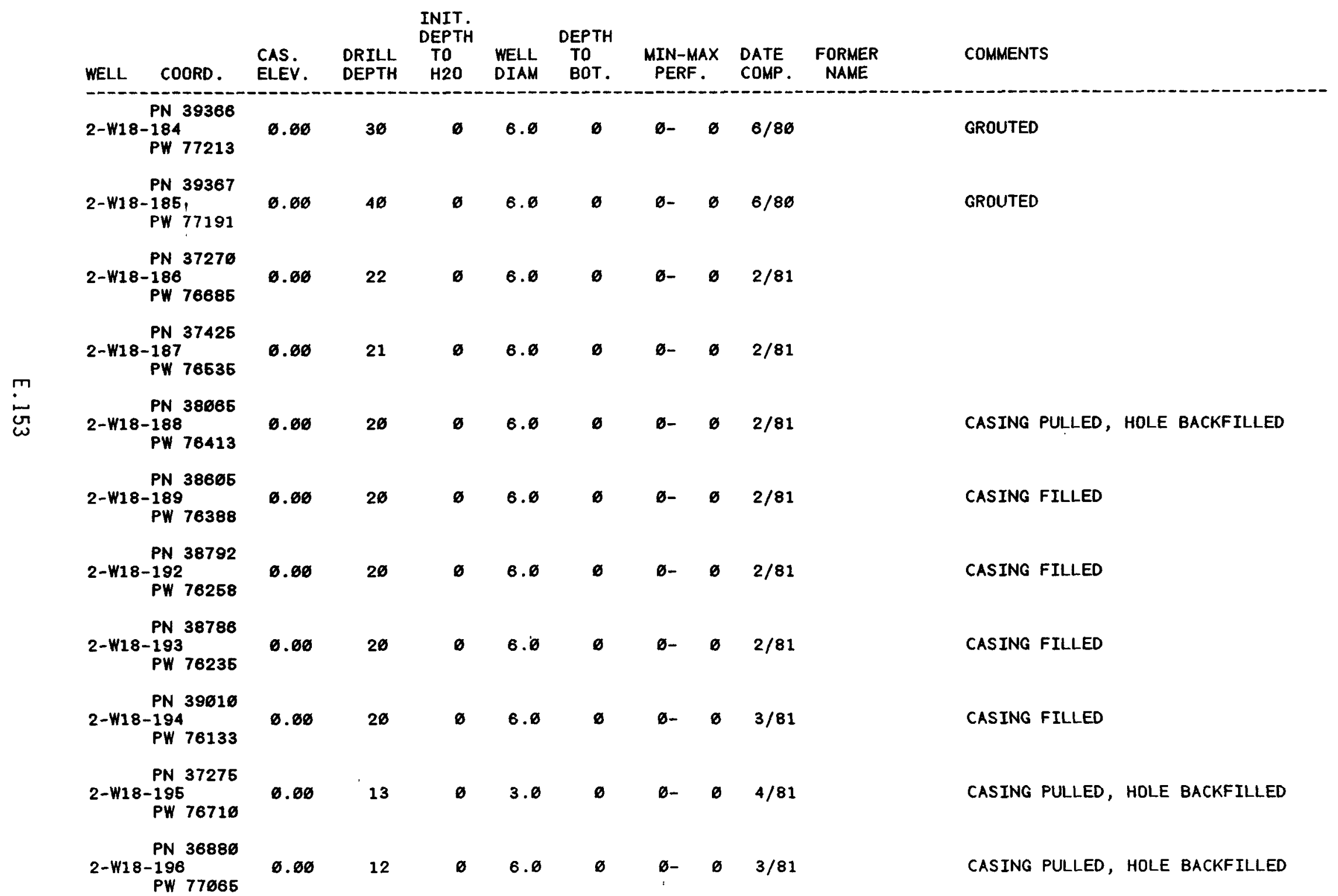




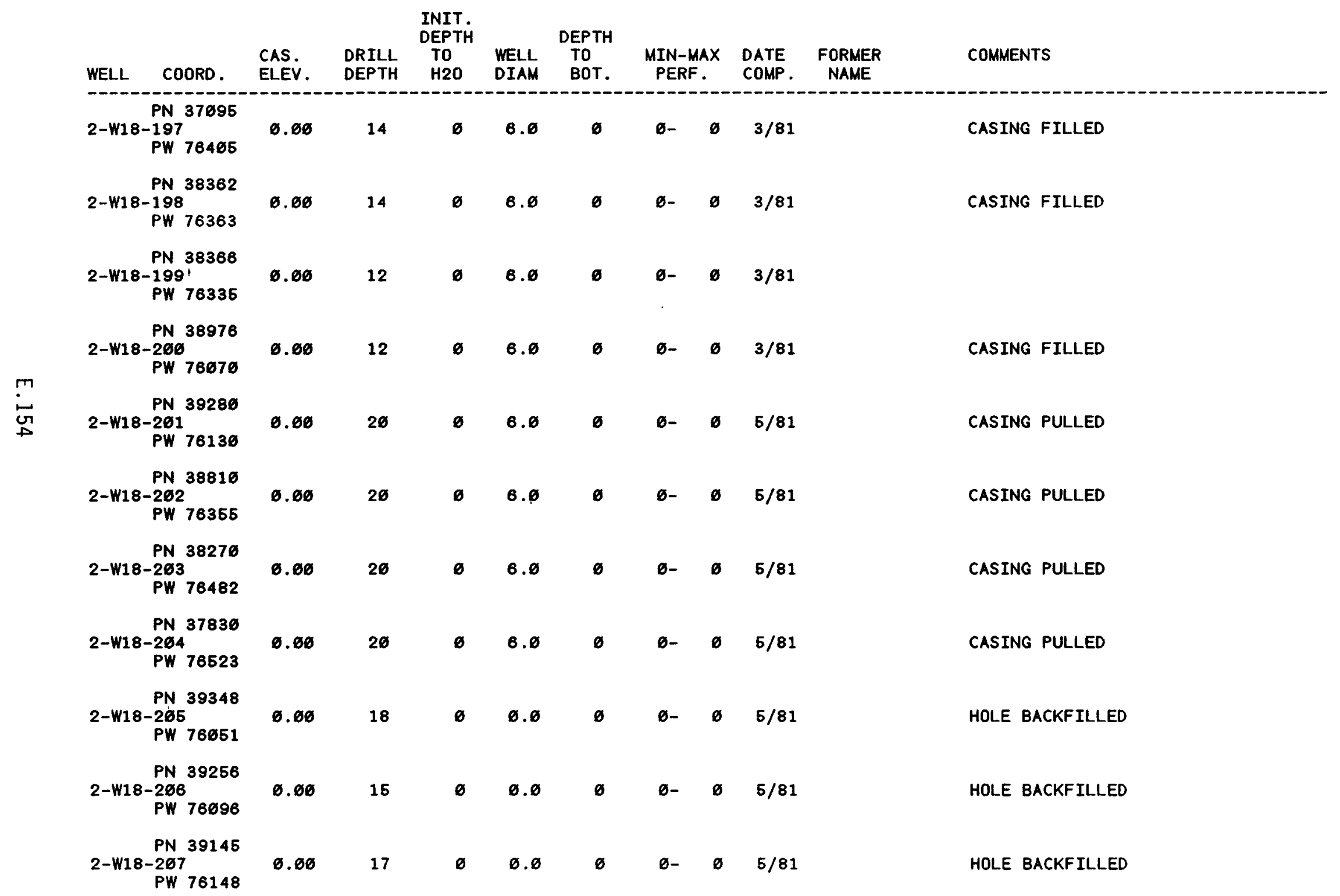




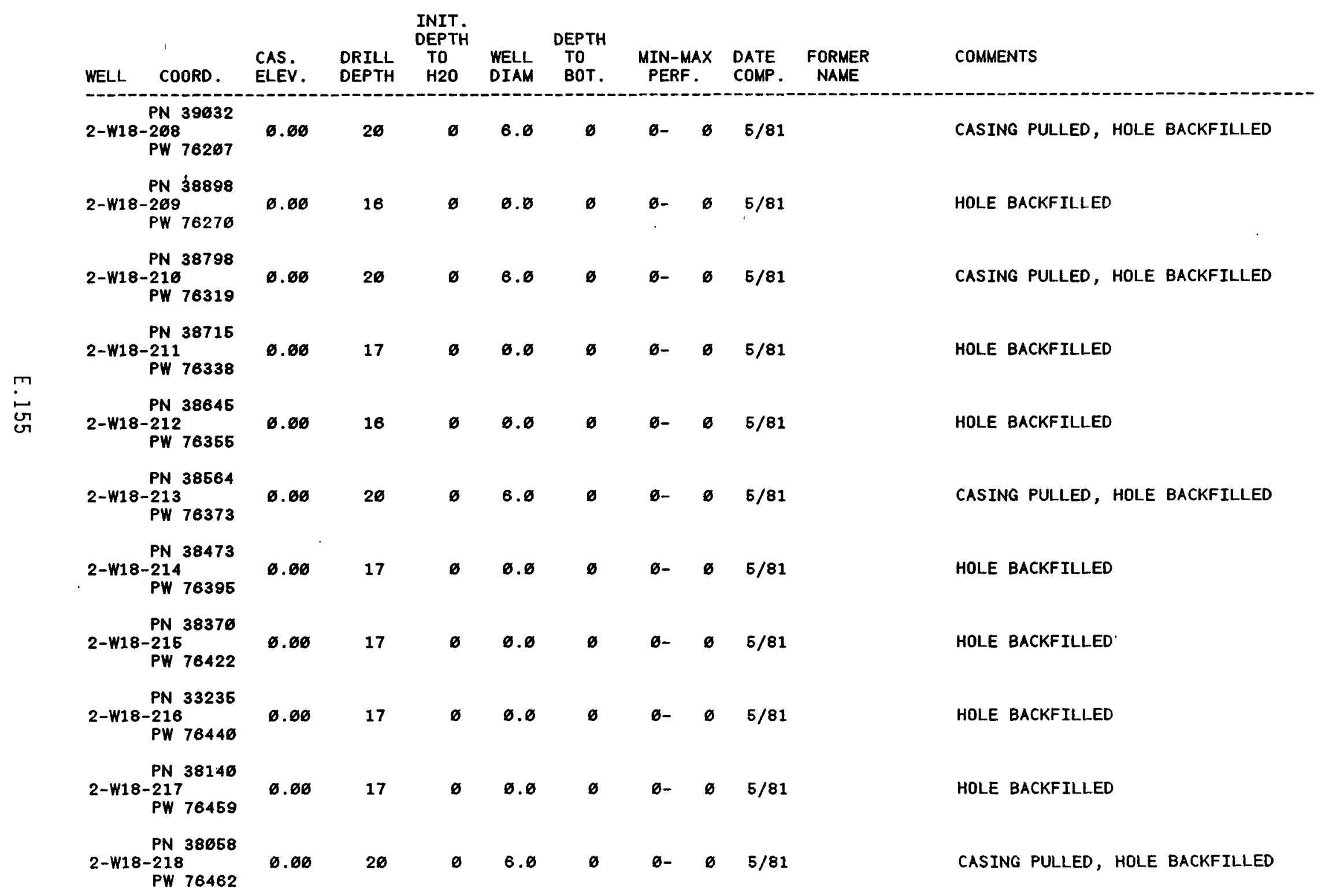




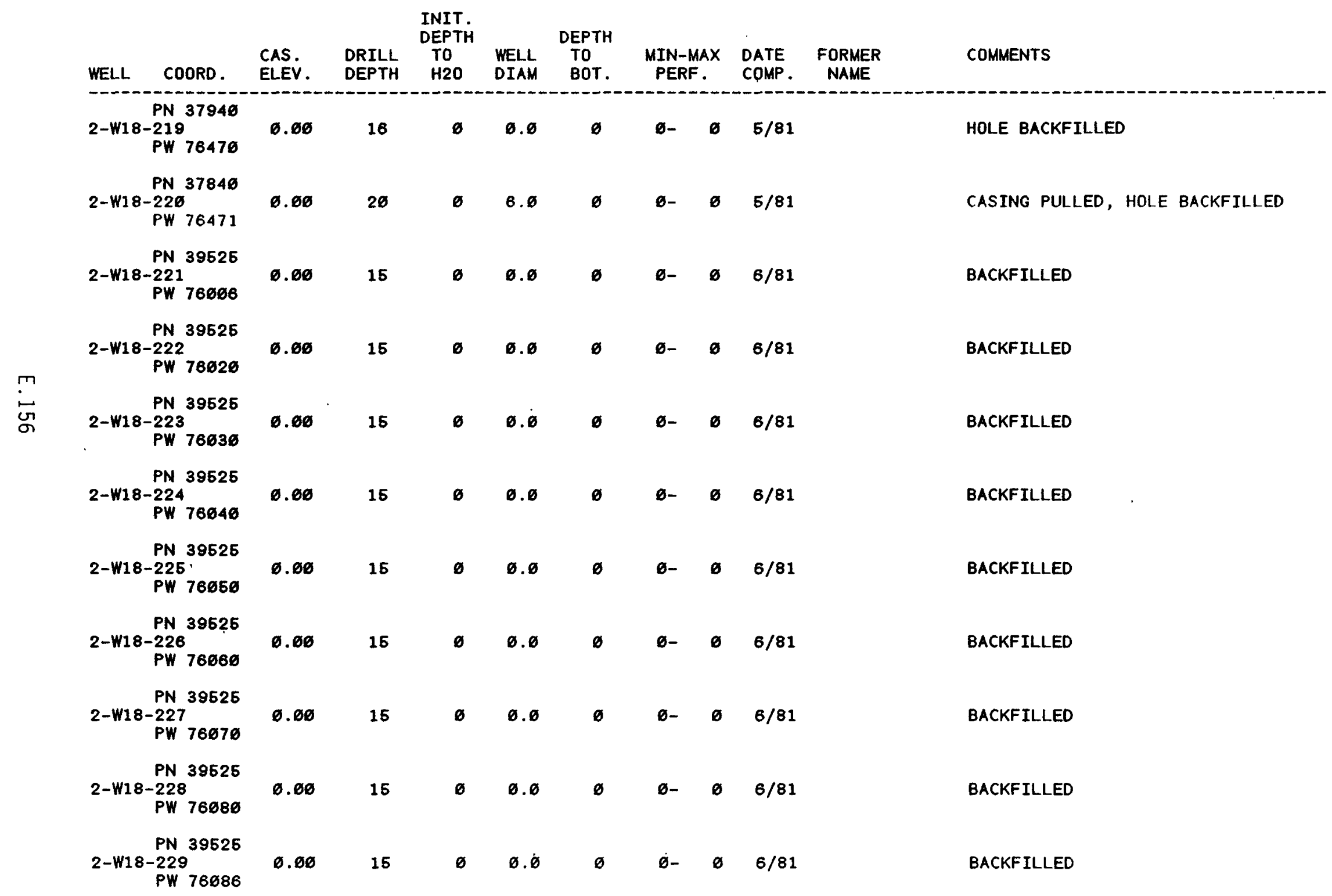




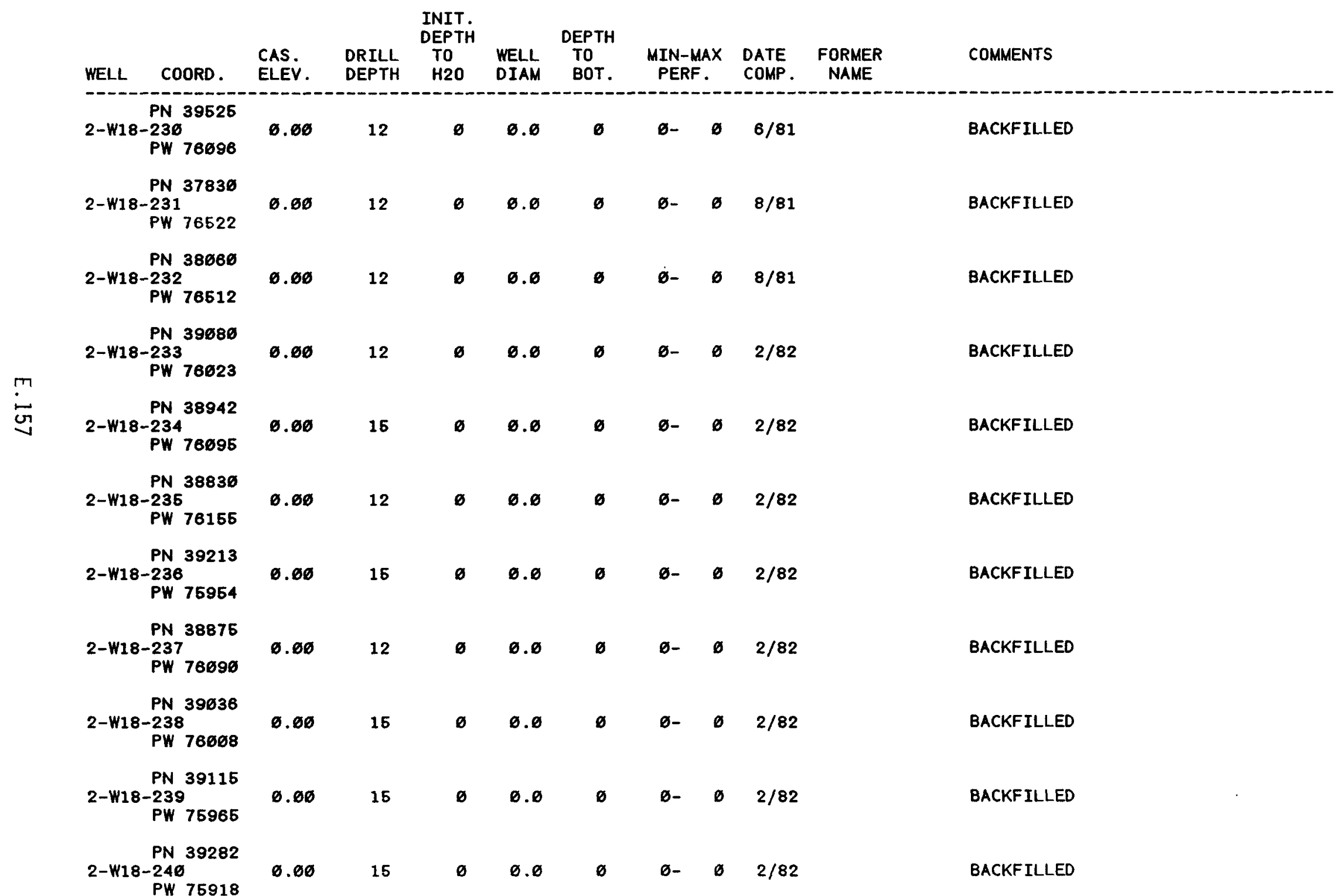




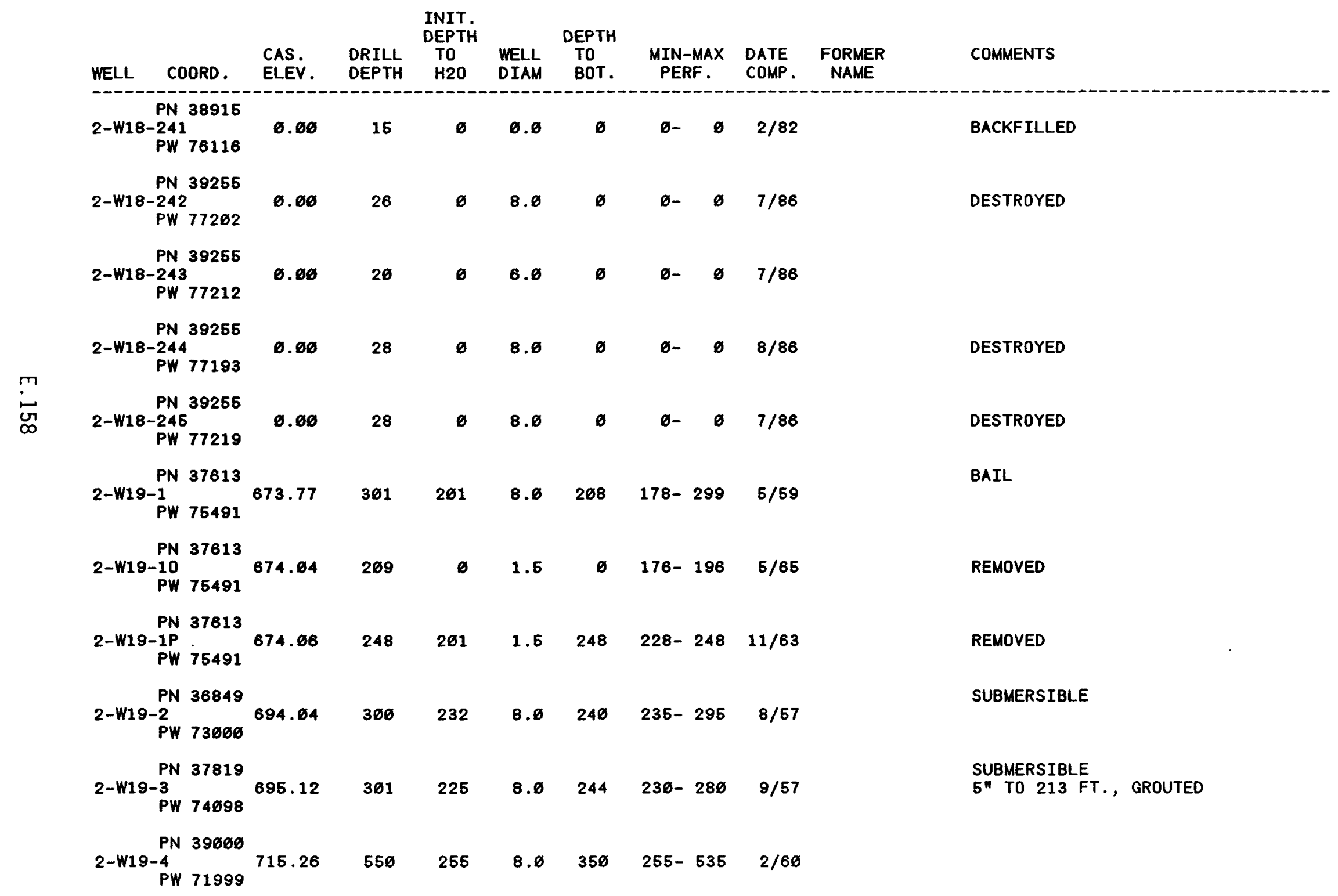




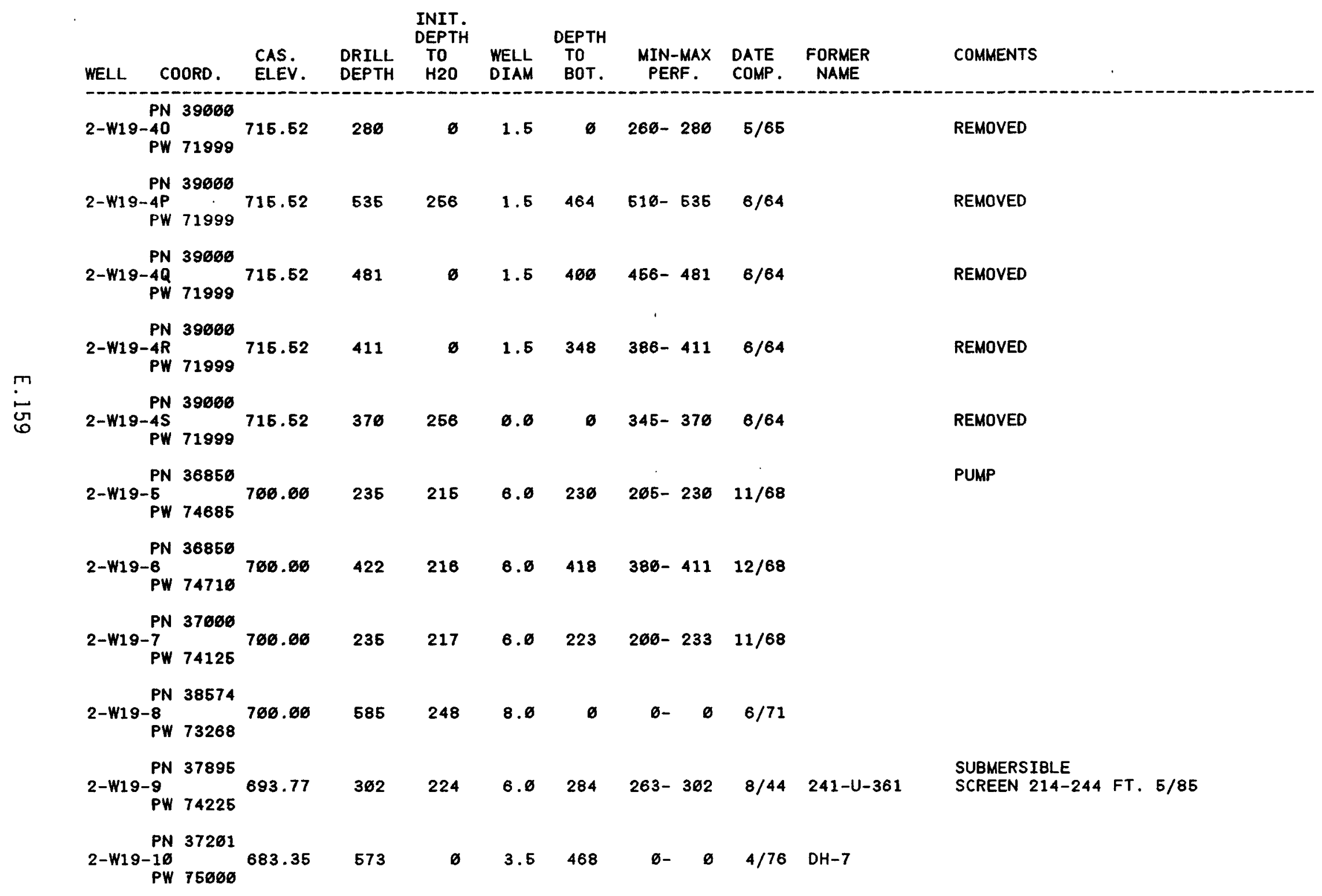




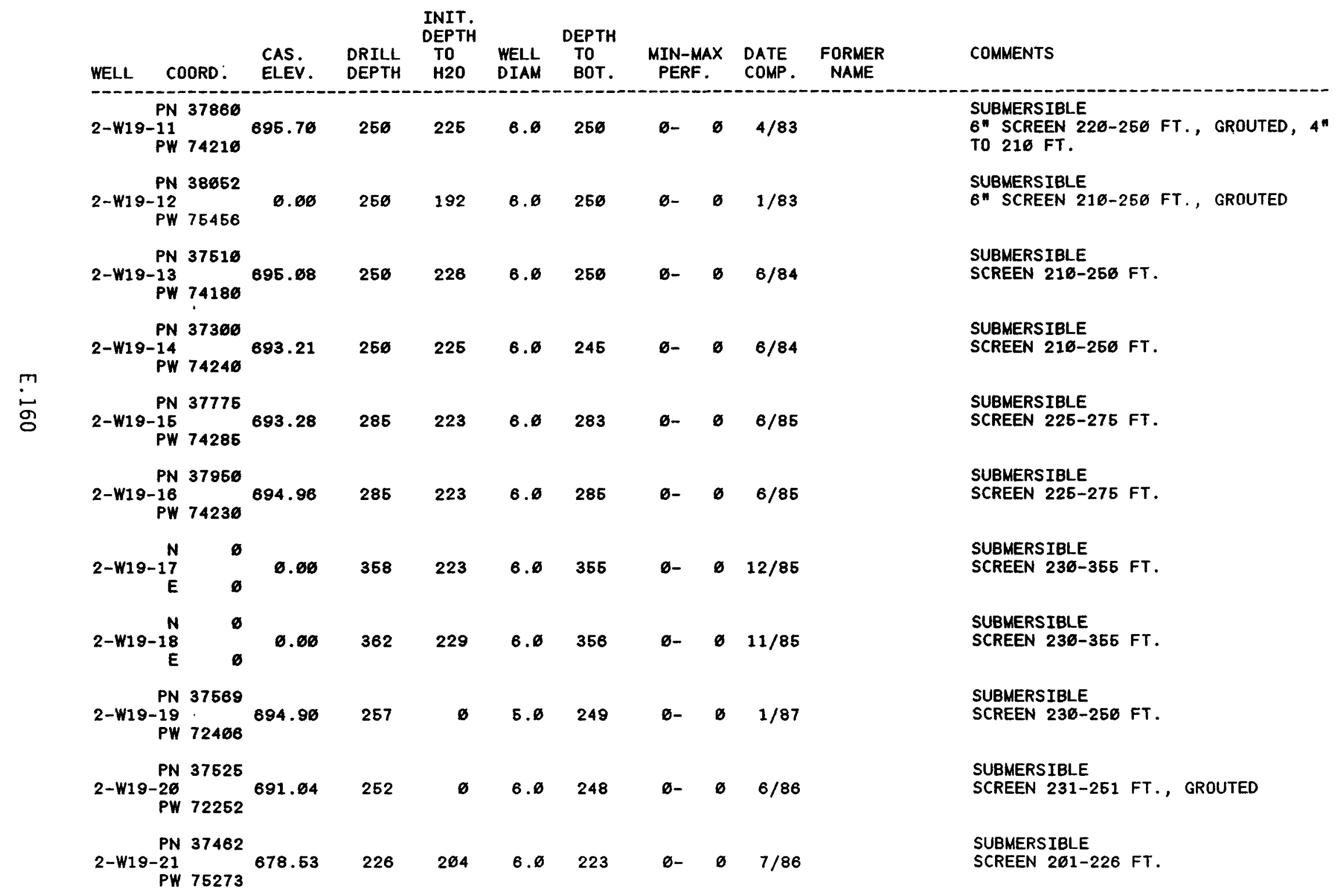




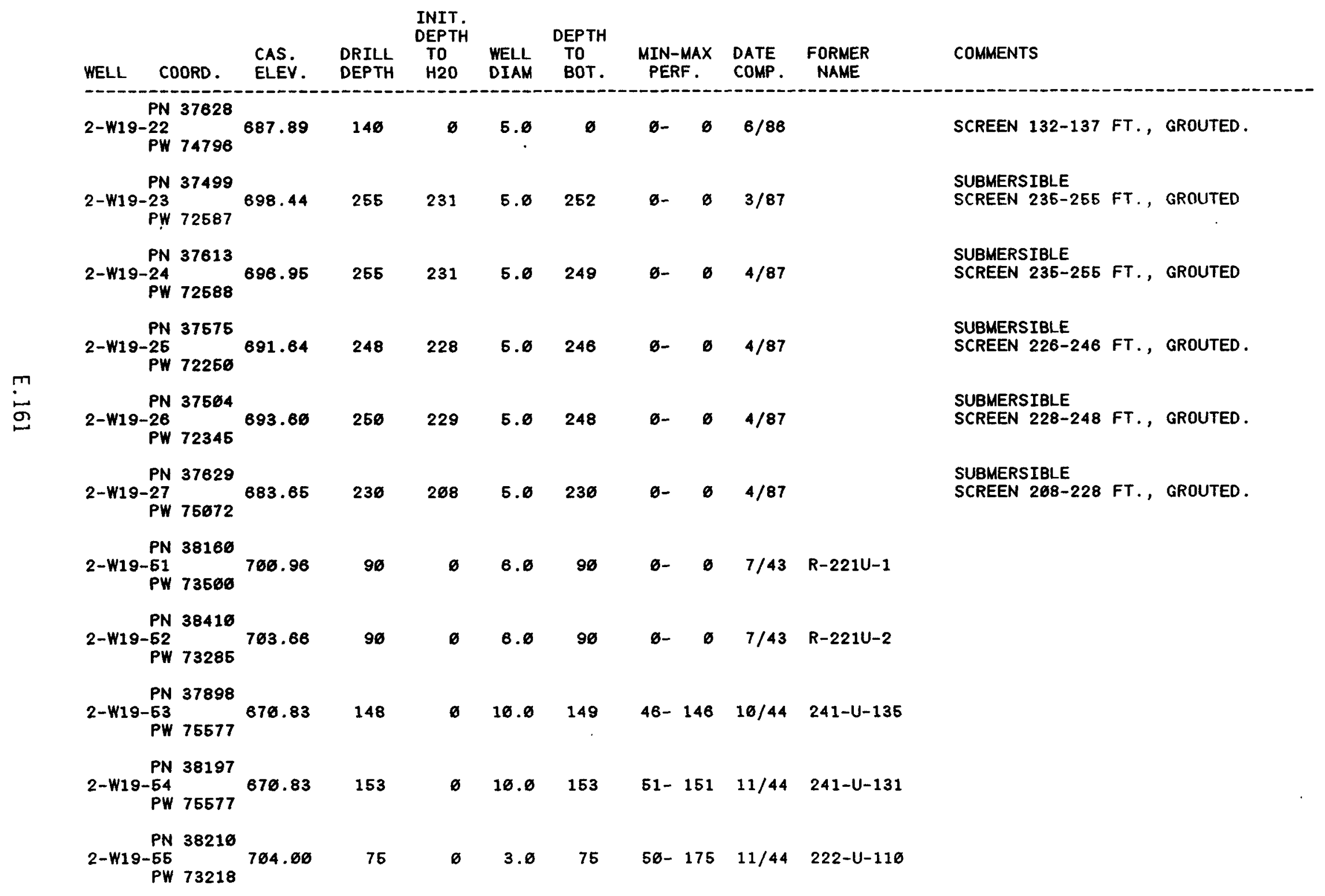




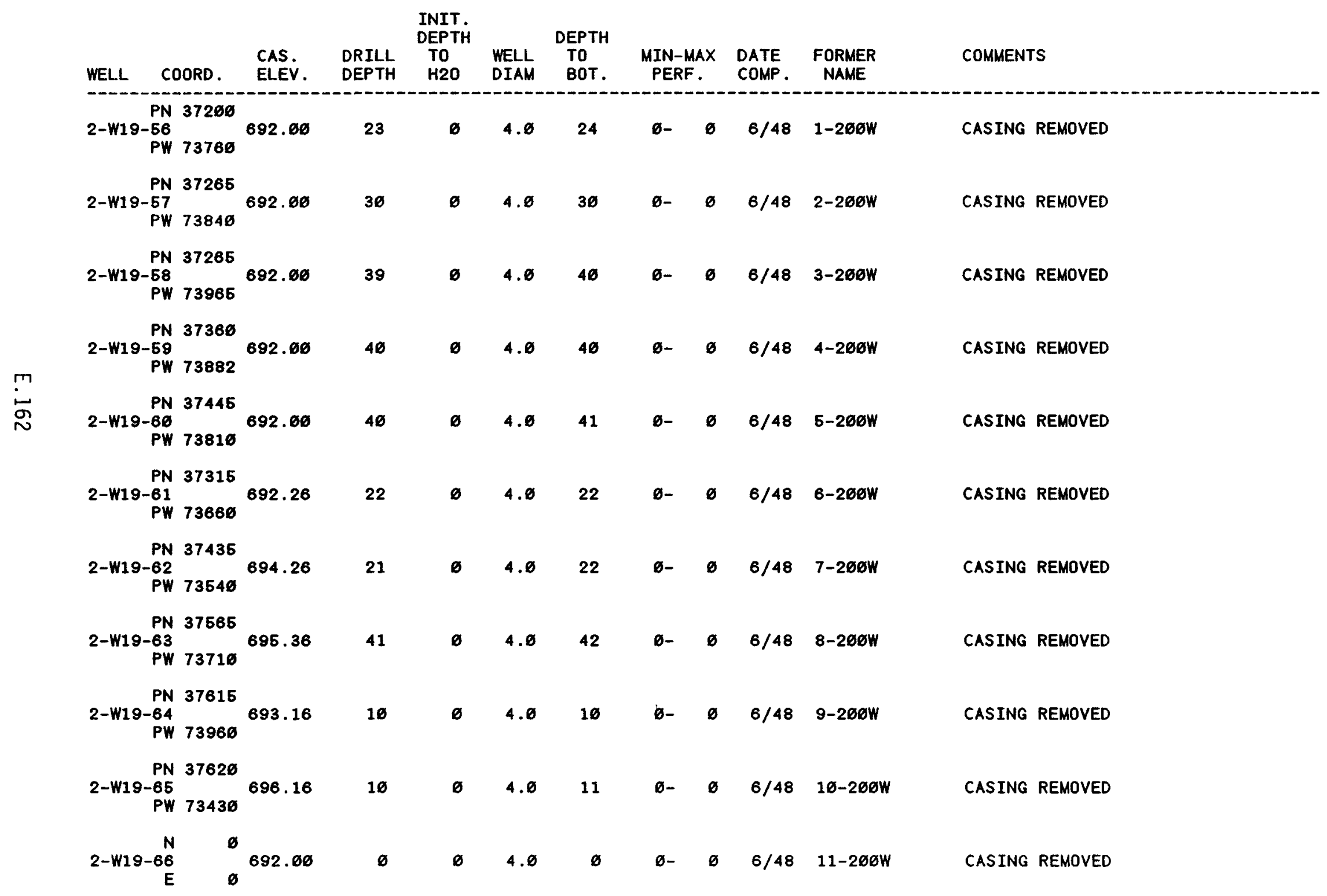




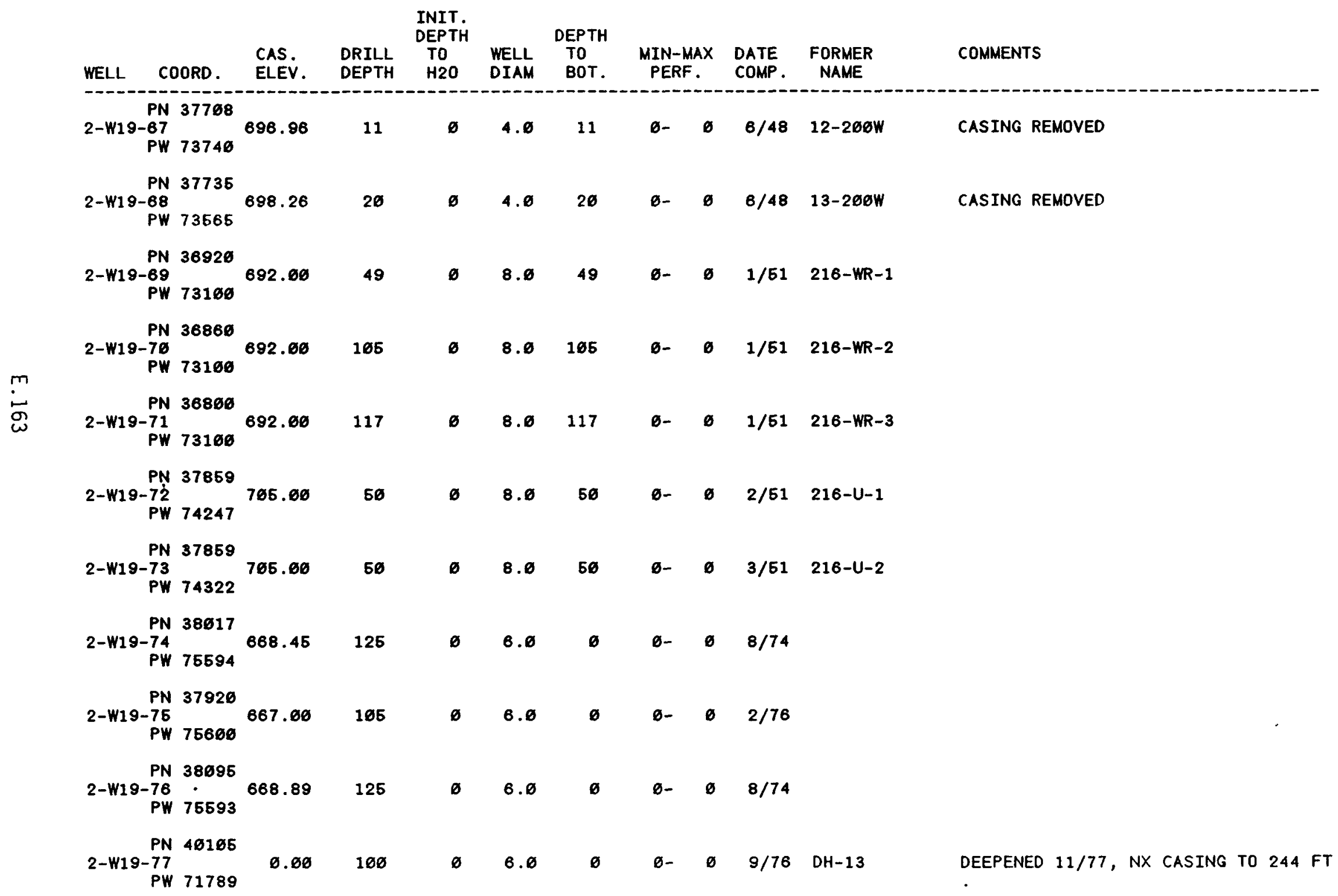




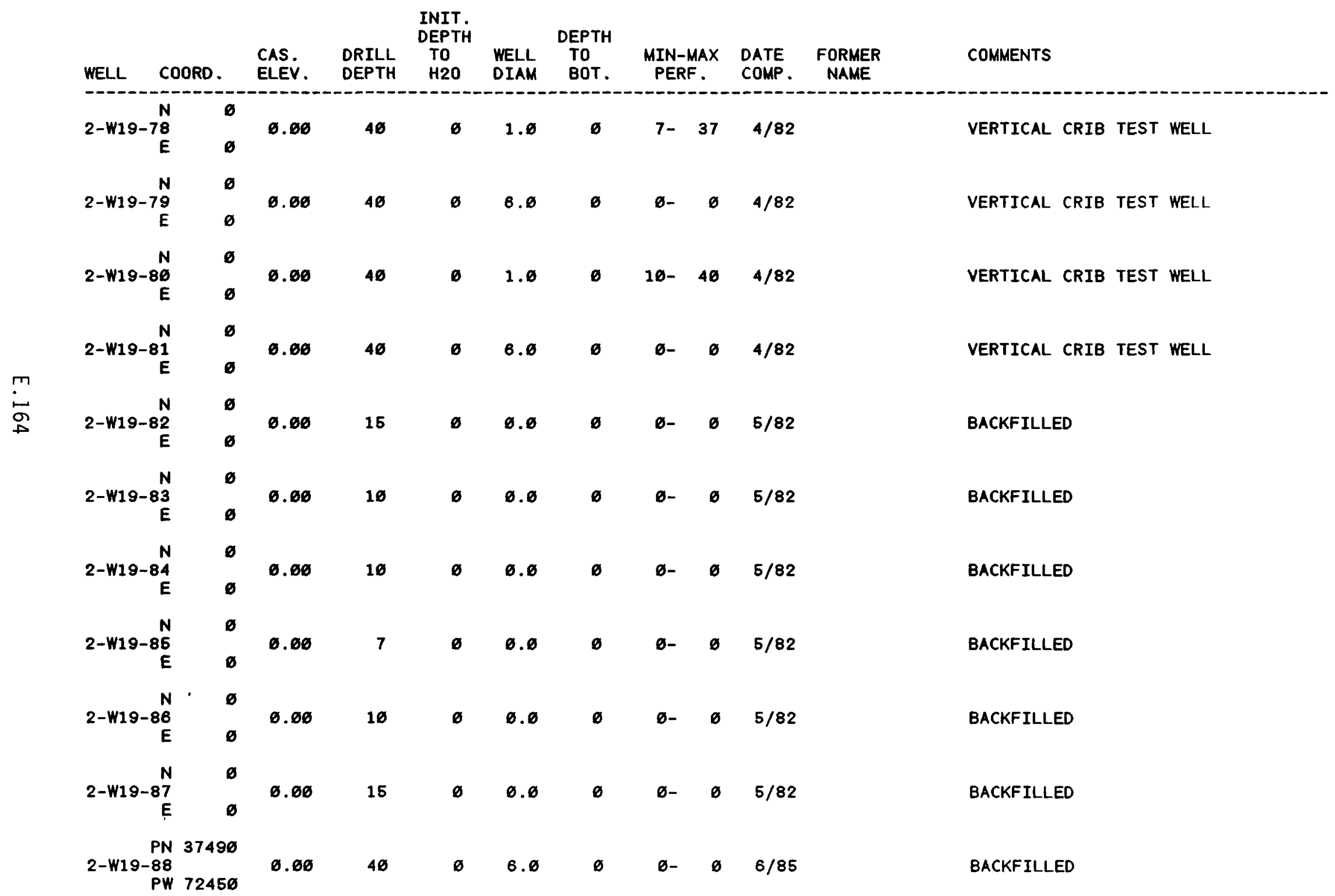




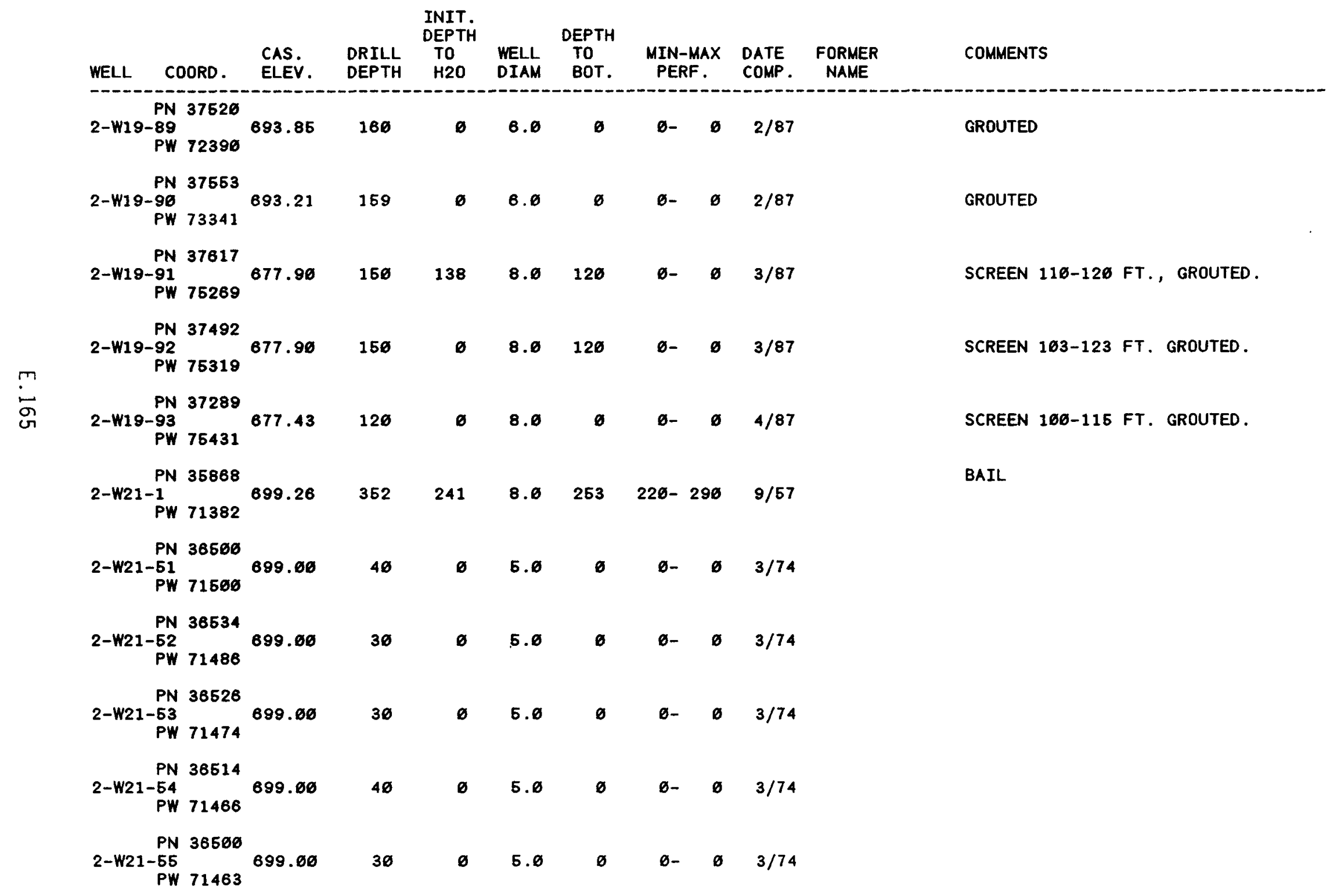




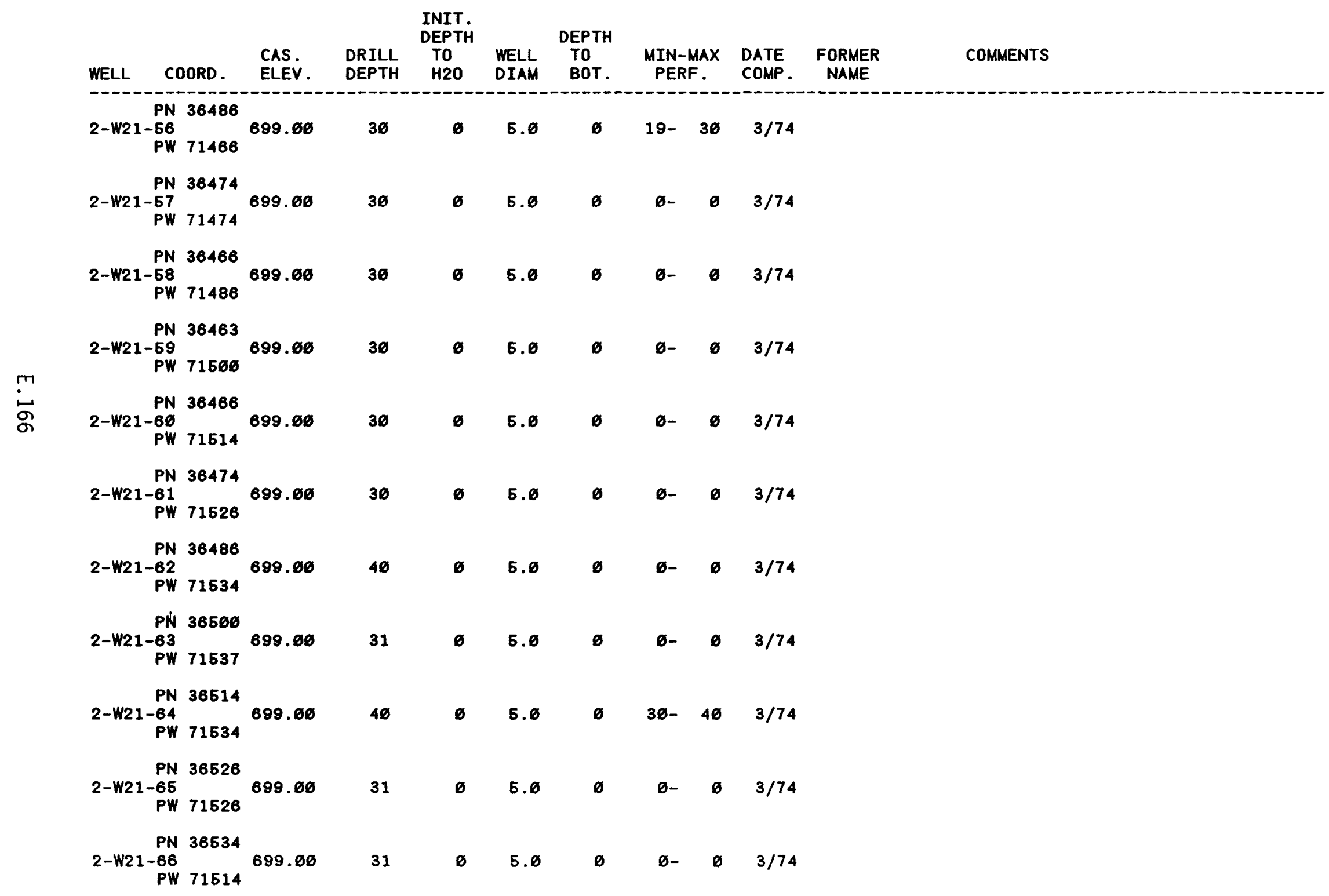




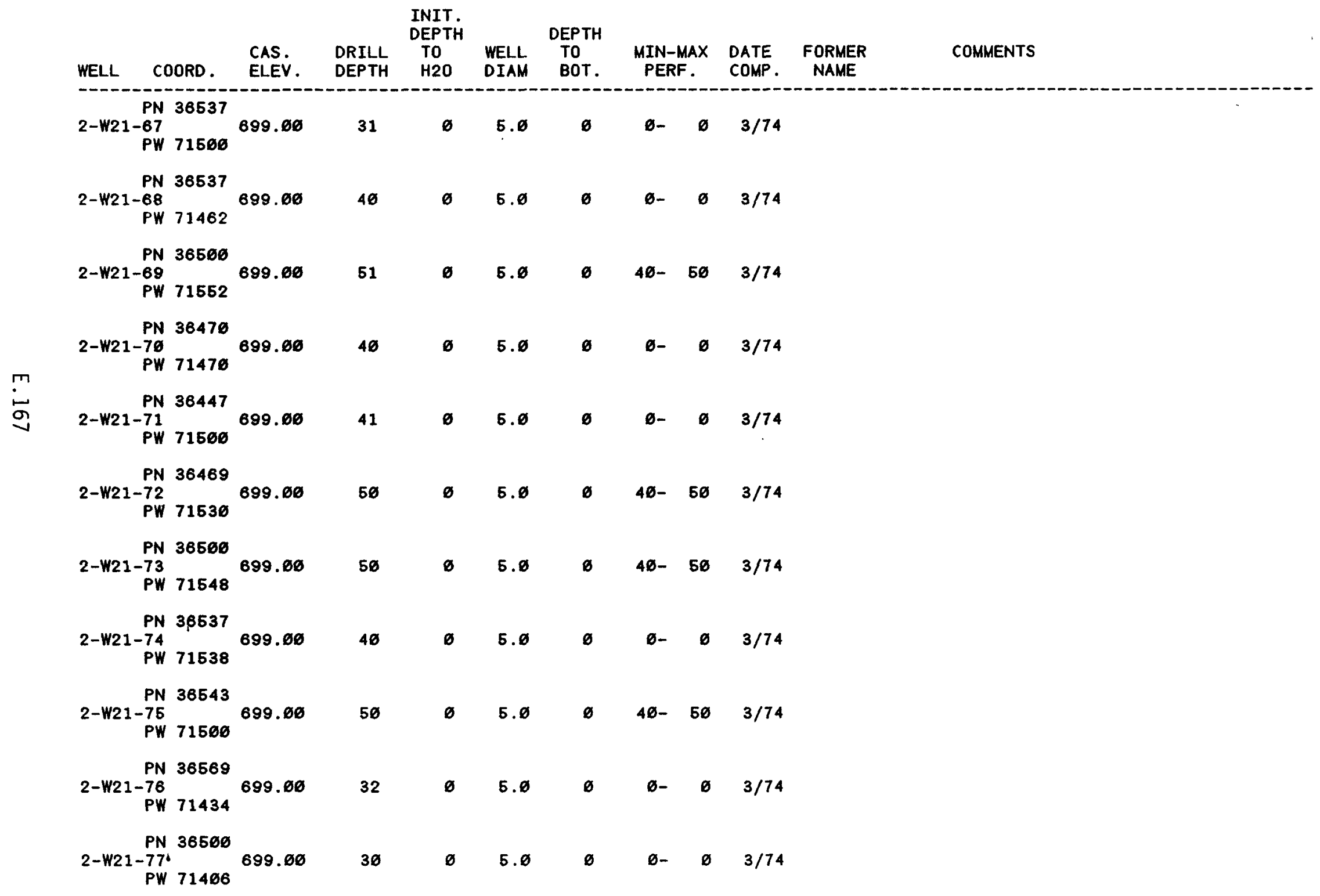




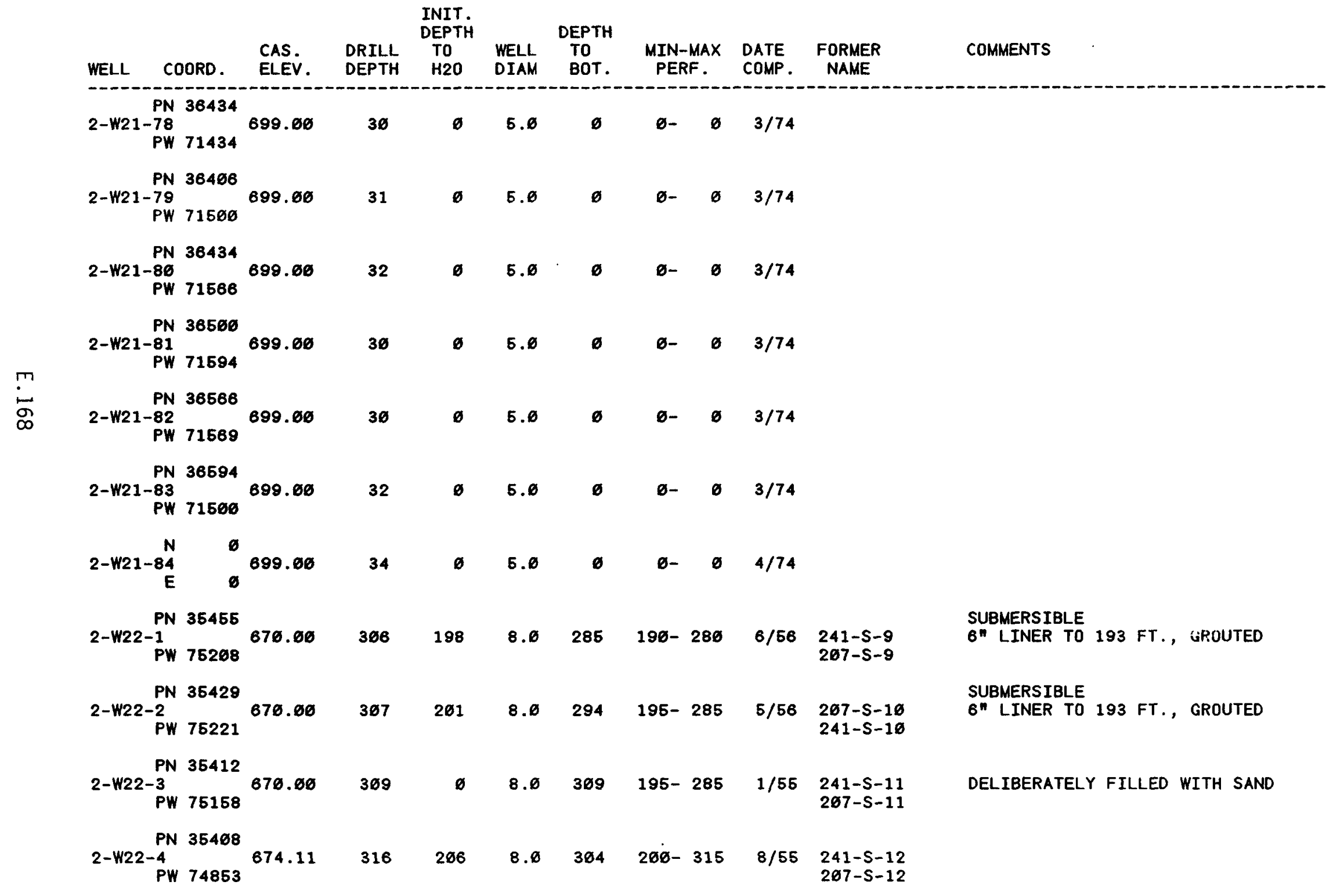




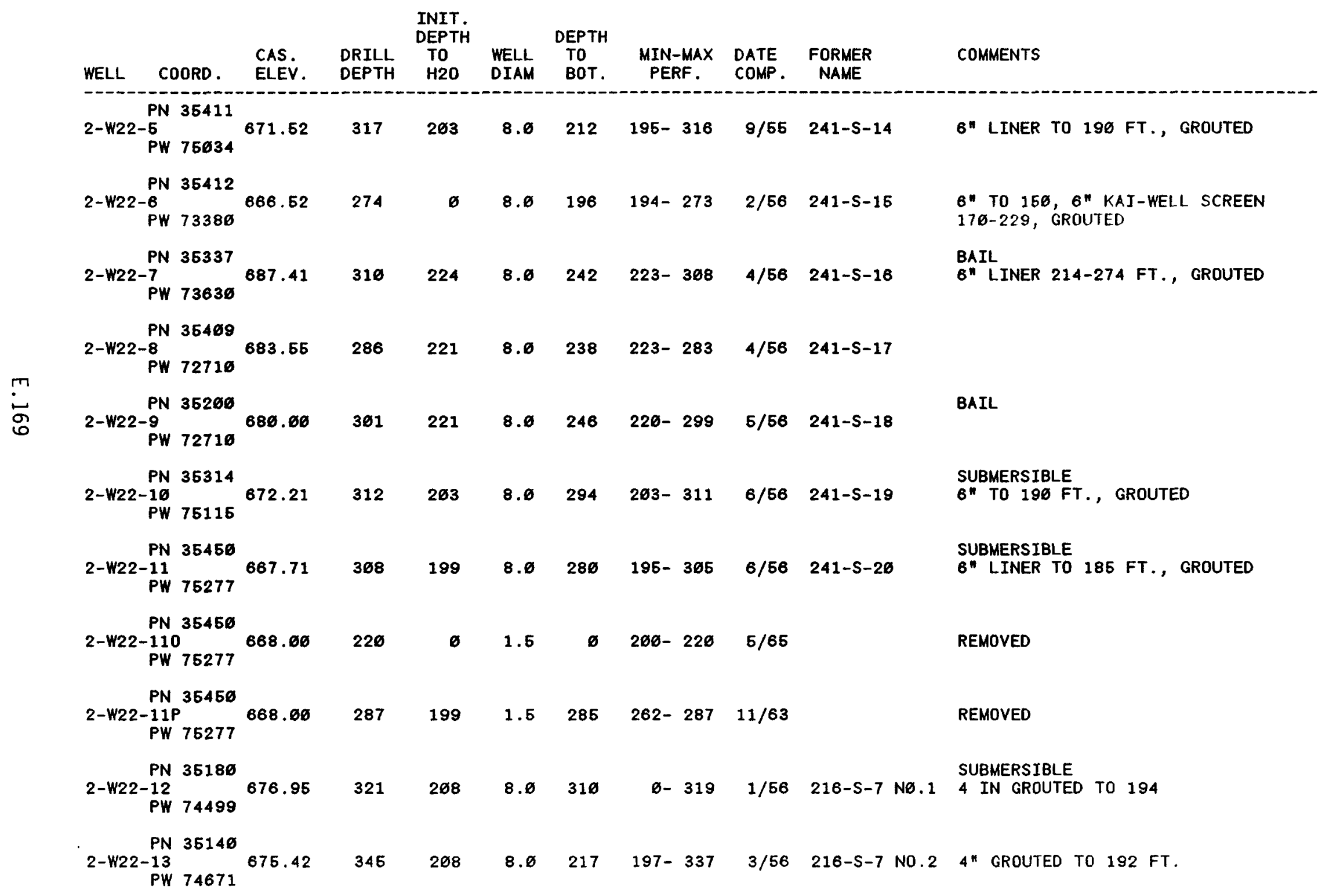




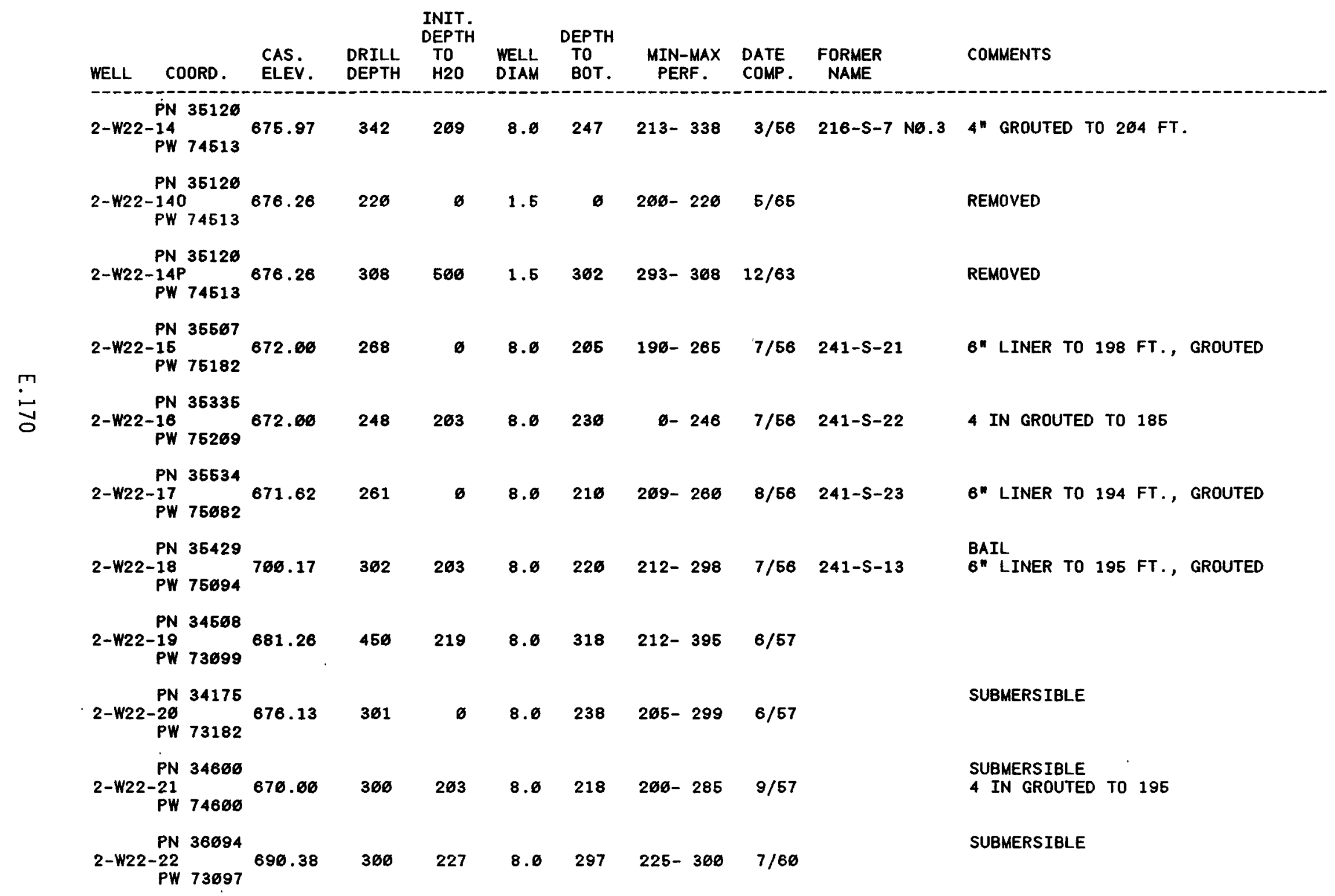




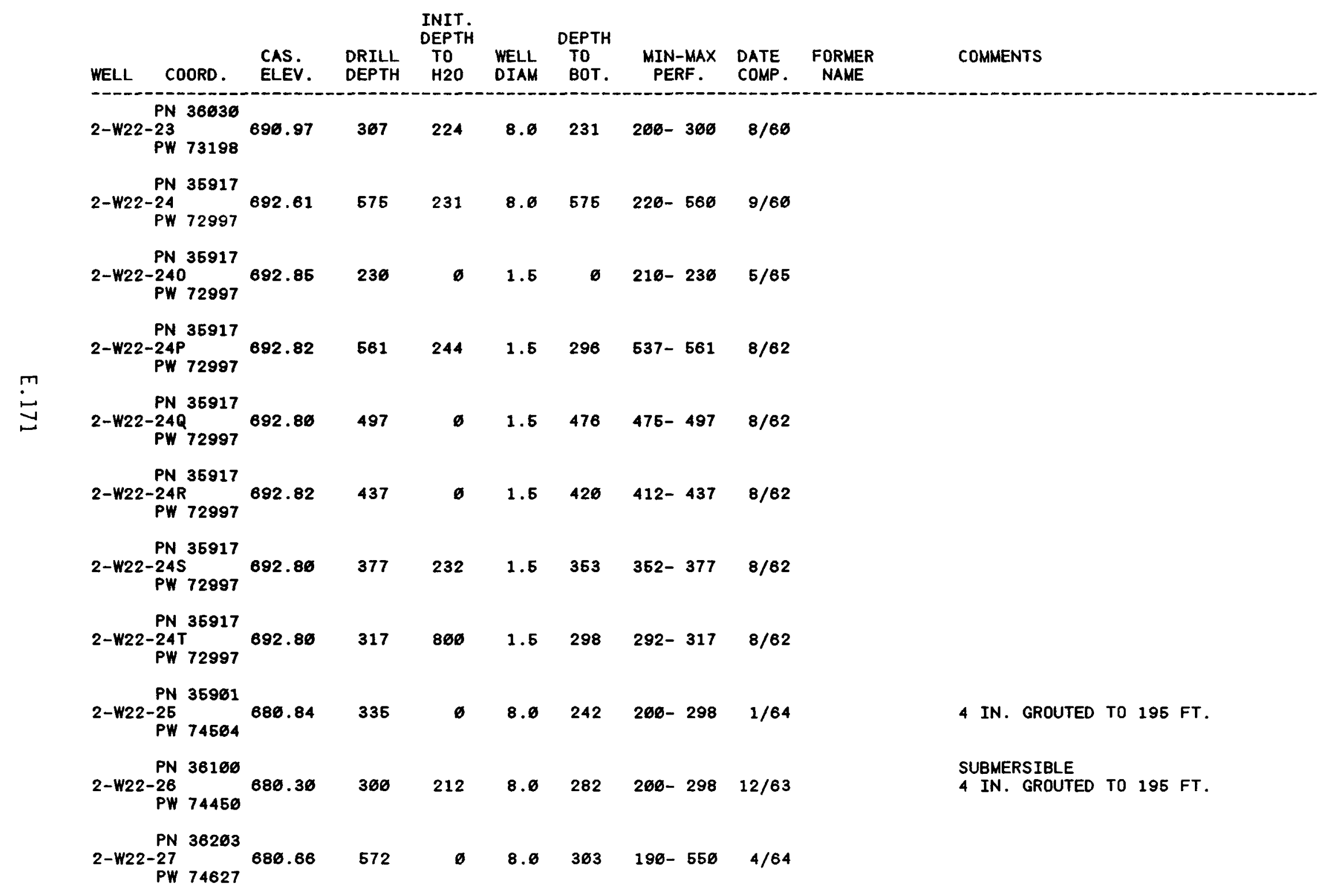




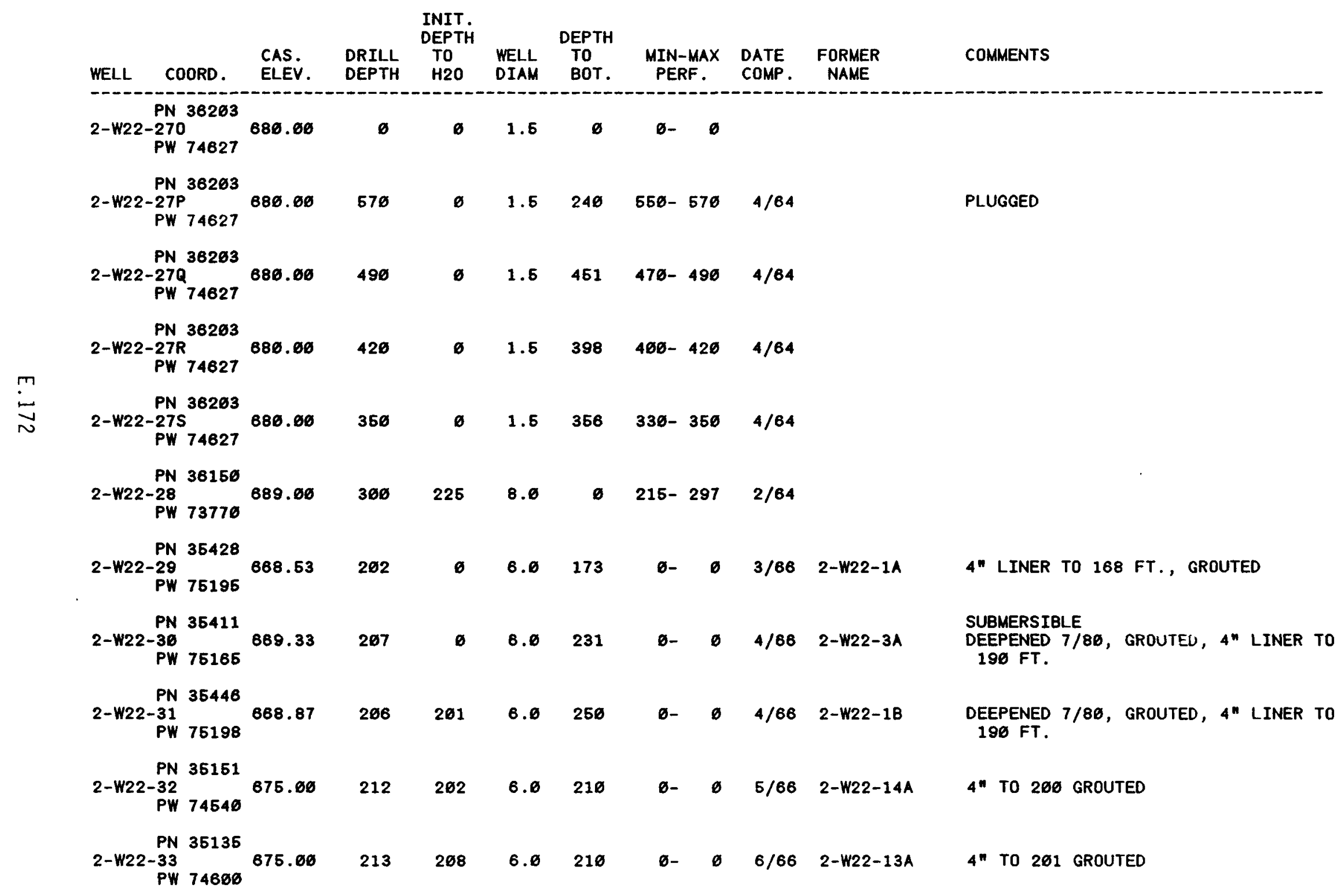




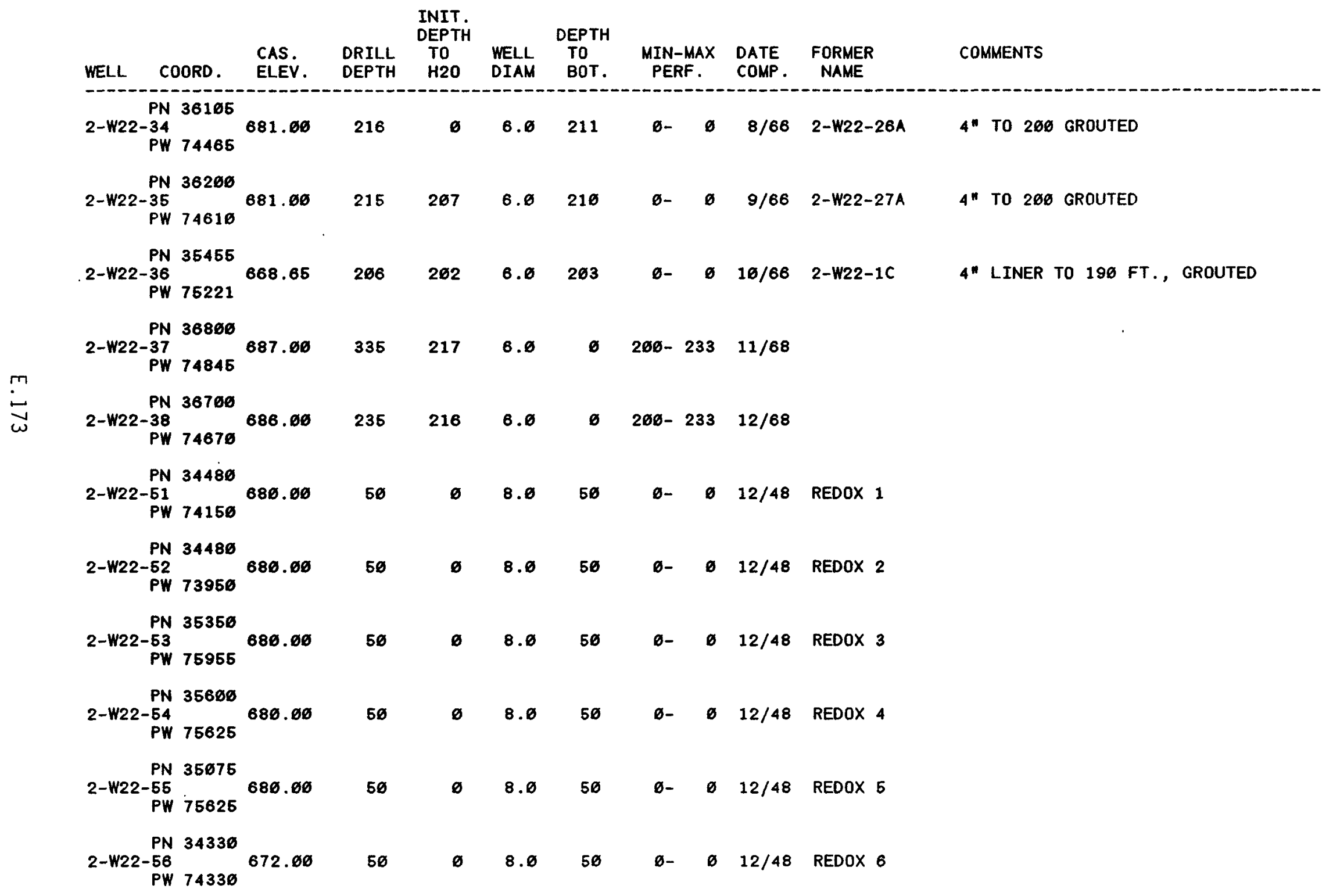




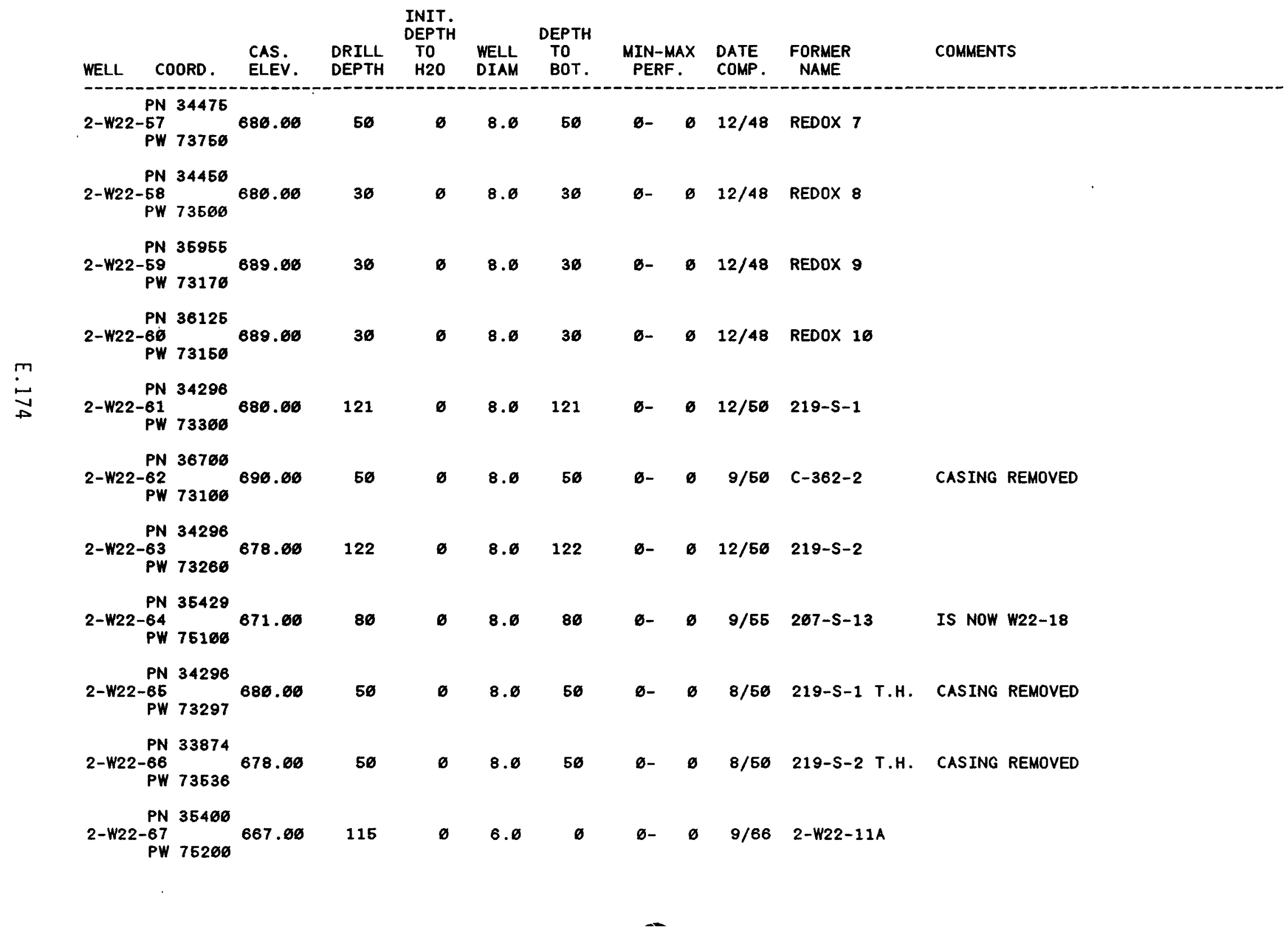




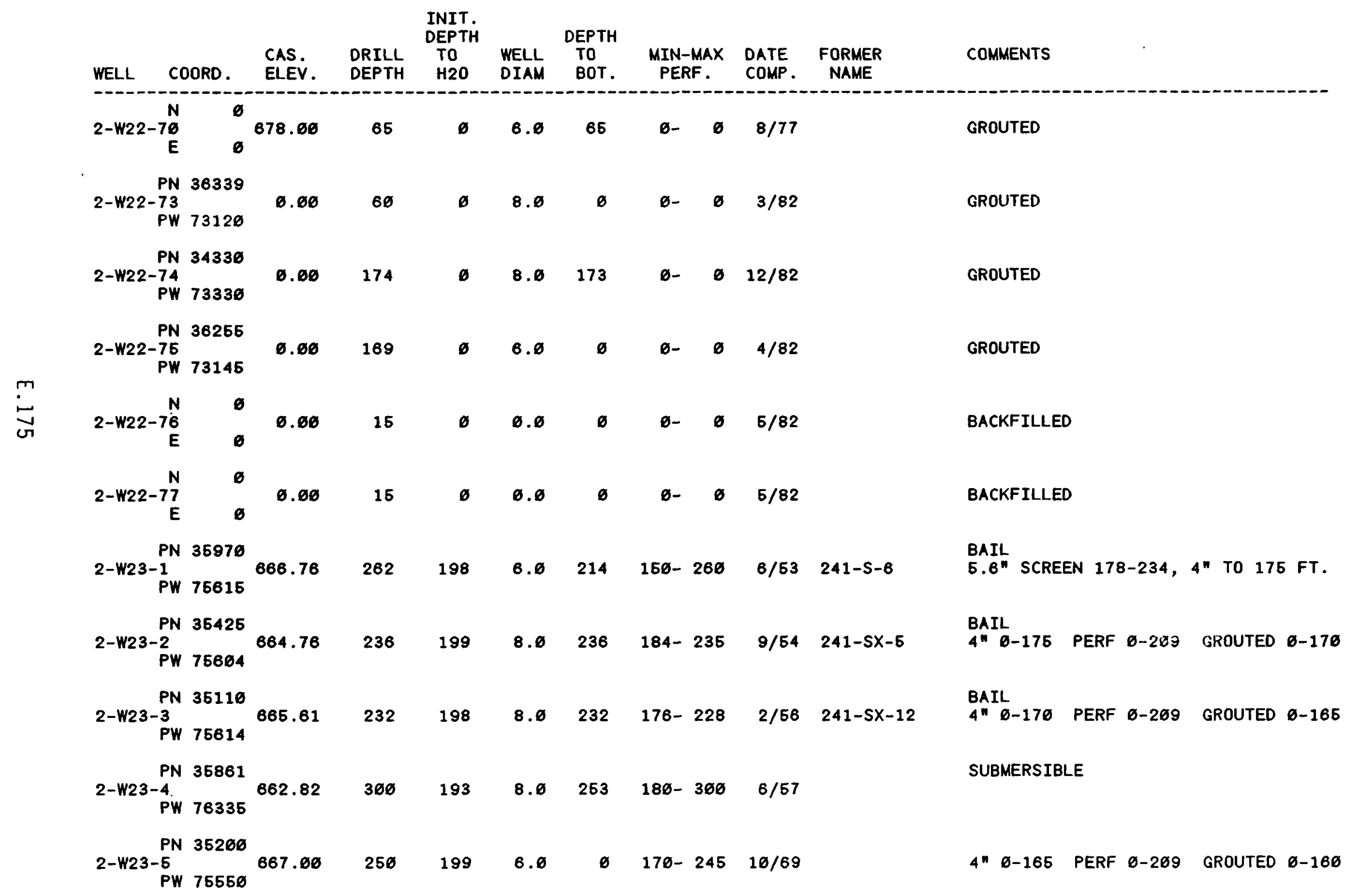




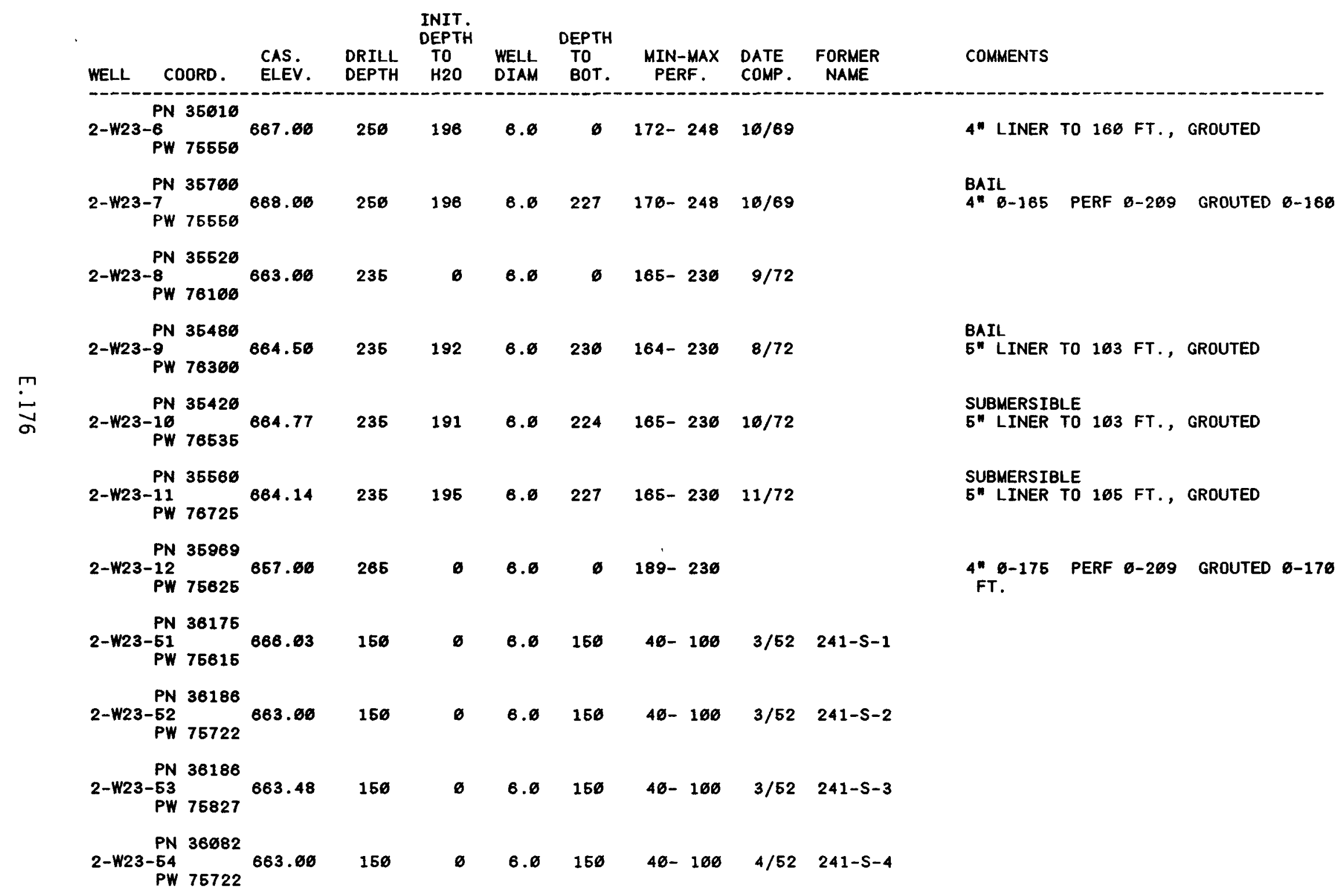




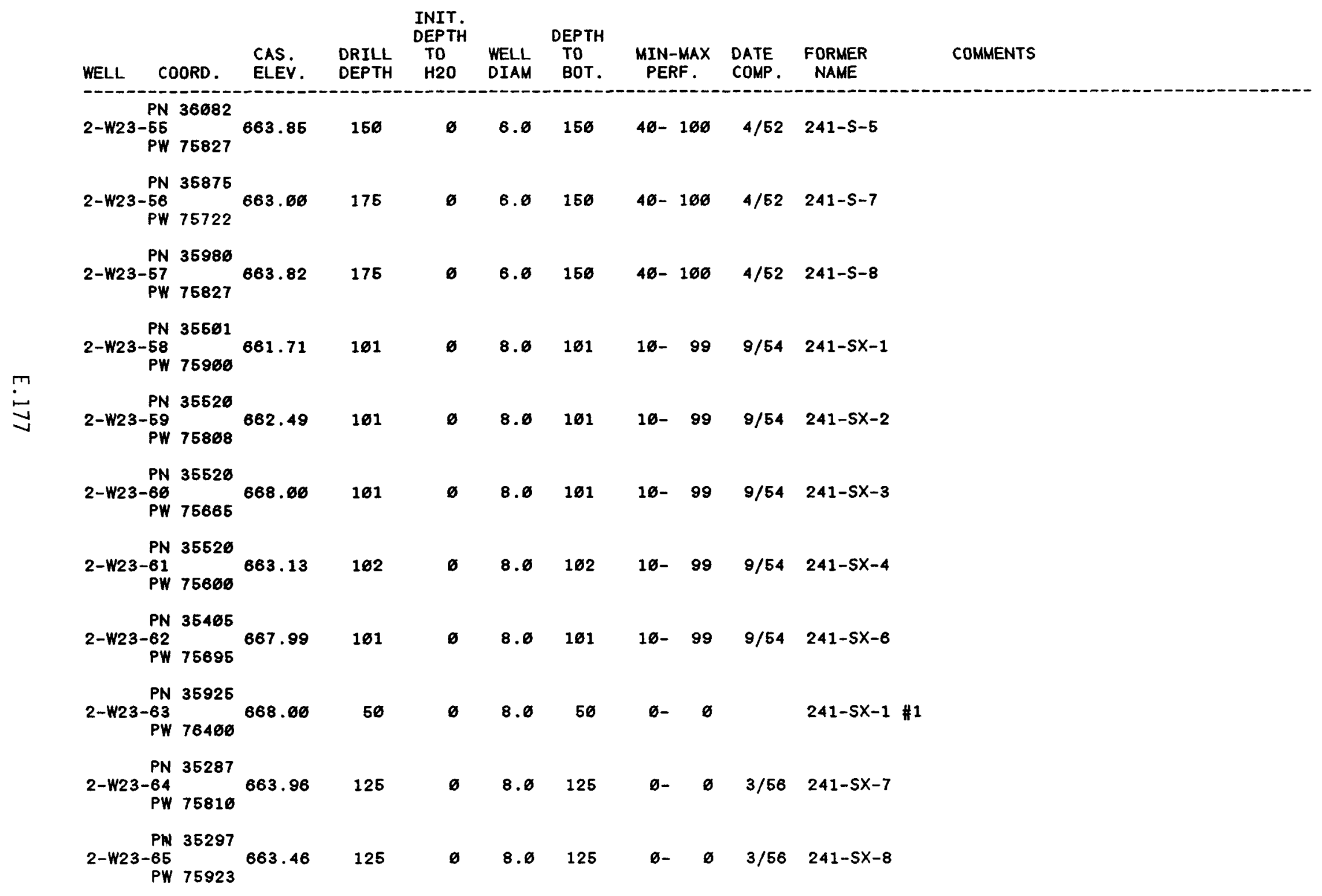




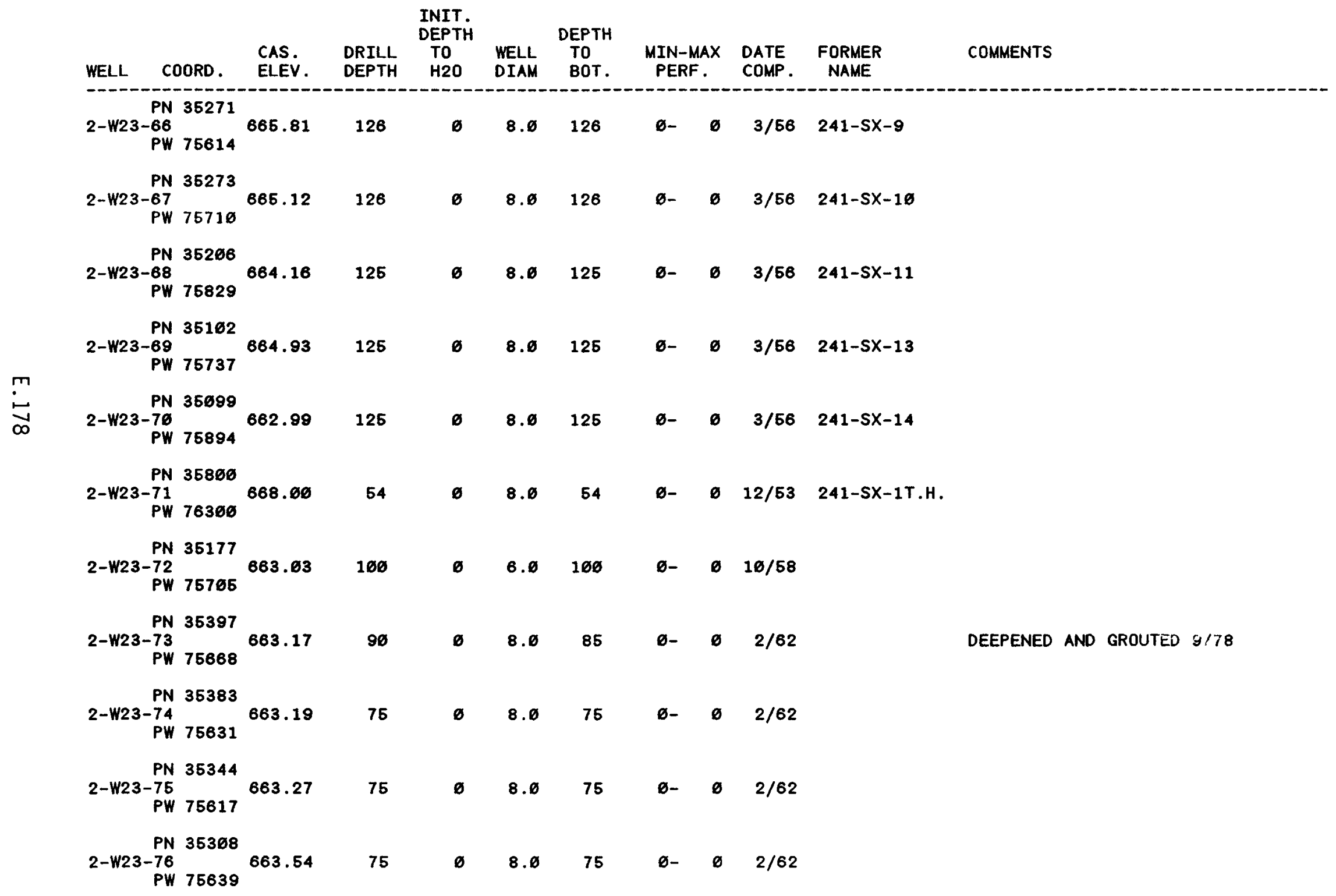




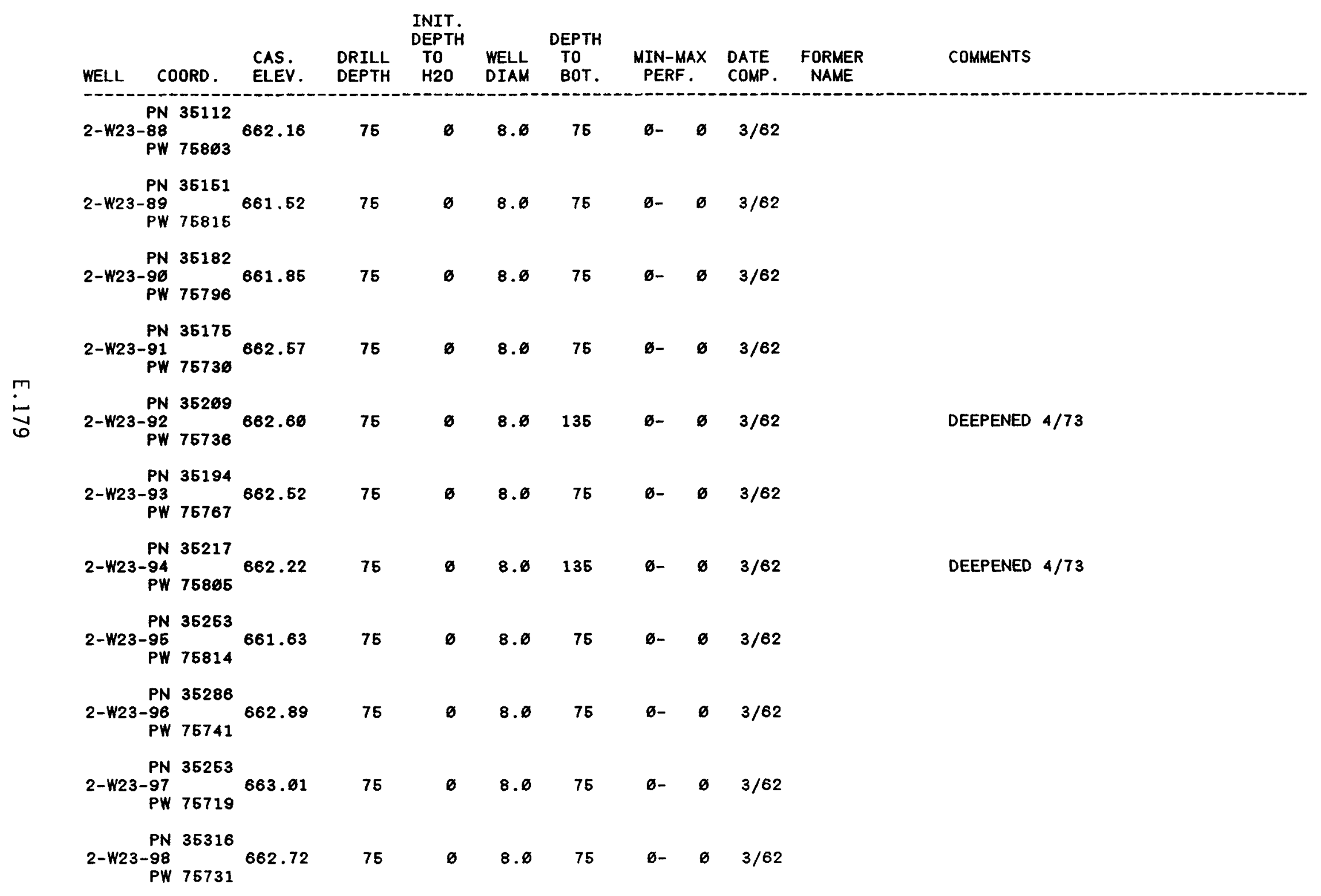




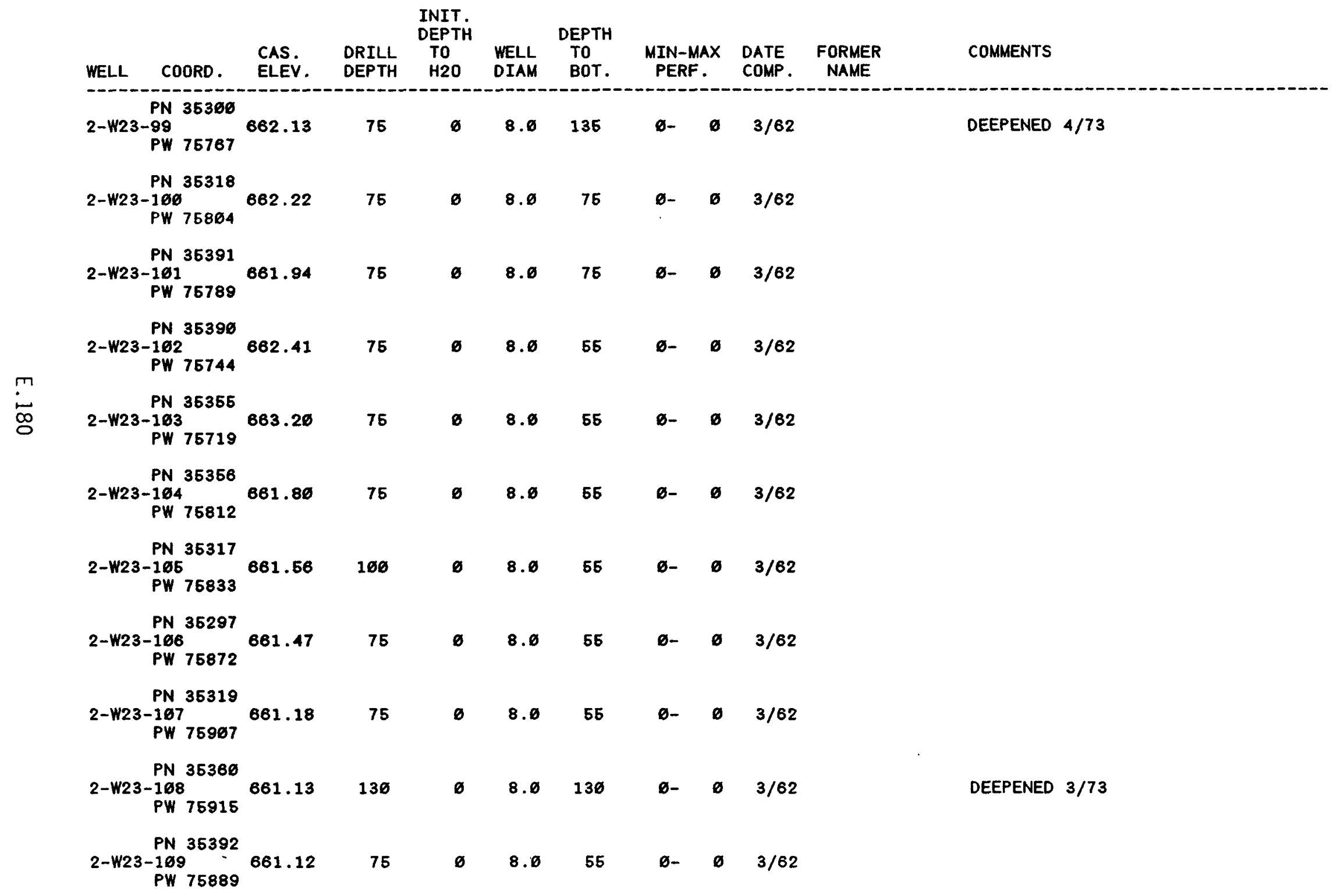




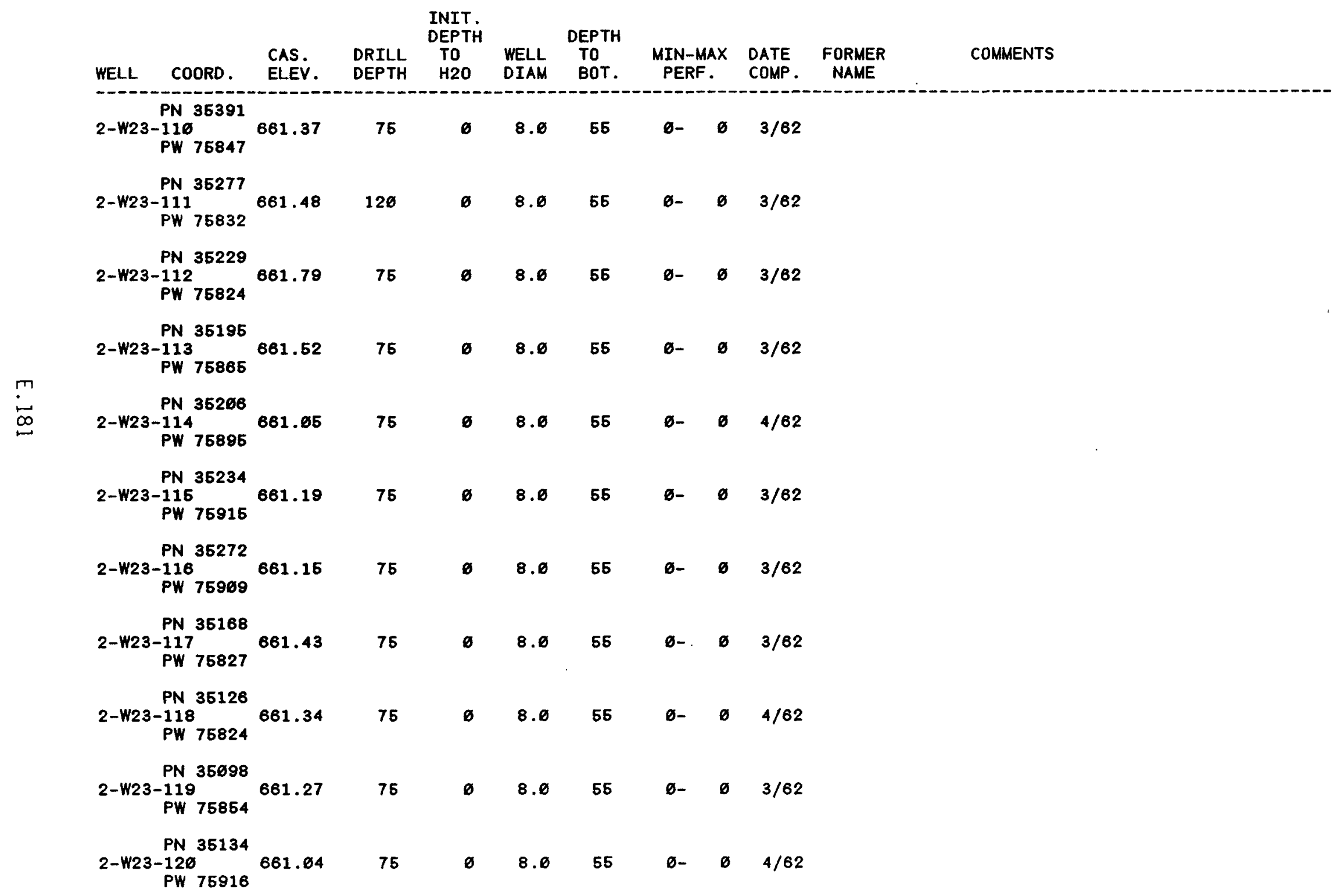




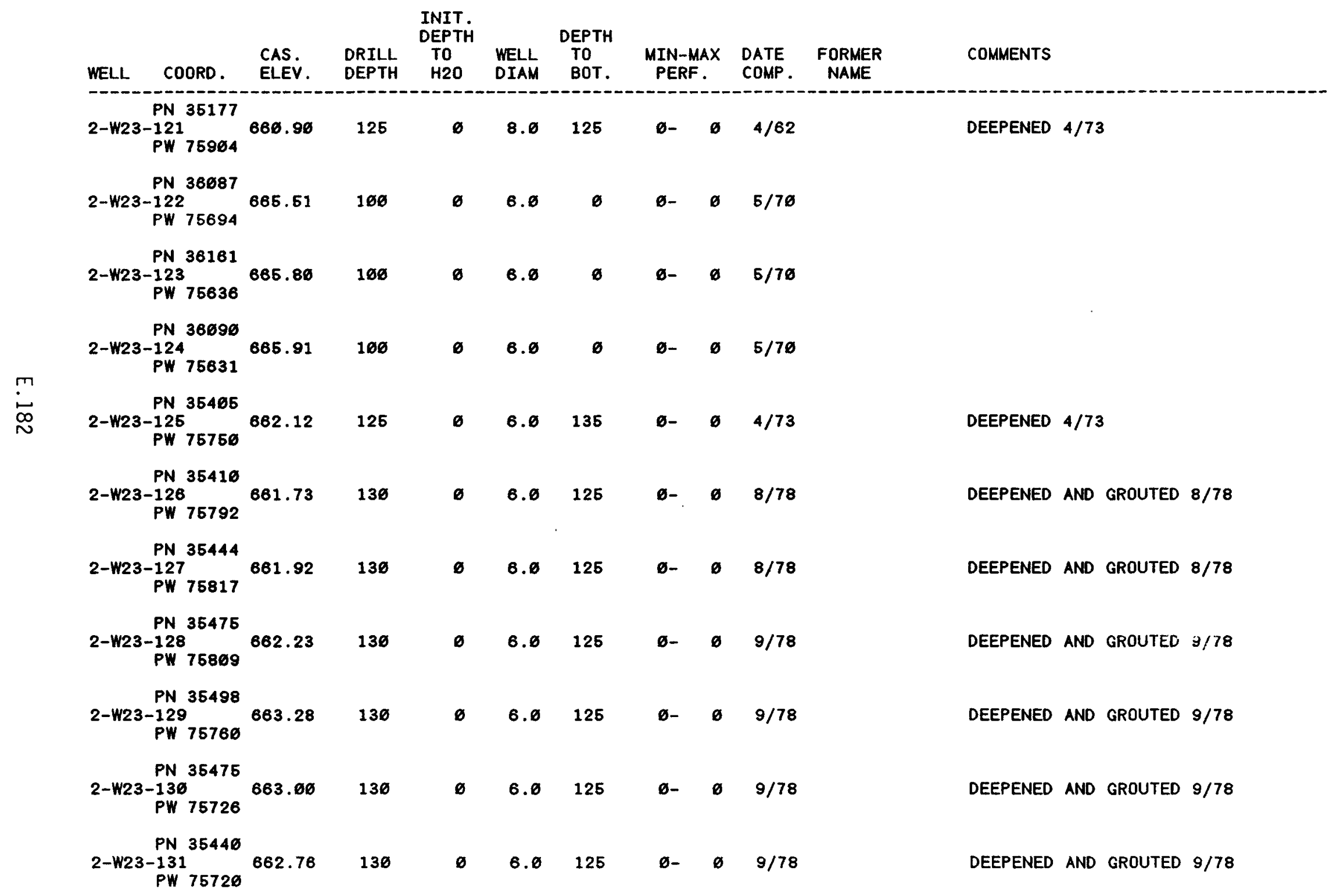




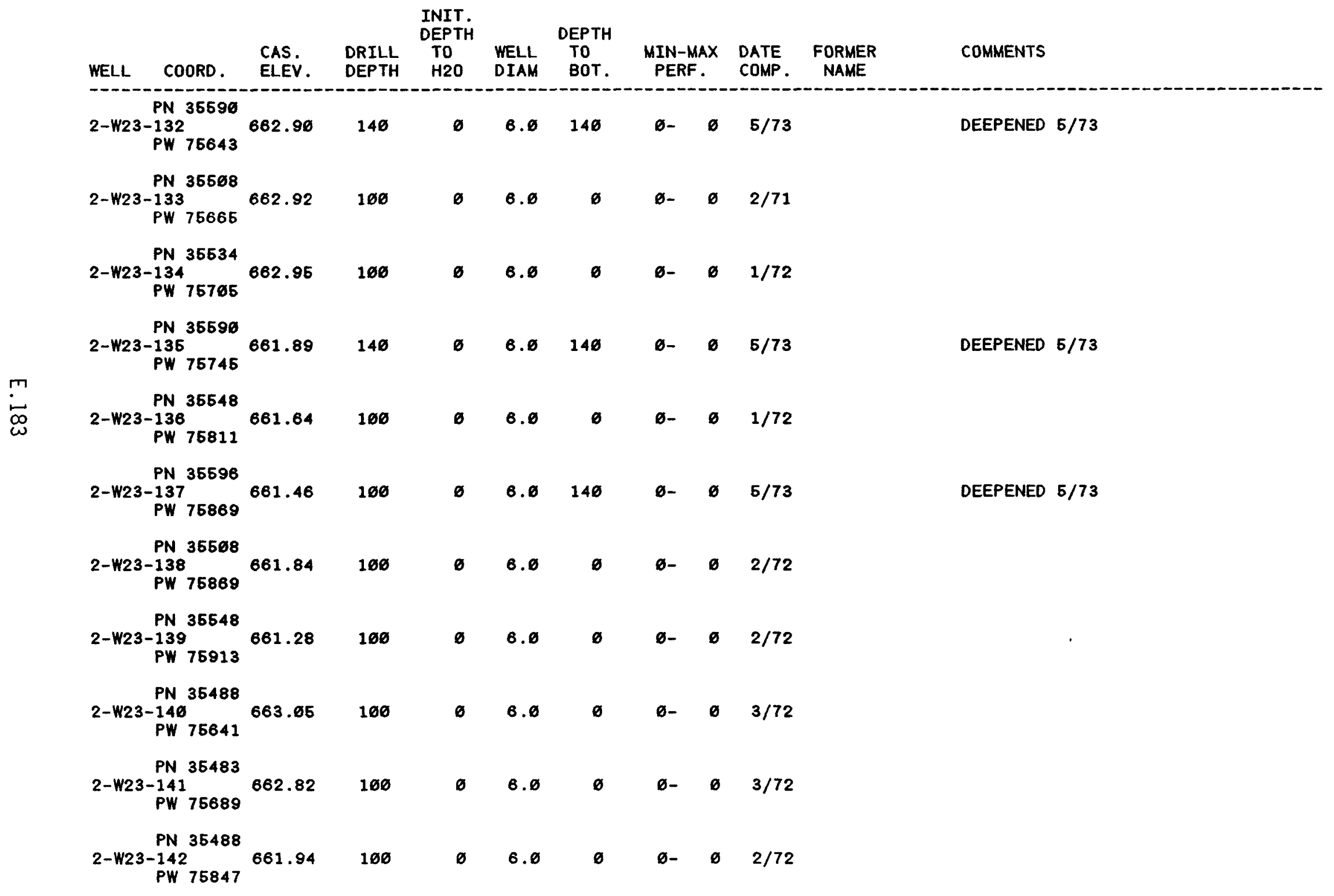




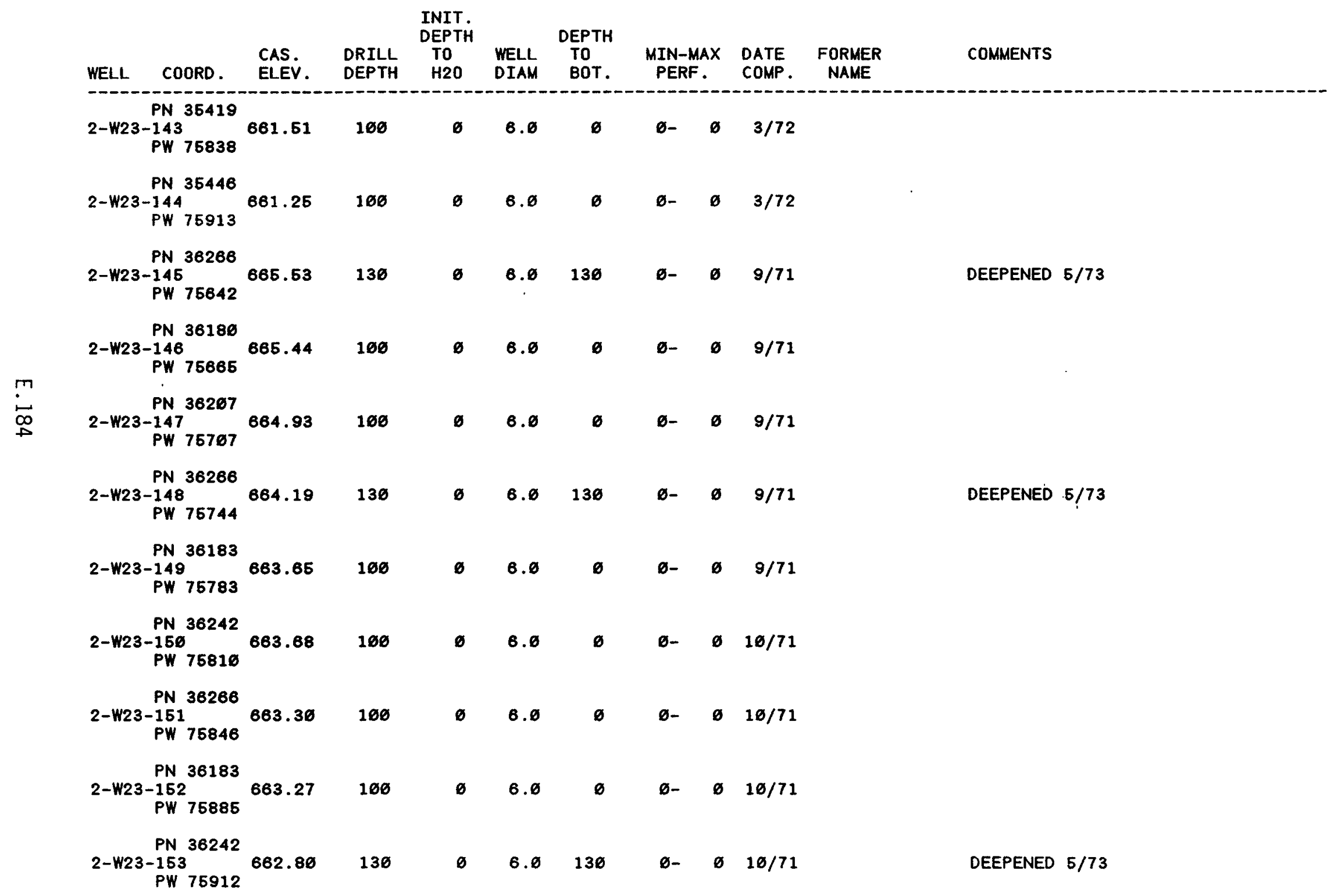




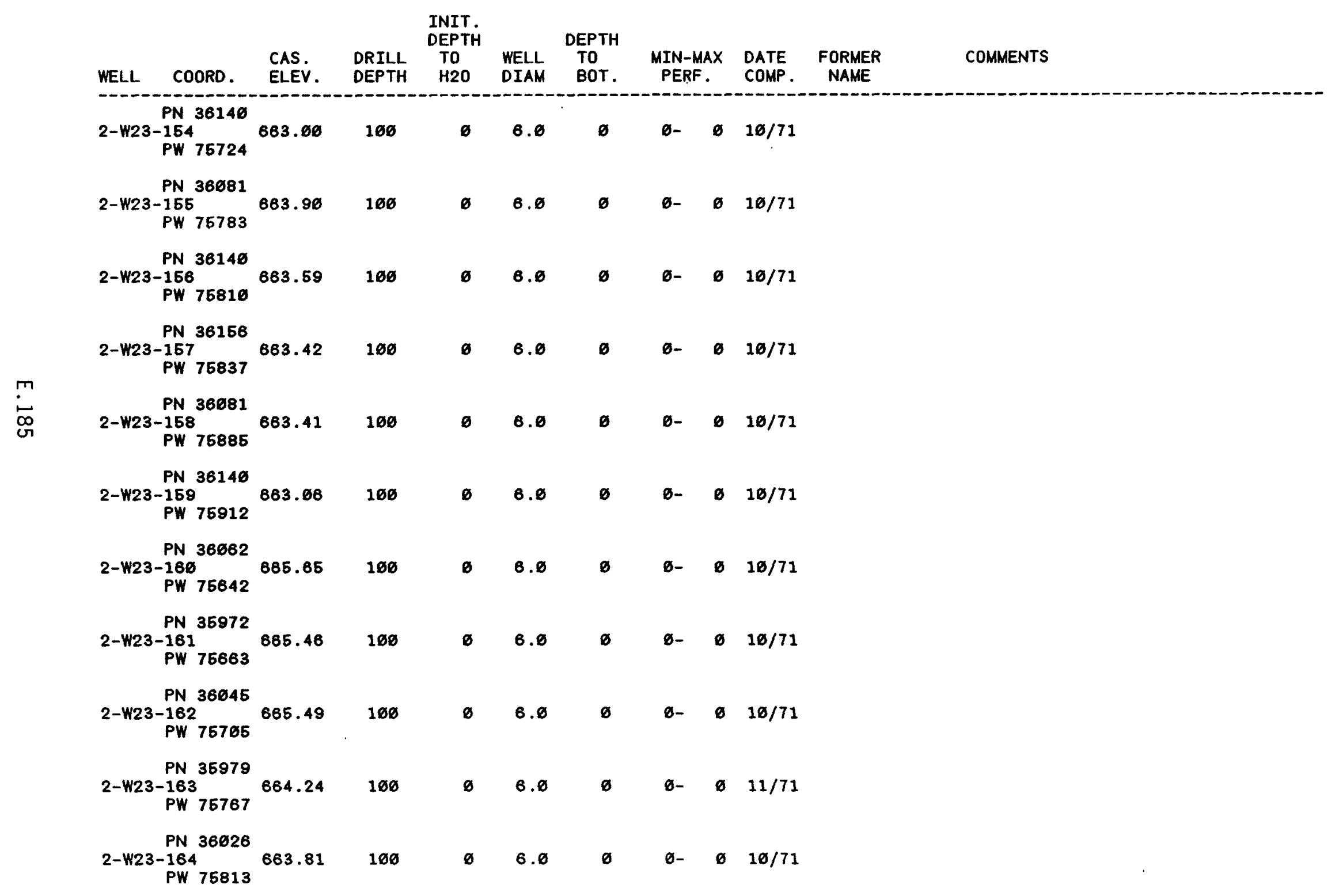




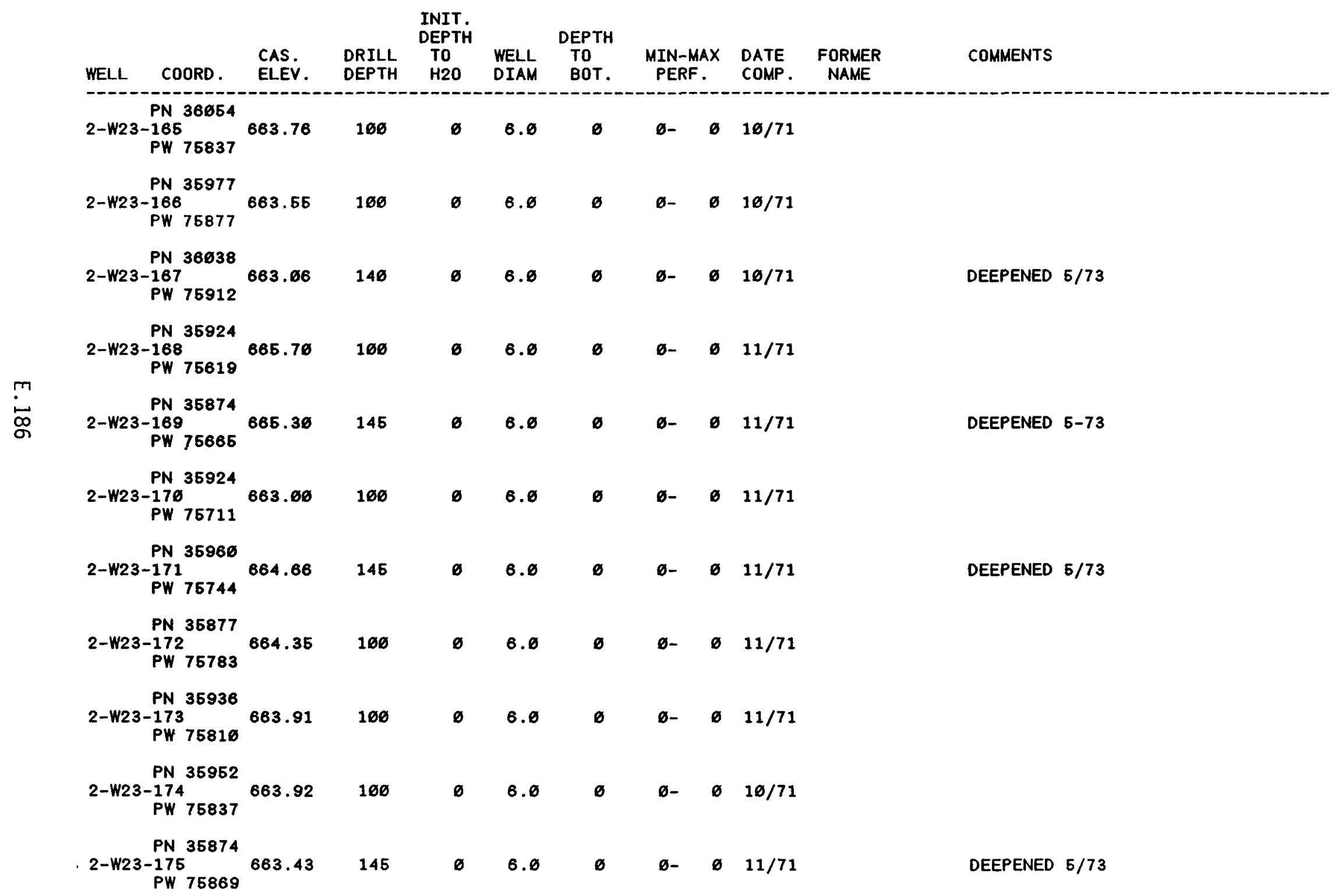




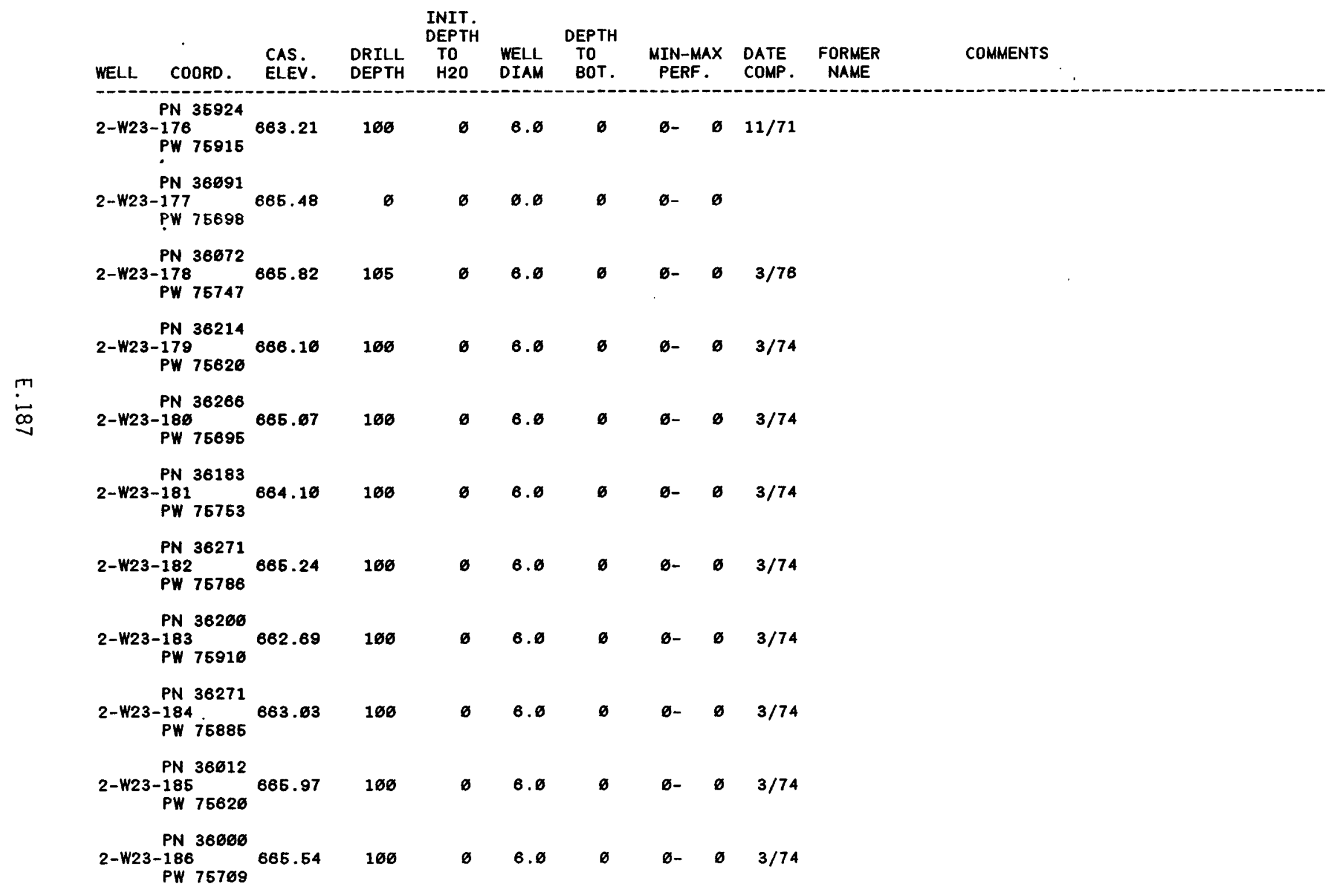




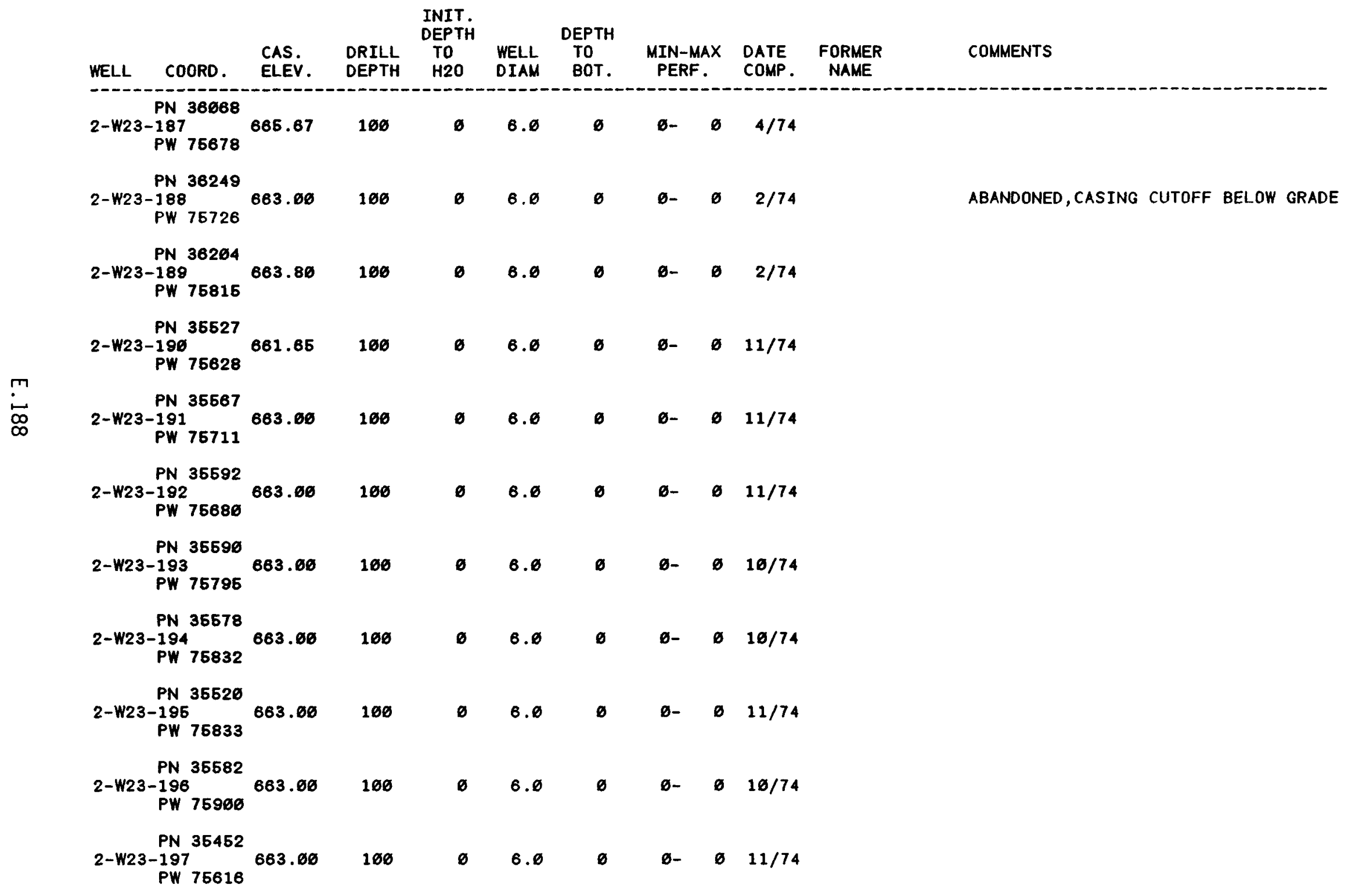




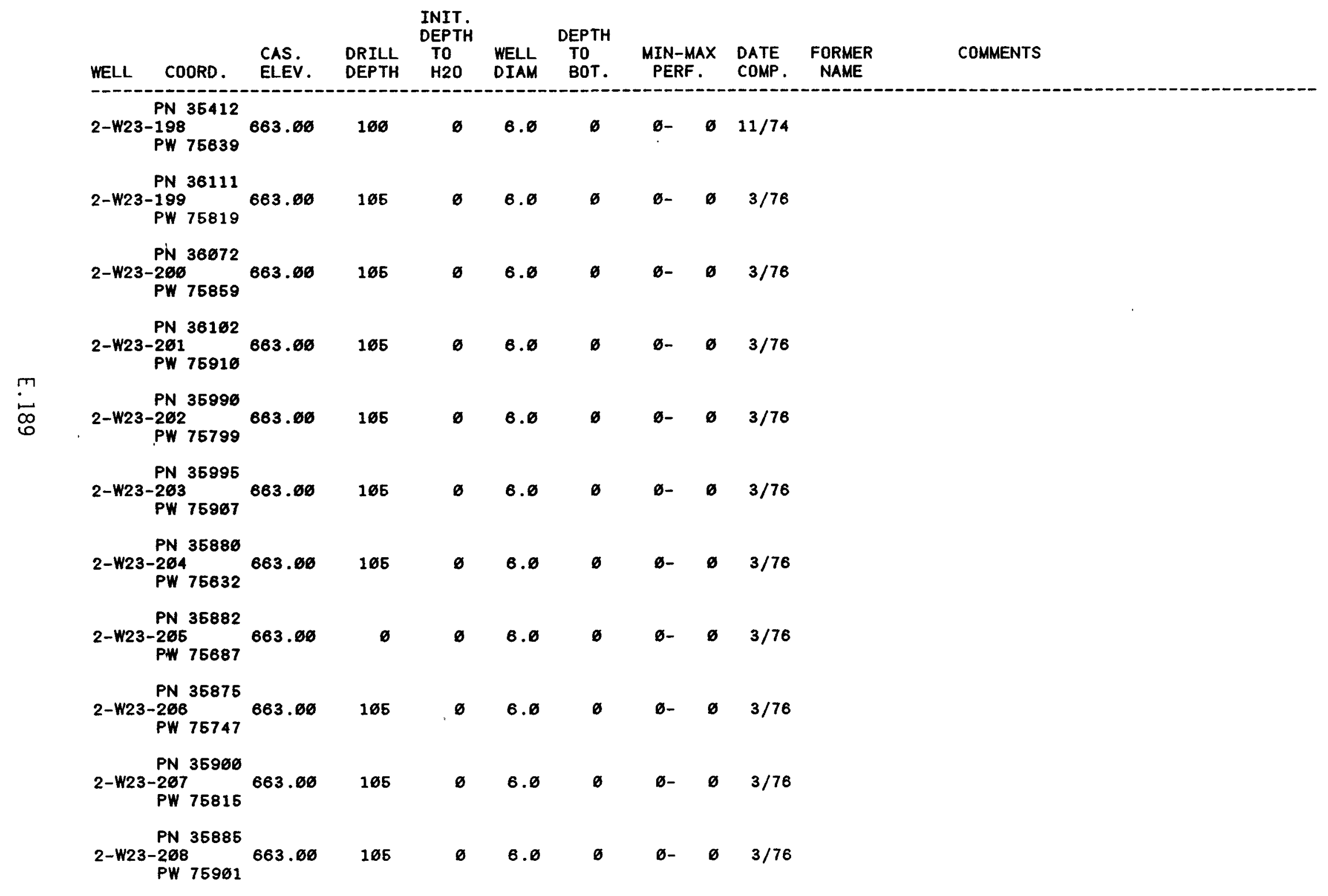




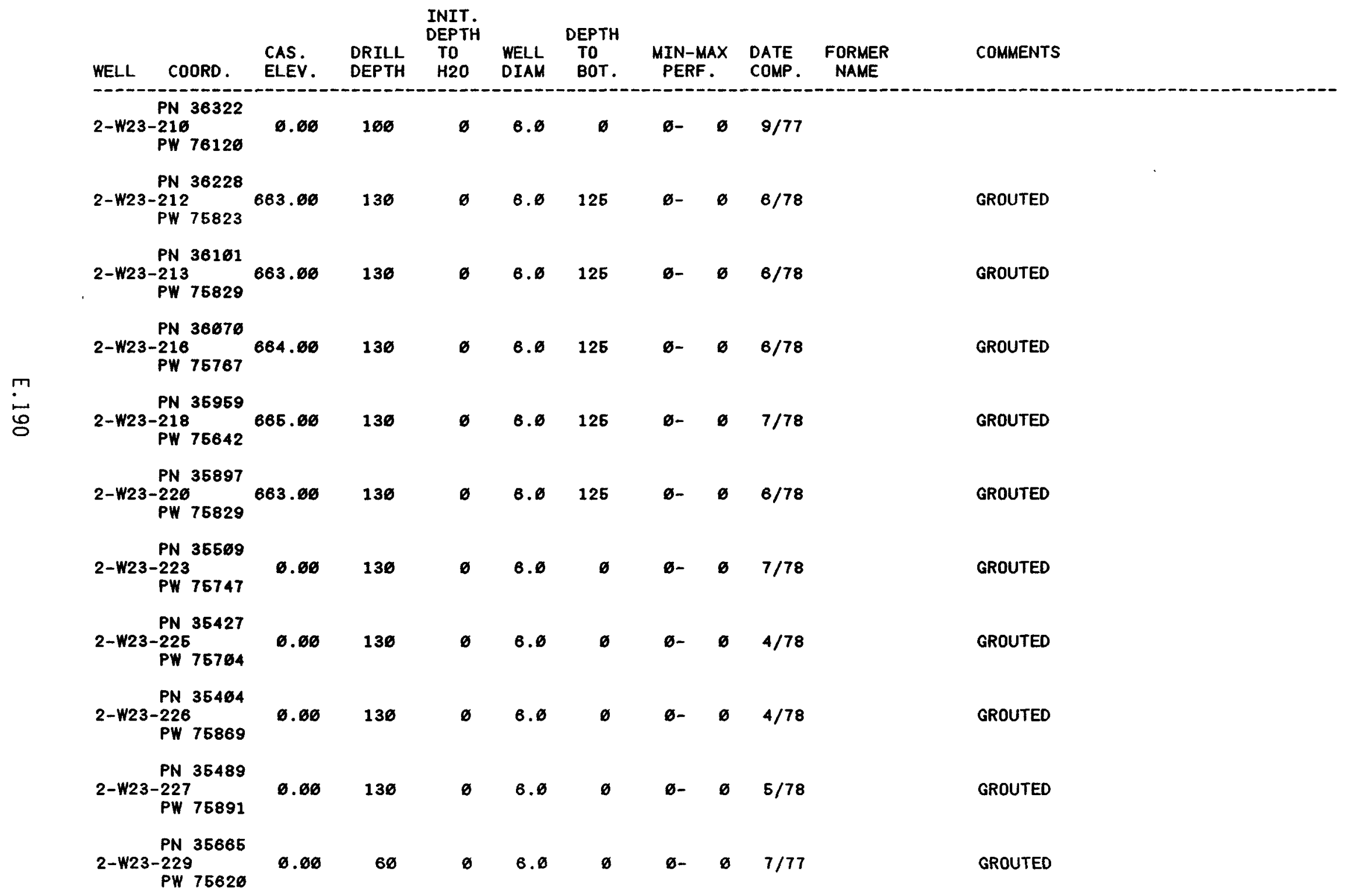




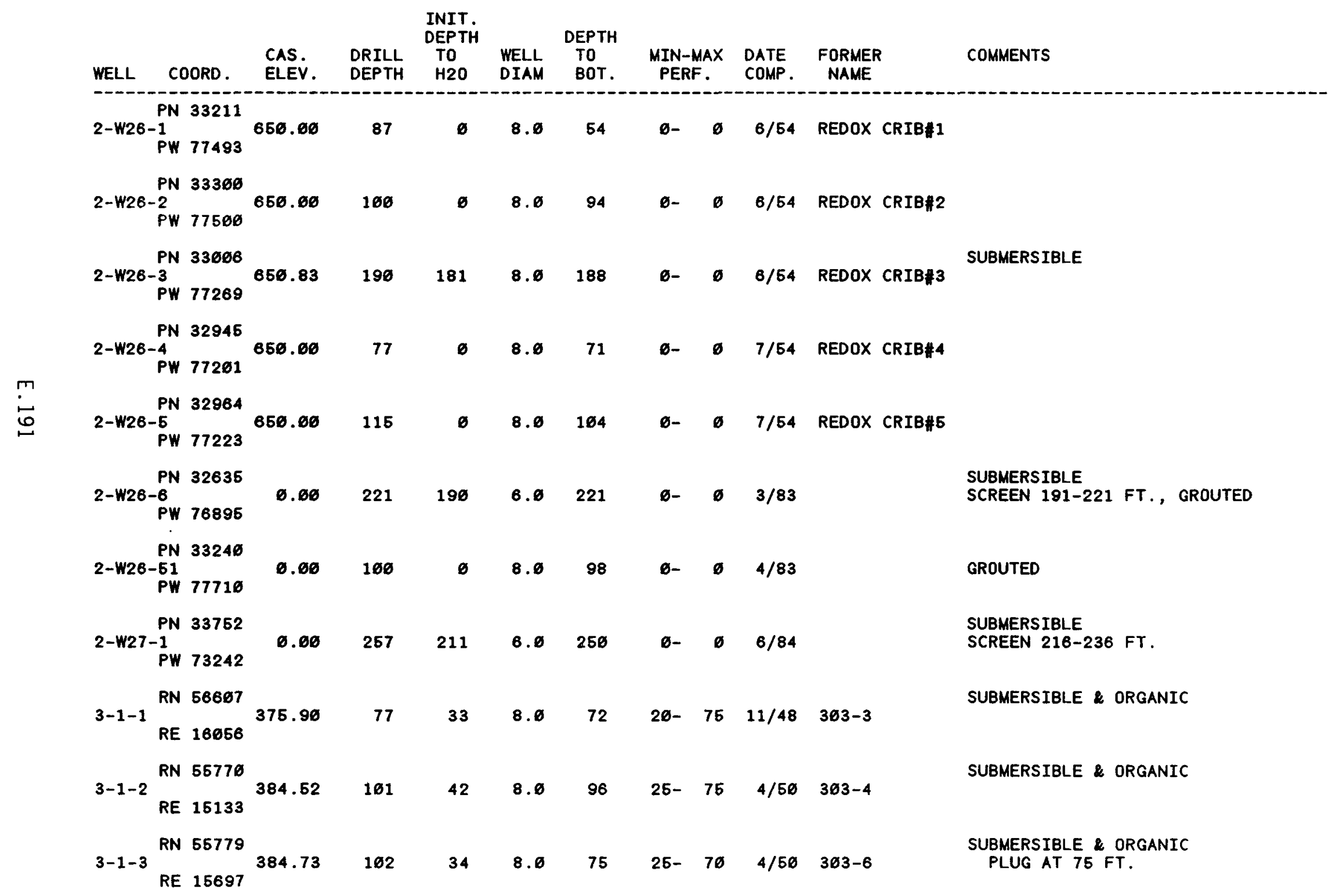




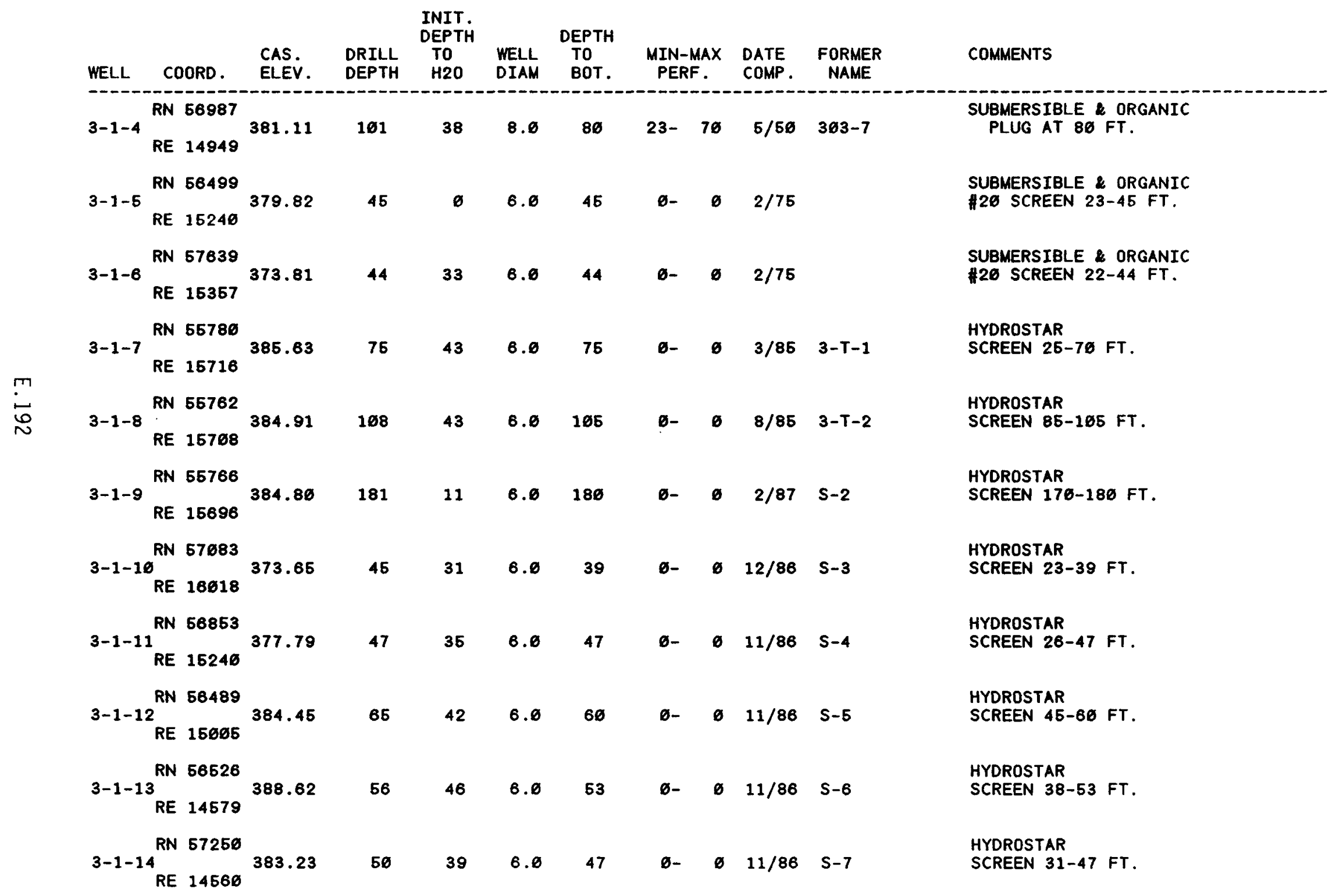




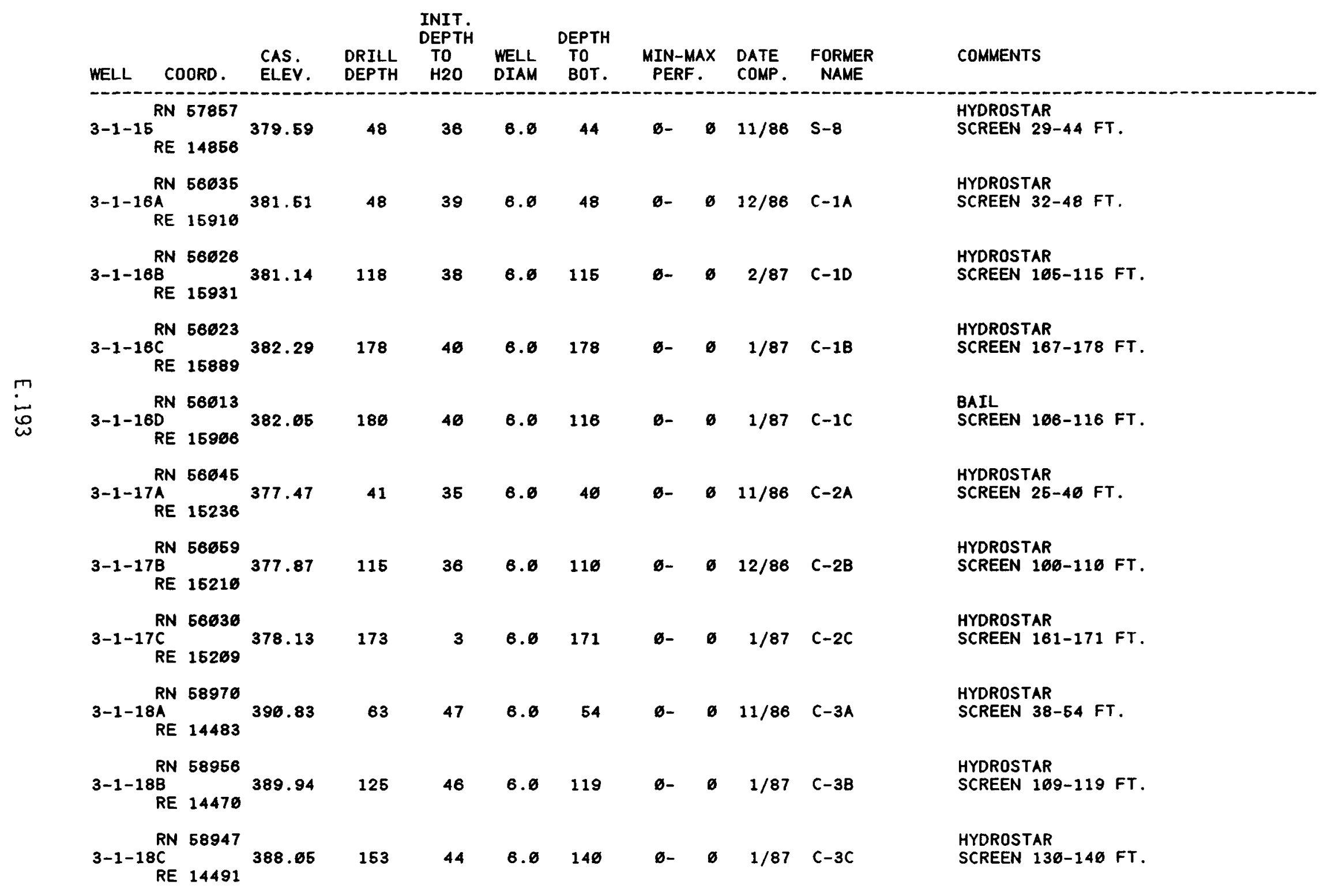




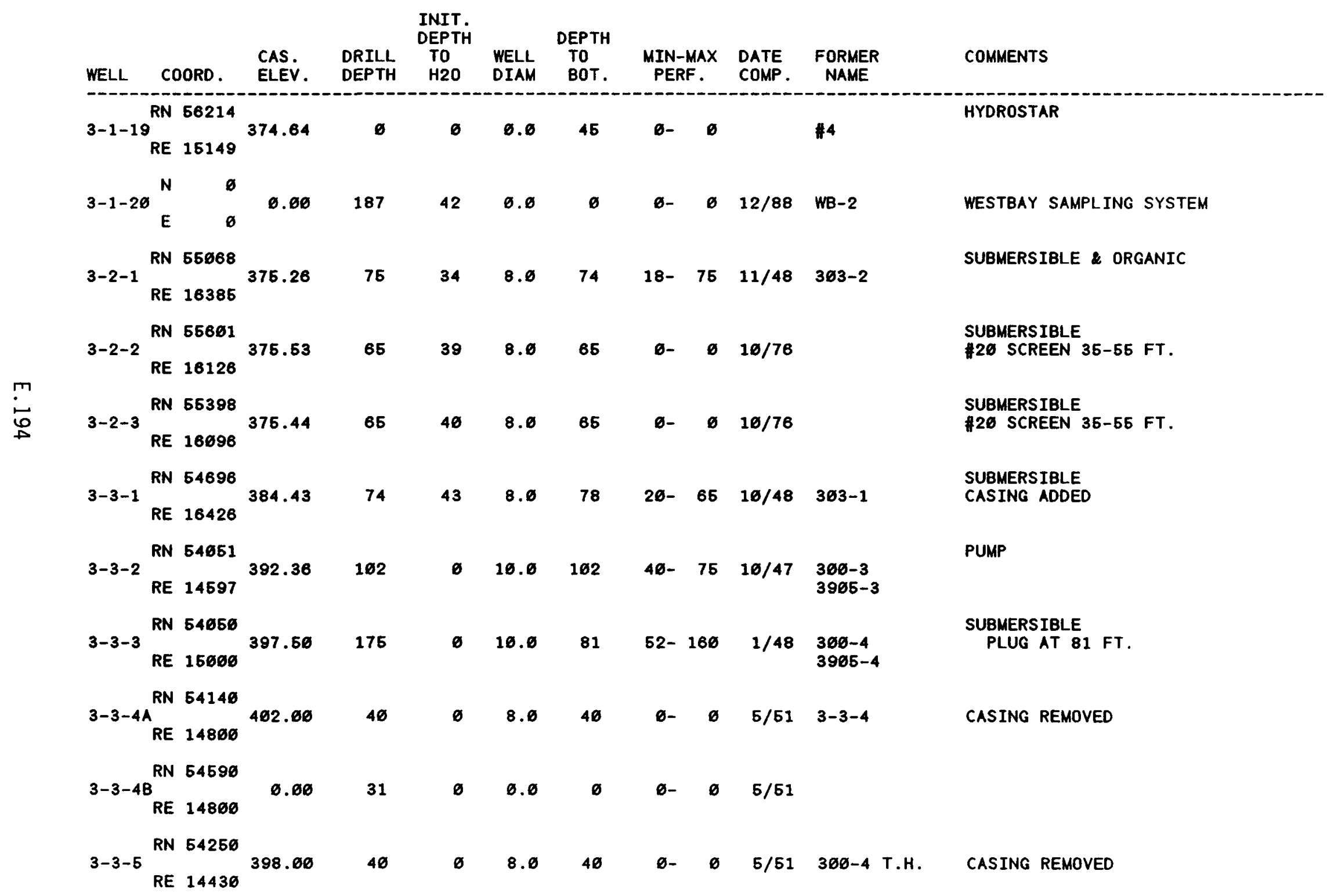




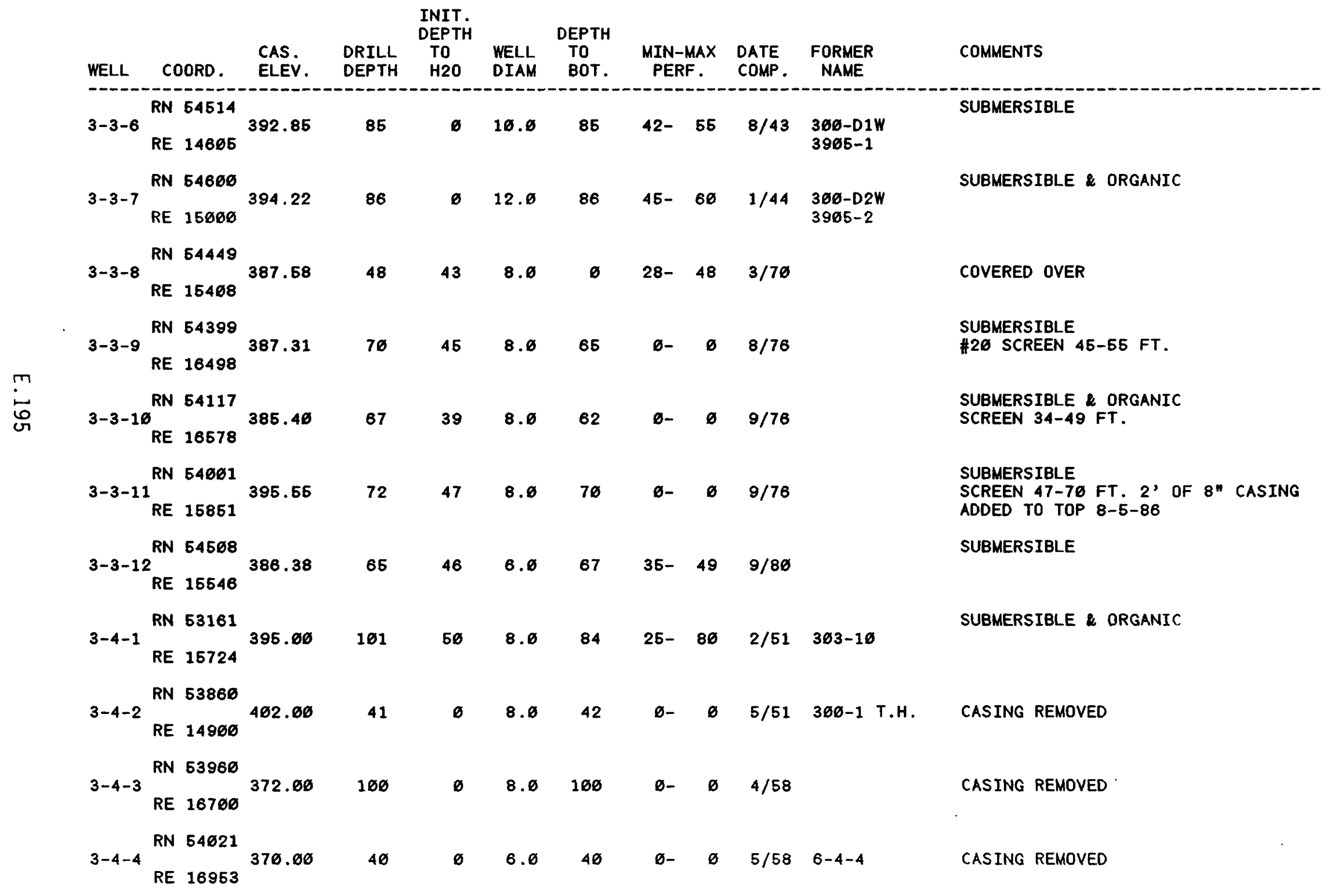




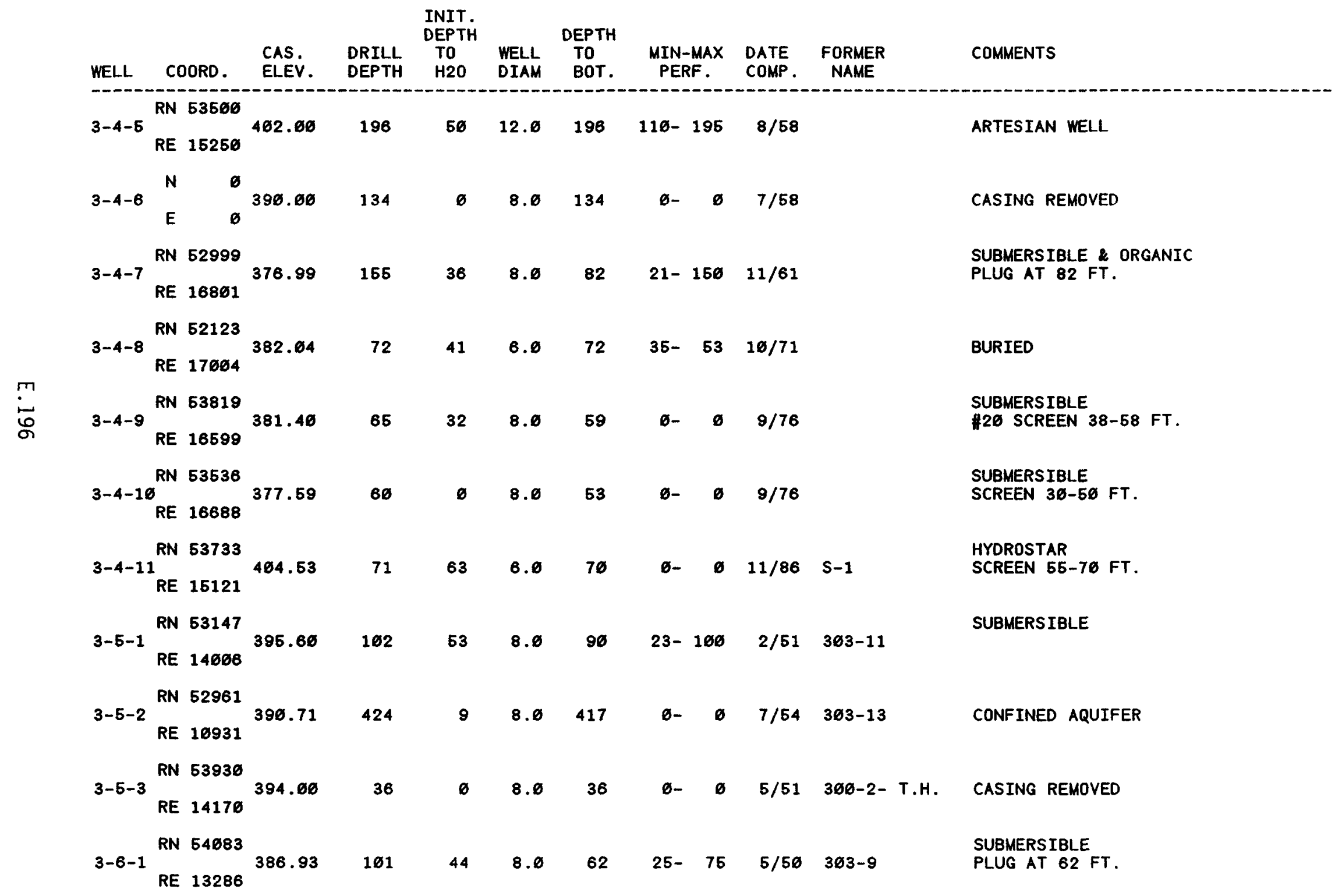




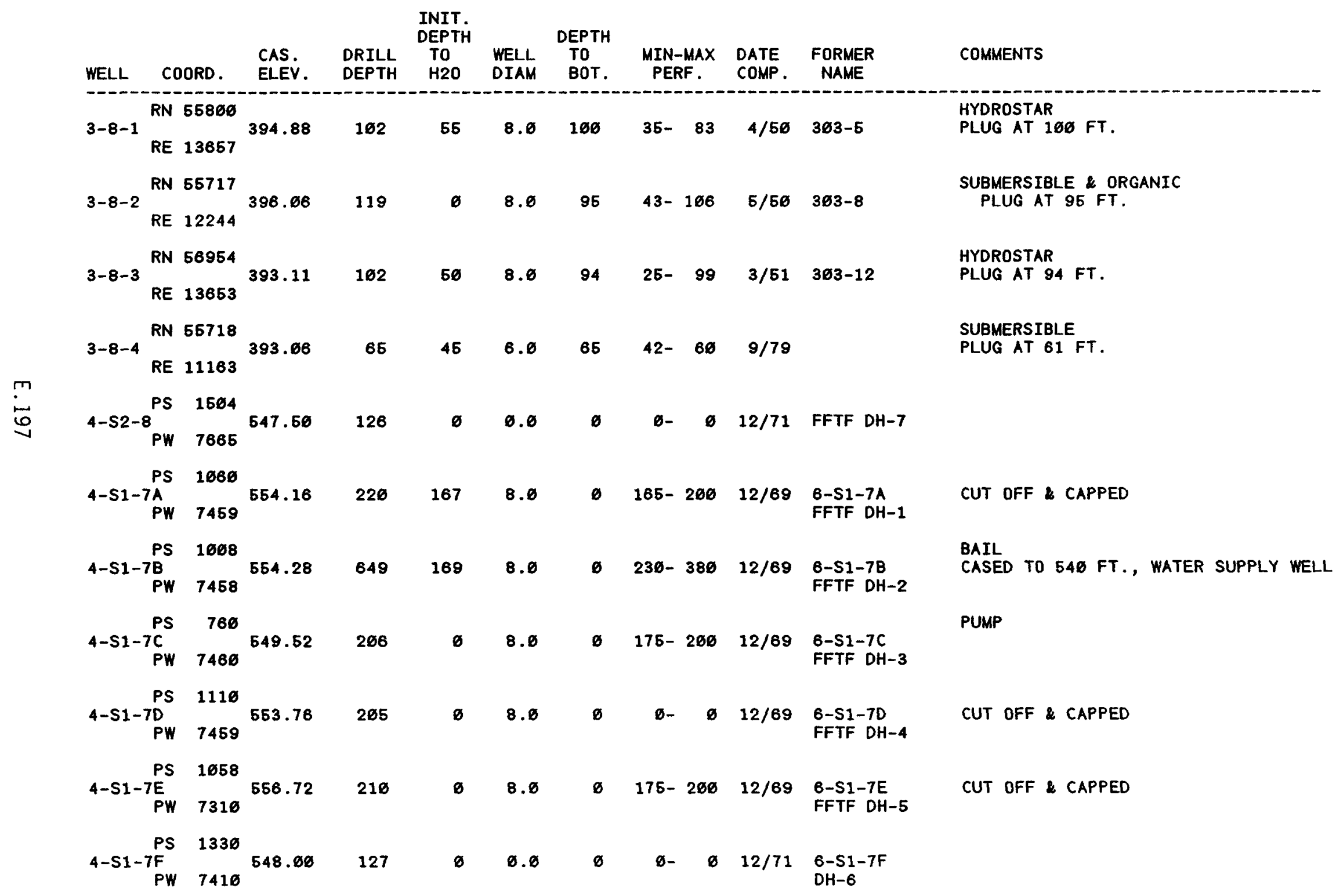




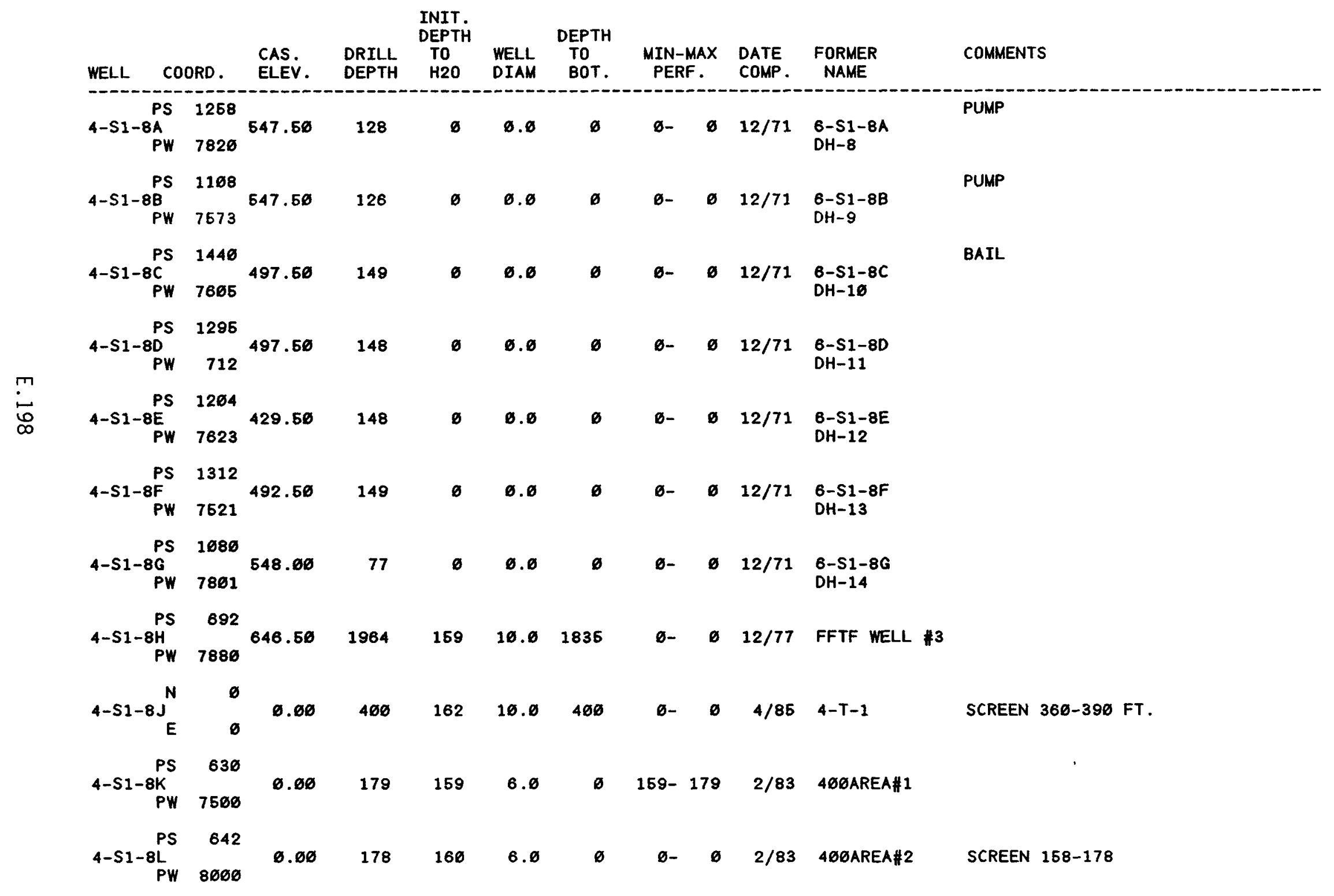




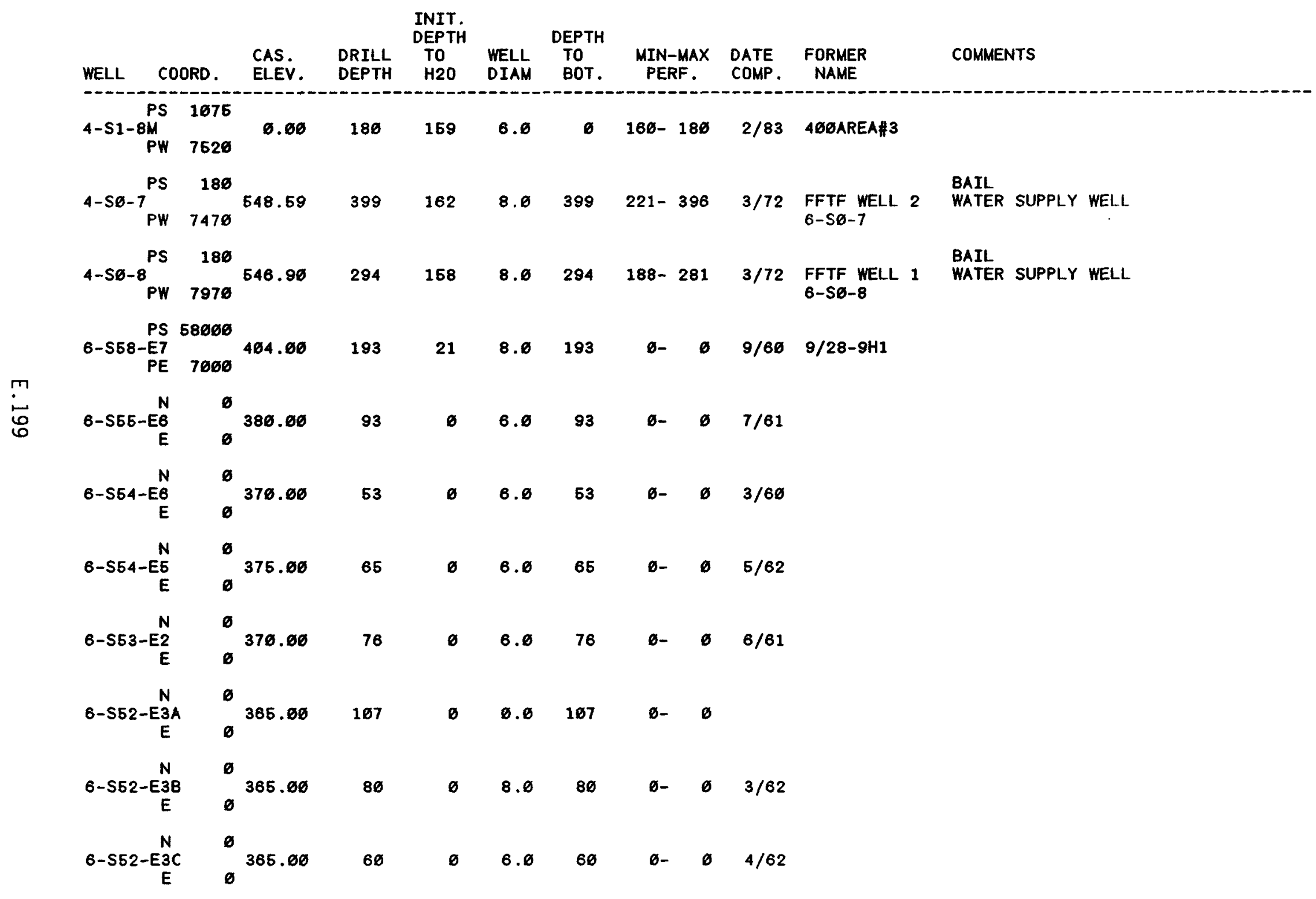




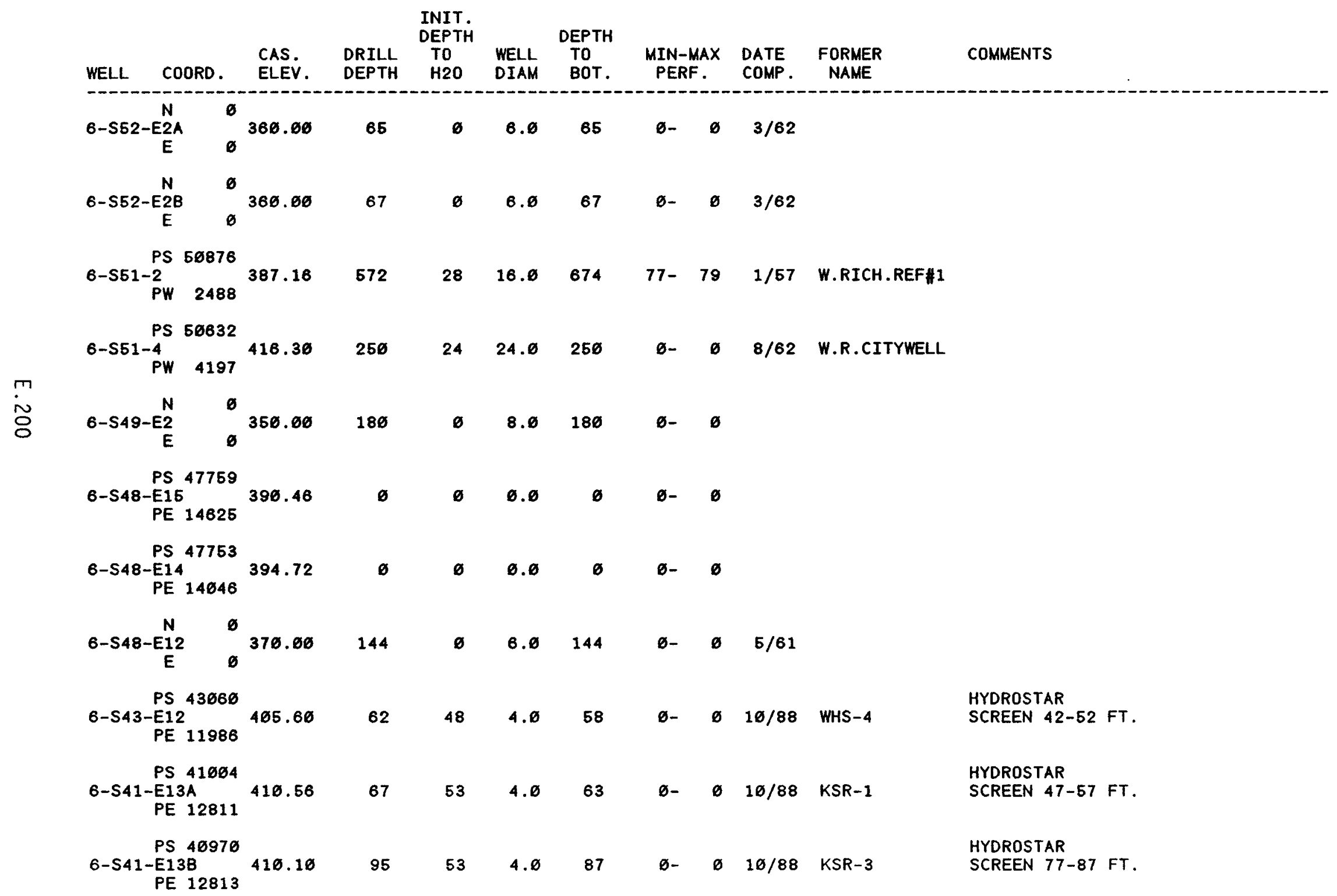




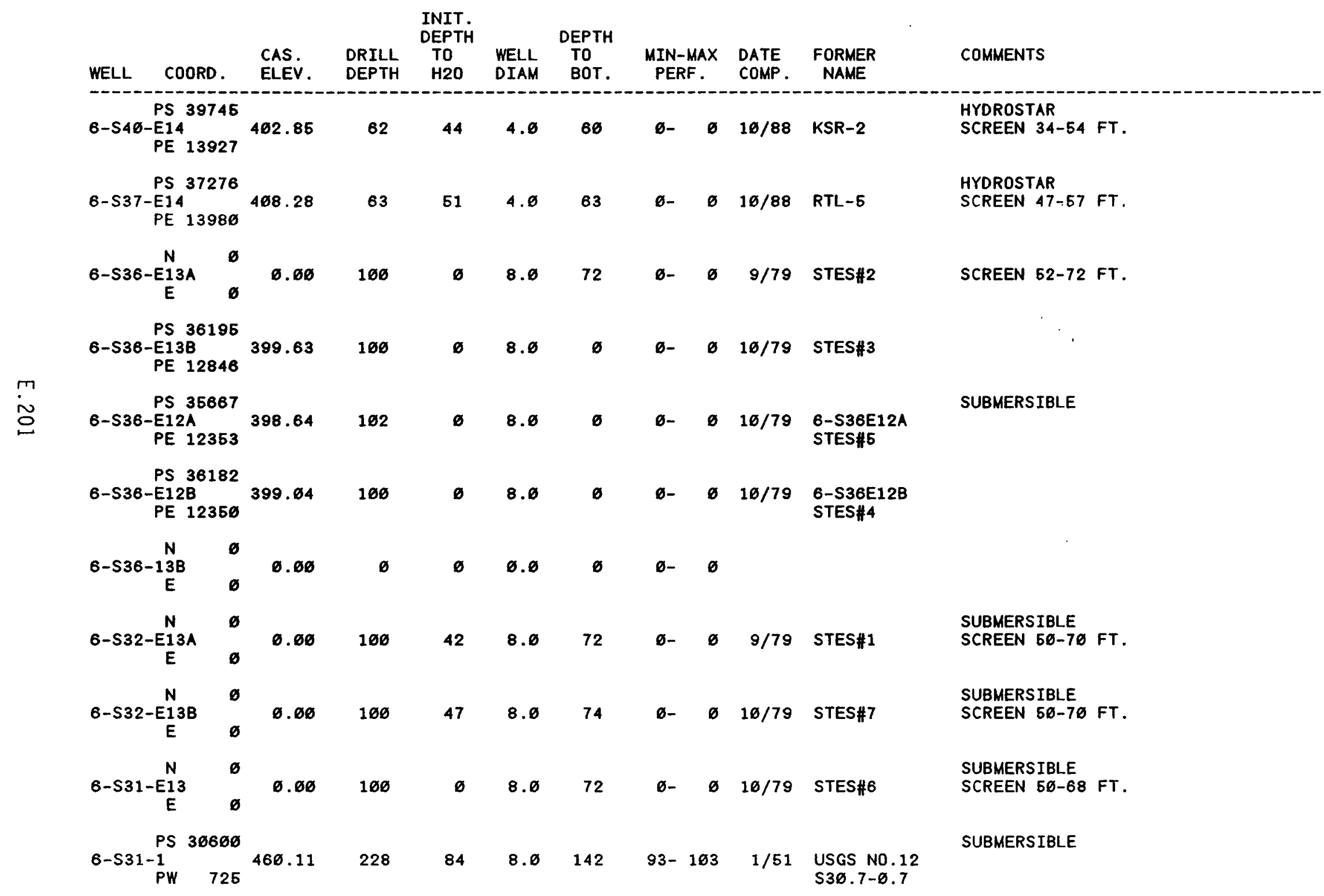




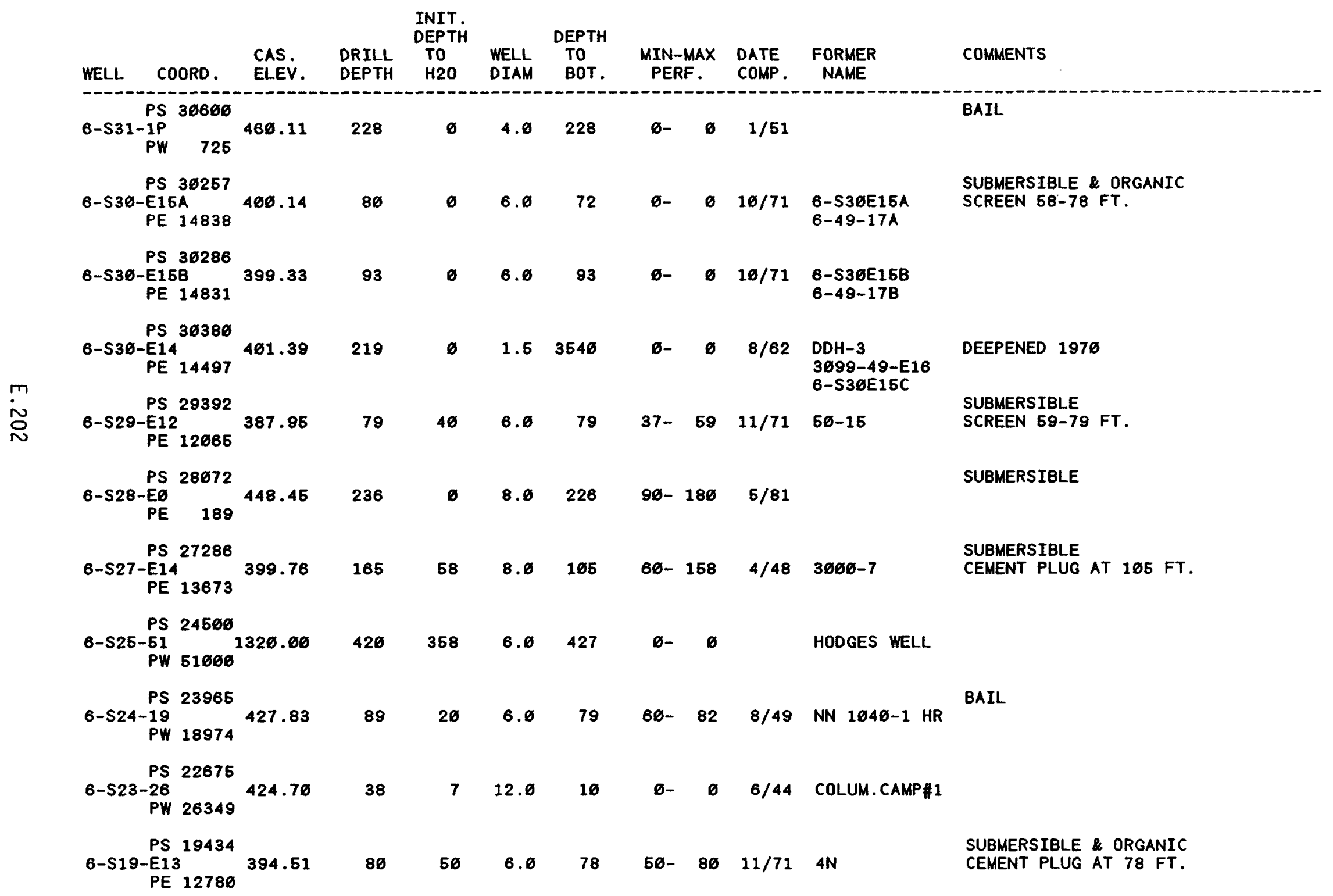




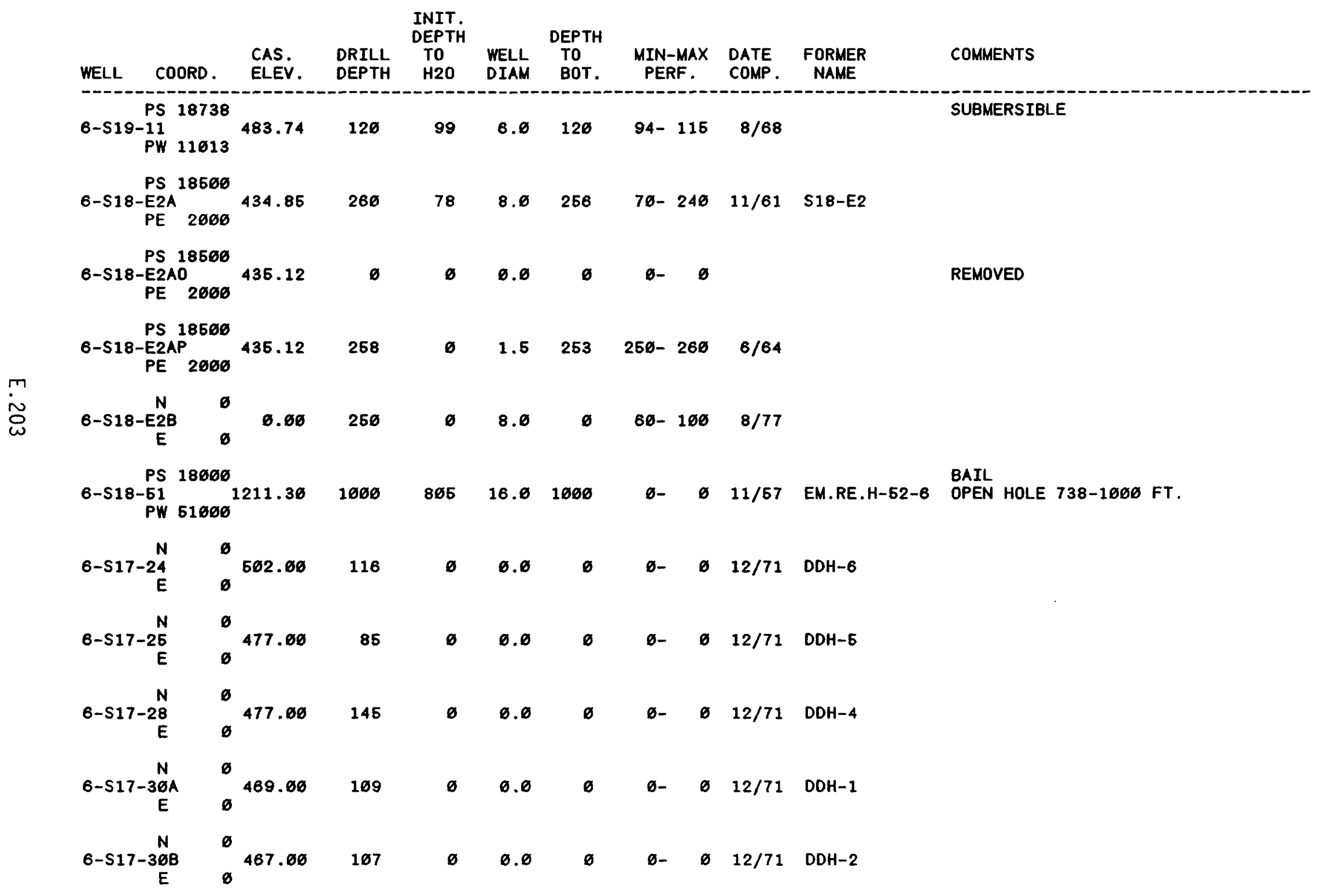




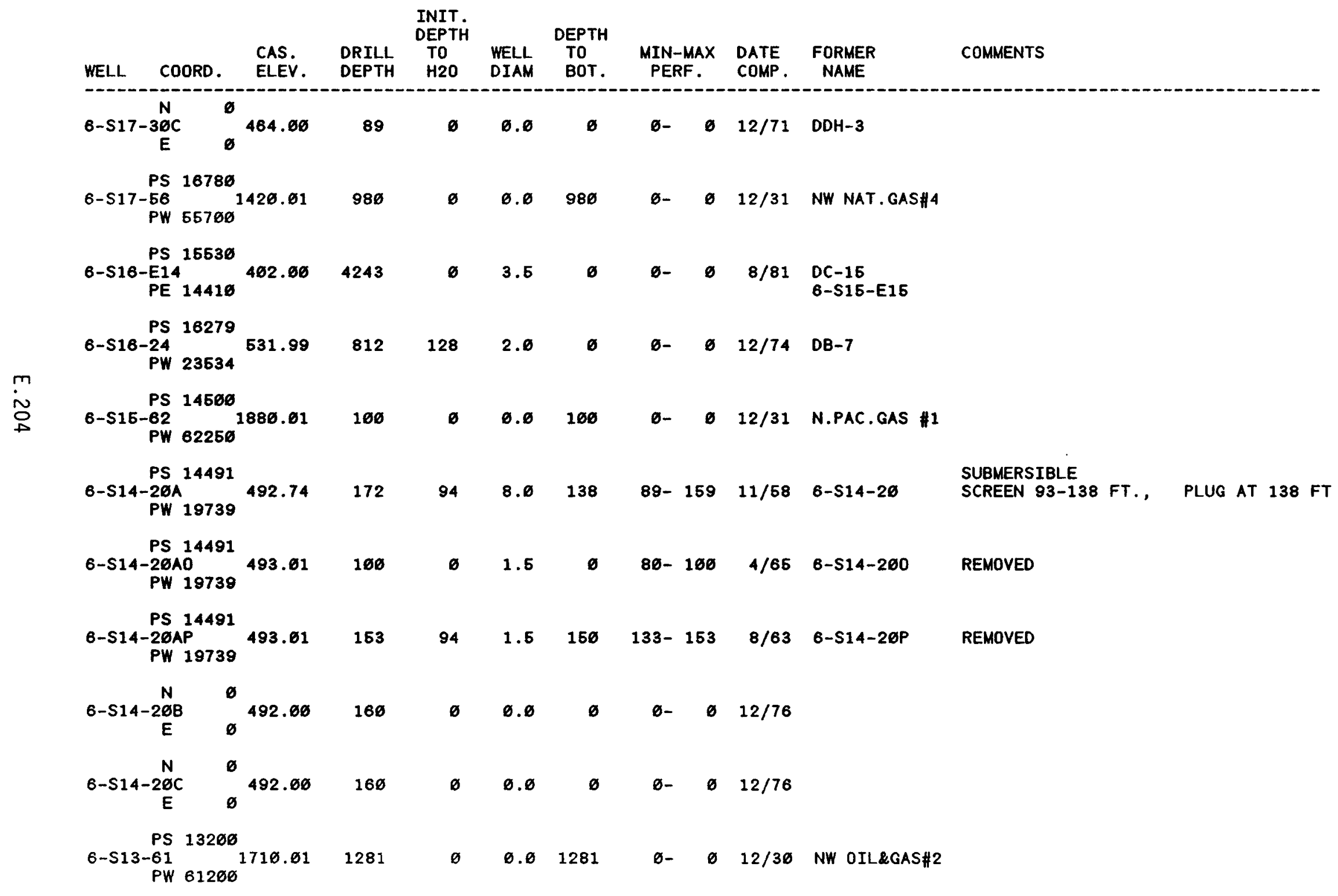




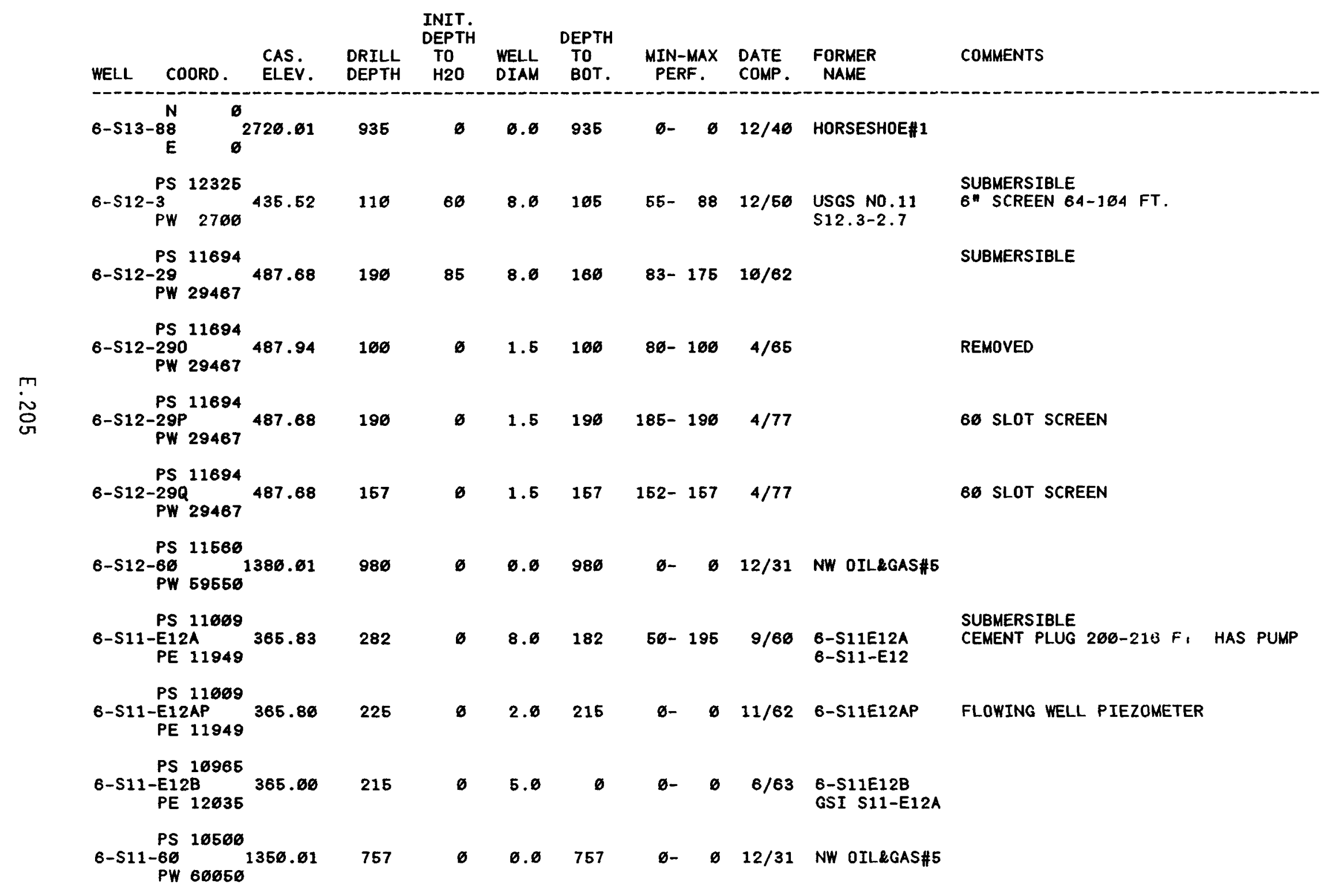




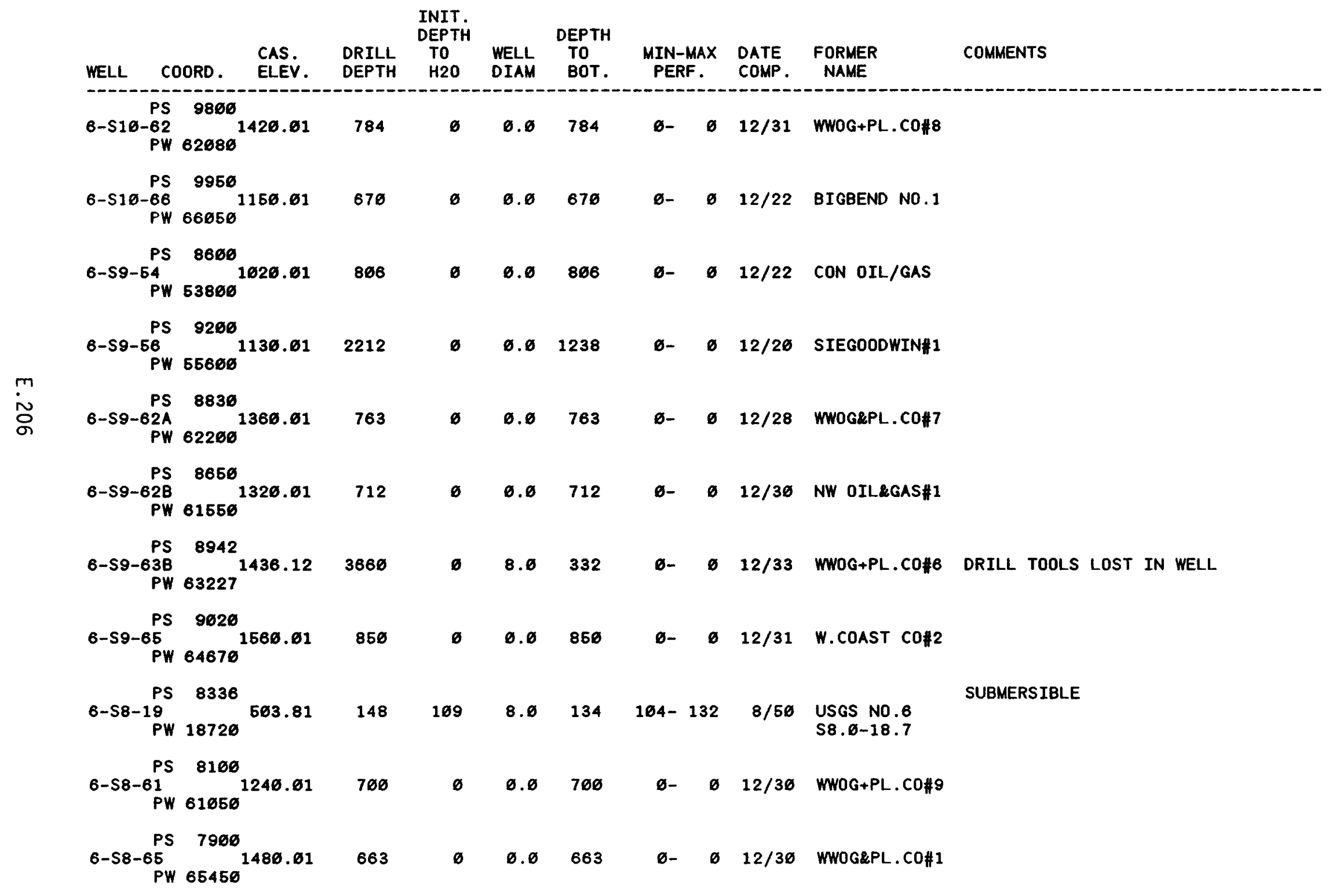




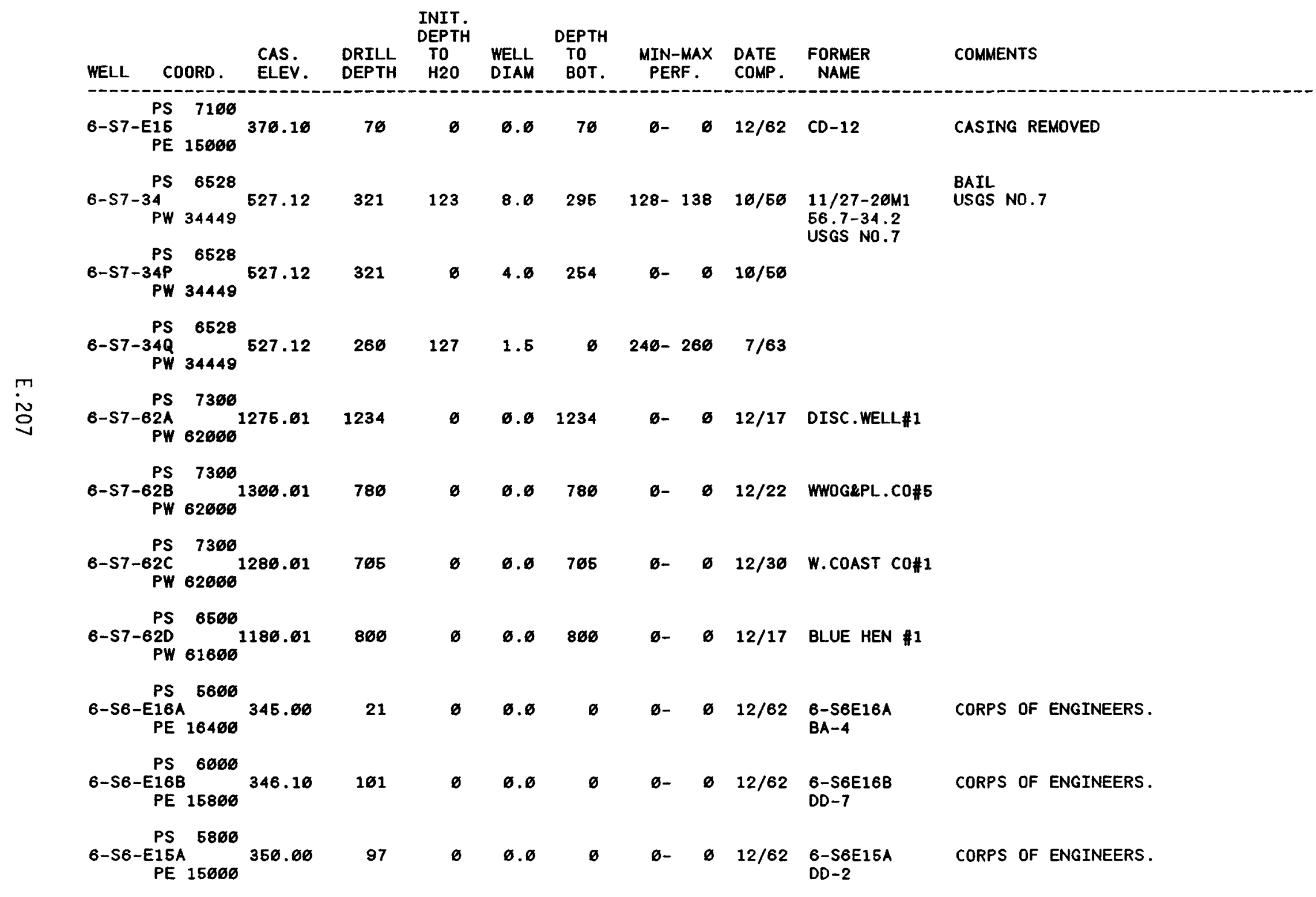




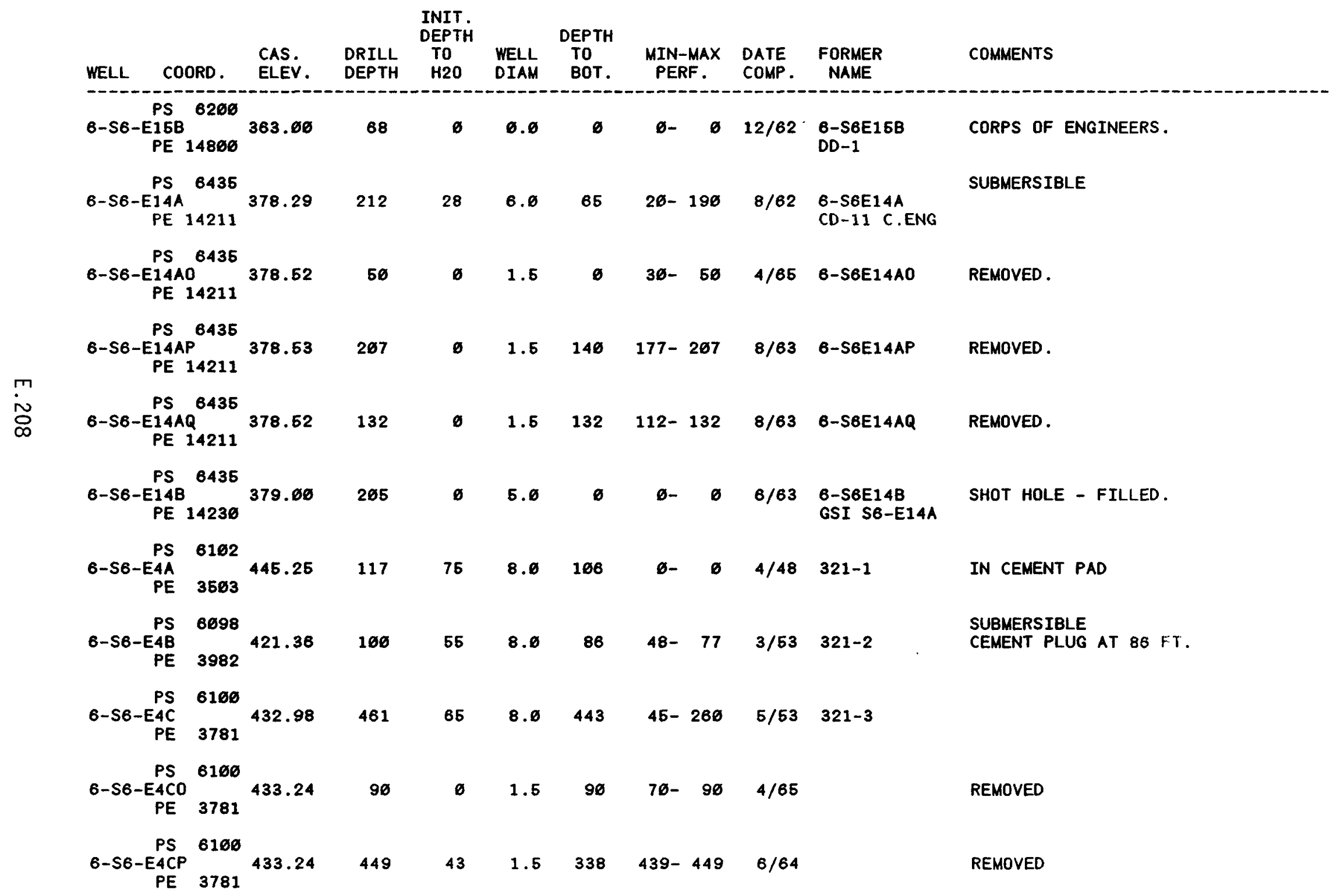




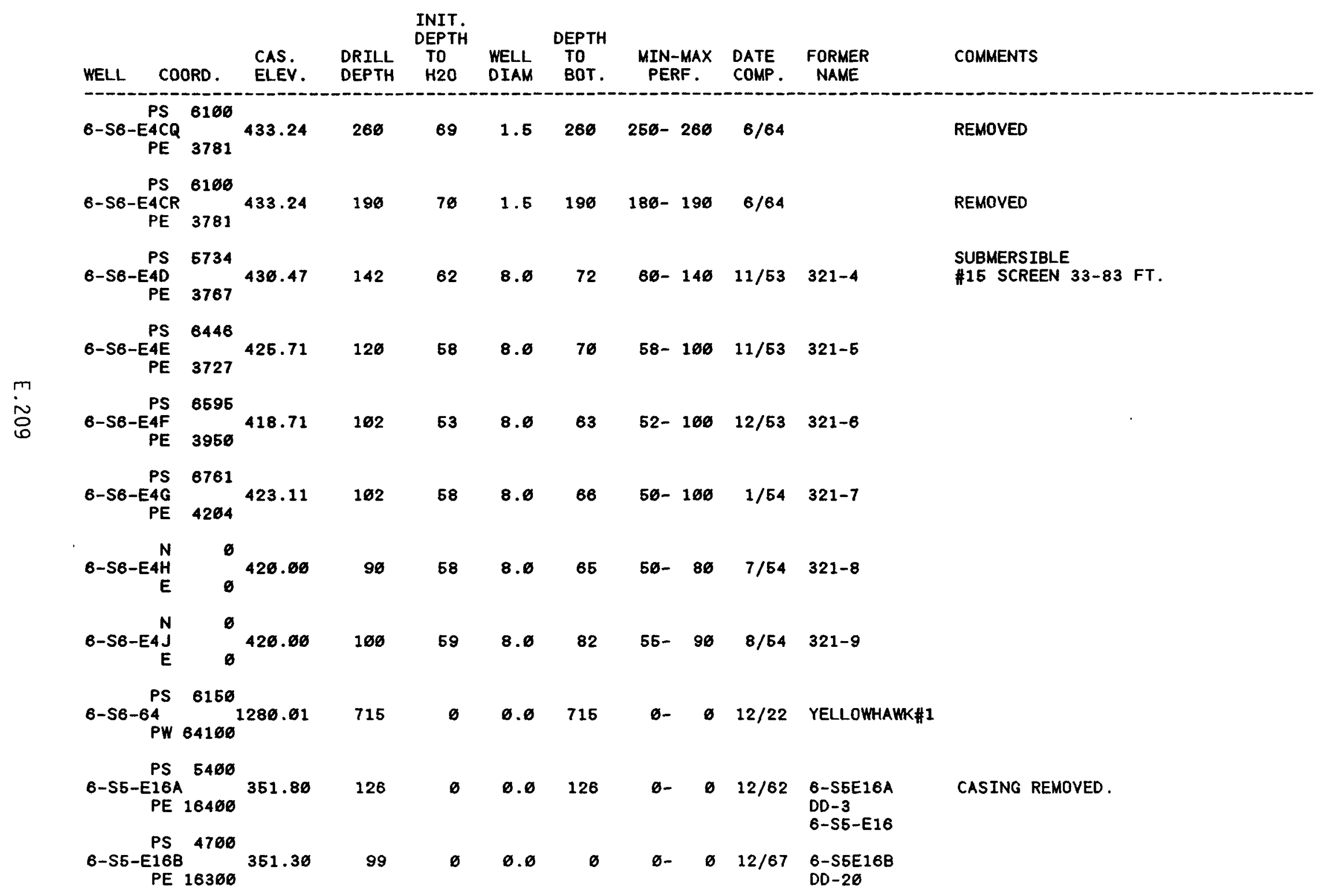




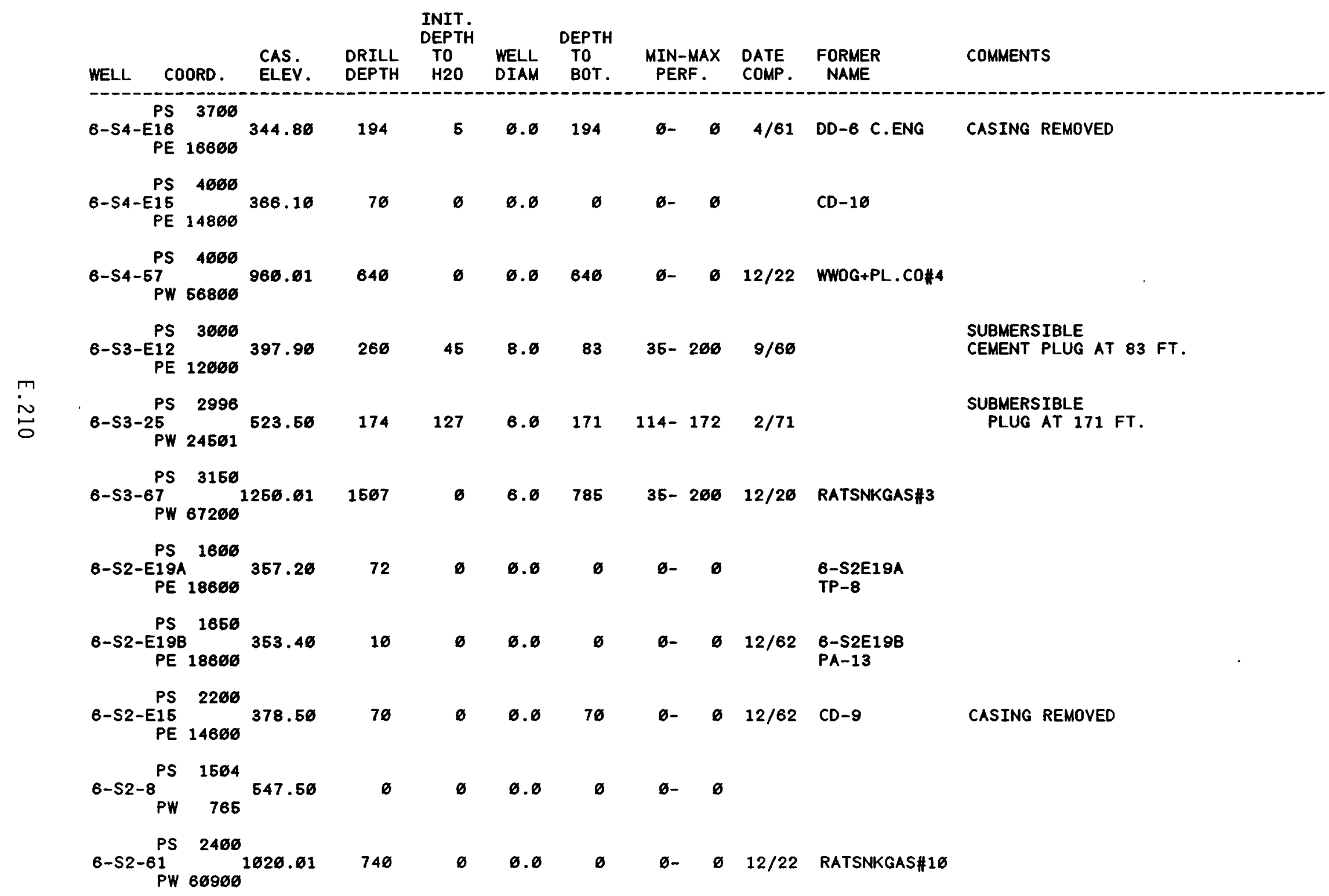




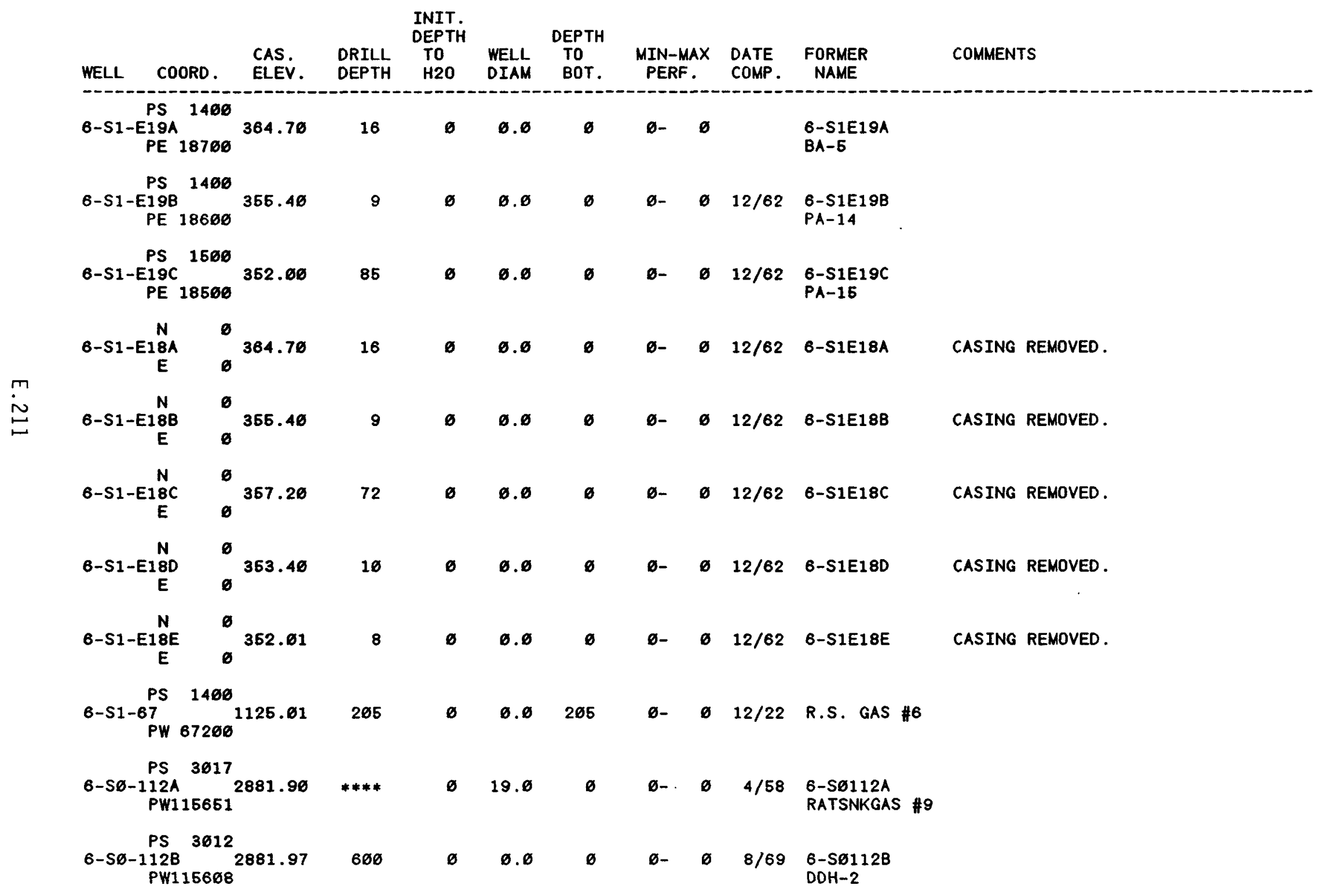




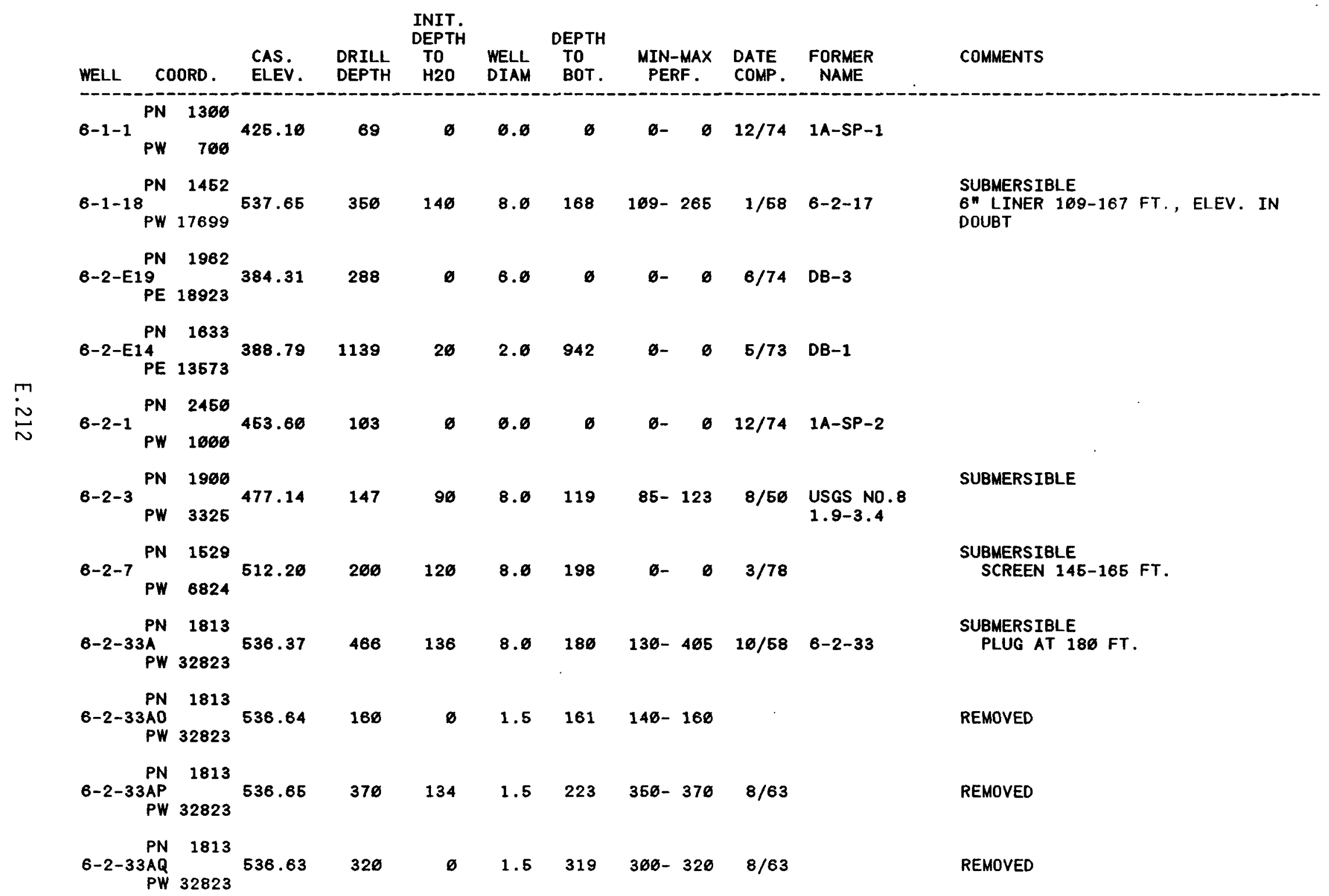




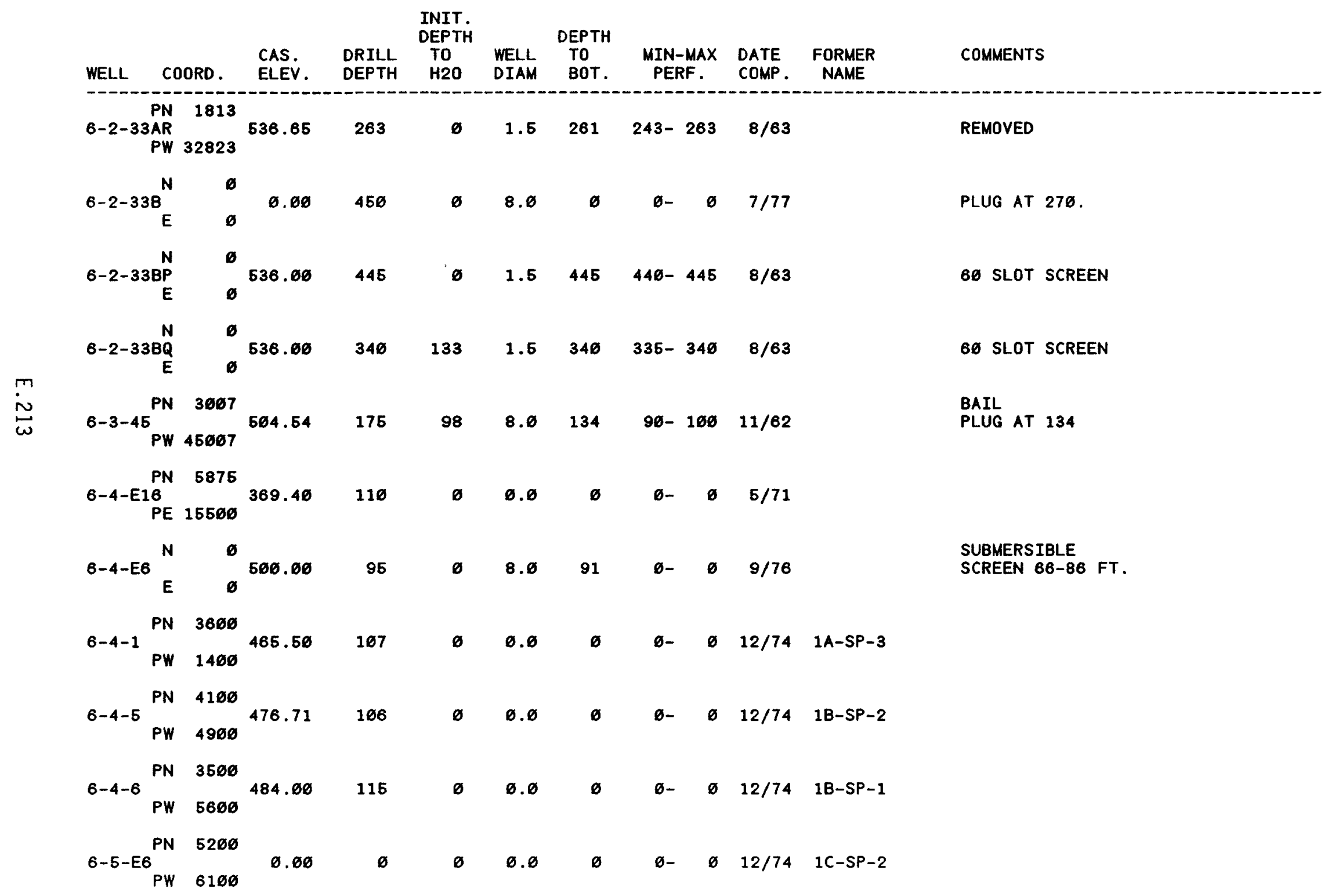




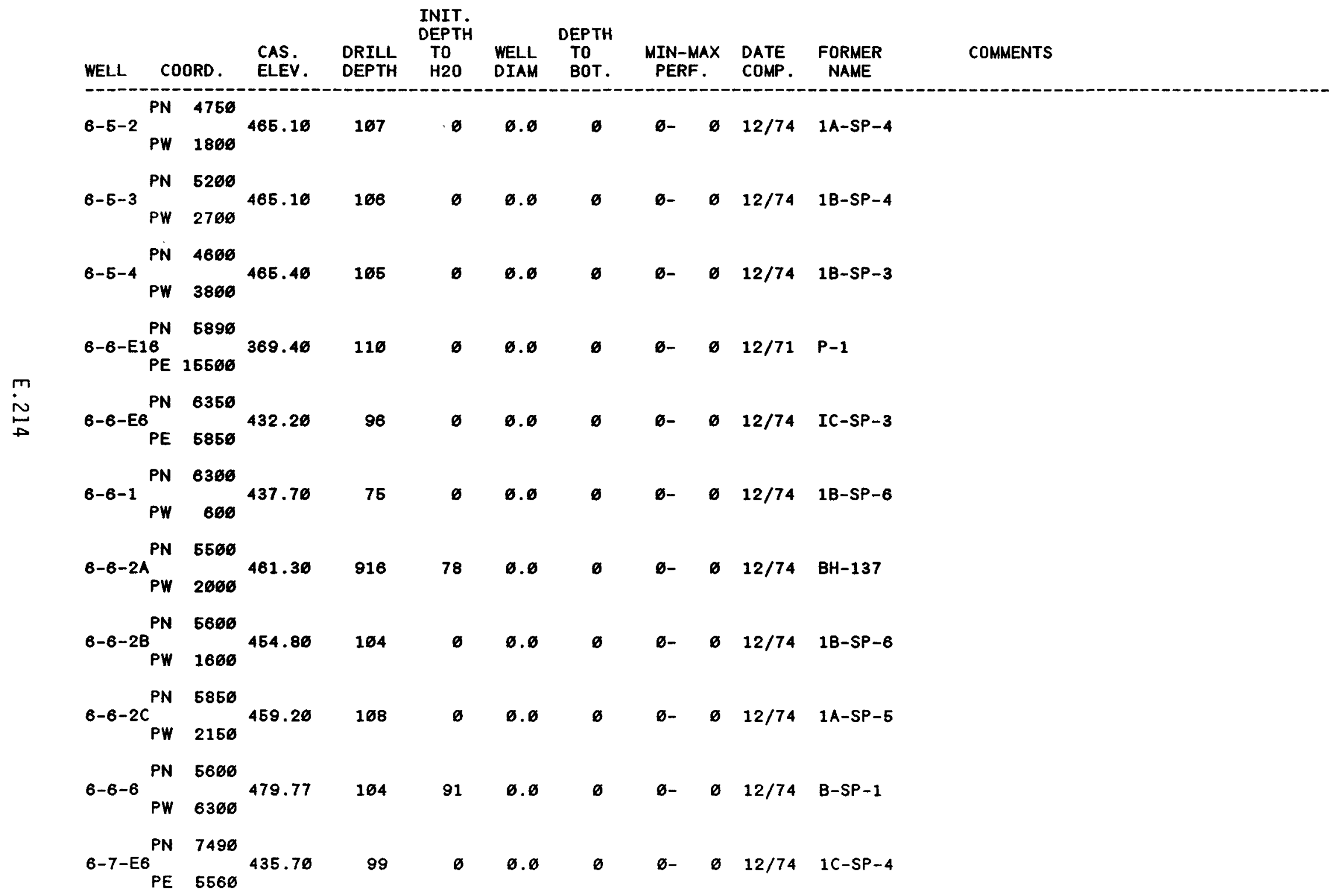




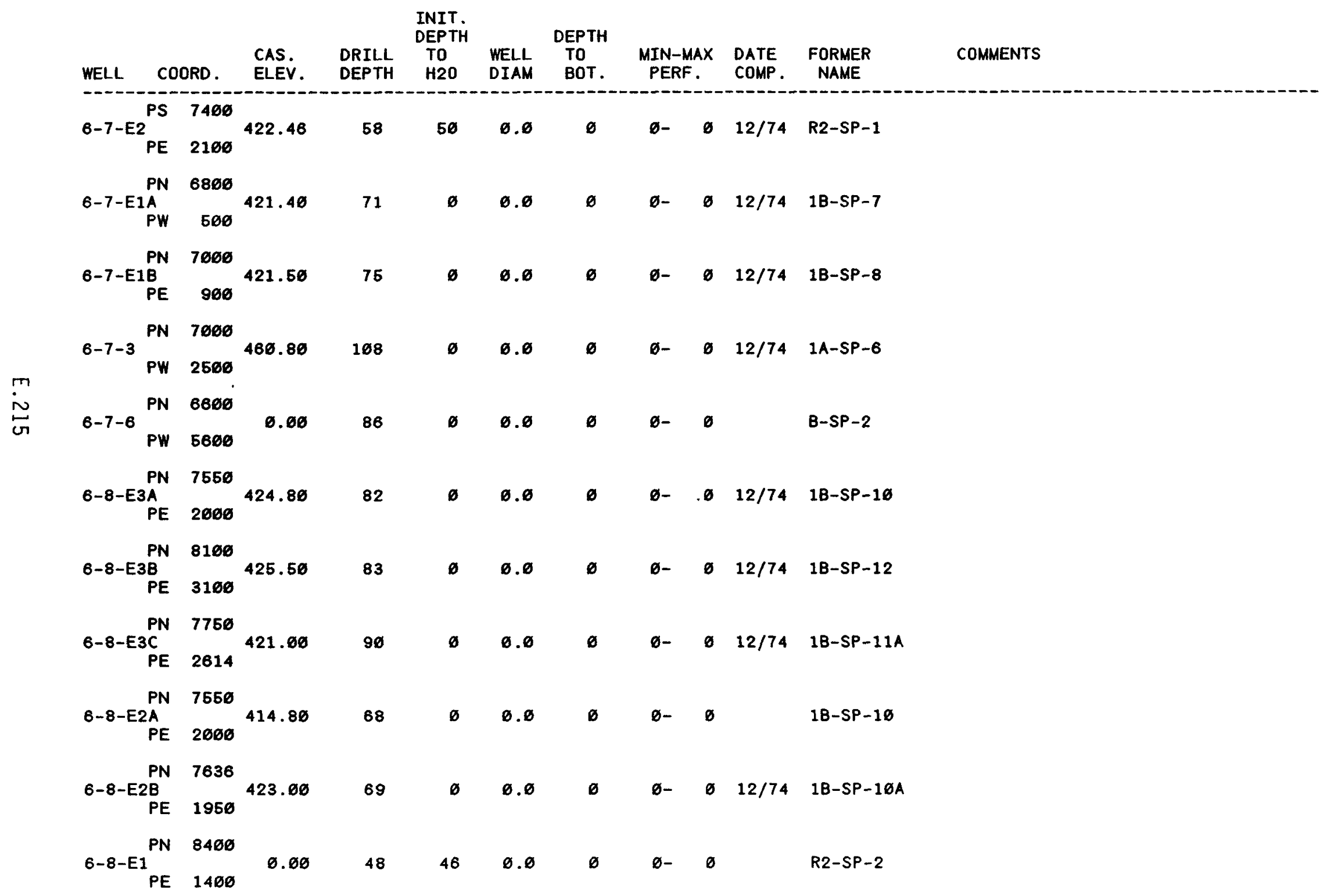




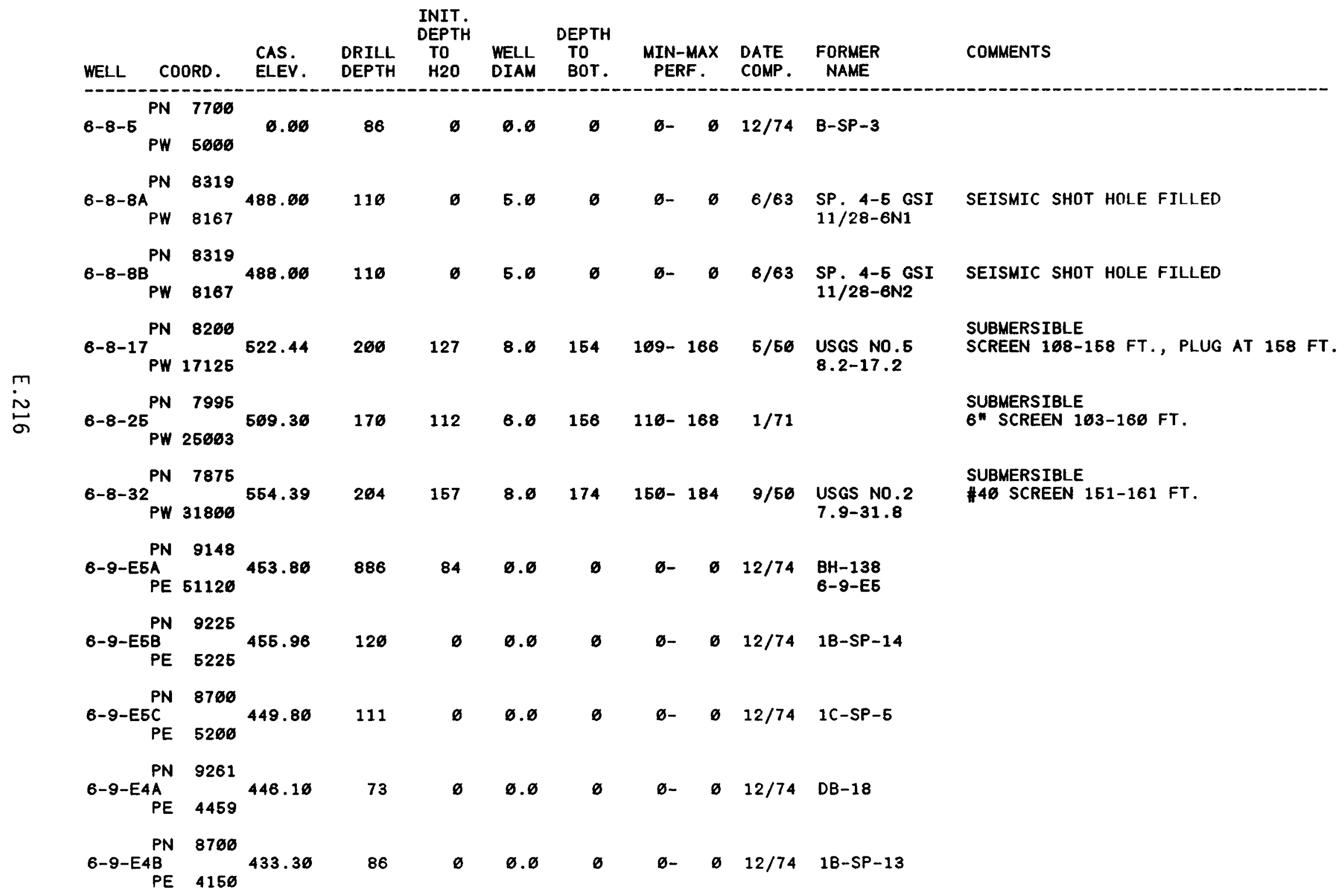




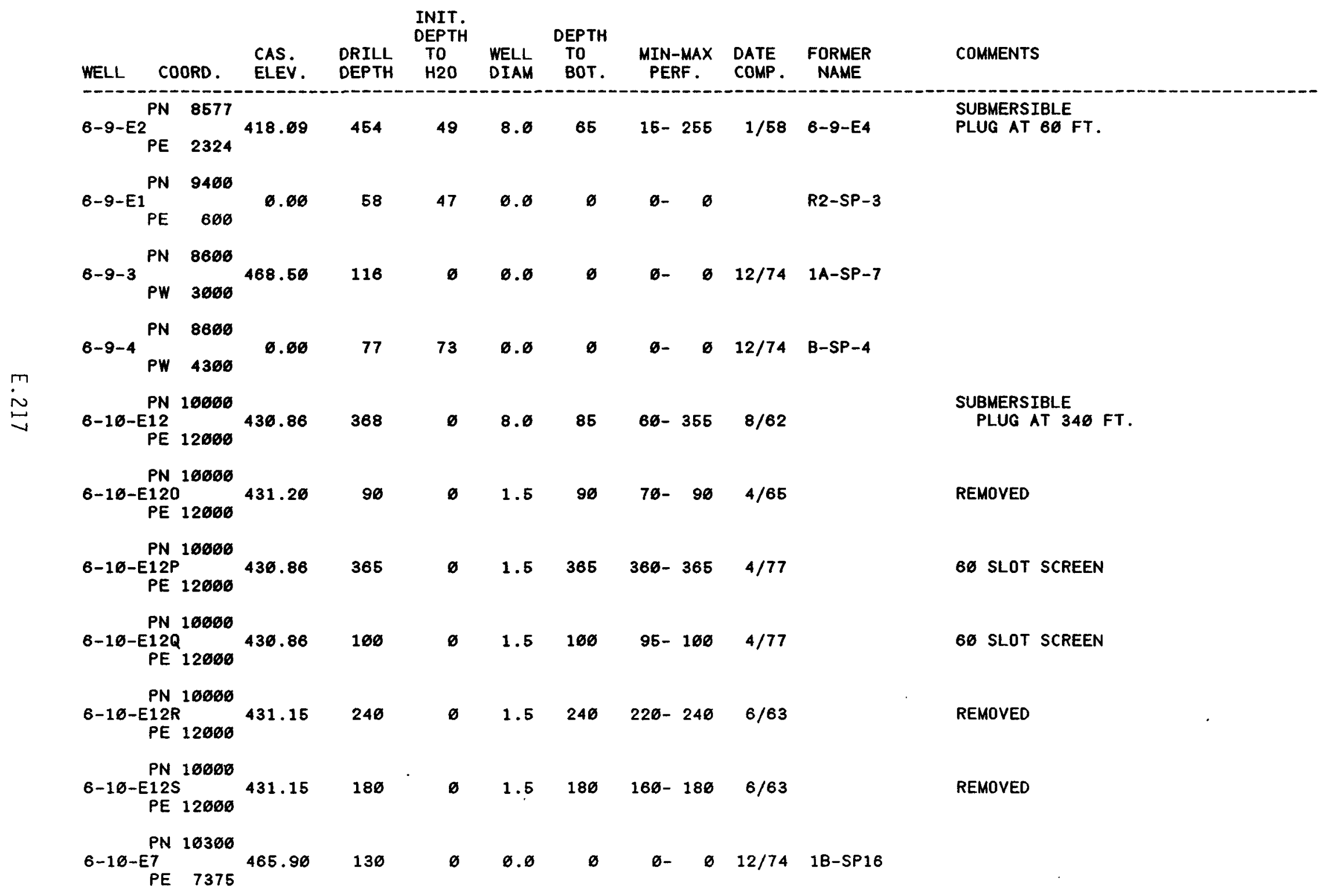




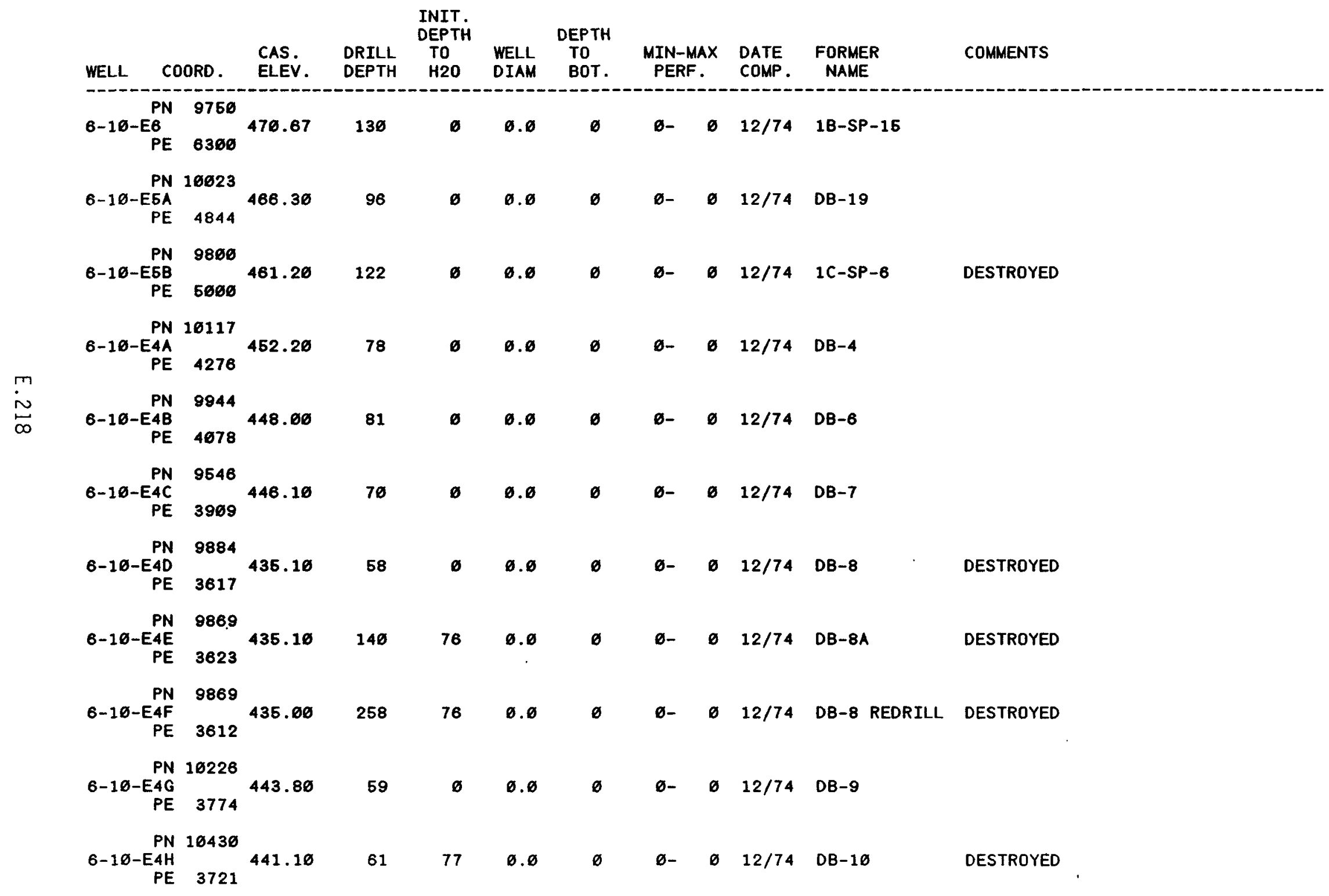




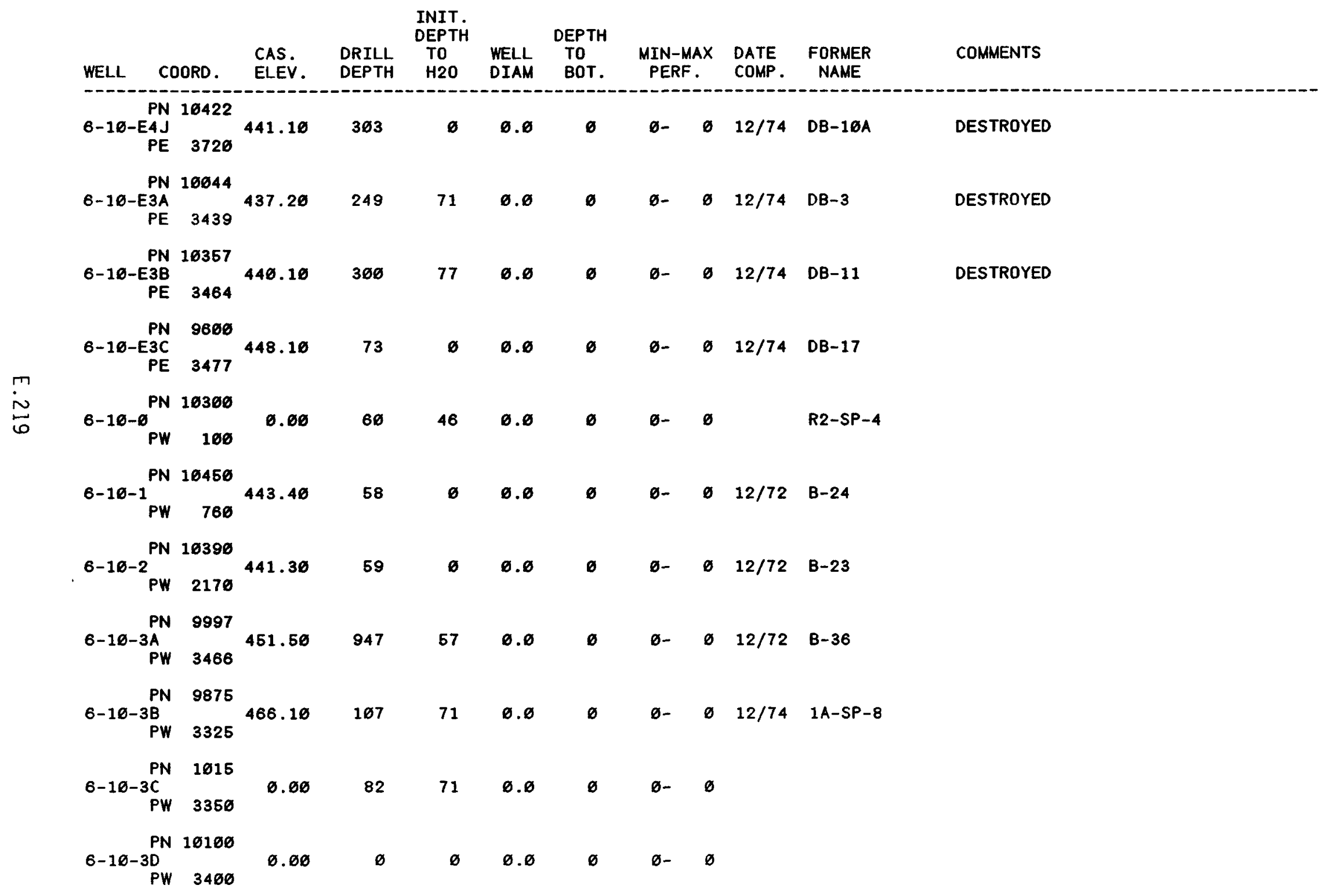




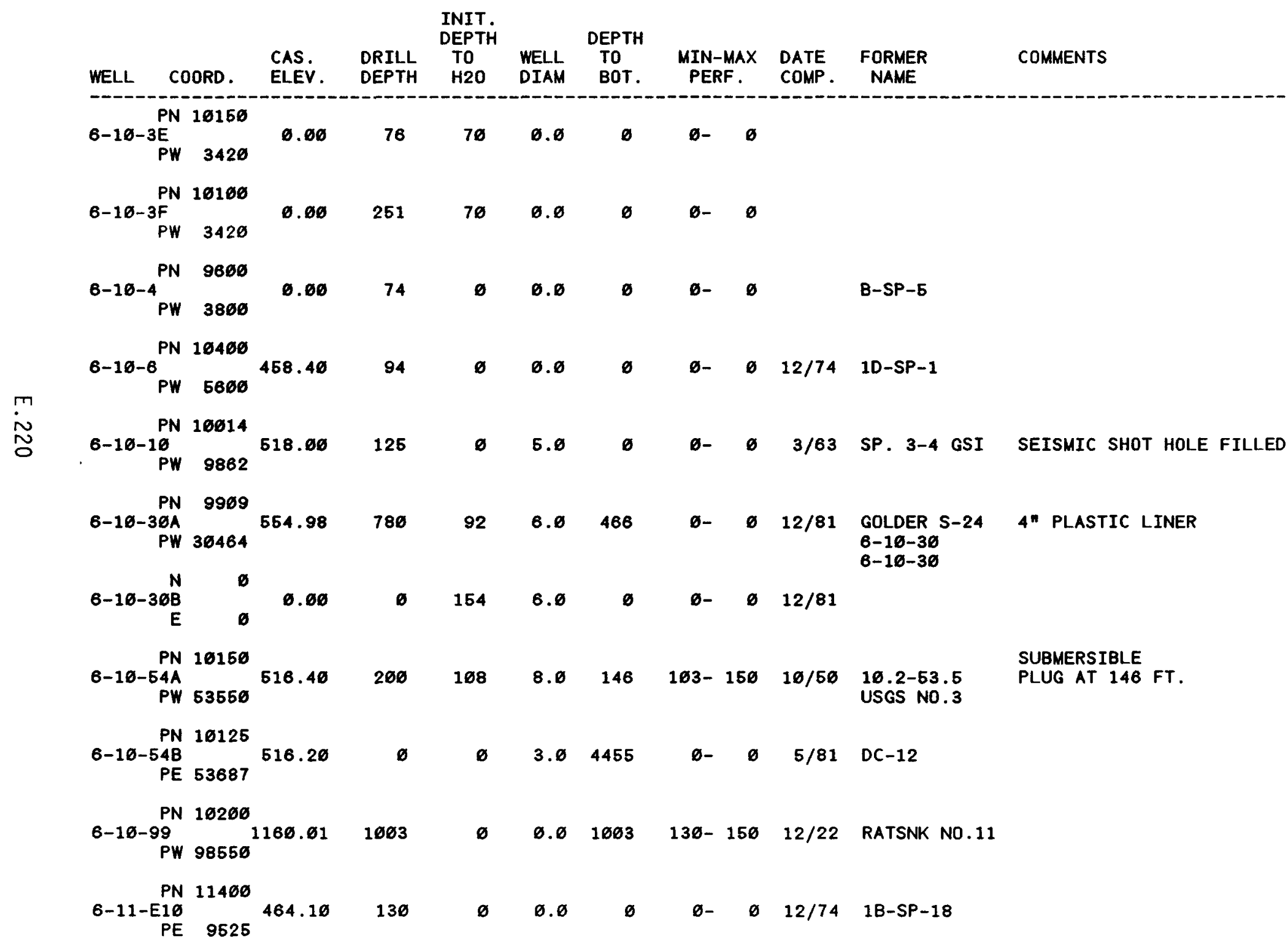




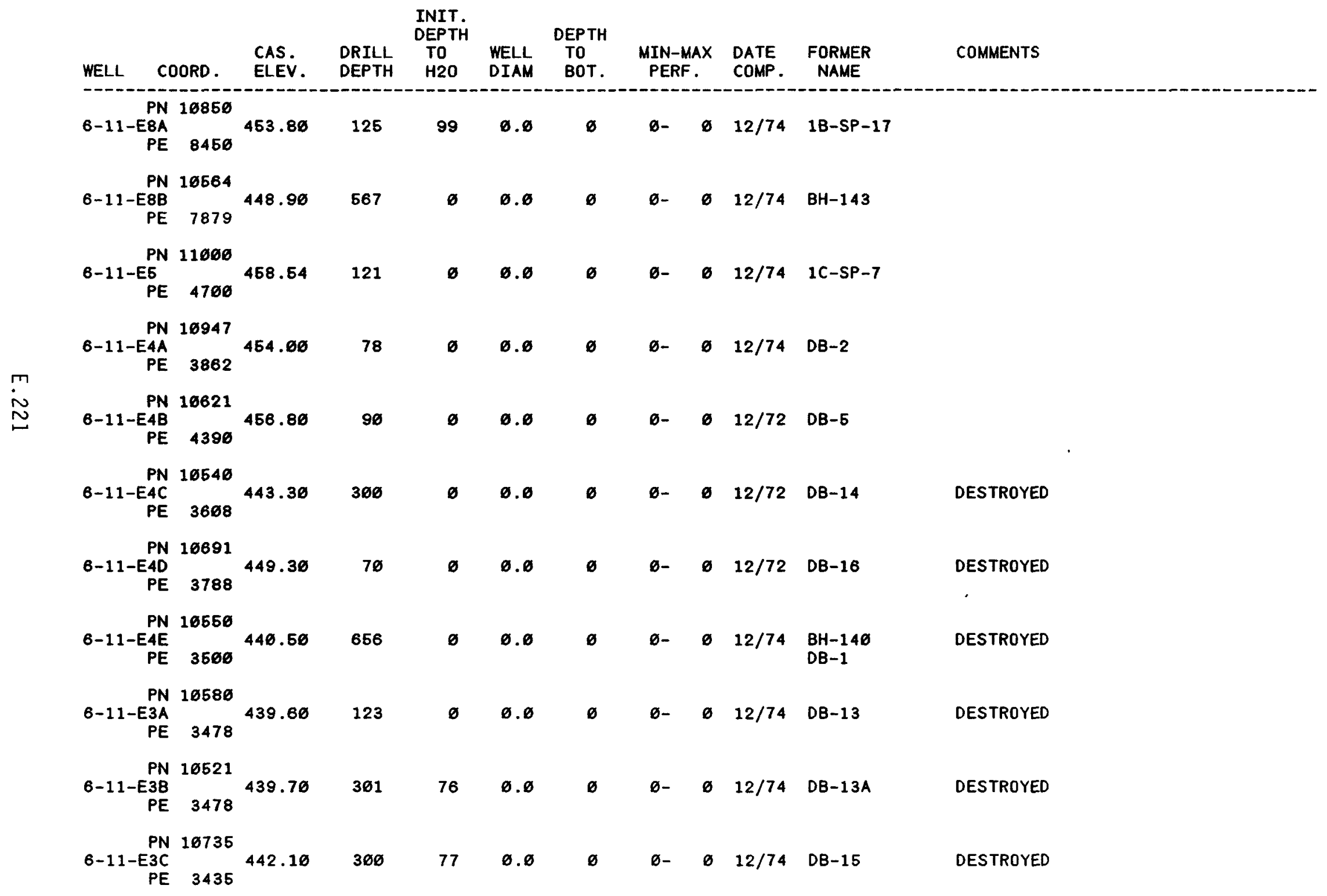




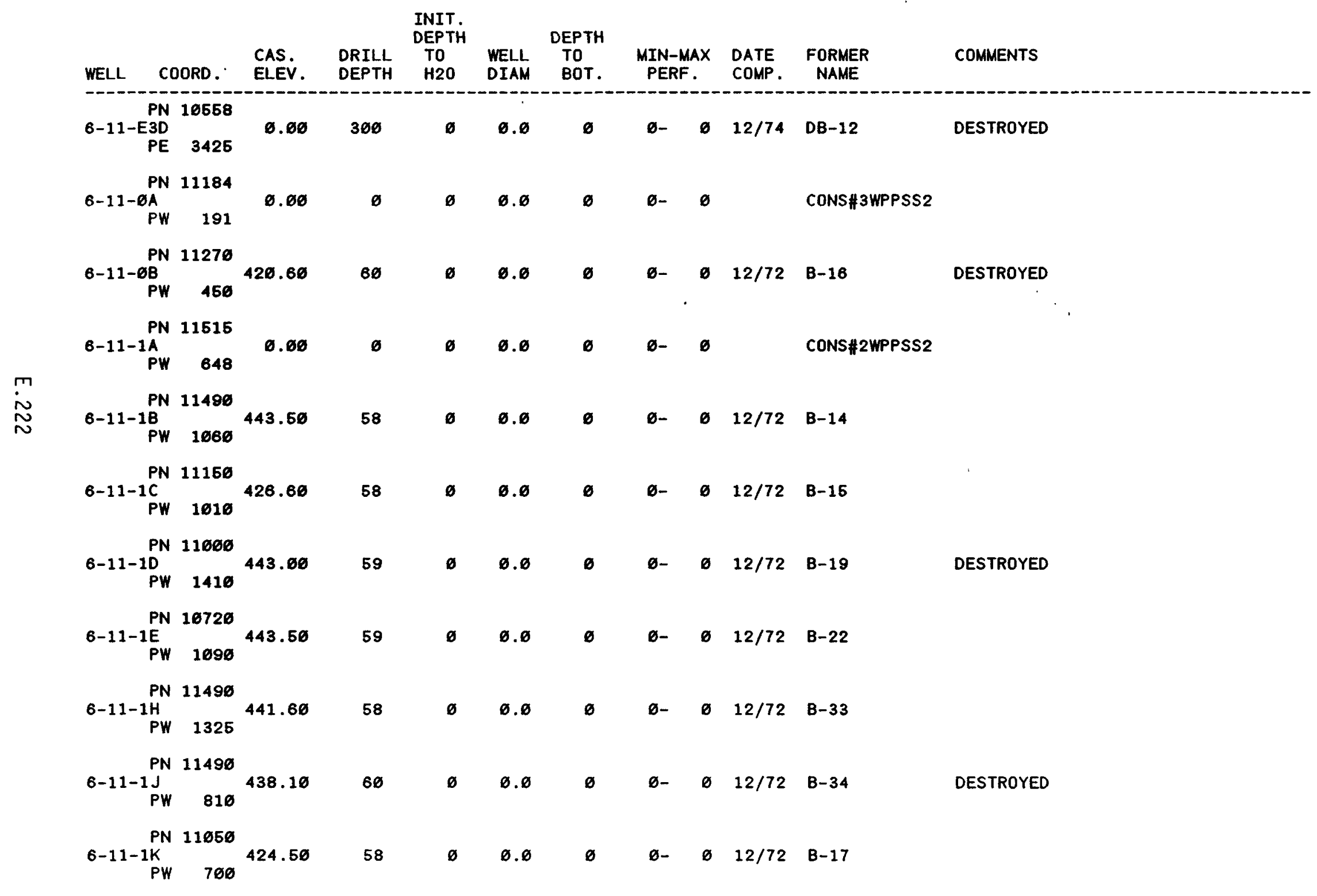




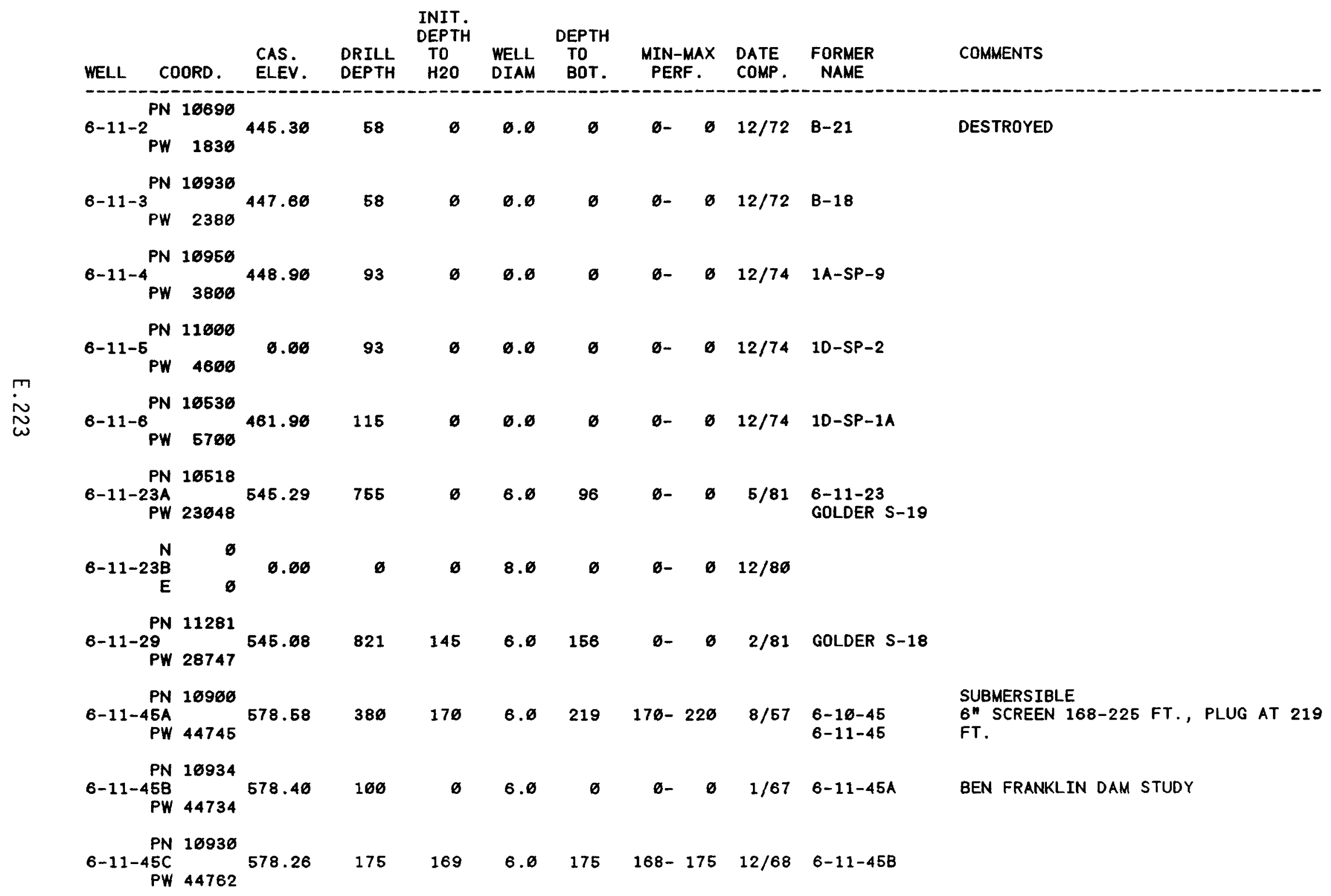




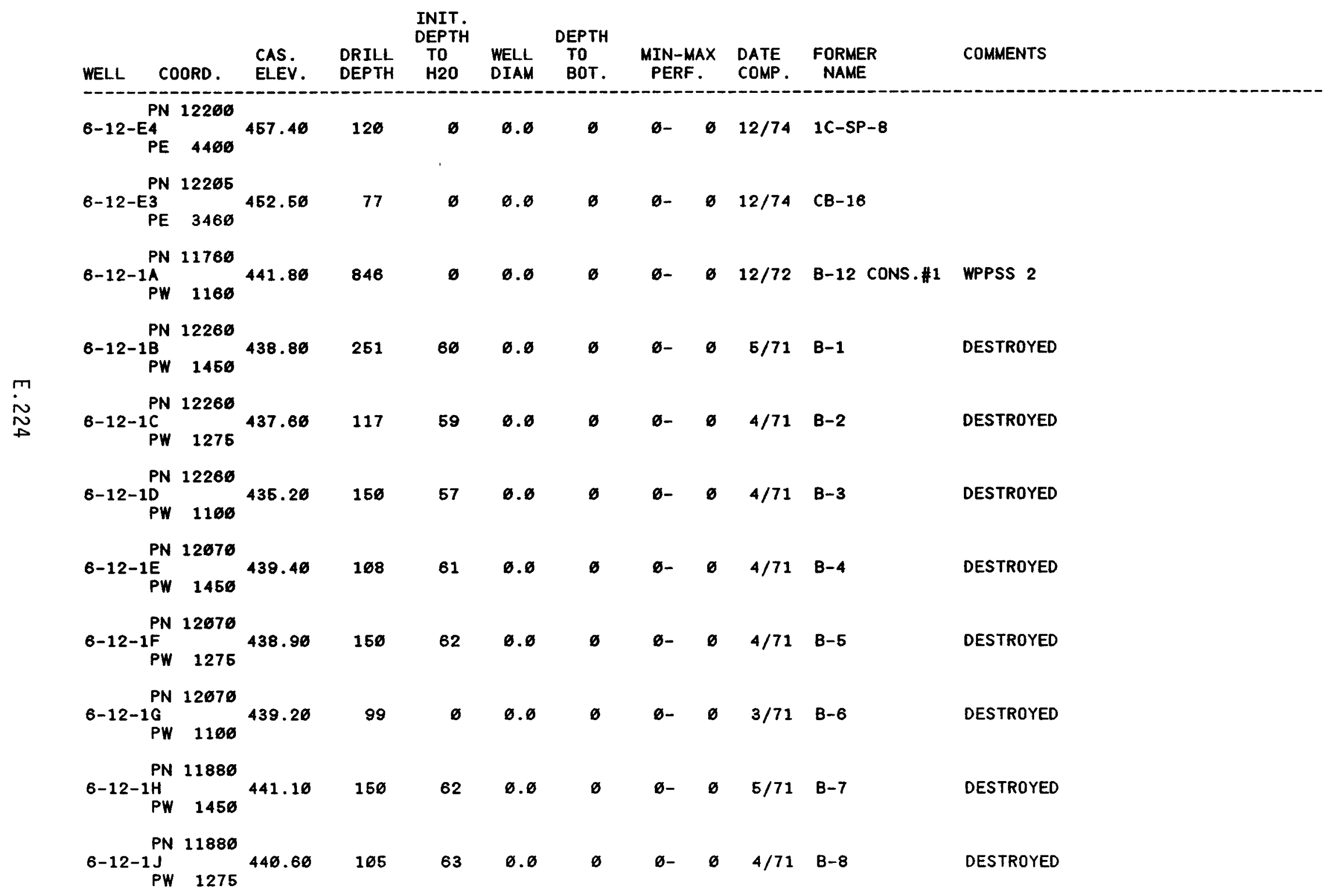




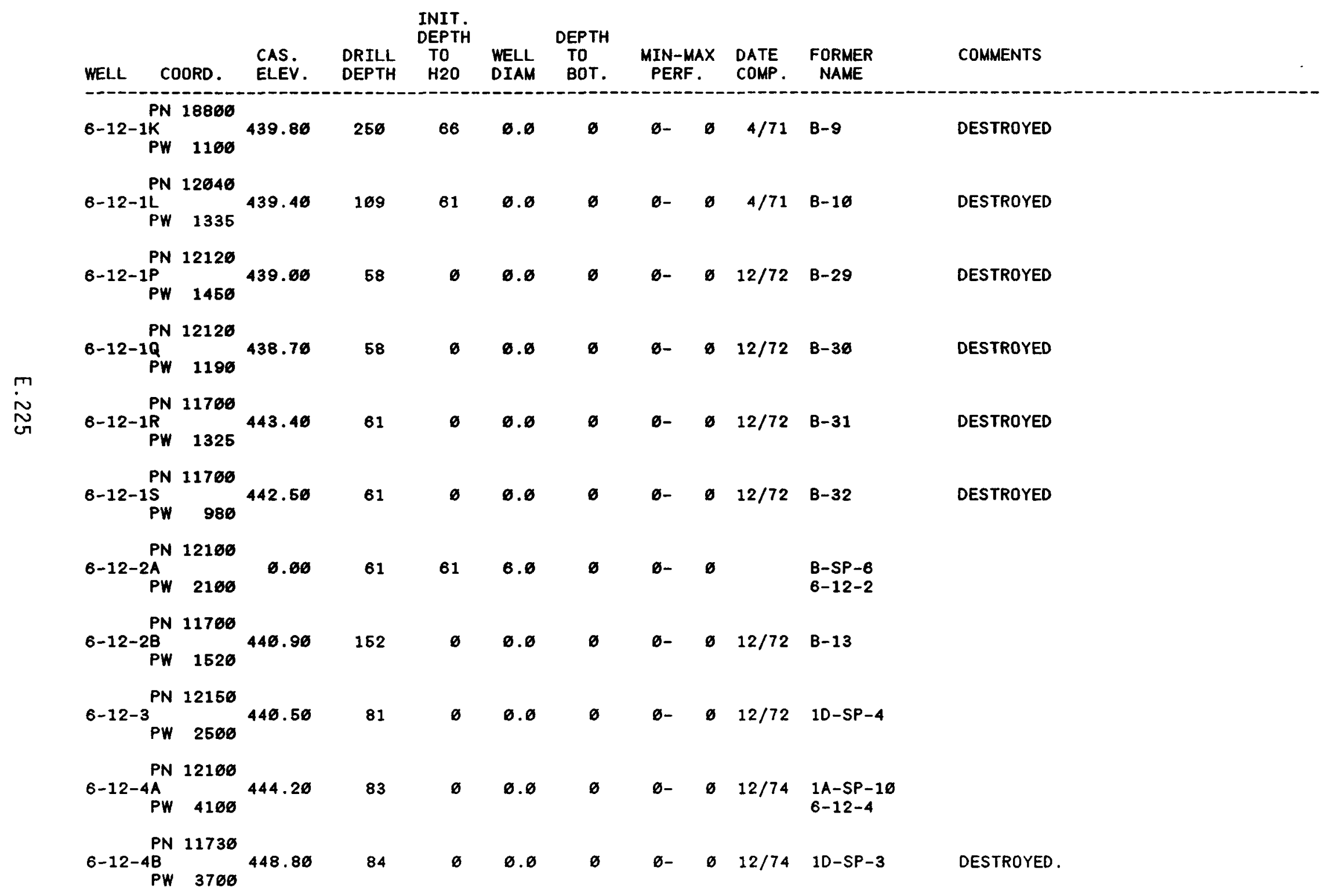




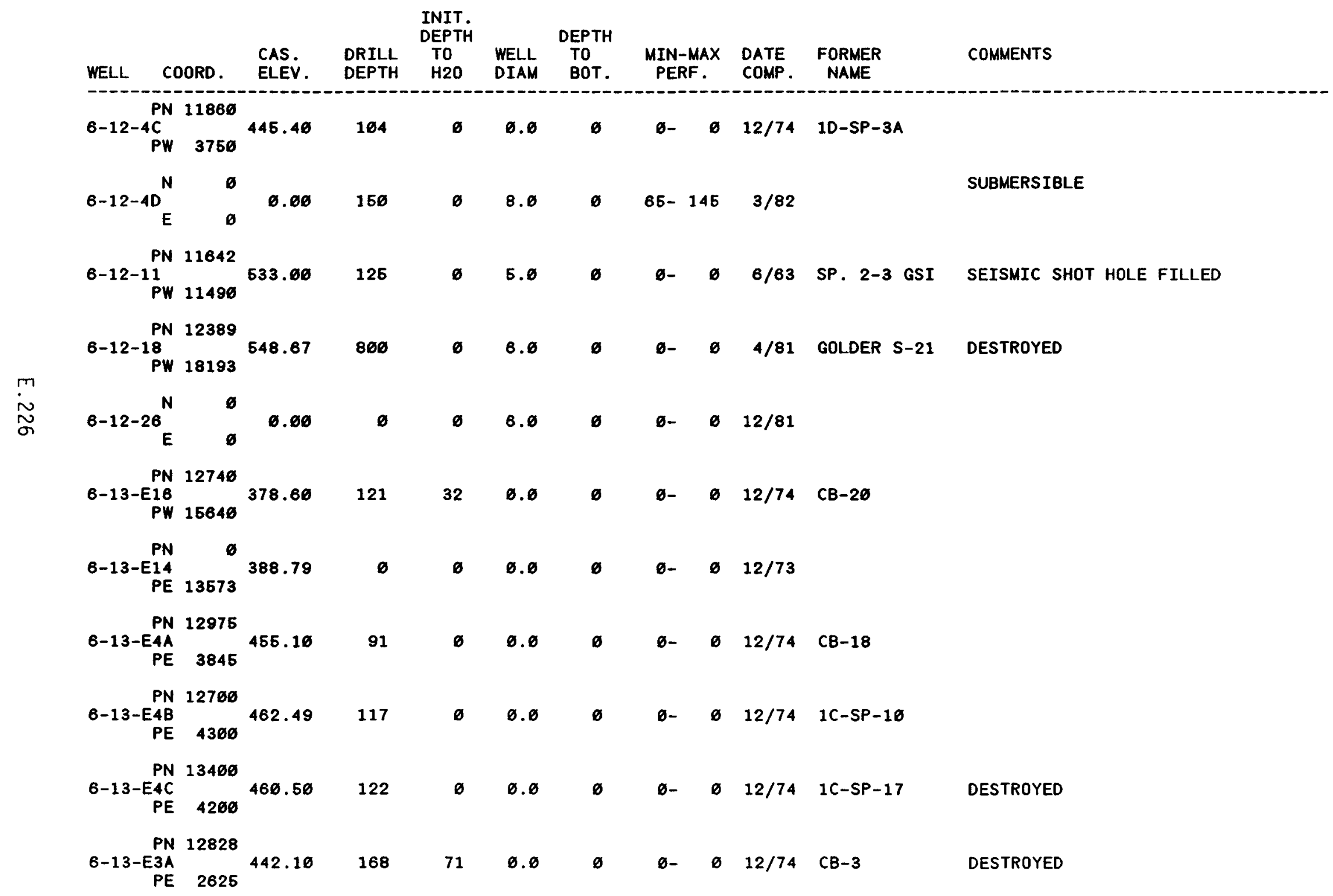




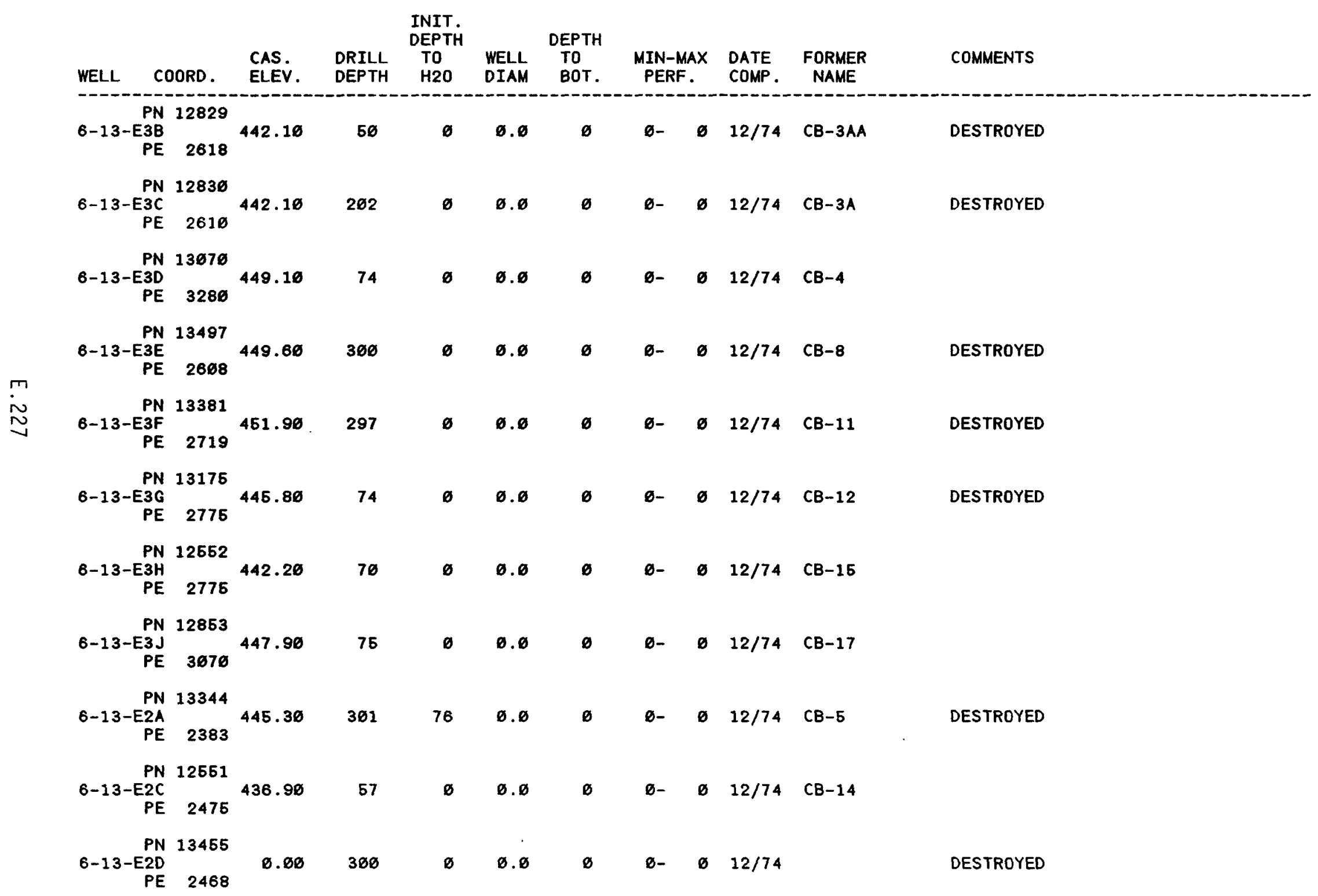




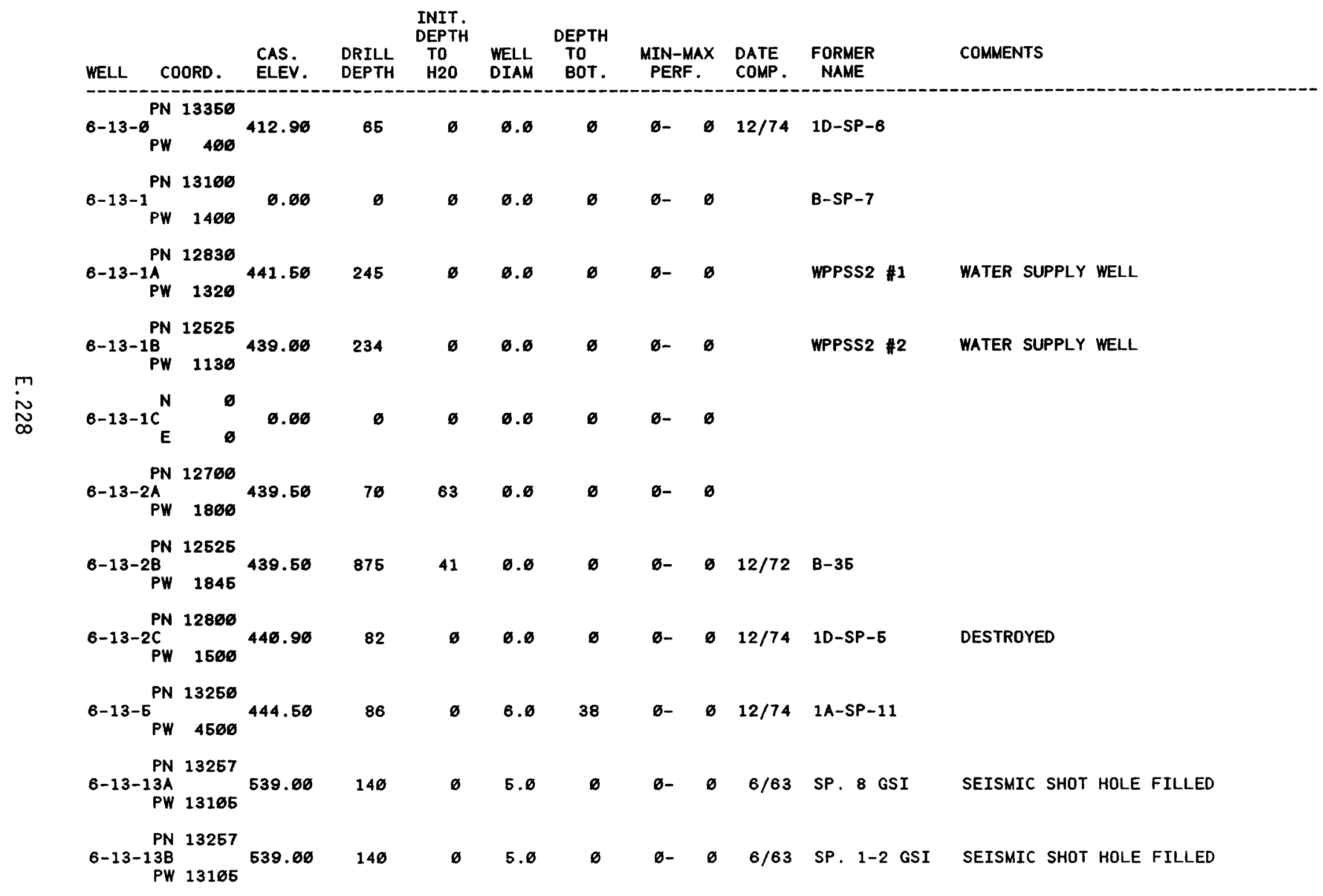




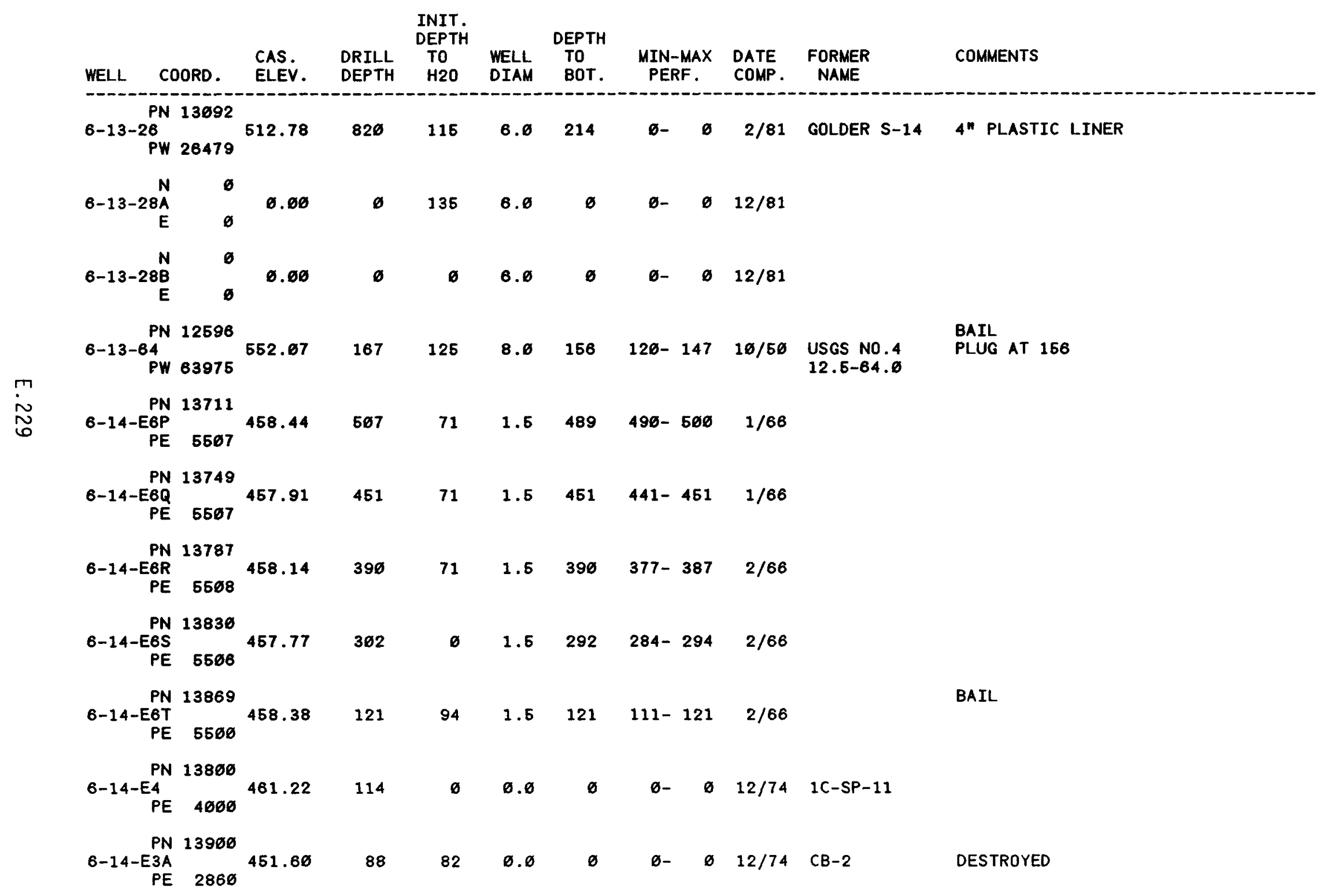




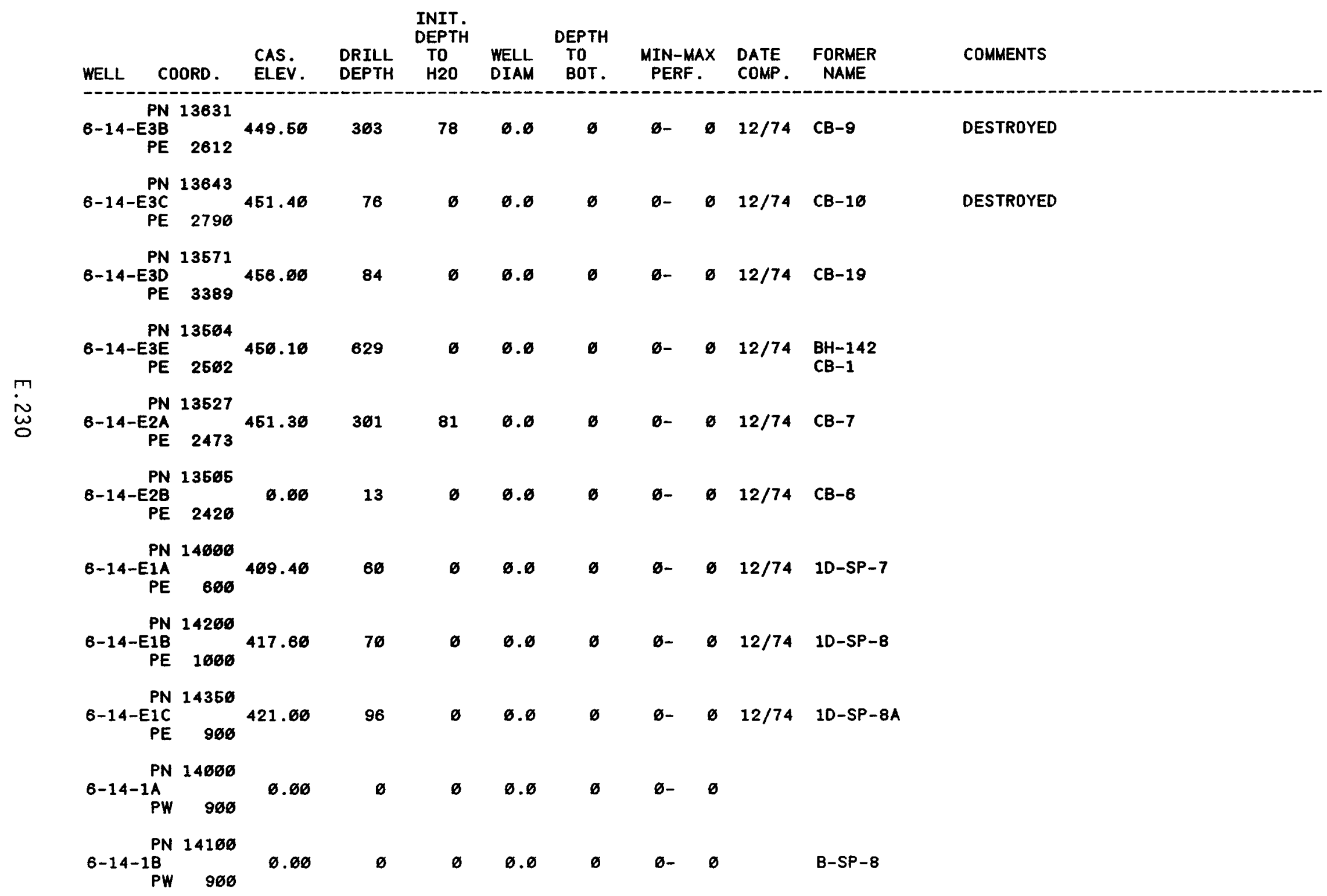




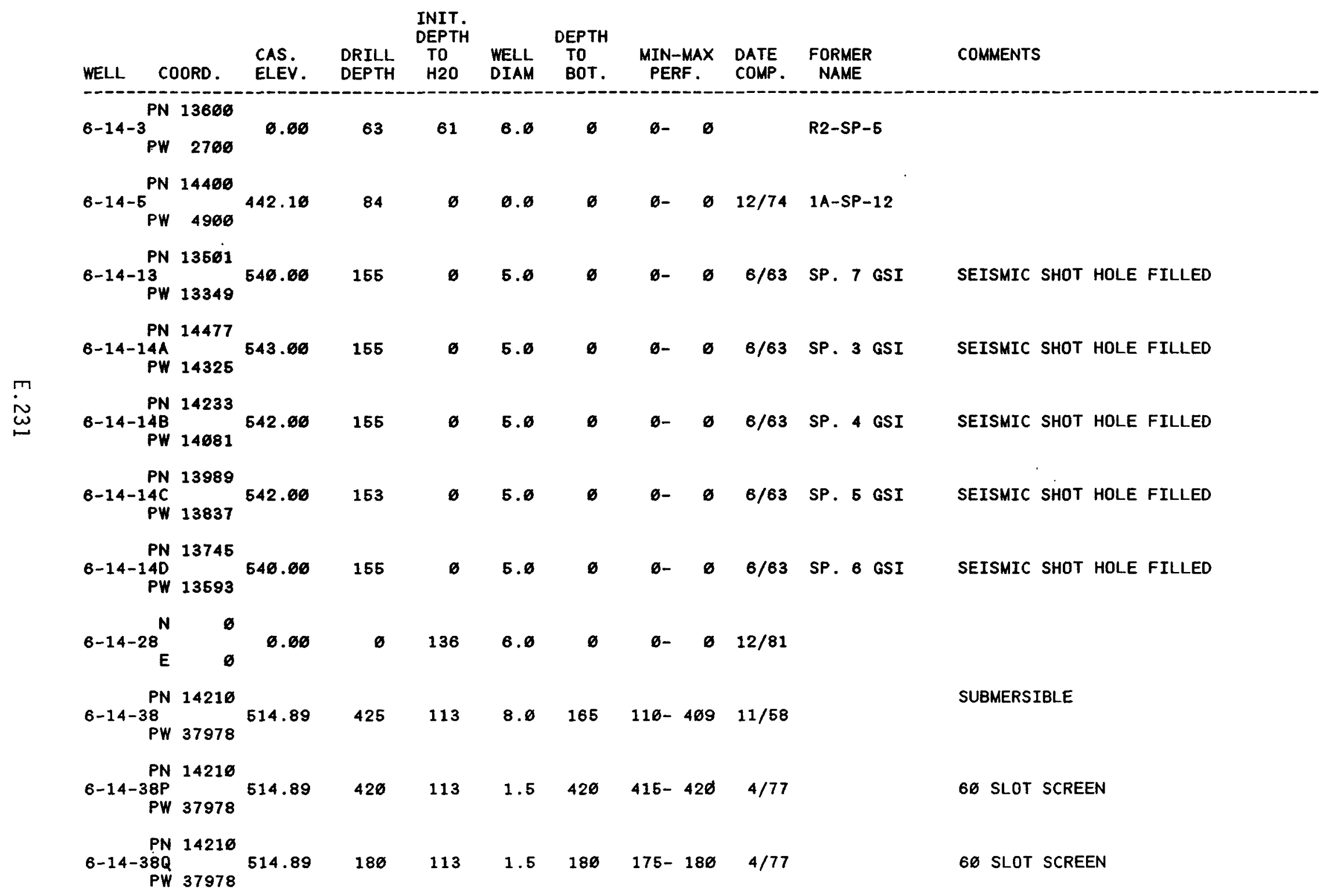




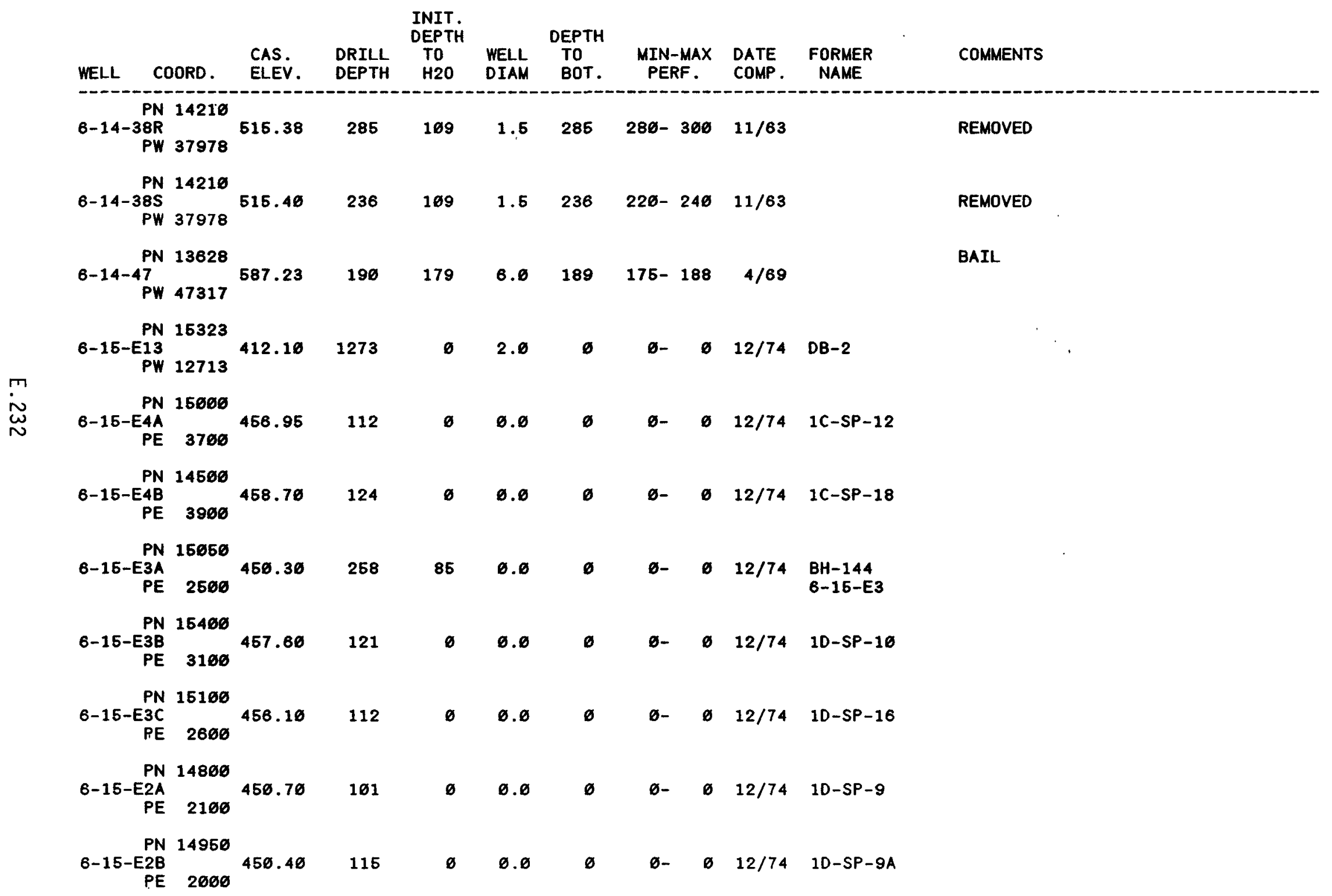




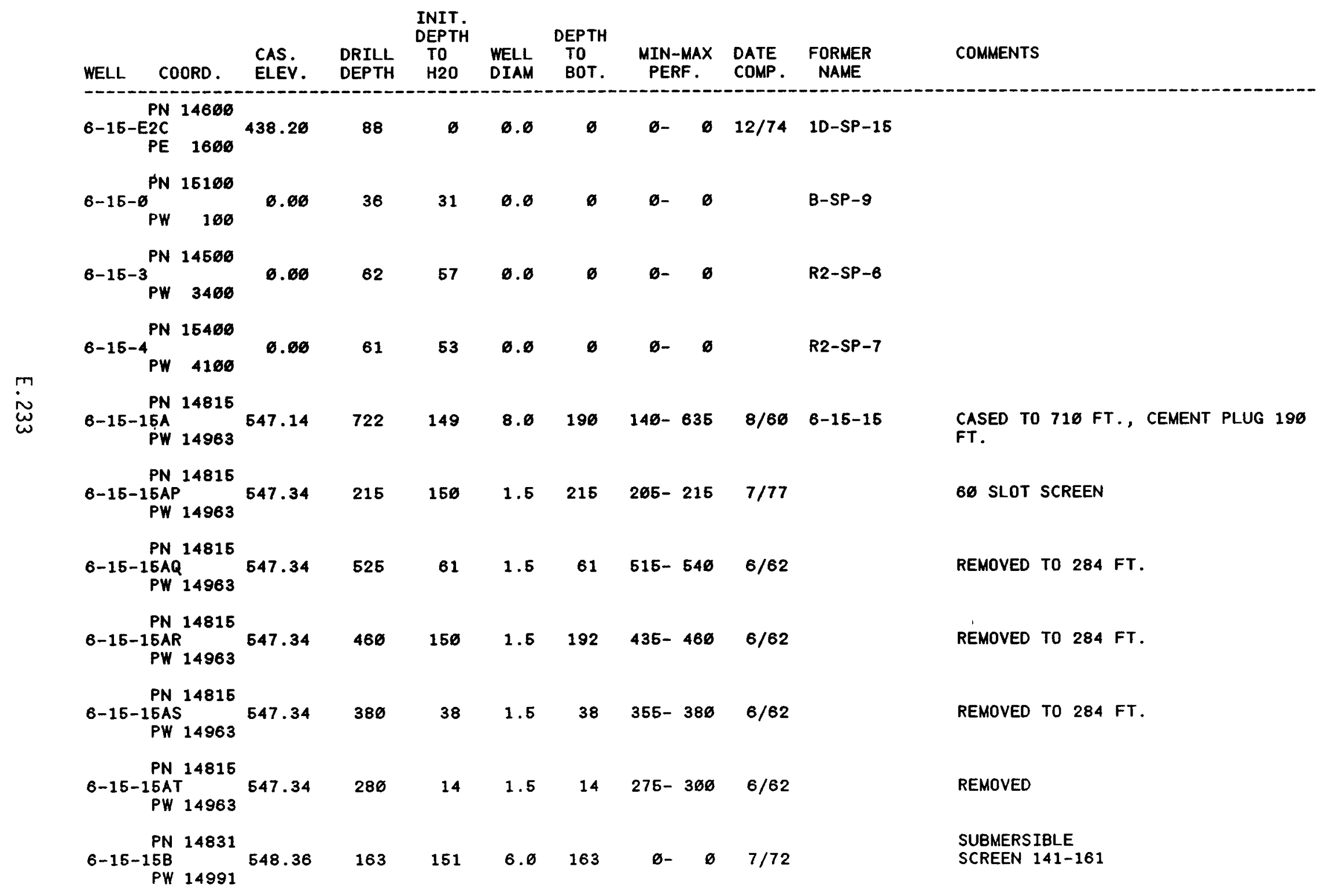




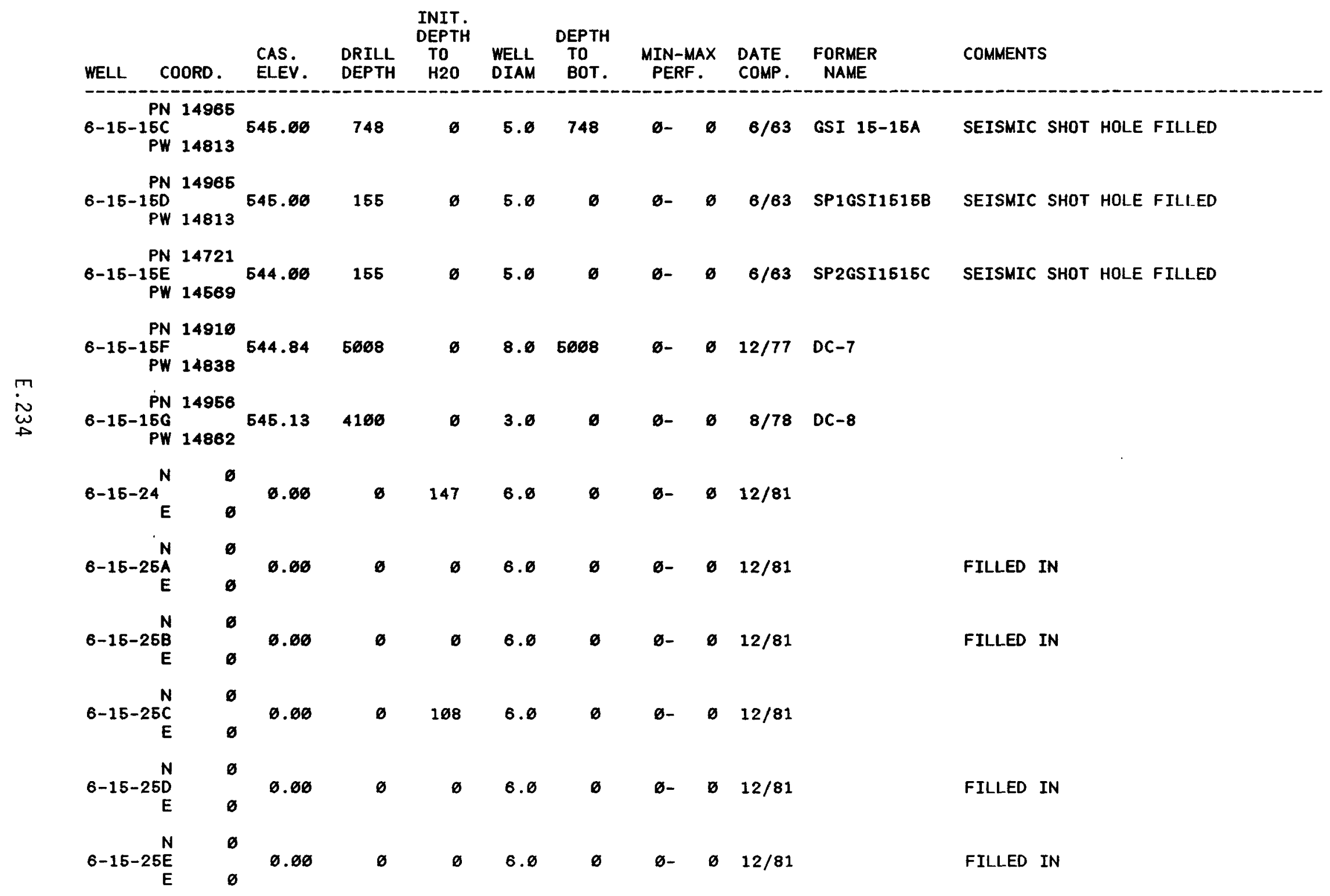




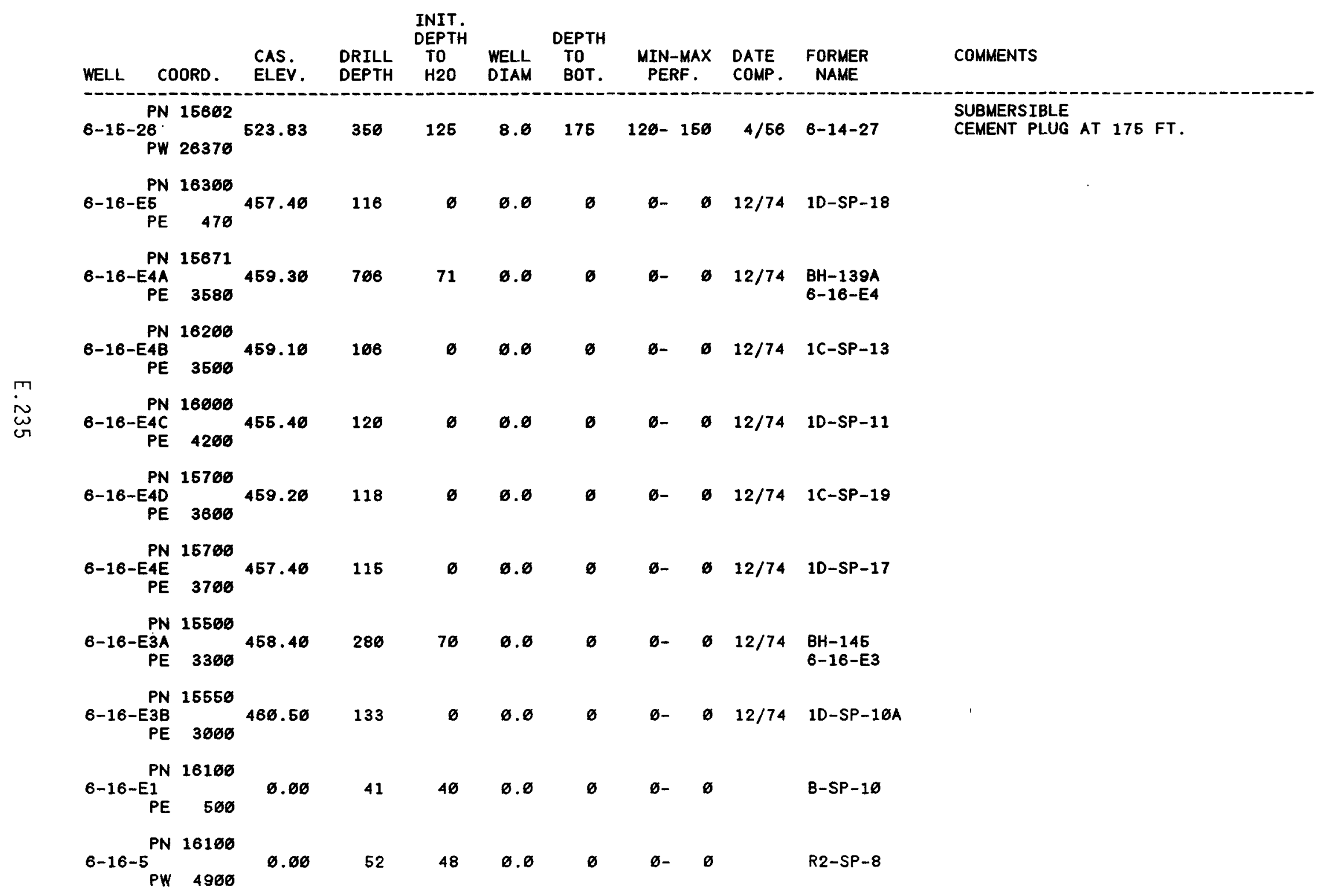




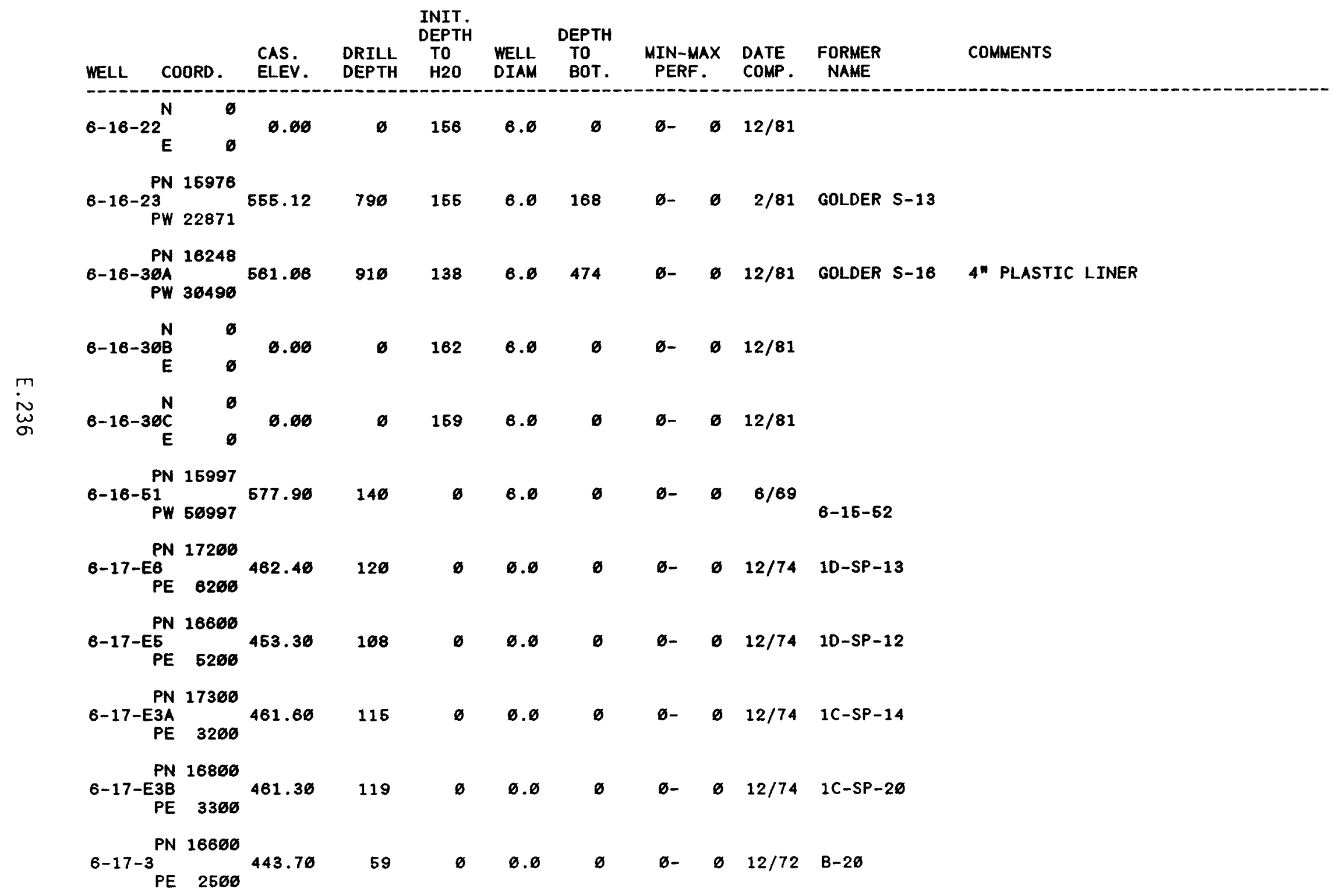




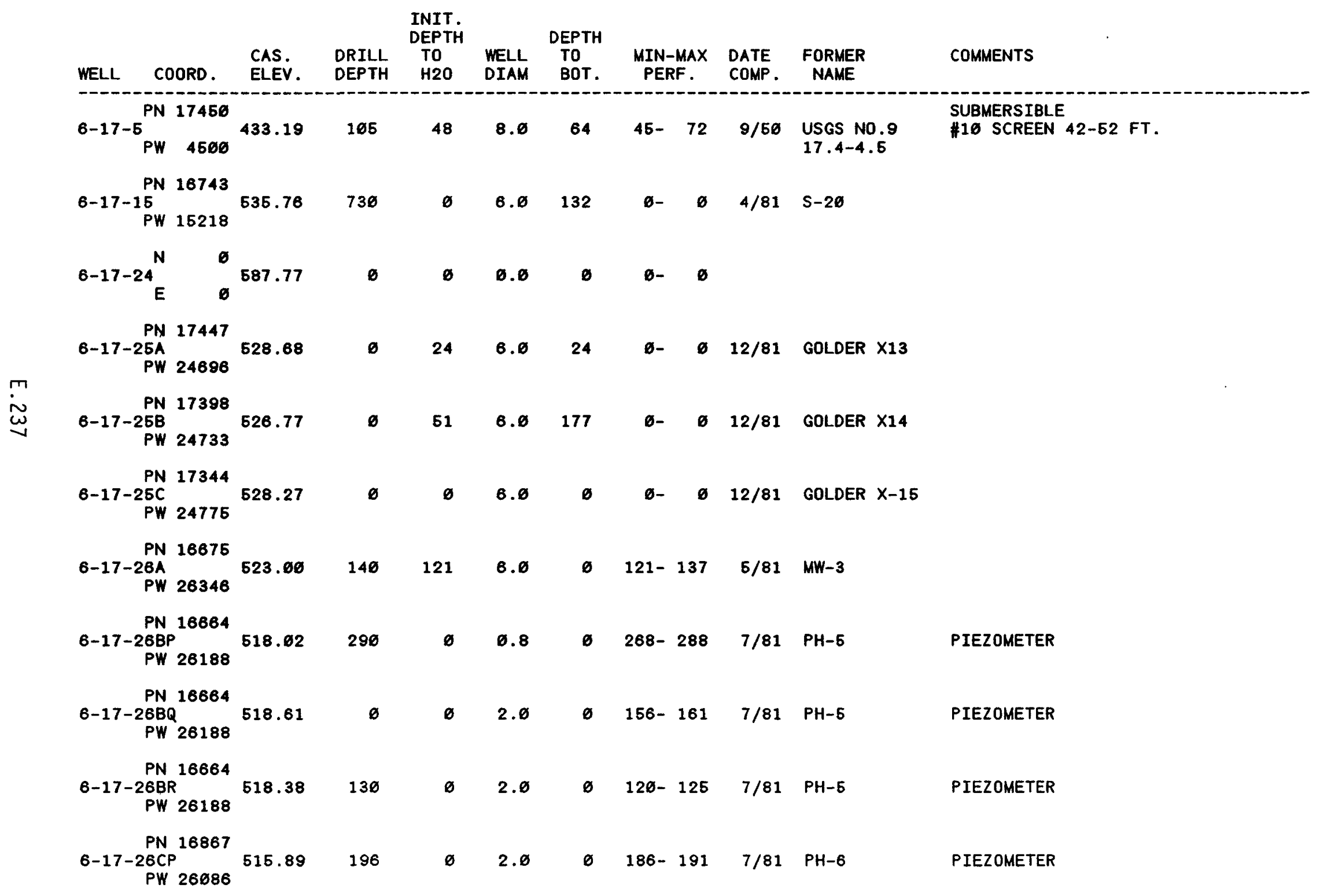




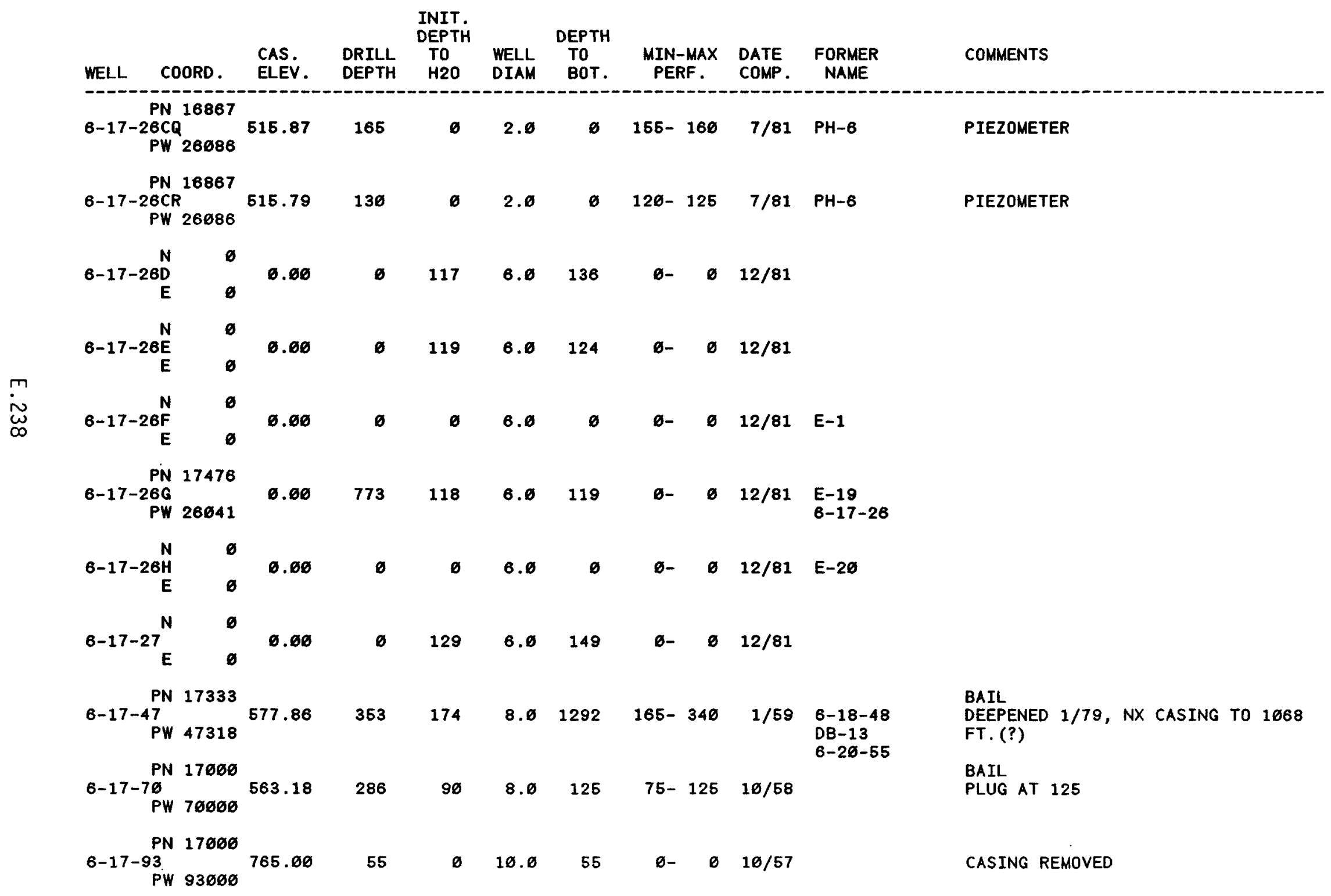




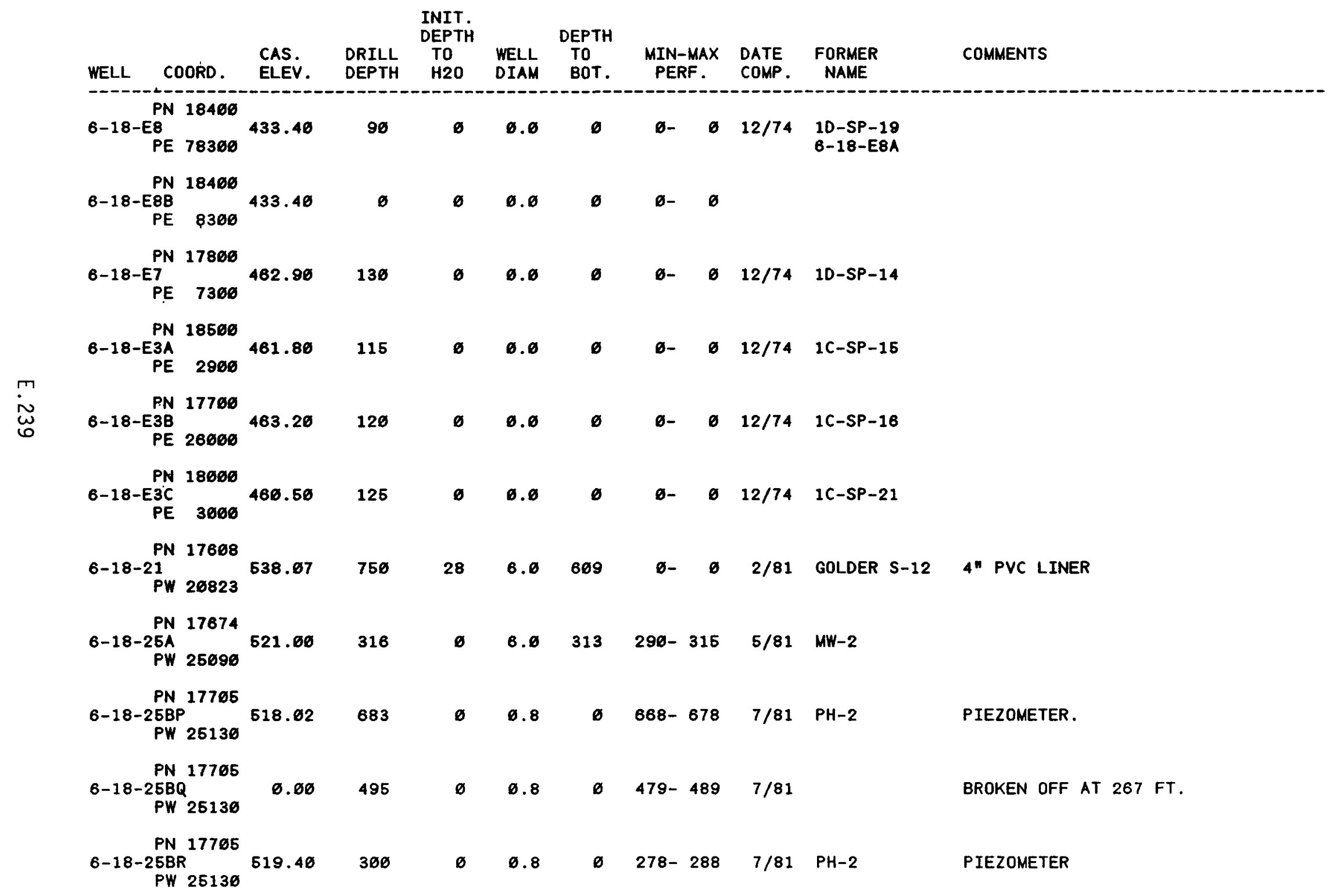




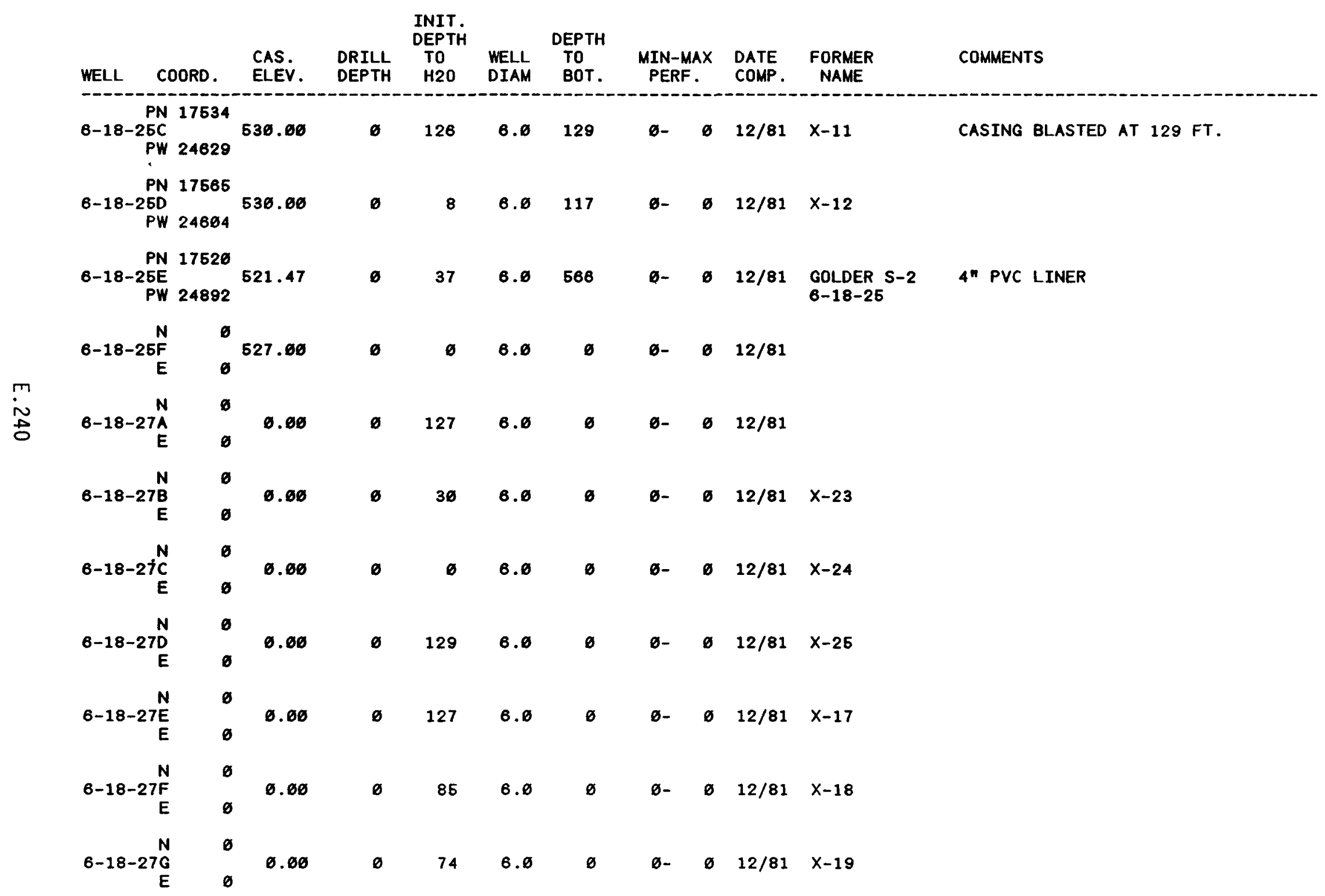




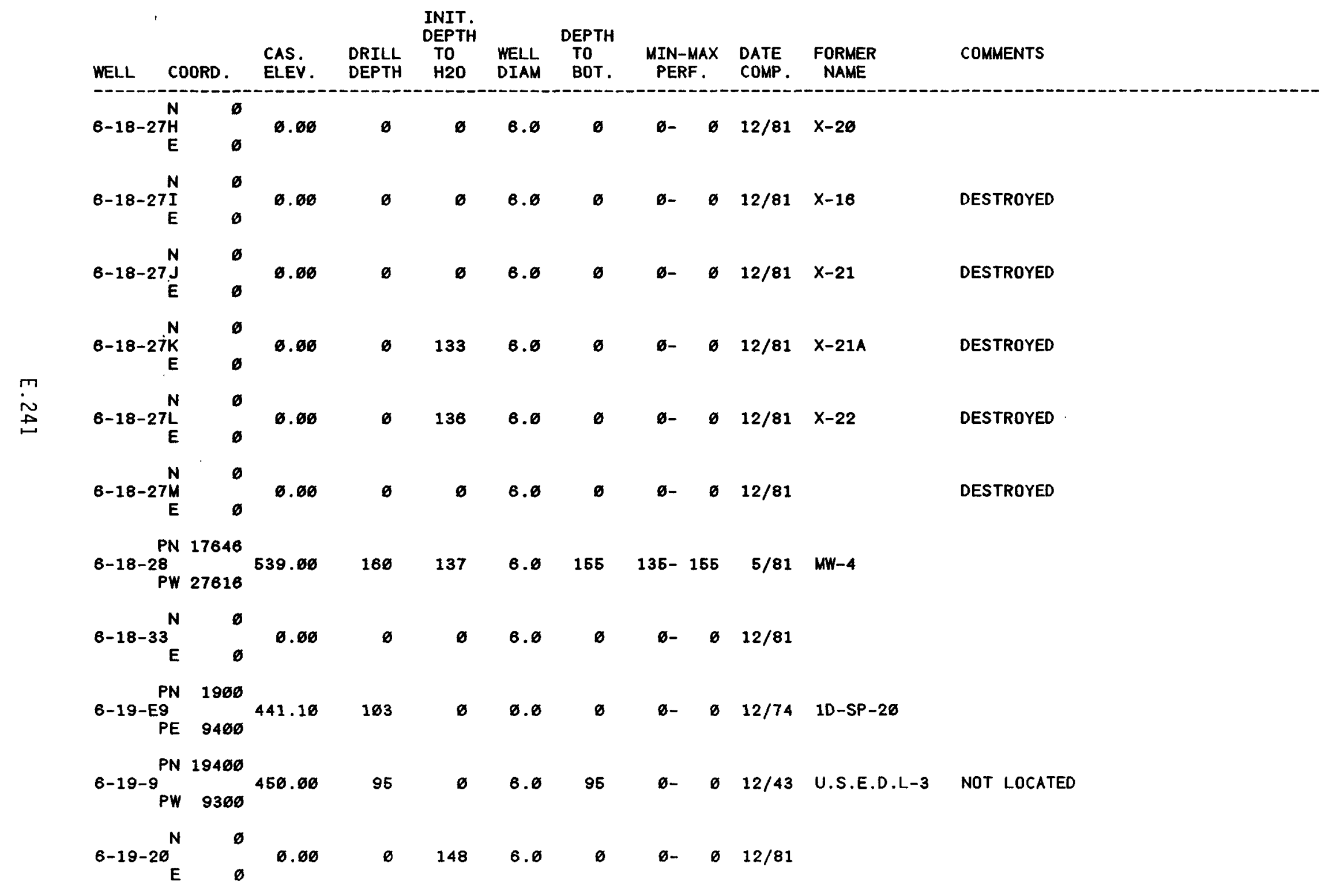




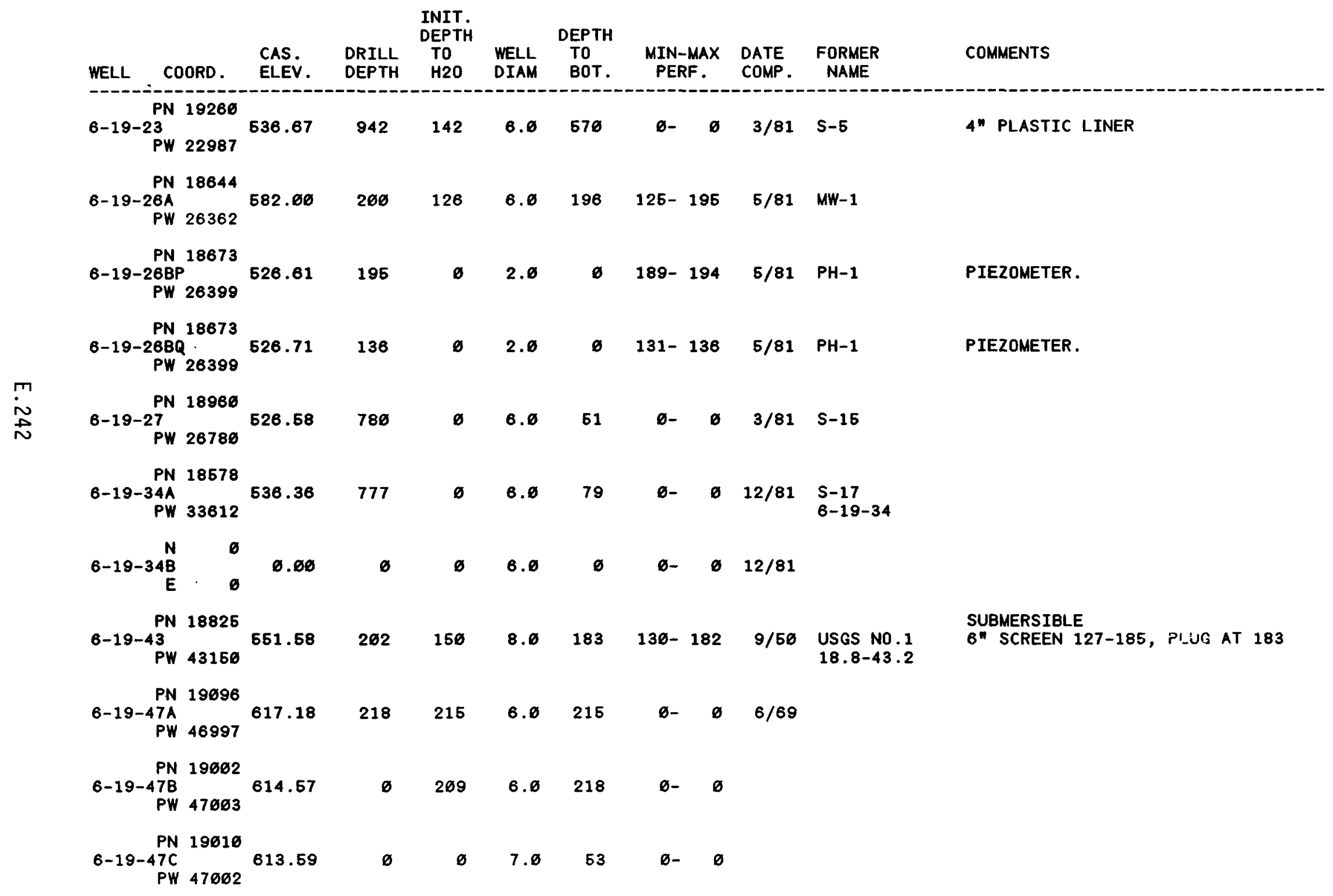




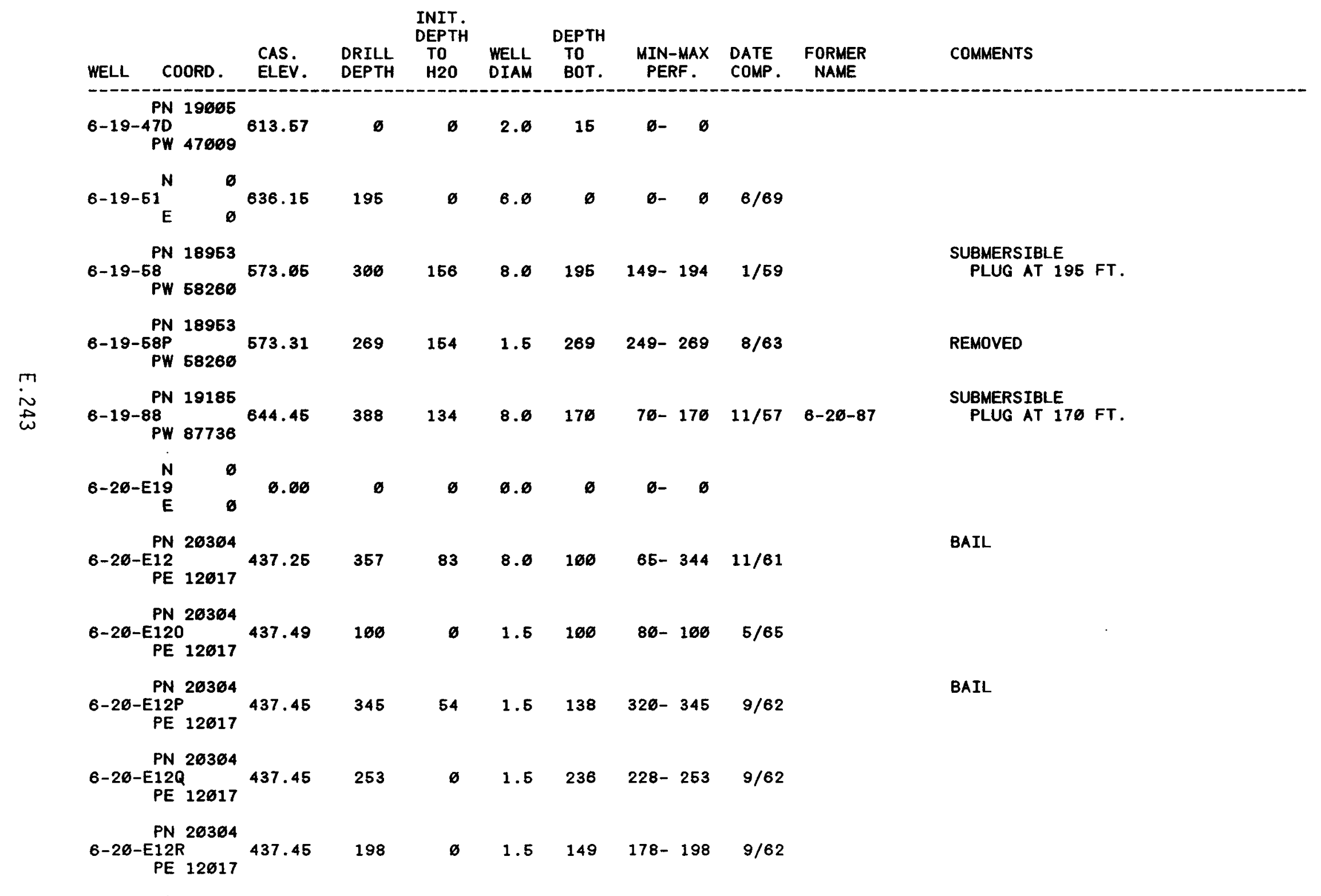




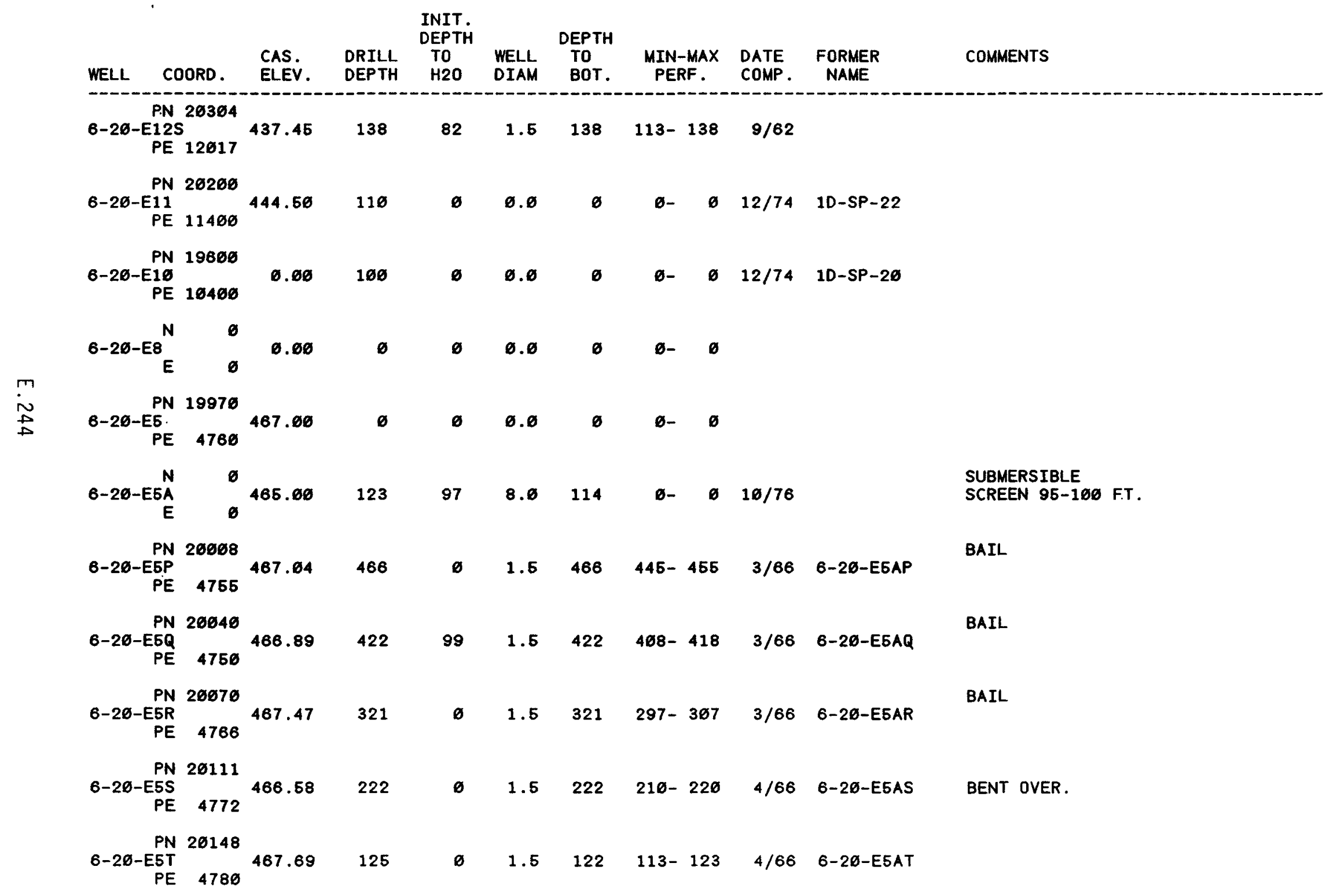




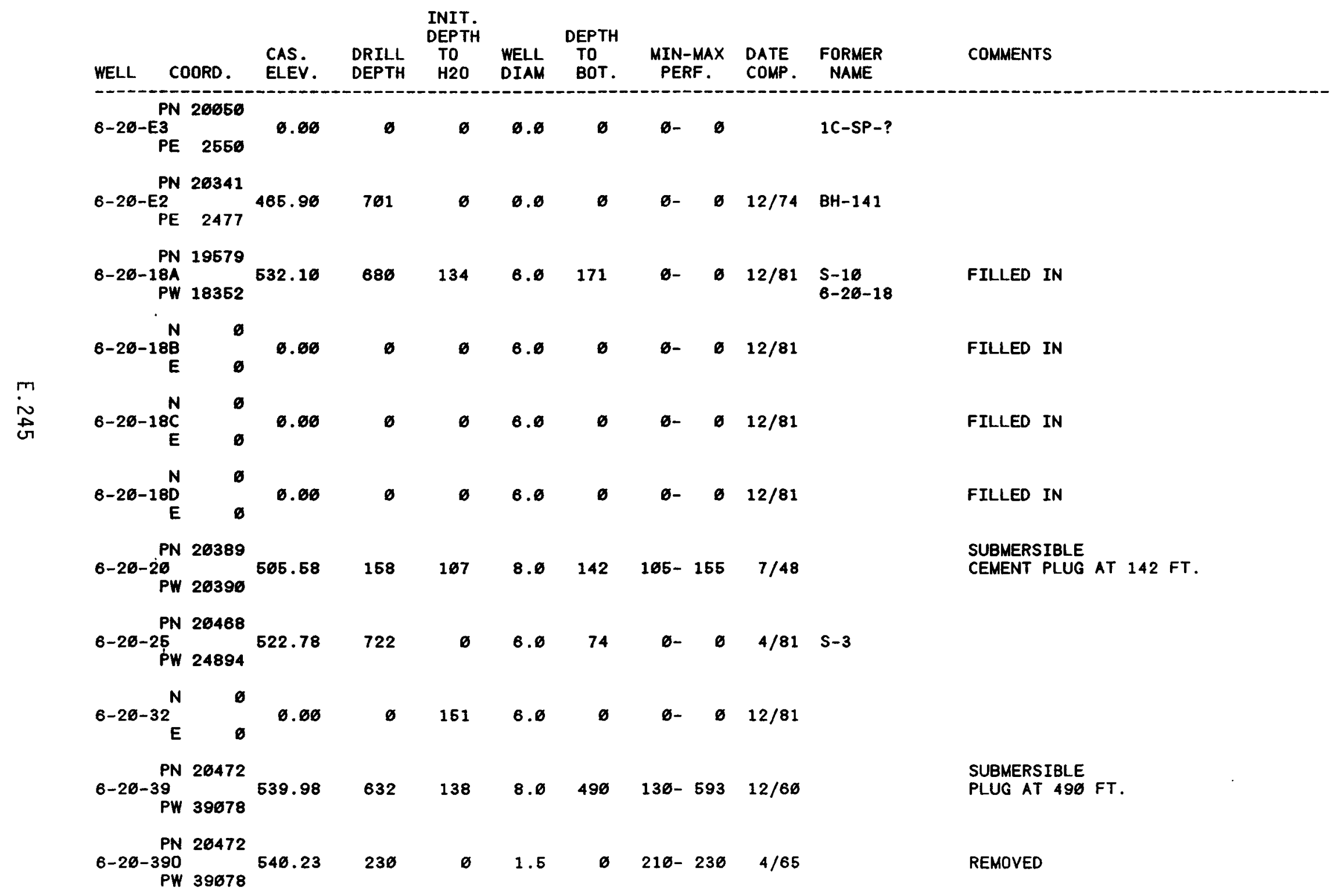




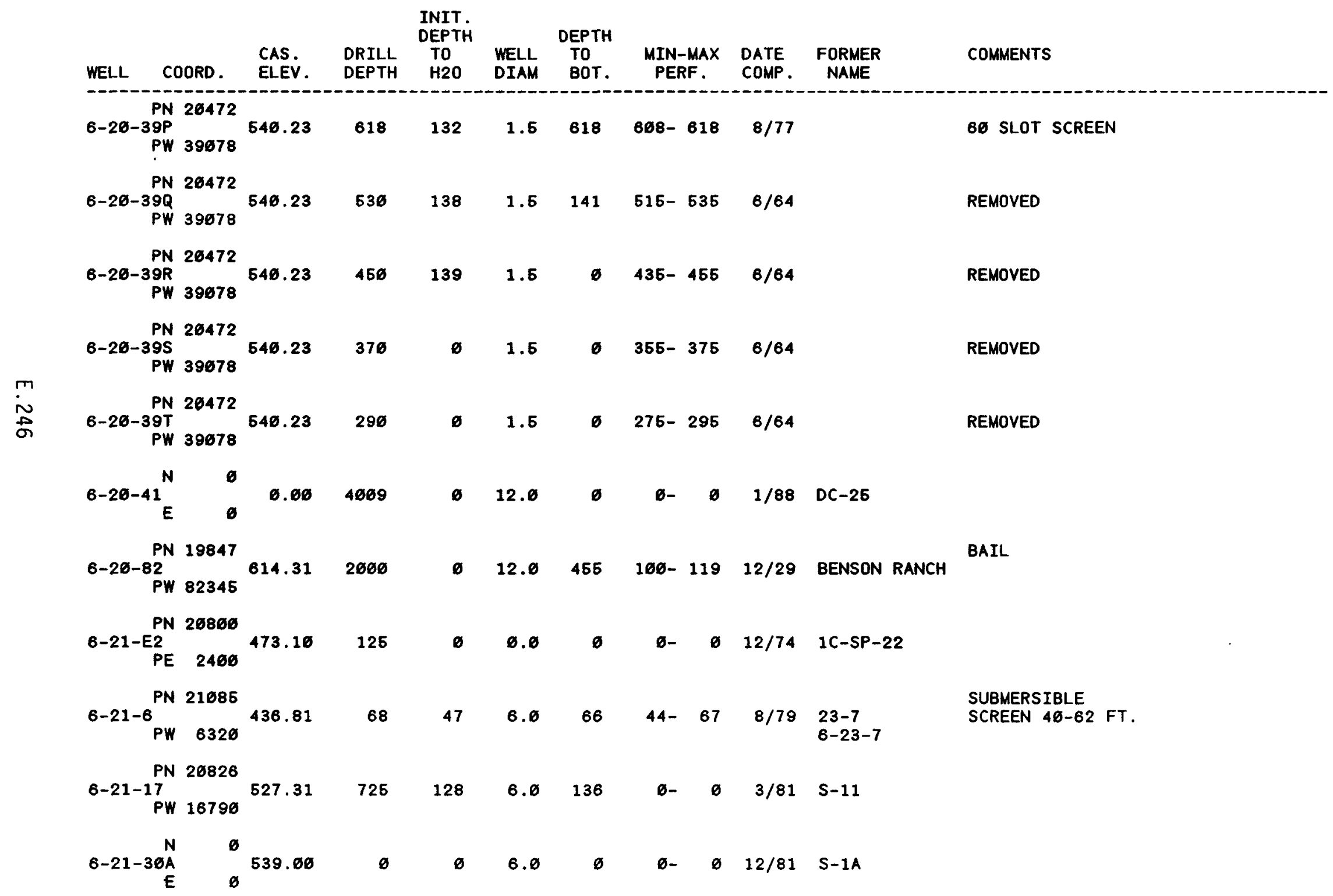




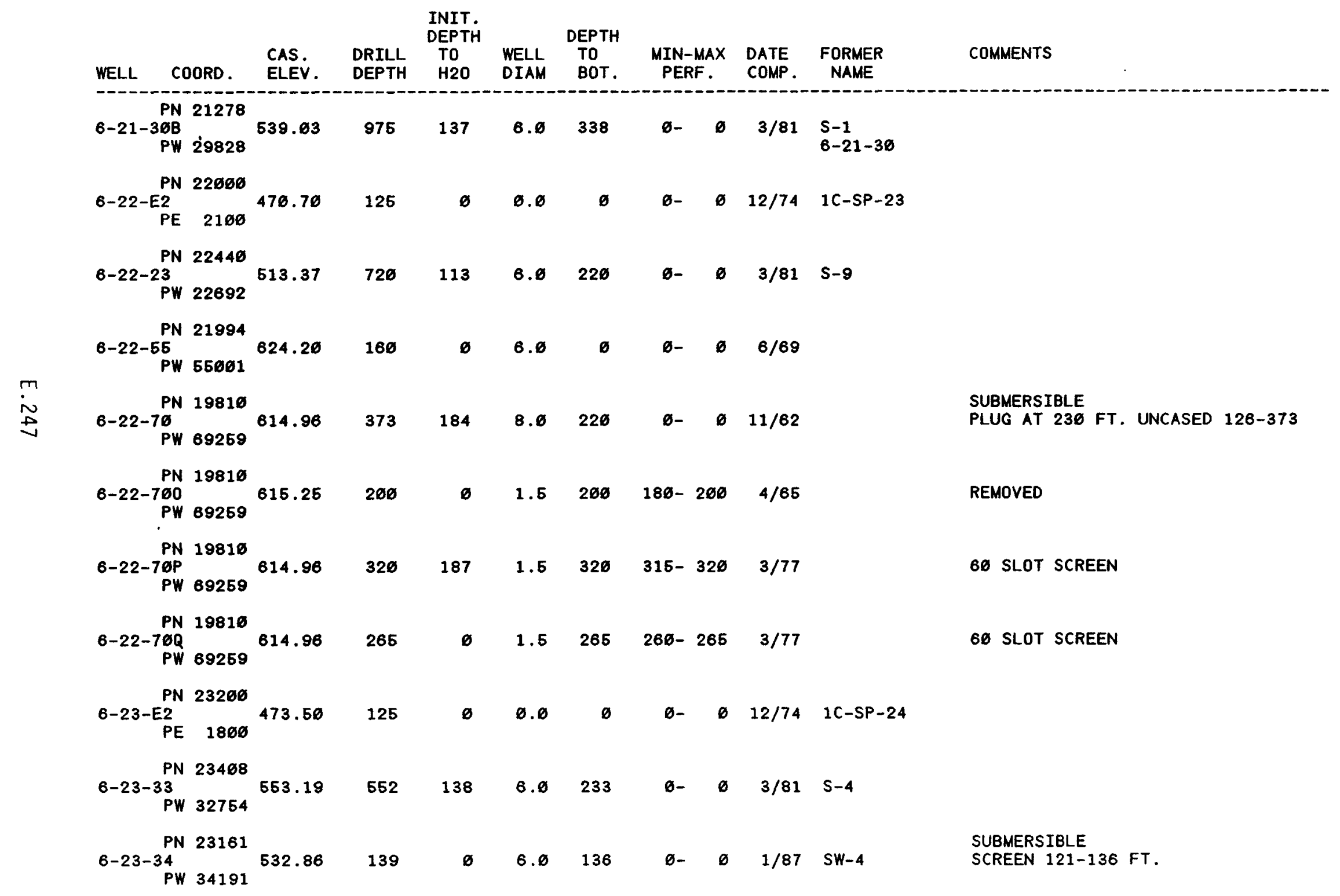




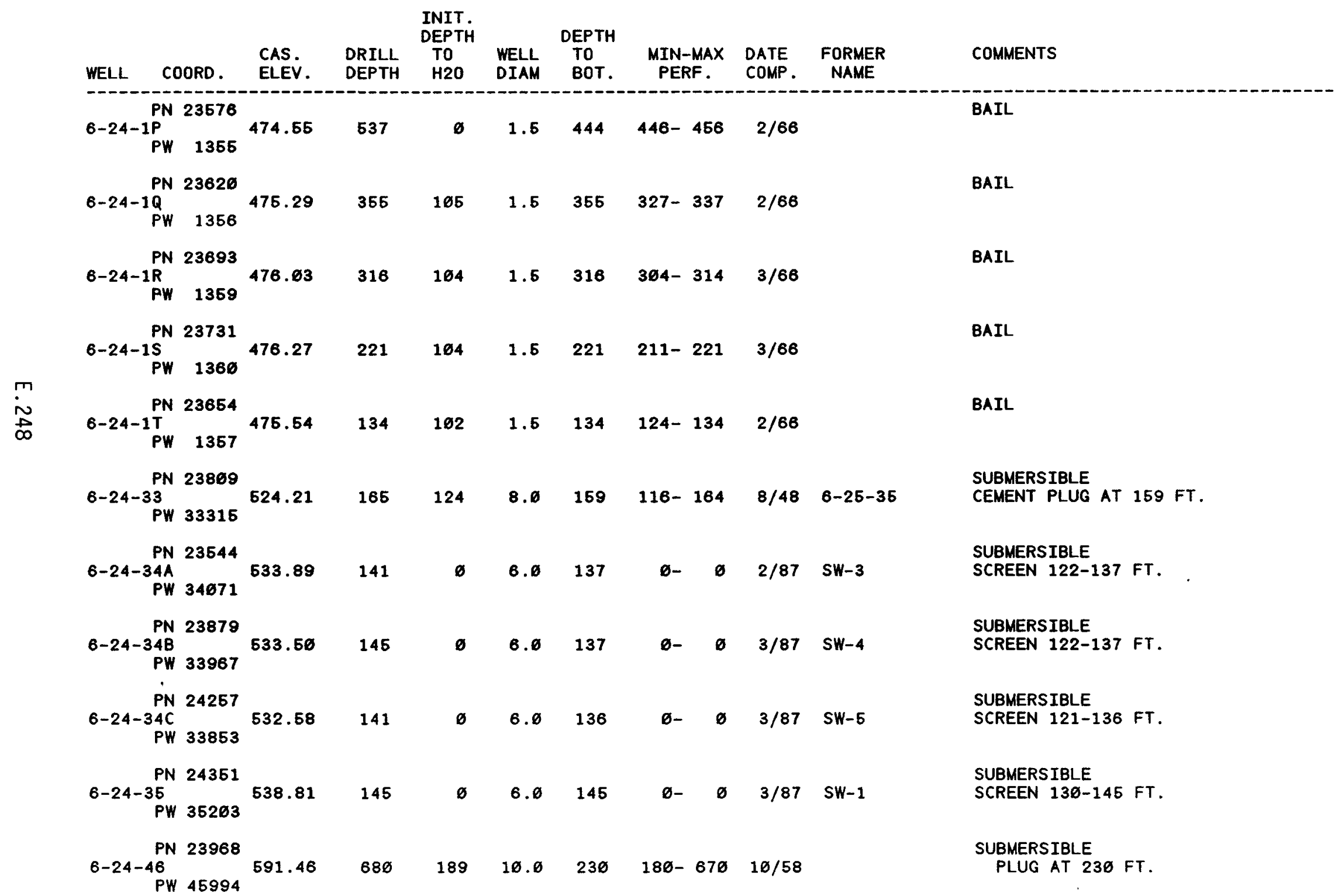




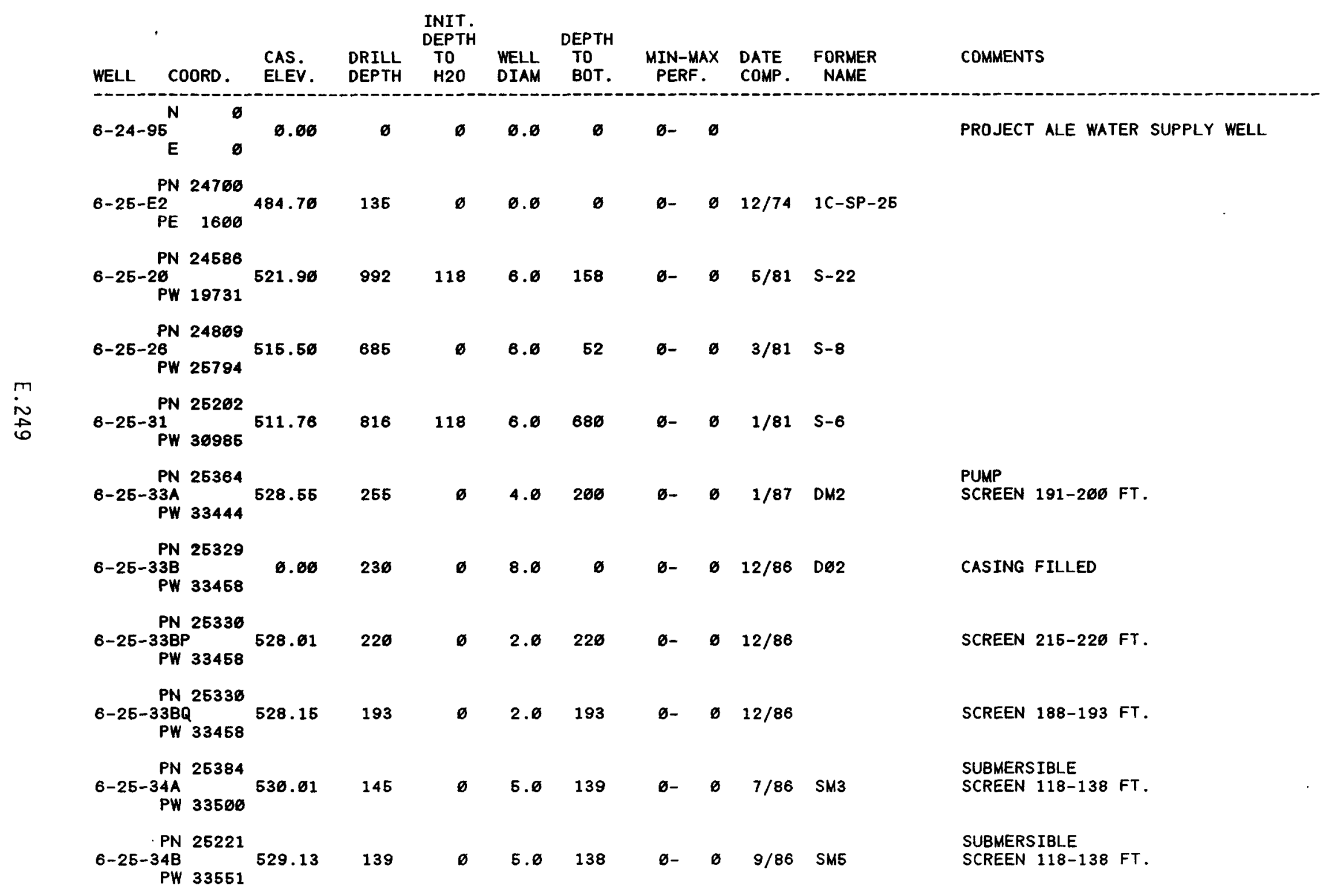




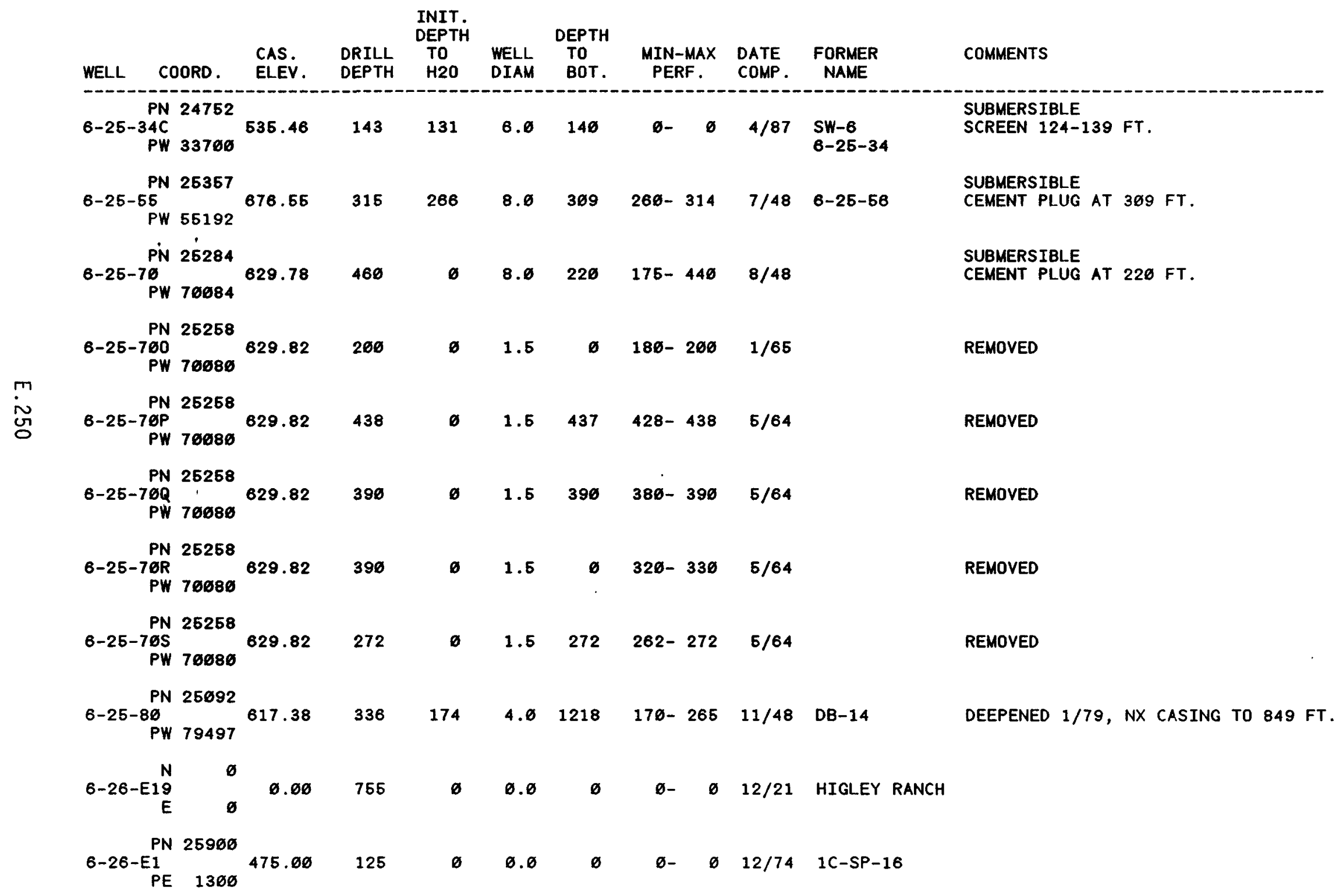




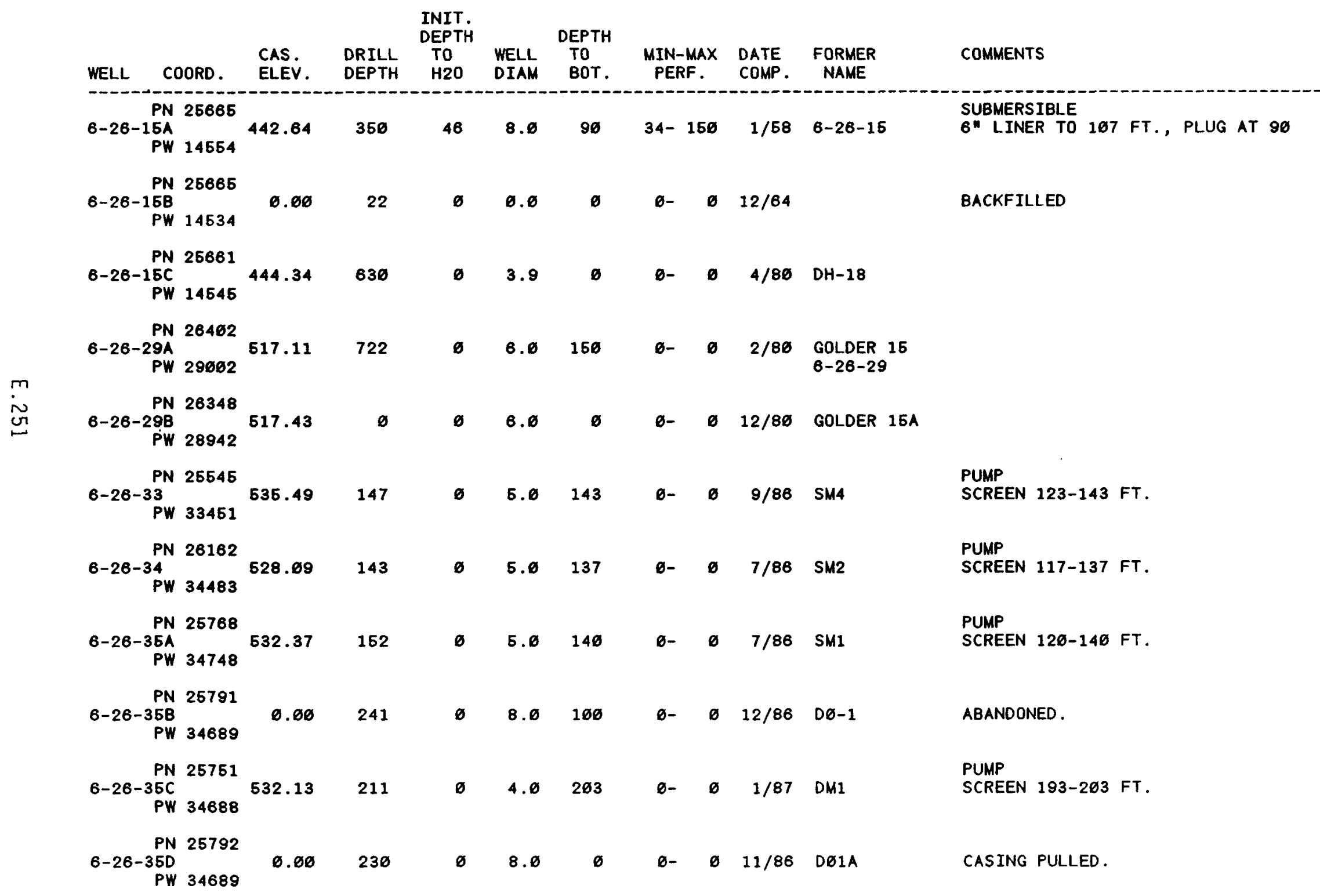




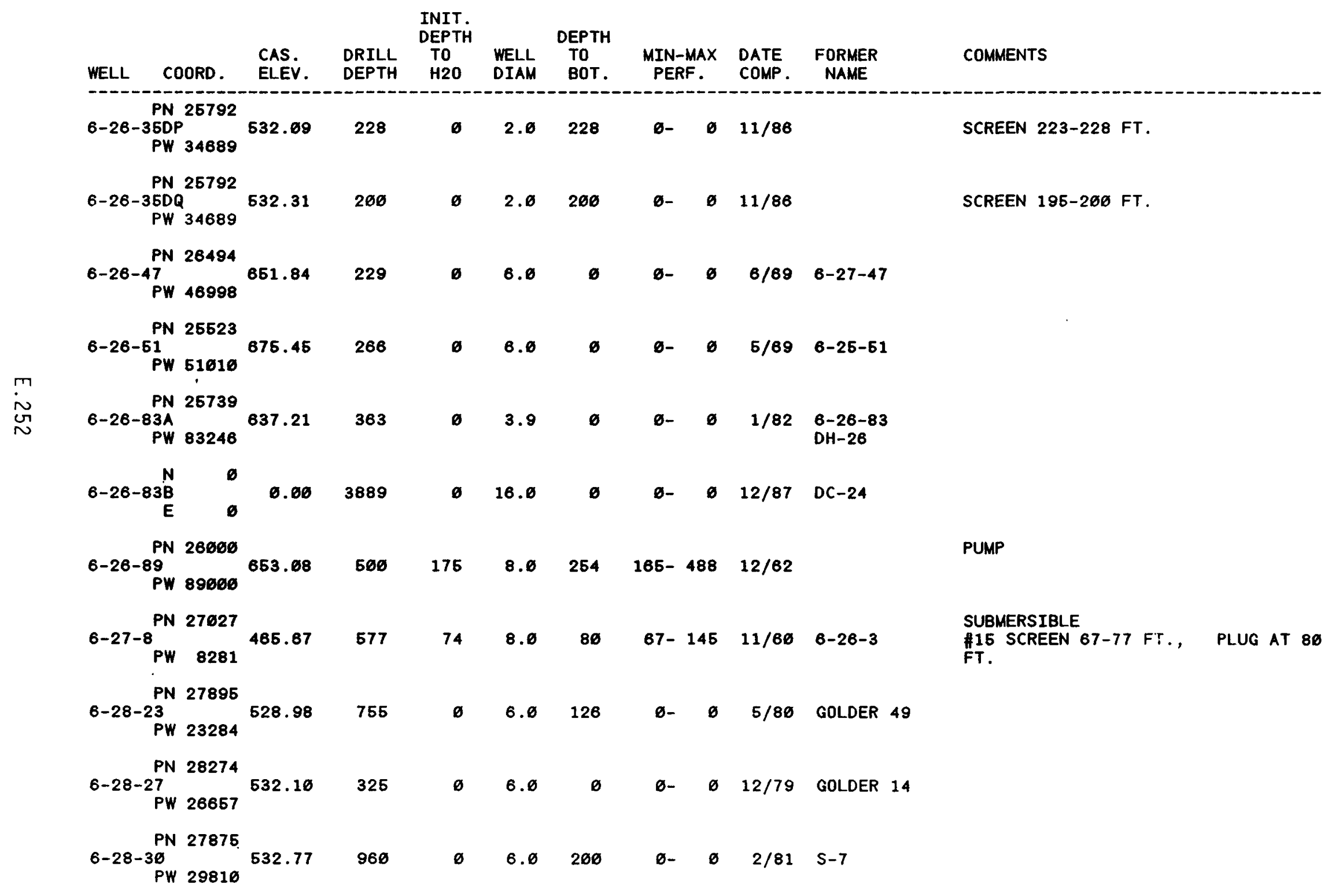




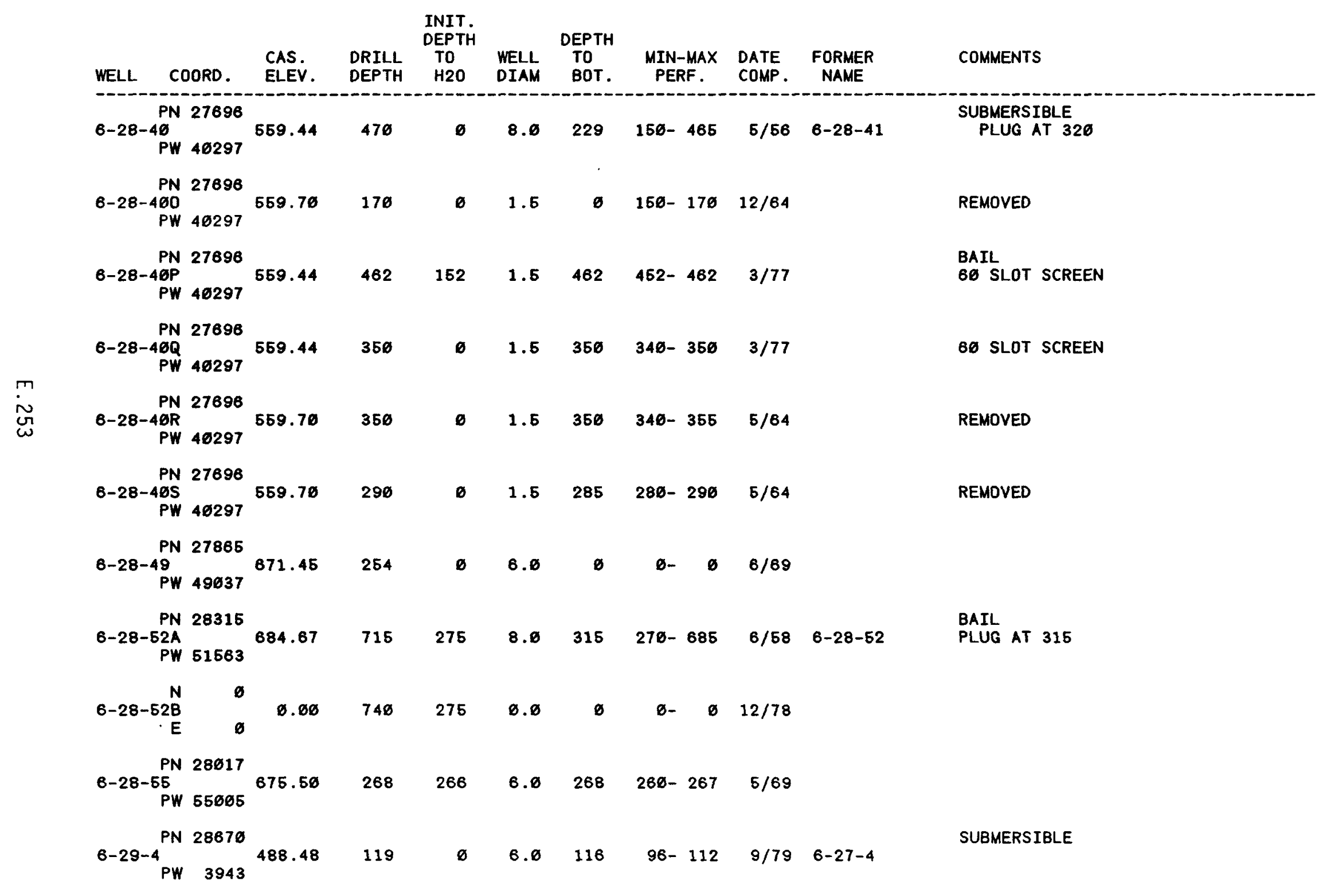




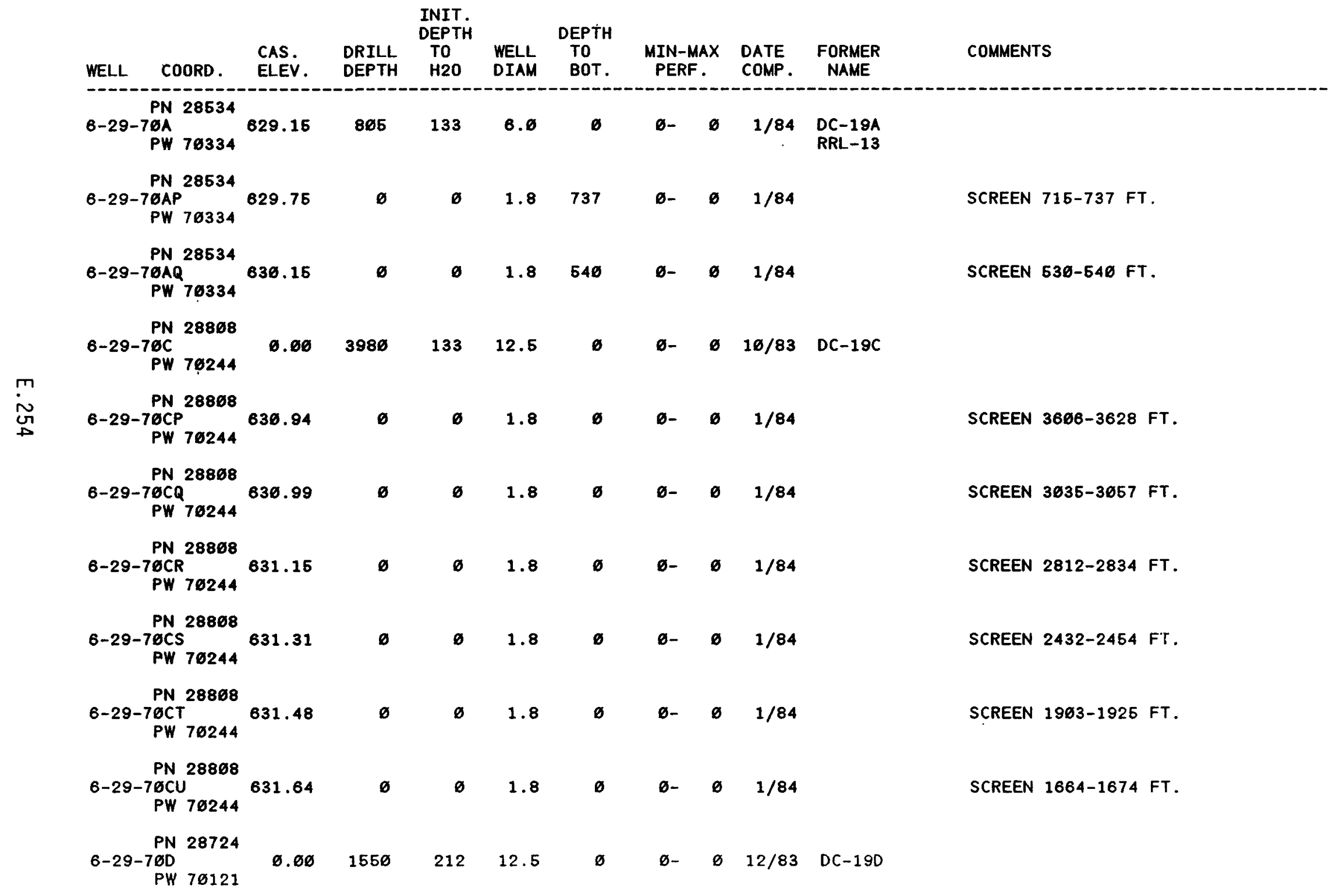




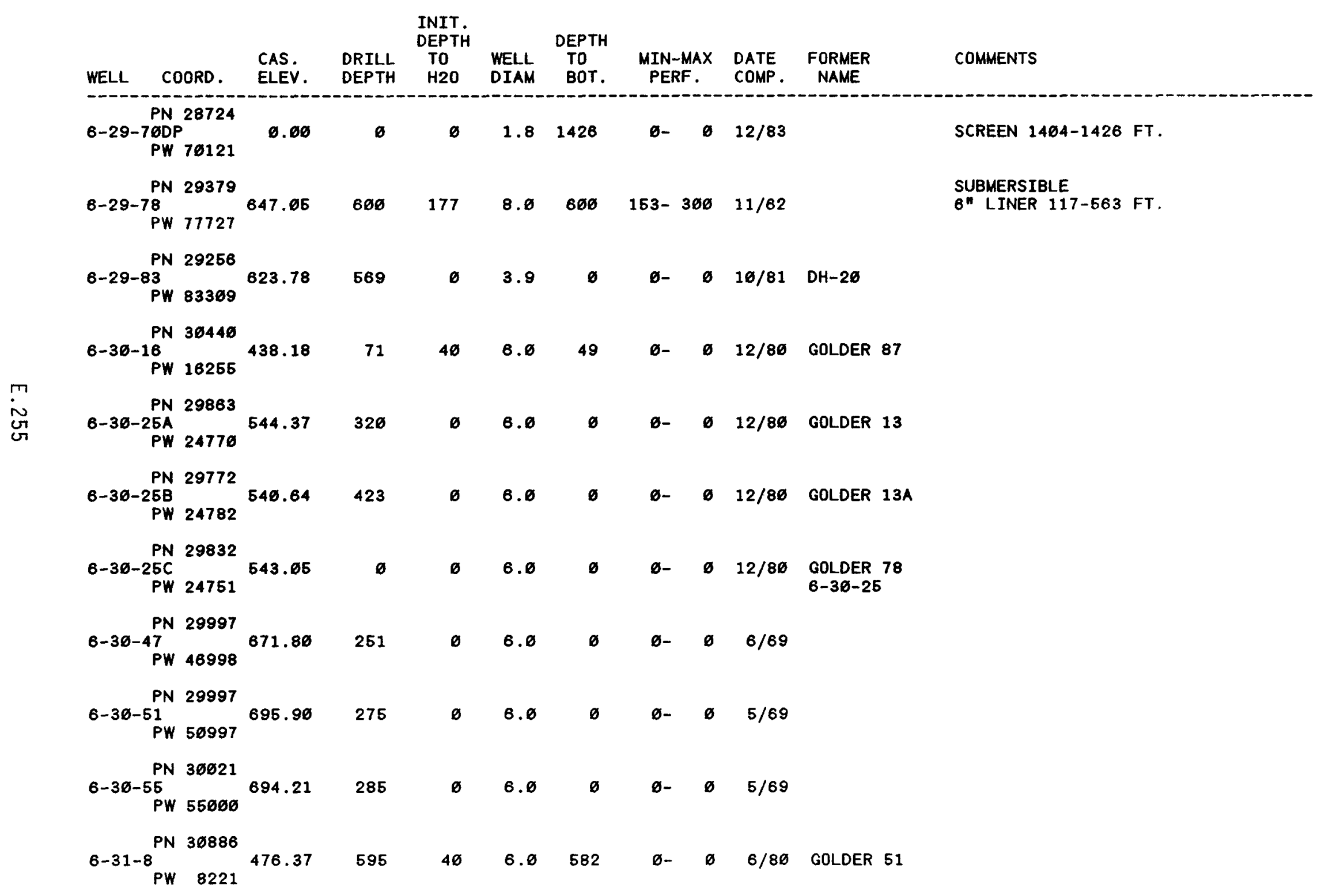




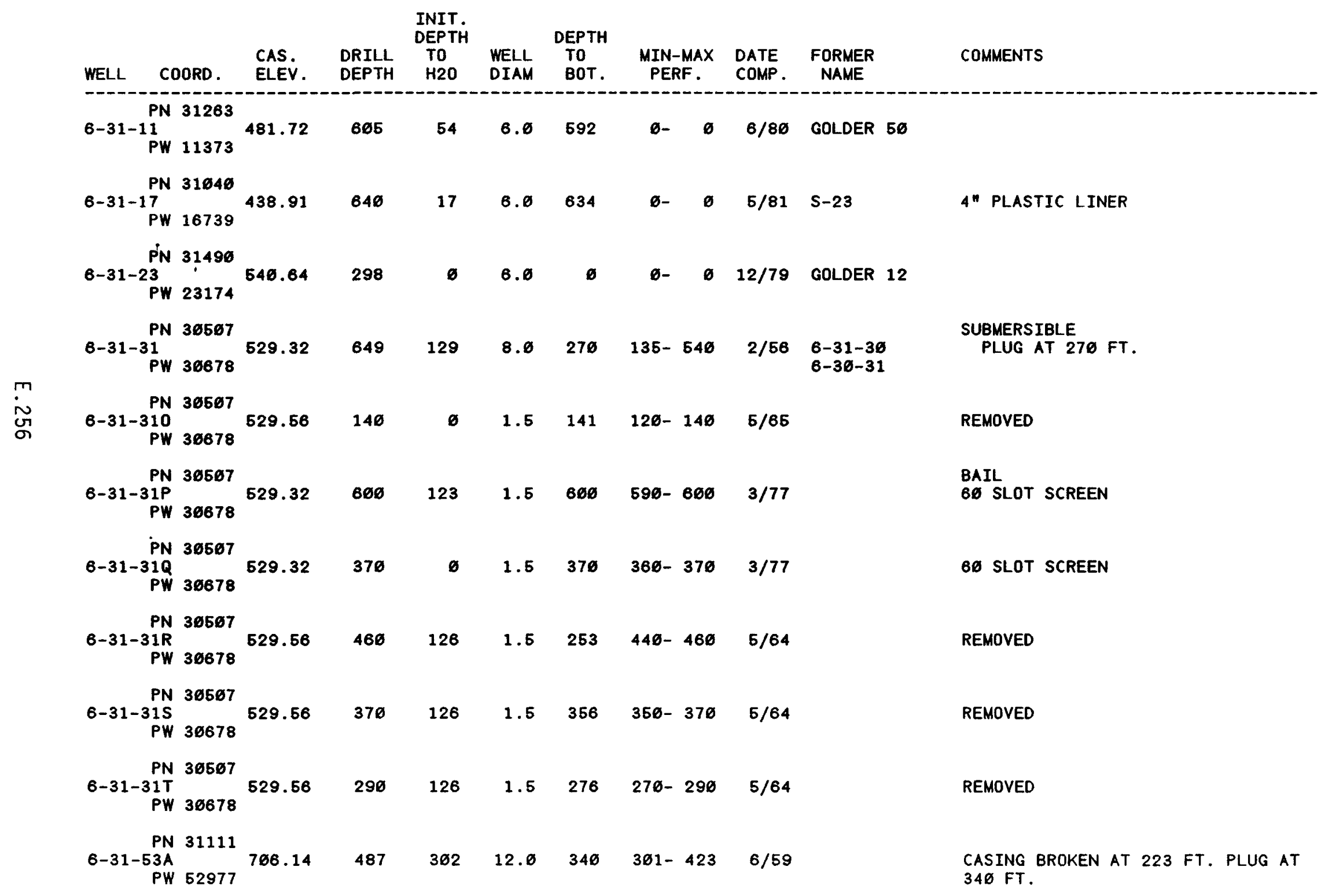




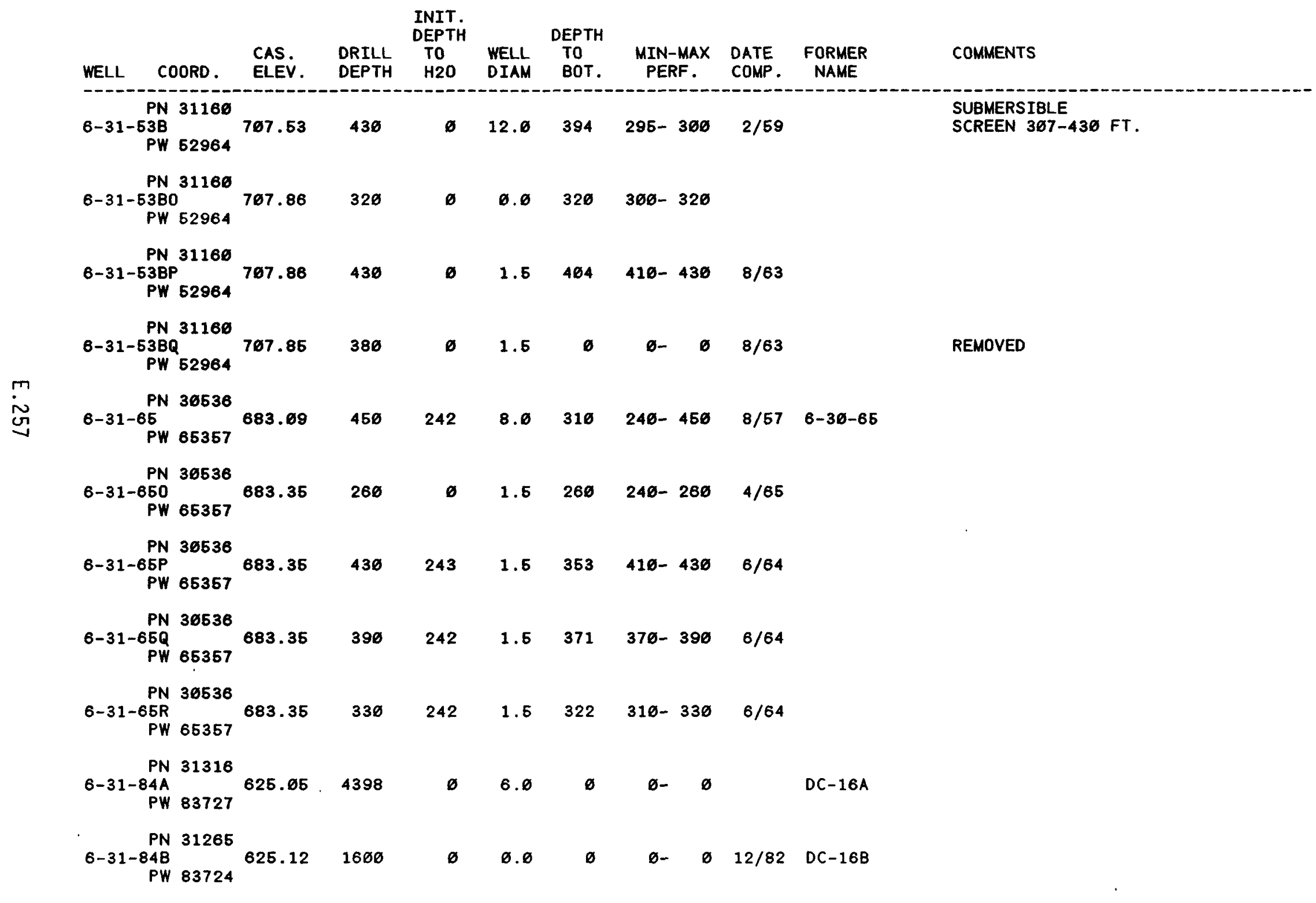




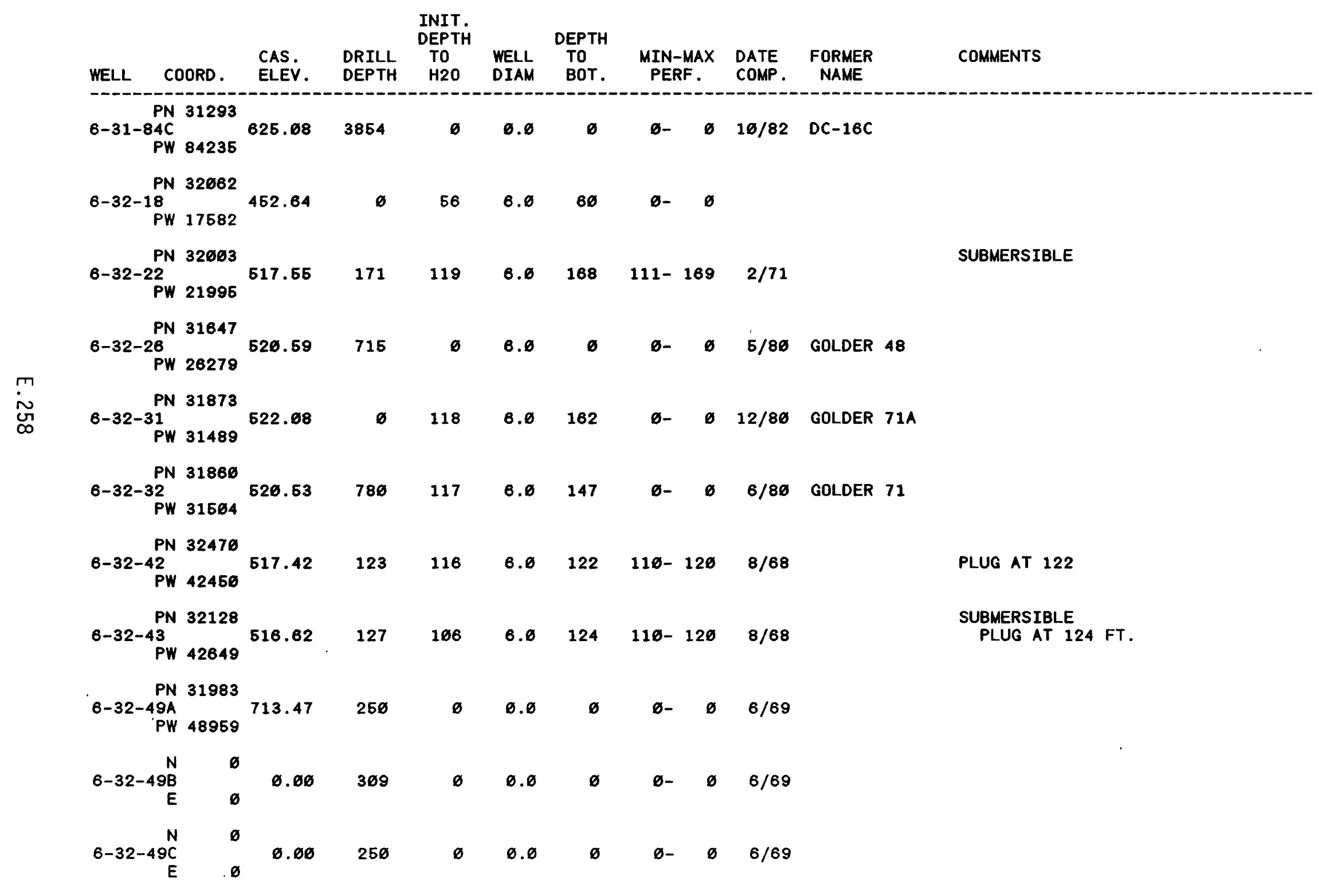




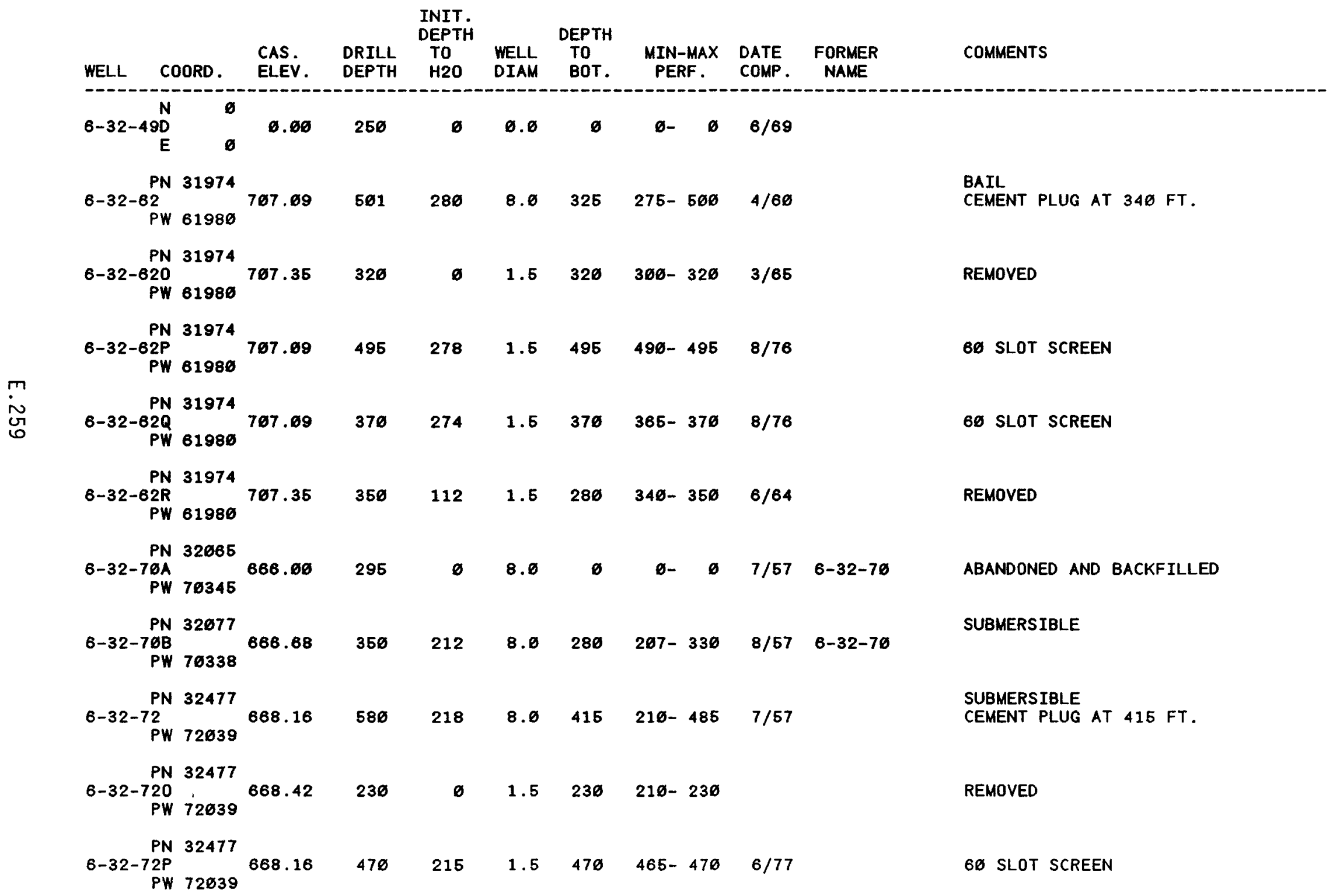




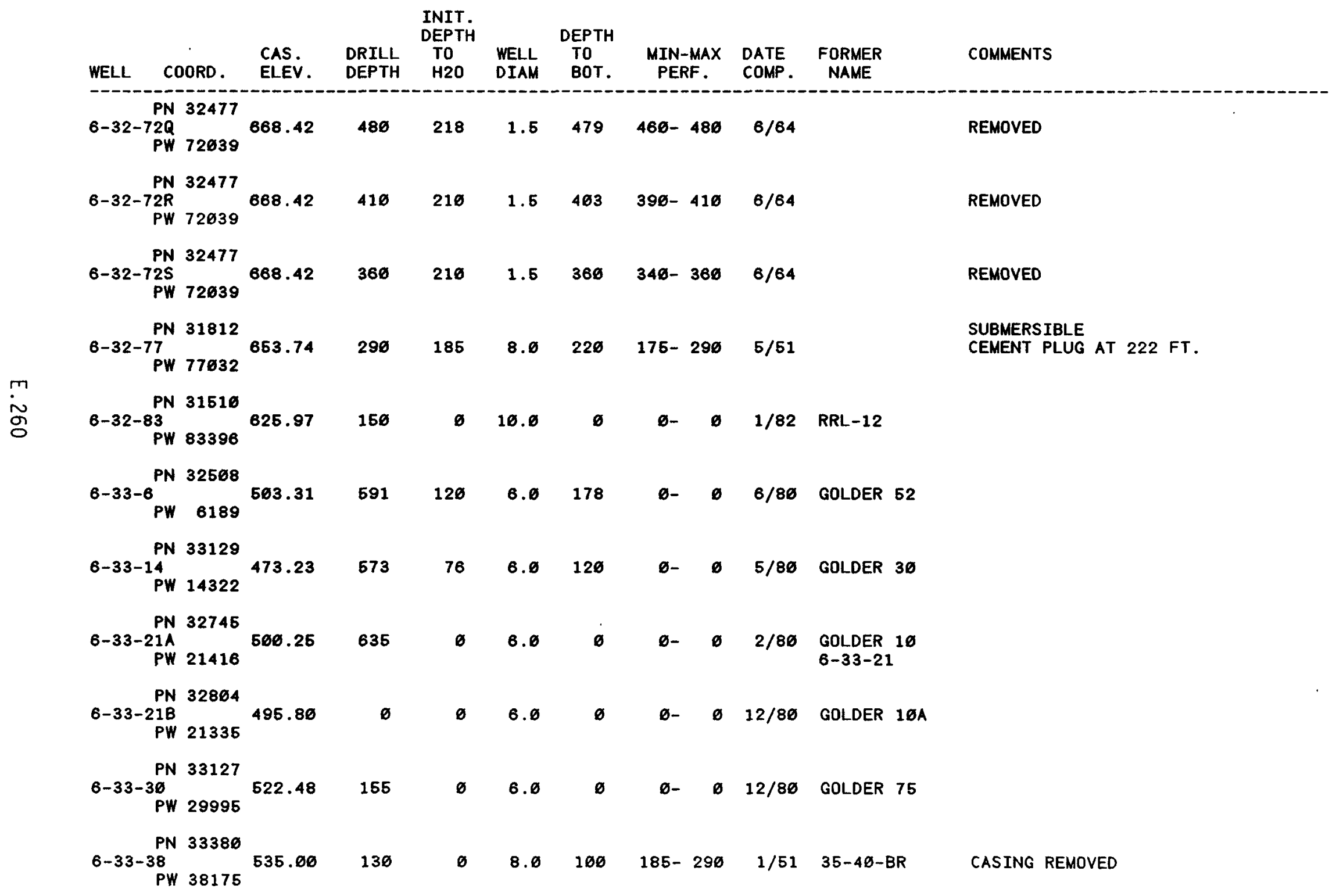




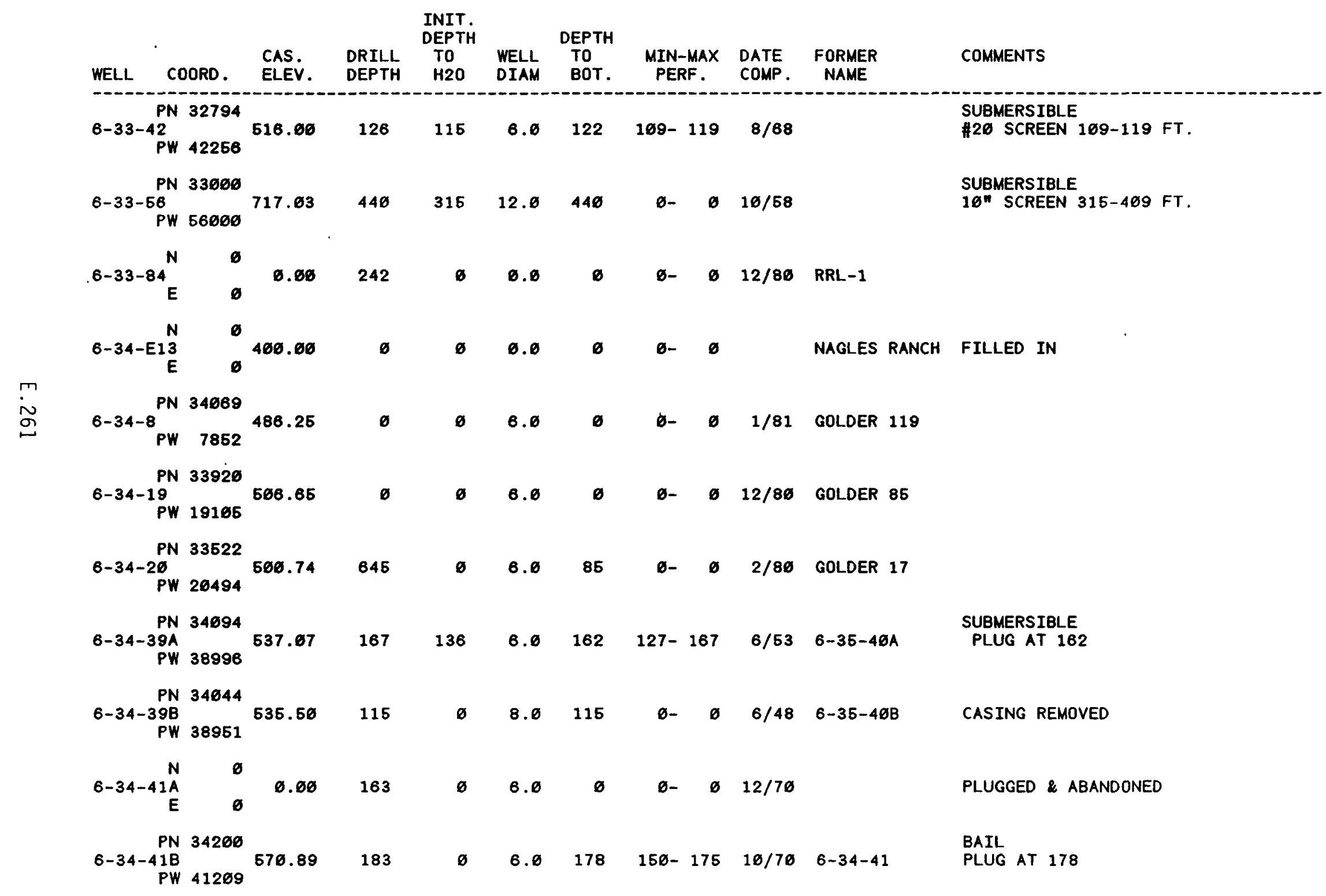




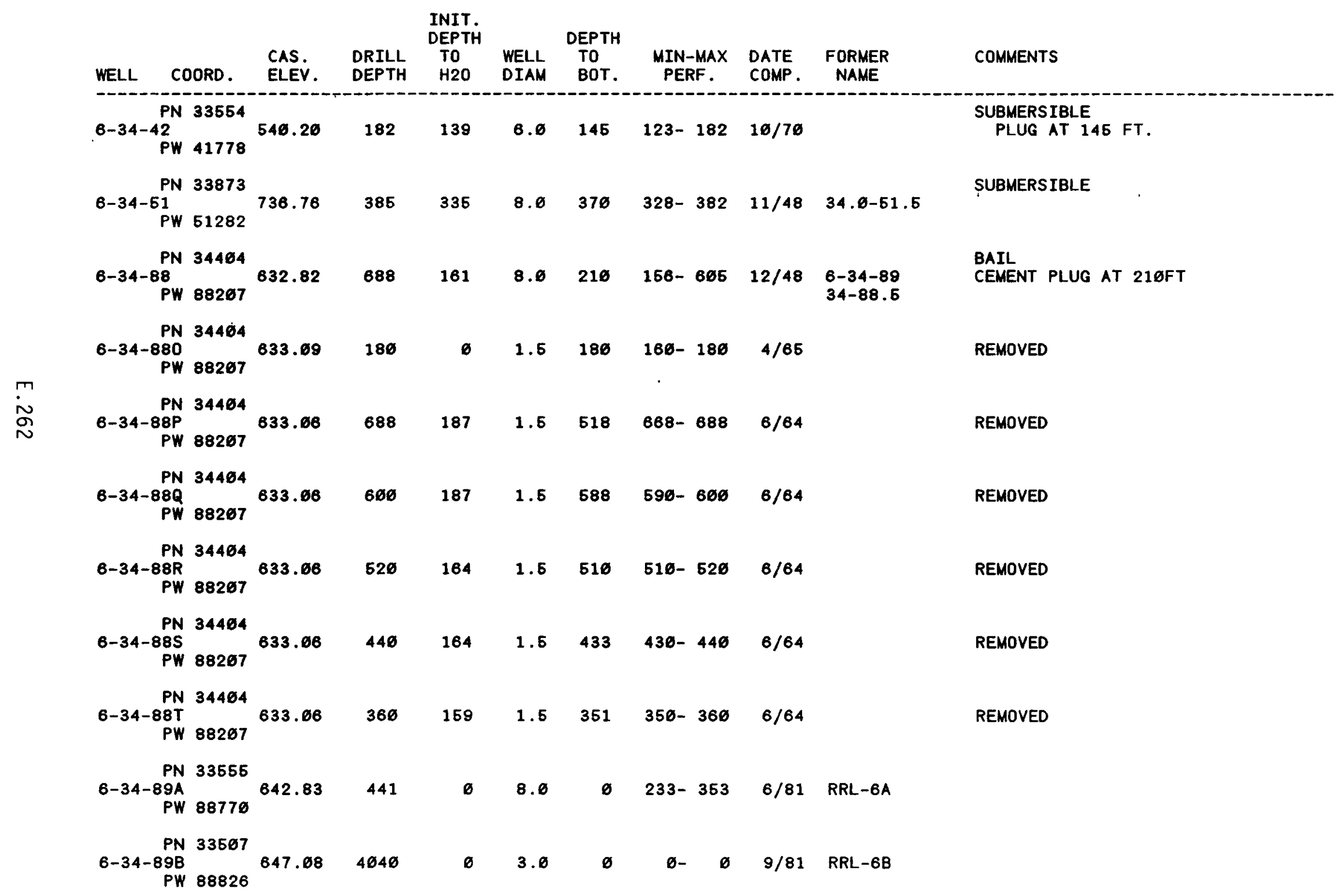




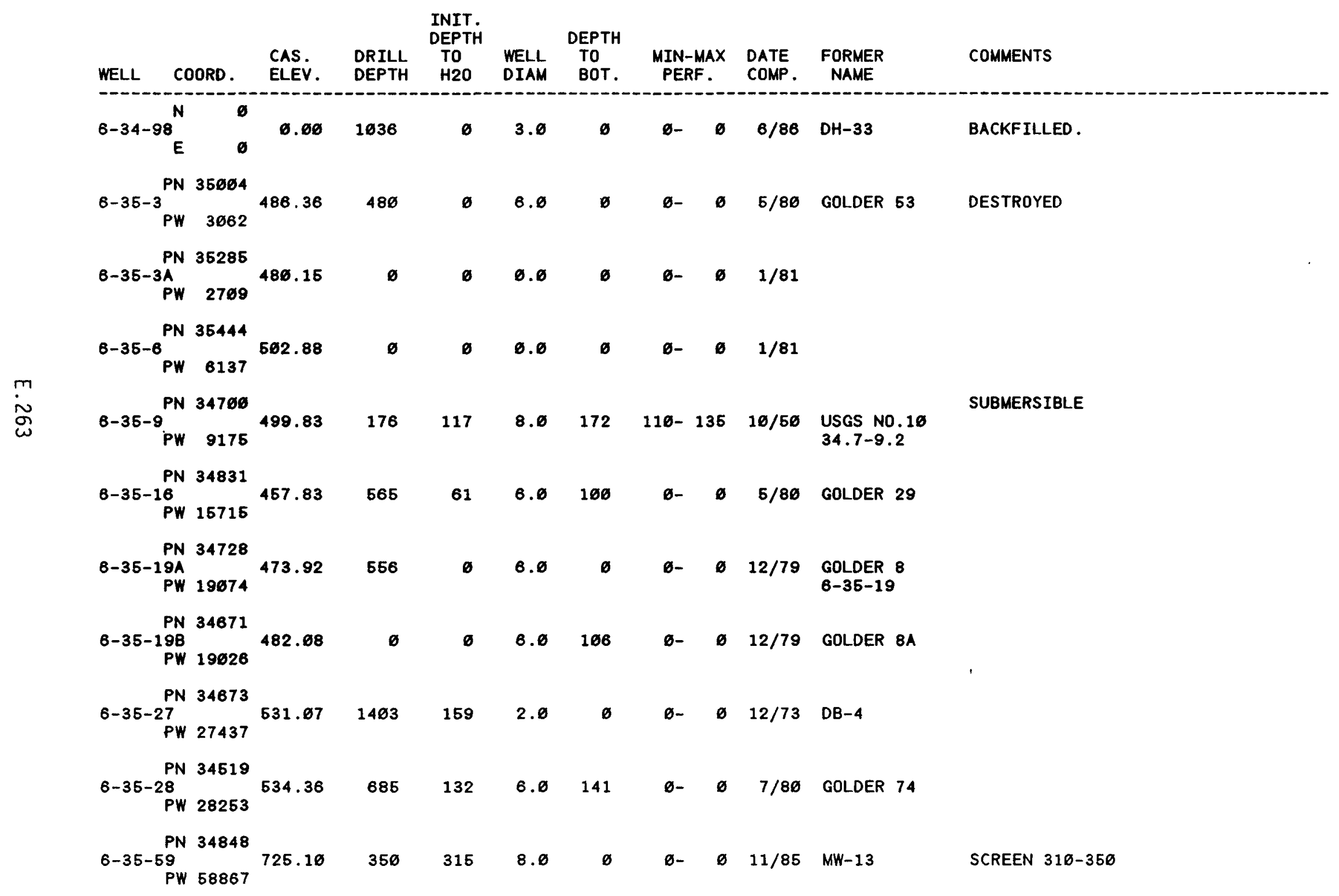




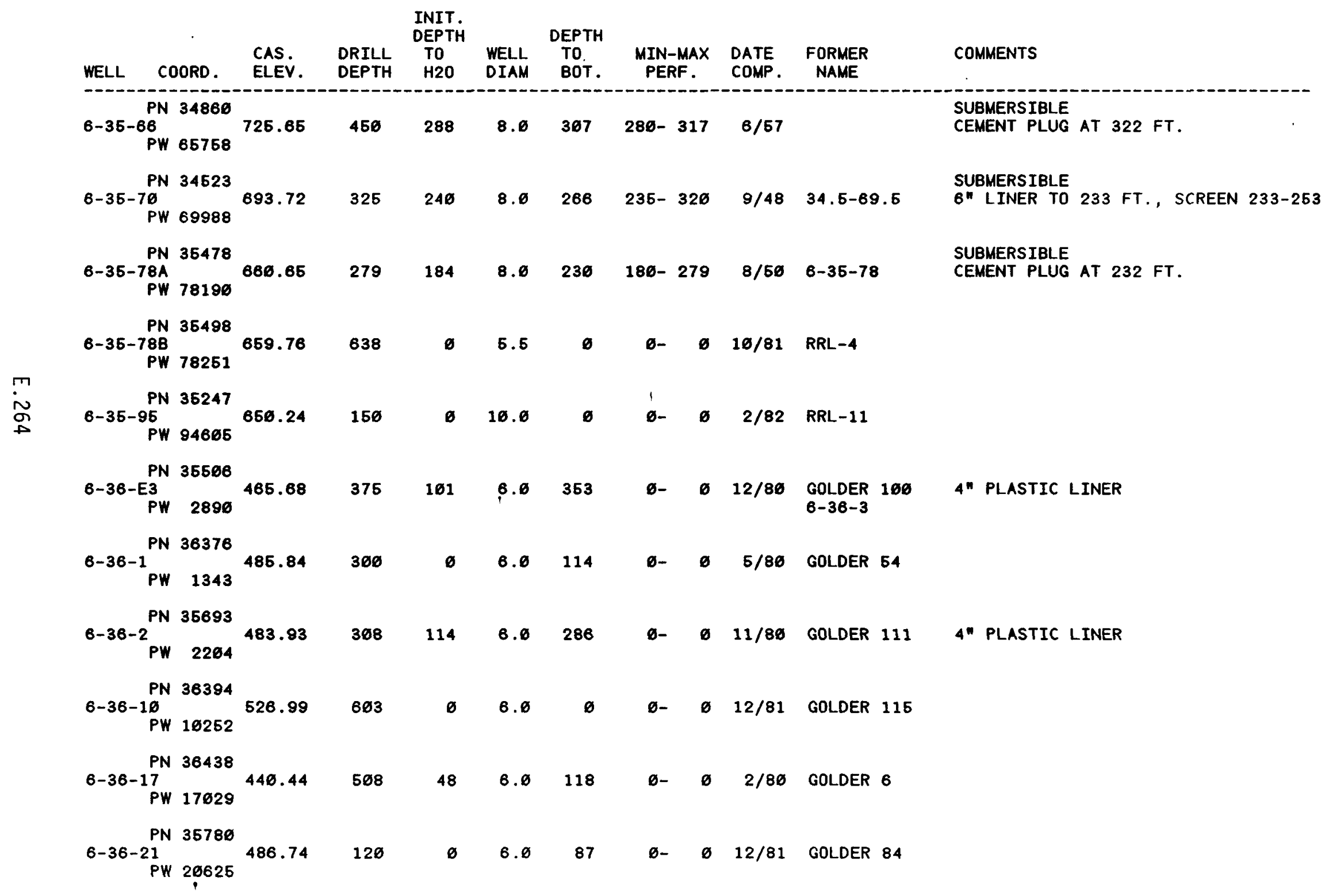




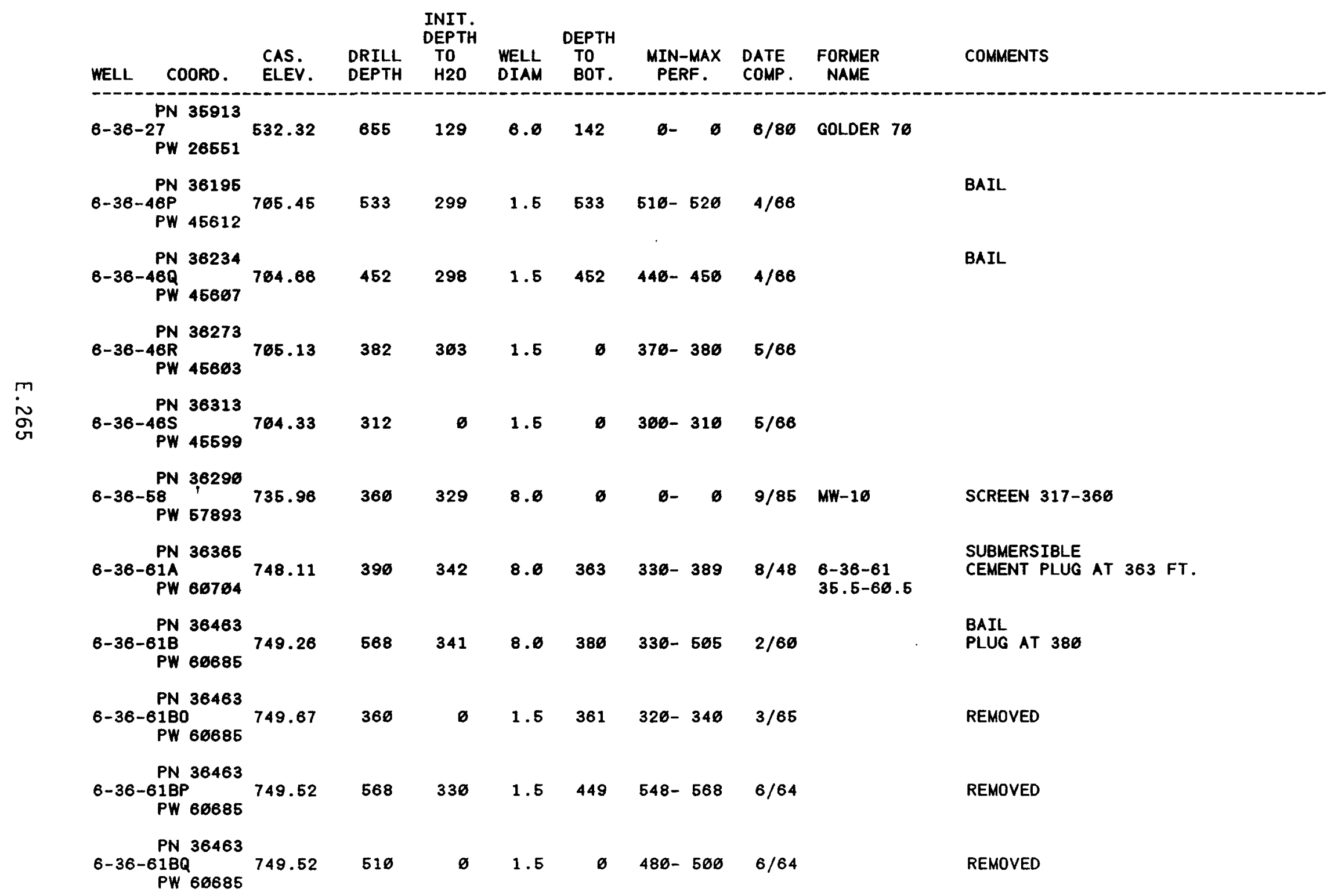




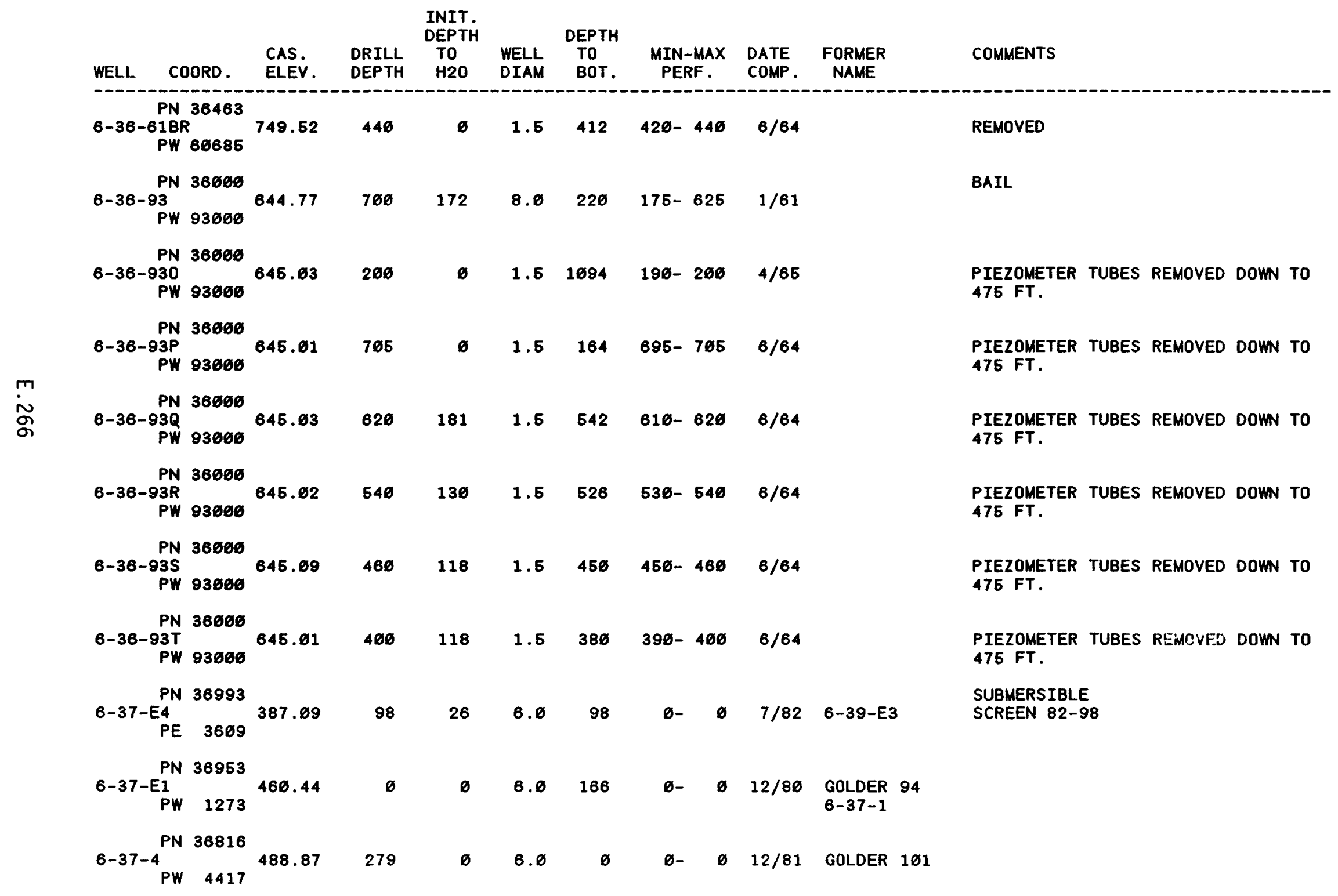




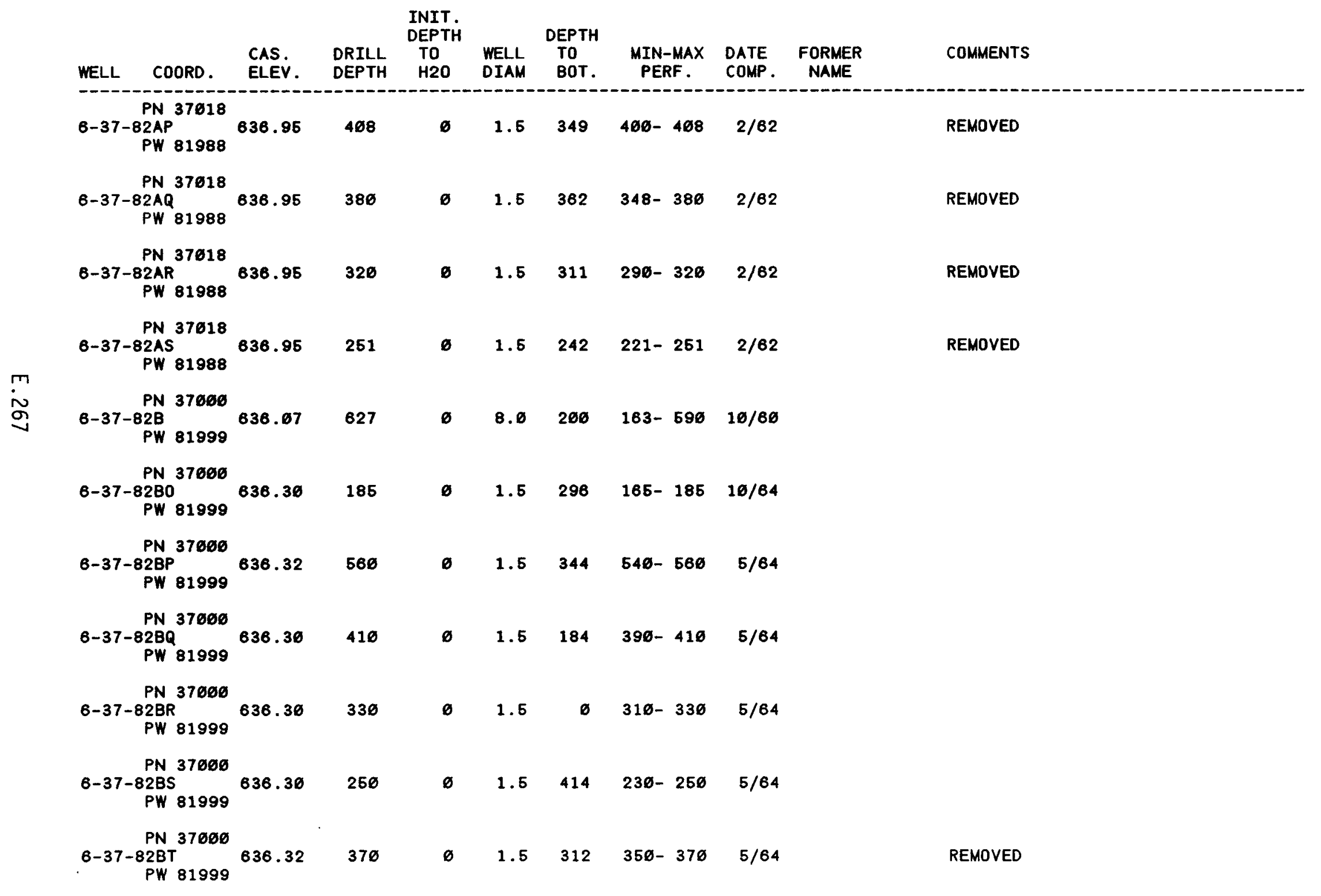




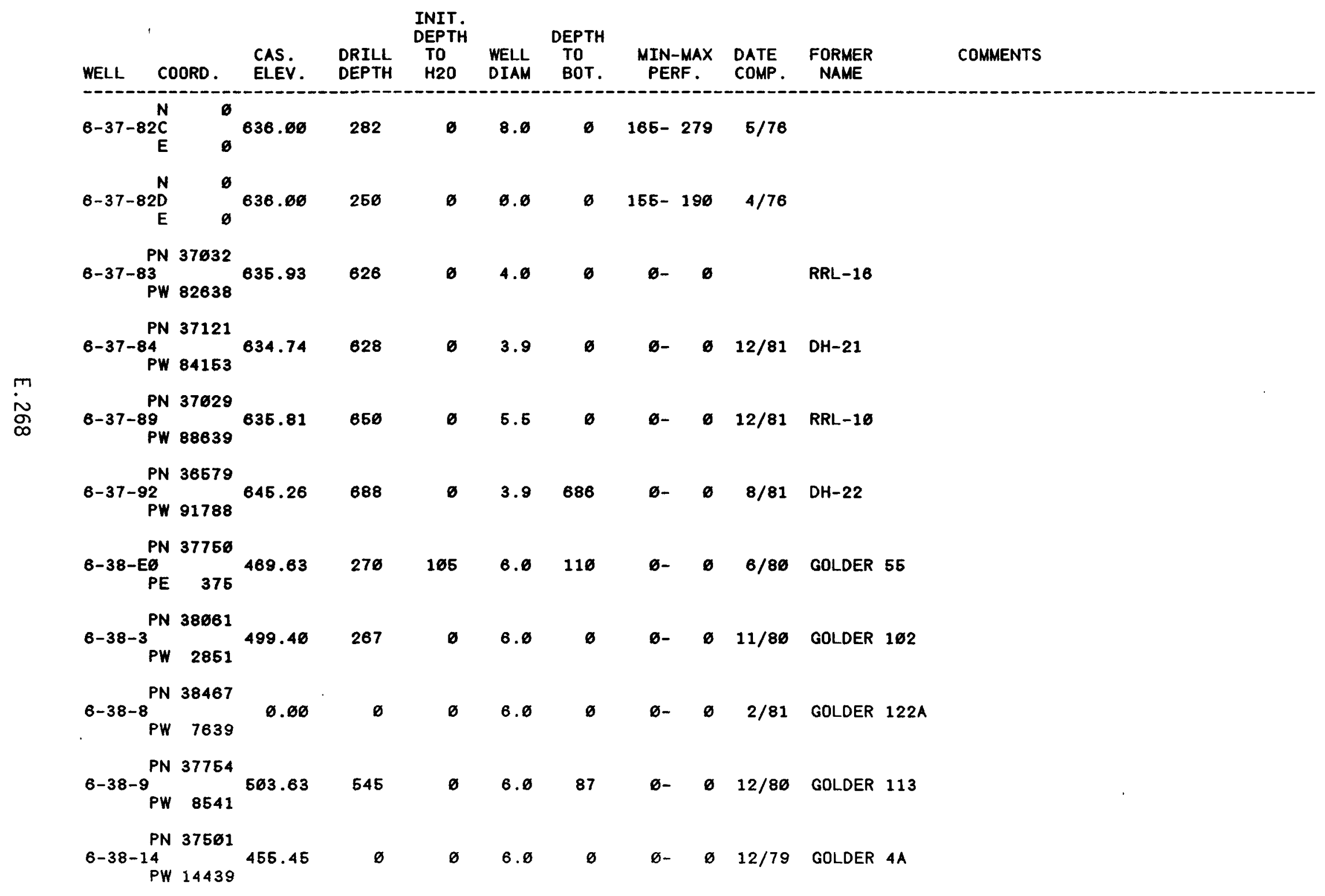




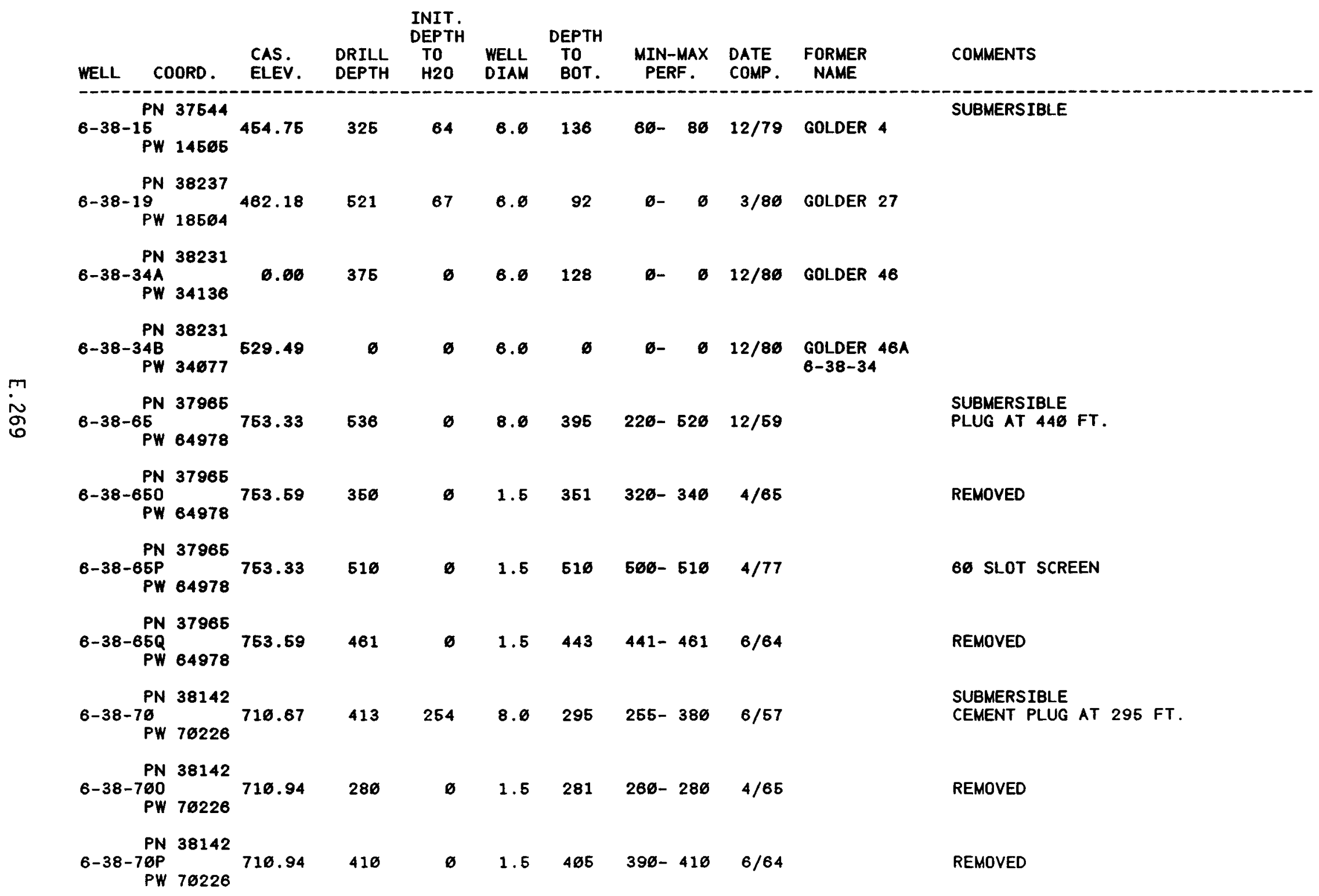




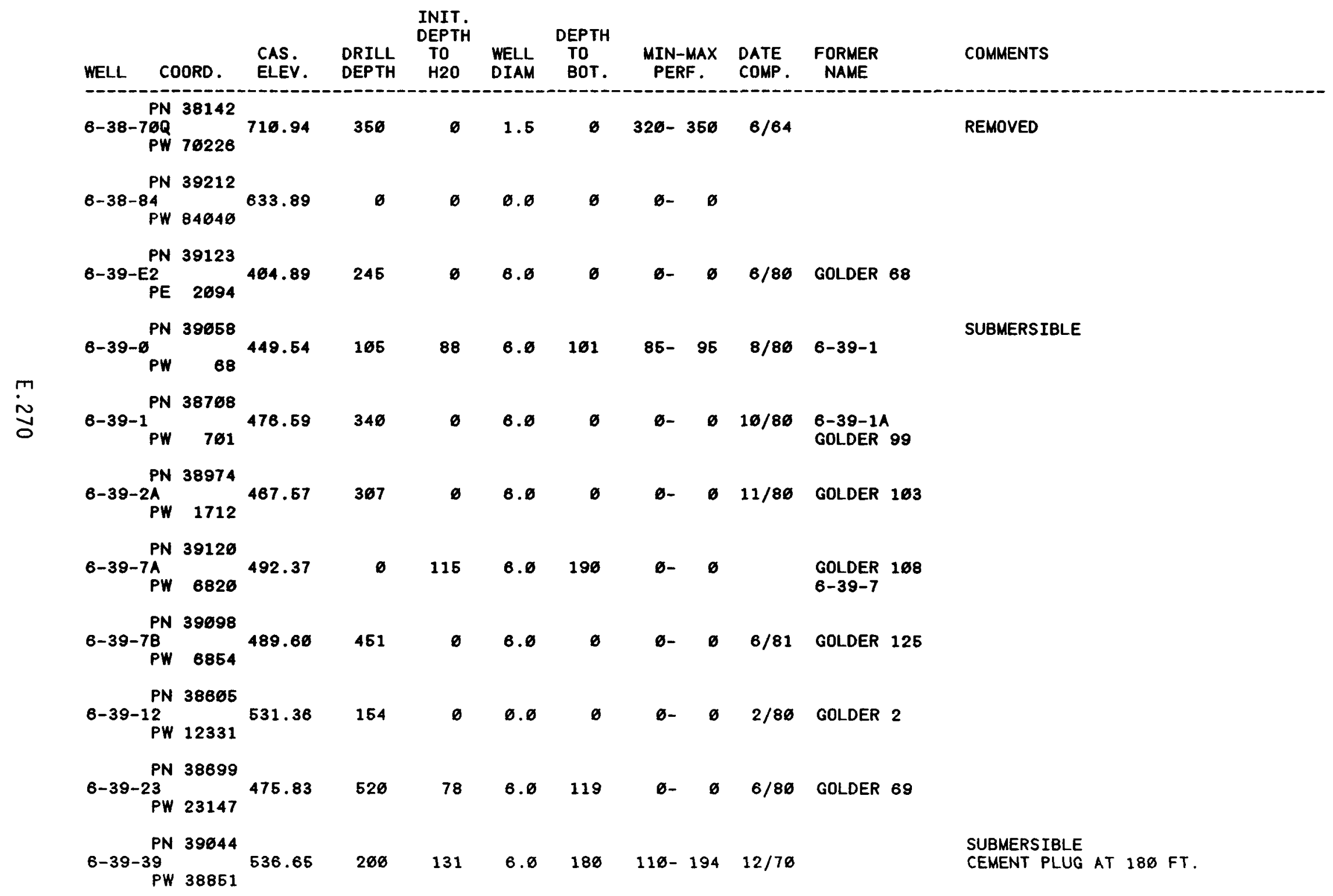




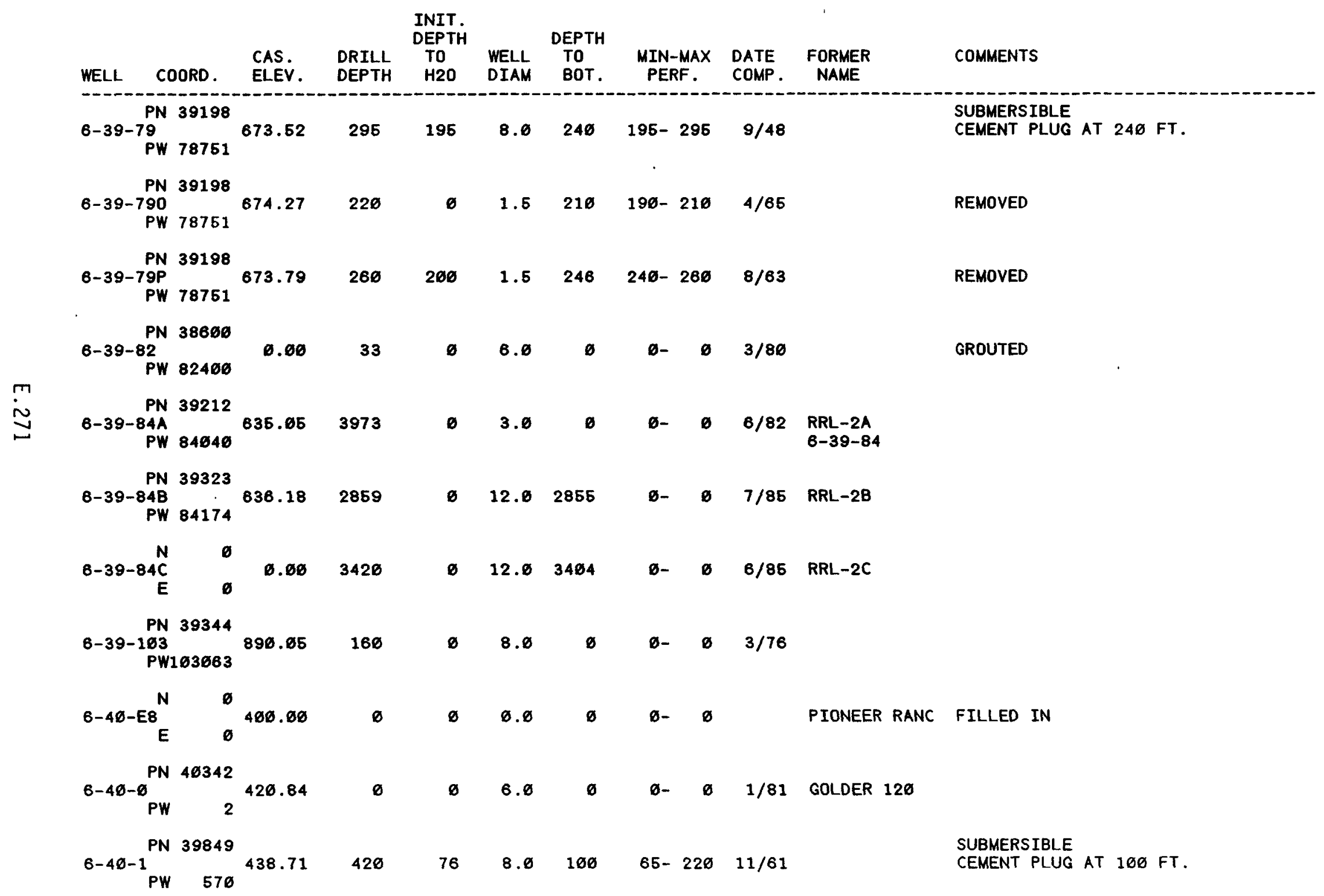




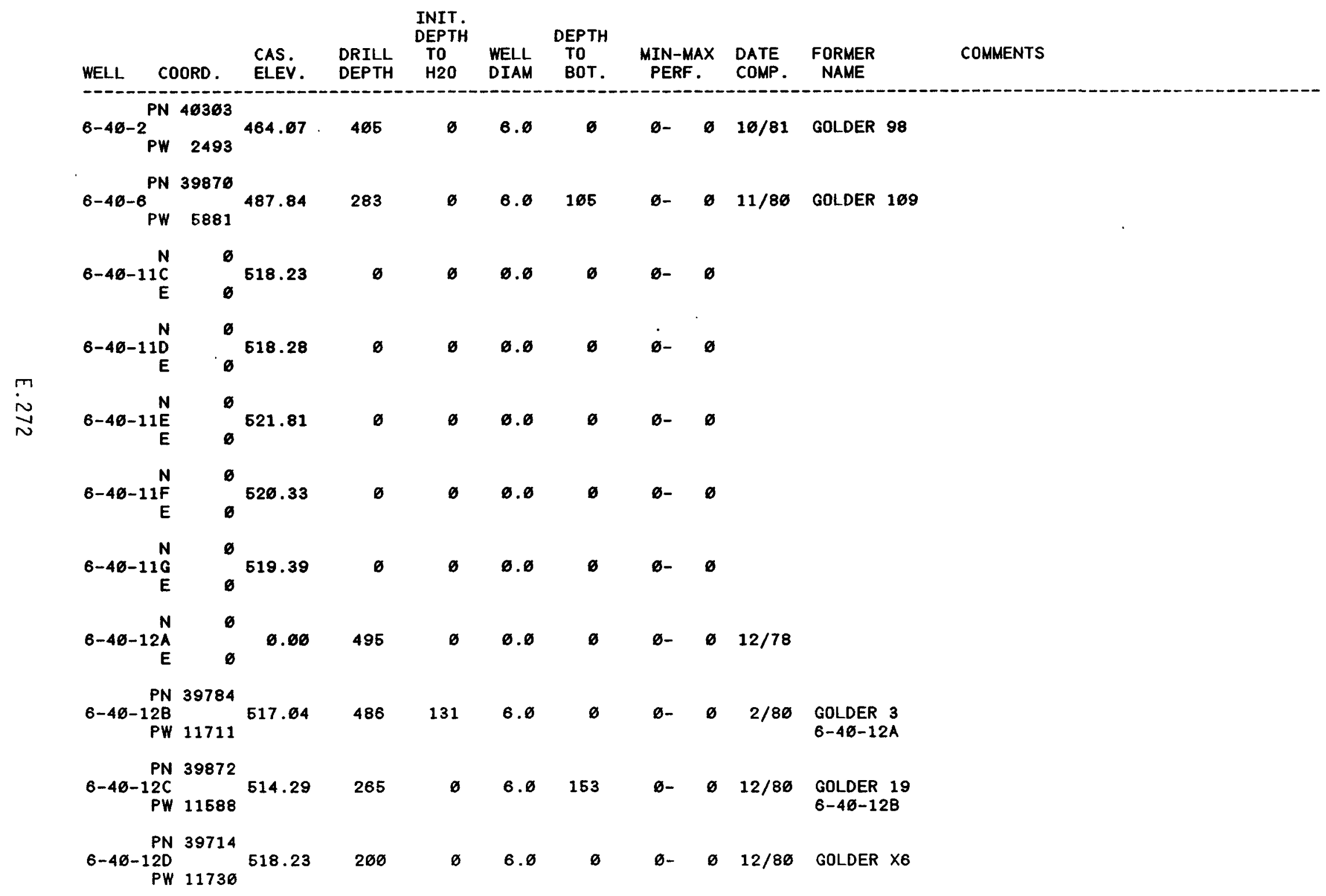




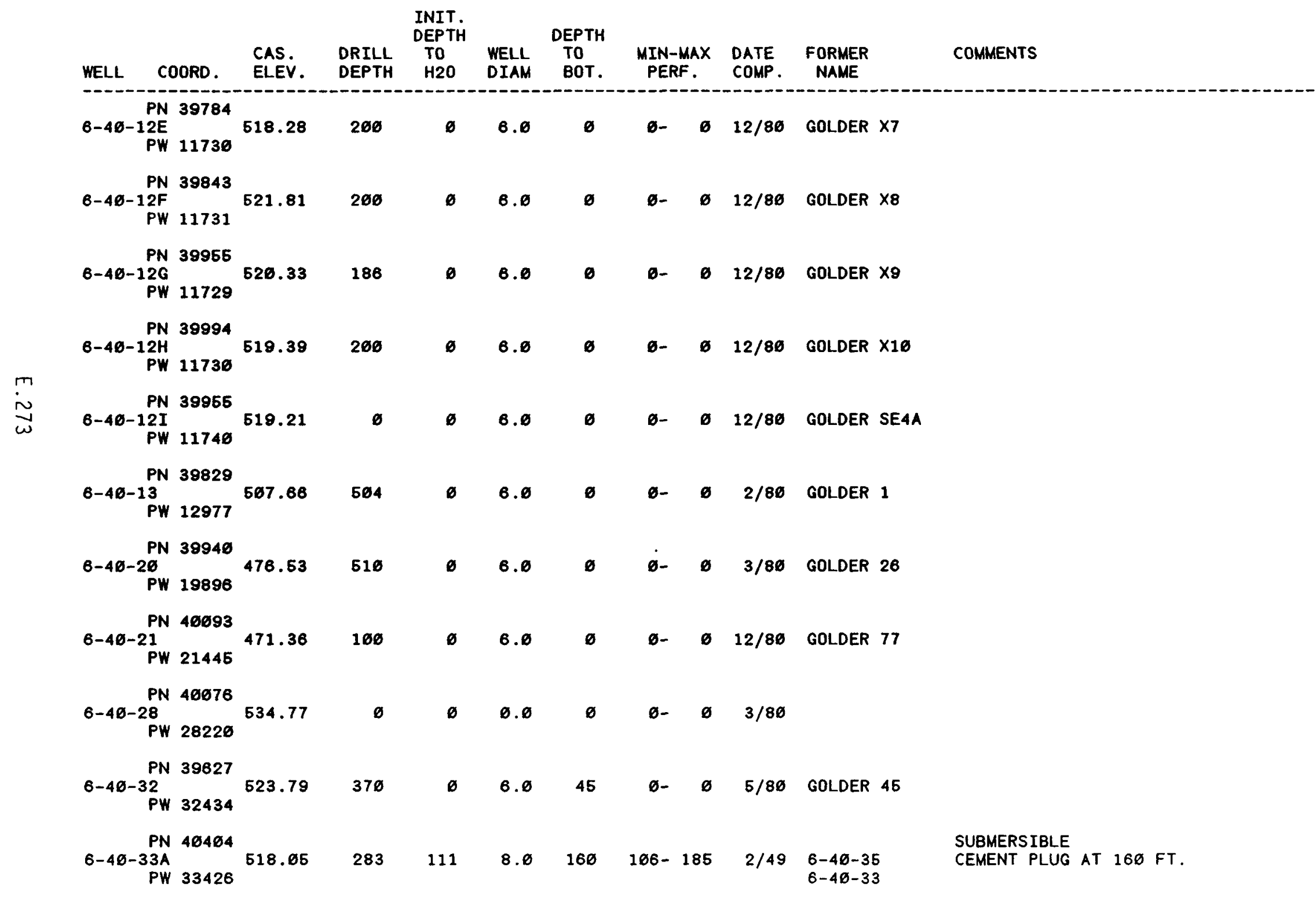




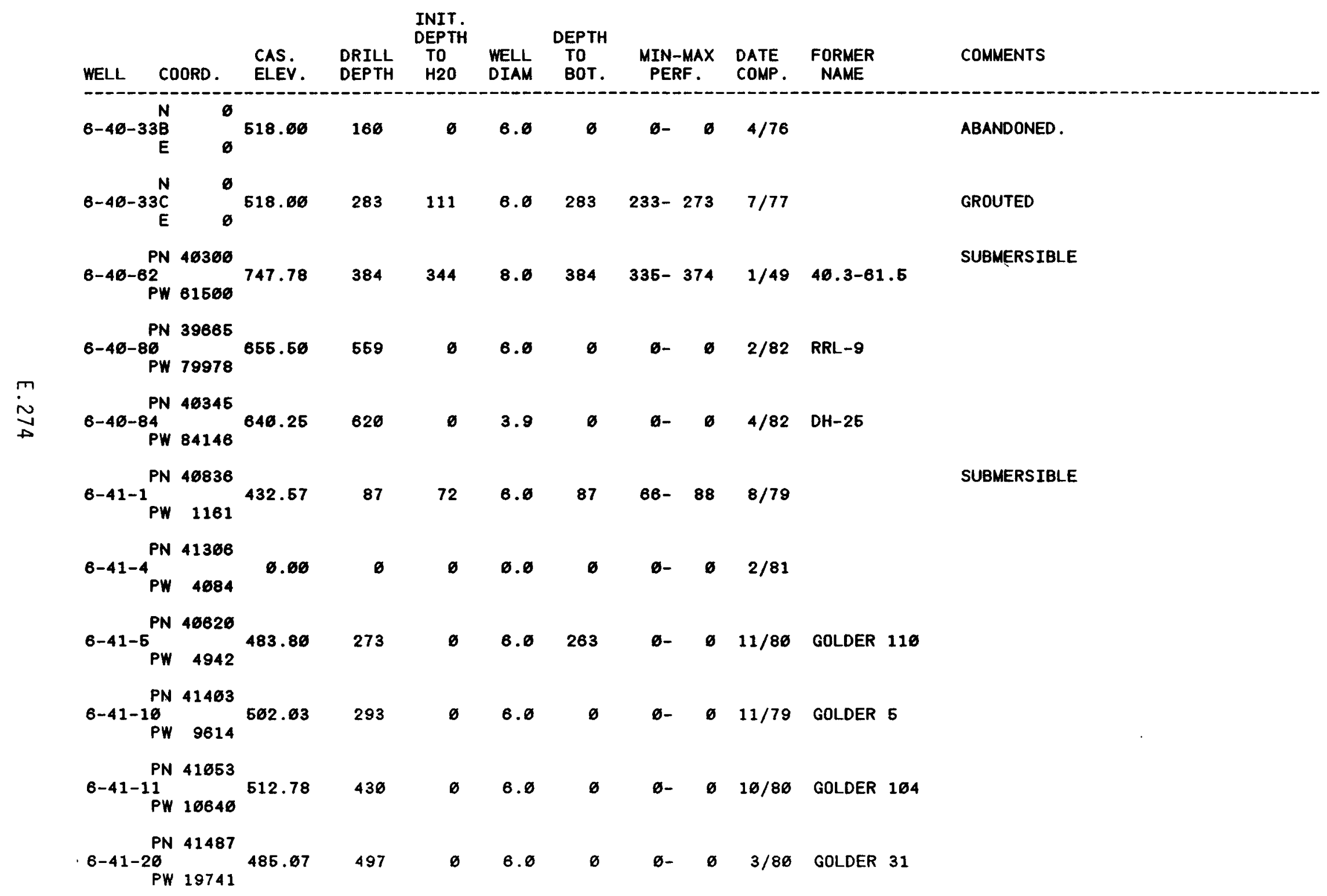




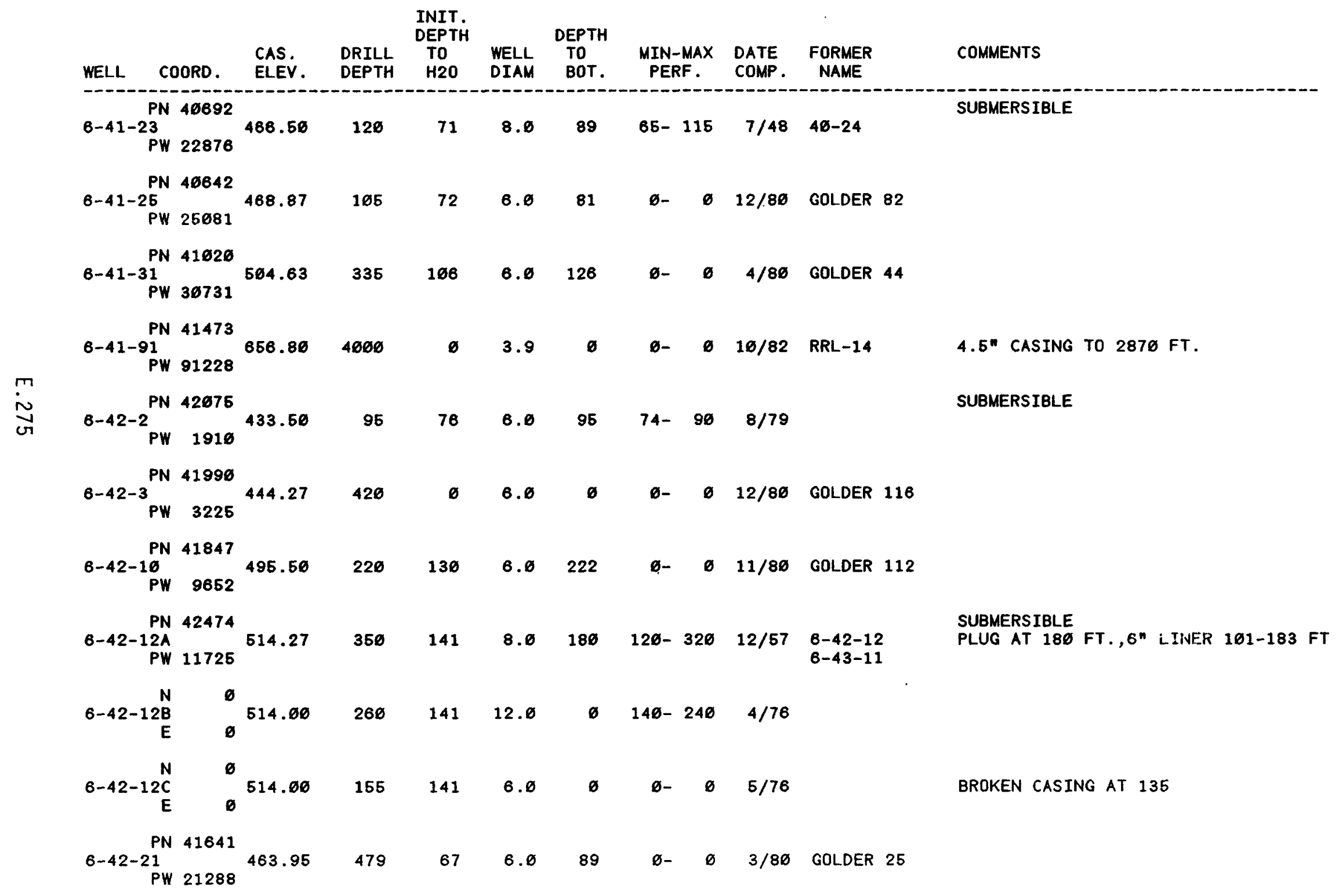




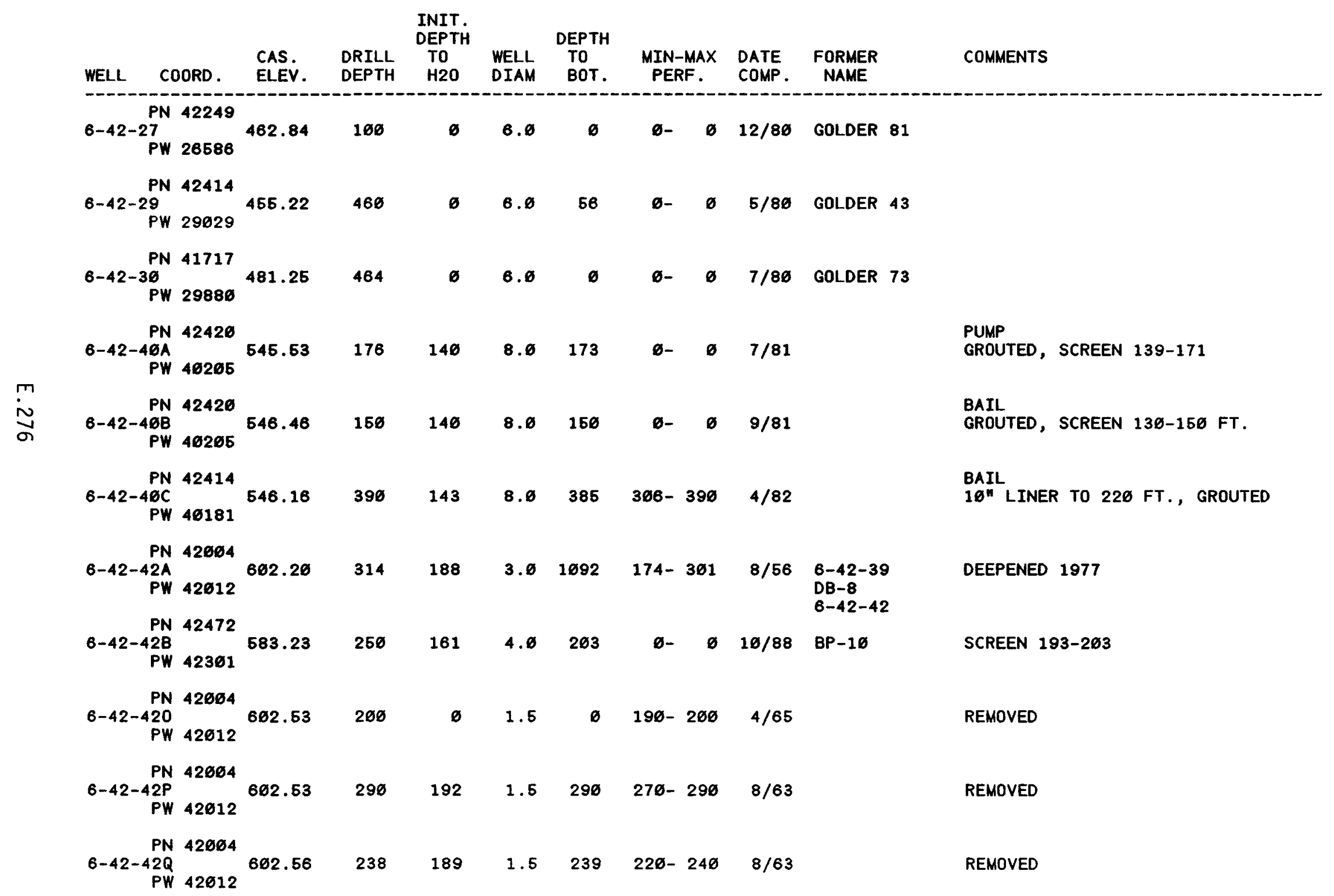




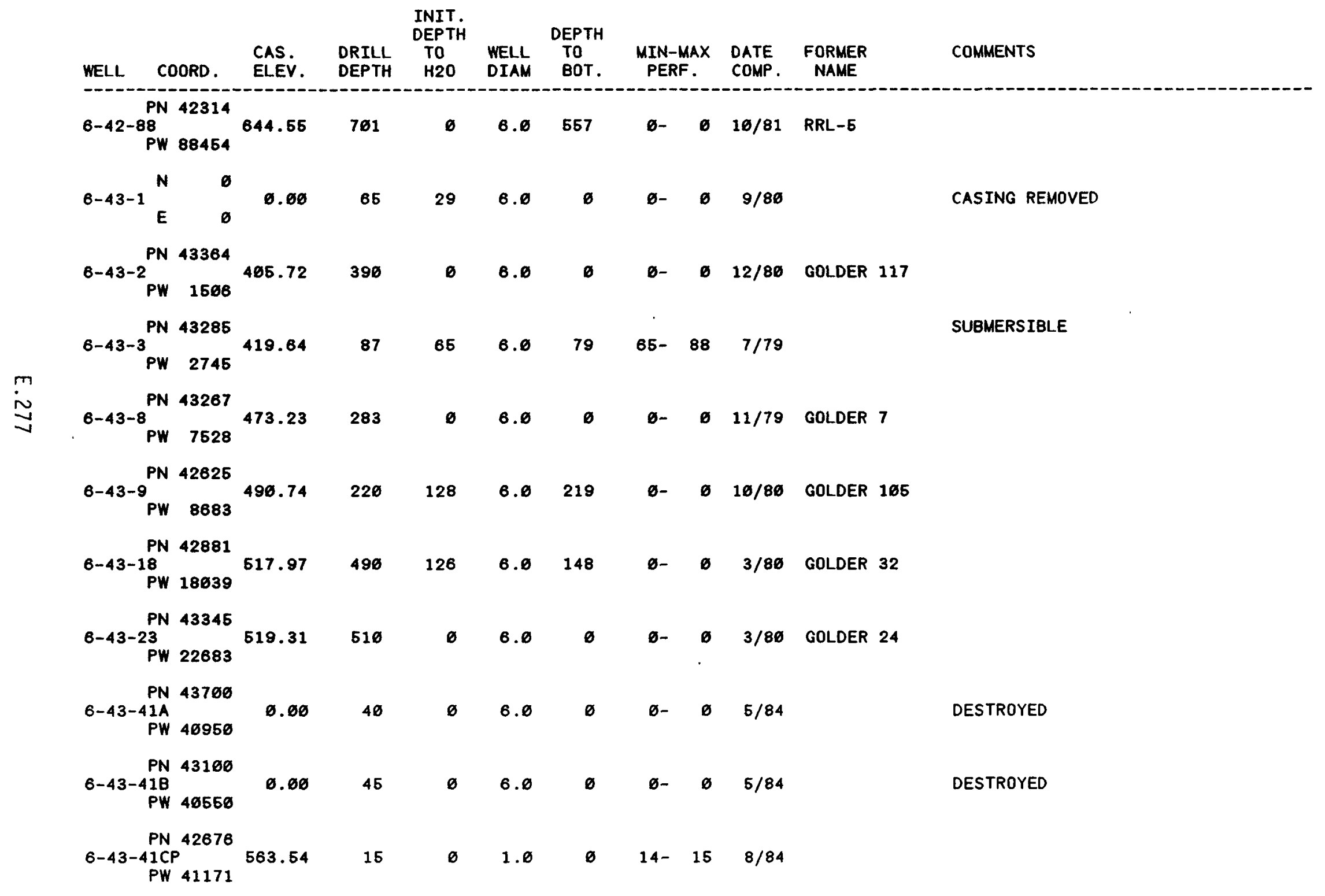




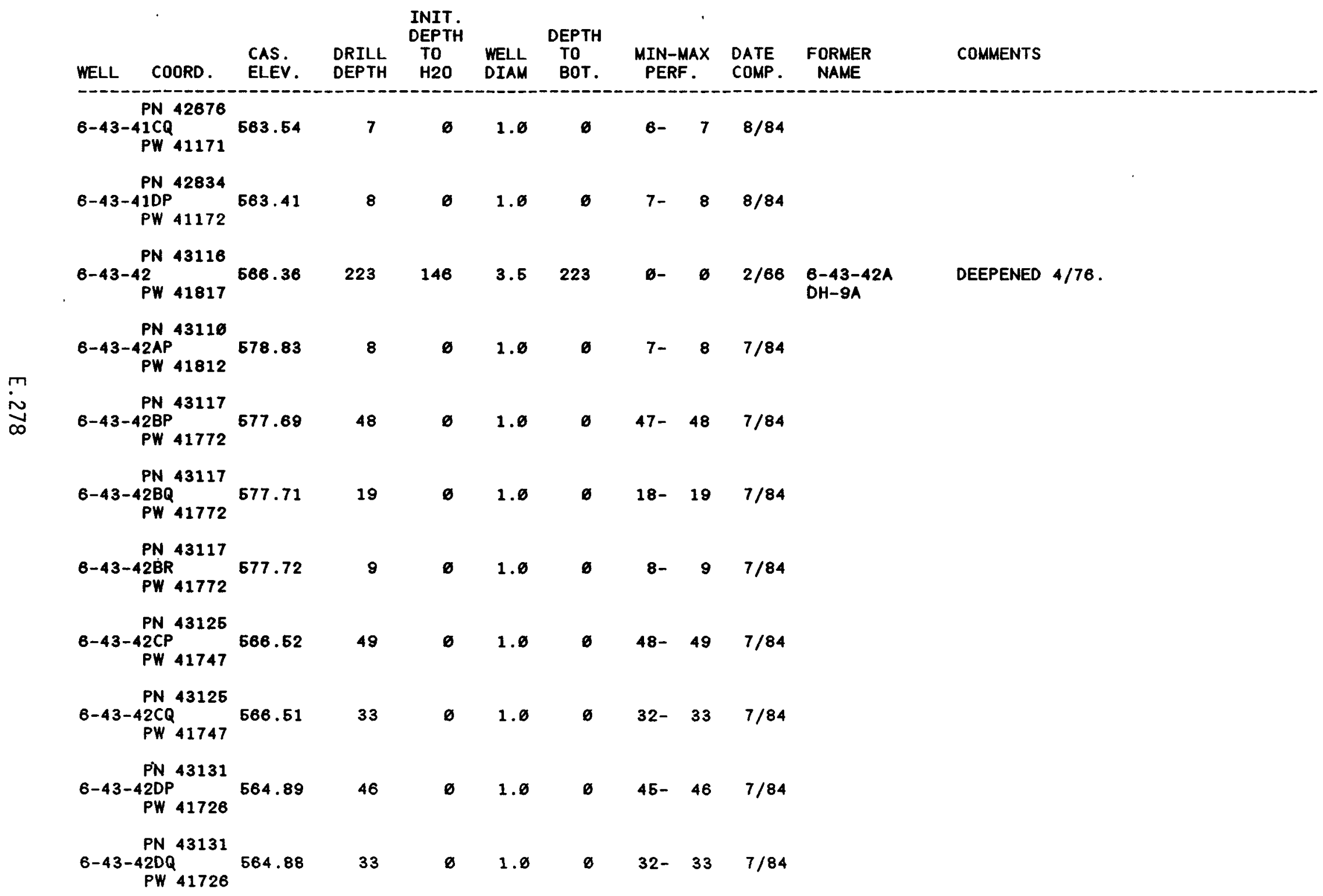




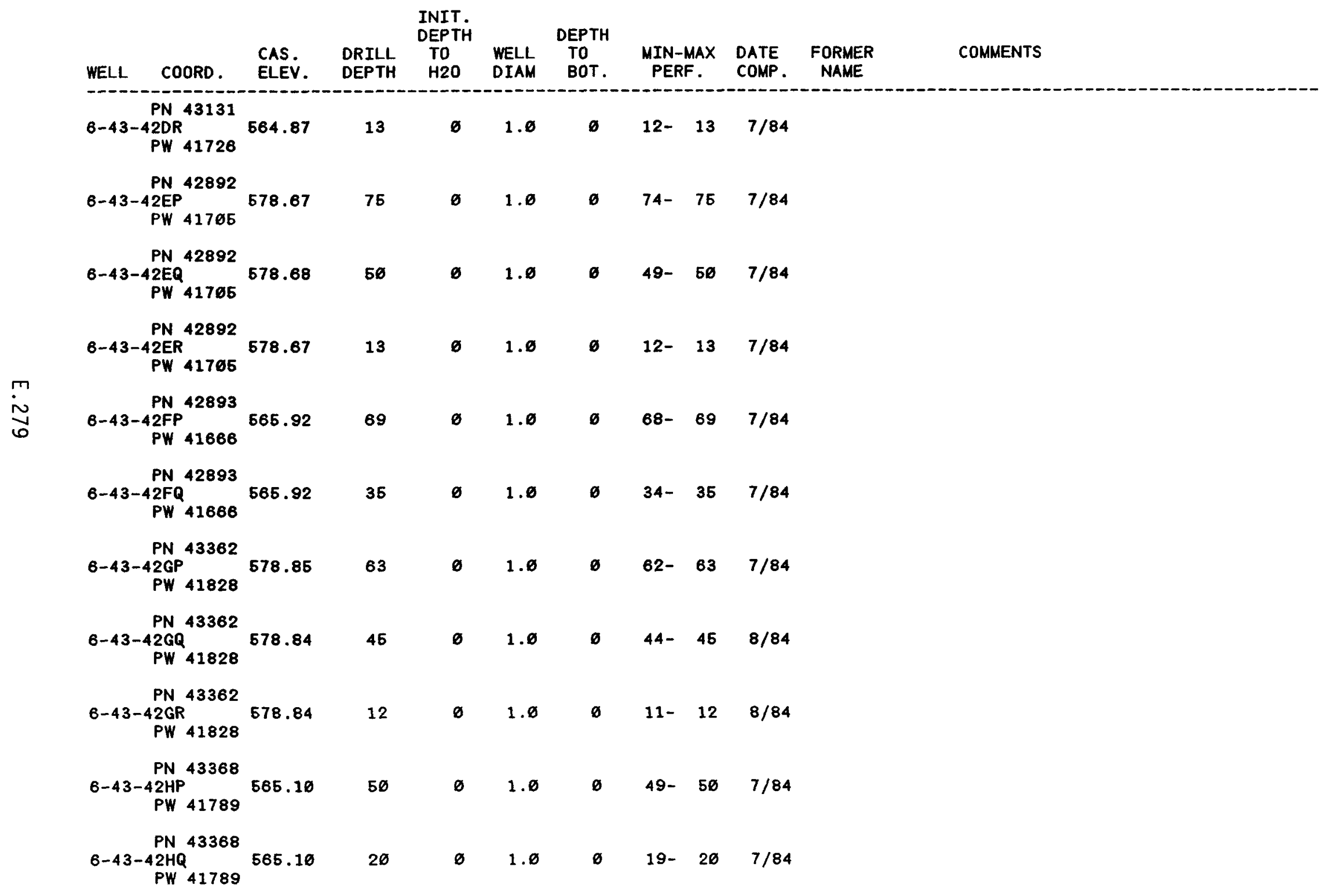




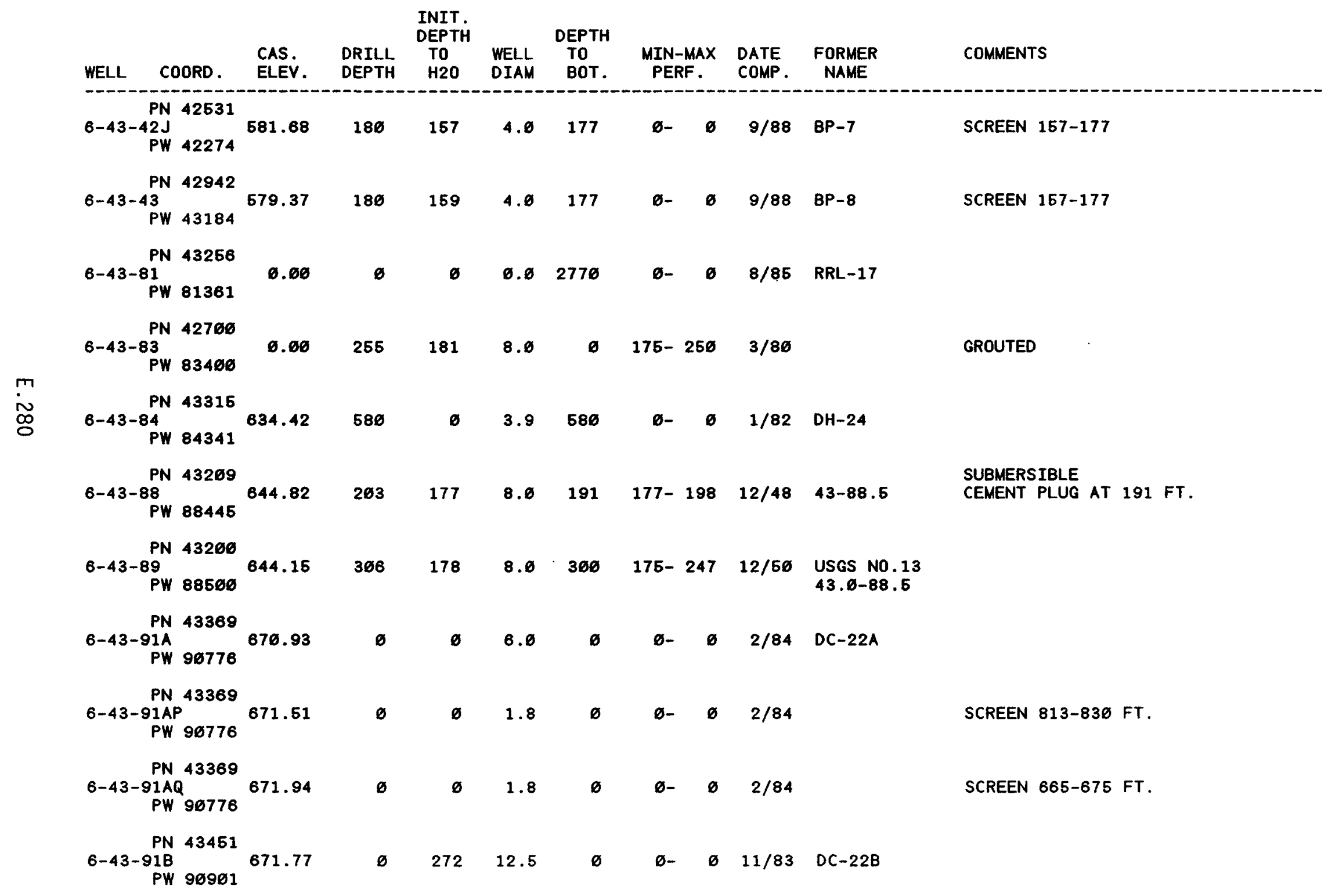




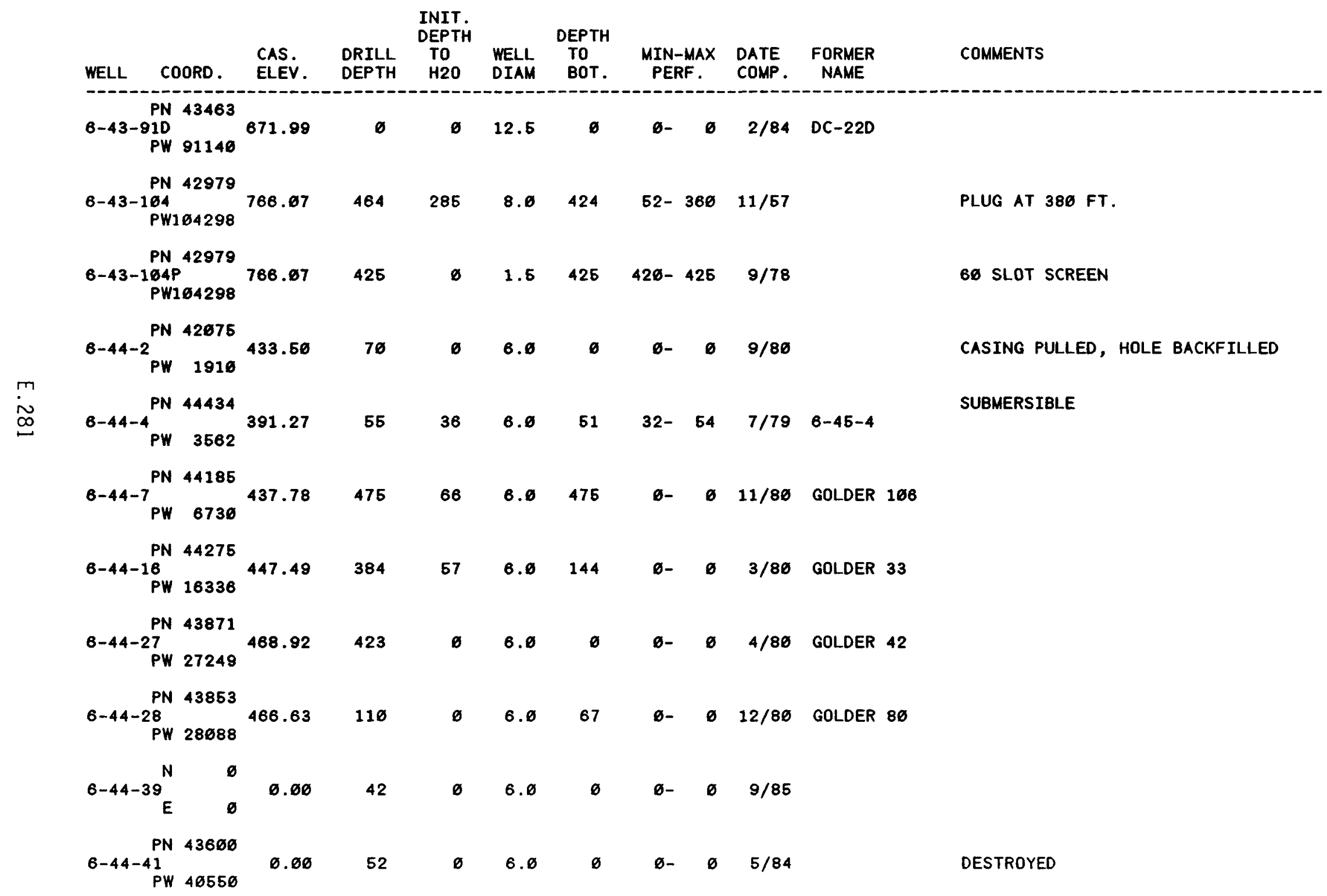




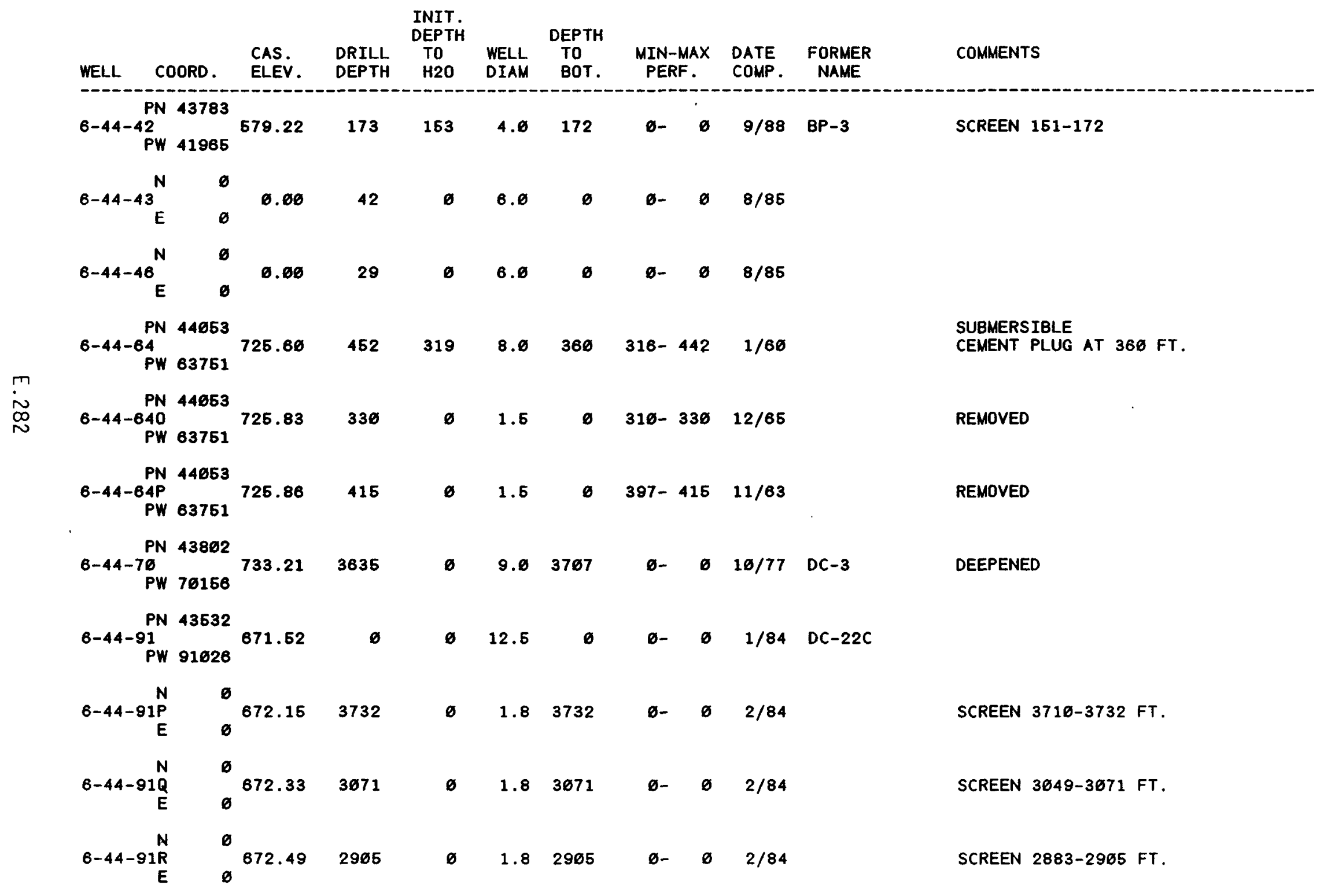




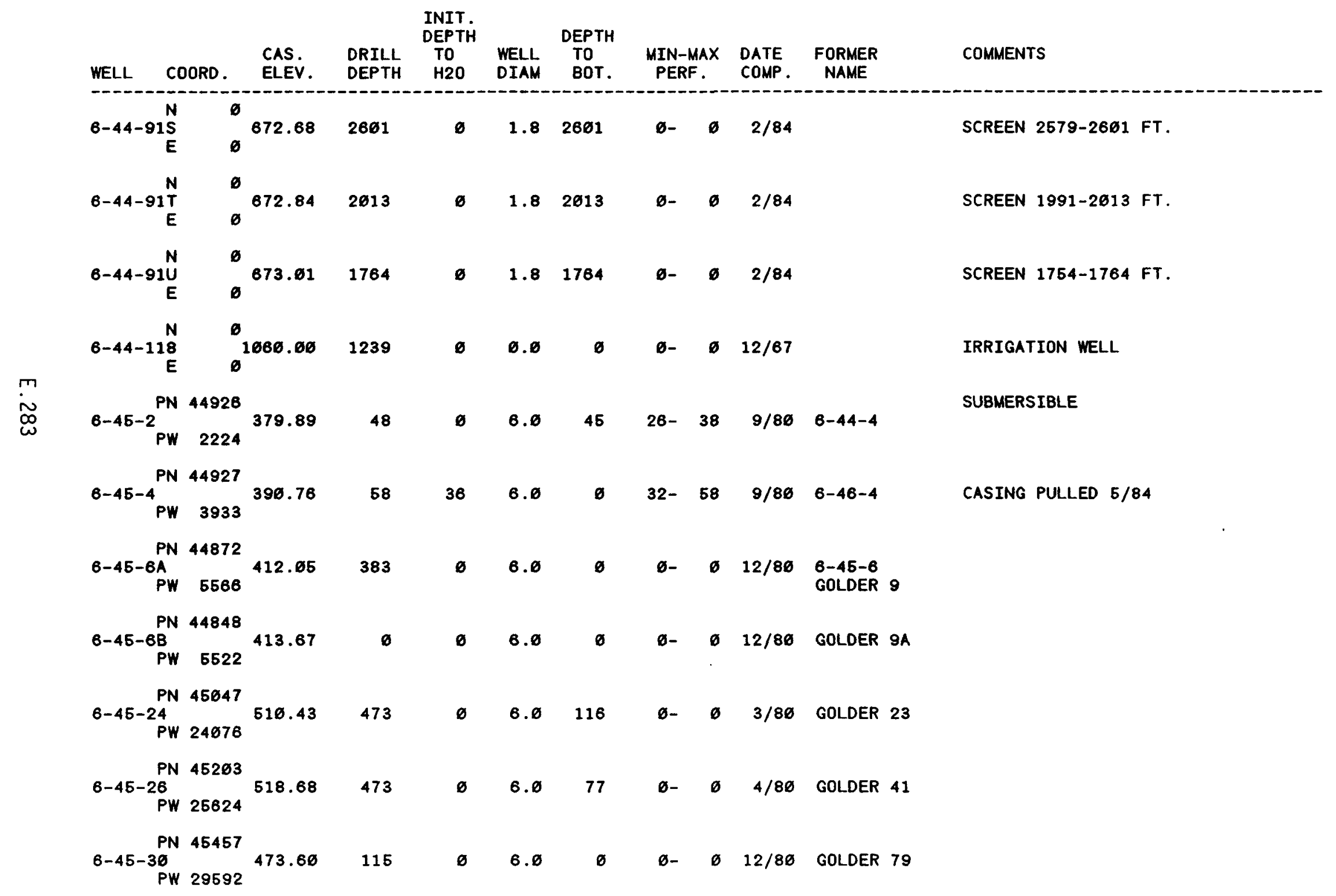




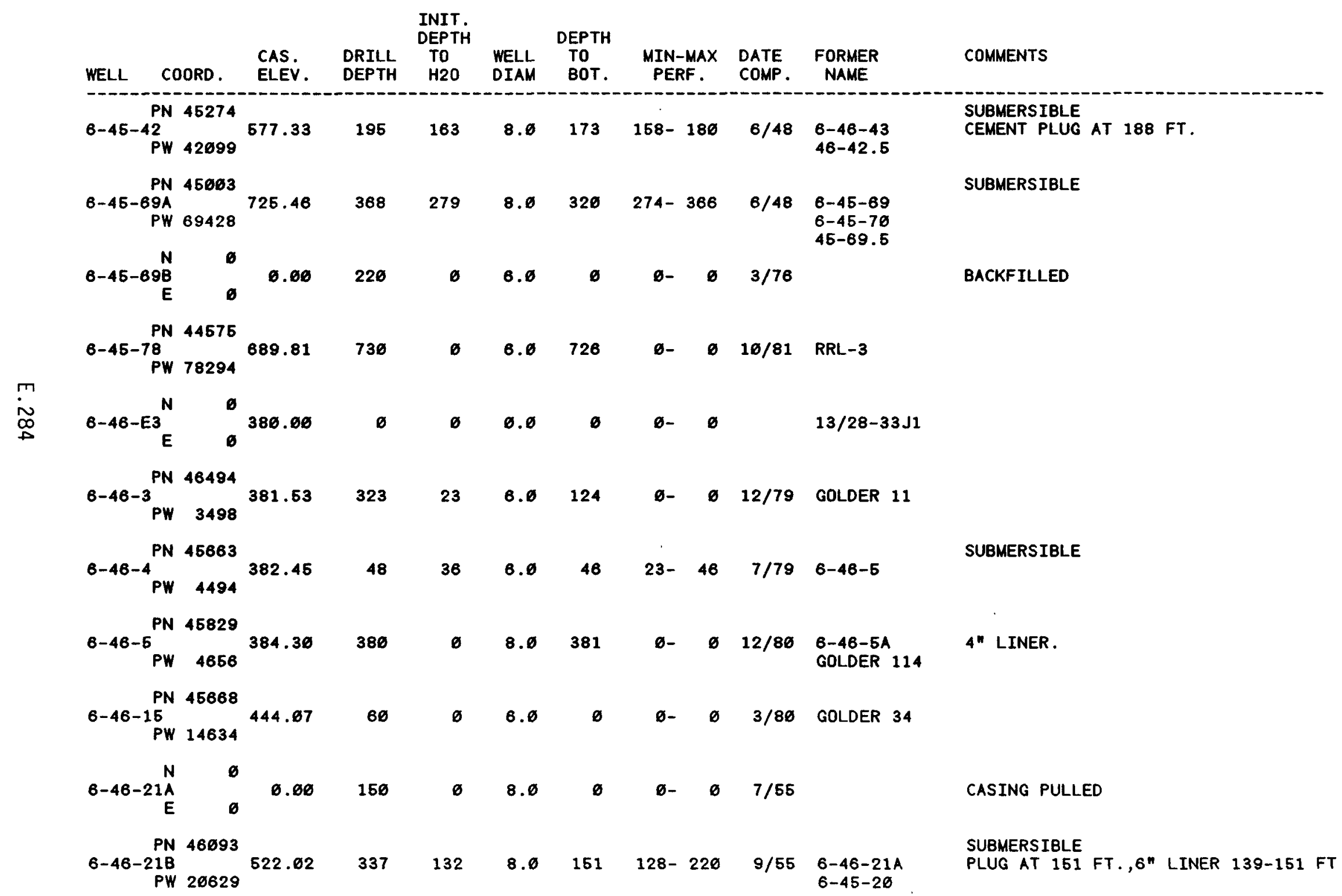




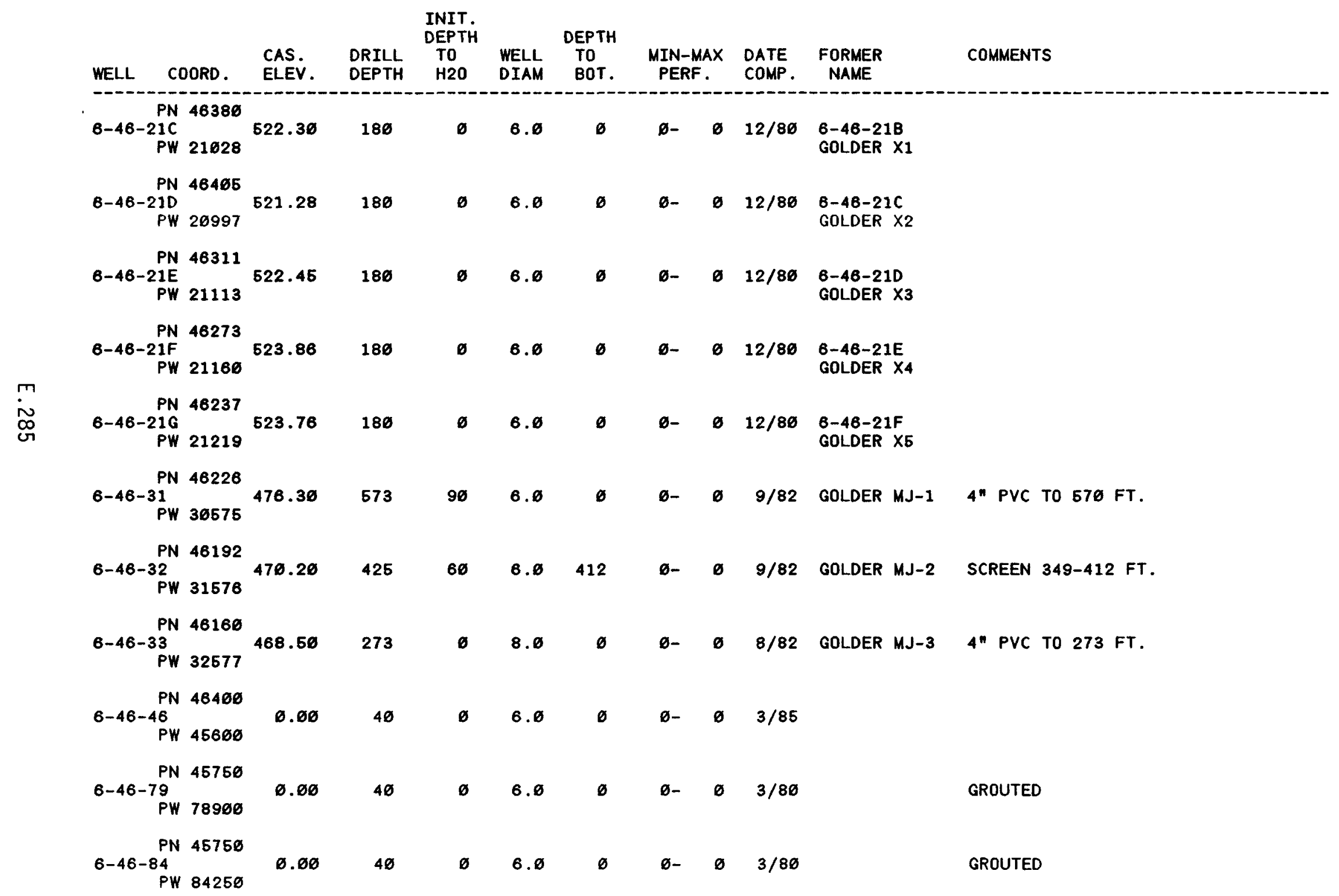




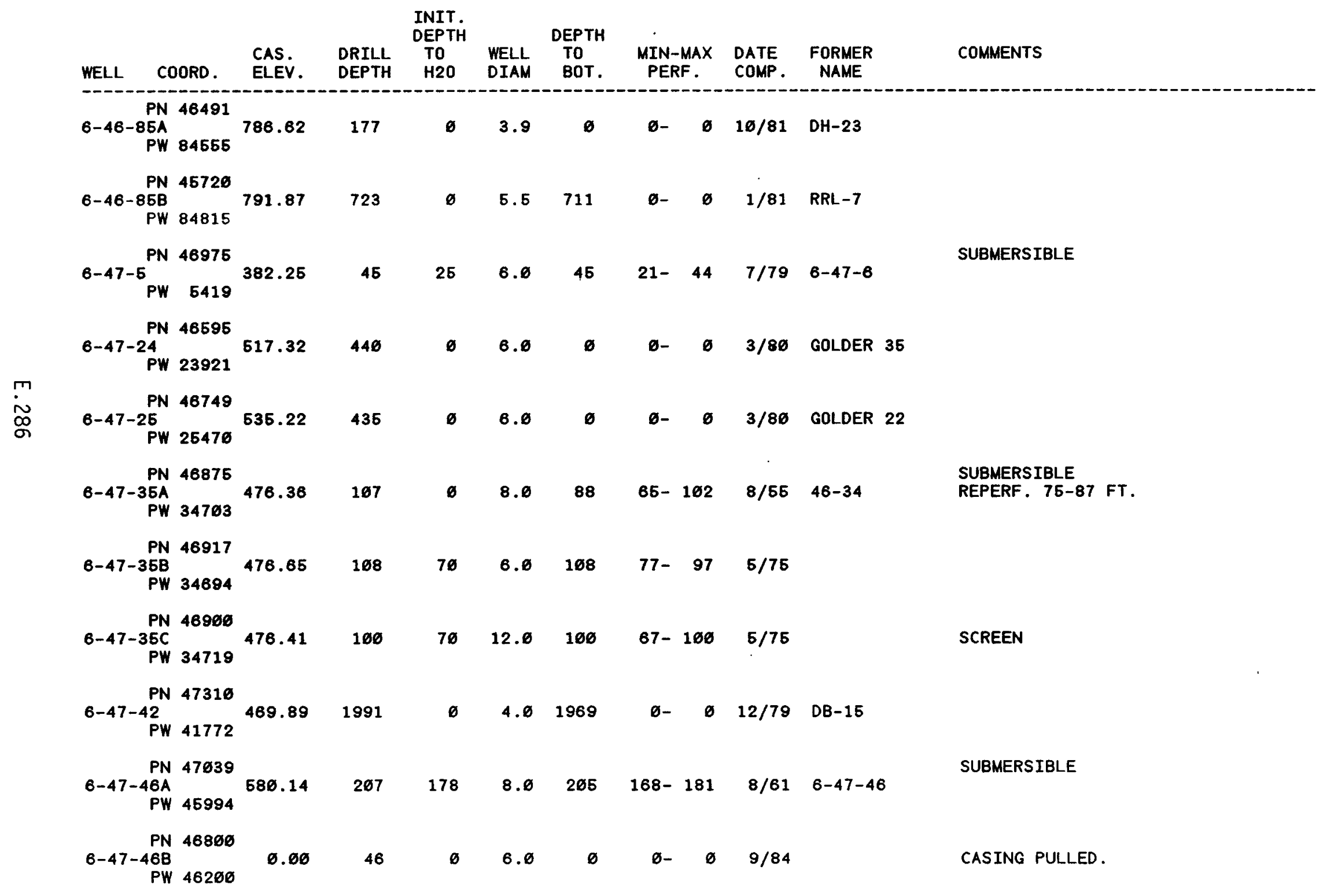




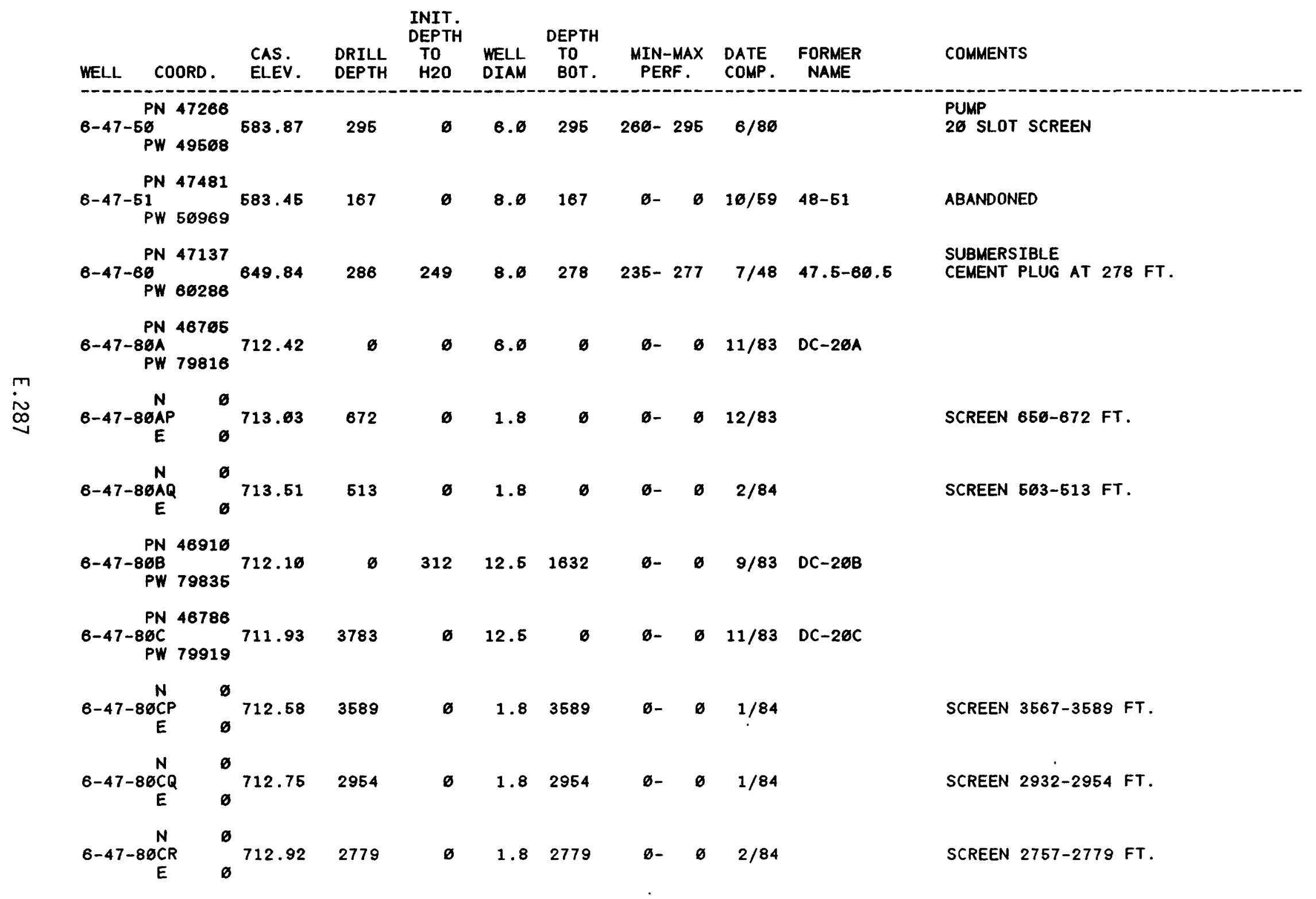




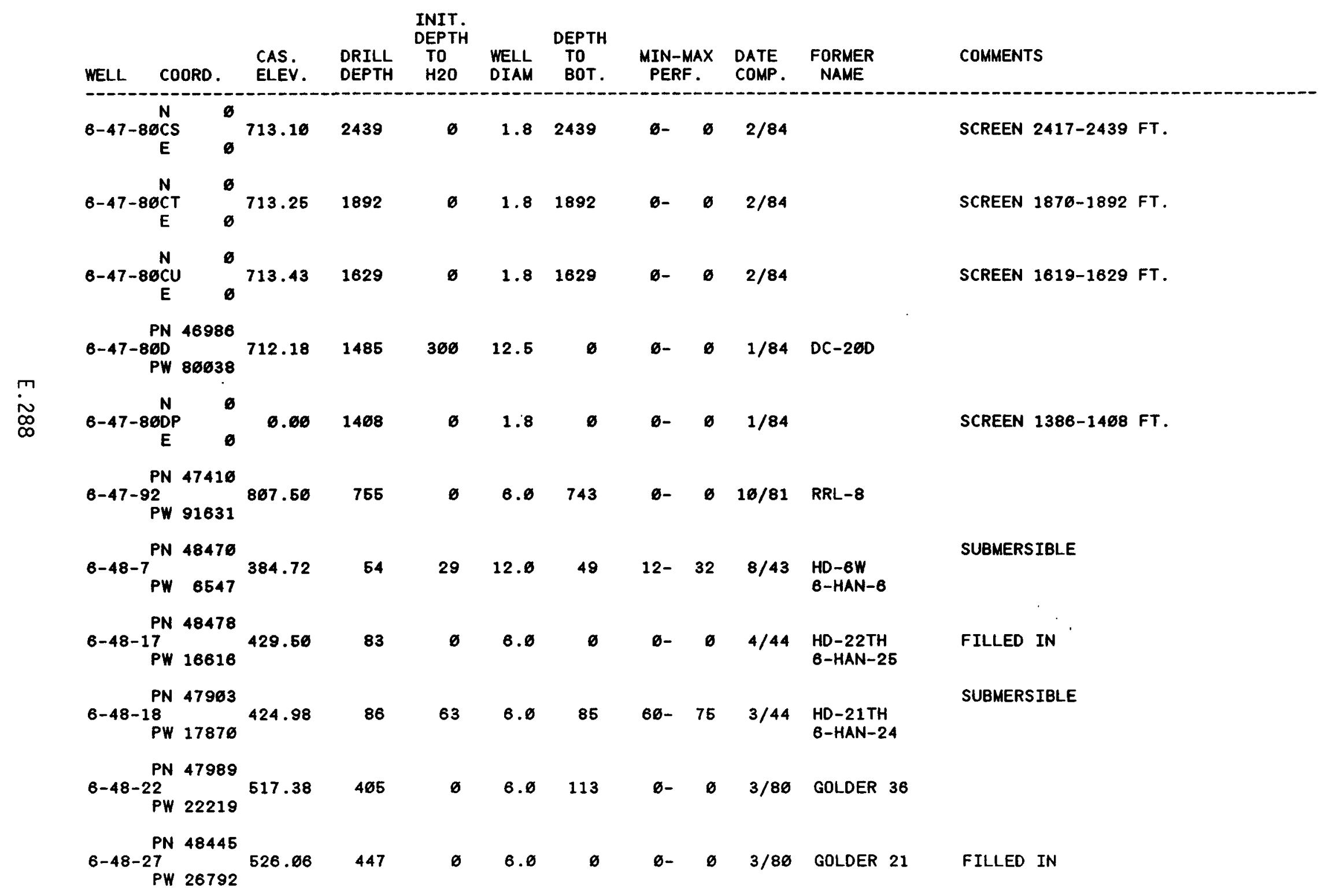




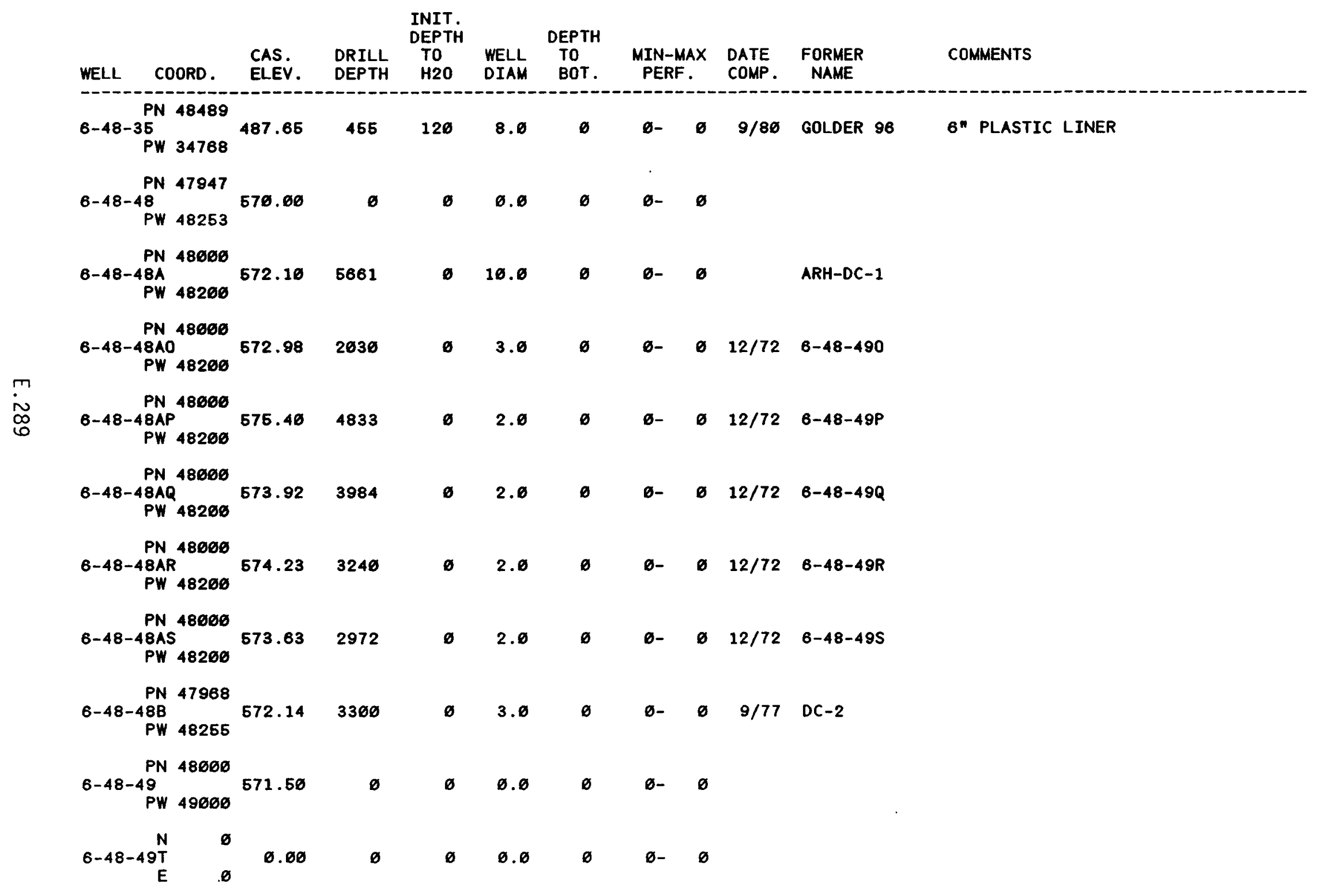




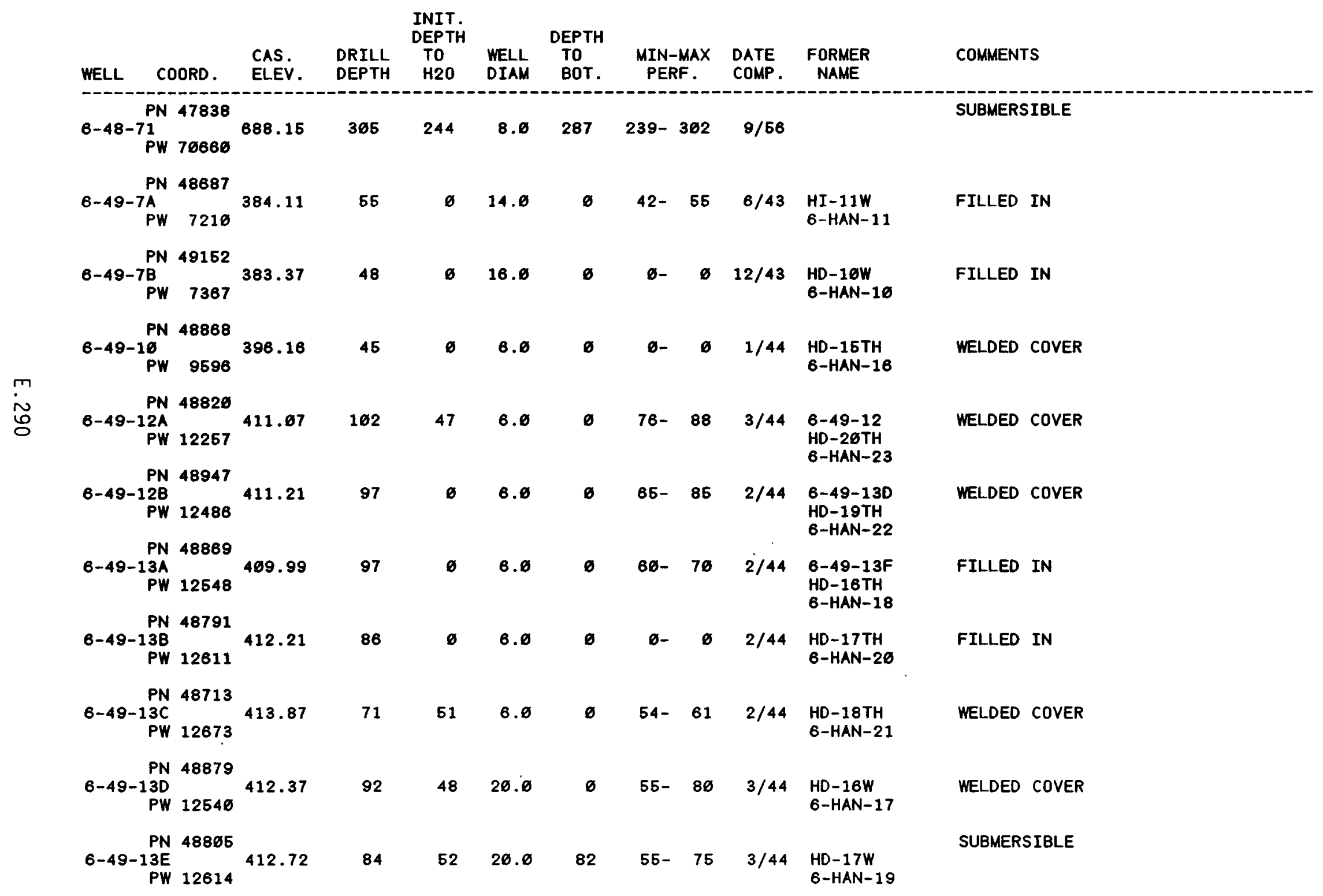




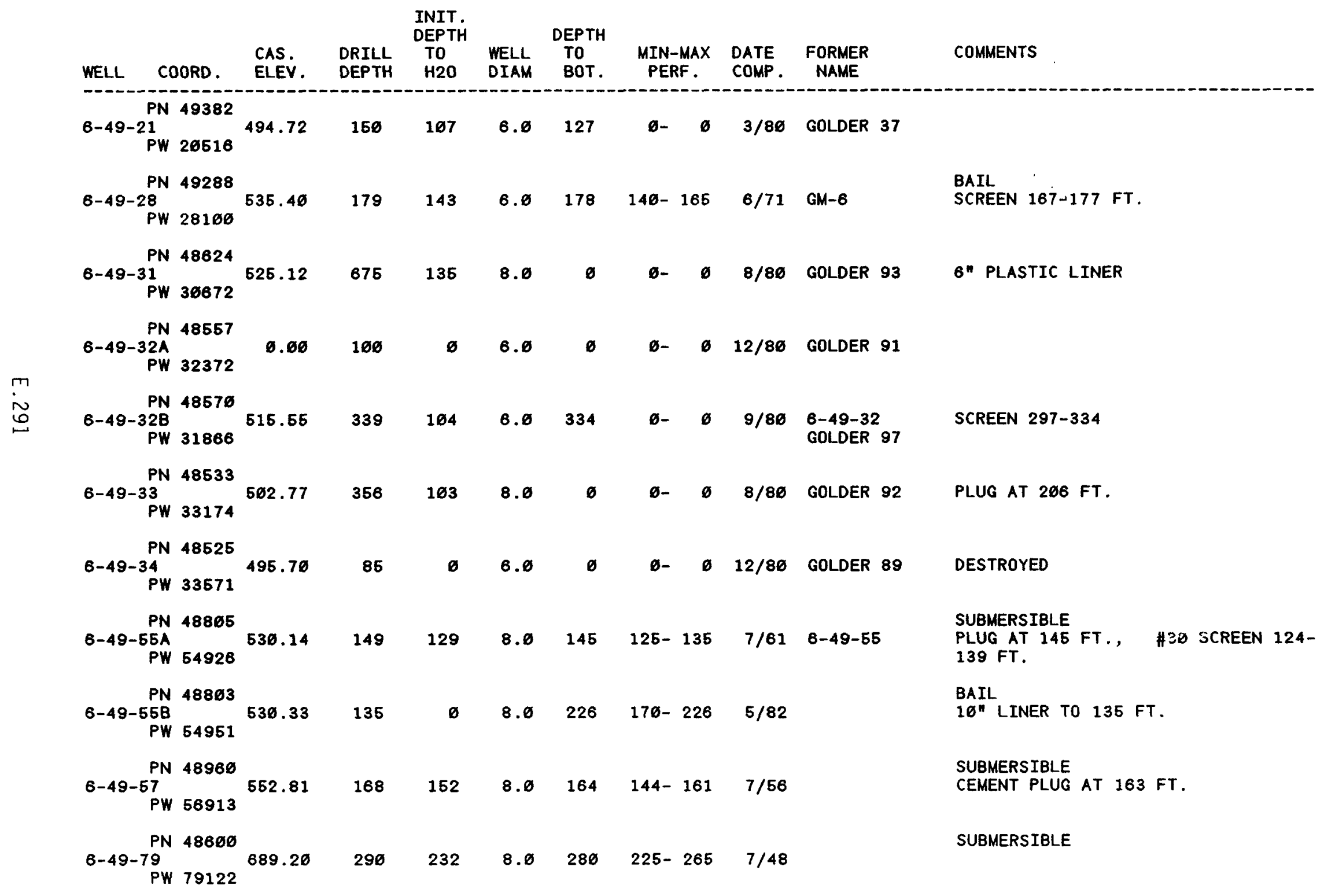




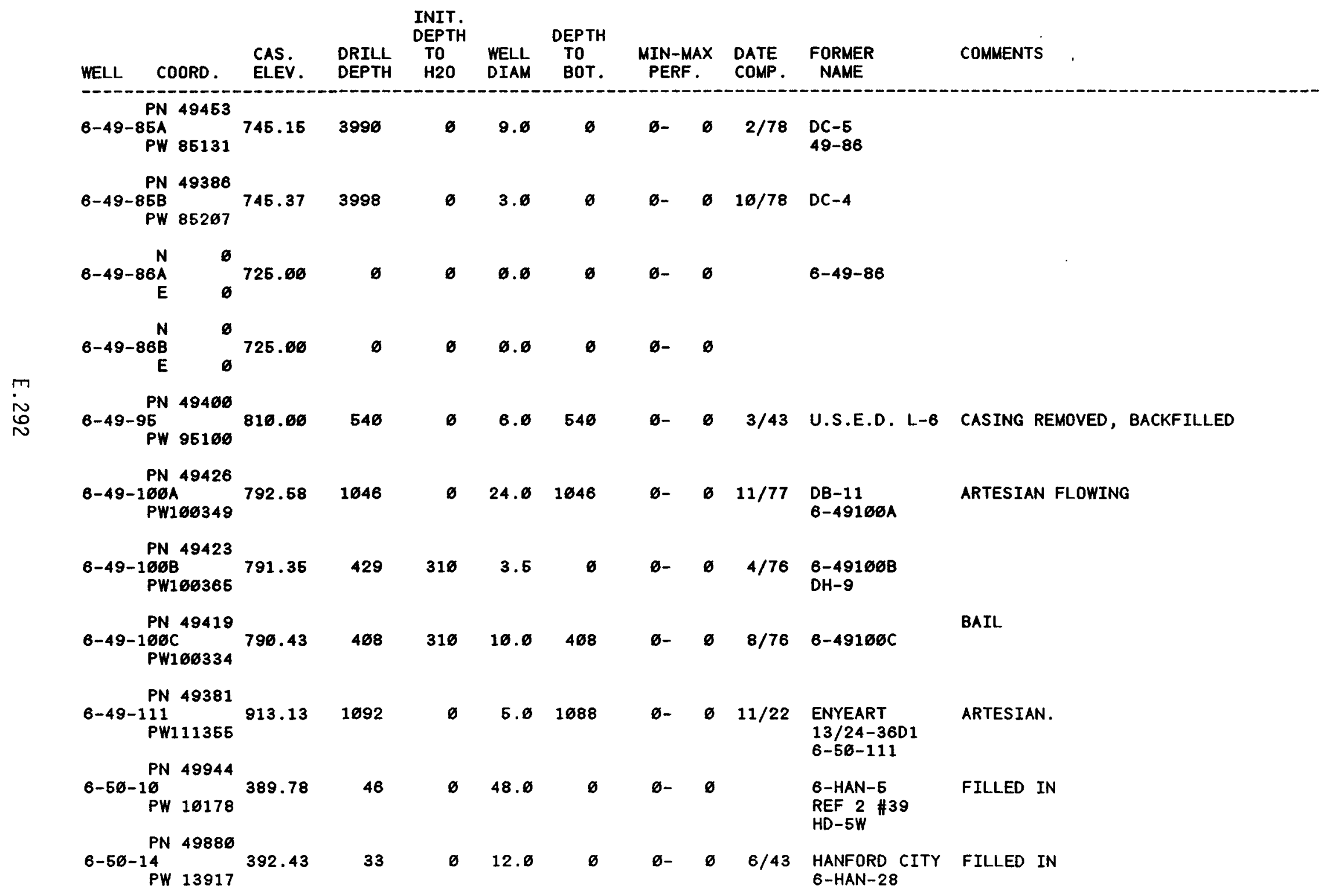




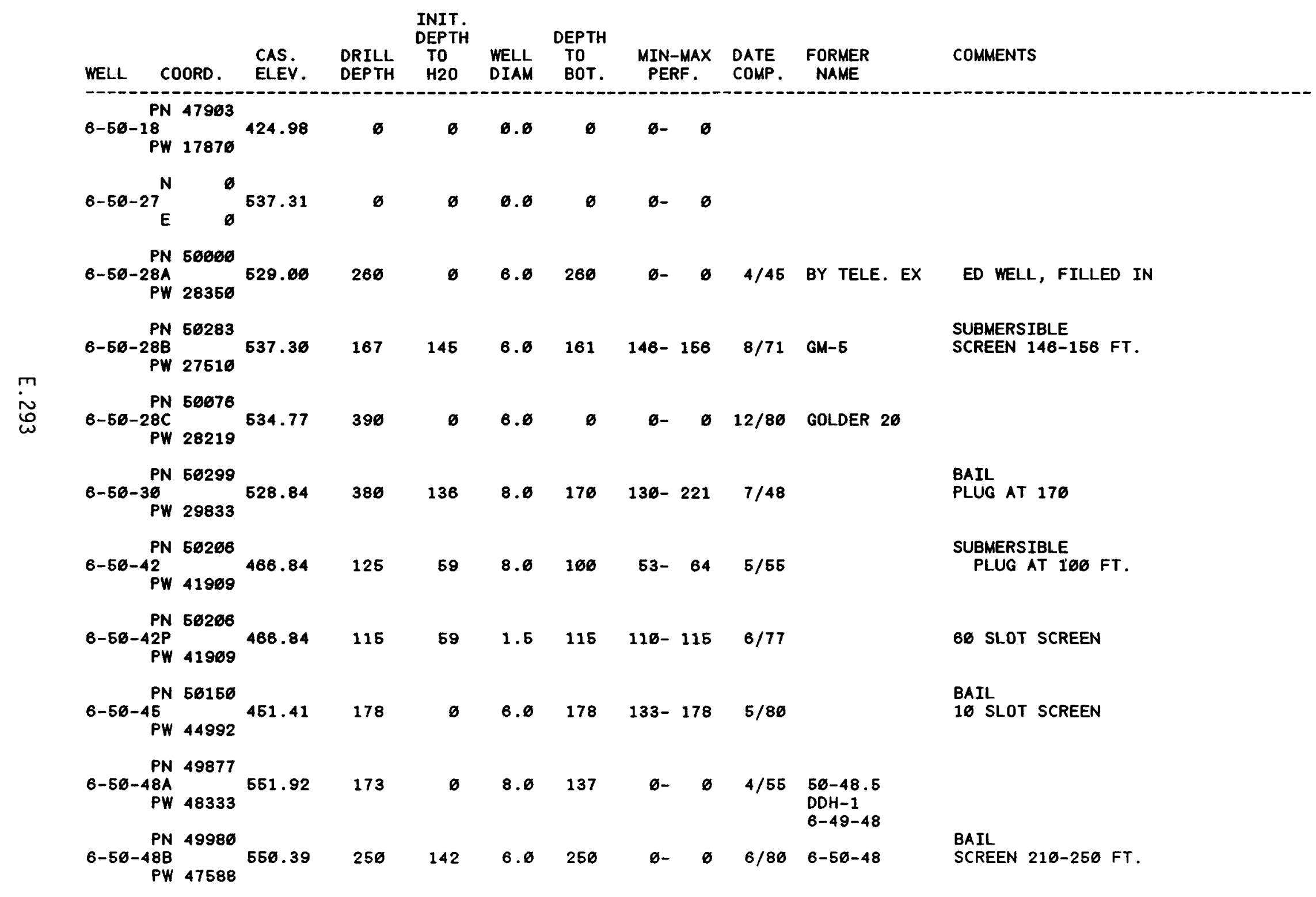




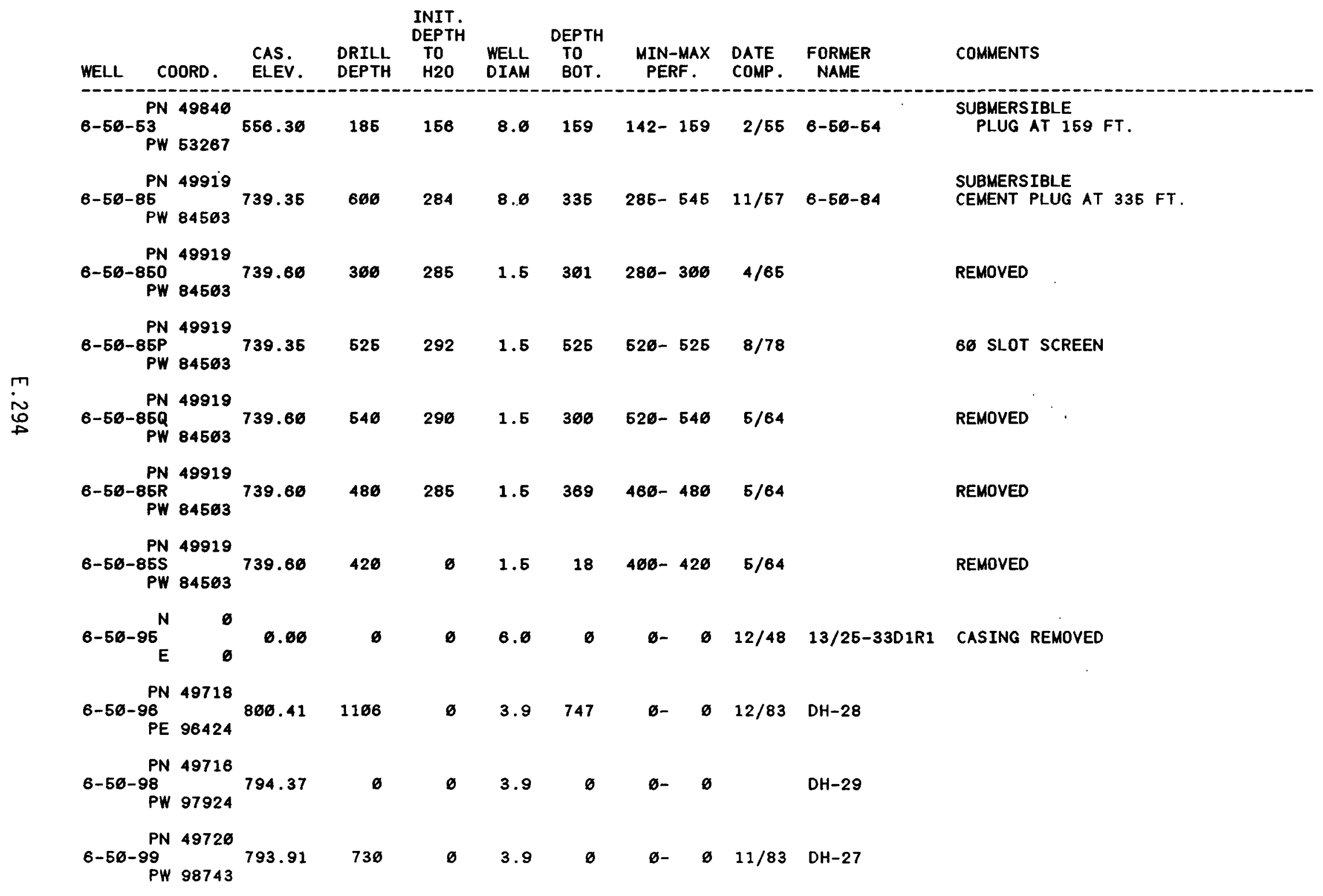




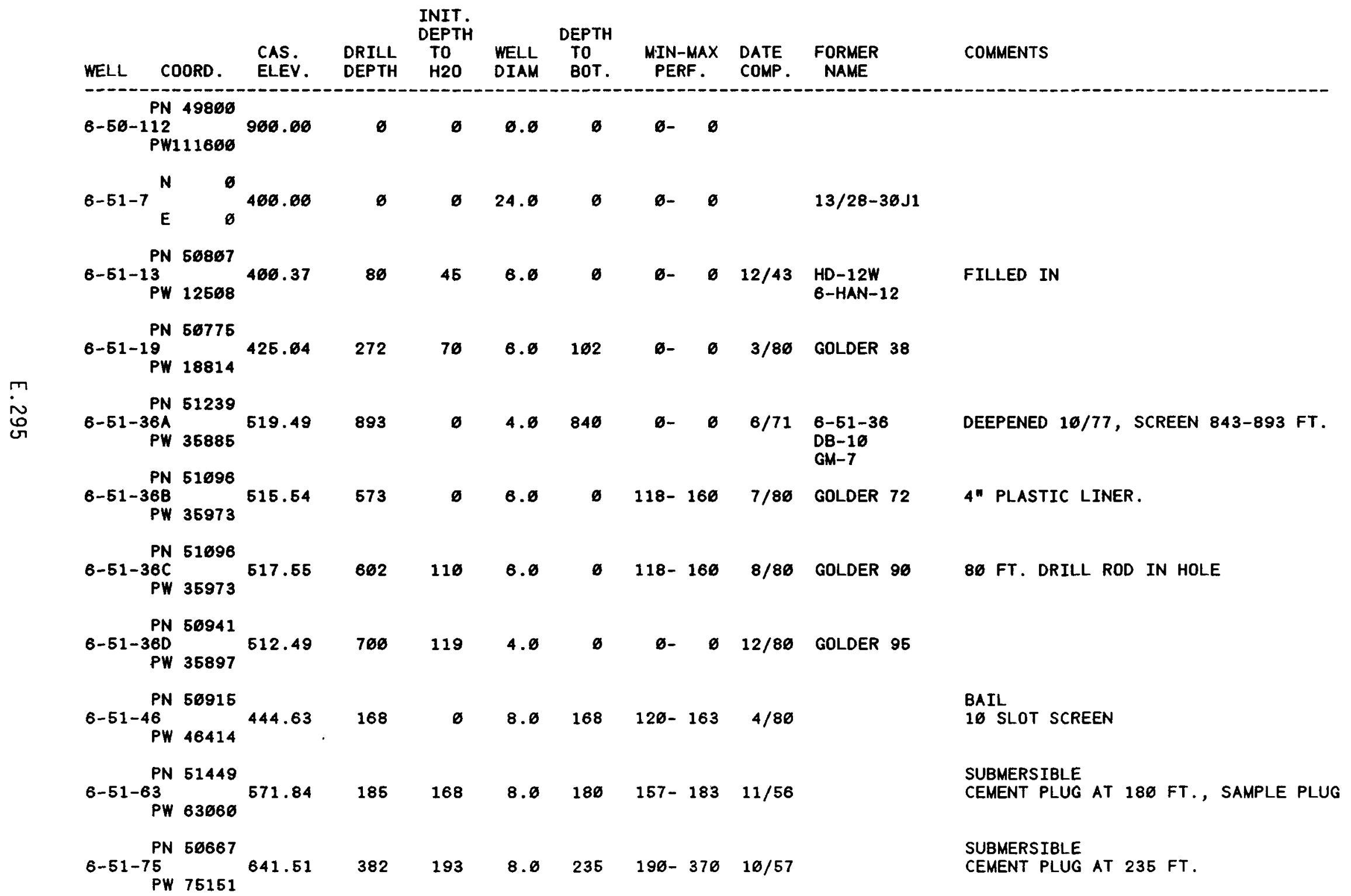




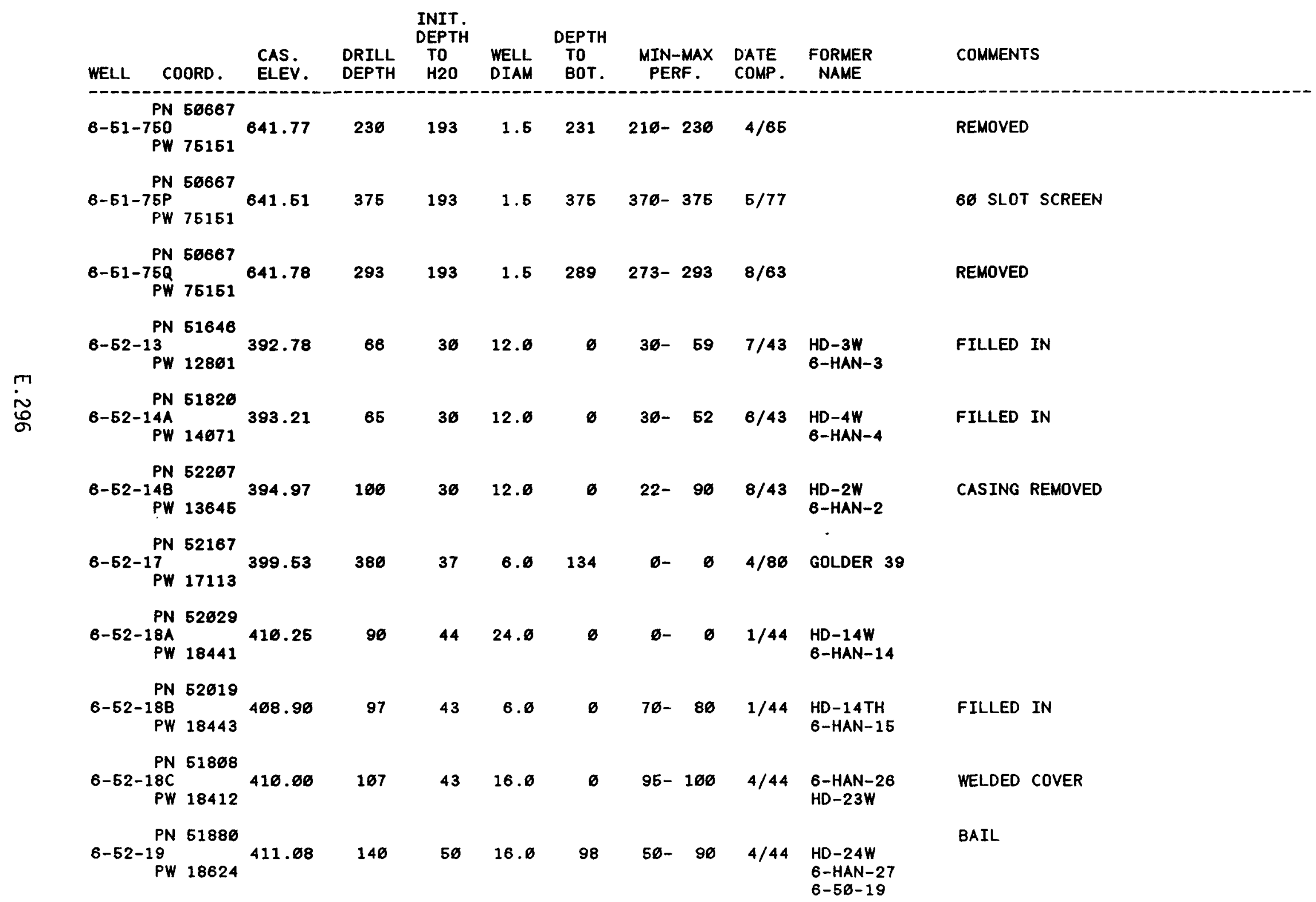




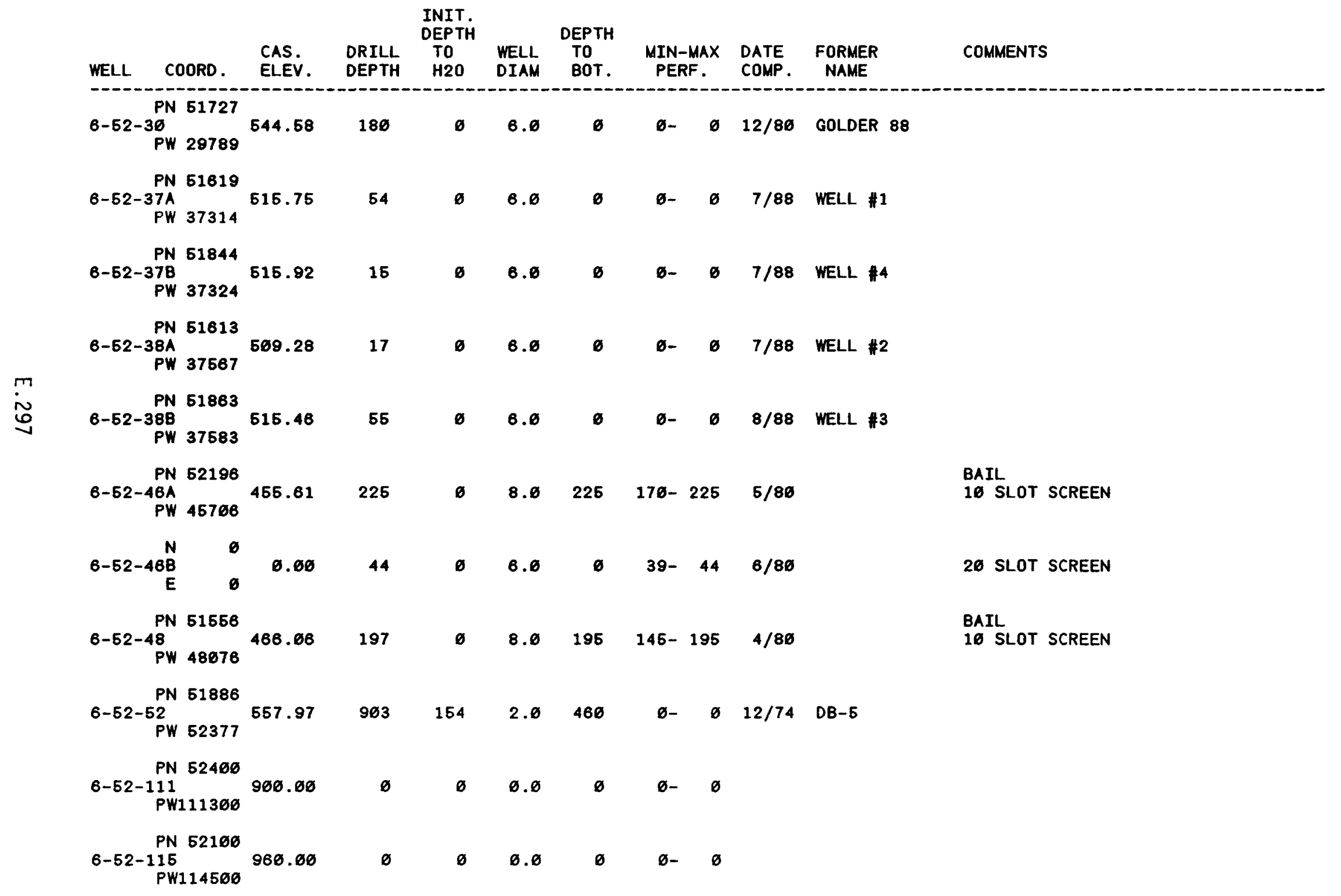




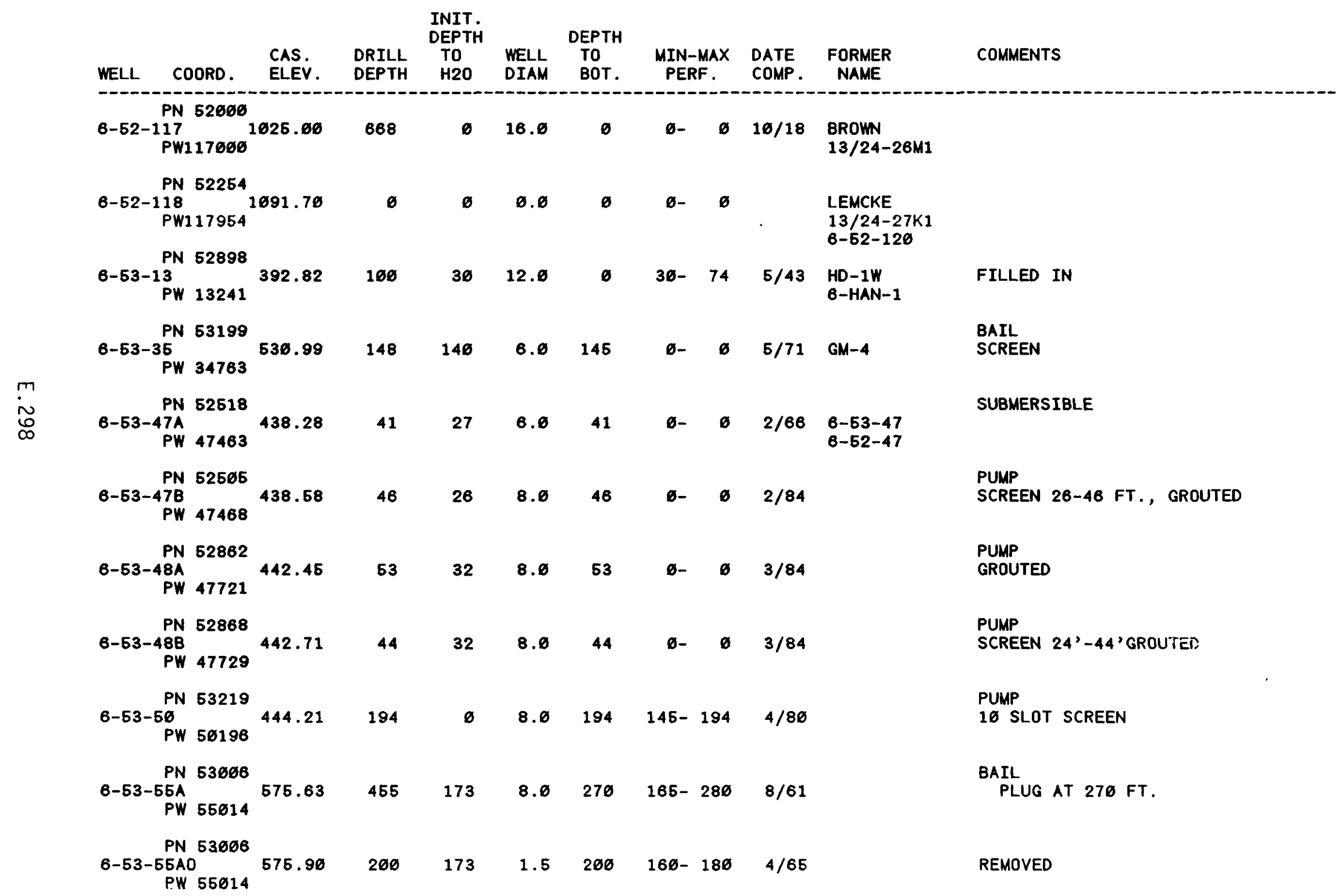




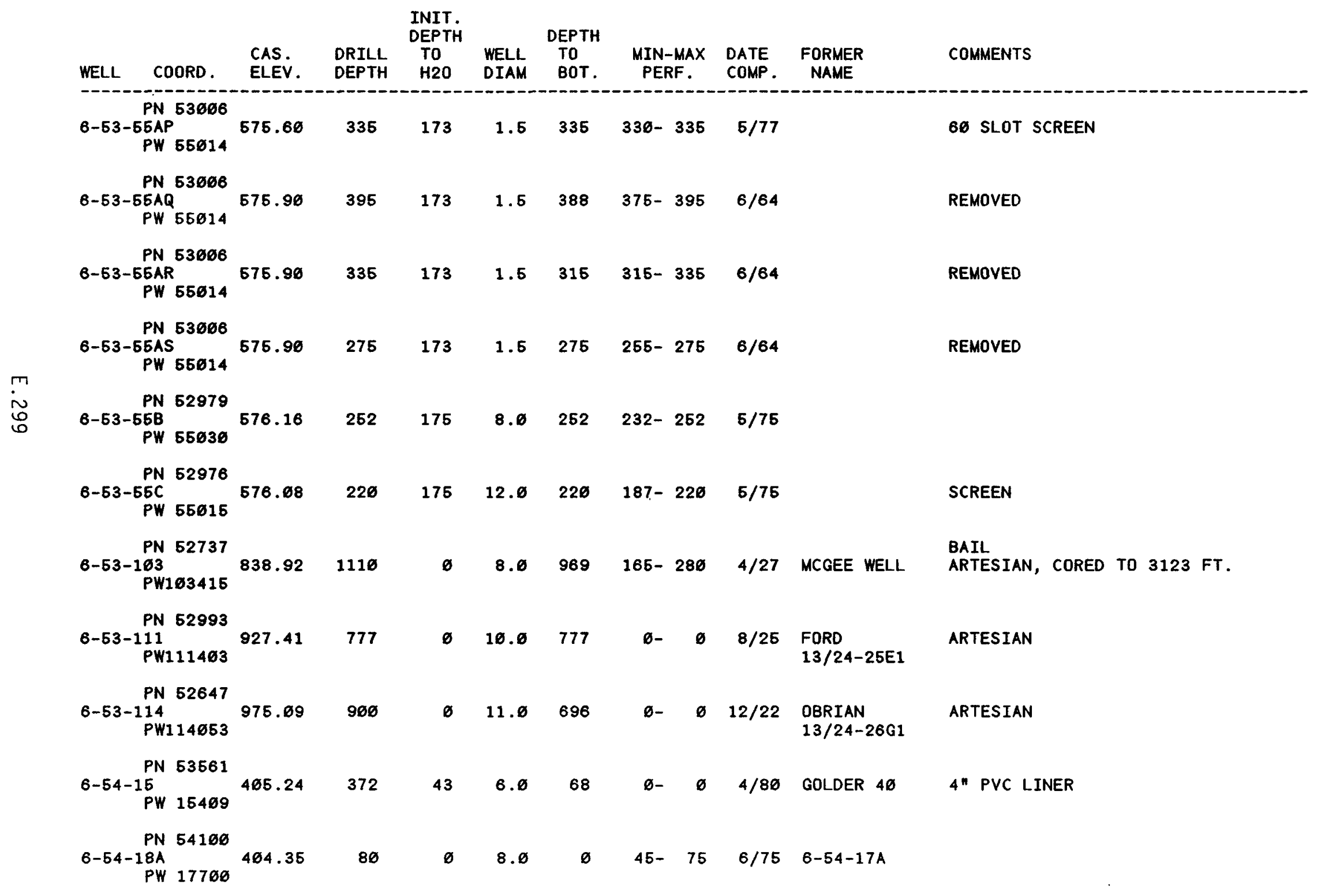




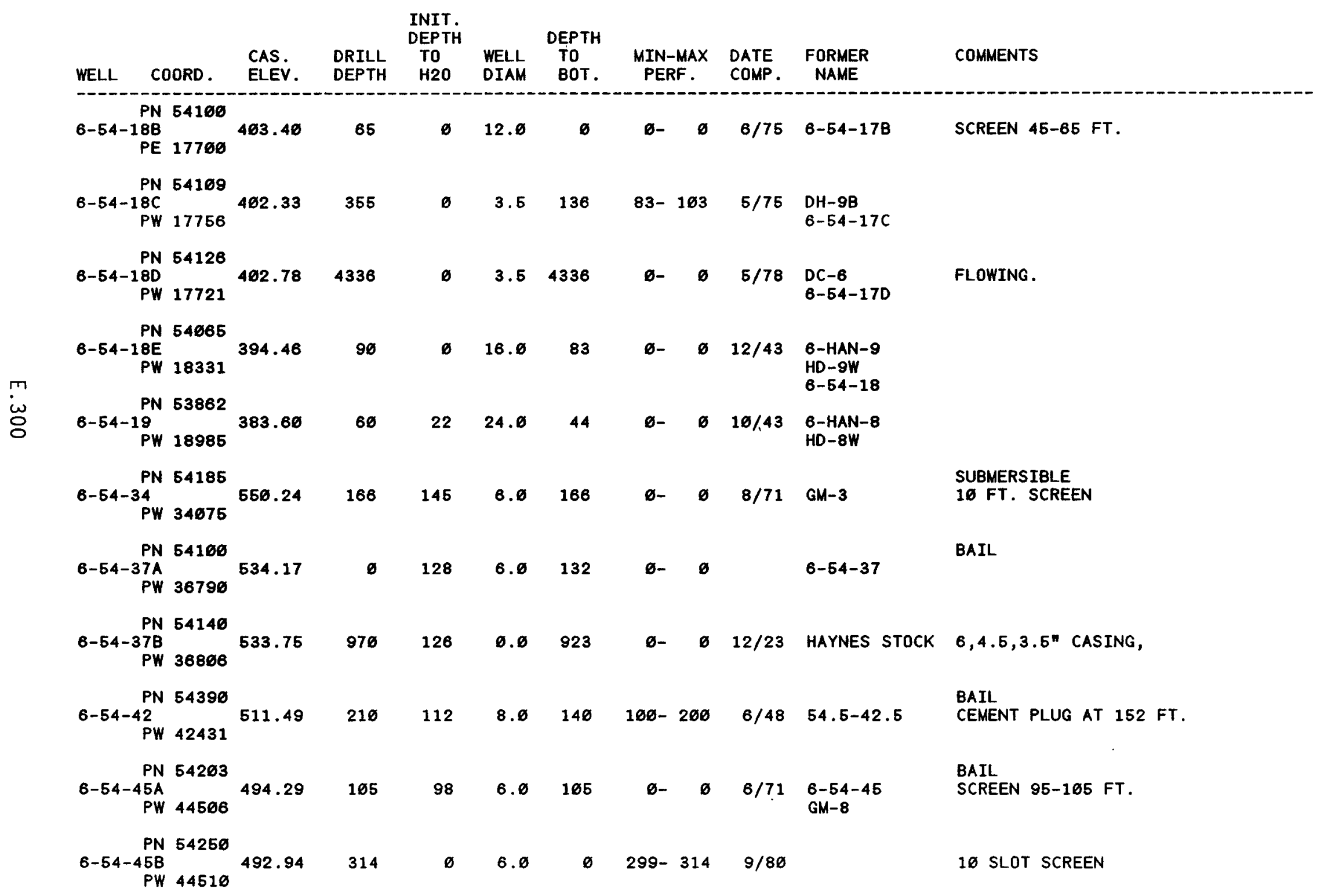




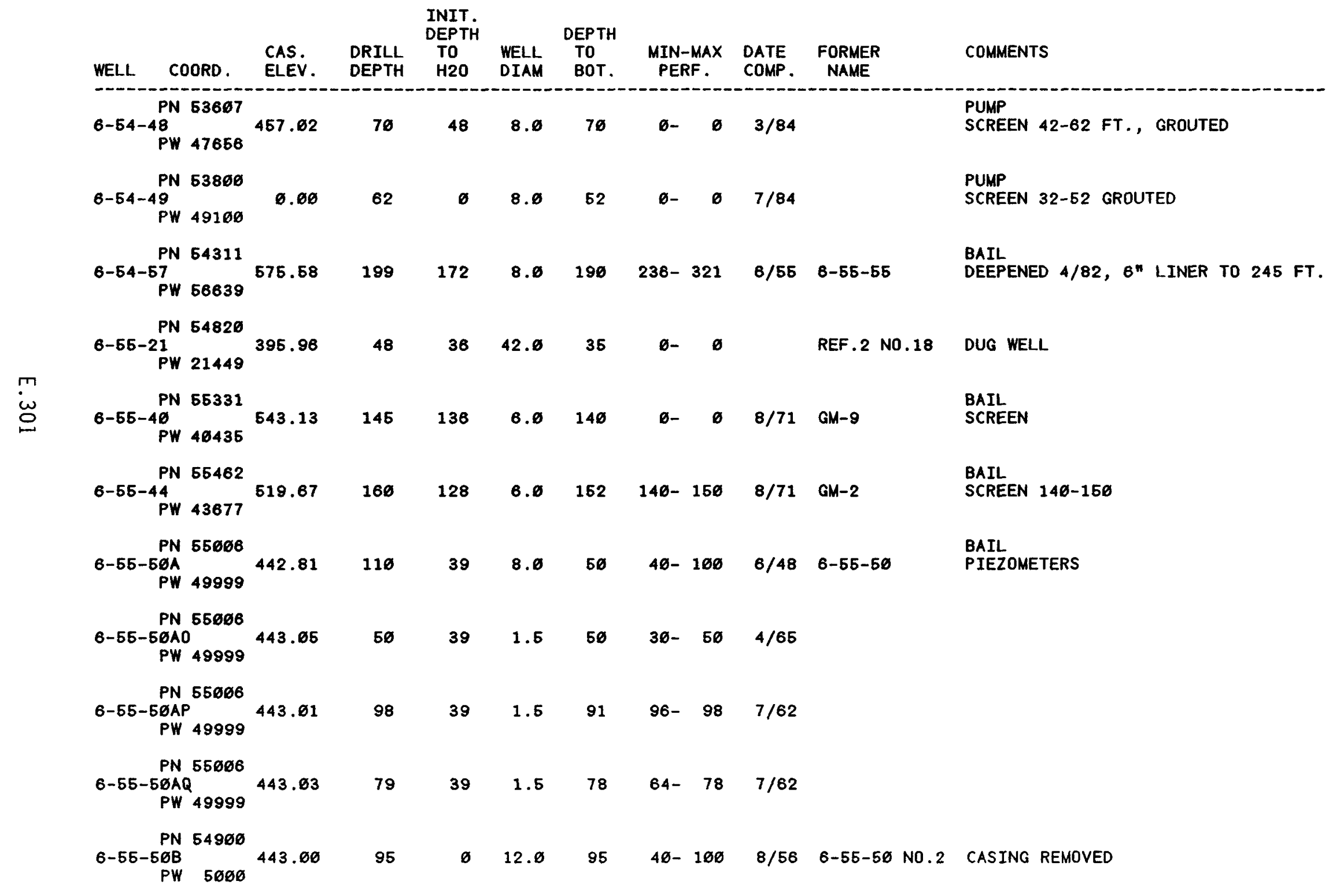




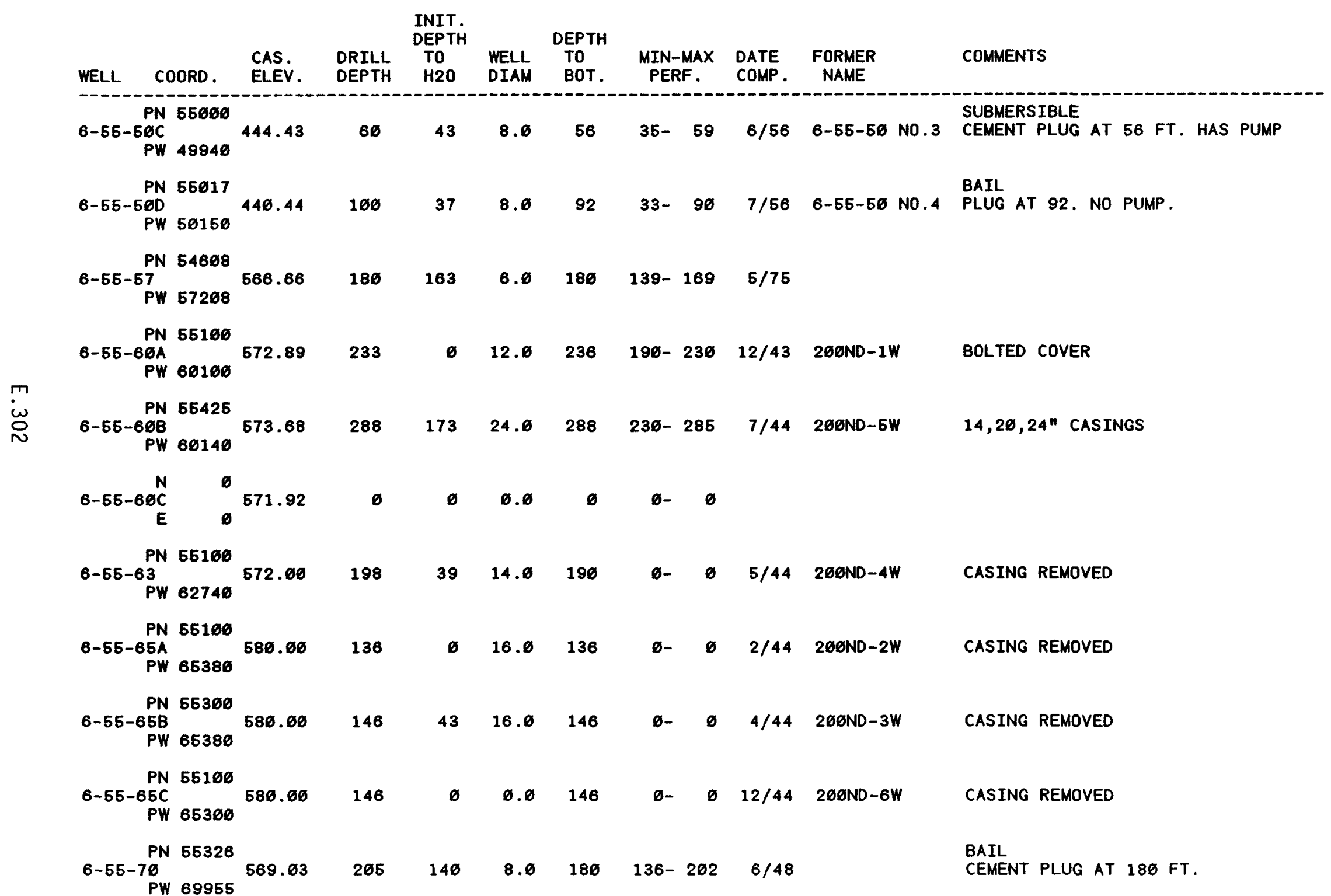




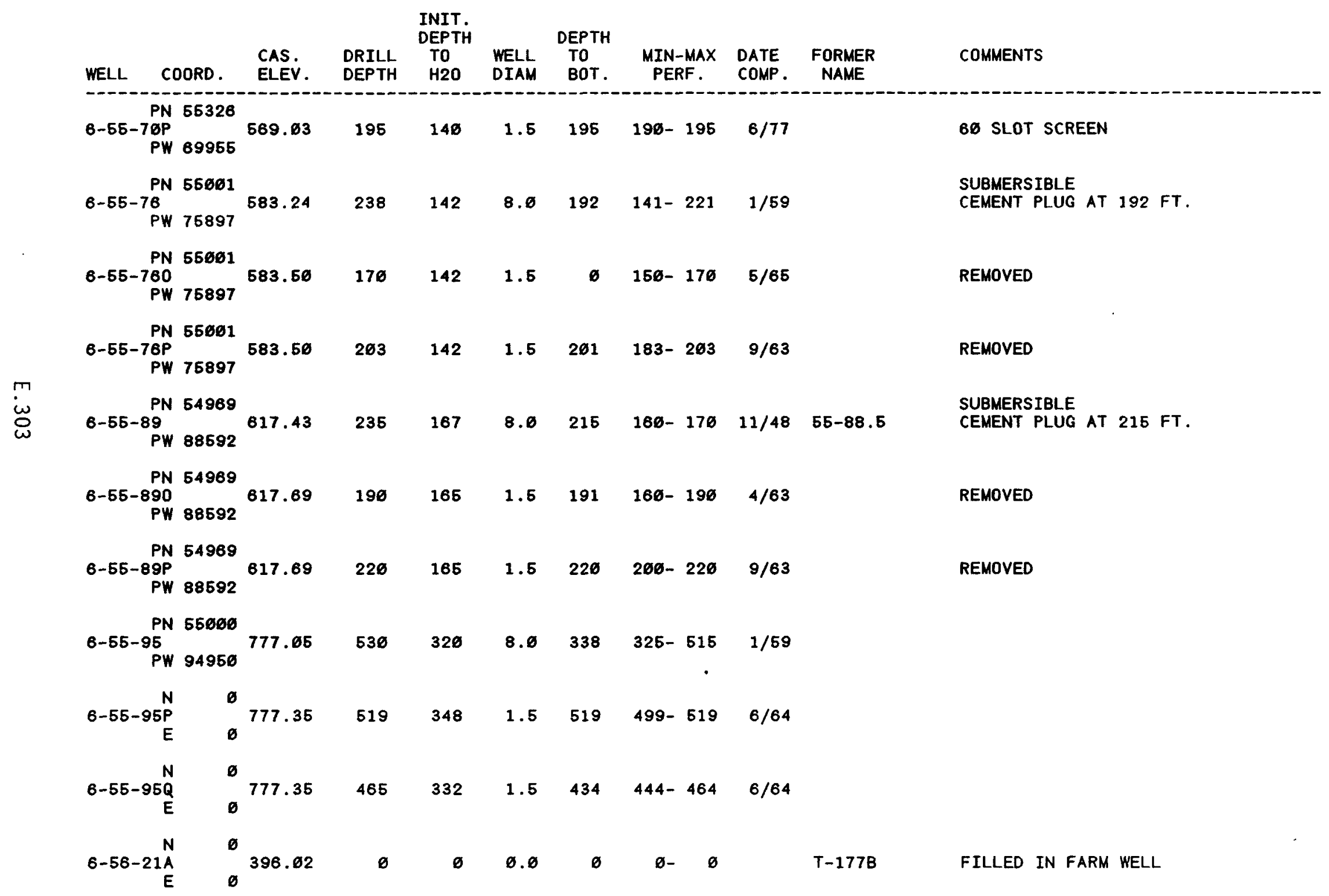




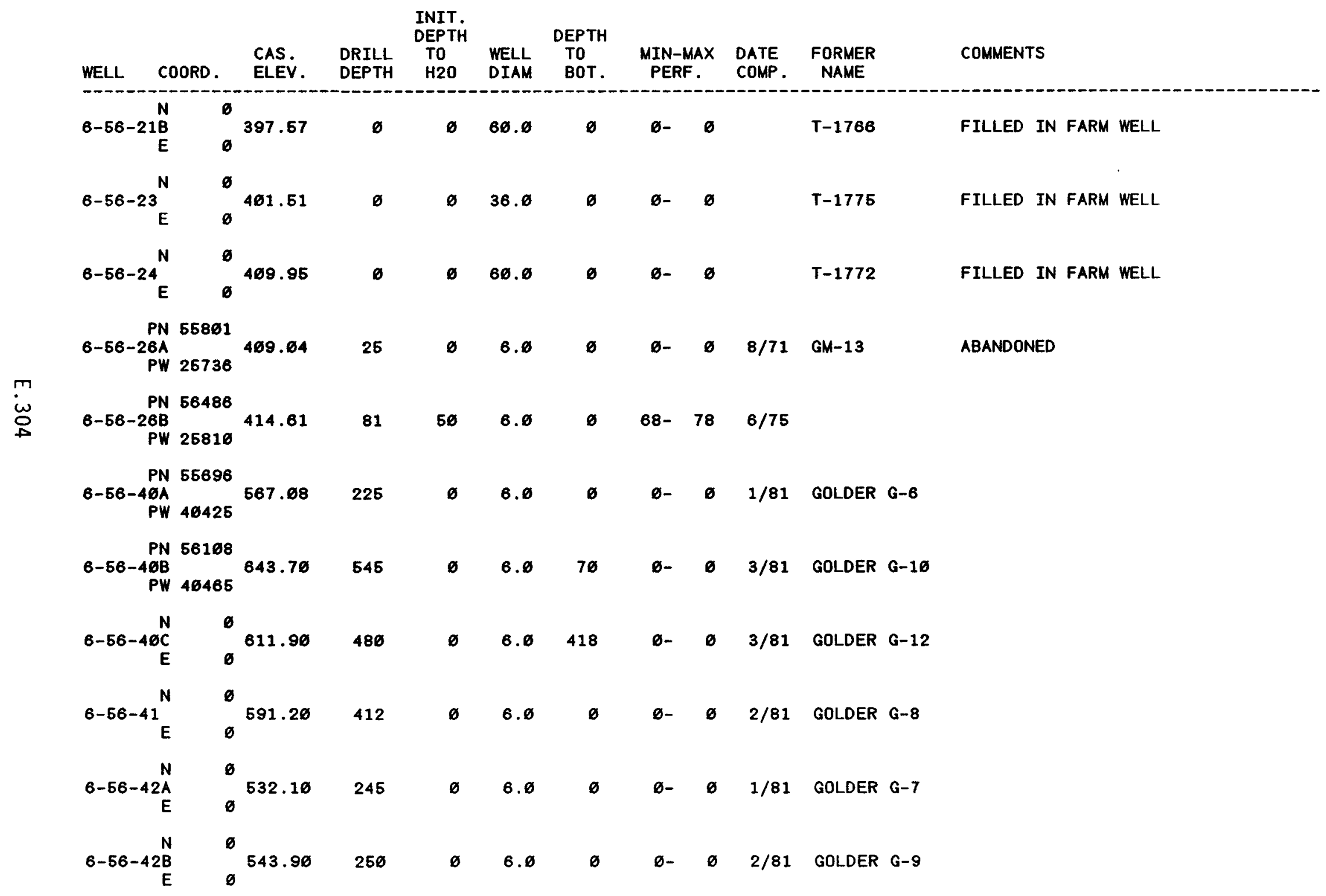




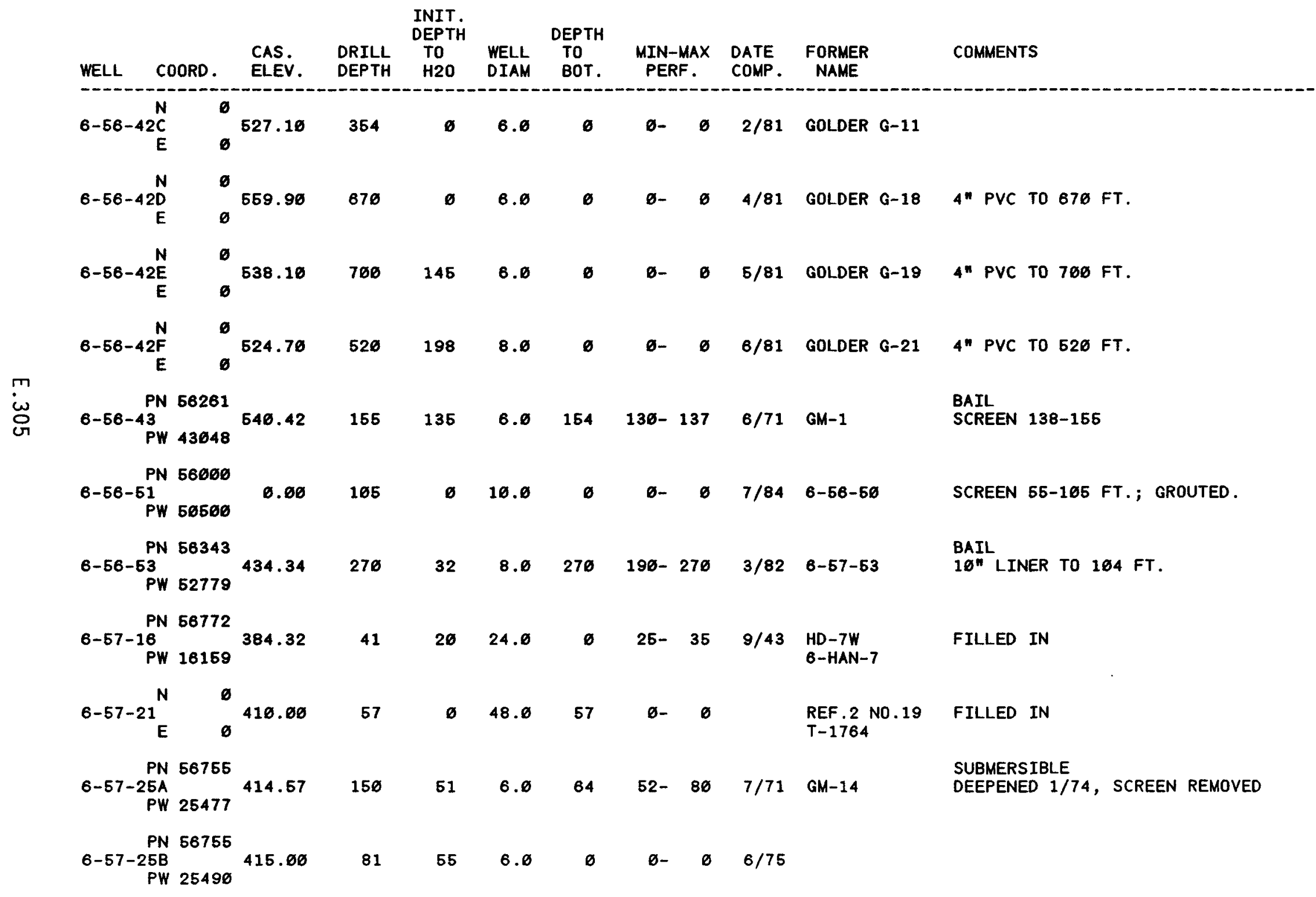




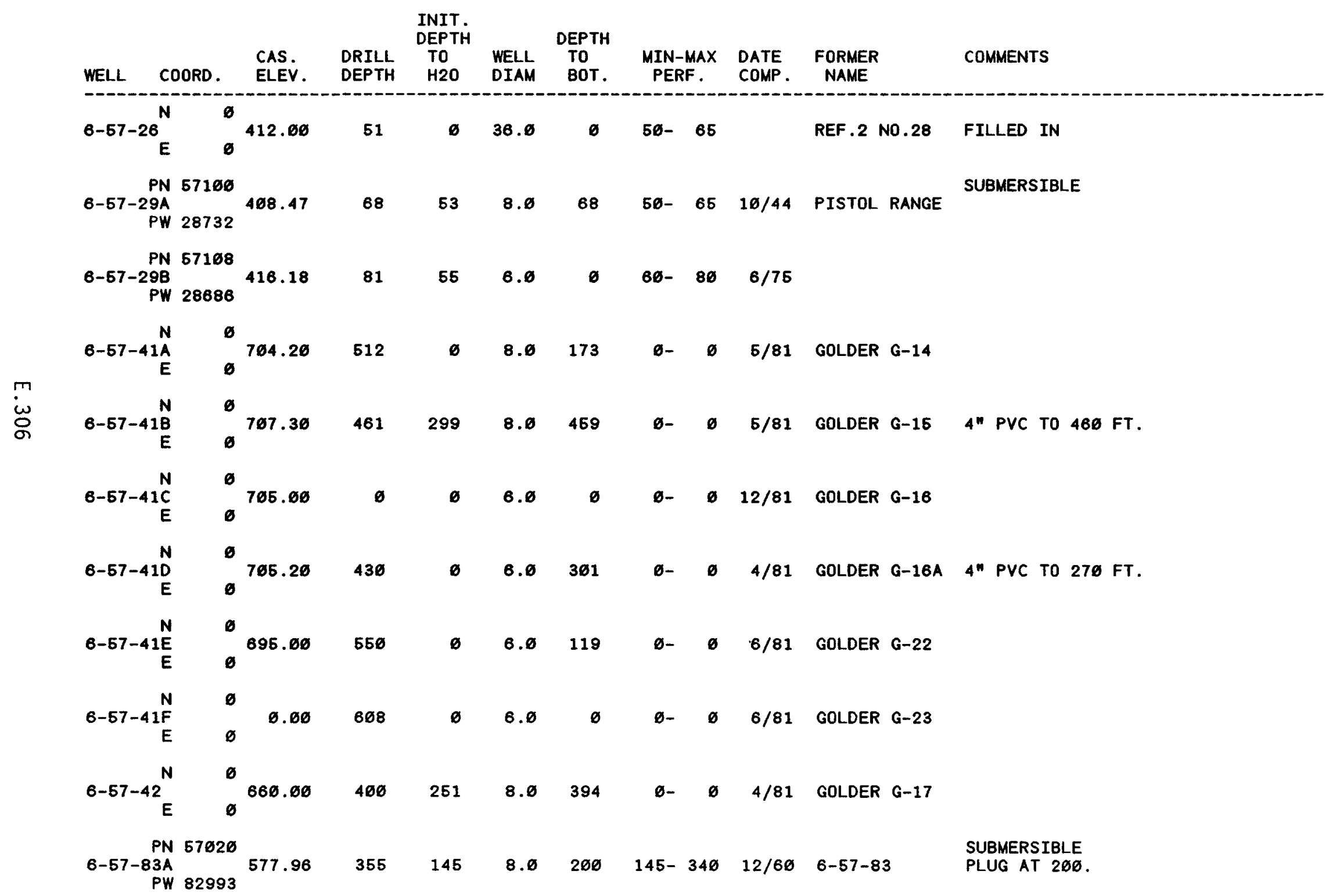




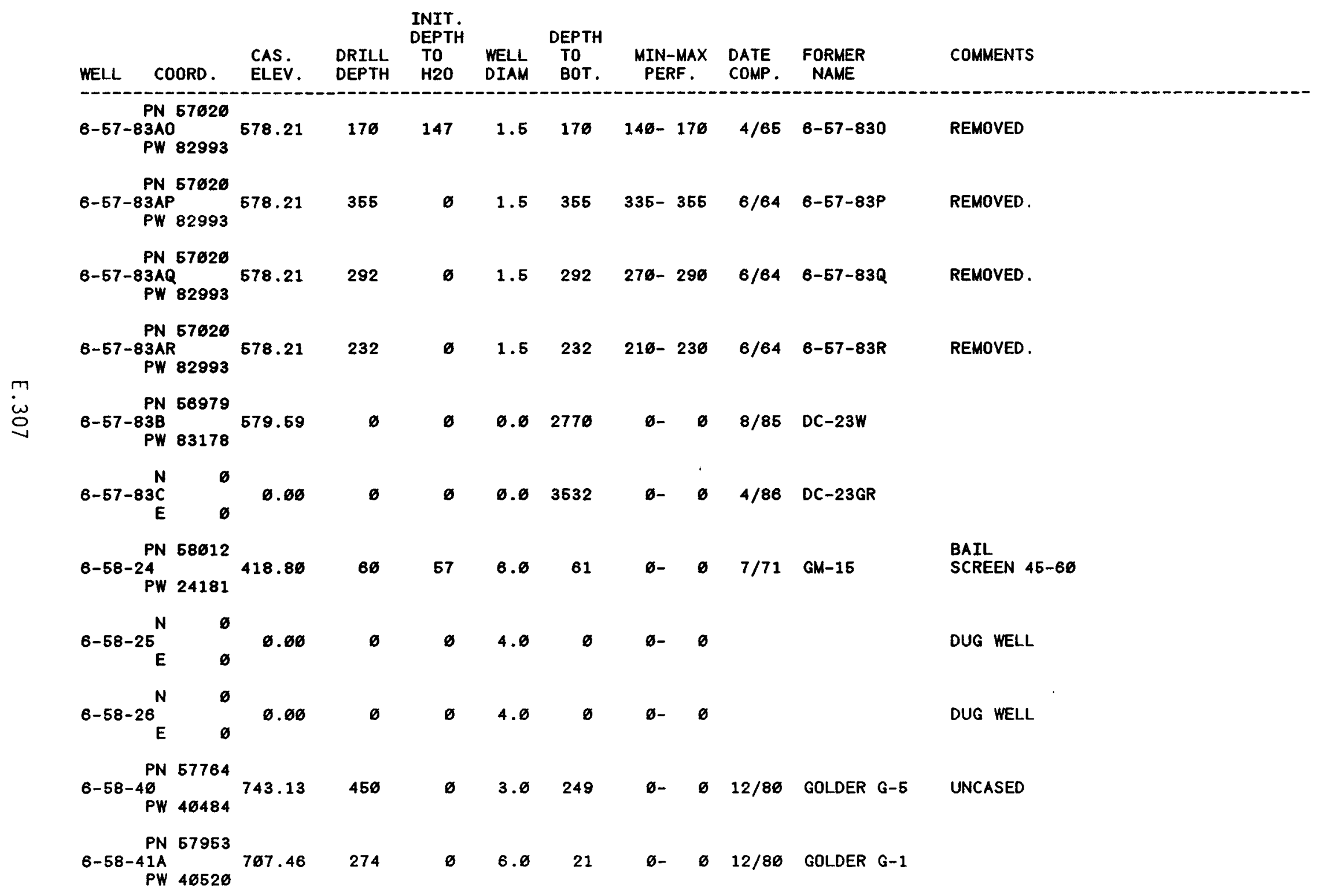




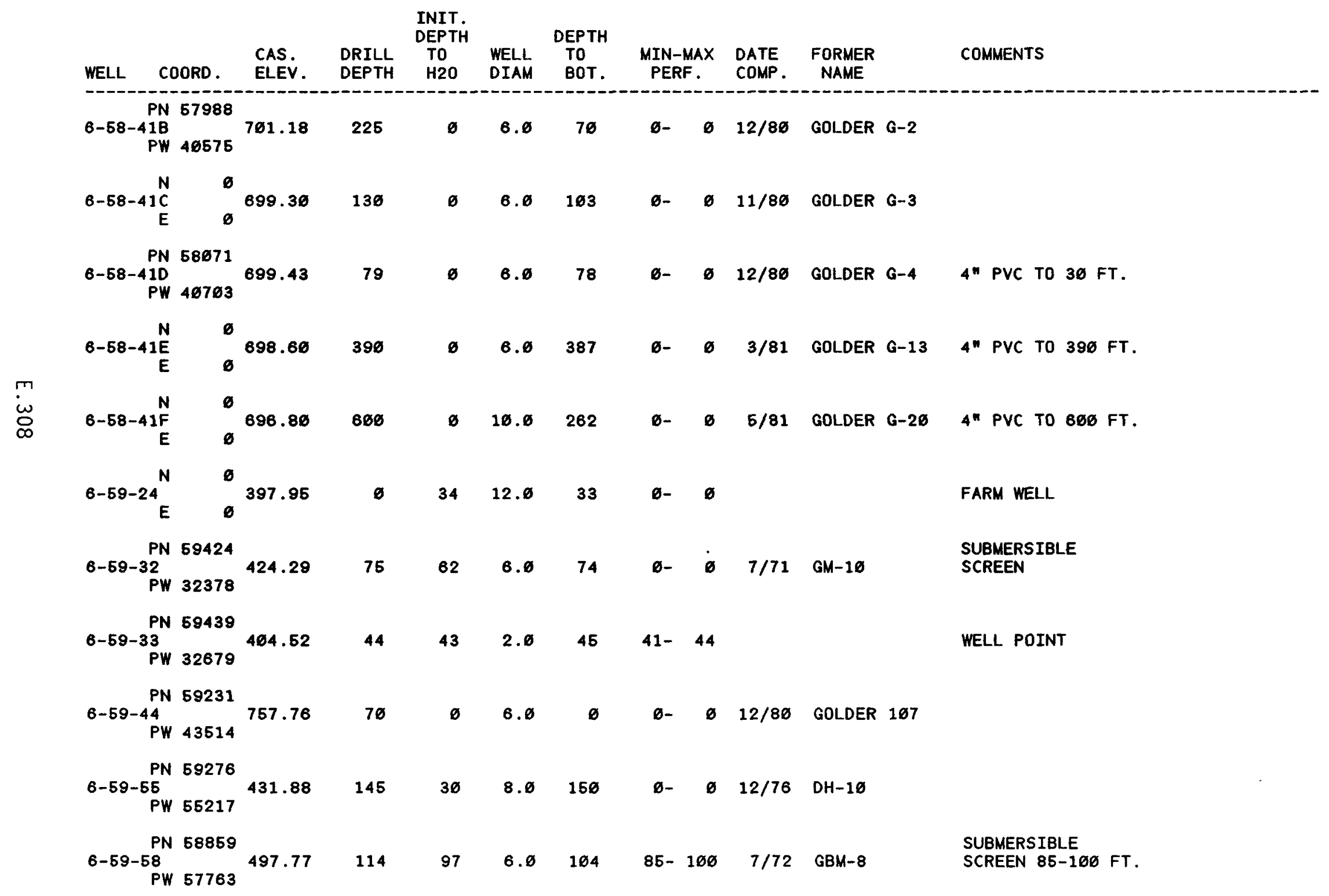




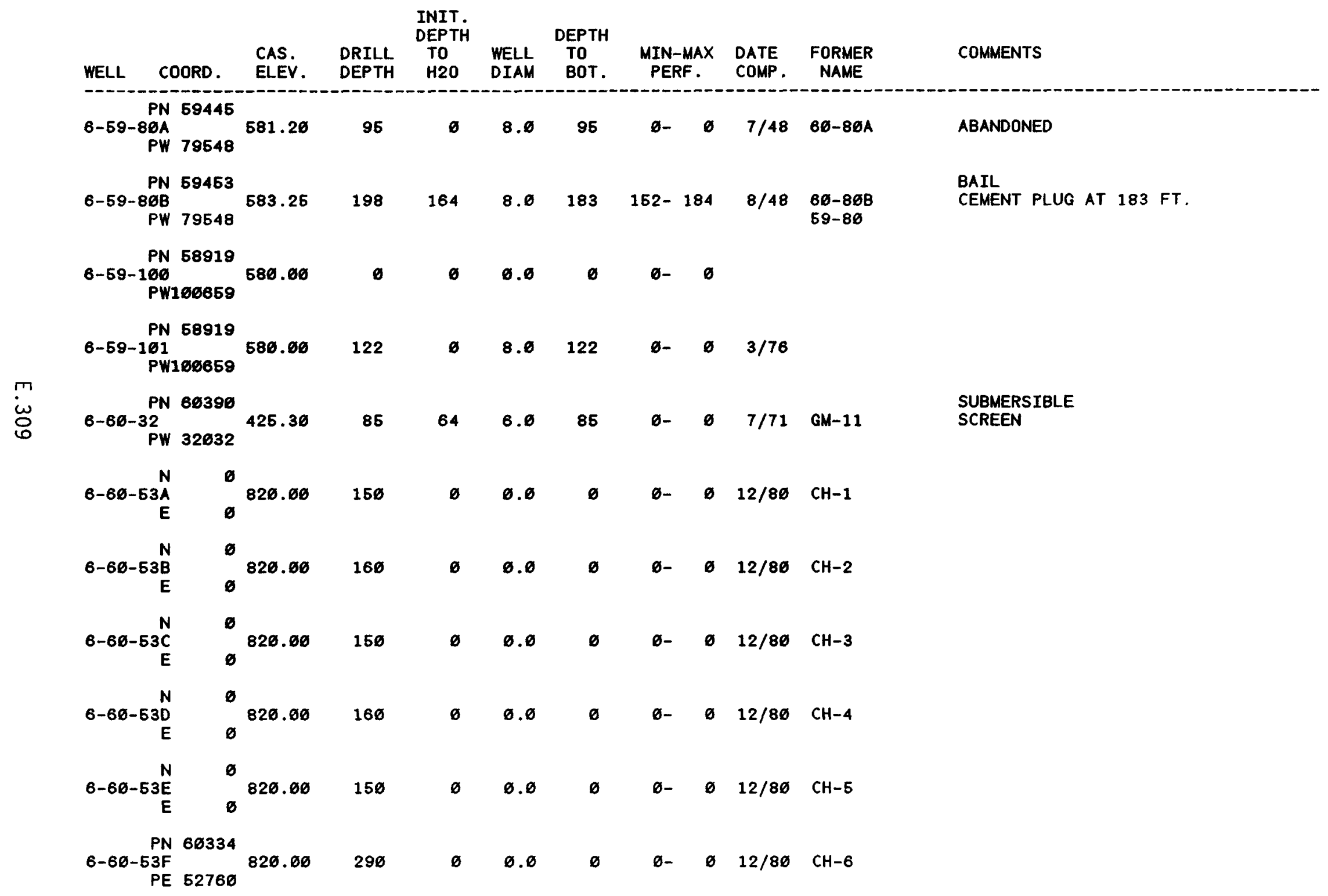




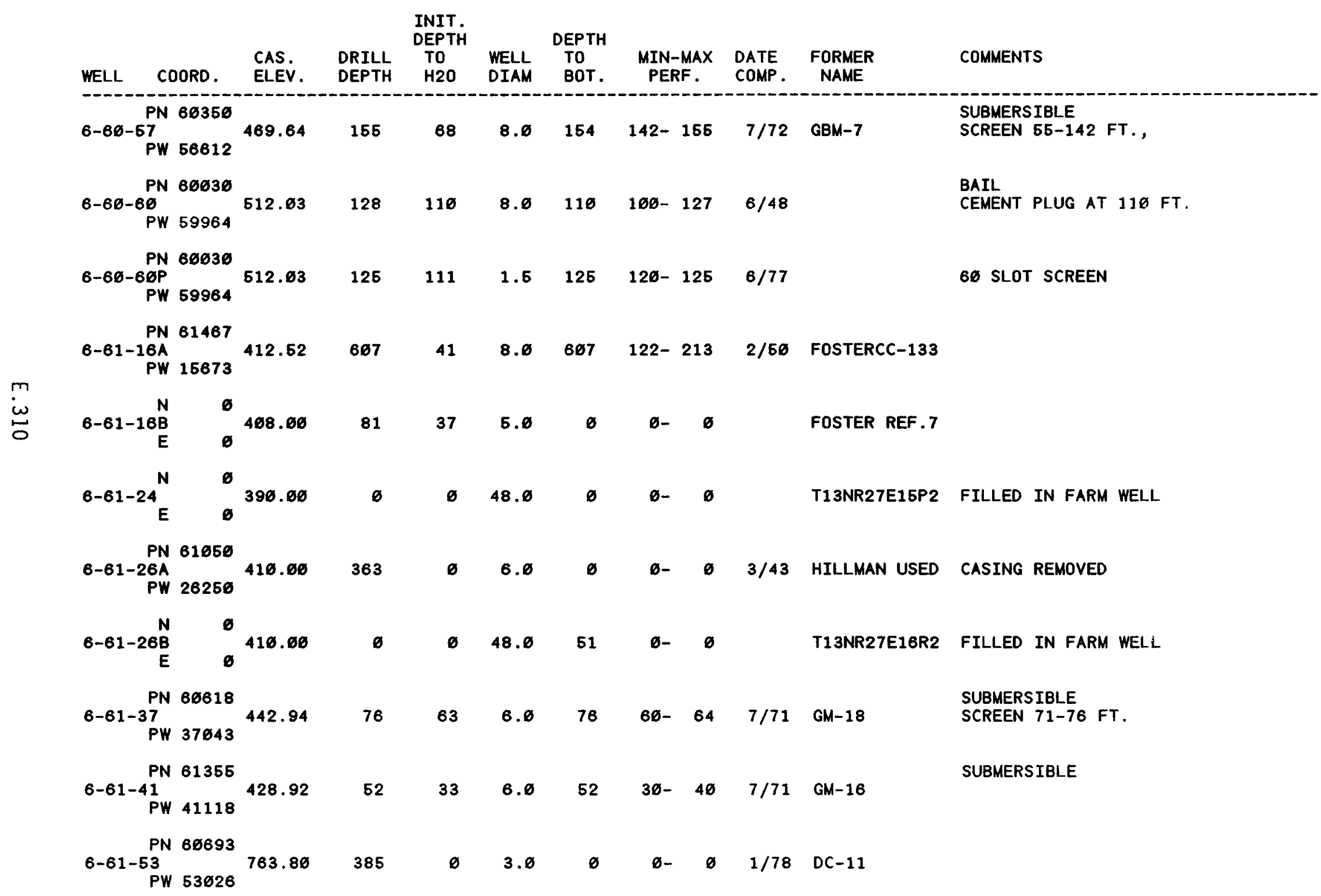




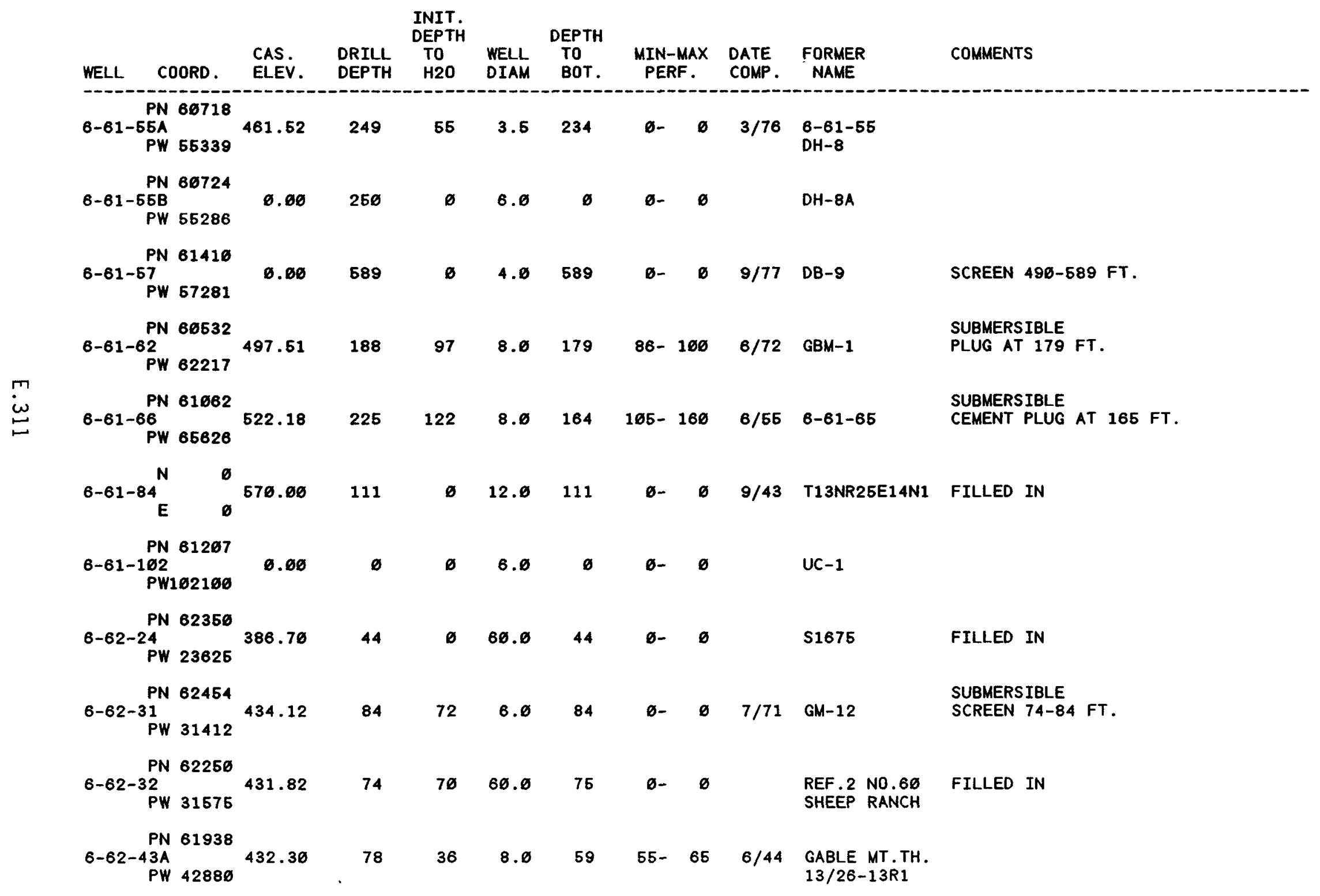




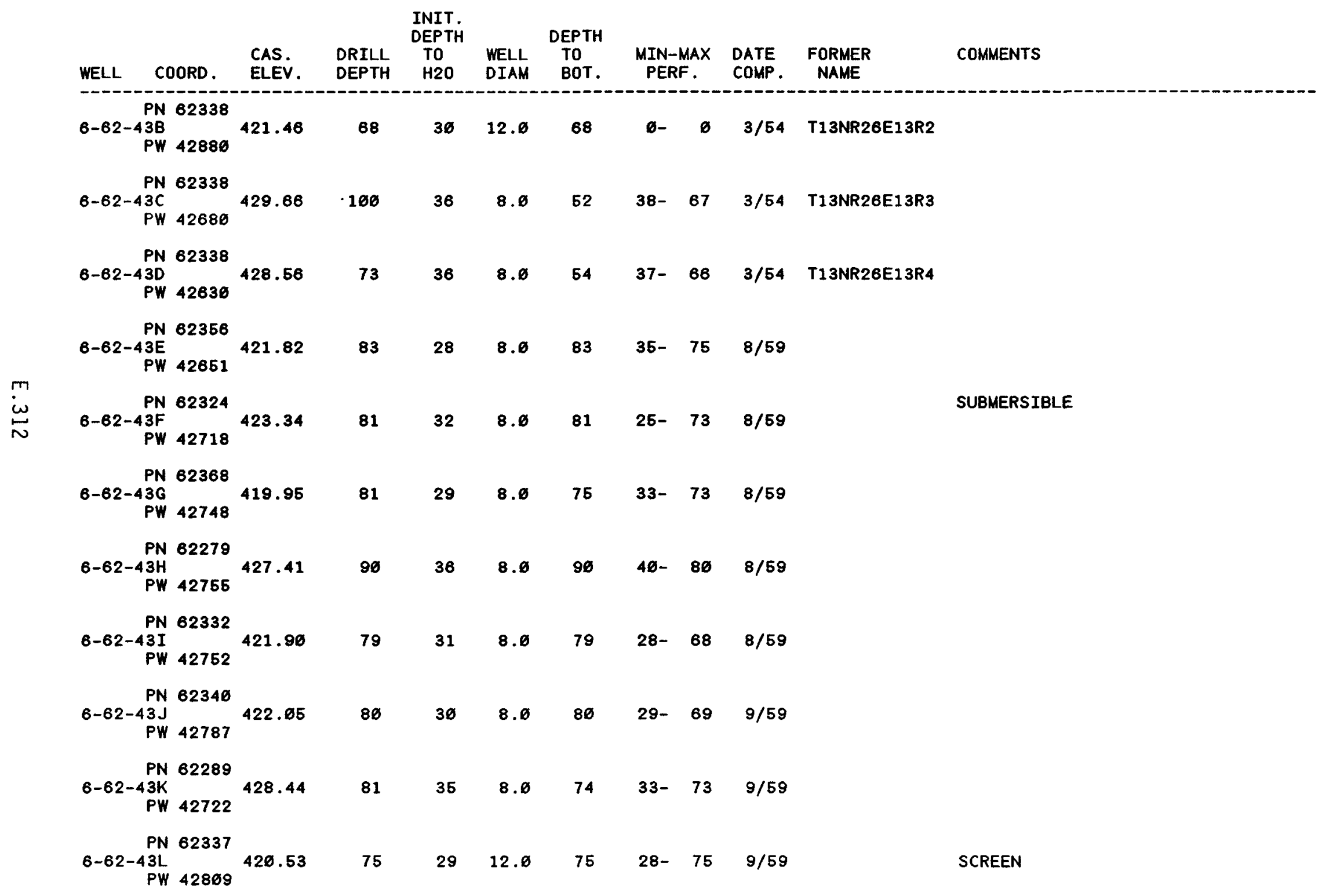




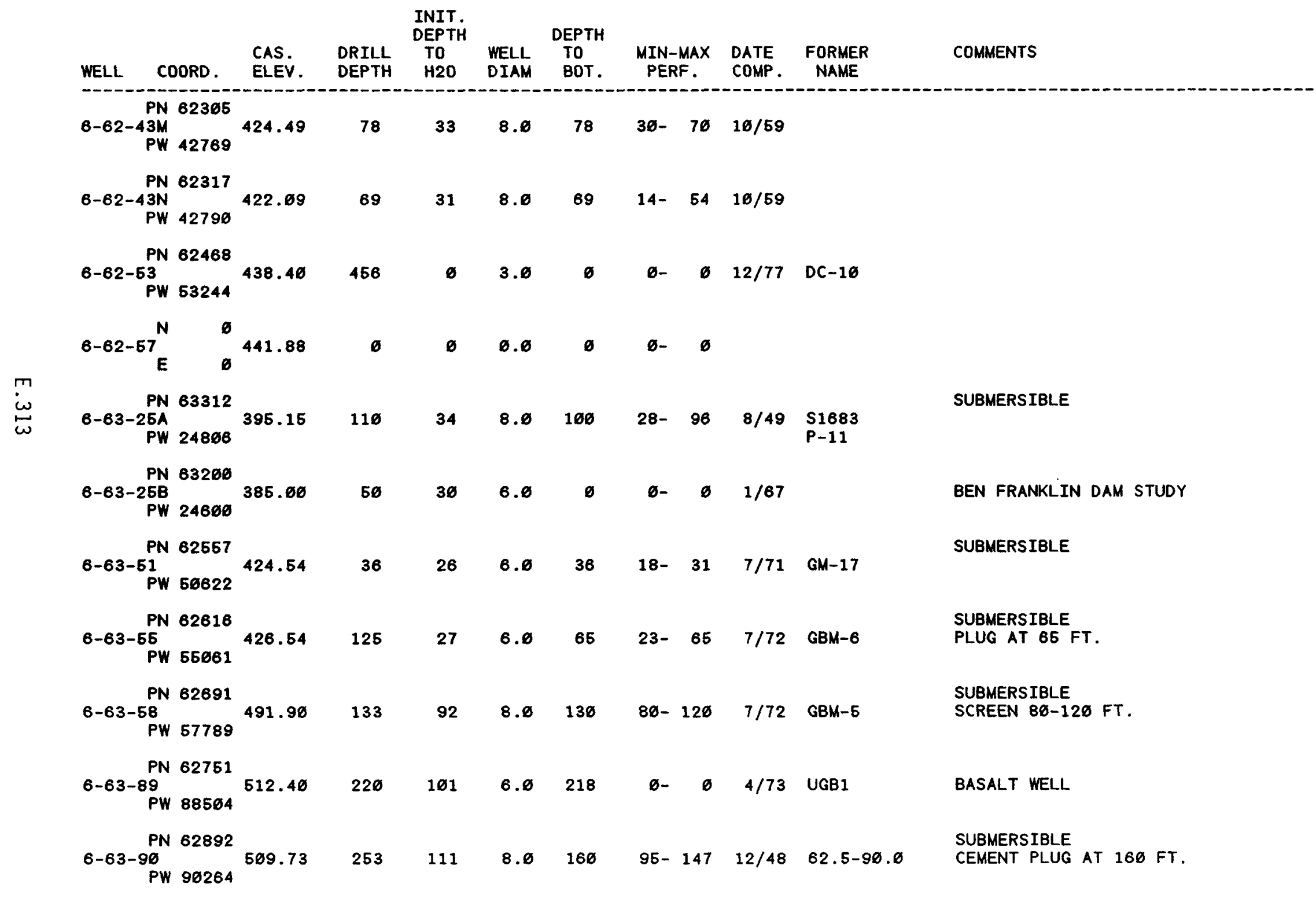




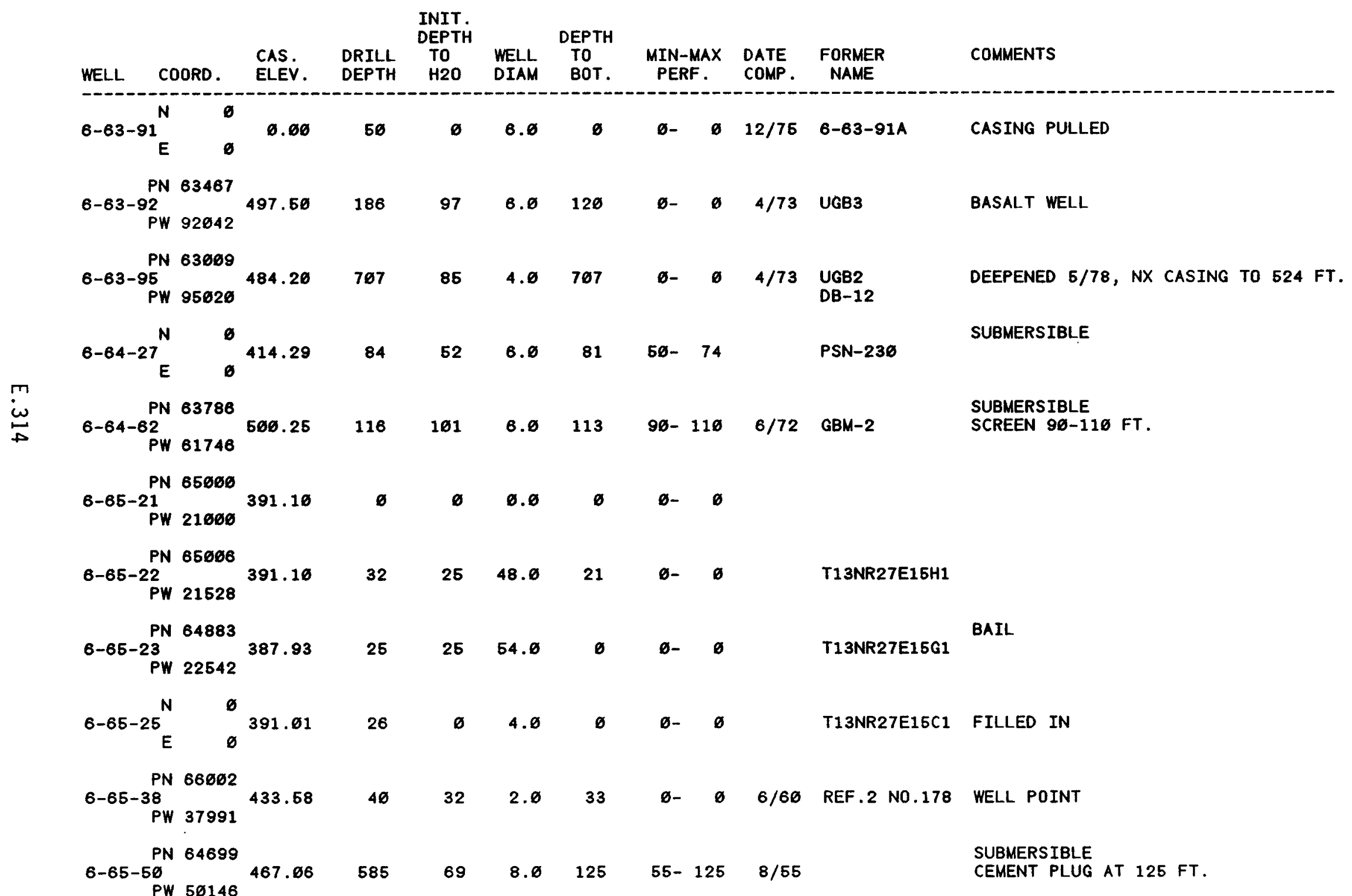




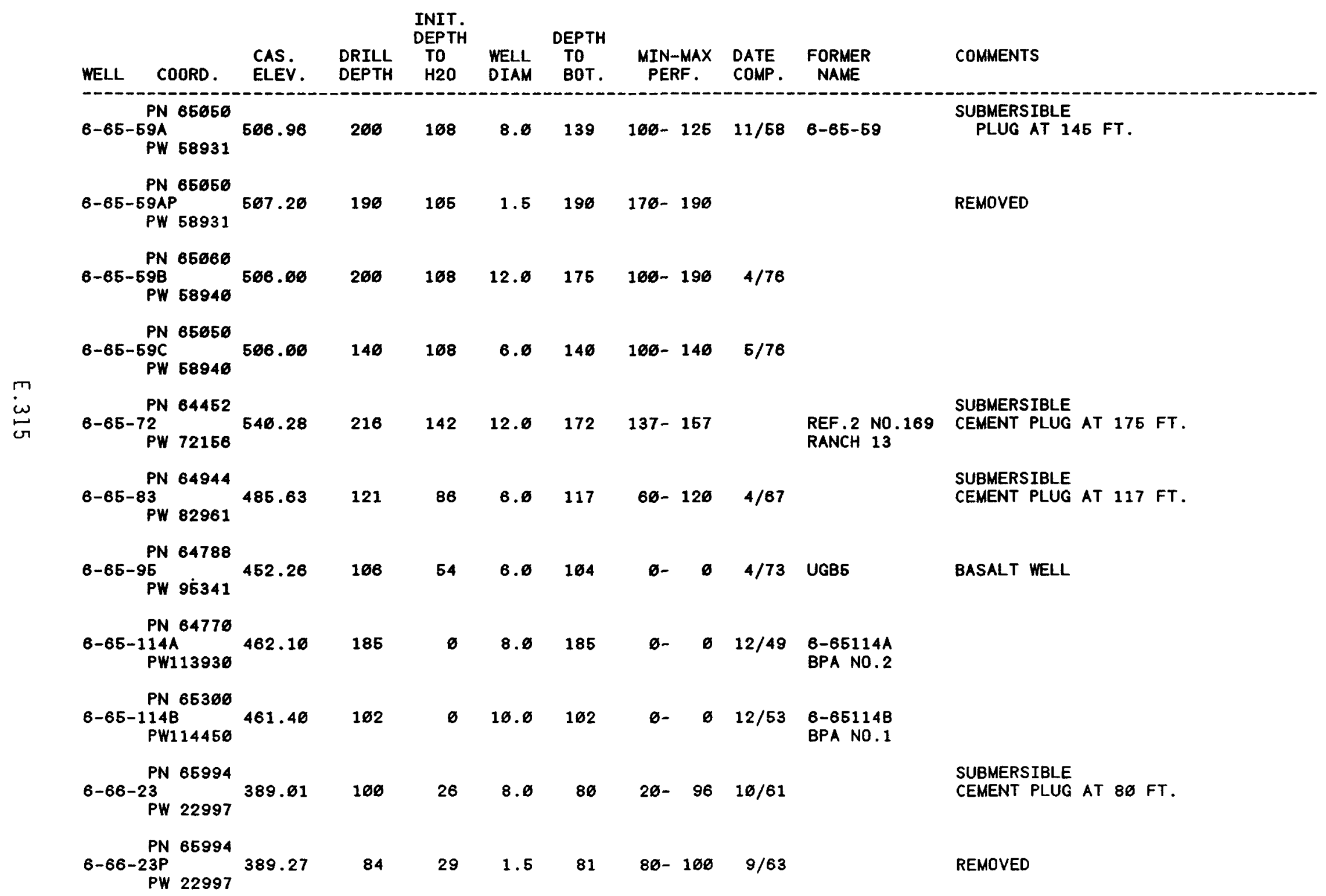




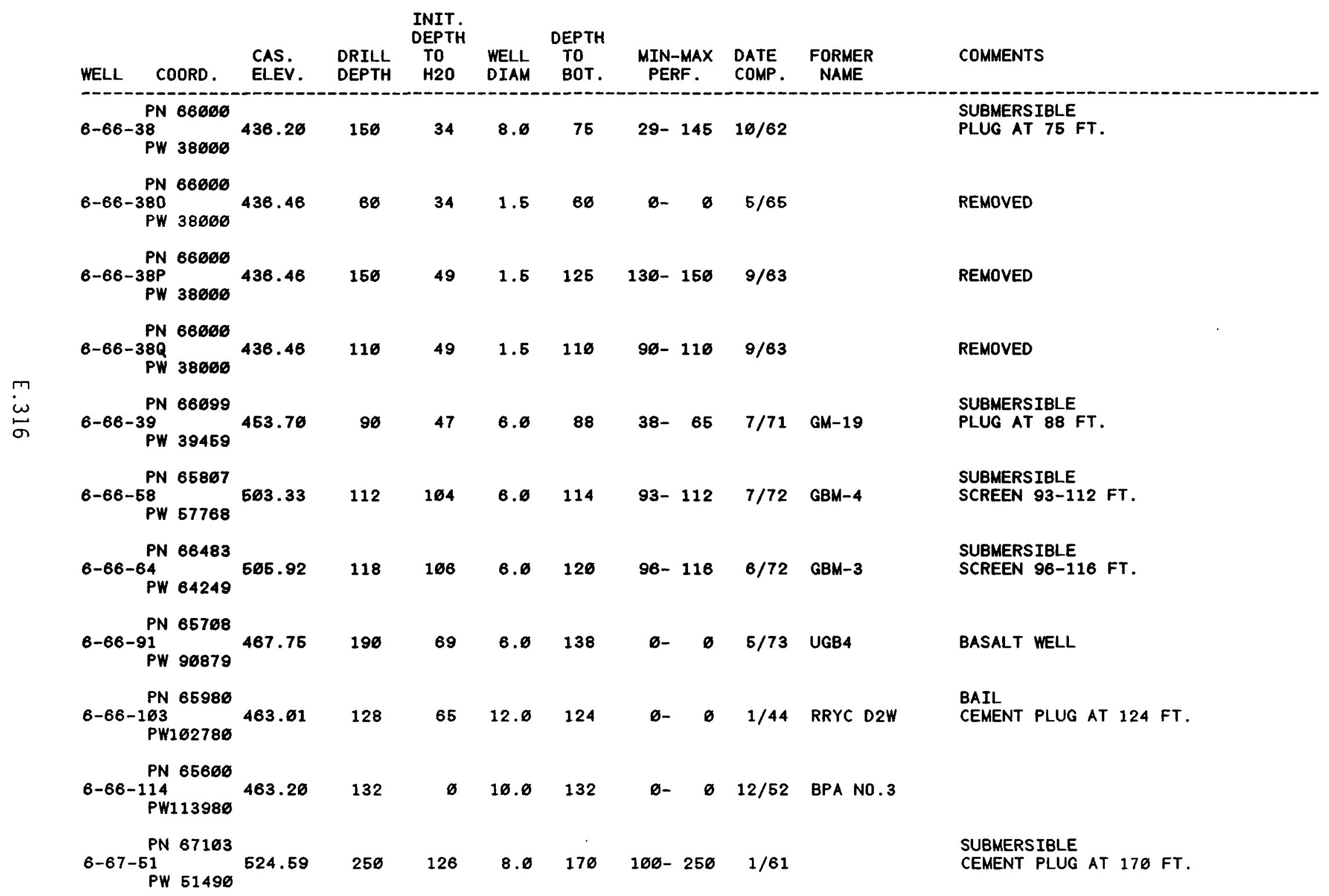




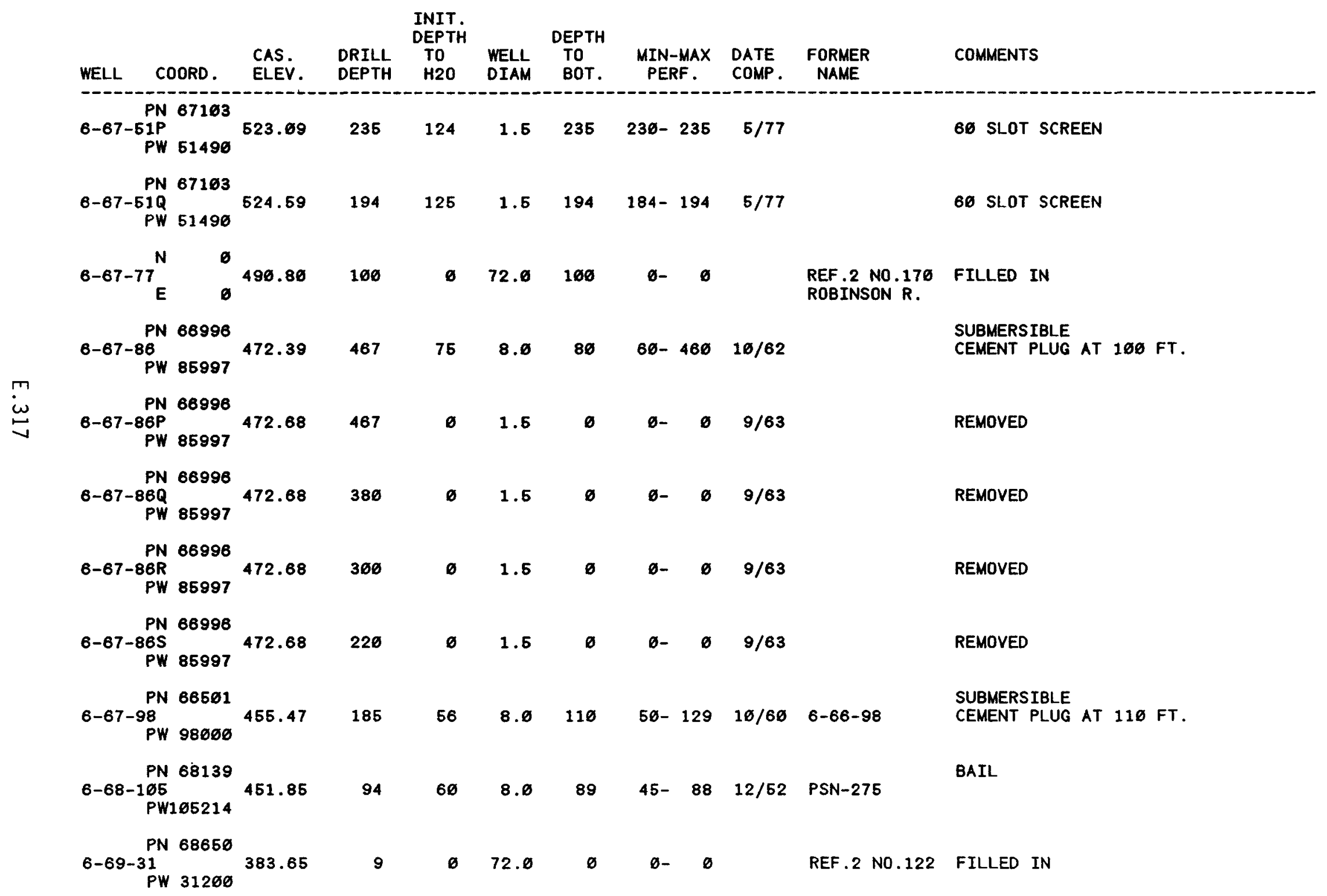




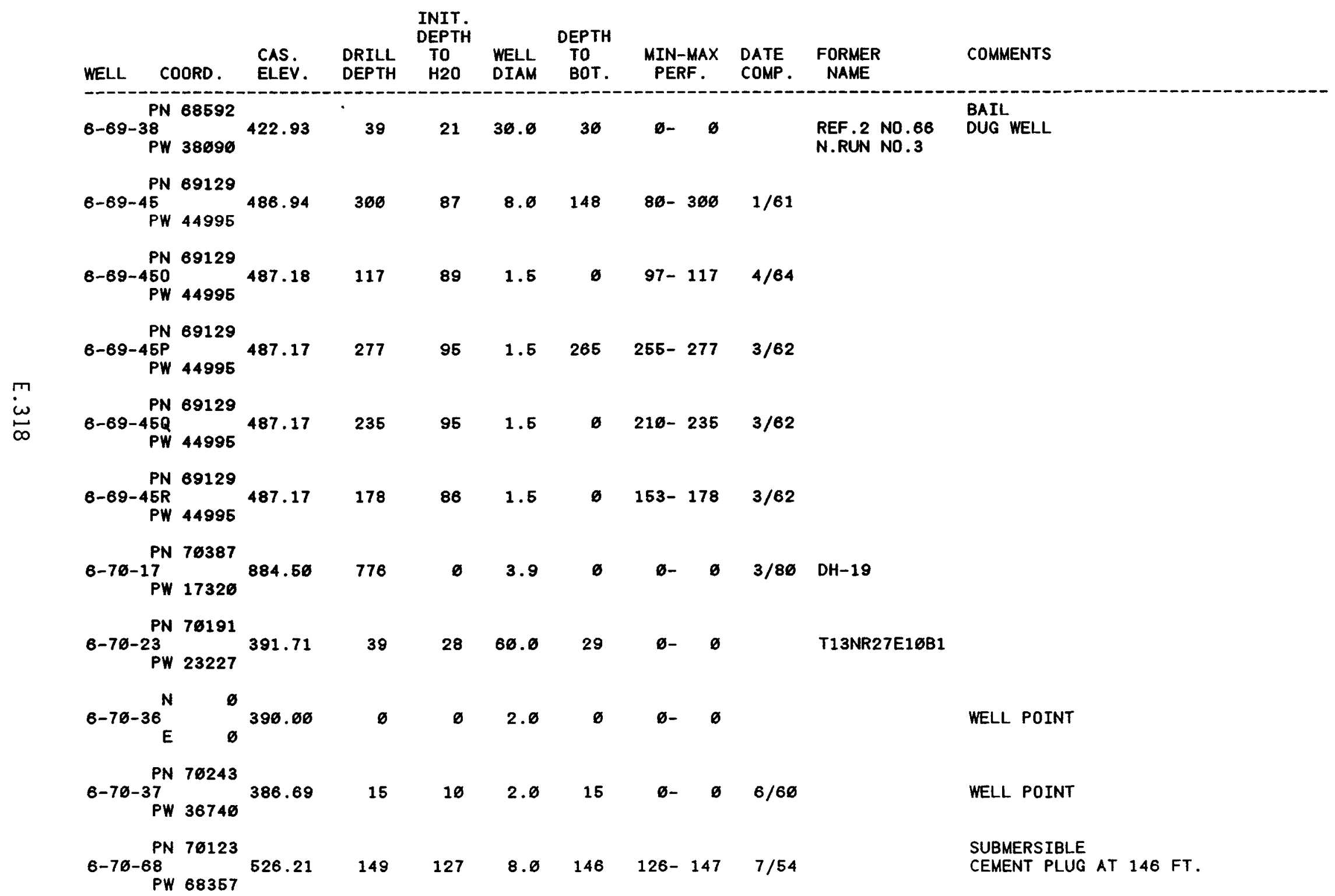




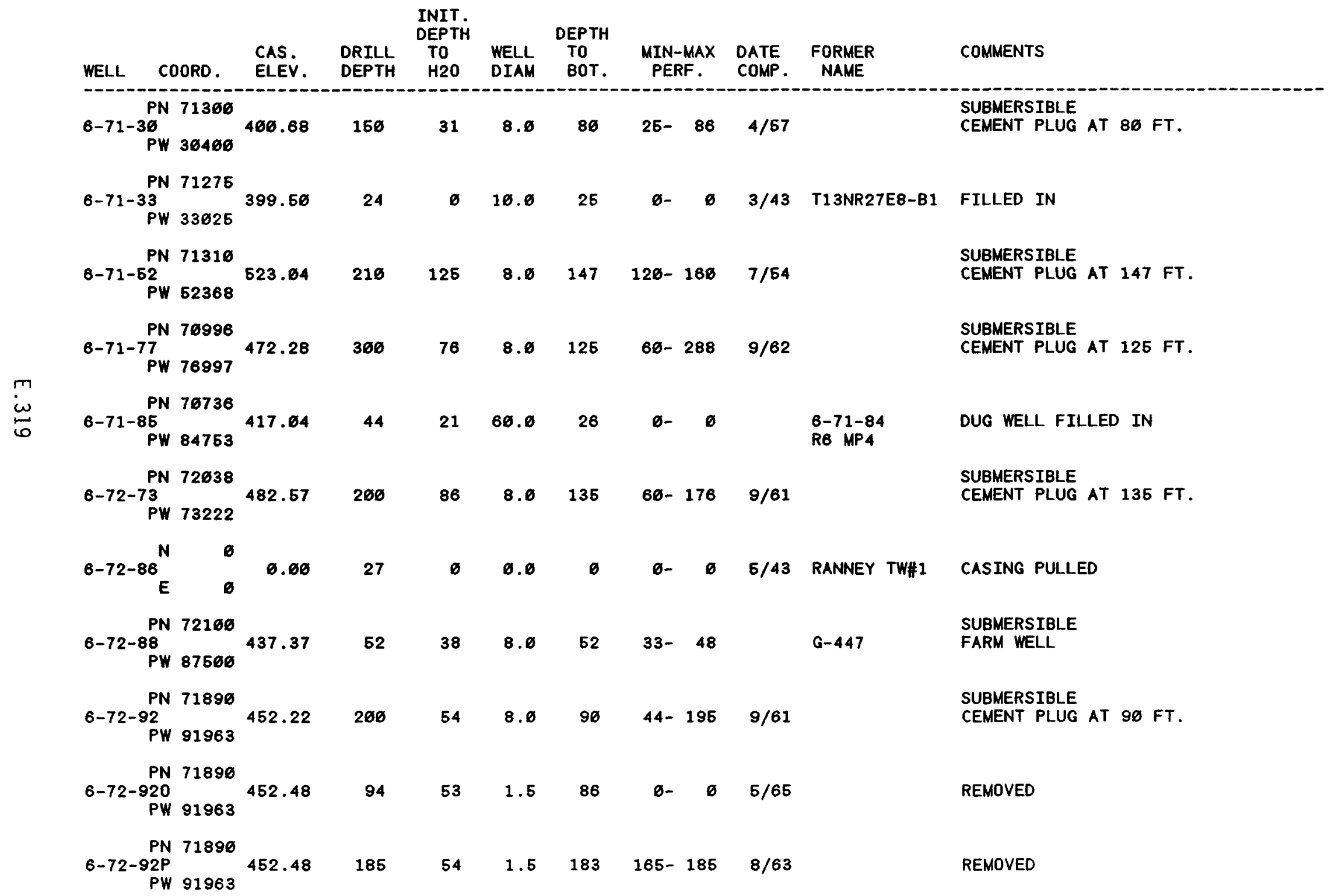




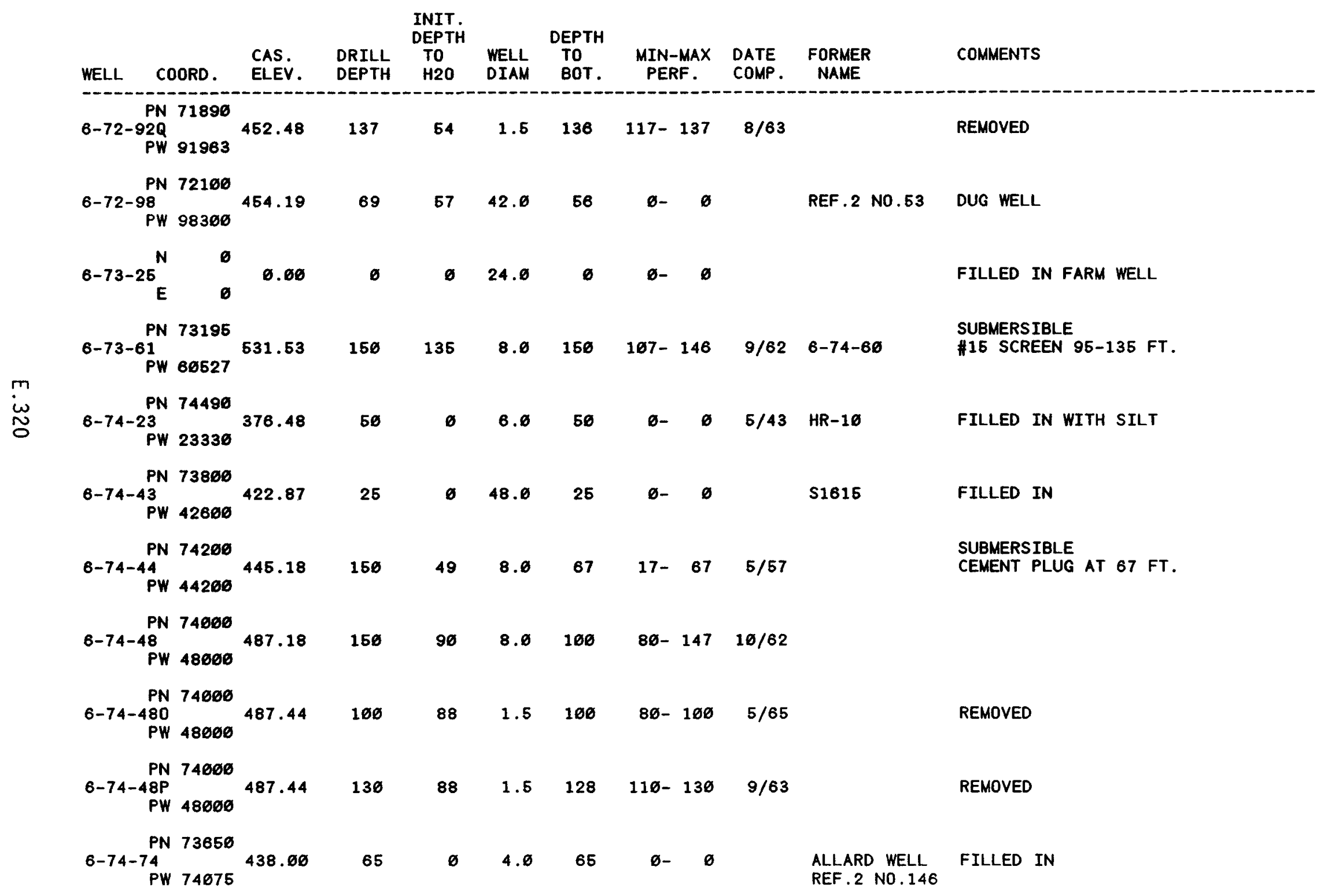




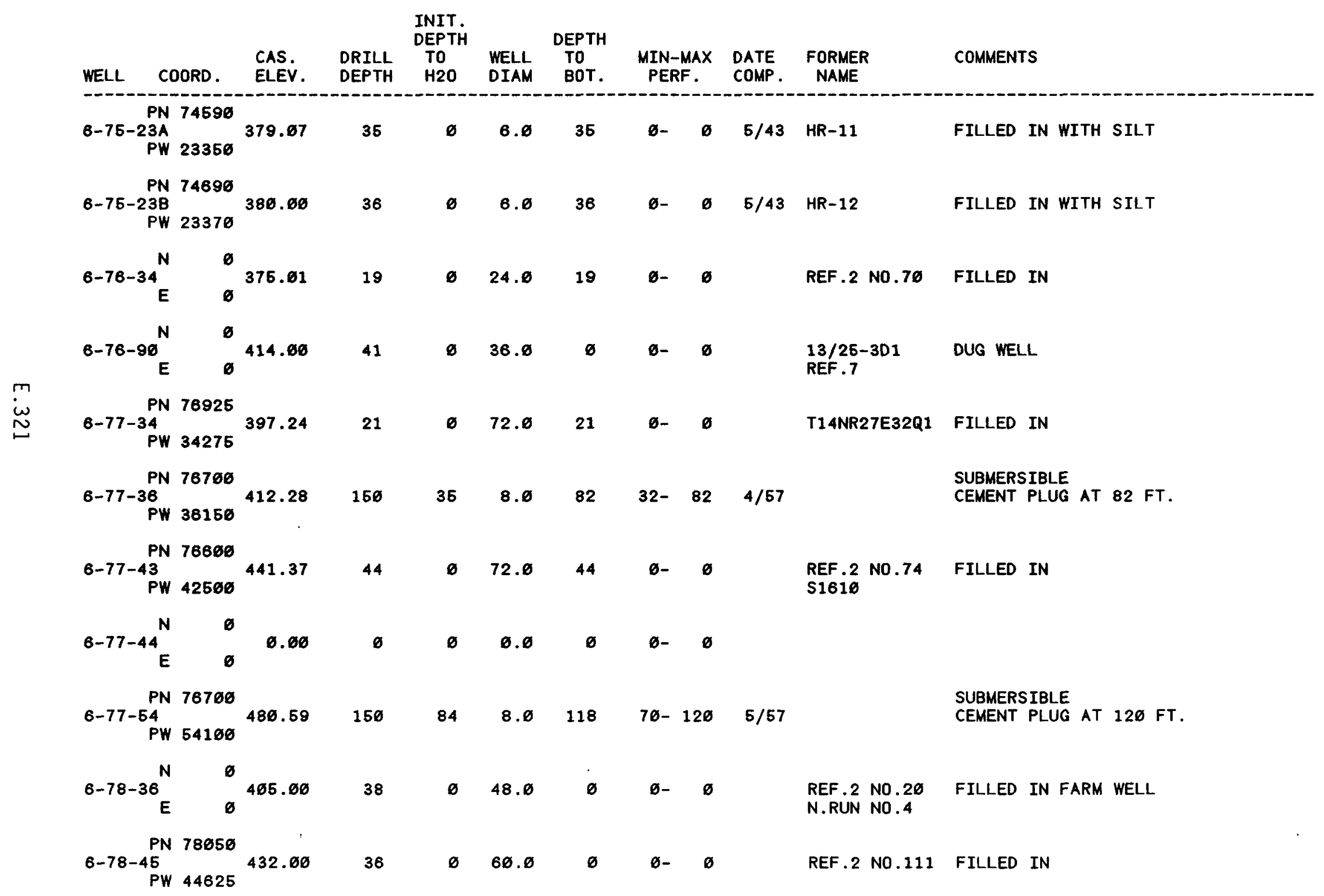




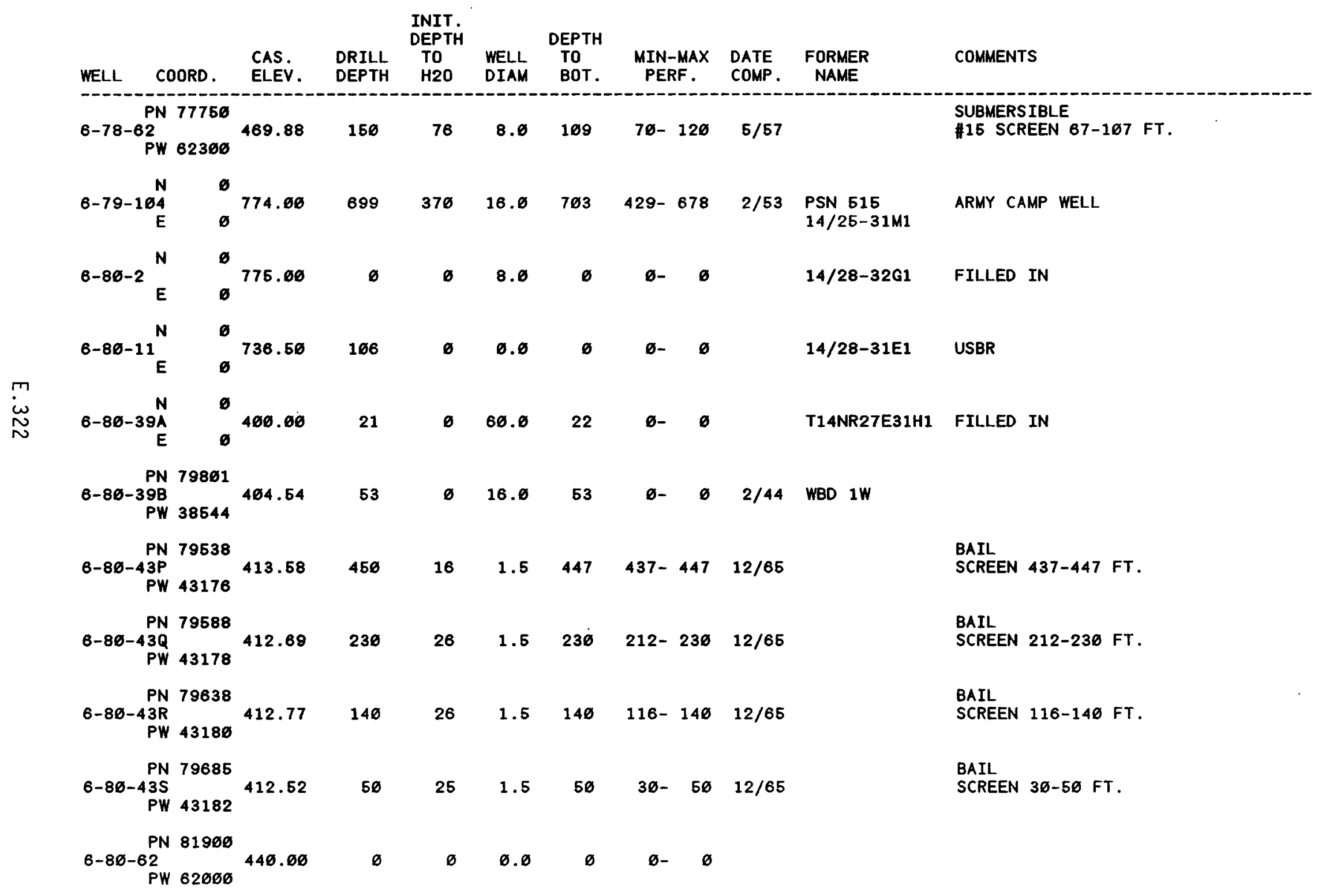




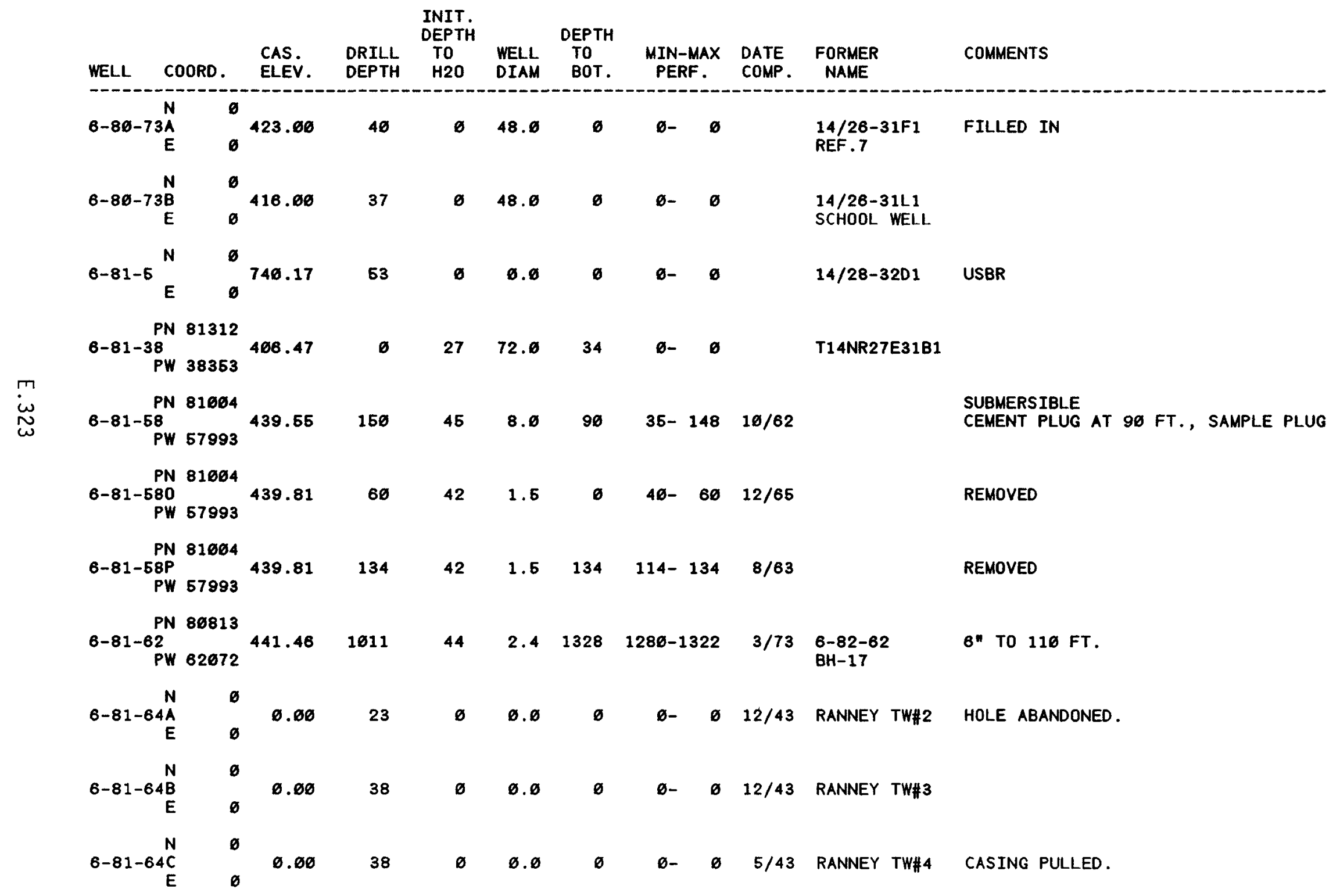




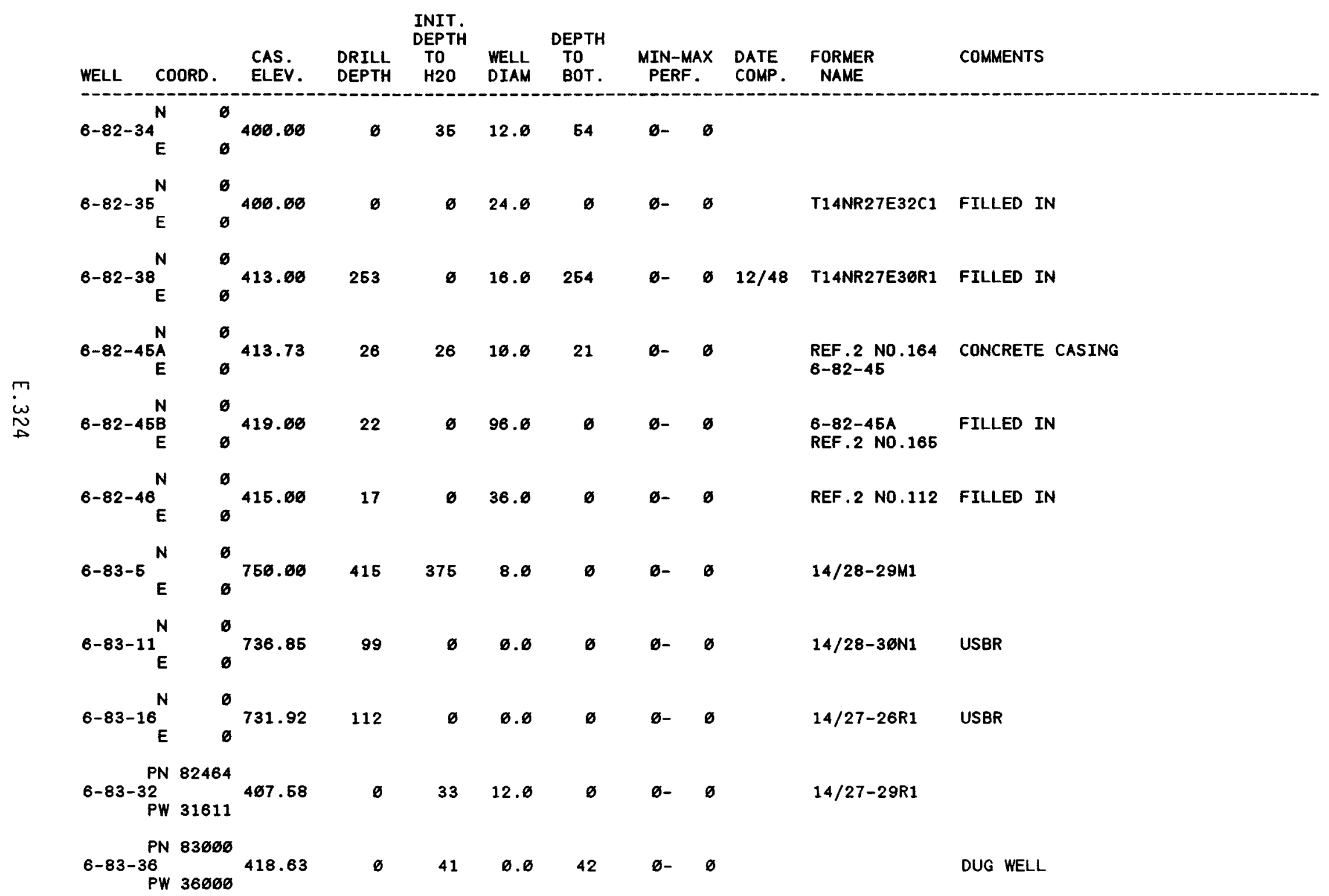




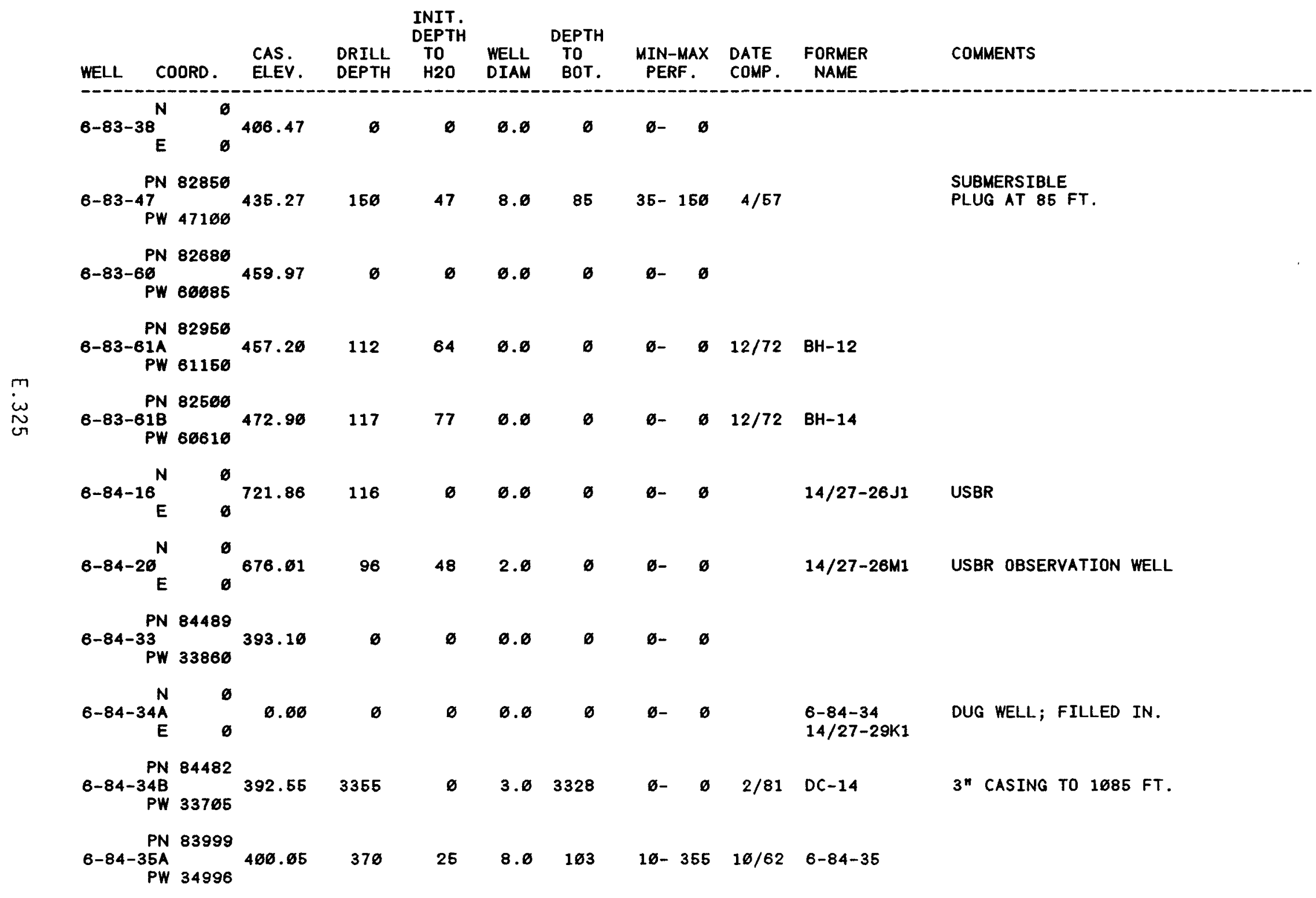




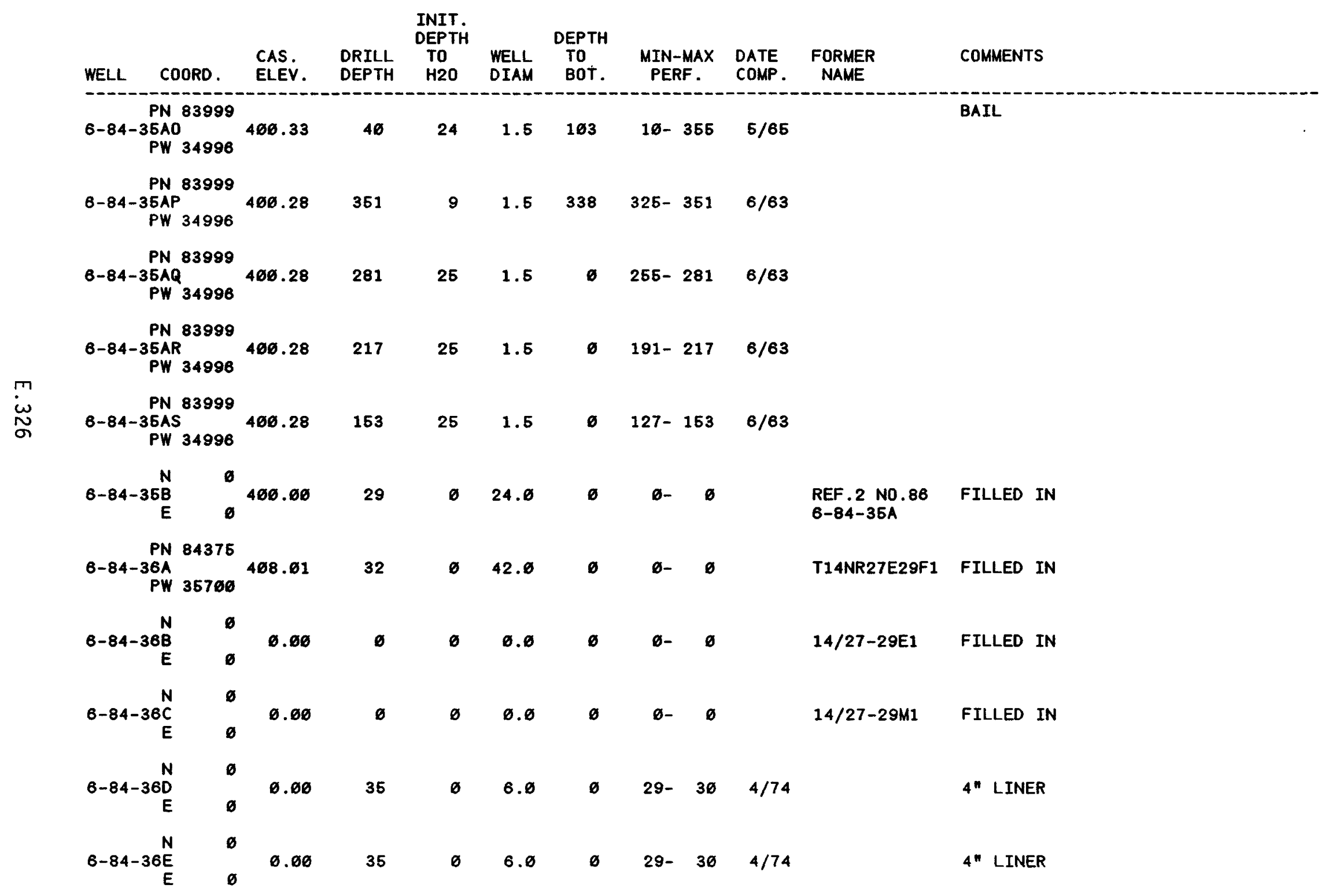




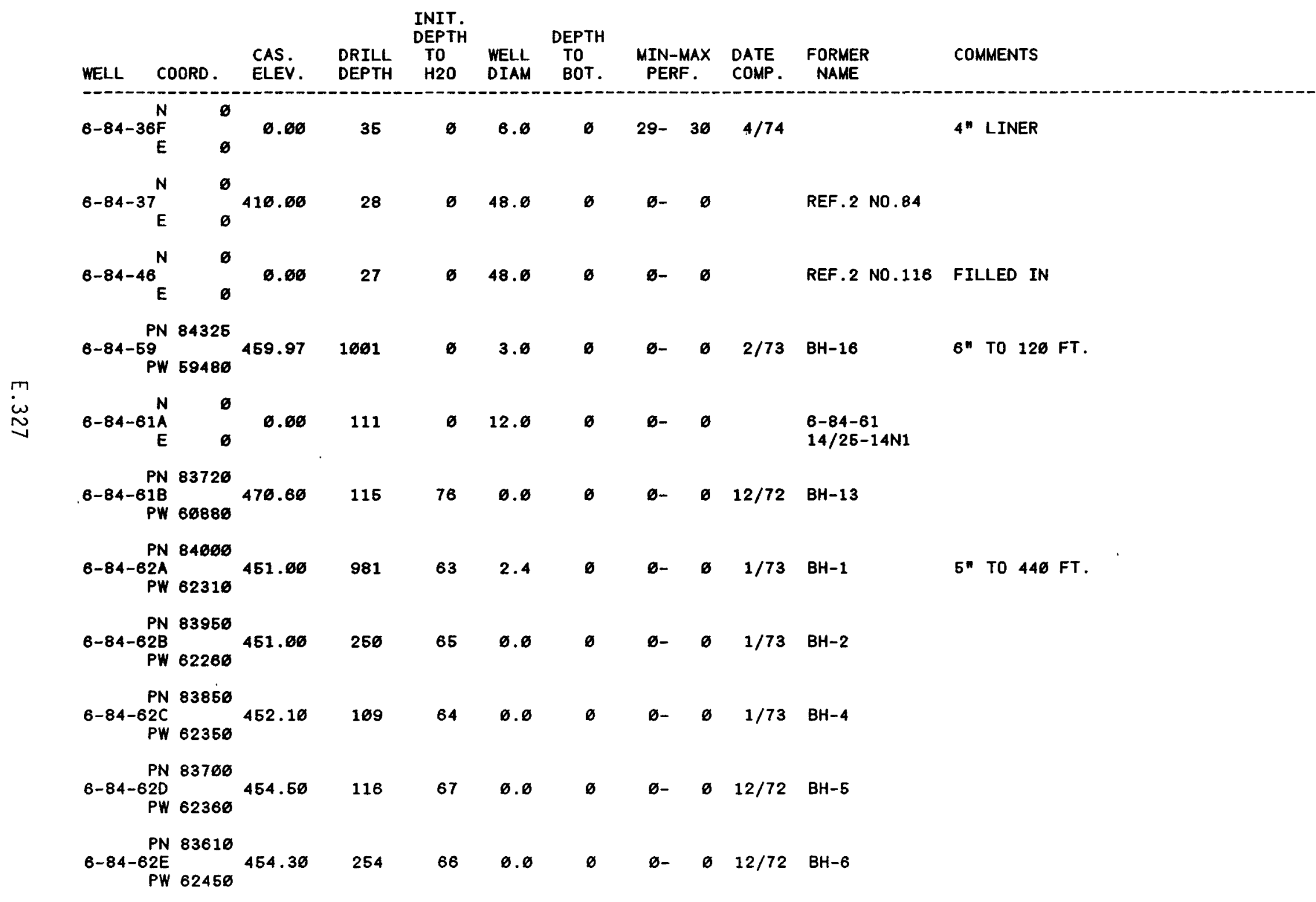




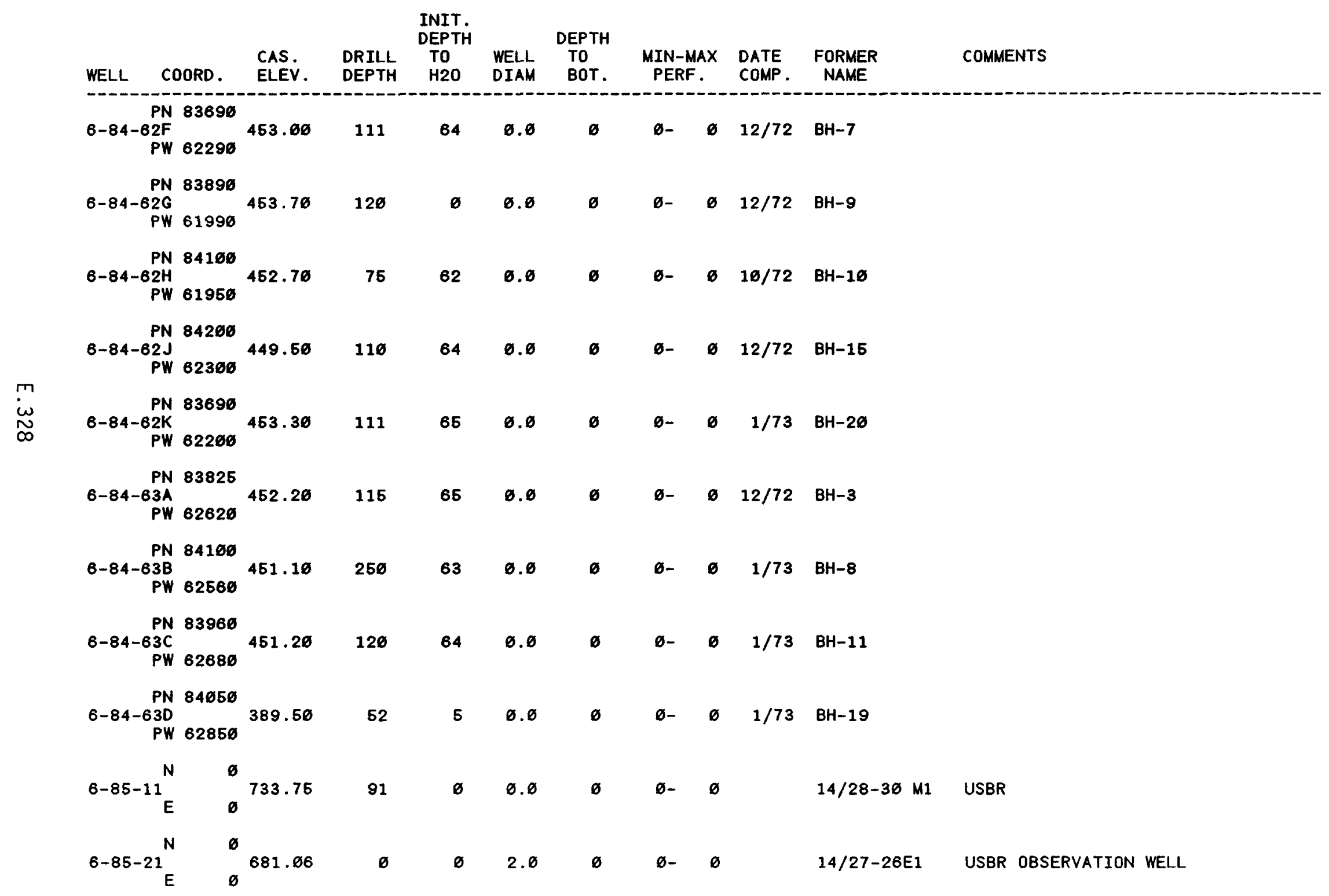




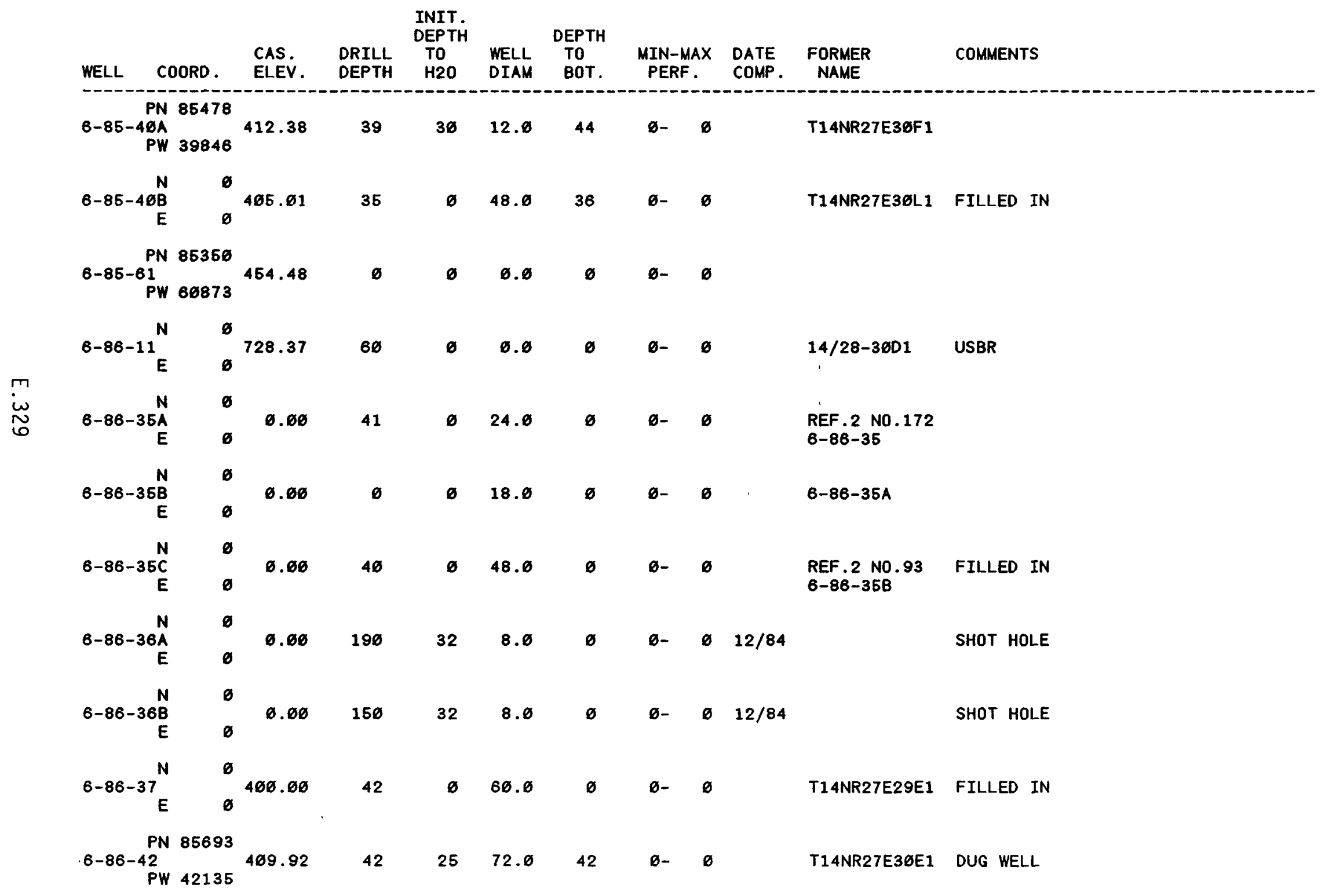




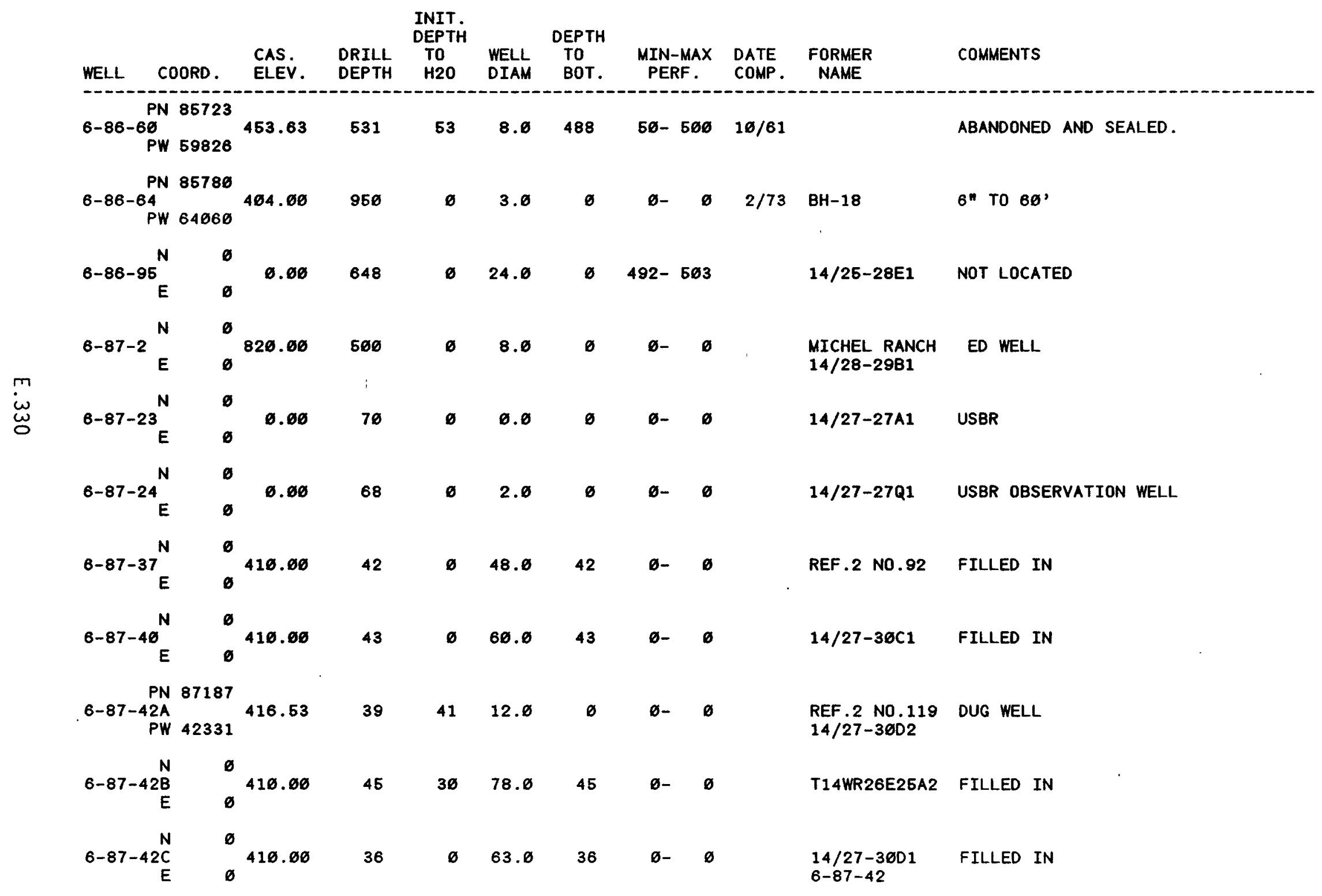




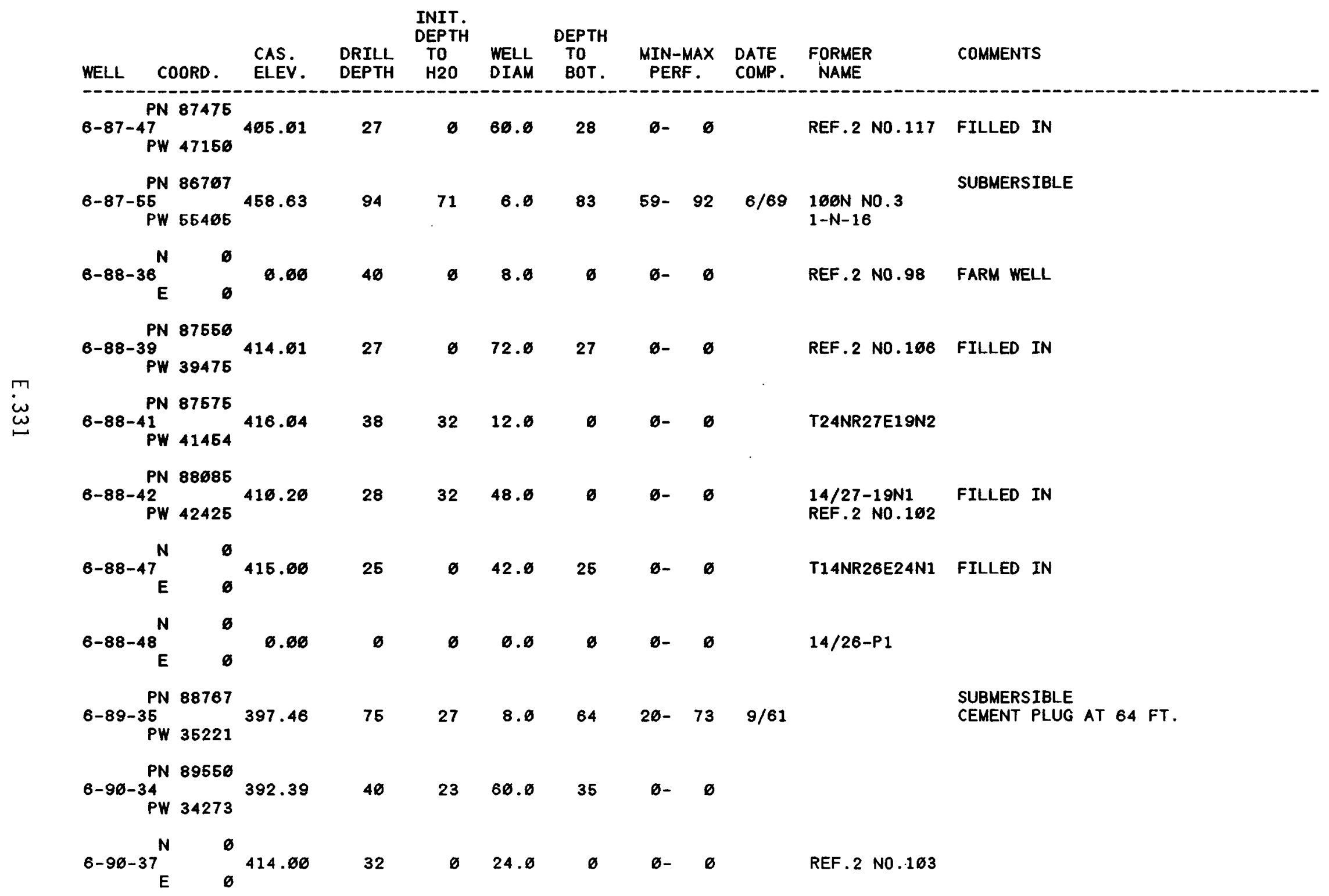




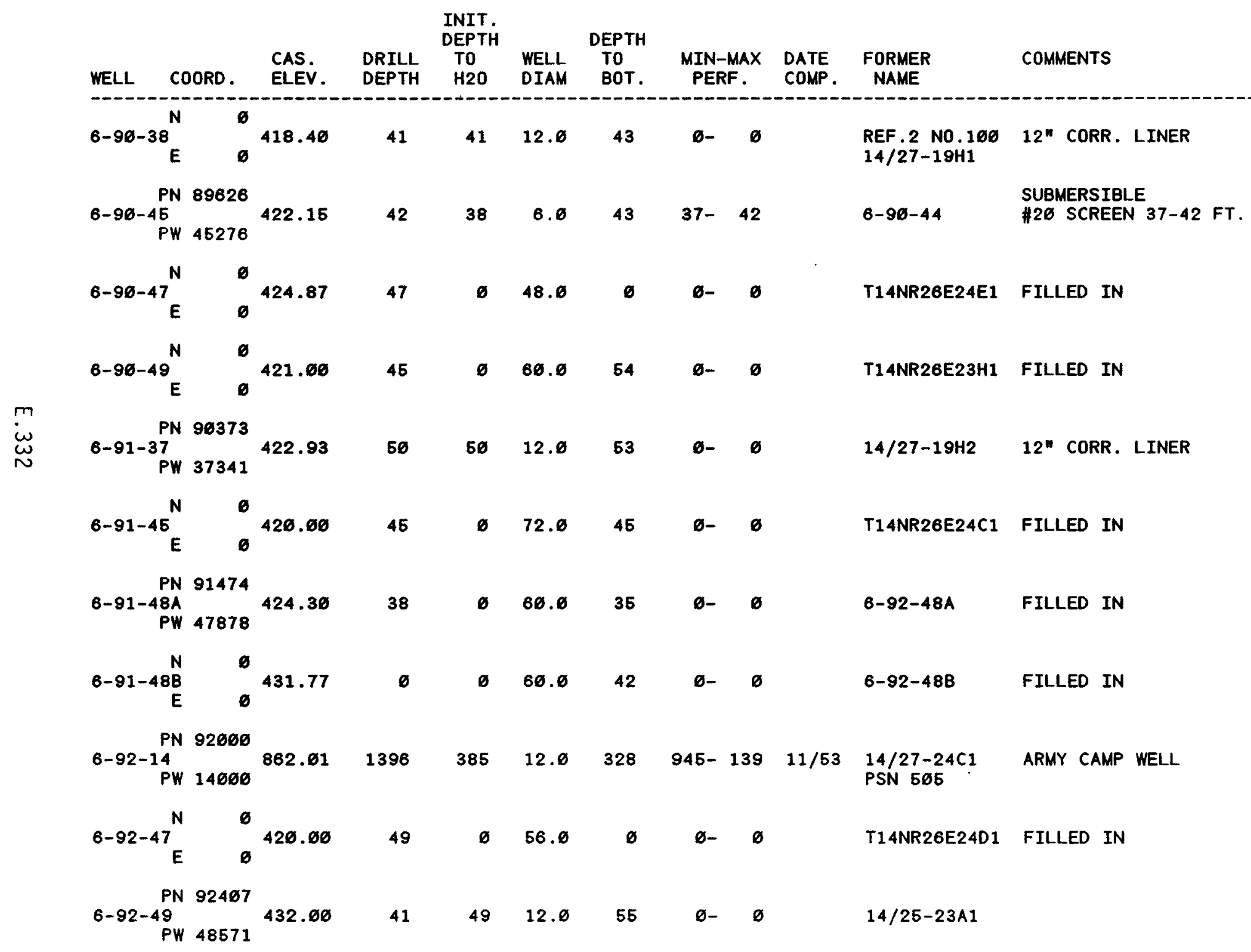




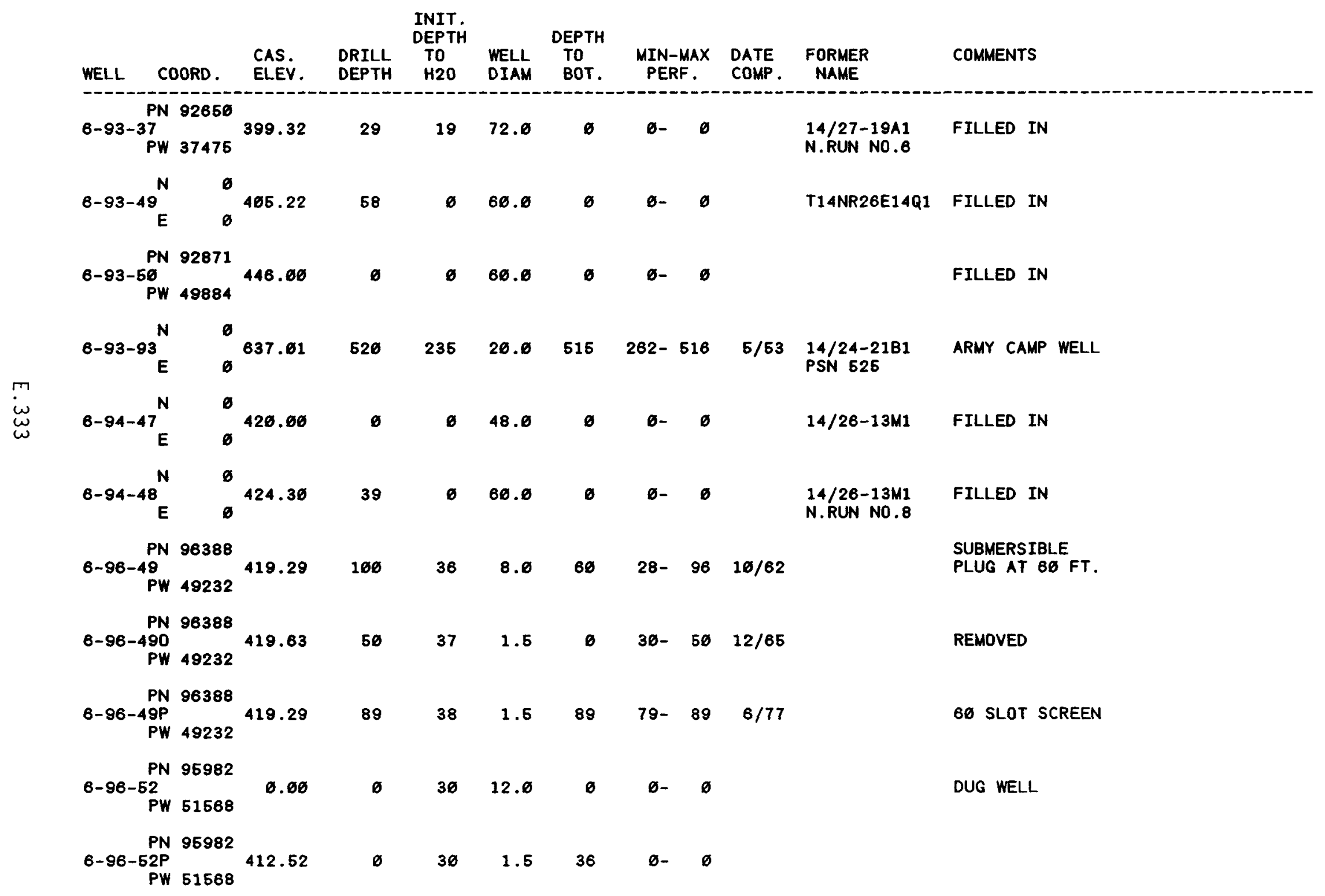




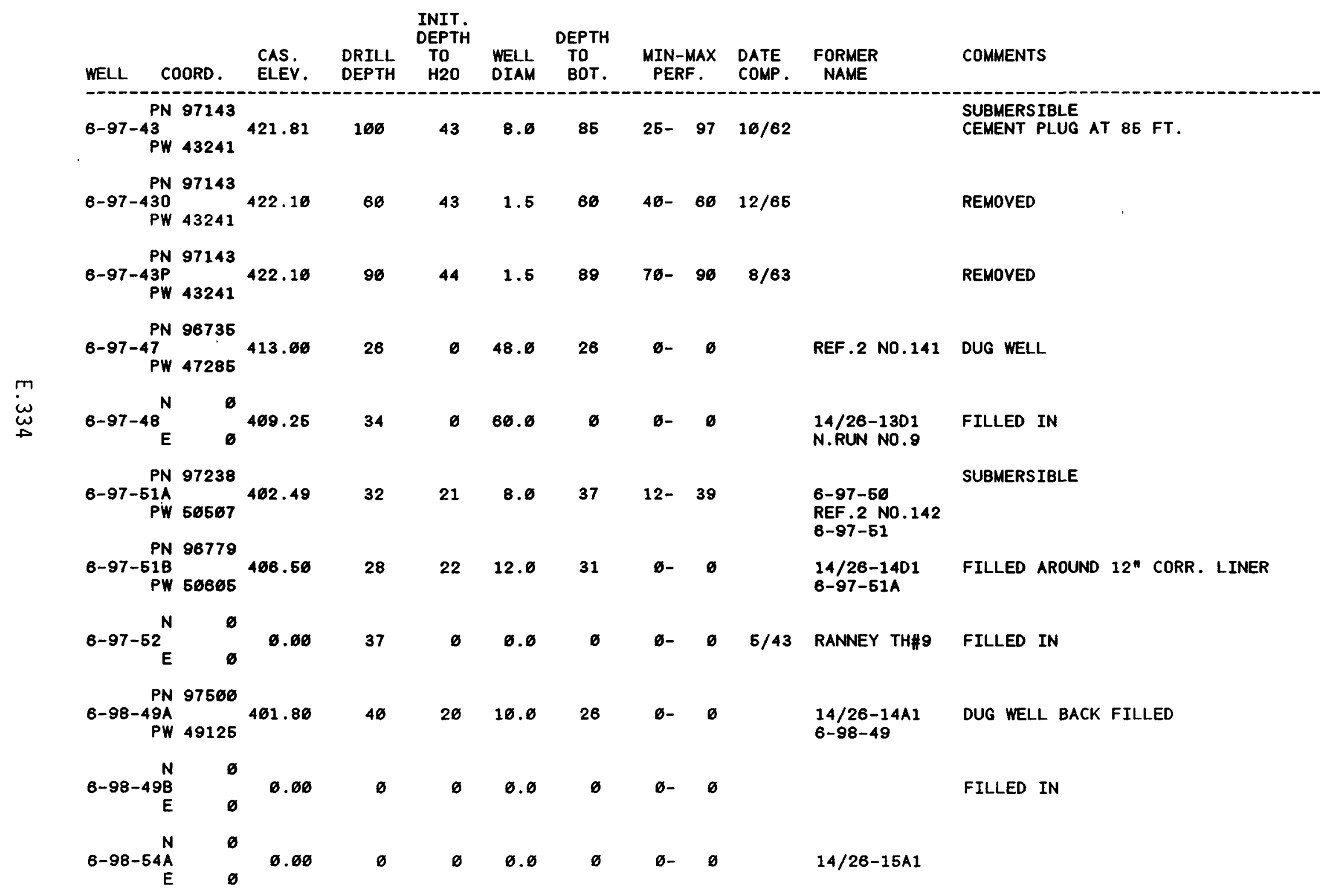




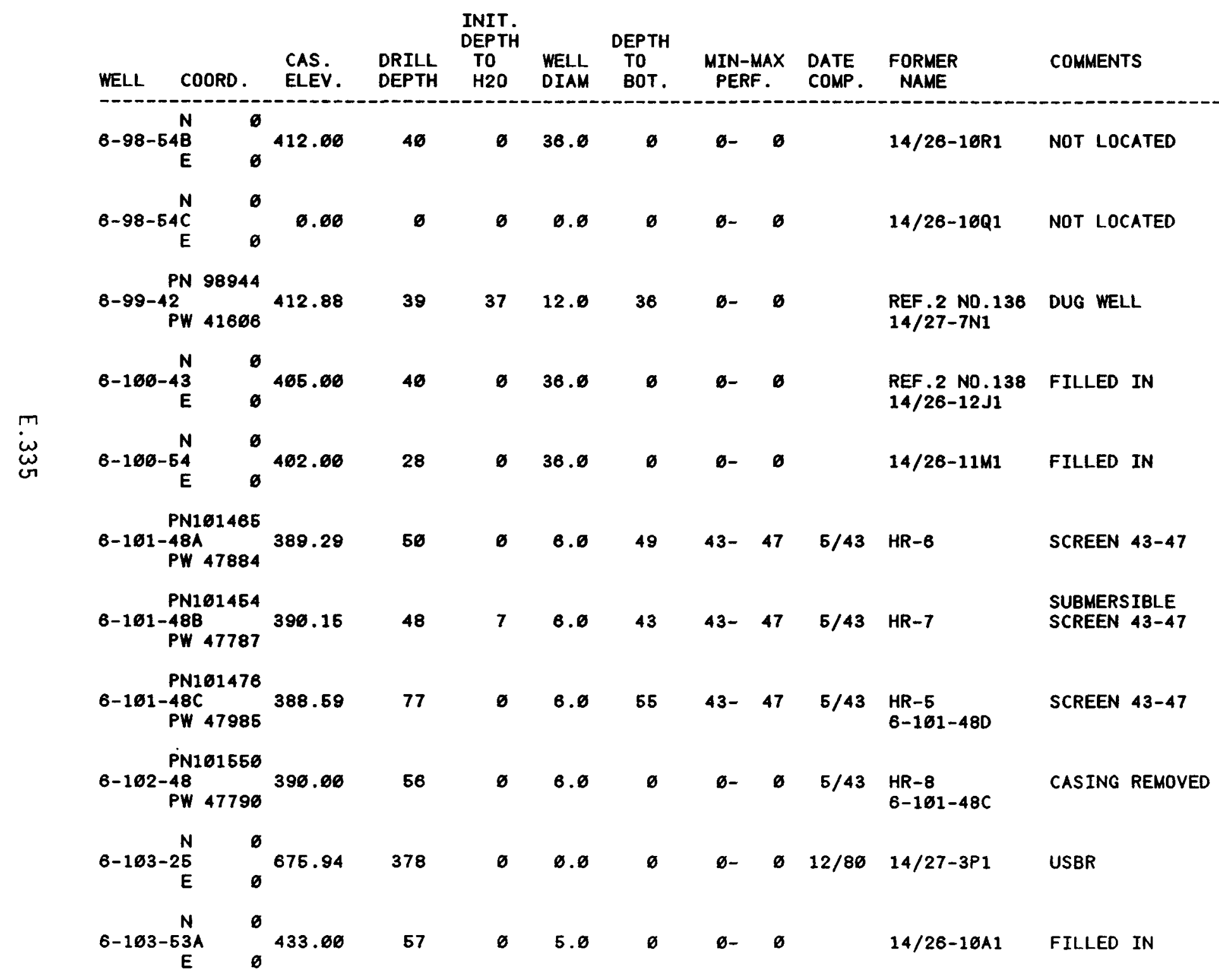




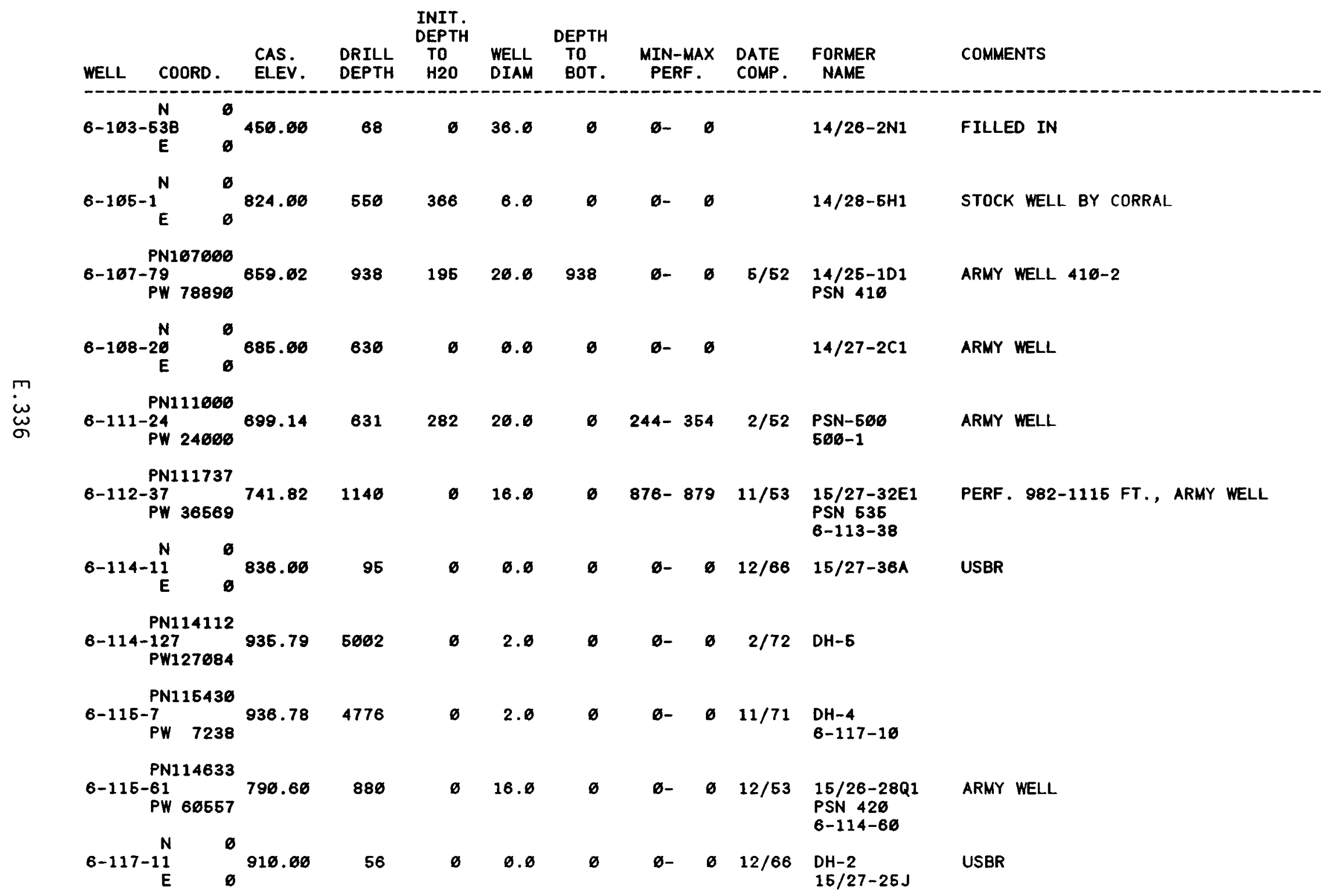




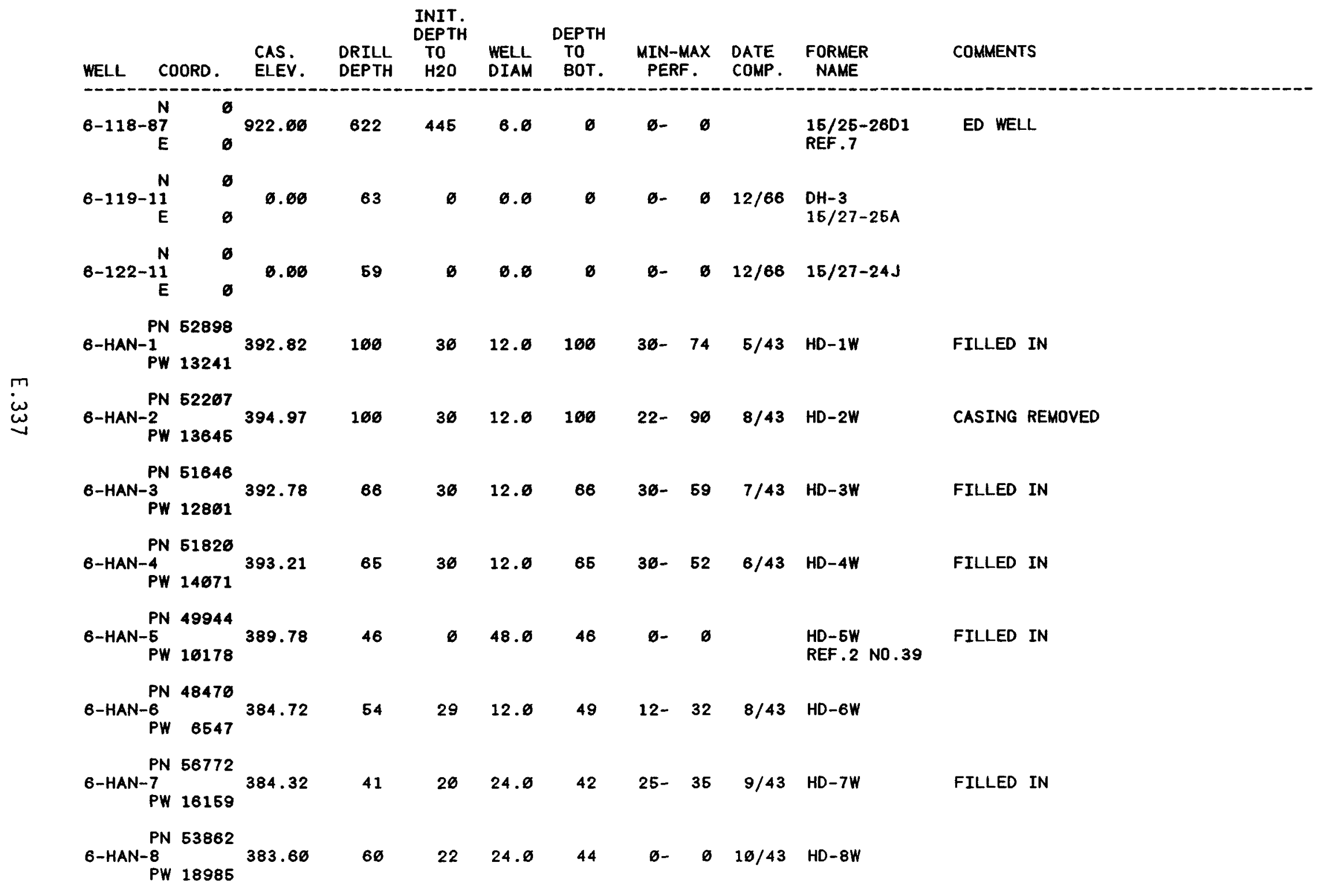




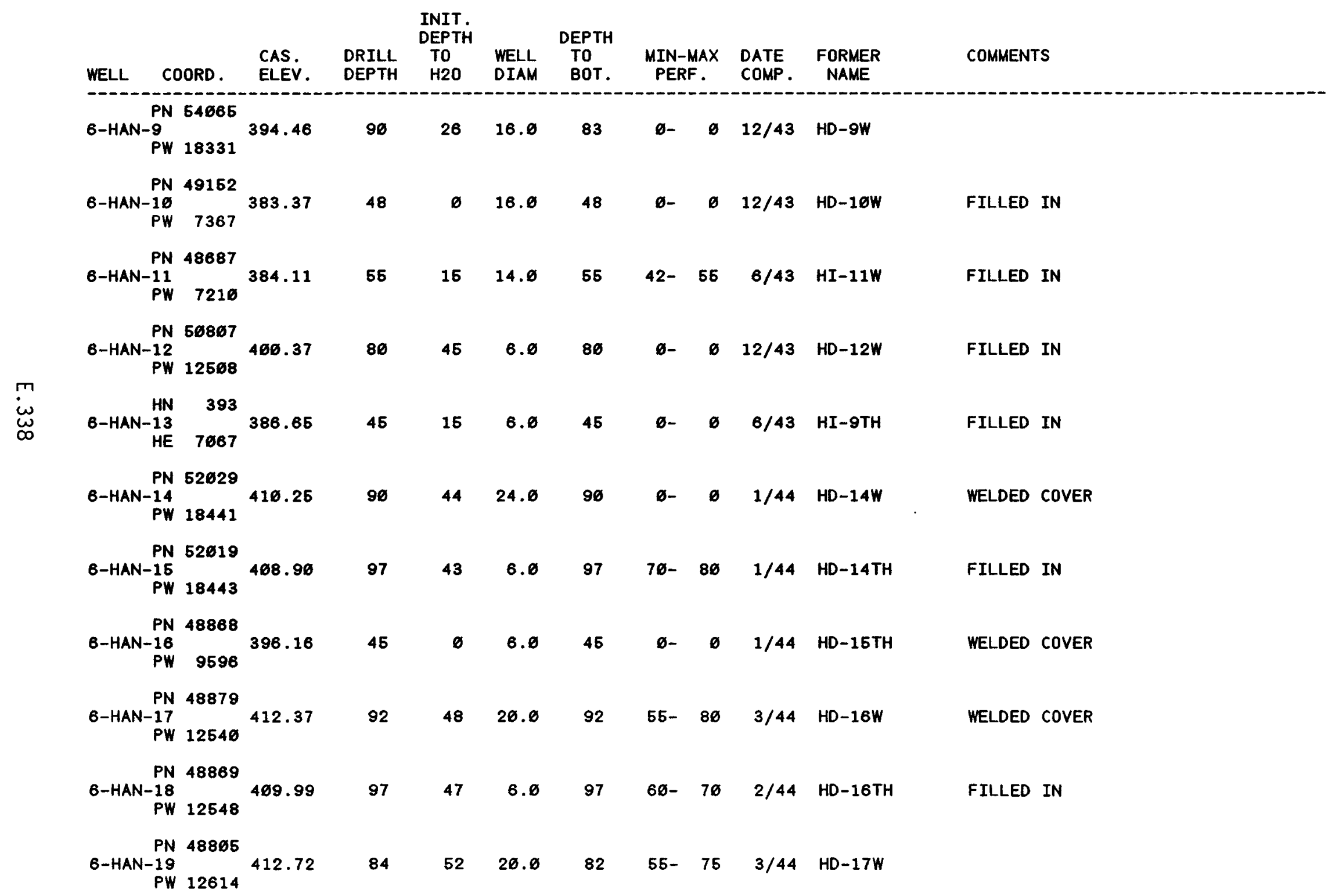




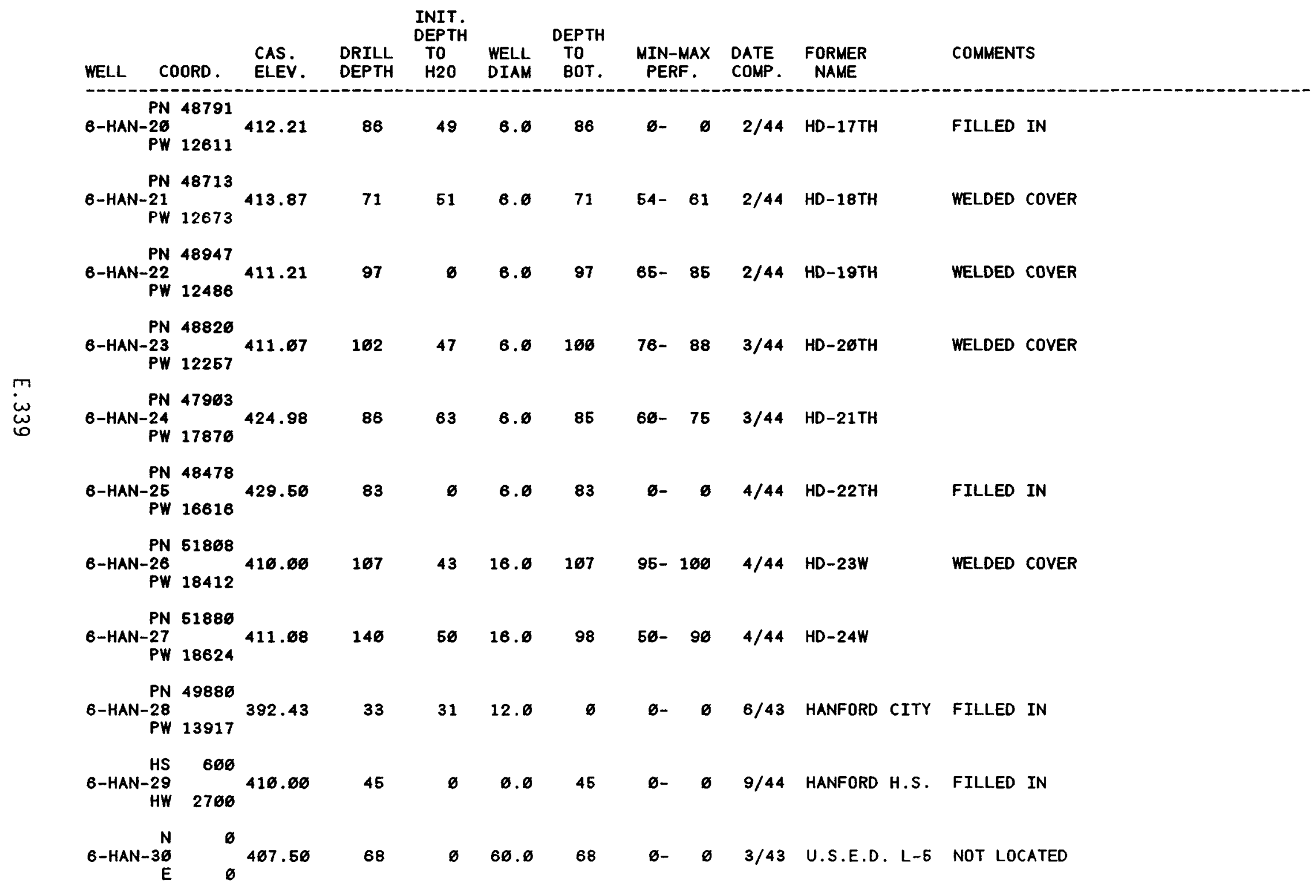




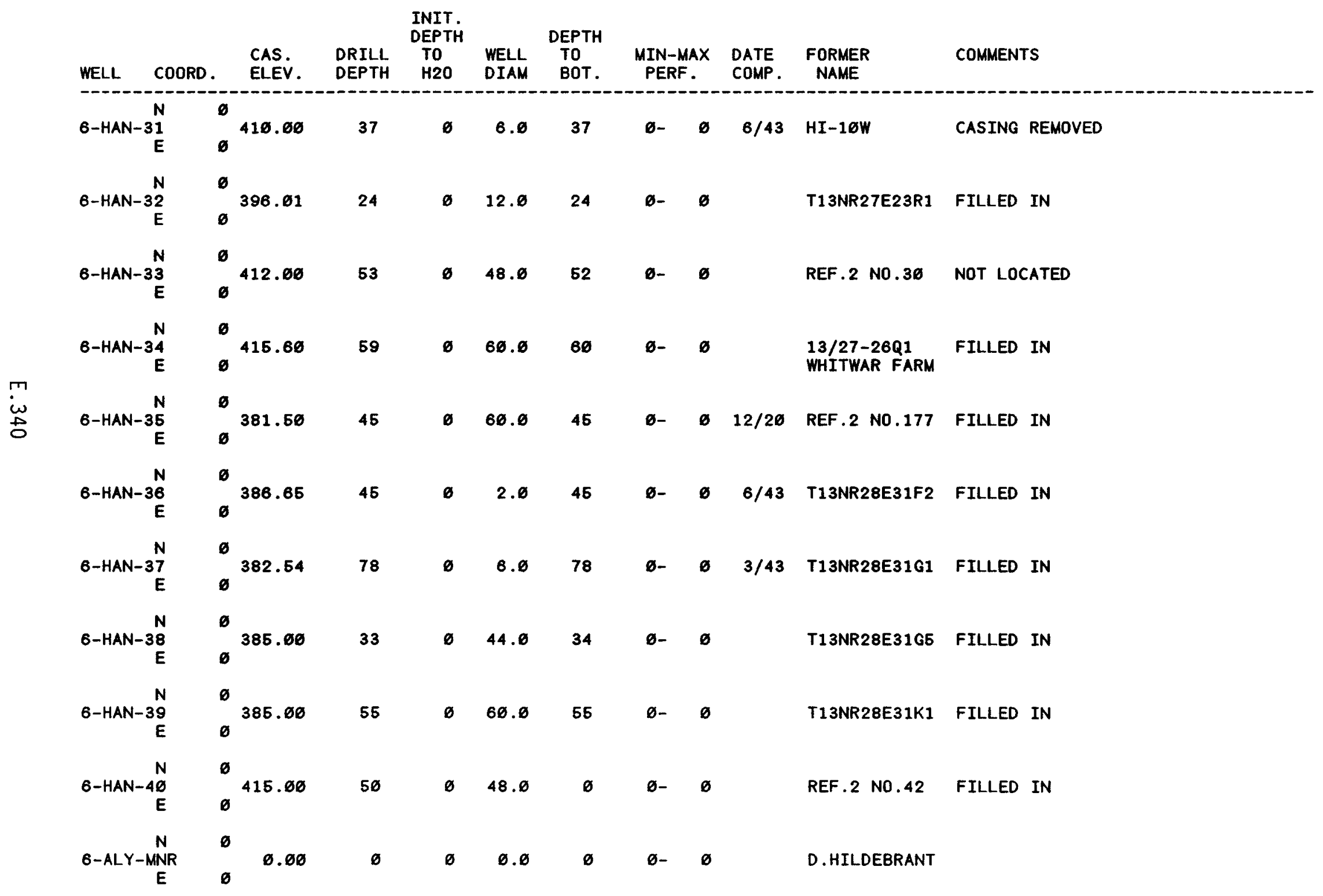




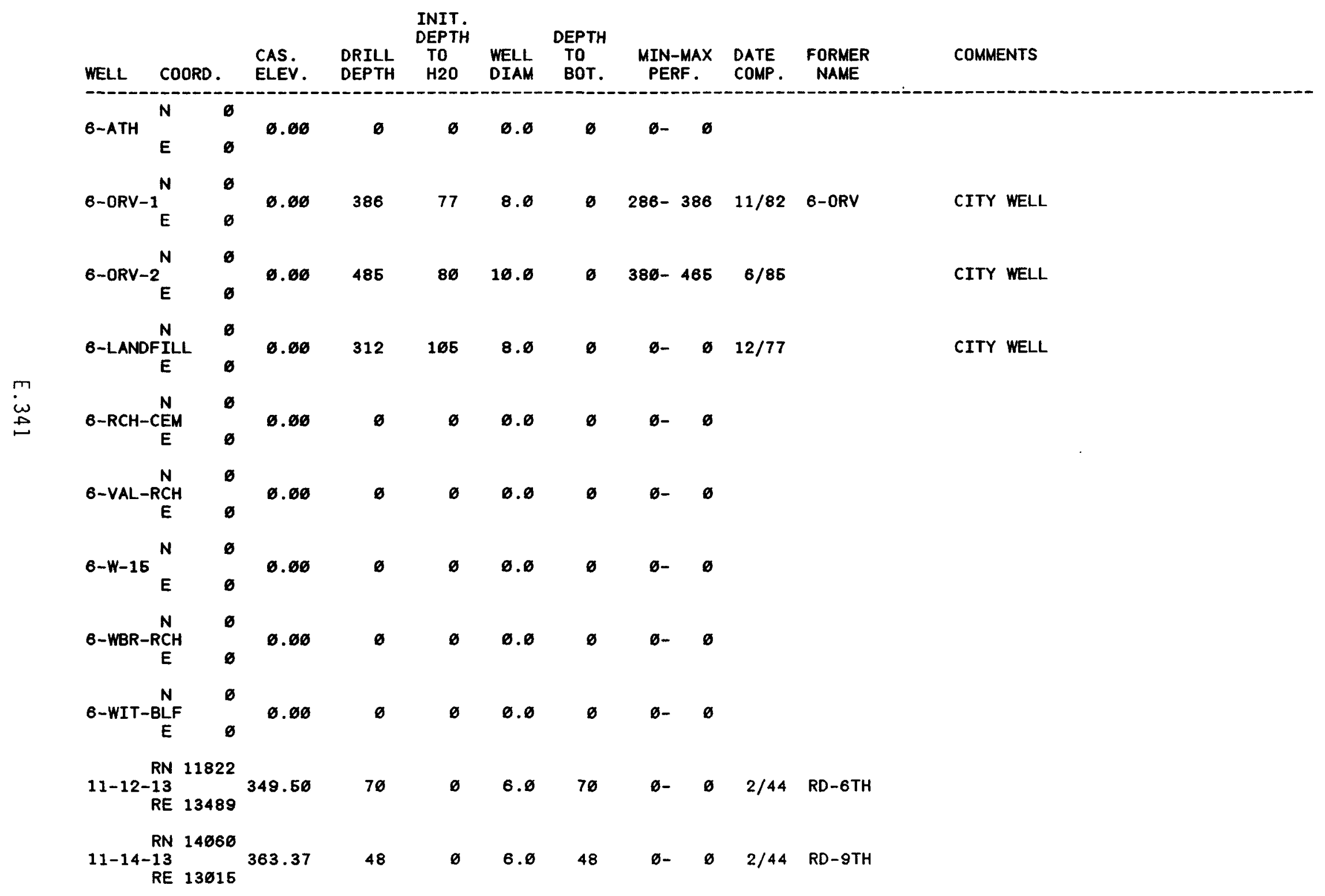




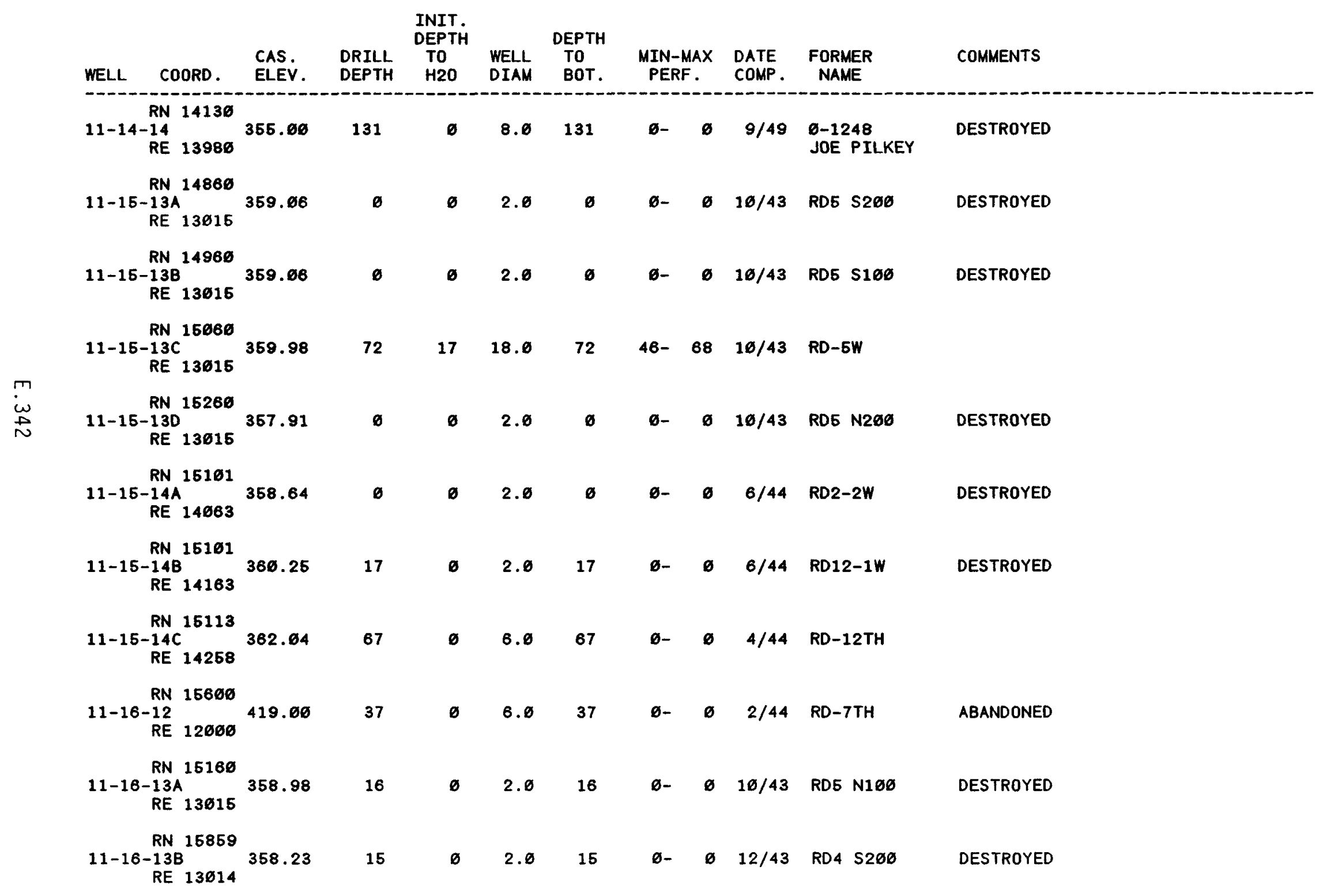




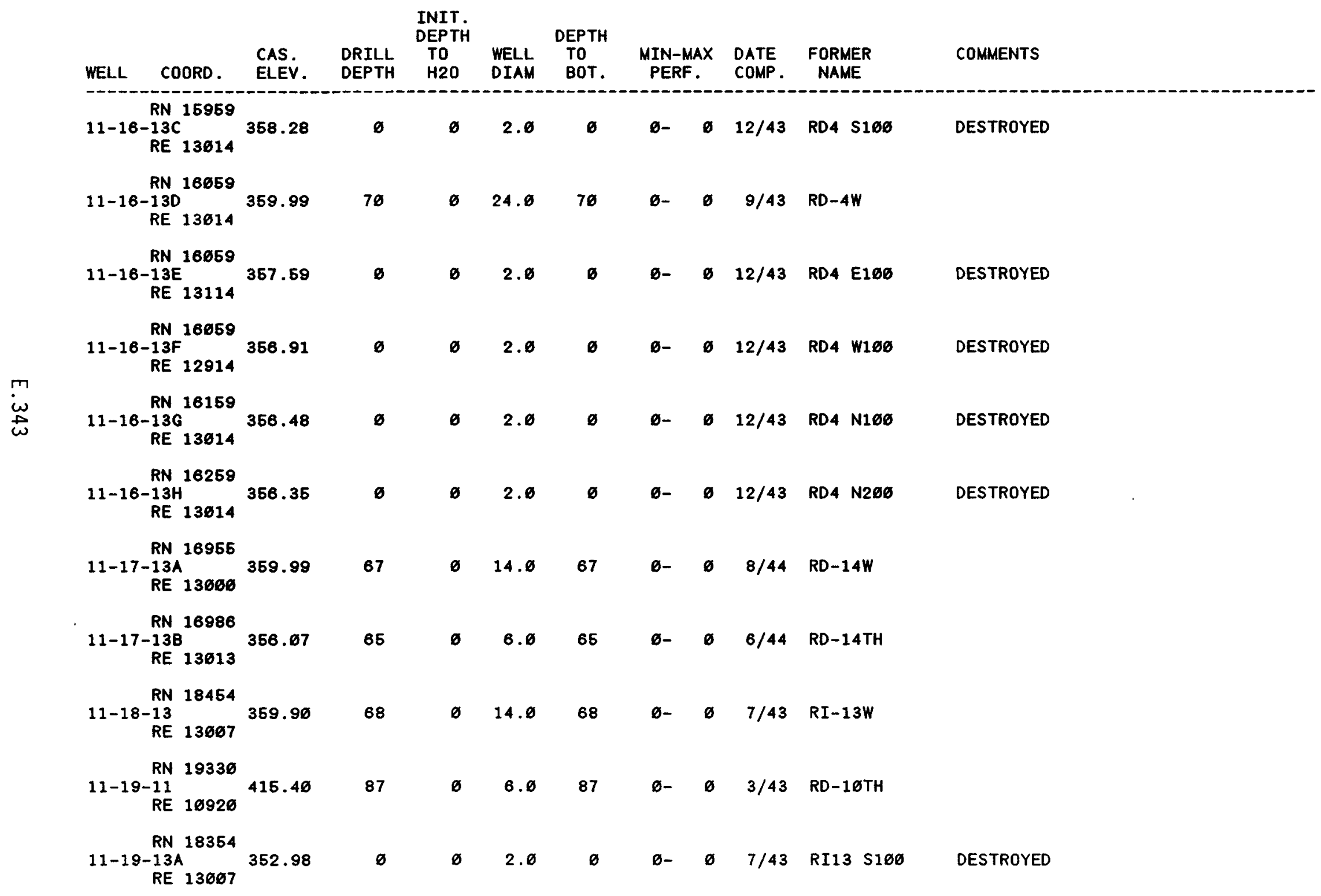




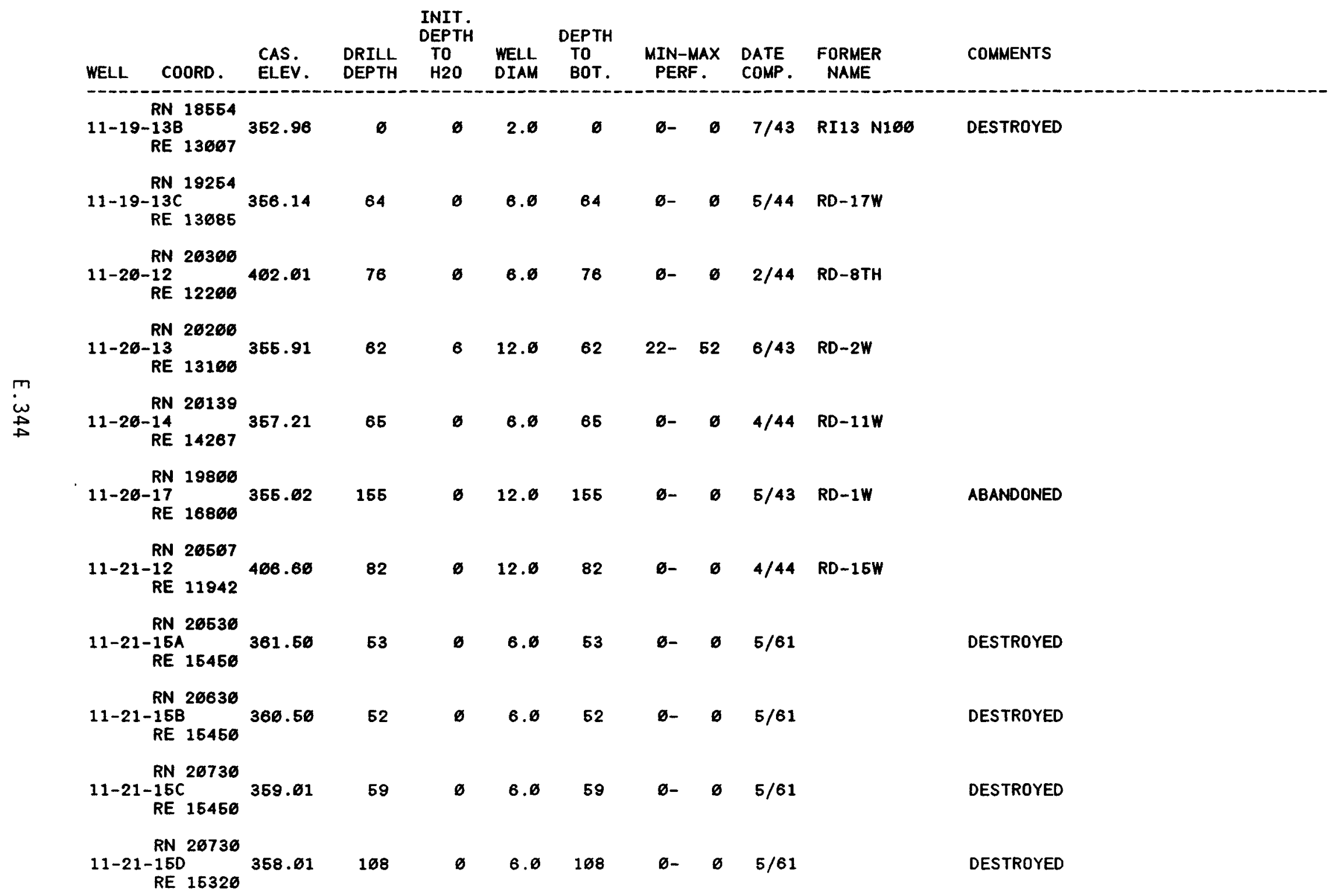




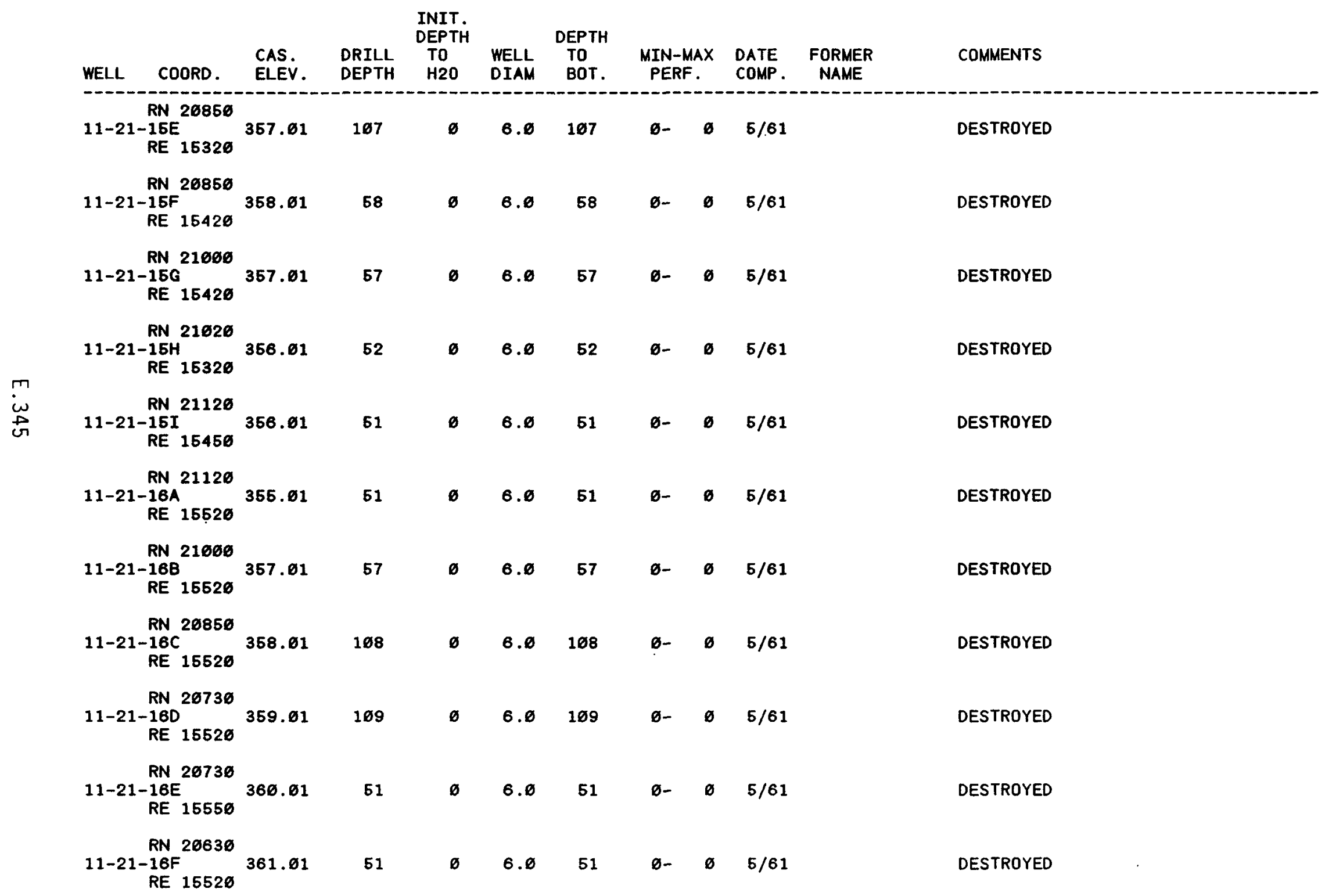




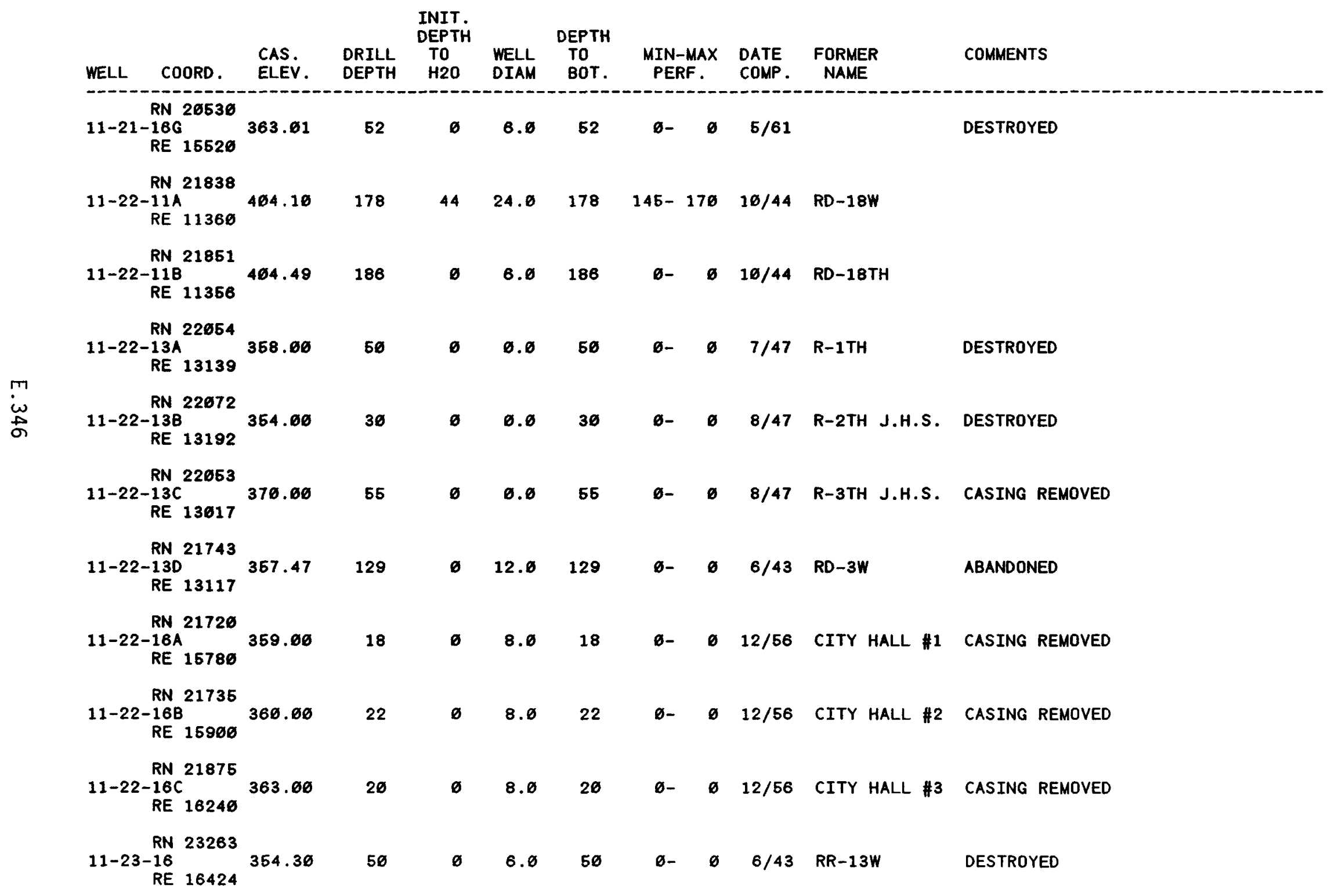




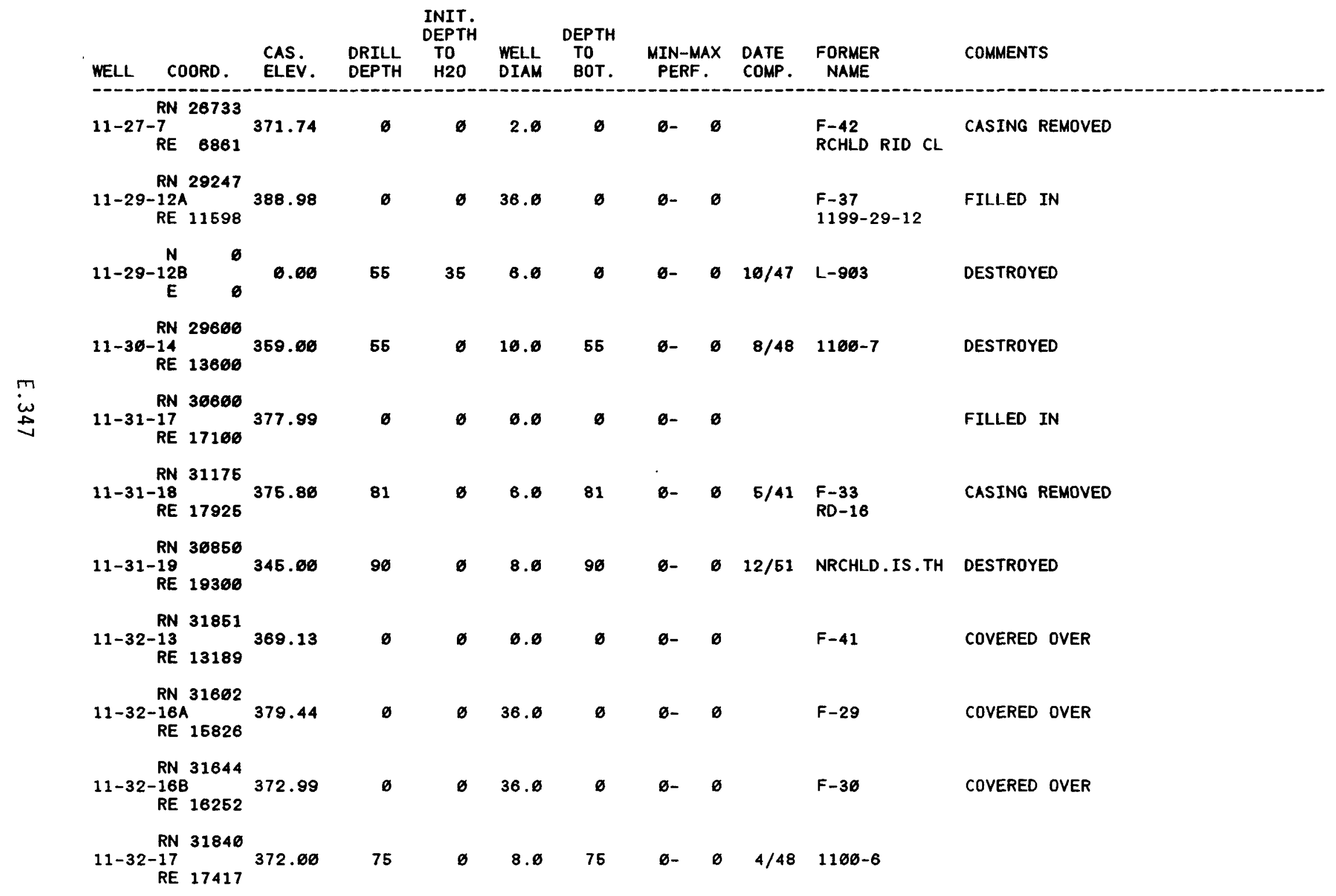




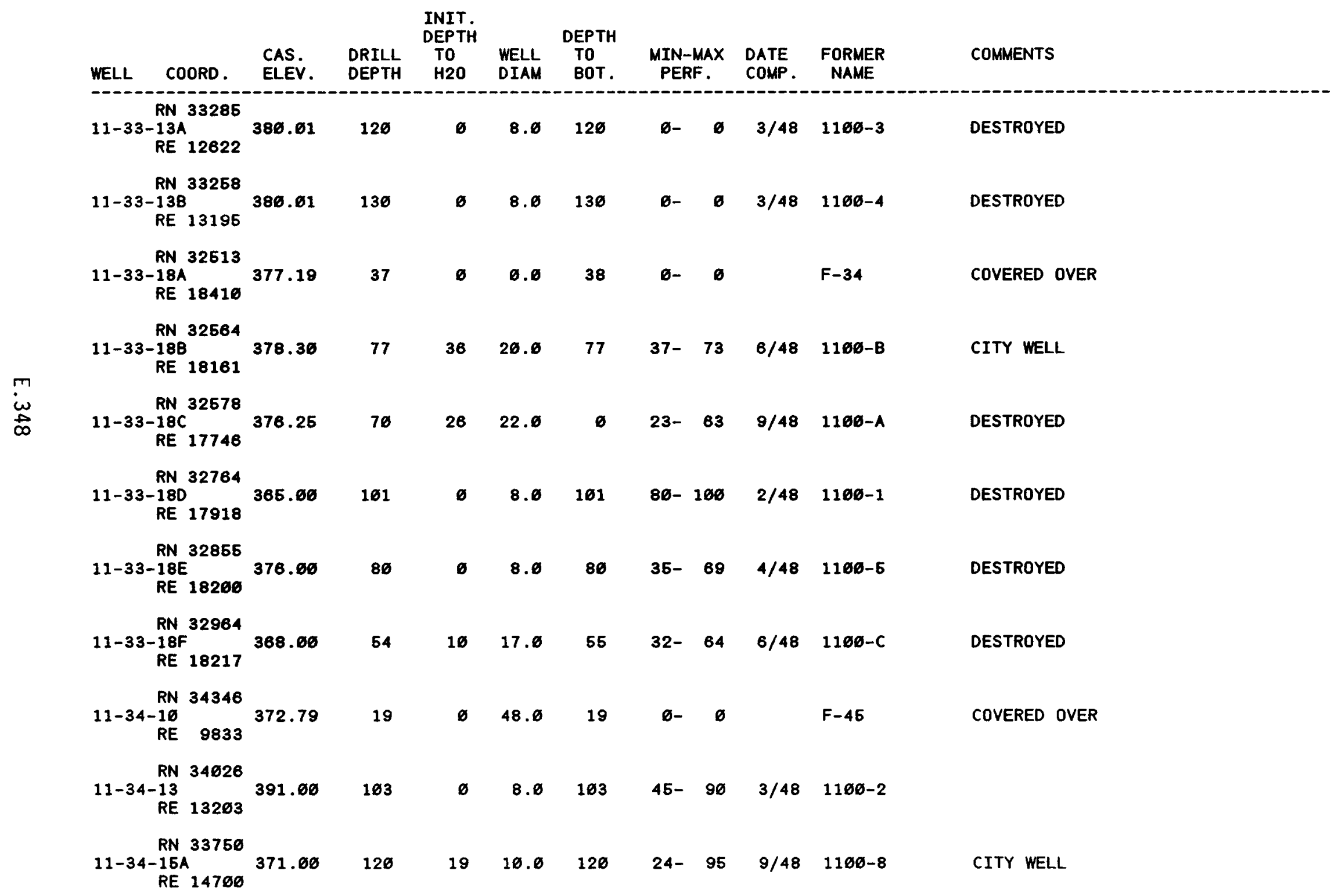




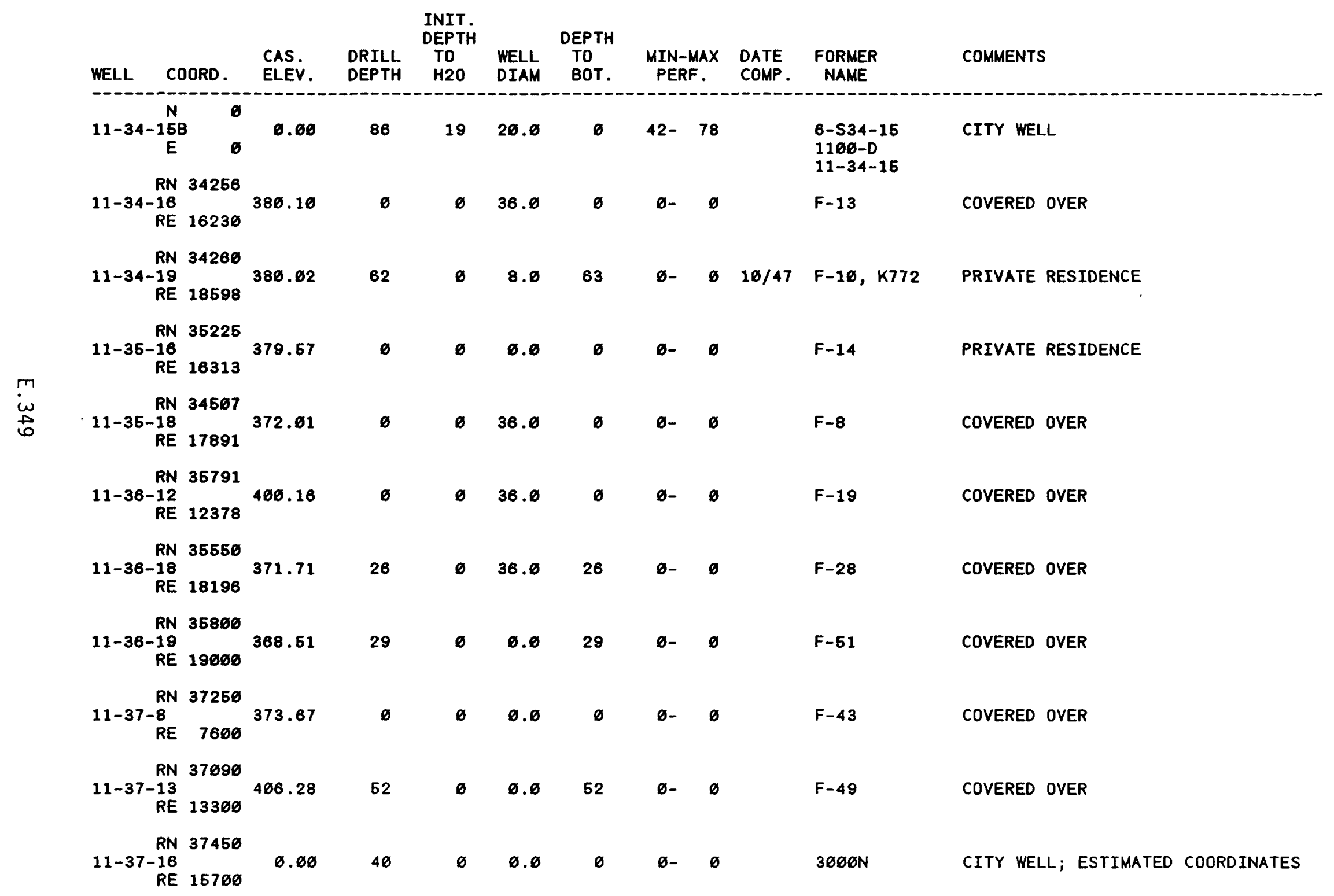




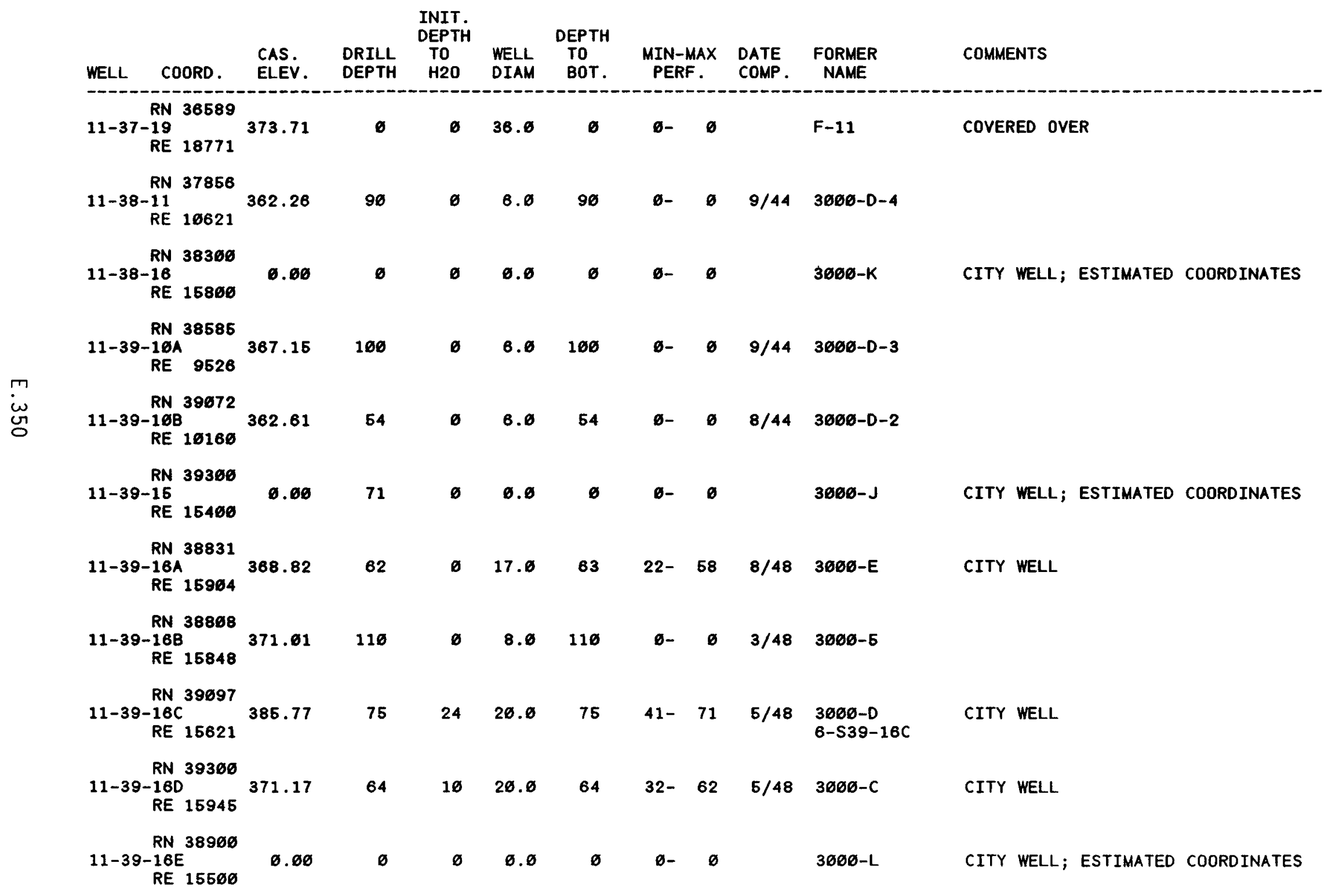




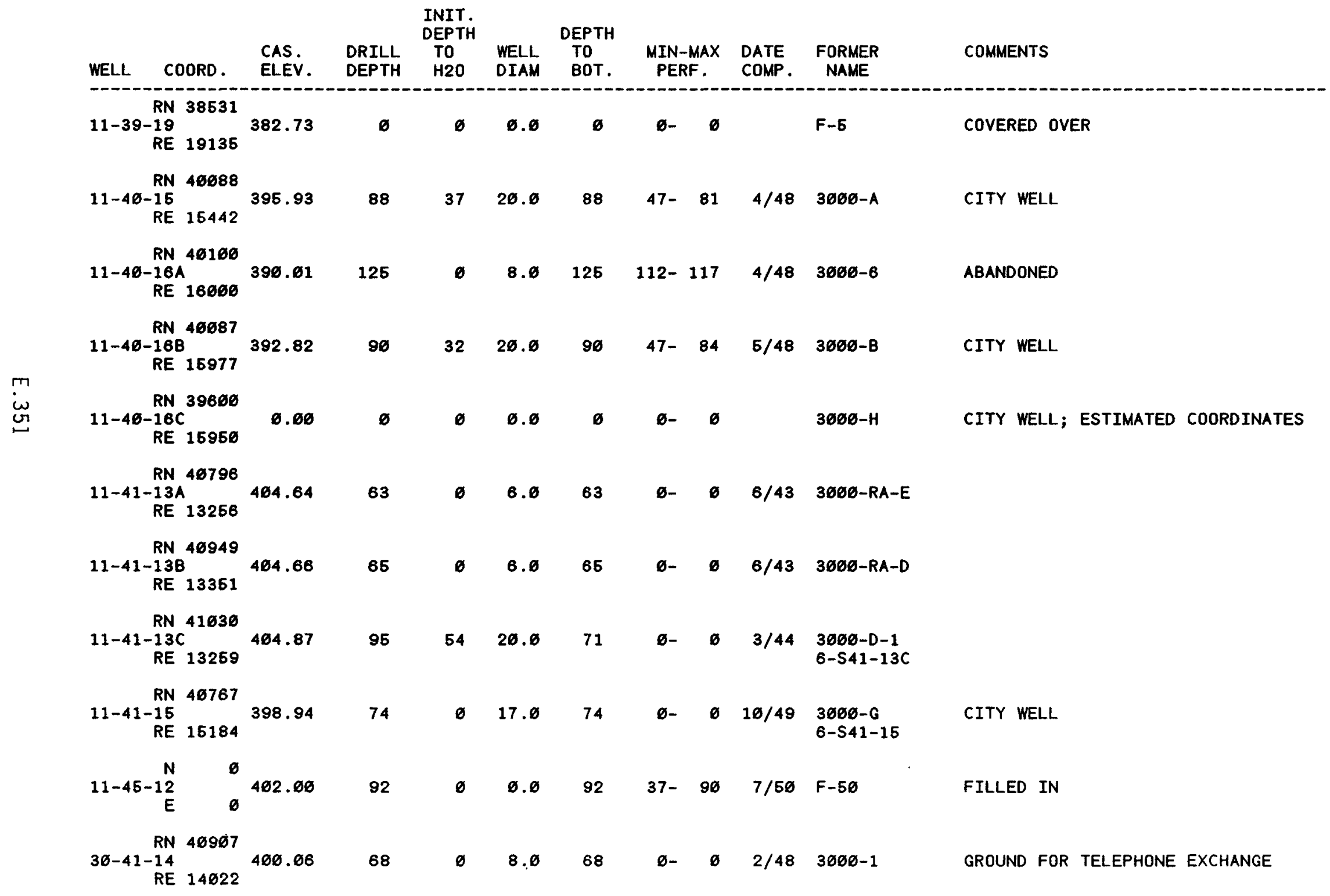




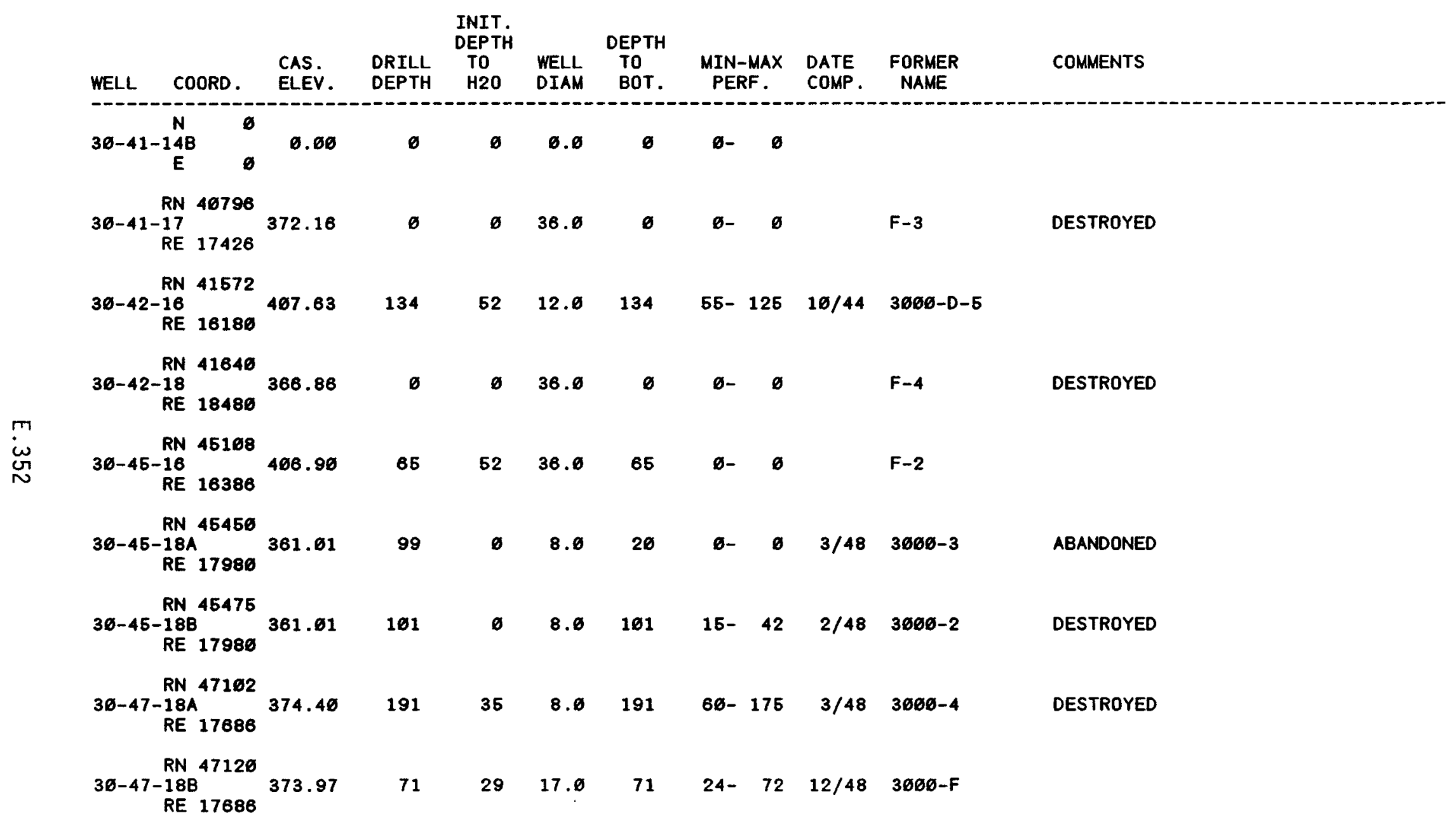


WELLS LISTED $=\mathbf{3 8 7 6}$

㩊 
APPENDIX $F$

ABBREVIATIONS AND ACRONYMS 


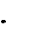




\section{ABBREVIATIONS AND ACRONYMS}

$\begin{array}{ll}\text { BH } & \text { A Washington Public Power Supply System (WPPS) Borehole } \\ \text { CB } & \text { A Term Used by WPPSS } \\ \text { CH } & \text { Core Hole } \\ \text { DB } & \text { Deep Borehole } \\ \text { DC } & \text { Deep Core Hole } \\ \text { DDH } & \text { Deep Drill Hole } \\ \text { DH } & \text { Drill Hole } \\ \text { E } & \text { Name Used by Golder and Associates } \\ \text { FFTF } & \text { Fast Flux Test Facility } \\ \text { GM } & \text { Gable Mountain } \\ \text { Golder } & \text { Golder Associates } \\ \text { GSI } & \text { Geophysical Services, Inc. } \\ \text { Han. } & \text { Former Hanford Townsite } \\ \text { HD } & \text { Hanford Durand (Driller's Name) } \\ \text { HI } & \text { Hanford International (Drilling Company Name) } \\ \text { HR } & \text { Hanford Ranney (Driller's Name) } \\ \text { MW } & \text { Term Used by Golder and Associates } \\ \text { N. Run } & \text { North Run (Well Measuring and Sampling Route) } \\ \text { PH } & \text { Piezometer Hole - Term Used by Golder } \\ \text { PSN } & \text { Army Gun Position } \\ \text { RD } & \text { Richland Durand (Driller's Name) } \\ \text { RI } & \text { Richland International (Drilling Company Name) } \\ \text { RRL } & \text { Reference Repository Location } \\ \text { RRYC } & \text { Railroad Yard } \\ \text { S } & \text { Term Used by Golder } \\ \text { Sea. Inl. Emp. } & \text { Seattle Inland Empire Gas Company } \\ \text { SP } & \text { Shot Point - Term Used by Golder } \\ \text { STES } & \text { Seasonal Thermal Energy Storage } \\ \text { TH } & \text { U.S. Geological Survey } \\ \text { USGS } & \end{array}$

\section{F.1}


WBD

WPPSS

WWOG

$X$
White Bluffs Durand (Driller's Name)

Washington Public Power Supply System

Western Washington $0 \mathrm{il}$ and Gas Company

Term Used by Golder 


\section{DISTRIBUTION}

No. of

Copies

OFFSITE

2 DOE Office of Scientific and Technical Information

\section{ONSITE}

2 DOE Richland Operations office

E. A. Bracken

M. L. Tiernan

Hanford Environmental Health Foundation

R. D. Gi1more

21 Westinghouse Hanford Company

M. R. Adams

J. R. Brodeur

J. A. Camman

D. R. Cowan

K. R. Fecht

T. R. Green

R. B. Hall

C. E. Hodge

R. L. Jackson

A. G. Law

R. G. Mikulecky

D. R. Myers

D. L. Parker

S. J. Phillips

W. H. Price

A. L. Schatz

J. A. Serkowski

S. J. Skurla

T. W. Spicer

D. C. Weeks

T. J. Wood

3 Kaiser Engineers Hanford

G. F. Brazil

A. K. Lewis

J. F. Keller
No. of

Copies

55 Pacific Northwest Laboratory

S. P. Airhart

D. J. Bates

B. N. Bjornstad

R. W. Bryce

J. V. Borghese

M. A. Chamness

R. L. Dirkes

J. C. Evans

J. W. Falco

M. D. Freshley

R. M. Fruland

T. J. Gilmore

S. M. Goodwin

J. M. Hales

S. H. Hall

P. C. Hays

E. L. Hilty

E. J. Jensen

G. V. Last

T. L. Liikala

S. P. Luttrell

J. P. McDonald

V. L. McGhan (10)

P. J. Mitchel1

R. W. Nelson

D. R. Newcomer

K. B. 01 sen

K. R. Oster

K. R. Price

M. R. Quarders

J. T. Rieger

R. Schalla

D. R. Sherwood

R. L. Skaggs

R. M. Smith

S. S. Teel

R. W. Wallace

E. J. Westergard

R. E. Wildung

R. K. Woodruff

Publishing Coordination

Technical Report Files (5) 
